Sabine Klinger

\title{
(De-)Thematisierung \\ von Geschlecht
}

Rekonstruktionen bei Studierenden der Erziehungs- und Bildungswissenschaften 


\section{Sabine Klinger \\ (De-)Thematisierung von Geschlecht}


Sabine Klinger

\section{(De-)Thematisierung von Geschlecht}

Rekonstruktionen bei Studierenden der Erziehungs- und Bildungswissenschaften

Budrich UniPress Ltd.

Opladen • Berlin • Toronto 2014 
Die vorliegende Arbeit entstand im Rahmen des interdisziplinären Promotionskollegs „Geschlechterverhältnisse im Spannungsfeld von Arbeit, Organisation und Demokratie" an der Philipps-Universität Marburg. Zugleich ist sie Dissertation an der Philipps-Universität Marburg im Fachbereich Erziehungswissenschaft. Die Erstellung der Dissertation und ihre Drucklegung wurde von der Hans-Böckler-Stiftung finanziell unterstützt. Zudem wurde die Publikation mit Unterstützung der KarlFranzens-Universität Graz gedruckt.

Bibliografische Information der Deutschen Nationalbibliothek

Die Deutsche Nationalbibliothek verzeichnet diese Publikation in der Deutschen Nationalbibliografie; detaillierte bibliografische Daten sind im Internet über http://dnb.d-nb.de abrufbar.

Gedruckt auf säurefreiem und alterungsbeständigem Papier.

Alle Rechte vorbehalten.

(C) 2014 Budrich UniPress, Opladen, Berlin \& Toronto

www.budrich-unipress.de

\section{ISBN 978-3-86388-057-6 (Paperback)}

eISBN 978-3-86388-238-9 (eBook)

Das Werk einschließlich aller seiner Teile ist urheberrechtlich geschützt. Jede Verwertung außerhalb der engen Grenzen des Urheberrechtsgesetzes ist ohne Zustimmung des Verlages unzulässig und strafbar. Das gilt insbesondere für Vervielfältigungen, Übersetzungen, Mikroverfilmungen und die Einspeicherung und Verarbeitung in elektronischen Systemen.

Lektorat: Andrea Lassalle, Berlin

Typografisches Lektorat: Anja Borkam, Jena

Umschlaggestaltung: Bettina Lehfeldt, Kleinmachnow - http://www.lehfeldtgraphic.de

Druck: paper\&tinta, Warschau

Printed in Europe 


\title{
Danksagung
}

\begin{abstract}
„Was wäre an intellektueller Kreativität möglich ohne die intensiven Gespräche mit ,Schwestern (und Brüdern) im Geiste', wo wildes Gedanken-Schweifen, freies Assoziieren unzensiert erlaubt ist, wo Gedanken bis zur Absurdität oder ,Vollkommenheit' auf die Spitze getrieben werden können, (... )“ (Maurer 1996, S. 202).
\end{abstract}

An dieser Stelle möchte ich all denjenigen danken, die mir während der Arbeit an der Dissertation in unterschiedlicher Weise geholfen haben, sei es durch konkrete Anregungen, Ideen und Ratschläge, oder durch moralische Unterstützung oder technische Hilfestellungen. Einige von ihnen möchte ich an dieser Stelle besonders hervorheben. Ein großer Dank geht an meine BetreuerInnen Prof. ${ }^{i n}$ Dr. ${ }^{i n}$ Susanne Maurer und Prof. ${ }^{i n}$ Dr. $^{\text {in }}$ Bettina Dausien. Sie haben mich bei meiner Arbeit an der Dissertation maßgeblich begleitet und gaben mir mit ihrem fundierten Fachwissen viele Anregungen. Ohne ihr Wissen, ohne ihre Ideen und ihre Kritik wäre mein Forschungsprojekt niemals soweit gekommen.

Mein Dank richtet sich auch an meine KollegInnen des Promotionskollegs „Geschlechterverhältnisse im Spannungsfeld von Arbeit, Organisation und Demokratie" an der Philipps-Universität Marburg, denn die Zusammenarbeit mit ihnen war ein Meilenstein bei der Erstellung meiner Doktorarbeit.

Mein Dank geht zudem an das Graduiertenkolleg „Repräsentation, Materialität und Geschlecht: gegenwärtige und historische Neuformierungen der Geschlechterverhältnisse" des Zentrums Gender Studies der Universität Basel und der Leiterin Prof. ${ }^{i n}$ Dr. ${ }^{i n}$ Andrea Maihofer. Dieser Forschungszusammenhang hat die Arbeit an meiner Dissertation wesentlich gefördert. Unterstützend war hier insbesondere der kritische und kollegiale Austausch innerhalb des Kollegs und die vielen bereichernden Diskussionen.

Für interessante und ertragreiche Diskussionen sowie konstruktive Beiträge danke ich auch Prof. ${ }^{i n}$ Dr. $^{\text {in }}$ Rita Casale und Prof ${ }^{i n}$ Dr. $^{\text {in }}$ Ingrid Miethe.

Als besonders bereichernd und unverzichtbar habe ich die Lernund Arbeitszusammenhänge der interdisziplinären Interpretationsgruppe „Qualitative Sozialforschung" der Universität Marburg und der Peermentoring-Gruppe „QualiZüri“ der Universität Zürich empfunden. Den Mitgliedern dieser Gruppe möchte ich meinen Dank aussprechen, da der 
wertschätzende und professionelle Umgang die Konzeption der empirischen Arbeit, die Auswertung und sowie die Erprobung und die Überprüfung der eigenen Arbeit und Thesen erst möglich machte.

Das DoktorandInnen-Kolloquium von Prof. ${ }^{i n}$ Dr. ${ }^{\text {in }}$ Susanne Maurer hat sich für mich als Raum für die Wahrnehmung von Differenz und Vielfalt ebenso erwiesen wie als Raum für Kontroversen und Verständigung. Und dafür möchte ich meinen KollegInnen danken.

Ich möchte mich auch ganz herzlich bei Julia Graf, Linda Kagerbauer, Kristin Ideler, Birgit Hofstätter, Agnes Trattner und Monika Götsch für den regen Austausch sowie für die vielen ermutigenden und wertschätzenden Gespräche bedanken. Auch meinen KorrekturleserInnen möchte ich für die kritischen Anmerkungen Danke sagen. Den InterviewpartnerInnen, die ich aufgrund der Anonymisierung leider nicht beim Namen nennen kann, möchte ich für ihre Offenheit, Bereitschaft und Unterstützung danken.

Besonderer Dank geht auch an meine Eltern, Franz und Margareta Klinger, die mich in allen meinen Entscheidungen und Vorhaben fortwährend unterstützen und an mich glauben.

Wesentlichen Anteil an der Fertigstellung meiner Dissertation hat mein Mann Stefan Rossegger. Er stand mir in allen Phasen - egal ob nah oder fern - als liebevoller und unterstützender Partner zur Seite. Ihm danke ich von ganzem Herzen für seinen Zuspruch, seine unermüdliche Unterstützung, seine Liebe und Motivation. 


\section{Inhaltsverzeichnis}

1 Einleitung: Die (De-)Thematisierung von ,Geschlecht" $\begin{array}{ll}\text { und Geschlechterfragen } & 9\end{array}$

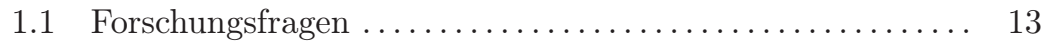

1.2 Aufbau der Arbeit.......................... 15

1.3 Begriffe und Begriffsklärungen .................. 19

1.4 Lesehinweise................................ 22

2 Erziehungs- und Bildungswissenschaften und,Geschlecht 62

2.1 Die Etablierung im universitären Erscheinungsbild ...... 27

2.2 (K)ein ,typischer Frauenstudiengang"? ............. 29

2.3 Die Relevanz von ,Geschlecht' und seine Konjunkturen ... 31

3 ,Geschlecht' und Geschlechterverhältnisse im Spiegel theoretischer Diskurse 43

3.1 Das Zwei-Geschlechter-Modell ................... 44

3.2 Die moderne Gesellschaft und die Geschlechterverhältnisse 47

3.3 ,Geschlecht' aus sozialisationstheoretischer Perspektive . . 52

3.4 Die Trennung von sex und gender ............... 57

3.5 Konstruktion und Dekonstruktion von ,Geschlecht ${ }^{6} \ldots . . . .58$

3.6 ,Geschlecht' aus intersektionaler Perspektive .......... 68

3.7 ,Geschlecht ${ }^{\star}$ als gesellschaftlich-kulturelle Existenzweise(n) 72

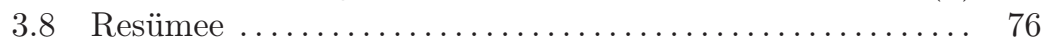

4 Die Relevanz einer praxeologischen Perspektive $\quad 79$

4.1 Das Habitus-Feld-Konzept von Pierre Bourdieu ........ 81

4.2 Die Dokumentarische Methode und praxeologische

Wissenssoziologie ........................ 99

4.3 Gesellschaftskritische und geschlechterreflektierende Analysefolien ........................... 105 
5 Methodischer Zugang und der Forschungsprozess

5.1 Zur Auswahl des Untersuchungsfeldes und den Universitäten ,Sonne' und ,Mond' ................ 130

5.2 Gruppendiskussionsverfahren als zentrale Erhebungsmethode .......................... 133

5.3 Datenerhebung und empirisches Material ........... 137

5.4 Auswertung auf Basis der dokumentarischen Methode ... 147

6 Datenauswertung: ,Geschlecht' zwischen und auf den Zeilen

6.1 Deskriptive Darstellung der Gruppendiskussionen: Themen und Inhalte.......................... 158

6.2 Ein divergenter Diskurs bei der Gruppe Holz an der Universität Sonne ............................. 168

6.3 Die (selbst)reflektierende Gruppe Feuer an der Universität Sonne .............................. 213

6.4 Komparative Analyse: Gruppenübergreifende Orientierung an der Universität Sonne ....................... 236

6.5 Die ,Ja, aber'-Mentalität der Gruppe Metall an der Universität Mond.......................... 239

6.6 Ein ,naturgegebener“ Zugang bei der Gruppe Wasser an der Universität Mond ....................... 280

6.7 Komparative Analyse: gruppenübergreifende Orientierungen bei der Universität Mond

7 Ergebnisdiskussion: Spielarten der (De-)Thematisierung von ,Geschlecht ${ }^{6}$ und Geschlechterfragen

7.1 Habituelle Konstruktionen bei der (De-)Thematisierung

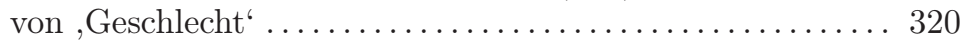

7.2 Universitätsbezogene (De-) Thematisierung von ,Geschlecht' 324

7.3 Universitätsübergreifende und studiumsbezogene (De-)Thematisierung von ,Geschlecht' ............... 331

7.4 Der neue Geschlechtervertrag und rhetorische Modernisierungsprozesse als konjunktiver Erfahrungsraum 332

7.5 Resümee und Ausblick: Plädoyer für einen geschlechterreflexiven Erfahrungsraum ............. 339

Literaturverzeichnis 


\section{Einleitung: Die (De-)Thematisierung von ,Geschlecht' und Geschlechterfragen}

Heutzutage erscheint es schon fast unzeitgemäß, unangebracht und altmodisch, ,Geschlecht' und Geschlechterverhältnisse als eine Ursache für gesellschaftliche Ungleichheit in den Blick zu nehmen (vgl. Rendtorff 2005, S. 34f.) oder diese zu thematisieren. Feministische Einwände gelten als überholt und unangemessen und der Gleichberechtigung von Frauen und Männern scheint nichts mehr im Weg zu stehen(vgl. Rendtorff 2005, S. 34f.). Elisabeth Kelan schreibt dazu: „People assume that gender no longer matters because the issue has long been solved" (Kelan 2009, S. 5ff). Barbara Rendtorff vermutet dahinter sowohl Resignation als auch Wunschdenken. Letzteres scheint mit der Hoffnung verbunden, dass die Problematik, über die nun so lange geredet und geschrieben worden ist, endlich von selbst verschwinden möge (vgl. 2005, S. 34f.). Es zeigt sich jedoch, dass in der alltägliche Lebenspraxis Naturalisierungen und biologistische Deutungen von Beziehungsmustern, Familienmodellen, Geschlechterrollen und sexuellen Orientierungen nach wie vor Konjunktur haben und die Grundlage für (Re-)Strukturierungsprozesse von Geschlechterdifferenzen und eine hierarchische Geschlechterordnung bilden (vgl. Friebertshäuser 2012, S. 112; Wetterer 2013, S. 246).

Vor diesem von Widersprüchen geprägten Hintergrund ist in der Frauen- und Geschlechterforschung in den letzten Jahren eine Diskussion darüber entbrannt, ob der Geschlechterdifferenz tatsächlich bzw. immer noch der Status einer Leitdifferenz zugesprochen werden kann (vgl. Kapitel 4.3). Angelika Wetterer legt als eine Vertreterin dieser Debatte nahe, dass der soziale Wandel und die Modernisierung von Geschlechterverhältnissen gegenwärtig einen Stand erreicht haben, der sich vor allem durch Widersprüche, Brüche und Ungleichzeitigkeiten kennzeichnet (vgl. Wetterer 2003, S. 288). Auch Barbara Friebertshäuser konstatiert:

„Wir können also von einer Gleichzeitigkeit von Veränderungen und Verfestigung bestehender Ungleichheit in Geschlechterverhältnissen ausgehen [...]. Alles deutet auf eine Diskrepanz zwischen reflexiver Aufklärung, praktischem Handeln und emotionaler Besetzung einerseits, den tradierten Bildern, medial vermittelten Vorbildern, Fremdzuschreibungen und Selbstbildern andererseits" (Friebertshäuser 2012, S. 112). 
Diese gegenwärtig diagnostizierte Diskrepanz zwischen einer Gleichheitsrhetorik, die eine scheinbare Gleichberechtigung von Frauen und Männern behauptet, und der sozialen Praxis, in der die Ungleichheit zwischen den Geschlechtern im Handeln und im handlungsleitenden Wissen weiterhin bestehen bleibt (vgl. Rendtorff 2005; Wetterer 2006; Friebertshäuser 2012), bildet den Ausgangspunkt dieser Arbeit, in der nach der (De-)Thematisierung und der Relevanz von ,Geschlecht' und Geschlechterfragen gefragt wird. Damit wird die Forderung von Barbara Rendtorff aufgegriffen, die Brisanz und insofern auch die Relevanz von ,Geschlecht und Geschlechterfragen auf der Ebene der Bedeutungszuschreibungen zu berücksichtigen (vgl. Rendtorff 2012). Mit der (De-)Thematisierung von ,Geschlecht' und Geschlechterfragen steht in dieser Arbeit nicht die „wissenschaftliche Thematisierung der Geschlechterdifferenz" (Hirschauer 2002, S. 212) im Zentrum, sondern die empirische Untersuchung studentischer Praxen in Bezug auf die (De-)Thematisierung und die Relevanz von ,Geschlecht' und Geschlechterfragen.

Mit dem Begriff der Thematisierung knüpfe ich an die Überlegungen von Angelika Wetterer an, die von der "Nicht-Thematisierung“ (Wetter 2002, S. 149) von ,Geschlecht' spricht. Bezugnehmend auf das Konzept des undoing gender ${ }^{1}$ präzisiert Wetterer, dass dieses eigentlich zwei unterschiedliche Formen der gerade nicht kritisch aktualisierten Geschlechterdarstellung und -zuordnung beinhalte: Sie unterscheidet aktive Strategien der „Neutralisierung“" von der eher passiven „Nicht-Thematisierung“ von Geschlecht. Bei der Nicht-Thematisierung von, Geschlecht' bleibt die Hintergrunderwartung der Geschlechtsdarstellung latent, da diese nicht thematisiert und problematisiert wird. Hingegen beziehen sich Neutralisierung und Neutralisierungsstrategien erkennbar auf die Normalitätserwartung der Geschlechtszuordnung und versuchen, ihr mehr oder weniger aktiv und absichtsvoll entgegenzuwirken (ebd.).

Im Vorgriff auf die Ergebnisse dieser empirischen Untersuchung kann hinsichtlich der Geschlechterdarstellung und -zuordnung gesagt werden, dass aktive Strategien der Neutralisierung nur am Rande rekonstruiert werden konnten. Das heißt, bei der Thematisierung von ,Geschlecht' und Geschlechterfragen blieb die Hintergrunderwartung der Geschlechtsdarstellung und -zuordnung zumeist latent und wurde nicht problematisiert. Eine Neutralisierung von ,Geschlecht' lässt sich somit nur auf einer rhetorischen Ebene, also auf einer kommunikativ-generalisierten Wissen-

1 Das Absehen von der Geschlechtszugehörigkeit bezeichnet Stefan Hirschauer als „eine Art soziales Vergessen“ (Hirschauer 1994, S. 678). Dabei handle es sich um eine konstruktive Leistung, um ein undoing gender (ebd.). 
sebene, im Sinne von Gleichheitsbekundungen feststellen. Im Gegensatz dazu wirken auf der Ebene der habituellen Praxis Hintergrunderwartungen an Geschlechtsdarstellung und -unterscheidung explizit und implizit weiter. Zudem zeigt sich, dass es trotz Thematisierung von Geschlecht und Geschlechterfragen zu einer Verschleierung und Verdeckung der geschlechtsbezogenen Ungleichheit und Benachteiligungen kommt.

Neben der von Wetterer angeführten Nicht-Thematisierung und Neutralisierung von Geschlechterdarstellungen lässt sich ausgehend von den Befunden aus den Gruppendiskussionen auf der Ebene des Diskurses eine weiteres Phänomen beschreiben, dass ich als (De-)Thematisierung bezeichnen möchte. Indem ich von der (De-)Thematisierung von Geschlecht und Geschlechterfragen spreche, verweise ich auf die paradoxe Gleichzeitigkeit der Thematisierung von Geschlecht und der NichtThematisierung von Geschlechterdarstellung und -zuordnung. Diese Differenzierung treffe ich deshalb, weil in der Untersuchung deutlich wird, dass nicht nur zwischen einer Neutralisierung und "Nicht-Thematisierung" von ,Geschlecht' unterschieden werden kann. Es zeigt sich, dass bei einer Thematisierung von ,Geschlecht' und Geschlechterfragen außerdem Dynamiken wirken, die zu einer De-Thematisierung führen: Implizit und explizit bleiben binäre Geschlechternormen und die Orientierung an einer kohärenten, lebenslang stabilen Geschlechtsidentität wirksam, während die Bedeutungszuschreibung von ,Geschlecht" und Geschlechterfragen an Relevanz und Legitimation verliert. Daher kann, dass die grundsätzliche Annahme bzw. die idealtypische Vorstellung, dass die Thematisierung von ,Geschlecht' und Geschlechterfragen, aber auch die Auseinandersetzung mit dem Thema ,Geschlecht', unwillkürlich zu einem reflektierten Umgang mit ,Geschlecht' führen, in dieser Arbeit nicht bestätigt werden (vgl. Kapitel 6). Bei der Untersuchung der Thematisierung von ,Geschlecht' und Geschlechterfragen bei Studierenden der Erziehungsund Bildungswissenschaften wurde außerdem deutlich, dass eine Verdeckung von sozialen Ungleichheiten und deren Wirkmechanismen über Geschlechterverhältnisse praktiziert wird.

Hinsichtlich der (De-)Thematisierung von ,Geschlecht' und Geschlechterfragen gehe ich von zwei Thesen ${ }^{2}$ aus, welche die Ausrichtung dieser

2 Beide Thesen verstehe ich als sensitizing concepts. Der Begriff sensitizing concepts geht auf Herbert Blumer (1954) zurück. Glenn Bowen fasst Blumers Verständnis wie folgt zusammen: ,contrasted definitive concepts with sensitizing concepts. [...] Social researchers now tend to view sensitizing concepts as interpretive devices and as a starting point for a qualitative study. [...] Sensitizing concepts draw attention to important features of social interaction and provide guidelines for research in specific settings" (Bowen 2009, S. 13f.). 
Arbeit maßgeblich bestimmen: Zum einen, dass (1) die (De-)Thematisierung in engem Zusammenhang mit gesellschaftlichen Prozessen steht. Sie wird maßgeblich beeinflusst von der gegenwärtigen diagnostizierten Diskrepanz zwischen einer Gleichheitsrhetorik und der sozialen Praxis, in der die Ungleichheit zwischen den Geschlechtern im Handeln und im handlungsleitenden Wissen weiterhin bestehen bleibt. Durch diese Mechanismen verliert die Ebene der Bedeutungszuschreibungen an Relevanz. Eine zweite These ist, dass (2) die (De-)Thematisierung und die Relevanz von ,Geschlecht' und Geschlechterfragen eng mit dem sozialen Feld (vgl. Bourdieu 1983, 1998), in dem es zur Thematisierung kommt, zusammenhängen. Somit bildet das soziale Feld eine wesentliche Grundlage für die (De-)Thematisierung von ,Geschlecht' und Geschlechterfragen und im Weiteren für die Ausbildung einer entsprechenden habituellen Praxis. Für diese Arbeit greife ich die Universität ${ }^{3}$ in ihrer Rolle als Bildungsinstitution, und hier im Speziellen das Studium der Erziehungsund Bildungswissenschaften, als soziales Feld heraus, um zu zeigen, in welcher Form Bewertungs-, Denkschemata und Handlungsorientierungen hinsichtlich der (De-)Thematisierung von ,Geschlecht' auf dem Wechselverhältnis zwischen Individuum und Gesellschaft gründen. Die Frage nach den Thematisierungsweisen von ,Geschlecht" und der geschlechterreflektierenden und gesellschaftskritischen (Aus-)Bildung der Einzelnen ist somit ganz konkret mit dem Feld der Erziehungswissenschaften verbunden.

Das universitäre Feld der Erziehungs- und Bildungswissenschaften ist für diese Arbeit relevant, weil ihr eine besondere Verantwortung für die Tradierung der hierarchischen (Geschlechter-)Differenz zukommt: Sind doch Familie und Schule als Vermittlungsinstanzen und Erziehung wie auch Bildung als Tradierungsfelder von Geschlechterbildern maßgeblich an deren Aufrechterhaltung beteiligt (vgl. Rendtorff/Moser 1999, S. 7, 16f.). Durch die beständig aktive Verknüpfung von Handlungen und Interaktionen mit der (Re-)Produktion von ,Geschlecht ${ }^{6}$ - innerhalb institutioneller Einrichtungen wie z. B. Schulen, Jugendvereinen, Universitäten - und die Nähe zu Subjekten und deren Identitätsfragen, scheint gerade in den Erziehungswissenschaften die Umsetzung und Übersetzung geschlechtertheoretischer und -reflektierter Überlegungen nahe zu liegen. Demnach ist davon auszugehen, dass es die Thematisierung von ,Geschecht" und Geschlechterfragen als soziales Feld Teil des Studiums und -aneignung. 
sind. Für Studierende ${ }^{4}$ der Erziehungs- und Bildungswissenschaften ist es heute üblich/möglich an Seminaren mit geschlechtersensiblen und geschlechterreflektierenden Inhalten teilzunehmen, auch wenn sie gar nicht beabsichtigen, einen beruflichen Weg im Bereich der geschlechtersensiblen und -reflektierenden Pädagogik und Erziehungswissenschaft ${ }^{5}$ einzuschlagen. Dennoch ist weitgehend unerforscht, wie Studierende ,Geschlecht" und Geschlechterfragen thematisieren und in welcher Weise sie dabei in ihren Wahrnehmungen, Denk- und Handlungsmustern sowie in ihrer Kommunikation von den Erfahrungen in der erziehungswissenschaftlichen Fachkultur und -umwelt geprägt werden. Daher wird in dieser Arbeit wird diese Forschungslücke mit der Frage nach den Praxen von Studierenden der Erziehungs- und Bildungswissenschaften in Bezug auf die (De-)Thematisierung und die Relevanz von ,Geschlecht' und Geschlechterfragen aufgegriffen.

\subsection{Forschungsfragen}

Die Forschungsfrage der habituellen Praxen in Bezug auf die (De-)Thematisierung und die Relevanz von ,Geschlecht' bei StudentInnen der Erziehungs- und Bildungswissenschaften ist auf zwei Ebenen angesiedelt: (1) sehe ich die (De-)Thematisierung von ,Geschlecht' und Geschlechterfragen in engem Zusammenhang mit dem gegenwärtigen Bruch zwischen der rhetorischen Geschlechtergleichheit und der sozialen Praxis der Geschlechterungleichheit. Desweiteren (2) nehme ich hier eine disziplinäre Perspektive ein, da die Machtfelder und das Gefüge der Disziplinen eine weitere starke Einflussgröße sind, wenn es um Thematisierungschancen und -dynamiken geht (vgl. Maurer 2008, S. 115). Demnach verstehe ich die (De-)Thematisierung nicht als Einzelleistung der Studierenden im Feld, sondern als habituell, fach- und feldspezifisches Handeln.

In der vorliegenden Arbeit soll gezeigt werden, (a) was und wie Studierende über ,Geschlecht' und Geschlechterfragen sprechen. Weiters (b) ob sich im Rahmen dessen habituelle Praxen rekonstruieren lassen und welche individuellen und kollektiven Orientierungen sich dabei offenbaren. Außerdem wird untersucht (c) ob eine geschlechterreflektierende

4 Um eine Reifizierung von Heteronormativität und Geschlechterbinarität in der Arbeit zu vermeiden, werden bewusst unterschiedliche Formen geschlechtergerechter Sprache verwendet. Zudem verweist die Verwendung des Gender Gaps z. B. Student_innen, auf gegenderte Existenzen, wie z. B. Intersexuelle oder TransgenderPersonen (vgl. Lesehinweise 1.4).

5 Beide werden in der Folge synonym verwendet. 
Haltung Teil des studentischen Habitus ist. Zudem stellt sich (d) die Frage, ob sich Muster der (De-)Thematisierung erkennen lassen, die auf eine Gleichheitsrhetorik hinweisen oder Ungleichheiten und Benachteiligungen zwischen Geschlechtern verdecken.

Hinsichtlich der ersten Ebene ist die Frage nach gesellschaftlichen Verdeckungszusammenhängen und rhetorischen Modernisierungprozessen, welche auf die (De-)Thematisierung von ,Geschlecht' einwirken, von Interesse. Hierfür beziehe ich mich auf eine Kombination von drei Analysefolien, deren Fluchtpunkt neben der (De-)Thematisierung von ,Geschlecht' der gemeinsame Fokus auf das Handeln der Akteur_innen innerhalb gesellschaftlicher Rahmenbedingungen und Prozesse legt. Angelika Wetterers Gegenwartsanalyse (1) der „rhetorischen Modernisierung" (2002) und ihrer These, dass es sich bei der Thematisierung von ,Geschlecht' um eine Modernisierung im Diskurs der Sprache handelt, die sich jedoch nicht in der Praxis zeigt, wird mit der (2) Analysefolie des Verdeckungszusammenhangs (Tübinger Institut für frauenpolitische Sozialforschung e.V. 2000) verknüft. Der Verdeckungszusammenhang identifiziert hinter den modernen Diskursen, z. B. die Modernisierung von Weiblichkeitsbildern und Männlichkeitsbildern, die Verdeckung struktureller Probleme. Dieser Perspektivenmix wird zudem durch die feministisch-politische Sichtweise von Angela McRobbie (2010) erweitert. Sie geht davon aus, dass jungen Frauen heute ein neuer Geschlechtervertrag angeboten wird, der die Desartikulation feministischer Forderungen und Inhalte bei der (De-)Thematisierung von ,Geschlecht' beinhaltet. Als Gegenzug wird Frauen das Angebot gemacht, öffentlich sichtbar zu werden, um am Arbeits- und Bildungsmarkt zu partizipieren und um an der Konsumkultur und Bürgergesellschaft teilnehmen zu können. Diese drei Analysefolien gehen (mehr oder minder dezidiert) davon aus, dass Denk-, Bewertungs- und Handlungsschemata von Individuen im Wechselverhältnis mit gesellschaftlichen Strukturen stehen. Ihre kombinierte Verwendung ermöglicht es zudem, den Blick auf die ,Bruchstelle zwischen habitualisiertem Handeln (im Sinne von Geschlechterdifferenz) und rational orientierten Überzeugungen, Vorstellungen und Normen (in Sinne von Geschlechtergleichheit und Gleichberechtigung) zu richten.

Bezüglich der zweiten Ebene wird nach den habituellen Praxen und den handlungsleitenden Orientierungen der Studierenden gefragt. Hierfür greife ich auf einen praxeologischen Ansatz zurück, der die Rekonstruktion handlungsleitender Orientierungen ermöglicht. Zum einen dient dieser Arbeit das Habitus-Feld-Konzept von Pierre Bourdieu (1983; 1992; 1993; 1998) als Folie, um Mechanismen, Dynamiken und Prozesse 
des wissenschaftlichen Feldes zu erfassen, welche auf die StudentInnen einwirken. Meine theoretischen Annahmen und Grundzüge sind somit von der Denkfigur Pierre Bourdieu's geprägt, derzufolge gesellschaftliche Strukturen und das Individuum selbst in einem konstitutiven Wechselverhältnis stehen. Zum anderen bietet die in der Tradition der Wissenssoziologie Karl Mannheims (1980) stehende dokumentarische Methode Ralf Bohnsacks $(1989 ; 2008 ; 2010)$ einen weiteren theoretischmethodologischen Hintergrund. Mit Hilfe dieser Sichtweise wird die habituelle Praxis beleuchtet und der Blick auf handlungsbezogenes und handlungsleitendes Wissen, d. h. auf das ,konjunktive Wissen“ (Bohnsack 2006, S. 280f.; Mannheim 1964) ins Zentrum zu gerückt.

Zusammenfassend kann gesagt werden, dass das Studium der Erziehungs- und Bildungswissenschaften, so die empirisch zu prüfende Annahme, einen „konjunktiven Erfahrungsraum“ (Bohnsack 2006, S. 280f.) bildet, innerhalb dessen die Studierenden im gemeinsamen Handeln kollektive Orientierungen ausbilden. Dabei ist von einer Überlagerung von studiums- und universitätsbezogenen Erfahrungsräumen bzw. Milieus auszugehen. Orientierungen lassen sich rekonstruieren, welche die Studierenden derselben Disziplin - so die These - auch maßgeblich mit gesellschaftlichen rhetorischen Modernisierungsprozessen und Verdeckungszusammenhängen verbunden sind. Ergänzend soll danach gefragt werden, inwieweit der von McRobbie postulierte „neue Geschlechtervertrag“ (McRobbie 2012) als möglicherweise konkurrierender konjunktiver Erfahrungsraum wirksam ist.

\subsection{Aufbau der Arbeit}

Die Arbeit ist in sechs übergeordnete Kapitel gegliedert, die zum einen die theoretische Fundierung und zum anderen die Darstellung der empirischen Untersuchung beinhalten und mit der Ergebnisdiskussion schließen.

Im Anschluss an die Einleitung, (Kapitel 1), findet im Kapitel 2 eine Annäherung an das Forschungsfeld statt. Hier wird die Ausgangsthese expliziert, dass die Machtfelder und das Gefüge der Disziplin Erziehungswissenschaften durchaus einen starken Einfluss haben, wenn es um Thematisierungschancen und -dynamiken von ,Geschlecht' und Geschlechterfragen geht, und dass es von Bedeutung ist, die disziplinären und institutionellen Abhängigkeiten zu reflektieren (vgl. Maurer 2012, S. 134). Das 2. Kapitel dient dazu, den bildungsmilieutypischen Erfahrungsraum 
darzustellen, in dem Studierende ,Geschlecht' (de-)thematisieren. Da die universitären Rahmenbedingungen (so die These) einen starken Einfluss auf die sozialen und kulturellen Räume der Studierenden haben und sich innerhalb disziplinärer Diskurse zu und Thematisierungsweisen von ,Geschlecht' und Geschlechterfragen gegenseitig beeinflussen, wird versucht, das Gefüge der Disziplin Erziehungswissenschaften darzustellen. Somit wird hier in Betracht gezogen unter welchen Bedingungen, in welchem disziplinären Umfeld und mit welchen Dynamiken die (De-)Thematisierung von ,Geschlecht" und Geschlechterfragen stattfindet. Zudem geht es darum, eine geschlechterkritische Perspektive auf das Fach einzunehmen und zu skizzieren, wie und in welcher Art und Weise ,Geschlecht ' - innerhalb des disziplinären Feldes, in dem sich die Studierenden bewegen und dessen Teil sie zugleich sind - zum Thema gemacht wird. Hierfür wird eine doppelte Reflexionsposition bzw. -perspektive vorgenommen: zum einen die historische Rekonstruktion der Thematisierung von geschlechterbezogenen Fragestellungen in der Disziplin (Kapitel 2.3.1) und zum anderen eine theoretische und praktische Perspektive auf die Kernkonzepte Bildung und Erziehung (vgl. Kapitel 2.3.2). Weiter wird die Etablierung der Erziehungswissenschaften an deutschen Hochschulen nachgezeichnet (Kapitel 2.1) und die statistische Repräsentation/Verteilung von Frauen und Männern genauer in den Blick genommen (Kapitel 2.2). Im Anschluss wird/werden die Konjunktur(en) der Thematisierung von ,Geschlecht' in den Erziehungswissenschaften auf theoretischer und empirischer Ebene besprochen (Kapitel 2.3).

Die theoretische Fundierung der Arbeit findet in den Kapiteln 3 und 4 statt. In Kapitel 3 werden verschiedene theoretische Konzepte zu Geschlechterkonstruktionen sowie Verständnisse und Ansätze dargestellt, welche darum bemüht sind, zur Sensibilisierung für die Konstruktion/Konstruktionsmechanismen von Geschlechterdifferenzen und die damit einhergehende Herstellung sozialer Ungleichheit beizutragen und jene, welche in der theoretischen Debatte dominier(t)en. Auch wenn im Zentrum dieser Arbeit nicht die Frage nach den unterschiedlichen ,Spielarten der Konstruktion" (Knorr-Centina 1989) von ,Geschlecht' steht, wird die Entwicklung der theoretischen Diskussion um ,Geschlecht' in groben Zügen nachgezeichnet. Damit soll dargestellt werden, mit welcher Intensität die gesellschaftlichen Geschlechterverhältnisse ins Zentrum wissenschaftlicher Überlegungen, vor allem in der (feministischen) Frauenforschung sowie den Gender Studies, gerückt sind. Hiermit findet über verschiedene theoretische Ansätze und Überlegungen, Begriffe und Konzepte eine Annäherung an den Forschungsgegenstand Thematisierung ,Geschlecht 
statt. In Abgrenzung zu der theoretischen Diskussion zeigt dieses Kapitel, dass diese überwiegend auf die situative Herstellung der Differenz von Geschlechtern beschränkt bleibt, aber Überlegungen und soziale Strukturen, die einen Einfluss auf die Interaktion haben und die Stabilität der Konstruktionen und Existenzweisen von ,Geschlecht' erklären, nur marginal erfassen. Diese Lücke soll mittels des praxeologischen Ansatzes, welcher für diese Arbeit gewählt wurde, bearbeitet werden.

Im Rahmen des Kapitels 4 wird dieser praxeologischer Ansatz vorgestellt, der über Fragen zur (Geschlechts-)Identität und -konstruktion hinausreicht und sich daher in theoretischer und methodologischer Weise als geeignet für die Rekonstruktion handlungsleitender Orientierungen, Bewertungs- und Denkschemata von StudentInnen erweist. Damit wird nun die Grundlage für die empirische Untersuchung habitueller Praxis in Bezug auf die (De-)Thematisierung von ,Geschlecht' und Geschlechterfragen bei StudentInnen der Erziehungs- und Bildungswissenschaften geschaffen. Dies knüpft unmittelbar an das zweite Kapitel an, indem das Forschungsfeld, in dem die Studierenden ,mitspielen' als soziales Feld und konjunktiver Erfahrungsraum der Studierenden eingeordnet wird. Das Habitus-Feld-Konzept von Pierre Bourdieu (Bourdieu 1983; 1992; 1993; 1998; vgl. Kapitel 4.1) dient hierbei der Verdeutlichung der Mechanismen und der Funktionsweisen des wissenschaftlichen Feldes und der Überwindung des (herkömmlichen) Dualismus von Individuum und Gesellschaft. Ziel dieses Kapitels ist es, die verschiedenen Perspektiven vorzustellen, aus denen ich die Problemstellung dieser Arbeit betrachte. Jede dieser Sichtweise umfasst einen anderen Aspekt und somit ist es möglich, auch verschiedene Bereiche auszuleuchten und nicht nur einen Teil in der Rahmenanalyse wie auch in der Auswertung des empirischen Materials zu erhellen. Meine Analysefolie ist somit nicht durch einen theoretischen Blickwinkel geprägt, sondern vielmehr von sich ergänzenden Überlegungen praxeologischer und methodologischer sowie gesellschafts- und geschlechterkritischer Perspektiven. Im zweiten Abschnitt dieses Kapitels wird auf die in der Tradition der Wissenssoziologie Karl Mannheims stehende dokumentarische Methode Ralf Bohnsacks (Bohnsack 1989; 2008; 2010; vgl. Kapitel 4.2) eingegangen. Die dokumentarische Methode bildet, wie ich in Kapitel 5 näher ausführen werde, auch die Grundlage der Analyse des empirischen Materials. In Kapitel 4.3 werden drei feministisch geprägte Analysefolien vorgestellt, die auf gesellschaftliche Rahmenbedingungen und Prozessen bei der (De-)Thematisierung von ,Geschlecht' und Geschlechterfragen fokussieren. Diese drei unterschiedlichen, aber sich ergänzenden Perspektiven die Gegenwartsanalyse der 
rhetorischen Modernisierung von Angelika Wetterer (2006; vgl. Kapitel 4.3.1), die Analysefolie des Verdeckungszusammenhangs des Tübinger Instituts für frauenpolitische Sozialforschung (2000; vgl. Kapitel 4.3.2) und die These von Angela McRobbie eines neuen Geschlechtervertrags und der Desartikulation feministischer Inhalte und Positionen (2012; vgl. Kapitel 4.3.3) beschäftigen sich mit derselben Frage bzw. haben dieselbe Problemstellung als Ausgangslage. Im Zentrum steht die Individualisierung von gesellschaftlichen Problemlagen, die aufgrund hierarchischer Geschlechterverhältnisse jenseits verbaler Beteuerungen von Gleichheit und Gleichberechtigung bestehen.

Das Kapitel 5 stellt den Übergang zur Empirie dar, da hier der methodische Zugang und der Forschungsprozess erläutert wird. Im Kapitel 5.1 wird der Aufbau der Untersuchung dargestellt und auf die Auswahl der Universitäten eingegangen, an denen sich 14 Studierende (2 männlich, 12 weiblich; 7 Bachelor- und 7 Diplomstudierende) an insgesamt vier Gruppendiskussionen beteiligt haben. Zudem wird das Gruppendiskussionsverfahren als zentrale Erhebungsmethode vorgestellt (Kapitel 5.2). Im nächsten Schritt steht die Datenerhebung und das empirische Material im Zentrum (Kapitel 5.3). Im Kapitel 5 wird zudem der Umgang mit dem Probleme der Reifizierung der Kategorie ,Geschlecht ${ }^{6}$ in dieser empirischen Forschung diskutiert und erläutert (Kapitel 5.3.3). Schließlich werden die Auswertungsschritte der dokumentarischen Methode expliziert (Kapitel 5.4).

Das Kapitel 6 ist der empirischen rekonstruktiven Auswertung der vier Gruppendiskussionen aus zwei Universitäten gewidmet. Als Einstieg in das empirische Material erfolgt die deskriptive Darstellung der Thematisierung von ,Geschlecht' in den Gruppendiskussionen (Kapitel 6.1). Danach wird die Rekonstruktion kollektiver Orientierungen bei (De-)Thematisierung entlang der einzelnen Gruppendiskussionen und gruppiert nach der jeweiligen Universität erläutert. Hier werden die entsprechenden Gruppen mit den für sie charakteristischen Orientierungen in Hinblick auf die (De-)Thematisierung von ,Geschlecht' und Geschlechterfragen dargestellt. Die fallinternen Homologien bzw. der Habitus der einzelnen Gruppen stehen dabei im Zentrum. Zudem werden gruppenübergreifende Vergleiche angestellt (Kapitel 6.2, 6.3, 6.5 und 6.6). In der gruppenübergreifenden komparativen Analyse jener Gruppen, die derselben Universität zugeordnet sind, werden die gemeinsamen universitäts- und studiumsbezogenen konjunktiven Erfahrungen bei der (De-)Thematisierung zur Geltung gebracht (Kapitel 6.7 und Kapi- 
tel 6.4). ${ }^{6}$ Um die habituelle Praxis und die kollektiven Orientierungen der Studierenden bei der (De-)Thematisierung von ,Geschlecht' und Geschlechterfragen zu typologisieren, greife ich auf die dokumentarische Auswertungsmethode (Bohnsack 2008) zurück.

Im abschließenden Kapitel 7 werden die Ergebnisse dieser empirischen Analyse im Sinne einer Typologie zusammengefasst. Dabei lassen sich Mechanismen und Muster herausarbeiten, die die (De-)Thematisierung von ,Geschlecht' und Geschlechterfragen auszeichnen (Kapitel 7.1). In der universitätsinternen und universitätsübergreifenden komparativen Analyse lassen sich Ähnlichkeiten und Differenzen identifizieren, welche in den Kapiteln 7.2 und 7.3 zusammengefasst werden. Im Anschluss daran wird im Kapitel 7.4 genauer herausgearbeitet, dass es neben studiumsbezogenen Erfahrungen und Erlebnissen und individuellen Erfahrungen auch gesellschaftliche Strukturen und Diskurse gibt, die Vorstellungen und Praktiken leiten. Es kann bereits vorweggenommen werden, dass das Geschlechterwissen und -verständnis der Student_innen die (De-)Thematisierung von ,Geschlecht' und Geschlechterfragen maßgeblich beeinflusst bzw. eine Thematisierung verhindert, daher muss zwischen Geschlechterwissen und Geschlechterhandeln unterschieden werden. Unter der Überschrift „Verdeckungszusammenhänge bei der (De-)Thematisierung von ,Geschlecht' und Geschlechterfragen" (Kapitel 7.4.2) werden Diskursmodi zusammengefasst, die eine Thematisierung erschweren und/oder zu einer (De-)Thematisierung führen. Resümee und Ausblick widmen sich schließlich dem Plädoyer für geschlechterreflektierende studiumsbezogene Erfahrungsräumen und Lehrkonzepten (Kapitel 7.5).

\subsection{Begriffe und Begriffsklärungen}

$\mathrm{Zu}$ Beginn dieses Buches sollen, relevante heuristische Begriffsfelder und Kategorierahmen kartiert werden, um eine Orientierungshilfe für den/die Leser_in zu schaffen und das Handlungsfeld bzw. den abstrakten Denkraum meiner Überlegungen abzustecken. Dabei handelt es sich weniger um eine systematische Herleitung der Begriffe als vielmehr um eine topographische Ordnung relevanter Begriffsfelder, die in meiner Arbeit einen besonderen Stellenwert einnehmen wie gender oder: ,Geschlecht' sowie geschlechterreflektierende und -reflektierte Haltung.

6 In diesem Sinne kann in allen Gruppen eine Thematisierung von ,Geschlecht' und Geschlechterfragen rekonstruiert werden, die Heteronormativität und eine eindeutige und kohärente Geschlechterbinarität als Norm reproduziert. 


\section{Gender oder: ,Geschlecht' und Geschlechterfragen}

In der deutschsprachigen Frauen- und Geschlechterforschung werden die Worte gender und ,Geschlecht' oftmals synonym verwendet, daher steht vielfach die Frage im Raum: Wann wird gender präferiert und wann ,Geschlecht' (vgl. Knapp 2008, S. 299). Zudem wird gender mittlerweile inflationär verwendet und verkommt so immer stärker zu einer Worthülse, die ihr gesellschaftskritisches Potential aufgrund von Vereinnahmungsprozessen zunehmend verliert. In diesem Sinne kritisiert auch Joan Scott gender und seine Karriere vom kritischen Konzept zum mainstream label (vgl. Knapp 2008, S. 301). Vor dem Hintergrund dieser Überlegungen habe ich mich deshalb dazu entschieden, in meiner Arbeit den deutschen Begriff Geschlecht zu verwenden. Dies verfolgt jedoch nicht aus Gründen der Abgrenzung zur im Englischen gemachten Unterscheidung zwischen sex (biologisches Geschlecht) und gender (soziales Geschlecht), auch wenn diese Unterscheidung spätestens seit den 1990er Jahren vom dekonstruktivistischen Feminismus kritisiert wird: Eine solche Trennung berge die Gefahr, das biologisches ,Geschlecht' zu essentialisieren und seine diskursive Verfasstheit zu übersehen (vgl. Kapitel 3.4). Im Gegenteil verstehe ich diese Diskussion als überaus produktiv, da sie zur Weiterentwicklung feministischer, macht- und gesellschaftskritischer Überlegungen angeregt hat. Mit der Präferenz des deutschen Begriffs Geschlecht möchte ich ebenfalls nicht die Diskussion und die Grundlagenkritik zum ,gender scepticism" (Knapp 2008, S. 297) unterstützen. ${ }^{7}$ Mir geht es vielmehr darum, mit der Verwendung von ,Geschlecht' auf die obsolete sex/gender

7 Gudrun-Axeli Knapp (2008) widmet sich in ihrem Artikel „Achsen der Differenz - Aspekte und Perspektiven feministischer Grundlagenkritik" der proklamierten ,Krise der Kategorie Geschlecht' und macht dabei deutlich, was alles bedacht werden muss, wenn vom Bedeutungsverlust von ,Geschlecht' die Rede ist. Ihre Ausführungen zeigen, dass die Behauptung des Relevanzverlustes differenzierter betrachtet werden muss und dass Aussagen dieser Grundlagenkritik einer klaren Verortung bedürfen. Hier unterscheidet sie zwischen sozialdiagnostischen und epistemischen Aussagen. Gleichzeitig zeigt Knapp, dass kritische Betrachtungen der Kategorie ,Geschlecht' nicht automatisch einen Relevanzverlust der Kategorie ,Geschlecht' bedeuten bzw. implizieren, sondern als Teil der Selbstreflexion der (feministischen) Frauen- und Geschlechterforschung/Gender Studies gesehen werden können. Knapp betont, dass es nicht generell darum gehe, die Bedeutung der Kategorie ,Geschlecht' zu delegitimieren, sondern auch das körperliche Geschlecht, also sex, verstärkt zu betrachten und den feministischen Blick nicht nur auf ,Geschlecht' (im Sinne von gender) zu richten. Knapp plädiert somit dafür, die Kritik an der Kategorie gender nicht zwangsläufig als grundlegende Krise zu werten, die zum Postfeminismus führe. Vielmehr sieht sie darin das Potenzial einer produktiven Wende, um das selbstreflexive Potential feministischer Theorien neu zu stärken (vgl. 2008, S. 291ff). 
Dichotomie hinzuweisen und ,Geschlecht' nicht nur mit gender gleichzusetzen, sondern daran zu erinnern, dass gender und sex eng miteinander verbunden sind und dass beide Konzepte gesellschaftlichen Vorstellungen entspringen. Zudem sei mit der Bevorzugung von ,Geschlecht ${ }^{6}$ an den ursprünglichen radikalen Impetus feministischer Gesellschaftskritik erinnert, aber auch auf die Objektivierung von ,Geschlecht' als Thema verwiesen.

Allerdings haben nicht nur epistemologische Überlegungen zu dieser Begriffswahl geführt, sondern auch die Erfahrungen aus der empirischen Untersuchung selbst. Bei der empirischen Erhebung zeigte sich, dass die Befragten mit gender einen sehr abstrakten und wissenschaftstheoretischen Begriff verbinden, der nur wenig mit den Erlebens- und Erfahrungswelten der Studierenden zu tun hat. Zudem wird gender oftmals mit Abwertungen und negativen Assoziationen verbunden (vgl. Kapitel 6). Der Begriff Geschlecht erscheint hingegen weniger negativ besetzt und weniger als ExpertInnenbegriff und scheint somit näher an den Erlebens- und Erfahrungswelten der Studierenden zu sein. In Bezug auf die spezifischen Fragestellungen wird im Folgenden nicht nur von ,Geschlecht' sondern auch von Geschlechterfragen gesprochen. Hierbei handelt es nicht um eine bloße Addition. Statt dessen soll darauf hinweisen werden, dass nach wie vor Fragen und Probleme nach, um und durch ,Geschlecht' bestehen und sich diese auch entlang des gesellschaftlichen Wandels verändern. Zudem möchte ich hervorheben, dass diese Fragen auch je nach gesellschaftlich-kulturellem Kontext differieren und die Erörterung dieser Fragen prozesshaft und kontextualisiert erfolgen muss, statt universell und abschließend beantwortet zu werden.

Auf der theoretischen Ebene verstehe ich ,Geschlecht' überwiegend als eine soziale Differenzkategorie und als gesellschaftlich-kulturelle Existenzweise(n). Mit dem Begriff der Existenzweisen ist die Anerkennung verschiedener Geschlechtlichkeiten und Geschlechtsidentitäten verbunden. Der Begriff lässt ein Kontinuum von Existenzweisen, jenseits von Männlichkeit und Weiblichkeit zu (vgl. Kapitel 3.7). Indem ich den Begriff ,Geschlecht' unter Anführungszeichen setze, soll hervorgehoben werden, dass Vorstellungen von ,Geschlecht" Ergebnis gesellschaftlicher Bedeutungszuweisungen sind und die binäre Kodierung in ,weiblich und ,männlich' bereits patriarchal hierarchisch strukturiert ist. Die Geschlechterdifferenz ist demnach keine natürliche Gegebenheit, sondern ein konstitutives Strukturelement des herrschenden heterosexuellen Geschlechterdiskurses und wird von diesem hervorgebracht. Auch wenn in dieser Arbeit sich als Männer und Frauen empfindende Individuen im 
Fokus stehen, möchte ich deutlich machen, dass ich Geschlechtsidentität nicht als etwas zeitlich Stabiles verstehe und in meinem Verständnis von ,Geschlecht' auch marginalisierte und fluide Geschlechteridentitäten im Blick habe. Demnach verweist, Geschlecht' nicht auf eine Geschlechterdualität, sondern auf eine Geschlechterpluralität.

\section{Geschlechterreflektierende und -reflektierte Haltung}

Im Zusammenhang mit der (De-)Thematisierung von ,Geschlecht' und Geschlechterfragen spreche ich in dieser Arbeit immer wieder von einer geschlechterreflektierenden und -reflektierten Haltung oder einem reflektierten und reflektierenden Umgang mit ,Geschlecht'. Ich favorisiere hier den Begriff der Geschlechterreflexion gegenüber Geschlechtersensibilität, da letzterer Geschlechterunterschiede als festgeschrieben suggeriert. Zudem ist das Besondere an einem reflektierten und reflektierenden Umgang mit ,Geschlecht', dass Positionen und Handlungen in Frage gestellt, Routinen und Automatismen immer wieder einer reflexiven Prüfung, Kontrolle und Kritik unterzogen werden. Es bedeutet aber auch, einen macht- und herrschaftskritischen Blick zu entwickeln und gesellschaftliche Normen zu hinterfragen. Eine geschlechterreflektierende Haltung verstehe ich auch als Selbstreflexion (vgl. Dietze 2006; 2008) und sie bedeutet demnach auch die (eigene) hegemoniale Position innerhalb gesellschaftlicher Diskurse zu erkennen und die jeweilige Positionierung im Feld zu begreifen (vgl. Kapitel 7.5).

\subsection{Lesehinweise}

Im Laufe dieses Buches werden Lesehinweise gegeben, die hier als Abschluss der Einleitung gesammelt angeführt werden:

- Um eine Reifizierung von Heteronormativität und Geschlechterbinarität in der Arbeit zu vermeiden, werden bewusst unterschiedliche Formen geschlechtergerechter Sprache verwendet. Zudem verweist die Verwendung des Gender Gaps, auch ,Unterstrich' genannt, (z. B. Student_innen), auf gegenderte Existenzen, wie z. B. Intersexuelle oder Transgender-Personen, die in dem dichotomen Zwangssystem keinen anerkannten und begrifflich markierten Ort haben. Mittels geschlechtlicher Benennung durch den Unterstrich soll außerdem herausgestellt werden, dass die Leerstelle ,Geschlecht‘ von unterschiedlichen Dominanzverhältnissen, Macht- 
praktiken und Diskursen erst gefüllt wird (vgl. Hausbichler 2008; Dietze et al. 2007). Die in dieser Arbeit durchmischte Schreibweise soll auf die Vielfalt der sprachlichen Möglichkeiten hinweisen und für einen kritisch-reflektierten Sprachgebrauch sensibilisieren. Gleichzeitig soll sie die Vielfalt von Existenzweisen widerspiegeln.

- Für eine bessere Lesbarkeit werden im Empirieteil zwischen Kurzzitaten und zitierten Passagen aus den Gruppendiskussionen unterschieden. Kurzzitate von den Studierenden werden im Fließtext mit Anführungszeichen hervorgehoben. Es wird zudem angegeben aus welcher Gruppendiskussion das Zitat stammt, jedoch ohne Angaben der jeweiligen Zeilennummer im Originaltranskript. Die längeren zitierten Passagen aus den Gruppendiskussionen heben sich vom restlichen Fließtext ab, indem sie mit der jeweiligen Originalzeilennummerierung gekennzeichnet sind. Zudem wurden die zitierten Passagen zur besseren Lesbarkeit leicht geglättet, d. h. für den Lesefluss störende ,ähs ${ }^{6}$, ,ehms ${ }^{6}$ und Wortwiederholungen wurden entfernt. Der Sinngehalt der Aussagen wurde durch diese Überarbeitung nicht verändert. 



\section{Erziehungs- und Bildungswissenschaften und ,Geschlecht'}

Wenn wir davon ausgehen, dass der Bereich unserer individuellen Erkenntnis ein Feld gesellschaftlicher, institutionell und disziplinär vermittelter Praxis ist, so gilt es auch hier sehr konkret die jeweiligen Kontexte $\mathrm{zu}$ bestimmen, in denen um Bedeutung und Artikulationsmöglichkeiten gekämpft wird. Demzufolge ist Erkenntnis nicht unabhängig von ihren Produktions-, Distributions- und Rezeptionsverhältnissen. Im Gegenteil sind die Machtfelder und das Gefüge der Disziplinen durchaus starke Einflussgrößen, wenn es um Thematisierungschancen und -dynamiken geht. Somit ist es von Bedeutung, die disziplinären und institutionellen Abhängigkeiten zu reflektieren (vgl. Maurer 2012, S. 134). Das folgende Kapitel dient dazu, den bildungsmilieutypischen Erfahrungsraum darzustellen, in dem Studierende, Geschlecht' (de-)thematisieren. Hier wird deutlich, dass Wahrnehmungs-, Denk- und Bewertungsschemata nicht losgelöst vom sozialen Feld erfasst werden können und dass der Habitus nicht mechanisch funktioniert, sondern nach einem Modell lebender Systeme (vgl. Krais/Gebauer 2010, S. 63). Für Studierende der Erziehungs- und Bildungwissenschaften bildet die gemeinsam erlebte Wissensvermittlung im sozialen Feld ${ }^{1}$ und die Zugehörigkeit zu einer Universität bilden den konjunktiven Erfahrungsraum ${ }^{2}$ der Studierenden. Da die universitären Rahmenbedingungen (so die These) einen starken Einfluss auf die sozia-

1 Der von Pierre Bourdieu entlehnte Begriff des sozialen Feldes bezieht sich auf ausdifferenzierte Bereiche einer Gesellschaft wie z. B. Kunst, Wissenschaft oder Politik. Dies gilt auch für die Hochschule in ihrer Rolle als Bildungsinstitution, die in dieser Arbeit als relativ autonomes Feld aufgefasst wird. Dabei werden soziale Felder als Kräftefelder gesehen, deren Dynamik in den Beziehungen der AkteurInnen zueinander liegt und die über eine innere Homogenität des Systems verfügen (vgl. Engler 2010, S. 262; vgl. Kapitel 4.1.1).

2 Karl Mannheim spricht vom konjunktiven Erfahrungsraum dort, wo Menschen eine bestimmte Handlungspraxis und Sozialisationsgeschichte teilen. Das Konzept des konjunktiven Erfahrungsraums fasst eine von der konkreten Gruppe gelöste Kollektivität grundlagentheoretisch. Es verbindet diejenigen, die an Wissensund Bedeutungsstrukturen teilhaben, welche in einem bestimmten Erfahrungsraum gegeben sind. Zugleich ist diese Kollektivität keine dem Einzelnen externe, ihn zwingende oder einschränkende, sondern eine, die Interaktion und alltägliche Praxis ermöglicht und die gemeinsamen Handlungsvollzüge ohne Umweg über den Subjektbegriff beschreibbar macht (vgl. Przyborski 2004, S. 24). Hierzu siehe auch Abschnitt 4.1.1. 
len und kulturellen Räume der Studierenden haben und sich innerhalb disziplinärer Diskurse zu und Thematisierungsweisen von ,Geschlecht gegenseitig beeinflussen, wird, das Gefüge der Disziplin Erziehungswissenschaften dargestellt und in die Überlegungen miteinbezogen. Denn auch die Praxis des Nachdenkens, Reflektierens und Thematisierens von ,Geschlecht' ist geprägt von der Kontextualisierung durch das akademische Universum, die feldinternen Kräfteverhältnisse und vom sozialen Umfeld der Subjekte (vgl. Rieger-Ladich 2009, S. 23). Somit muss bei der Frage danach, wie Studierende der Erziehungs- und Bildungswissenschaften ,Geschlecht' und Geschlechterverhältnisse thematisieren, in Betracht gezogen werden, unter welchen Bedingungen, in welchem universitären Umfeld und mit Hilfe welcher Dynamiken dies stattfindet.

In dieser qualitativen empirischen Untersuchung zeigen sich die befragten Studierenden bei der (De-)Thematisierung von ,Geschlecht' stark von dem disziplinären Gefüge und den Rahmenbedingungen ihres Studienfachs beeinflusst. Die befragten Studierenden hadern mit den Vorurteilen und Stereotypen, die dem Fach entgegengebracht werden. So wird in der Gruppe ,Holz' problematisiert, dass das „Bild vom gutbezahlten, im Labor arbeitenden Wissenschaftler" (GD_Holz) ${ }^{3}$ nicht mit dem eines „Pägagogen“ überein stimmt: „Der Pädagoge ist entweder eine Pädagogin, oder einer mit Birkenstock ${ }^{4}$ und Gitarre an der Hand" (GD_Holz). Zudem beeinflusst auch die hohe Zahl der weiblichen StudentInnen die (De-)Thematisierung von ,Geschlecht'. Demnach scheint es keinen Grund zu geben, sich mit Geschlechterfragen im universitären Kontext auseinander zu setzen. Da Männer nicht typischerweise dieses Studium wählen, werden sie als Ausnahme dargestellt, die von der Normalität im Studium abweicht. Des Weiteren werden, die ${ }^{6}$ Männer im Studium als abweichend von der heterosexuellen Geschlechternorm konstruiert, indem über die homosexuelle Orientierung der (wenigen) Männer spekuliert wird (vgl. Kapitel 6). Das Ziel dieses Kapitels besteht nicht darin, unterschiedliche Studiengänge zu vergleichen, nur nach dem heutigen Stand der Ge-

Die im Folgenden angegebenen Kurzzitate stammen aus dem, für diese Arbeit erhobenen Datenmaterial. Es wurden vier Gruppendiskussionen, ,Holz', ,Feuer', ,Metall' und ,Wasser', an zwei Universitäten geführt (vgl. Kapitel 5.1).

4

„Birkenstock“ ist eine Markenbezeichnung, unter der vor allem Hausschuhe und Sandalen aus Naturmaterialien mit einem ausgeprägten Fußbett (sogenannte, Gesundheitsschlappen') bekannt sind. Mit dieser Marke wird ein ,alternativer' Lebensstil verbunden, die mit der Hippiekultur assoziiert wird. Die Kombination von Birkenstock und Gitarre verweist auf eine nicht-hegemoniale und marginalisierte Lebensweise, zugleich wird damit eine ,unterlegene' Weiblichkeit und Männlichkeit ausgedrückt. 
schlechterforschung in der Erziehungswissenschaften zu fragen oder die Implementierung von geschlechterreflektierten Inhalten zu überprüfen. Vielmehr geht es darum, eine geschlechterkritische Perspektive bezüglich des Fachs einzunehmen und zu skizzieren, ob und in welcher Art und Weise ,Geschlecht ${ }^{6}$ - innerhalb des disziplinären Feldes, und dessen Teil sie zugleich sind - zum Thema gemacht wird. Dies erfordert eine doppelte Reflexion: zum einen die historische Rekonstruktion der Thematisierung von geschlechterbezogenen Fragestellungen in der Disziplin (vgl. Kapitel 2.3.1) und zum anderen eine theoretische und praktische Perspektive auf die Kernkonzepte Bildung und Erziehung (vgl. Kapitel 2.3.2). Bevor ich jedoch damit beginne, die Thematisierung von ,Geschlecht' in den Erziehungswissenschaften historisch zu umreißen (vgl. Kapitel 2.3), werde ich die Etablierung der Erziehungswissenschaften an deutschen Hochschulen nachzeichnen (vgl. Kapitel 2.1) und die statistische Repräsentation/Verteilung von Frauen und Männern genauer in den Blick nehmen (vgl. Kapitel 2.2). Im Anschluss daran werden die Konjunkturen der Thematisierung von ,Geschlecht" auf theoretischer und empirischer Ebene dargestellt.

\subsection{Die Etablierung im universitären Erscheinungsbild}

In den letzten 50 Jahren hat die Pädagogik oder die Erziehungswissenschaften, ${ }^{5}$ wie das Fach an den bundesdeutschen Hochschulen mittlerweile genannt wird, einen weitreichenden Gestaltungswandel vollzogen (vgl. Otto/Rausenbach/Vogel 2002, S. 7). Dieser Wandel zeigt sich ab den 1960er Jahren nicht nur in der Etablierung der Erziehungswissenschaften im universitären Erscheinungsbild, sondern auch in innerdisziplinären Veränderungen. Auf beide Aspekte der Veränderungen wird im Folgenden in groben Zügen eingangen

Bis in die 1960er Jahre war das Fach durch die Tradition der geisteswissenschaftlichen Pädagogik gekennzeichnet, die sich als eine philosophische Disziplin und nicht als eine empirische Wissenschaft verstand. Diese Auffassung hat sich innerhalb weniger Jahre stark geändert und die Erziehungswissenschaften wurde gewissermaßen auf ,den empirischen Weg gebracht" (Garz 2012, S. 30). ${ }^{6}$ Neben der theoretischen

5 Beide Bezeichnungen werden in dieser Arbeit synonym verwendet.

6 Bereits zu Beginn des 20. Jahrhunderts gab es eine erfahrungswissenschaftlich orientierte (experimentelle) Pädagogik (vgl. Garz 2012, S. 30). 
Reflexion im Hinblick auf bestimmte Vorstellungen (z. B. Mündigkeit, Bildung, Geschichtlichkeit) trat eine methodische Komponente hinzu. Es wurde anerkannt, dass Erziehungswissenschaften unverzichtbar eine erfahrungsbezogene Komponente benötigt. Anfangs wurde diese Lücke von quantitativer Forschung gefüllt, zunehmend jedoch auch von qualitativ, rekonstruktiv-interpretativ verfahrender Methoden (vgl. ebd., S. 40). Insgesamt gesehen haben sich mit der Etablierung empirischer Arbeiten in der Erziehungswissenschaften auch deren Gestalt und damit die Struktur der Disziplin verändert. Neben philosophischen und theoretischen Überlegungen sind Studien getreten, die faktenorientiert sind und sich mit der Interpretation und Rekonstruktion sozialer Wirklichkeit(en) auseinandersetzen. Auf diese Weise soll das pädagogische Denken, also das theoretische Wissen, auf gesicherte, nicht spekulative Weise zunehmen (vgl. ebd., S. 43).

Ungefähr zur selben Zeit hat sich eine Veränderung auf institutioneller Ebene vollzogen. Bis in die 1960er Jahre war das Fach eingebettet in die LehrerInnenbildung, die Erziehungswissenschaft selbst war damals an den Universitäten marginal vertreten. Zentrale Bedeutung erlangte das Fach durch die LehrerInnenbildung an den damals noch flächendeckend vorhandenen Pädagogischen Hochschulen (vgl. ebd.). Diese Veränderung begann ab Mitte der 1960er Jahre durch drei einschneidende Entwicklungen: erstens durch den rasant steigenden LehrerInnenbedarf in Deutschland aufgrund der demographischen Entwicklung einerseits, und andererseits durch die Erhöhung der Bildungsbeteiligung ab Mitte der 1960er bis Ende der 1970er Jahre; zweitens durch die explosionsartig steigende Nachfrage nach erziehungswissenschaftlichen Studienplätzen im Laufe der bundesweiten Einführung des Diplomstudiengangs ab Anfang der 1970er Jahre; drittens durch die strukturellen Veränderungen in der Universitätslandschaft in den 1970er und frühen 1980er Jahren. In dieser Zeit kam es zur sukzessiven Transformation der Pädagogischen Hochschulen in wissenschaftliche Hochschulen bzw. deren Integration in bestehende Universitäten. Alle drei Entwicklungen zusammen haben die Erziehungswissenschaften grundlegend verändert (vgl. ebd.).

Eine weitere einschneidende Veränderung in den letzten Jahren ist der Umstieg vom Diplom hin zum Bachelor/Master-Modell. Diese Umstellung auf Bachelor- und Master-Programme führte zu standortspezifischen Schwerpunktsetzungen und unterschiedlich gestalteten Studienangeboten (vgl. ebd., S. 77). Auch wenn der Umstieg nicht unumstritten ist, kann dieser seit Wintersemester 2011/2012 als weitgehend komplettiert gelten (vgl. Stisser/Horn 2012, S. 7). Heute sind die Erziehungs- und Bil- 
dungswissenschaften aus dem universitären Erscheinungsbild nicht mehr wegzudenken. Dennoch wird die Disziplin oftmals noch in ihrer tradierten Form wahrgenommen oder sieht sich mit langlebigen Vorurteilen konfrontiert: Noch immer wird die Pädagogik vielerorts gleichgesetzt mit der Lehrer_innenbildung und ausgebildete PädagogInnen gleichgesetzt mit dem Berufsbild der Lehrerin/des Lehrers. ${ }^{7}$ Nach wie vor erliegen viele ,Außenstehende ${ }^{6}$ der falschen Annahme, dass die Mehrzahl der AbsolventInnen des Faches entweder arbeitslos sind oder in fachfremde Tätigkeitsbereiche ausweichen (vgl. Otto/Rausenbach/Vogel 2002, S. 8). Daher leidet die Disziplin der Erziehungs- und Bildungswissenschaften an einem Reputationsproblem.

Eine weitere gängige Annahme ist, dass die Erziehungs- und Bildungswissenschaften ein ,typischer Frauenstudiengang“ (GD_Holz) sei. ${ }^{8}$ Es ist zwar ein Studiengang, der von weiblichen Studierenden dominiert wird, aber kann deshalb von einem „typischen Frauenstudium“(FaulstichWieland 2002, S. 173) gesprochen werden? Dieser Frage werde ich anschließend nachgehen.

\section{2 (K)ein ,typischer Frauenstudiengang“?}

Frauen als Studierende sind mittlerweile eine normale Erscheinung an den Hochschulen. Seit einigen Jahren stellen sie sogar mehr als die Hälfte der Erstsemestrigen. Die Verteilung auf die verschiedenen Studienfächer ist jedoch sehr ungleich. Erziehungs- und Bildungswissenschaften gehört zu den weit überproportional von Frauen gewählten Studienfächern. Mehr als drei Viertel aller eingeschriebenen Studierenden in der Erziehungs- und Bildungswissenschaften sind weiblich (vgl. FaulstichWieland/Horstkemper 2012, S. 195). ${ }^{9}$ Das Fach Erziehungs- und Bildungswissenschaften (einschließlich der Lehrer_innenbildung) besitzt ei-

7 In den Gruppendiskussionen ,Holz', ,Feuer' und ,Wasser' wird das Reputationsproblem der Erziehungswissenschaften problematisiert. Die Studierenden besprechen auch Vorurteile, mit denen sie konfrontiert sind.

8 In den geführten Gruppendiskussionen wird der hohe Anteil weiblicher Studierender diskutiert und nach den Gründen und Auswirkungen auf das Feld gefragt.

9 Bislang lassen sich insbesondere drei Erklärungsansätze für geschlechtsbezogene Segregation in Ausbildungs- und Bildungsverläufe finden. Eine Vielzahl von Arbeiten fokussiert auf den Moment der Berufsfindung und konzeptualisiert diesen primär als individuelle Entscheidung und Präferenzen. Andere Studien sehen die Gründe für die berufliche Segregation vor allem in institutionellen Mechanismen. Hier wird untersucht, inwiefern Institutionen geschlechtsbezogene Unterschiede voraussetzen, diese reproduzieren und verstärken. Des Weiteren wird häufig auf normative Erwartungen, insbesondere auf Geschlechternormen und -vorstellungen, 
ne herausragende Rolle in der Hochschulausbildung von Frauen. Im Wintersemester 2010/11 lag der Anteil der weiblichen Hauptfachstudierenden deutschlandweit bei 77,6 \% (vgl. ebd., S. 194). Frauen sind in den letzten Jahren im Schnitt zu drei Viertel an den Abschlüssen des Diplomund Magisterstudiums der Erziehungs- und Bildungswissenschaften beteiligt. Der geringe Anteil von männlichen Studierenden ist unter Gleichstellungsaspekten eine Ungleichheit zuungunsten von Männern. Professionspolitisch bedeutet dies zum einen, Maßnahmen zu ergreifen, die dazu beitragen, den Anteil der Männer bei den Studierenden zu erhöhen, ohne damit die Studienchancen von Frauen zu beeinträchtigen (vgl. ebd., 211f.).

Wenn man sich jedoch mit der Frage, ob Erziehungs- und Bildungswissenschaften ein ,typischer Frauenstudiengang ${ }^{\text {"10 }}$ sei, genauer beschäftigt, fällt auf, dass sich das Blatt wendet, sobald man sich mit den folgenden wissenschaftlichen Qualifikationsschritten der Studierenden beschäftigt. Frauen studieren in den Erziehungs- und Bildungswissenschaften zwar erfolgreicher als Männer, bei der wissenschaftlichen Fortsetzung des Studiums in Form einer Promotion bzw. einer Habilitation ändert sich die Geschlechterverteilung jedoch drastisch. Liegt ihr Anteil bei Promotionen noch bei mehr als der Hälfte, unterschreitet die Anzahl der Habilitationen bereits 50 \% Marke. Erscheint dieser Trend vorerst nicht gerade negativ, ändert sich der Eindruck jedoch, wenn bedacht wird, dass Männer mit nur einem Viertel an den Abschlüssen des Diplomoder Magisterstudiums beteiligt sind, und dann mit knapp der Hälfte an den Promotionen und mit über der Hälfte an den Habilitationen teilhaben. Bei genauer Betrachtung sind Frauen bei den Mittelbaustellen, die zu Promotionen führen, mit zwei Drittel vertreten. Führt man sich das Verhältnis der promovierten Frauen und Männer vor Augen bedeutet dies jedoch, dass von den verhältnismäßig wenigen männlichen Absolventen wesentlich mehr eine Promotionsstelle erhalten (vgl. ebd., S. 212). Somit nimmt bei steigender Qualifikation der Frauenanteil ge-

als Ursache für geschlechtersegregierte Ausbildungs- und Bildungsverläufe verwiesen. Danach prägen Geschlechterstereotype in Bezug auf vermeintlich natürlich unterschiedliche Fähigkeiten und individuelle Lebensziele von Männern und Frauen die Selbstbilder und Präferenzen von jungen Erwachsenen ebenso wie die Erwartungen und Zuschreibungen von Eltern, Berufscoaches oder Arbeitgebenden (vgl. Schwiter/Wehner/Maihofer/Huber2011, S. 21ff).

10 In den geführten Gruppendiskussionen wird zum einen über die Geschlechterverteilung bei den Studierenden gesprochen und zum Teil die weibliche Dominanz problematisiert. Zum anderen wird aber auch festgestellt, dass es verhältnismäßig viele männlich besetzte Professuren im Vergleich zu den männlichen Studenten gibt. 
messen an den weiblichen Studierendenzahlen deutlich ab. Dieser Eindruck verstärkt sich in der Betrachtung von Lehrenden und ProfessorInnen. Zwar stieg der Frauenanteil beim erziehungswissenschaftlichen Personal in den vergangen Jahren kontinuierlich, und liegt bei den Professuren 2011 bei $43 \%$ (vgl. ebd.). Doch auch wenn dieser Anteil deutlich höher ist als in den meisten anderen Fächern, ist damit noch längst keine Parität erreicht.

Zieht man die quantitative Besetzung des Studienfachs bei den Lehrenden als Kriterium für die Vergeschlechtlichung des Studiums heran, kann man nicht zu der Einschätzung gelangen, dass Erziehungs- und Bildungswissenschaften ein „Frauenstudium“ (Faulstich-Wieland 2002, S. 176) sei. Zusammenfassend kann gesagt werden, dass auf quantitativstatistischer Ebene die Frage, ob Erziehungs- und Bildungswissenschaften ein „typischer Frauenstudiengang " ${ }^{\text {11 }}$ ist, unterschiedlich beantwortet werden muss. Gemessen an den Studierendenzahlen lautet die Antwort „Ja“. Gemessen am universitären Mittelbau und an den Professuren ist die Frage mit einem klaren „Nein“ zu beantworten. Für die Betrachtung der Untersuchung der Erziehungs- und Bildungswissenschaften bedeutet dies, nicht rein auf der Basis von Studierendenzahlen auf ein „Frauenstudium“ zu schließen, sondern den Ausbildungsweg und die Disziplin in seiner Gesamtheit wahrzunehmen, da es auf allen Ebenen einer stärkeren Berücksichtigung der Kategorie ,Geschlecht' bedarf (vgl. FaulstichWieland 2002, S. 174). Dies gilt für die paritätische Verteilung der Geschlechter sowie für theoretische und praktische Perspektiven auf die Kernkonzepte Bildung und Erziehung und die damit verbundenen marginalen und hegemonialen Diskurse. Deshalb wird/werden im nächsten Abschnitt die Konjunktur(en) der Thematisierung von geschlechtsbezogenen Fragestellungen nachgezeichnet.

\subsection{Die Relevanz von ,Geschlecht' und seine Konjunkturen}

Die Frage nach der Thematisierung von ,Geschlecht' und Geschlechterfragen in den Erziehungs- und Bildungswissenschaften geht vor allem

11 Diese Bezeichnung eines Studiengangs als „typischer Frauenstudiengang“ (GD Holz) oder als „Frauenstudium“ (Faulstich-Wieland 2002, S. 176) sind alltagsweltliche Beschreibungen eines Studiums, dass fast ausschließlich von Frauen studiert wird. Damit ist auch eine implizite Abwertung verbunden, von der ich mich hier distanzieren möchte. Deshalb ist der Begriff im Folgenden unter Anführungszeichen gesetzt. 
Hand in Hand mit der Frage nach den Institutionalisierungserfolgen der Frauen- und Geschlechterforschung. ${ }^{12}$ Beide Fragen sind nicht unabhängig voneinander zu beantworten und sind deshalb im Anschluss in ihrer Verwobenheit dargestellt. Mittlerweile blickt die Frauen- und Geschlechterforschung auf etliche fruchtbare Jahre zurück, in denen sie sich durch theoretische und empirische Arbeiten auszeichnen und Anstoß für zahlreiche und innovative Debatten war. In der erziehungswissenschaftlichen Moderne hat die pädagogische Thematisierung von ,Geschlecht', im Gegensatz zu anderen Disziplinen, eine lange Tradition. Jedoch werden sie häufig nicht als solche problematisiert bzw. erfolgt die Thematisierung von ,Geschlecht" und Geschlechterfragen oftmals abseits vom pädagogischen und erziehungswissenschaftlichen Mainstream erfolgt(e) (vgl. Faulstich-Wieland 2002, S. 177). Demzufolge scheint die Thematisierung von ,Geschlecht ${ }^{6}$ und Geschlechterfragen keinen hegemonialen, sondern eher einen marginalisierten Diskurs darzustellen. Somit bleibt die Integration der Kategorie ,Geschlecht' in die Arbeiten an den systematischen Grundlagen und den Forschungsfragen der Erziehungswissenschaft lückenhaft und fragmentarisch. Innerhalb hegemonialer Kämpfe wurde/wird auch versucht, das Projekt der feministischen Pädagogik, mittlerweile als erziehungswissenschaftliche Frauen- und Geschlechterforschung bezeichnet, sukzessive im akademischen Feld zu etablieren (vgl. ebd.). Es finden Auseinandersetzungen darüber statt, was innerhalb der Erziehungs- und Bildungswissenschaften bei welchen FachvertreterInnen als wahrheitsfähig gilt (und was nicht) und was von welchen Gruppen als ernstzunehmendes Forschungsthema eingeschätzt wird (und was nicht), was von den Autorisierungsinstanzen zum kanonischen Wissensbestand der Disziplin gezählt wird (und was nicht) (vgl. Rieger-Ladich 2009, S. 18). Festzuhalten bleibt, dass Interventionen für eine feministische Pädagogik oder erziehungswissenschaftliche Frauen- und Geschlechterforschung nie auf einen neutralen diskursiven Raum treffen, sondern von der Kontextualisierung durch das akademische Universum und feldinterne Kräfteverhältnisse geprägt sind (vgl. ebd., S. 23). Deshalb ist im Folgenden die Ordnung des pädagogischen Diskurses als Hintergrundfolie zu sehen, vor welcher Studierende über Geschlechterthemen reden und Thematisierungsmodi hierfür ausbilden.

12 Hier soll festgehalten werden, dass es abseits vom wissenschaftlichen Diskurs und historisch gesehen bereits vor den Fragen zur Institutionalisierung von Frauen- und Geschlechterforschung eine an Geschlechterfragen und Geschlechterverhältnissen orientierte Pädagogik und Frauenbewegung gab. 


\subsubsection{Die historische Rekonstruktion der Thematisierung von geschlechterreflektierten Fragestellungen}

Die moderne Geschlechterordnung und die Formierung der Erziehungswissenschaft als Disziplin haben historisch dieselben Wurzeln. Das 18. Jahrhundert, welches als das pädagogische Jahrhundert gilt, ist gleichzeitig die Epoche, in der die Polarisierung der ,Geschlechter' ihren Anfang nimmt. ${ }^{13} \mathrm{Zu}$ dieser Zeit verdichtet sich der Wandel von der feudalen Ständegesellschaft zur modernen, bürgerlichen Gesellschaft vor dem Hintergrund der Philosophie der Aufklärung und den politischen Forderungen nach bürgerlichen Rechten. Auch der sozioökonomische Wandel in Richtung einer industriellen Gesellschaft setzt Veränderungsprozesse in Gang (vgl. Dausien/Thon 2009, S. 339).

Die Familie als wirtschaftliche Einheit verliert an Bedeutung und mit der Trennung zwischen öffentlichen und privaten Räume geht eine Unterscheidung zwischen ,männlicher' und ,weiblicher ${ }^{6}$ Sphäre einher. Gleichzeitig verändert sich auch das Wissenschaftsverständnis in Bezug auf die Frage nach dem Wesen des Menschen. Diesbezüglich etabliert sich das naturwissenschaftliche Paradigma. Metaphysische Vorstellungen werden durch rationale, auf Beachtung der ,Natur des Menschen" basierende Konzepte abgelöst (vgl. ebd.). Gesellschaftstheoretisch liefert diese Entwicklungen das Argument für eine vorgebliche Gleichheit aller Menschen. Gleichzeitig jedoch wird in dieser Zeit durch das naturwissenschaftliche Paradigma die Annahme einer Hierarchie zwischen den Geschlechtern aufgrund der als biologisch postulierten Zweigeschlechtlichkeit legitimiert. Diese Entwicklung zeigt sich auch in den Erziehungstheorien der Aufklärung. So ist das Erziehungsziel der Unabhängigkeit

13 „In akademischen Texten wird zuweilen die Fiktion erzeugt, alles könne an seinen „Ursprung“ zurückgeführt werden“ (Maurer 1996, S. 204). Wird jedoch „Erkenntnis als soziales Produkt" verstanden (ebd. S. 202), das ohne die soziale Einbettung und den kollektiven Austauschprozessen nicht, ent- und bestehen' könnte und davon ausgegangen, dass Erkenntnis auch den Raum und die Aufmerksamkeit einer (bestimmten) Öffentlichkeit braucht, ist dieser Anspruch wohl kaum möglich (vgl. Maurer 1996, S. 203f.). Dies ist speziell bei der Aufarbeitung der Thematisierung von geschlechterreflektierten Fragestellungen zu bedenken. Feministisches Erkenntnisinteresse, Theoriebildung, Entwicklung von Themenstellungen und Untersuchungsmöglichkeiten wurden/werden überwiegend in selbst organisierten Arbeitsgruppen außerhalb der Wissenschaftsinstitution betrieben (ebd., S. 427) und fanden/finden demnach nicht (nur) im Kontext der scientific community und der ,dokumentierten' Wissensproduktion statt. So können die folgenden Ausführungen den „Mythos der Originalität“ (ebd., S. 202) und der Vollständigkeit nicht erfüllen. 
und das Eigenrecht des Kindes den Jungen vorbehalten, z. B. in „Emile oder Über die Erziehung“" (1762) von Jean-Jacques Rousseau (vgl. Dausien/Thon 2009, S. 339). In Abgrenzung zu der Nützlichkeitspädagogik der Aufklärung wird im neuhumanistischen Bildungsideal ein Anspruch auf allgemeine Bildung formuliert. In den Schriften von Wilhelm von Humboldt wird universelle Bildung als Basis der Selbstkonstituierung des modernen, emanzipierten, bürgerlichen Subjekts postuliert.

Dieser Universalitätsanspruch der Bildungsidee wird aber unterlaufen, indem Humboldt Frauen auf spezifische Weise ausnimmt. Er konzipiert das Verhältnis des sich bildenden Subjekts zur Welt als abstrahierendes und objektivierendes Erkenntnisstreben. Zu der dafür nötigen distanzierten Unterscheidung von Ich und Welt seien Frauen allerdings aufgrund der ihnen unterstellten mütterlichen Tendenz zur harmonischen Verschmelzung mit der Welt weniger in der Lage. In dieser Beziehung bleibt das Subjekt von Bildung und damit von Menschwerdung ein männliches Subjekt. Die damit verbundenen Männlichkeitskonstruktionen sind allerdings schon seit Rousseau auf Rationalität, Aktivität und Stärke ausgerichtet und in ihrer Polarisierung als einseitig zu identifizieren. Insofern stehen diese damit im Widerspruch zur Idee eines universellen Konzepts menschlicher Subjektivität (vgl. ebd., S. 340). Die Universalisierung von Bildung fand somit unter Aussonderung des Weiblichen statt.

Im Kampf um Gleichberechtigung wurde die aufklärerische Idee einer angeborenen Freiheit, Gleichheit und Vernunftbegabung aller Menschen von Frauen (und Männer) in Anspruch genommen. Gleiches gilt für den emphatisch emanzipatorischen und universellen Bildungsbegriff des Neuhumanismus, entgegen der Engführung, die er bei Theoretikern der Erziehung und Bildung erfuhr. Dies zeigt sich an den Kontroversen, welche durch die beginnende Frauenbewegung in der zweiten Hälfte des 19. Jahrhunderts entfacht wurde und die Frage der Frauenbildung vorantrieb. Eine beruflich anspruchsvolle und gesellschaftlich akzeptierte Tätigkeit abseits einer männerdominierten Welt war Mitte der 1920er Jahre nur im Bereich der allgemeinbildenden höheren Mädchenbildung oder der sozialen Frauenbildung möglich. Auch für die Sozialarbeit schufen Frauen aus der ersten Frauenbewegung ein Ausbildungswesen, in dem akademisch gebildete Frauen gebraucht wurden (vgl. Jacobi 1990, S. 259).

Die radikale Forderung nach Bildung für Frauen und Gleichheit der Geschlechter wird auch in der ,Zweiten Frauenbewegung. ${ }^{64}$ Mitte der

14 Susanne Maurer hat 1996 in ihrem Buch „Zwischen Zuschreibung und Selbstge- 
1960er Jahre aufgegriffen (vgl. Dausien/Thon 2009, S. 340f.). Elisabeth Blochmann ${ }^{15}$ hat eine umfassende Studie zur Mädchenbildung in Deutschland vorgelegt. Ihre Arbeit „Das ,Frauenzimmer' und die ,Gelehrsamkeit', eine Studie über die Anfänge des Mädchenschulwesens in Deutschland", ist 1966 erschienen und ist ein bemerkenswerter Versuch, die Frühphase der deutschen pädagogischen Klassik unter dem Aspekt der Geschlechterpolarität bildungstheoretisch zu erfassen. Erstaunlich ist jedoch ihr Nachwort, in dem sie in durchaus unüblicher Form Mitte der 1960er Jahre persönliche Hoffnungen und Wünsche für die Frauenbildung darlegt (vgl. Jacobi 1990, S. 261). In diesem Nachwort hat Blochmann auf die Schwierigkeiten der Selbstdefinition von Frauen aufmerksam gemacht: „Die bloße Anpassung an den männlichen Maßstab oder die Übertreibung des rein Femininen sind aktuelle Gefahren" (Blochmann 1966, S. 125 zit. n. Jacobi 1990, S. 261). Die Thematisierung von Differenz, das Sichtbarmachen und Skandalisieren der Benachteiligung von Frauen im Bildungssystem haben seit den 1970er Jahren zu einer Reihe von Entwürfen einer feministischen Pädagogik geführt. Diese setzten zunächst an den unterstellten und angeblichen Defiziten von Mädchen und Frauen ${ }^{16}$ an, orientierten sich damit aber implizit an einer männlichen Norm und wurden in den 1980/90ern durch eine Betonung der Differenz und des Eigenwerts weiblicher Kultur abgelöst (vgl. Dausien/Thon 2009, S. 342).

Mitte der 1980er Jahre fokussierte diese Diskussion dann maßgeblich auf die Sichtbarmachung von Frauen. In Deutschland gaben z. B. Astrid Kaiser und Monika Oubaid 1986 einen Sammelband zu den deutschen Pädagoginnen der Gegenwart heraus. Ilse Brehmer ergänzte die Aufarbeitung 1990 mit einem Sammelband über Lebensläufe deutscher Pädagoginnen in der ersten Hälfte des Jahrhunderts. Zudem wurde die Arbeitsgemeinschaft zu „Frauen und Schule“ 1981 gegründet. Immer wieder

staltung. Feministische Identitätspolitiken im Kräftefeld von Kritik, Norm und Utopie" herausgearbeitet, dass die Frauenbewegungen eine ,Soziale ErkenntnisPraxis unter Frauen' ermöglichen, welche eine der Verschränkungen zwischen den Bereichen ,Individualität - Kollektivität - Erkenntnis' markiert (vgl. S. 427).

15 Elisabeth Blochmann erhielt 1952 einen Ruf an die Philipps Universität Marburg. Sie war die einzige Lehrstuhlinhaberin im Fach Pädagogik an einer westdeutschen Universität nach dem Zweiten Weltkrieg und blieb dies für fast zwei Jahrzehnte (vgl. Jacobi 1990, S. 261).

16 Dieser Defizitansatz hat bei geschlechtsbezogenen Bildungsfragen nach wie vor Aktualität - sowohl in der Mädchenbildung (Technik, Mathematik, Naturwissenschaften) als auch bei der Jungenbildung (Sprache, Soziale Kompetenz). Ich möchte hervorheben, dass die Defizite immer mit der gesetzten Norm entstehen und insofern hat die Feststellung von Defiziten nie wirklich Legitimität und ist Teil einer gesellschaftlich konstruierten (männliche) Norm. 
behandelte Themen in den durchgeführten Kongressen waren Inhalte der Lehrbücher, Interaktion in der Schule, sexistische Sprache und die Personalstruktur in den Schulen und Schulleitungen (vgl. Faulstich-Wieland 2002, S. 177f.). Auch an den verschiedenen Hochschulen ging die Debatte um ,Geschlecht' weiter und es wurde versucht, eigene feministische Positionen zu entwickeln. Im Zuge dessen entstanden erziehungswissenschaftliche Arbeitskreise oder Arbeitsstellen, die eine Institutionalisierung von Frauenforschung und Frauenstudien anstrebten. Die erste dieser Initiativen gab es an der Technischen Universität Berlin, an der Barbara Schaeffer-Hegel 1980 eine „Arbeitsstelle Sozial-, Kultur- und Erziehungswissenschaftliche Frauenforschung" etablierte. Am Fachbereich Erziehungswissenschaften der Johann Wolfgang Goethe-Universität in Frankfurt gründete sich 1984 ein „Arbeitskreis Frauenstudien“, der 1991 als „Arbeitsstelle Frauenforschung und Frauenstudien“ institutionalisiert wurde (vgl. ebd., S. 179). Dieser Fortschritt der Institutionalisierung lässt sich auch in der erziehungswissenschaftlichen Standesorganisation, der „Deutschen Gesellschaft für Erziehungswissenschaft (DGfE)“ nachvollziehen. Hier wurde 1984 ebenfalls eine „Arbeitsgruppe Frauenforschung“ gegründet. Diese wurde 1991 zu einer „Kommission Frauenforschung" und 1999 zur "Sektion Frauen- und Geschlechterforschung“. Diese Entwicklung der Adressierung dokumentiert den zunehmenden Akzeptanz- und Integrationsprozess: Während Arbeitsgruppen nur auf Zeit gegründet werden konnten, weil sie für die Bearbeitung vorübergehender Probleme gedacht waren, sollten Kommissionen für Daueraufgaben stehen. Mit der Neustrukturierung der DGfE in Sektionen und darin untergliederten Kommissionen wurde die Frauen- und Geschlechterforschung zu einem institutionellen Bestandteil der Erziehungs- und Bildungswissenschaften (vgl. ebd., S. 179). Die Frauen- und Geschlechterforschung in der Erziehungs- und Bildungswissenschaften ist heute ein ausdifferenzierter Wissenschaftsbereich, der zu den Disziplinen quer steht und doch ein Zentrum ihrer Arbeit betrifft (vgl. Glaser/Andresen 2009, S. 7). Engagierte WissenschafterInnen machten sich mit der Entwicklung der Frauen- und Geschlechterforschung daran, den Androzentrismus der Wissenschaft zu überwinden.

\subsubsection{Die erziehungswissenschaftliche Frauen- und Geschlechterforschung}

Die erziehungswissenschaftliche Frauen- und Geschlechterforschung gibt es, bereits seit Jahrzehnten. Die Arbeiten und Untersuchungen sind viel- 
fältig und stellen die Geschlechterthematik in unterschiedlicher Weise und in verschiedenen Bereichen ins Zentrum des Interesses. Schwerpunkte bilden Rekonstruktionen spezifischer Probleme weiblicher Bildungsund Professionsentwicklung oder empirisch geleitete Untersuchungen zu geschlechterstereotypen Sozialisationseffekten in pädagogischen Institutionen. ,Geschlecht' wird in der Folge des Umbaus der geisteswissenschaftlichen Pädagogik in die empirisch orientierte Bildungswissenschaft zu einer Perspektive empirischer Forschung. Dabei stehen zunächst nur ,weibliche Wirklichkeiten' und pädagogische Konzepte und Methoden im Zentrum sowie Fragen nach institutionellen und gesellschaftlichen Kontexten. Flankiert wird diese Entwicklung durch die interdisziplinäre Sozialisationsforschung (vgl. Kapitel 3.3). Damit geraten gesellschaftliche Strukturen und differenzproduktive Bedingungen des Aufwachsens in den Blick. Neben schichts- und geschlechtsbezogener Sozialisation stehen auch zunehmend Fragen der Sozialisation in Migrationsgesellschaften im Zentrum (vgl. Dausien/Thon 2009, S. 343).

Charakteristisch für die erziehungswissenschaftliche Frauen- und Geschlechterforschung in diesem Rahmen ist die Erweiterung der Aufmerksamkeit auf Sozialisationsprozesse bei Jungen und Männern. Dabei lehnt sich die männliche Sozialisationsforschung zunächst argumentativ und methodisch an die Mädchen- und Frauenforschung an. Als Reaktion auf die Entwicklung einer feministischen Mädchenarbeit sind Ansätze antisexistischer Jungenarbeit zu verstehen, die mittlerweile ebenfalls aus (de-) konstruktivistischer Perspektive kritisch revidiert und reformuliert werden (vgl. ebd., S. 345; Forster 2005). Versuche, individuelle Sozialisationsprozesse mit gesellschaftlichen Strukturen theoretisch zu verknüpfen, werden häufig mit Rekurs auf Pierre Bourdieus Habitustheorie entworfen (Meuser 1998; Meuser 2006). Der Blick auf die androzentrische Konzeption von Bildung wirft hier erneut die Frage nach dem Subjekt von Bildung auf, die bislang erst von wenigen TheoretikerInnen bearbeitet wurde. Aktuell sind für den deutschsprachigen Raum neben den psychoanalytischen Ansätzen, wie dem von Barbara Rendtorff (2006) und dem an die Kritische Theorie anknüpfenden Ansatz von Eva Borst (2003), vor allem poststrukturalistisch inspirierte Arbeiten zu nennen. Die theoretische Dekonstruktion und Dezentrierung des Subjekts im Poststrukturalismus konfrontiert die Pädagogik vor allem mit Problemen, die sich gerade im Hinblick auf die Kategorie ,Geschlecht ' produktiv wenden lassen, wie etwa die kritischen Arbeiten von Gesa Heinrichs (2001), Melanie Plößer (2005) und die Diskussion um eine „dekonstruktive Pädagogik“ (Frische et al. 2001) oder „Lesarten des Geschlechts" (Lemmermöhle et 
al. 2000; vgl. Dausien/Thon 2009, S. 342f.). Es werden zunehmend auch ethnografische (Kelle 1999) oder biografische (Dausien 2008) Methoden genutzt. Diese Forschungsstrategien sein ein Versuch, der Gefahr, die binäre Kategorie durch die Forschung zu reifizieren, systematisch zu begegnen. Die Ansätze werden auch als rekonstruktive Geschlechterforschung verstanden. Kritisch zu hinterfragen bleibt, inwiefern diese, häufig auf mikrosoziale, konkret situierte Praktiken bezogenen Forschungsstrategien in der Lage sind, überdauernde institutionelle Kontexte und gesellschaftliche Machtstrukturen zu erfassen (vgl. Dausien/Thon 2009, S. 343).

Spätestens ab den 1990er Jahren weitete sich der Blick von den Frauen und ihren Benachteiligungen auf beide Geschlechter und queere Identitäten (vgl. Friebertshäuser 2012, S. 99). Neben ,Geschlecht' geraten auch weitere Kategorien sozialer Differenzierung in das Blickfeld der Debatte, vor allem Klasse und Ethnizität. Einen Versuch der Integration unternimmt hier die „Pädagogik der Vielfalt" von Annedore Prengel (1995). Diese verfolgt empanzipatorische Impulse aus der Integrationspädagogik, feministischen Pädagogik und interkulturellen Pädagogik. In der Erziehungs- und Bildungswissenschaften lassen sich mittlerweile unterschiedliche Begriffe zur Thematisierung und Problematisierung von ,Geschlecht' identifizieren. ,Geschlecht' wird dabei als eine von verschiedenen sozialen Differenzen (Herkunft, Alter, ,Rasse'/Ethnizität, Klasse) gefasst. Hierfür werden die Begriffe Heterogentität und Vielfalt verwendet (vgl. grundlegend Prengel 1995; 2007; außerdem dazu z. B. Weber 2003; Naumann 2008; Nohl 2007). In der erziehungswissenschaftlichen Debatte zu Heterogenität werden auch Dimensionen wie Leistungsheterogenität, Arbeitstempo, Motivation oder Lernstile verhandelt (vgl. Becker et. al 2004; Altrichter/Messner 2004). Die Begriffe Heterogentität und Vielfalt fokussieren jedoch weniger auf die Analyse von Machtund Herrschaftsverhältnissen. Ein Begriff, der an die angloamerikanische Diskussion anknüpft, ist diversity. Besonders im betriebswirtschaftlichen Kontext ist von diversity oder diversity management die Rede (vgl. Andresen/Koreuber/Lüdke 2009). Unter diversity werden nicht nur soziale Positionierungen verstanden, die traditionell mit Ungleichheit verbunden sind, sondern auch unternehmensrelevante Unterschiede wie Dauer der Firmenzugehörigkeit, unterschiedliche Denk- und Arbeitsweisen usw. Deshalb ist es vor allem jenseits wirtschaftlicher Kontexte ratsam, differenziert nach der jeweiligen Auslegung des diversity-Begriffs zu fragen. ${ }^{17}$ Aktuell werden unter den Stichworten Intersektionalität und In-

17 Die Frage nach „Gender und Diversity: Albtraum oder Traumpaar" wird im gleich- 
terdependenz die Möglichkeiten der Verknüpfung der Perspektiven auf unterschiedliche Kategorien sozialer Differenzierung diskutiert (Walgenbach 2007; Degele/Winker 2009). In den letzten dreißig Jahren hat die Frauen- und Geschlechterforschung bereits einige Veränderungen in den erziehungswissenschaftlichen Feldern erreicht und in erheblichem Maße dazu beigetragen, die strukturelle Bedeutung der Geschlechterordnung, die historische Gewordenheit geschlechtsbezogener gesellschaftlicher Bedingungen, pädagogischer Maxime und die geschlechtstypisierenden Einflüsse in Erziehungs- und Bildungsprozessen zu analysieren sowie in die öffentliche Diskussion hinein zu tragen (vgl. Andresen et al. 2008, S. 10).

Auch wenn sich die erziehungswissenschaftliche Frauen- und Geschlechterforschung erst einmal wie eine Erfolgsgeschichte liest, werden die Thematisierung von ,Geschlecht" und die Auseinandersetzung mit feministischen Theorieansätzen in den bisherigen allgemeinen Debatten der Erziehungs- und Bildungswissenschaften nur mit wechselnder Konjunktur miteinbezogen (vgl. Casale/Rendtorff 2008, S. 9) oder bei der Übernahme in die allgemeinen Diskurse der Erziehungs- und Bildungswissenschaften domestiziert und neutralisiert (vgl. Andresen et al. 2008, S. 10). Catherine Marshall und Michelle F. Young beschreiben diese Effekte der Neutralisierung und Installierung neuer Barrieren im ,SAGEHandbook for Gender and Methodologie“ (2006) folgendermaßen:

„We see retrenchment from the academy's support of women's studies and, we are told, our research is biased, that everything about girls and women has already been studied and fixed“" (S. 74).

Sie schreiben weiter:

„[...], the lack of legitimacy of most research on gender issues has made it difficult for some to have their findings acknowledged, appreciated and acted upon by public officials. It is likely that this condition moved feminist researchers to monitor and, in some cases, exploit value shifts in society" (ebd., S. 66).

Auch in Deutschland lässt sich seit etwa Mitte der 1990er Jahre eine Veränderung in der öffentlichen Stimmung gegenüber Frauen- und Geschlechterthematiken feststellen: Zum Teil ist gereizte Zurückweisung zu vernehmen, teilweise offensives Desinteresse oder gender-Müdigkeit (,Das Thema ist durch. '), und stellenweise wird die Geschlechterperspektive als ,Spiegelstrich', als nur in Maßen wichtiger Zusatzaspekt eingeordnet. Somit wächst die Gefahr, dass das Bewusstsein für die strukturelle 
Bedeutung von Geschlechterbeziehungen, für die nachhaltige Wirkung von Geschlechterordnungen auf die Subjektentwicklungen wie auch auf die institutionelle Dimension von Erziehung und Bildung wieder zurückgedrängt wird (vgl. Andresen et al. 2008, S. 12). Zudem wird auch hier zu Lande die Geschlechterperspektive von den KritikerInnen als scheinbar privates Spezialinteresse einiger WissenschafterInnen an den Rand geschoben (vgl. ebd., S. 10). Für feministisch engagierte WissenschafterInnen, so schreibt Hannelore Faulstich-Wieland, bestehe zusätzlich die Notwendigkeit, sich als Person und mit ihrer Arbeit zugleich in anderen erziehungswissenschaftlichen Feldern - wie z. B. der Schulpädagogik oder der Erwachsenenbildung - zu verorten (vgl. Faulstich-Wieland 2002, S. 180f.). Durch die bisherigen Ausführungen wird deutlich, dass die Interventionen für eine feministische und kritische Geschlechterperspektive in der Erziehungs- und Bildungswissenschaften auf keinen neutralen diskursiven Raum treffen, und dieser statt dessen nach wie vor von Widerstand und Zurückweisung geprägt ist. Um so stärker bedarf es im Anschluss an die dargelegten Entwicklungen und der damit einhergehenden Reduzierungen einer verstärkten Integration der Kategorie ,Geschlecht" in die Arbeiten an systematischen Grundlagen und den Forschungsfragen der Erziehungs- und Bildungswissenschaften (vgl. Rendtorff 2005, S. 29). Heinz-Hermann Krüger stellt 2006 in seiner „Einführung in die Theorien und Methoden der Erziehungswissenschaft" fest, dass es in Zukunft darum gehen muss,

„[D]ie Perspektiven für eine erziehungswissenschaftliche Geschlechterforschung weiter zu präzisieren, die die aktuellen gesellschaftlichen Entwicklungen der Geschlechterhierarchisierungen und der gleichzeitig stattfindenden Prozesse der Entdifferenzierung von Geschlechtsrollen ebenso untersuchen wie die Frage, welchen Stellenwert soziale Interaktionsprozesse in pädagogischen Institutionen bei der sozialen Konstruktion von Geschlechtlichkeit haben" (vgl. Krüger 2006, S. 158).

Für die erziehungswissenschaftliche Frauen- und Geschlechterforschung gilt es sich zukünftig folgenden Herausforderungen zu stellen: Zum einen geht es darum, Fragestellungen und Forschungsstrategien zu entwickeln, die für die Komplexität von Geschlechterkonstruktionen sensibel sind, ohne dabei die Stabilität überdauernder Strukturen auf Seiten der Gesellschaft und der Subjekte zu verfehlen. Zum anderen steht die Frage im Raum, wie pädagogische Praxiskonzepte entwickelt und bewertet werden können und wie sich die theoretisch, empirisch und praktisch entwickelten Geschlechtersperspektiven in das Konzept einer kritischen, 
differenzreflexiven Pädagogik einfügen und für eine Praxis des demokratischen Umgangs mit Differenzen und Identitäten fruchtbar machen lassen (vgl. Dausien/Thon 2009, S. 346). Die Herausforderung besteht hier vor allem darin, dass sich die Relevanz für die Berücksichtigung der Kategorie ,Geschlecht" in den Erziehungs- und Bildungswissenschaften und in der pädagogischen Praxis nicht von einer herrschaftskritischen, politischen Analyse abkoppelt und sich auf einen pädagogisch individualisierten und eindimensionalen Bildungsbegriff reduziert.

In der pädagogischen Praxis lässt sich eine ähnliche Verschiebung der Thematisierung von ,Geschlecht' beobachten wie in der Forschung: Wurden zunächst Frauen und Mädchen, später auch Jungen und Männer, als Zielgruppe von Institutionen und Bildungspraxen betrachtet, die spezifische Konzepte und Methoden verlangen, so geht es in jüngerer Zeit um eine grundlegende Verankerung geschlechterreflektierender Pädagogik (vgl. Dausien/Thon 2009, S. 345). Die praktischen Arbeitsfelder bieten zudem zahlreiche Aspekte, die für eine verstärkte Thematisierung von ,Geschlecht' im erziehungswissenschaftlichen Studium sprechen. Für Patricia Yancey Martin ist die Arbeitswelt ein Ort, wo ,Geschlecht' permanent reproduziert wird:

„People practise gender in all work settings, including boardrooms, insurance offices, fast-food restaurants, law firms, restaurants, banks, construction sites, churches and factories" (Martin 2006, S. 275).

Diese Feststellung kann m. E. auch auf die pädagogische Praxis und Wissensproduktion übertragen werden. Für pädagogische Kontexte und Settings kommt noch hinzu, dass diese häufig implizit (aber auch explizit) von hierarchischen Strukturen und Abhängigkeitsverhältnissen geprägt sind (z. B. LehrerInnen-SchülerInnen-Verhältnis, Beratungssituationen, Soziale Arbeit, Jugendarbeit etc.). In Interaktionen werden Geschlechterstereotype fortwährend von allen Beteiligten unreflektiert reproduziert und manifestiert. Martin schreibt hierzu:

„Gender is extensively practised at work [...]. The practising of gender is fluid and collective, interpersonal and interactional [...], individual and collective. [...] [A]nd much of this dynamic is unreflexive. Unreflexive practising is thus consequential" (2006, S. 260, S. 268).

Dies beeinflusst Frauen/Mädchen wie auch Männer/Jungen oftmals in unerwarteter Art und Weise (vgl. ebd., S. 254). ProfessionalistInnen wie auch AdressatInnen pädagogischen Handelns sind davon betroffen. Zum 
Beispiel kann ein unreflektierter Umgang mit ,Geschlecht' in Berufs- und Studienberatung, Beurteilung von deviantem Verhalten (im schulischen Kontext), Angebotspalette in Jugendzentren oder Vereinsarbeit für die AdressatInnen weitläufige Folgen haben.

Die Thematisierung von ,Geschlecht' und Geschlechterverhältnissen hat das Ziel, von einer unreflektierten Geschlechterpraxis zu einem reflektierten Umgang mit ,Geschlecht` zu gelangen, also die Veränderbarkeit der tradierten und als natürlich gewerteten Geschlechterrollen und -hierarchien wie auch deren Anteil an gesellschaftlichen Strukturen ins Zentrum zu rücken und die eigene Verstricktheit zu reflektieren. Dies ist als Erweiterung einer geschlechterreflektierten Haltung und als Teil der erziehungswissenschaftlichen Professionalität zu verstehen, die eine systematische Wahrnehmung und Berücksichtigung unterschiedlicher Lebenssituationen und -bedingungen von Frauen und Männern betont (vgl. Hornberg et al. 2008). Auf diesen reflektierten und reflektierenden Umgang mit dem Thema, Geschlecht' wird im Kapitel 7.5 genauer verwiesen. Im folgenden Kapitel werden theoretische Konzepte zu Geschlechterkonstruktionen sowie -verständnissen und Ansätze dargestellt, welche darum bemüht sind, zur Sensibilisierung für die Konstruktion/Konstruktionsmechanismen von Geschlechterdifferenzen und die damit einhergehende Herstellung sozialer Ungleichheit beizutragen. Außerdem wird auf diejenigen eingegangen, welche in der theoretischen Debatte dominier(t)en. 


\section{3 ,Geschlecht' und Geschlechterverhältnisse im Spiegel theoretischer Diskurse}

Wie bereits deutlich wurde, steht im Zentrum dieser Arbeit die Kategorie ,Geschlecht'; ich frage jedoch nicht nach den unterschiedlichen „Spielarten der Konstruktion“ (Knorr-Centina 1989) von ,Geschlecht", sondern vielmehr nach der Thematisierung von ,Geschlecht'. Dennoch werde ich im folgenden Kapitel theoretische Konzepte zu Geschlechterkonstruktionen und -verständnissen und Ansätze darstellen, die in der theoretischen Diskussion um die Konstruktion der Geschlechterverhältnisse dominieren. Die Entwicklung der theoretischen Diskussion um ,Geschlecht" werde ich in groben Zügen nachzeichnen, um darzustellen, mit welcher Intensität die gesellschaftlichen Geschlechterverhältnisse ins Zentrum wissenschaftlicher Überlegungen, vor allem in der Frauen- und Geschlechterforschung, ${ }^{1}$ gerückt sind.

Hier findet über verschiedene theoretische Ansätze und Überlegungen, Begriffe und Konzepte eine Annäherung an den Forschungsgegenstand statt. Darüber hinaus dient dieser Teil mit Blick auf die Empirie zur Sensibilisierung für die Konstruktion und -smechanismen von Geschlechterdifferenzen und die damit einhergehende Herstellung sozialer Ungleichheit. Die Darstellung der theoretischen Überlegungen steht in der Bemühung aufzuzeigen, dass sich die theoretischen Diskussionen überwiegend auf die situative Herstellung der Differenz von Geschlechtern beschränken und damit soziale Strukturen, die einen Einfluss auf die Interaktion haben und die Stabilität der Konstruktionen und Existenzweisen von ,Geschlecht‘ erklären, nicht erfasst werden können. Zudem

1 Während die Bezeichnungen Frauen- und/oder Geschlechterforschung sowie Gender Studies eher auf den Gegenstandsbereich verweisen, auf den sich das Erkenntnisinteresse richtet, stellt das Adjektiv feministisch einen politischen Impetus dieser wissenschaftlichen Strömung in den Vordergrund. Feministische Theorie beschreibt keine Festlegung auf einen bestimmten Analyseansatz, jedoch das Festhalten an einer kritischen Perspektive in Bezug auf androzentrische Züge in den Wissenschaften und auf sozialen Disparitäten im Verhältnis der ,Geschlechter'. In diesem Sinne ist nicht jede wissenschaftliche Beschäftigung mit der Geschlechterthematik als feministisch zu bezeichnen, ebenso wenig sind aber nicht-feministische Analysen zur Geschlechterthematik zwangsläufig unkritisch. Ich verwende den Begriff feministisch im Weiteren als ein plurales Perspektivenspektrum kritischer Analysen von Geschlechterverhältnissen und deren Vermittlung mit anderen Formen von Ungleichheit und Differenz (vgl. Knapp 2008, S. 291f.). 
wird deutlich, dass sie sich lediglich auf die öffentliche bzw. definitorische Ebene der Wirklichkeitskonstruktion richtet. Es wird nur eine spezifische Form des Wissens erfasst, nämlich kommunikativ-generalisiertes Wissen. Unberücksichtigt bleibt das unmittelbar handlungsbezogene und handlungsleitende, d. h. das konjunktive Wissen (vgl. Bohnsack 2006, S. 280f.). Bei diesen Konzepten wird auf die Ebene sozialer Identität fokussiert und die Ebene des Habitus, der habituellen Praxis, bleibt unbeleuchtet. Es ist jedoch von Bedeutung, zwischen (Selbst-)Darstellungen, (Fremd-)Konstruktionen und Fremdzuschreibungen auf der einen Seite und der habitualisierten Handlungspraxis der Akteure auf der anderen Seite zu unterscheiden.

Zuerst wird auf das Zwei-Geschlechter-Modell eingegangen (vgl. Kapitel 3.1) und die moderne Gesellschaft mit ihren Geschlechterverhältnissen im Spannungsfeld ,rhetorischer Modernisierungsprozesse“ (Wetterer 2006) und struktureller Persistenz skizzieren (vgl. Kapitel 3.2). Danach wird die Entwicklung von ,Geschlecht' als Gegenstand wissenschaftlichen Interesses nachgezeichnet, ein Ein- und Überblick über theoretische Positionen gegeben und verschiedene Perspektiven dargestellt. $\mathrm{Zu}$ den wichtigsten Eckpfeilern gehören zum einen die Bedeutung geschlechtsspezifischer Sozialisation und zum anderen deren Kritik (vgl. Kapitel 3.3). Eine daraus entstandene wichtige Entwicklung ist die Unterscheidung des biologischen Geschlechts (sex) und des sozialen Geschlechts (gender) (vgl. Kapitel 3.4), das Verständnis von ,Geschlecht' als soziale Konstruktion (vgl. Kapitel 3.5.1) und deren Dekonstruktion (vgl. Kapitel 3.5.2). Danach wird die Intersektionalitätsdebatte schlaglichtartig dargestellt (vgl. Kapitel 3.6). Abschließend werden Überlegungen von Andrea Maihofer, ,Geschlecht' als gesellschaftlich-kulturelle Existenzweise zu sehen, dargestellt und ihre Forderung nach der Anerkennung von Geschlechterdifferenz und Vielfalt unter Berücksichtigung von Herrschafts- und Machtverhältnissen expliziert (vgl. Kapitel 3.7). Diese Ausführungen zu den komplexen Positionen und Denkansätzen bilden den Ausgangspunkt für die weiteren theoretischen Überlegungen.

\subsection{Das Zwei-Geschlechter-Modell}

In der bürgerlichen Gesellschaft Mitte des 18. Jahrhunderts entwickelte sich eine - zunächst nur für die eigene Klasse geltende - neue Arbeitsteilung zwischen den Geschlechtern (vgl. Roer 1994, S. 20f.): Es wurde zwischen der produktiven Männer- und der reproduktiven Frauenarbeit 
unterschieden. Diese Konstruktion wurde im 19. Jahrhundert ideologisch abgesichert, indem die weiblich-bürgerliche Lebensweise als in der Natur der Frau liegend definiert wurde. Mit der Biologisierung gesellschaftlicher Anforderungen an Frauen wurden auch die Ansprüche an Männer naturalisiert. Diese Geschlechterordnung wurde somit als eine natürliche bzw. naturgegebene konstruiert sowie inszeniert und die Differenz wurde/wird als eine qualitative zwischen ,weiblichen' und ,männlichen Geschlechtsmerkmalen behauptet (vgl. Maihofer 1994, S. 238).

Die sexuelle Differenz wie Vagina und Penis sind relativ junge gesellschaftlich-kulturelle Phänomene und entstanden erst mit der bürgerlichen Moderne. Das heißt, die Art und Weise, wie wir heute über unsere Körper als biologisch distinkte Tatsache reden und als solche wahrnehmen, ist erst im 18. Jahrhundert entstanden (vgl. Maihofer 1995, S. 238). Davor wurden die Geschlechter graduell unterschieden, so sind beim „Ein-Geschlecht-Modell“ (vgl. Laqueur 1992, S. 21 zit. n. Maihofer 1994, S. 238) die männlichen und weiblichen Geschlechtsmerkmale gleichförmig beschaffen vorgestellt, nur einmal nach außen und einmal nach innen gestülpt. Zwar fungiert auch hier die männliche Morphologie als Norm und die Vagina wird als innerer Penis imaginiert - und nicht umgekehrt - jedoch wird der Unterschied als graduell gesehen und nicht als qualitative Differenz. Erst im Zwei-Geschlechter-Modell wird die Differenzierung und die grundlegende Unvergleichbarkeit von Männern und Frauen behauptet. Die Differenz wird nun als eine qualitative zwischen weiblichen und männlichen Geschlechtsmerkmalen gesetzt (vgl. Maihofer 1994, S. 238). Die Vagina wird als ein völlig verschiedenes körperliches Merkmal angesehen, das ausschließlich dem ,weiblichen' ,Geschlecht' entspricht. Diese Veränderung in der äußerlichen Wahrnehmung des Körpers bzw. der Rede vom Körper hat sich mit der Art und Weise verbunden, wie der weibliche und männliche Körper erlebt und empfunden wird (vgl. Maihofer 1995, S. 92). Im Diskurs des Zwei-Geschlechter-Modells ist der Mann mit ,Mensch' identisch, während die Frau eine engere und abweichende Bestimmung erfährt. Die Frau ist in allen Aspekten ihres Seins, Denkens und Fühlens durch ihre körperliche Geschlechtlichkeit bestimmt, d. h. sie wird mit ihrem Körper gleichgesetzt. Damit entsteht die für uns heute so selbstverständliche Vorstellung von der fundamentalen biologisch-anatomischen Differenz der Geschlechter (vgl. Maihofer 1994, S. 238 und 1995, S. 92). Diese Entwicklung vollzog sich nicht in erster Linie aufgrund neuer naturwissenschaftlicher Erkenntnisse über den Körper, sondern ist im Zusammenhang mit der Etablierung der bürgerlichkapitalistischen Gesellschaft zu sehen (vgl. ebd.). Zweifellos wurden be- 
reits vor der bürgerlichen Moderne Frauen und Männer unterschieden. Diese Unterscheidung wurde ebenfalls an körperlichen Merkmalen fest gemacht, allerdings spielten die körperlichen Geschlechtsmerkmale keine derart dominante und eindeutig verifizierende Rolle. Zudem wurde der körperliche Unterschied zwischen den Geschlechtern als nicht so grundsätzlichd deterministisch angesehen. Erst für den hegemonialen bürgerlichen Geschlechtskörper wurde diese binäre, patriarchal-hierarchische Codierung derart zentral (vgl. ebd., S. 92). Die oben beschriebenen Veränderungen müssen, so Maihofer, als Teil der Etablierung der modernen kapitalistischen Gesellschaften und ihrem bürgerlich-patriarchalen Arrangement der Geschlechter gesehen werden. In einer Zeit, in der auf die Gleichheit aller Menschen insistiert wurde und mit dieser Idee die Emanzipation des Bürgertums eingefordert wurde, wurde gleichzeitig der Diskurs der Geschlechterdifferenz etabliert. Beide Diskurse laufen parallel und sind aufs engste historisch verknüpft. Mit dem Diskurs um die Geschlechterdifferenz wird die Gleichheit aller Menschen relativiert und der Ausschluss der Frauen aus den Menschen- und Bürgerrechten und ihr Einschluss in die familiäre Sphäre legitimiert (vgl. Maihofer 1994, S. 239f.). So schuf die Erklärung der allgemeinen Menschen- und Bürgerrechte eine neuartige Begründung für den Ausschluss der Frauen aus dem öffentlichen Leben. Indem die Frau als fundamental verschieden zum Mann dargestellt wurde, konnte sie von dem Anspruch der Gleichheit aller Menschen ausgenommen werden. Ab der Etablierung der bürgerlichen Gesellschaft im 18. Jahrhundert wurden die Unterschiede in binär-hierarchischen Oppositionen einander entgegengesetzt und der Mann wurde zum Maßstab und die Frau zum ,Anderen` (vgl. Maihofer 1995, S. 99). Der moderne Geschlechterdiskurs, wie er im Zuge der Etablierung der bürgerlichen Gesellschaft im 18. Jahrhundert entstanden ist, konstituiert jedoch nicht nur die gegenwärtig hegemoniale Art und Weise, ,Geschlecht' in der Distinktion von männlich und weiblich materiell körperlich zu leben, sondern konstituiert darüber hinaus überhaupt die hegemoniale Art und Weise, dass und wie wir gegenwärtig zu Frauen und Männern gemacht werden und als solche existieren (vgl. ebd., S. 98). Die Geschlechterdifferenz ist ein zentrales Element des modernen heterosexuellen Geschlechterdiskurses, daher erfahren marginalisierte Geschlechteridentitäten wie gegenderte Existenzen (etwa z. B. Intersexuelle oder Transgender-Personen), wenig bis keine Anerkennung. ${ }^{2}$

2 Insbesondere die Queer Studies beschäftigen sich mit (marginalisierten) Geschlechtsidentitäten. Ihr Ziel besteht darin, Normalitäten sowie daran geknüpfte Mechanismen und Prozesse gesellschaftlicher Normierungen und Ausschlusskrite- 


\subsection{Die moderne Gesellschaft und die Geschlechterverhältnisse}

Die eben skizzierten Veränderungen sind Teil der Etablierung der modernen kapitalistischen Gesellschaften und ihrem bürgerlich-patriarchalen Arrangement der Geschlechter. Die Frau erfährt nun eine engere und von der Norm abweichende Bestimmung, der Mann dagegen ist mit ,Mensch gleichgesetzt. In diesem Diskurs wird Frau mit ihrem Körper ineinsbzw. gleichgesetzt. Die Frau ist in allen Aspekten ihres Seins, Denkens und Fühlens durch ihre körperliche Geschlechtlichkeit bestimmt (vgl. Maihofer 1994, S. 239). Suggeriert wird eine klare Trennung von Natur (weiblich) und Kultur (männlich) (vgl. Wesely 2000, S. 31). Dem kritischen Hinterfragen, Aufbrechen, Umwandeln, Verändern etc. dieser beschriebenen, etablierten, heteronormen Geschlechterdualität haben sich feministische Frauenbewegungen und Wissenschaften verschrieben. Vor dem Hintergrund dieser politisch-feministischen Frauenbewegung(en) als ein kritisches Erkenntnisprojekt, haben sich auf wissenschaftlichakademischer Ebene die Frauen- und Geschlechterforschung bzw. die Gender Studies herausbildet. Diese gehen u. a. der Frage nach, welche Rolle das Geschlechterverhältnis und das ,Geschlecht' in verschiedenen gesellschaftlichen Diskursen spielen (vgl. Rendtorff/Moser 1999, S. 57) und wie ,Geschlecht' in den komplexen gesellschaftlichen Verhältnissen hergestellt, reproduziert und verändert werden kann. Bis heute haben feministische Bewegungen und Wissenschaften gesellschaftliche Veränderungen und Veränderungstendenzen in zahlreichen Lebensbereichen bewirkt, jedoch scheint die Entwicklung auf halber Strecke stecken geblieben zu sein (vgl. Klaus 2008, S. 181).

Unsere heutige Gesellschaft ist gekennzeichnet von Problemen und Herausforderungen, die aus den Brüchen und Umbrüchen der Geschlechterverhältnisse hervorgehen. Die modernen Geschlechterverhältnisse sind geprägt von Persistenz und Wandel. Auf der einen Seite verlieren die Dualismen konstruierter Zweigeschlechtlichkeit, d. h. die Charakterisierungen von ,Männlichkeit‘ und ,Weiblichkeit', durch Überlappungen

rien sichtbar zu machen und zu kritisieren. Sie verstehen sich als eine kritische und ,verunsichernde' Denkströmung, die sich gegen essenzialisierendes, festschreibendes und damit ausschließendes Denken richtet. Die Queerbewegung zeichnet aus, dass sie bewusst nicht im Namen einer Identität (,die Lesben', ,die Frauen`) agiert, sondern dass vielmehr die Norm selbst (Heteronormativität, Identität und Begriffskategorien) zum Ausgangspunkt der Kritik gemacht wird (vgl. Degele 2008, S. 40ff und Jagose 2001). 
und Grenzüberschreitungen tendenziell ihre Polarität (vgl. Bilden 1991, S. 299). Dies bietet Frauen und Männern (scheinbar) mehr Offenheit für die eigene Gestaltung ihrer Leben und mehr Möglichkeiten in individuellen Entscheidungen wie Lebensausrichtung, Selbst- und Beziehungskonzepten (vgl. Breitenbach/Hagemann-White 1994, S. 256f.). Auf der anderen Seite jedoch entstehen damit strukturell bedingte geschlechtsbezogene Ungleichheiten, welche sich in der schlechteren Stellung von Frauen in der Berufswelt, ihrem niedrigeren Einkommen oder ihrer - im Vergleich zu Männern - miserablen sozialen Absicherung im Alter ablesen lassen (vgl. Gerhard 2007, S. 99). Weiter hat die Mehrheit der Frauen heutzutage den Anspruch, Familie und Beruf zu vereinen, allerdings wird dies institutionell nicht abgestützt und somit müssen individuelle Lösungen von den Frauen selbst gesucht werden. Zwar lassen sich auch im Bereich der Kindererziehung und der Hausarbeit im Vergleich zu den letzten dreißig Jahren Veränderungen feststellen: Männer beteiligen sich heutzutage verstärkt in diesen Domänen. Trotzdem liegen die Verantwortung und Zuständigkeit für die familiäre Arbeit oftmals bei den Frauen, während der Terminus der guten Mutter mehr den je zum Diktat wurde (vgl. Bilden 1991, S. 297f.).

Die unstrittige These ist, dass gegenwärtig Familie immer weniger als eine selbstverständliche gesellschaftliche Konvention gelebt wird. Für Paare, die Kinder bekommen, bedeutet dies, dass sie individuell herausfinden, wie sie die Haus-, Erziehungs- und Erwerbsarbeit aufteilen und organisieren (vgl. Maihofer/König 2004, S. 228). In modernisierten Gesellschaften ergeben sich somit neue Widersprüche zwischen Ansprüchen und Realisierbarkeit, die neue Varianten von Unfreiheit und Ungleichheit (vgl. King 2004, S. 82) hervorbringen. Anders gesagt, bieten moderne Lebensbedingungen theoretisch viele Möglichkeiten hinsichtlich Berufswahl, PartnerInnenwahl, Konsum- und Freizeitverhalten sowie der Weltanschauung und Lebensstilführung. Daraus ergibt sich allerdings eine neue Dialektik von Entscheidungszwang und Gestaltungsfreiheit (vgl. Erster Gleichstellungsbericht der Deutschen Bundesregierung 2011, S. 80). Für die meisten Frauen und Männer in den Gesellschaften der westlichen Hemisphäre gilt heute Gleichberechtigung zwischen den Geschlechtern als umgesetzt bzw. zum Greifen nahe (vgl. Hark 2008, S. 218). Junge Frauen und Mädchen setzen die Beurteilung ihrer Chancen und Lebenspläne meist mit jenen von Männern gleich, auch wenn die Realität eine andere Sprache spricht: Die Analyse des „Ersten Gleichstellungsberichts der Deutschen Bundesregierung“" (2011) zeigt, dass der Weg zur Gleichstellung zwischen Frauen und Männern in 
Deutschland weit ist und heute noch keine echte Wahl- und Gestaltungsfreiheit herrscht. Institutionelle und rechtliche Rahmenbedingungen setzen starke Anreize in Richtung einer ungleichen Verteilung von Erwerbsund Sorgearbeit zwischen verheirateten wie unverheirateten Partnern. Auch wenn junge Paare häufig mit ähnlichen Vorstellungen von gleichberechtigter Aufteilung der Erwerbs- und Sorgearbeit in ihr gemeinsames Leben starten, stellt sich spätestens mit dem ersten Kind eine Retraditionalisierung der Geschlechterrollen ein. In der Folge stecken meist die Frauen beruflich zurück. Die Analyse des „Ersten Gleichstellungsberichts der Deutschen Bundesregierung“ (2011) zeigt, dass auch institutionelle Rahmenbedingungen die Eingrenzung biografischer Entwicklungen und der Gestaltungsfreiheit im Besonderen von Frauen forcieren (vgl. ebd., S. 4f., 80f.). Dennoch erleben sich Mädchen und Frauen im jüngeren Erwachsenenalter bzw. in Geschlechterbeziehungen weitgehend gleich und in ihrer Lebensführung demonstrieren sie Unabhängigkeit (vgl. Gerhard 2007, S. 99). Frauen wollen nicht mehr, dass ihre Probleme primär auf ihr Frausein zurückgeführt werden (vgl. Stauber 2004, S. 34). Geschlechtsbezogene Benachteiligungen existieren in der Wahrnehmung von Frauen und Männern kaum noch, beziehungsweise werden sie als Einzelfälle und nicht als Teil eines allgemeinen Musters gewertet (vgl. Hark 2008, S. 218). ${ }^{3}$ Doch die Vorstellung, dass alles bestens sei, werden persönliche, berufliche und familiäre Zufriedenheit an die Selbstverantwortung von Frauen delegiert. Die Botschaft lautet: Frau ist selbst schuld, wenn diese einen Karriereknick erleidet oder über die Doppelbelastung, Überforderung und Unzufriedenheit klagt (vgl. Klaus 2008, S. 181f.). Der scheinbare Zugewinn von Optionen individualisiert und privatisiert damit gesellschaftliche Zwänge sowie strukturelle Diskriminierungen und verhandelt diese subtil als Fragen individueller Anstrengung (vgl. Hark

3 Der moderne Gleichheitsdiskurs, so problematisiert auch Andrea Maihofer (2009), basiert auf der „Anerkennung gleicher Rechte, auf der An-/Erkennung als Gleiche. Wird auf eine Verschiedenheit insistiert oder wird diese ,nachgewiesen' [...] wird diese ,qualitative' Differenz zur Legitimation von Ausgrenzung und Diskriminierung" (ebd., S. 33). In Anlehnung an Theodor W. Adorno und Max Horkheimer (1971) kritisiert sie, dass mit dem Gleichheitsanspruch auch ein Zwang zu Konformität und Anpassung, ein Negieren von Verschiedenheiten verbunden sein kann. Wer die Differenzen, das Nicht-gleich-Sein oder die eigene Individualität betont, läuft Gefahr, ausgegrenzt zu werden. Maihofer plädiert nun nicht dafür, auf Gleichheit zu bestehen, sondern Verschiedenheiten anzuerkennen, Differenzen und Differenzlinien - wie die zwischen Geschlechtern - als Lebenswirklichkeit wahrzunehmen, ohne in einen Essenzialismus zu verfallen. Das bedeutet, sie einerseits als sozial hergestellte und möglicherweise durch hegemoniale Diskurse bedingte Differenzen und andererseits als konkret erfahrene Existenzweise zu akzeptieren (vgl. Maihofer 1995; Götsch/Klinger/Thiesen 2012, Abs. 38/39; Kapitel 3.7). 
2008, S. 218). Dies führt zu spannungs- und konfliktreichen individuellen wie gesellschaftlichen Ungleichzeitichkeiten. Andrea Maihofer beschreibt das individuelle Dilemma wie folgt: Da sich die veränderten Anforderungen erst allmählich als neuer kultureller Habitus und neue mentale und psychische Strukturen herauszubilden beginnen, eignen sich die Individuen nach wie vor alte Identitätsmuster an bzw. werden sie weiterhin in diesem Sinne sozialisiert. Gleichzeitig werden jedoch durch die gesellschaftlichen Veränderungen von den Individuen bereits neue Denk-, Handlungs- und Gefühlsweisen gelebt und/oder gefordert, ohne dass sie über diese bereits in ausreichendem Maße verfügen. Das hat starke Verunsicherungen, regressive Sehnsüchte, Defiziterfahrungen und nicht zuletzt den zwanghaften Wunsch zur Harmonisierung innerer und gesellschaftlicher Widersprüche zur Folge (vgl. Maihofer 2002, S. 21).

Angelika Wetterer verweist hier hingegen auf die Gleichzeitigkeit von Modernisierungsprozessen. Sie fokussiert weniger auf die individuelle Ebene wie Maihofer, sondern auf die strukturelle Ebene. Wetterer verweist auf den Wandel von Geschlechterverhältnissen und der Reformulierung von Geschlechterdifferenzierungen in ihrer strukturellen Persistenz. Diese Prozesse fasst Wetterer mit dem Begriff der rhetorischen Modernisierung zusammen und beschreibt dabei eine Modernisierung, „die sich im Diskurs und der Sprache, kaum jedoch in der Praxis zeigt" (Wetterer 2006, S. 12). Hinzu kommt, dass modernisierte Geschlechterverhältnisse zwar den Mythos erreichter Gleichstellung beinhalten, jedoch sind diese Geschlechterverhältnisse weiterhin von hierarchischen Strukturen durchzogen (vgl. Stauber 2004, S. 34). Maria Bitzan und das Autorinnenkollektiv des Tübinger Instituts für frauenpolitische Sozialforschung haben für diese Mechanismen die Analysefolie des Verdeckungszusammenhangs entworfen:

„Typische aus dem Geschlechterverhältnis resultierende Konflikte sind durch Erscheinungsformen der Moderne verdeckt - und gerade nicht gelöst. Sozialpolitische Definitions- und Umgangsweisen (denen auch Sozialpädagogik, Bildungs- und Soziale Arbeit zuzurechnen sind) tragen zur Verdeckung und Normalisierung bei" (Bitzan 2002, S. 30).

Die Verdeckung von gesellschaftlichen, geschlechtlich formierten Herrschaftsverhältnissen, die durch eine Dichotomisierung der Geschlechter und den damit verbundenen Widersprüchen gestützt werden, geschieht dadurch, dass sie vor dem Hintergrund von Gleichheitspostulaten als solche nicht mehr benannt werden können und damit als Individualbelastungen definiert und auch erlebt werden. Dieser Erklärungsversuch, 
der bei struktur- wie handlungstheoretischen Überlegungen ansetzt, wird später nochmals aufgegriffen (vgl. Kapitel 4.3.2).

Die Debatte um den Gegenstand ,Geschlecht' ist in ihrer disziplinären und inter- bzw. transdisziplinären Ausdifferenzierung kaum mehr überschaubar. Die wissenschaftlichen Suchbewegungen zur Bestimmung des Gegenstands ,Geschlecht' greifen auf bestehende Theorierichtungen zurück, verknüpfen diese neu, verwerfen etablierte Positionen und/oder führen diese weiter bzw. entwickeln neue Denkansätze. Es gibt kein einheitliches Paradigma, doch vielzählige verschiedene forschungstheoretische Ausrichtungen. Daher hat sich die Begriffsbestimmung von ,Geschlecht' als ein kaum entwirrbarer Komplex an Positionen und Denkansätzen entwickelt. Aus verschiedenen Blickwinkeln passiert eine Annäherung an einen Gegenstand, der nicht einheitlich gefasst, sondern in der Spannung zwischen den Polen von Geschlechtergleichheit und differenz bis hin zur Aufhebung der Kategorie ,Geschlecht' diskutiert wird (vgl. Glaser/Andresen 2009, S. 7). Deshalb werden im Folgenden wichtige Meilensteine dieser Entwicklung dargestellt. Dabei handelt es sich um eine Auswahl aus der Vielfalt und Breite der Diskussion. Zu den wichtigsten Eckpfeilern gehört die Bedeutung geschlechtsbezogener Sozialisation und deren Kritik.

Ein weiterer wichtiger Abschnitt bei der Suche nach der Bestimmung des Begriffes Geschlecht war die Unterscheidung des biologischen Geschlechts (sex) und des sozialen Geschlechts (gender). Ebenso wird auf das Verständnis von ,Geschlecht' als soziale Konstruktion und die Dekonstruktion der Geschlechterordnung eingegangen. Danach werden Überlegungen von Andrea Maihofer, ,Geschlecht' als gesellschaftlichkulturelle Existenzweise zu sehen, und ihre Forderung nach der Anerkennung von Geschlechterdifferenz und Vielfalt unter Berücksichtigung von Herrschafts- und Machtverhältnissen dargestellt. Abschließend wird als jüngstes Beispiel für die produktiven Auswirkungen der Diskussion über Ungleichheit und Differenzen unter Frauen schlaglichtartig auf die Intersektionalitätsdebatte eingegangen. Diese Ausführungen zu den komplexen Positionen und Denkansätzen bilden den Ausgangspunkt für die weiteren theoretischen Überlegungen. Meine Kritik zu theoretischen Diskussionen ist, dass diese überwiegend auf die situative Herstellung der Differenz von ,Geschlecht' beschränkt sind und damit soziale Strukturen, welche einen Einfluss auf die Interaktion haben und die Stabilität der Konstruktionen und Existenzweise erklären, nicht erfasst werden können. Zudem bleibt die (Be-)Deutungszuschreibung in den Relevanzsystemen der einzelnen Individuen außen vor. Es wird oftmals die Ebene 
sozialer Identität fokussiert und die Ebene des Habitus, der habituellen Praxis bleibt unbeleuchtet.

\section{3 ,Geschlecht' aus sozialisationstheoretischer Perspektive}

Auch wenn die Frage der Geschlechterdifferenz innerhalb der Frauenund Geschlechterforschung divers diskutiert wird und von einer gemeinsamen Problemstellung, Methode und Schlussfolgerung nicht die Rede sein kann, geht doch ein breiter Strang des feministischen Denkens und der institutionalisierten Frauen- und Geschlechterforschung von dem Beauvoir'schen Leitmotiv aus: „Man wird nicht als Frau geboren, man wird es" (Beauvoir 2003, S. 334). Dieser Beauvoir'sche Grundgedanke richtet die Aufmerksamkeit auf soziale Aspekte des Geschlechts - die alltagsweltliche Annahme, dass ,Geschlecht' objektiv, faktisch und unveränderlich gegeben ist, wird damit herausgefordert (vgl. Villa 2008, S. 201). Das war auch das Ziel der sozialisationstheoretischen Überlegungen. Zu Beginn der Frauenforschung im deutschsprachigen Raum in den 1970er Jahren bildet der Sozialisationsbegriff den politisch-programmatischen Rahmen, um Frauenfragen kritisch zu diskutieren. Das Sozialisationsparadigma lieferte eine wissenschaftliche Begründung für die Verbindung des Subjekts mit den gesellschaftlichen Verhältnissen. Wissenschaftlich und politisch ging es darum, zu zeigen, dass ,Geschlecht' kein biologisches Schicksal, sondern eine gesellschaftlich-kulturelle Zuschreibung ist. In den 1980er Jahren folgte eine empirische und theoretische Differenzierung des Ansatzes, welche in das Konzept der geschlechtsbezogenen Sozialisation mündete (vgl. Dausien 2006, S. 22f.).

In den 1990er Jahren begann auch die kritische Männerforschung damit, die geschlechtsbezogene Sozialisation des Jungen und die Konstruktion des Mannes herauszuarbeiten. David Gilmore (1993) vertritt in seinem Buch „Mythos Mann: Wie Männer gemacht werden“ die äquivalente These: „Männer werden gemacht, nicht geboren“ (Gilmore 1993, S. 118). Demnach bildet jede Gesellschaft spezifische Männlichkeitsideologien aus und junge Männer werden zur Übernahme der geforderten Elemente animiert oder durch Verachtung und soziale Ausgrenzung dazu gezwungen sowie durch rituelle Praxen darauf vorbereitet (vgl. Friebertshäuser 2012, S. 99). Mit ihrer Studie „Der gemachte Mann“ lenkt Raewyn Connell (vormals Robert W. Connell) (1999) den Blick auf die Hegemonie eines spezifischen, heterosexuell geprägten Männlichkeitsmo- 
dells. Sie betont, dass dieses vom Machismo dominierte Männlichkeitsmodell andere Männlichkeiten, insbesondere homosexuelle Männer, diskriminiere. Die Entwicklung bzw. die Sozialisation eines Individuums wird durch Eltern und Familie, Kindergarten, Schule, Peers und Medien etc. geprägt. Mädchen und Jungen werden unterschiedlich behandelt und es werden jeweils andere Verhaltensweisen von ihnen erwartet (vgl. Klees/Marburger/Schumacher 2004, S. 19f.). Trotz oberflächlich egalitärer Einstellung machen Mädchen und Jungen auch heute noch unterschiedliche Erfahrungen und lernen differenzierte Sichtweisen kennen, die ihr späteres Leben und Verhalten als Frauen und Männer prägen und mitbestimmen (vgl. Bilden 1991, S. 281f.). Bereits Säuglingen gegenüber gibt es Erwartungen und Verhaltensinterpretationen, die mit dem angenommenen ,Geschlecht' der Kinder in Zusammenhang stehen. Verschiedene „Baby-X-Studien“ haben gezeigt, dass Erwachsene mit einem unbekannten Säugling, der einmal als Mädchen, ein anderes Mal als Junge vorgestellt wurde, unterschiedlich interagieren. Mädchen und Jungen werden in ein patriarchales System hineingeboren und das ,Geschlecht' einer Person löst - bewusst oder unbewusst - bestimmte Erwartungen, Deutungsmuster, Reaktionstendenzen usw. aus (vgl. Bilden

1991, S. 281f.). Herrschende Verhaltensmuster, typisierte Einstellungen und unterschiedliche geschlechtsbezogene Vorgaben für die Entwicklung von Mädchen und Burschen verfestigen bestehende Ungleichheiten. Dabei bleibt viel Potenzial verborgen und die Entwicklung ,gegengeschlechtlicher Anteile" (Klees/Marburger/Schumacher 2004, S. 19f.) wird behindert. Geschlechtsbezogene Sozialisation differenziert zwischen ,weiblich' und ,männlich' mit dem Ziel, die Polarisierung der Geschlechter abzubauen. Dies führt(e) zur Kritik, die unterschiedliche Bewertung der Geschlechter würde intensiviert und reproduziert, anstatt dem Anspruch, sie aufzulösen, Folge zu leisten (vgl. Breitenbach/HagemannWhite 1994, S. 256).

\subsubsection{Kritik an der geschlechtsbezogenen Sozialisation}

Ende der 1980er Jahre begann die Distanzierung der kritisch-feministischen Frauenforschung vom Sozialisationsparadigma. Zur Kritik führte der Eindruck, dass sich Phänomene wie die geschlechtsbezogene Segregation des Arbeitsmarktes mit sozialisations- bzw. subjekttheoretischen Ansätzen nicht ausreichend erklären lassen. Kritisiert wurde vor allem die These vom „weiblichen Arbeitsvermögen“ von Elisabeth Beck-Gernsheim und Ilona Ostner. Hiernach besitzen Frauen aufgrund ihrer Sozialisation 
ein spezifisch weibliches Arbeitsvermögen und dies würde dazu führen, dass Frauen eher typisch weibliche Berufe wählen bzw. innerhalb der Berufe eher nach weiblichen Mustern agieren (vgl. Maihofer 2002, S. 13). Diese These, dass Frauen aufgrund ihrer Sozialisation ein spezifisch weibliches Arbeitsvermögen haben, wurde kritisiert, da sie den widersprüchlichen sozialisatorischen Anforderungen an Frauen aufgrund ihrer ,doppelten Vergesellschaftung" (vgl. Becker-Schmidt 2003, S. 57) in Erwerbsund Familienarbeit nicht gerecht wird. Zudem kritisiert Gudrun-Axeli Knapp, dass ,Geschlecht' vor allem über Eigenschaften und ein bedingtes Verhalten bestimmt wird. Diese Vereigenschaftlichung führe aber zu einer Verfestigung traditioneller Geschlechterstereotypisierungen und dazu, die Frauen selbst für ihr Verhalten, ihre geschlechtsbezogenen Eigenschaften und für ihre Diskriminierung auf dem Arbeitsmarkt verantwortlich zu machen. Knapp zufolge sollte deshalb ,Geschlecht' nicht als ein biologisches Phänomen oder als Bündel typischer Eigenschaften verstanden werden, sondern als ein positionelles Phänomen, das etwas über die spezifische Verortung von Frauen und Männern im Kontext eines hierarchischen Systems aussagt. Sie sieht ,Geschlecht" vor allem in seiner „Platzanweiserfunktion“ und als ,soziale Strukturkategorie“ (Knapp 1987, S. 292 zit. n. Maihofer 2002, S. 14). Knapp plädiert zwar dafür, die sozialisationstheoretische Erklärungsperspektive nicht aufzugeben, auch wenn diese grundlegend neu überdacht und das Schwergewicht zugunsten einer strukturtheoretischen Perspektive verschoben werden sollte (vgl. Maihofer 2002, S. 14).

Eine andere prominente Kritikerin der geschlechtsbezogenen Sozialisation ist Angelika Wetterer. Sie tritt für eine völlige „theoretische Umorientierung" (Wetterer 1993, S. 9 zit. n. Maihofer 2002, S. 14) ein. Für sie tritt die Frage nach der Geschlechterdifferenz als mögliche Ursache der Marginalität von Frauen in hochqualifizierten Berufen mehr und mehr in den Hintergrund. Hingegen gewinnt, für sie die Analyse des Geschlechterverhältnisses als eine hierarchische Organisationsform sozialer Beziehungen an Bedeutung. Damit kommt es zu einer theoretischen und empirischen Schwerpunktverschiebung. ,Geschlecht" wird als Statuskategorie, als Allokationsmechanismus oder als Platzanweiser bei der Reproduktion von Hierarchien in den Geschlechterverhältnissen verstanden. Das heißt, an die Stelle subjekttheoretisch orientierter Ansätze treten gesellschafts- und strukturtheoretisch orientierte Konzepte. Somit sei die Diskriminierung von Frauen im Arbeitsbereich wird jetzt ausdrücklich nicht mehr sozialisationstheoretisch erklärt, sondern machttheoretisch. Die Diskriminierung von Frauen am Arbeitsmarkt Ausdruck 
und Reproduktion patriarchaler Machtverhältnisse. Diese machttheoretische Perspektive schließst für Angelika Wetterer sozialisationstheoretische Ansätze aus (vgl. Maihofer 2002, S. 15). Die soziale Konstruktion von Geschlechterdifferenzen verstelle den Blick, dass es sich z. B. bei der beruflichen Diskriminierung von Frauen, letztlich um nichts anderes handle, als um die Reproduktion patriarchaler Macht- bzw. Geschlechterverhältnisse. In ihrem Aufsatz „Geschlecht und Sozialisation“ konstatiert Andrea Maihofer, dass diese These zwar nachvollziehbar und überzeugend sei, Maihofer problematisiert jedoch deren Verabsolutierung. Denn damit gehe die Annahme einher, dass es in Wirklichkeit keine Geschlechterdifferenzen gebe, jedenfalls keine, die für die Forschung relevant wären. Zudem lege diese These nahe, dass jede Theorie, die sich auf Geschlechtsunterschiede beziehe, unvermeidlich an der Konstruktion von Geschlechterdifferenzen und damit an der Reproduktion der bestehenden patriarchalen Geschlechterverhältnisse beteiligt sei. Maihofer meint zwar, dass diese Gefahr zweifelsohne bestehe und dass es notwendig sei, diesen Effekt ständig kritisch zu reflektieren. Allerdings sei es unproduktiv, wenn ein ständiger Verweis auf diese Gefahr jede weitere theoretische und empirische Thematisierung von Geschlechterdifferenzen für untauglich erklärt (ebd.). Zudem reduziere Wetterer, aus Maihofers Perspektive, das Handeln von Individuen auf einen bloßen Vollzug einer Interaktionslogik. Sie blende die subjekttheoretischen Perspektive zugunsten einer machttheoretischen Perspektive aus den eigenen theoretischen und empirischen Überlegungen aus, statt diese miteinander $\mathrm{zu}$ verbinden (vgl. ebd., S. 17).

Neben dieser Kritik des Sozialisationsparadigmas und der damit verbundenen subjekttheoretischen Perspektive hat sich auch innerhalb der geschlechtsbezogenen Sozialisationstheorie eine ähnliche Kritik entwickelt. So problematisiert bereits Anfang der 1980er Jahre Carol Hagemann-White den Versuch, biologistische Erklärungen geschlechtlicher Unterschiede durch deren sozialisatorische Bedingtheit zu entkräften. Hagemann-Whites zentraler Kritikpunkt ist, dass dabei noch immer davon ausgegangen werde, Frauen und Männer hätten bestimmte geschlechtsbezogene Eigenschaften und darin begründete Verhaltensweisen. Auch wenn diese nun sozialisatorisch und nicht mehr biologisch erklärt werden, bleibe, so Hagemann-White, der Anschein der Natürlichkeit der Geschlechterverhältnisse bestehen und genau darin sieht sie den entscheidenden Fehler der geschlechtsbezogenen Sozialisationstheorien. Die weitere Abkehr von geschlechtsbezogenen Sozialisationstheorien wurde verstärkt durch den Artikel von Helga Bilden im „Neuen Hand- 
buch der Sozialisationsforschung“ von 1991. Bildens Kritik richtet sich zunächst ganz generell gegen die unterstellte Trennung zwischen Individuum und Gesellschaft und die damit verbundene Neigung, die Individuen vor allem als passive Objekte von Sozialisationsprozessen zu verstehen. Zudem wendet sie sich gegen das Konzept der stabilen Persönlichkeit und des mit sich identischen Individuums. Dieses kenne, jedenfalls bei gelungener Sozialisation, keine schwerwiegenden inneren Widersprüche, Spannungen oder Brüche (vgl. Bilden 1991, S. 270ff).

Ein weiterer Problempunkt ist der Begriff der Aneignung. Bilden hält diesen für zu kognitivistisch und kritisiert, dass er immer etwas fest Umrissenes voraussetze, das es anzueignen gelte und die Aneignung, so Bilden, wenn sie je angemessen wäre, angesichts des aktuellen gesellschaftlichen Wandels und der damit einhergehenden Veränderungen im Geschlechterverhältnis wenig Plausibilität hätte. Zentrum der Kritik ist die These, dass eine geschlechtsbezogene sozialisationstheoretische Perspektive zwangsläufig auf die Konstruktion eines, weiblichen' und ,männlichen' Sozialcharakters hinausläuft (vgl. ebd.). So lebt das Paradigma der geschlechtsbezogenen Sozialisation Bilden zufolge davon, Geschlechtsunterschiede als individuelle Persönlichkeitsunterschiede zu verdinglichen (vgl. Maihofer 2002, S. 15). Nach Maihofer gerät mit dieser Kritik jedoch ein zentraler, äußerst vielschichtiger und umfangreicher Bereich (fast) völlig aus dem Blick. Die wichtige Frage würde entweder gar nicht mehr oder nur mehr in sehr reduzierter Form gestellt: Wie werden in diesen konkreten Gesellschaftsverhältnissen unter Bedingungen eines hegemonialen Diskurses qualitativer, heterosexueller Geschlechterdifferenz aus kleinen Wesen erwachsene Frauen oder Männer und wie modifiziert sich dies im Laufe des Lebens (ebd.). Von Bettina Dausien wird die Entwicklung der Kritik an der geschlechtsbezogenen Sozialisation mit folgenden drei Punkten zusammengefasst:

- das Problem der Re-Naturalisierung und Reifizierung auf methodologischer Ebene

- die theoretische Konstruktion und Überfokussierung der Geschlechterdifferenz

- die Annahme einer eindeutigen, stabilen geschlechtlichen Identität

Aus der Kritik folgte ein Paradigmenwechsel, weg von der geschlechtsbezogenen Sozialisation hin zu den bis heute rezipierten konstruktivistischen und dekonstruktivistischen Ansätzen. Beide Richtungen versuchen auf unterschiedliche Weise auf die Vorstellungen des Sozialisations- 
paradigmas korrigierend einzuwirken (vgl. Dausien 2006, S. 25). ${ }^{4}$ Ein wichtiger Schritt in diese Richtung war die Unterscheidung der Kategorie ,Geschlecht' in biologisches Geschlecht (sex) und soziales Geschlecht (gender).

\subsection{Die Trennung von sex und gender}

Die begriffliche Unterscheidung zwischen sex und gender hat seinen Ursprung in der Transsexualitätsforschung der 1950er Jahre und wurde von der Frauen- und Geschlechterforschung aus strategischen Gründen übernommen (vgl. Knapp 2003, S. 69f.). ${ }^{5}$ Die Unterscheidung zwischen sex und gender kritisiert die Vorstellung vom ,natürlichen' Wesen von Frauen und Männern (vgl. Wesely 2000, S. 16) mit dem Ziel, die Unterschiede zwischen den Geschlechtern als Ergebnis von Geschichte aufzuzeigen und nicht als Effekt ,natürlicher' Unterschiede zu erklären (vgl. Gildemeister/Wetterer 1992, S. 205). Es besteht kein kausaler Zusammenhang zwischen biologischem und sozialem Geschlecht, daher müssen Geschlechterrollen als historisch-gesellschaftliches Produkt begriffen werden (vgl. Maihofer 1994, S. 237).

Der Begriff gender wurde aufgenommen, um die soziale Konstruktion der Geschlechterunterschiede erkennbar zu machen (vgl. Wesely 2000, S. 15). Mit gender wird der sozial oder kulturell geprägte Geschlechtscharakter benannt, welcher im Verlauf von Sozialisationsprozessen erworben wird und mit geschlechtsbezogenen Merkmalen korrespondiert (vgl. Gildemeister/Wetterer 1992, S. 205). Gender bezeichnet somit die Persönlichkeit und das Verhalten und bezieht sich auf kulturelle und sozialisierte Geschlechtsmerkmale. Es handelt sich um ein soziales Attribut, eine Strukturkategorie, welche vom historischen und kulturellen

4 Heute werden Sozialisation wie auch ,Geschlecht' nicht mehr als unausweichlich geprägt, sondern jeweils flexibel, interaktiv und prozesshaft gedacht (vgl. Götsch 2012, S. 12). Im Anschluss an die Geschlechter- und die Sozialisationsforschung führt z. B. Monika Götsch (2012) geschlechtlich-sexuelle bzw. heteronormative Sozialisation als soziale Praxis an.

5 In den 1950er Jahren verwendeten die Sexualwissenschafter John Money, Joan und John Hampson die begriffliche Unterscheidung von sex und gender. Der Psychoanalytiker Robert Stoller hat in seiner Studie ,Sex and Gender: On the Development of Masculinity and Femininity“ (1968) diesen Begriff weiter ausgeführt. Er hat darauf hingewiesen, dass das Verhältnis zwischen psychologischen Geschlechtsmerkmalen und den gängigen Weiblichkeitskonzepten sehr viel komplexer sei als bislang angenommen (vgl. Hof 2005, S. 12). Die Anthropologin Gayl Rubin (1975) machte ebenfalls auf die Existenz eines sex-gender-Systems aufmerksam (vgl. Wesely 2000, S. 16). 
Kontext abhängig ist (vgl. Wesely 2000, S. 31). ,Geschlecht' ist demnach „keine anthropologische Konstante, keine Essenz oder Wesenheit [...], die dem Menschen als Menschen qua Natur unveränderlich zukommt" (Maihofer 2004, S. 34).

Sex hingegen beschreibt die biologische und physiologische Klassifikation von Frauen und Männern. Diese Kriterien können die Genitalien bei der Geburt sein, Anatomie, Hormone, oder auch die Chromosomen (vgl. Gildemeister/Wetterer 1992, S. 205). Trotz dieser scheinbar, biologisch begründeten Unterschiede zwischen Männern und Frauen gilt es zu bedenken, dass deren Zuverlässigkeit nicht ohne Zweifel ist (vgl. Wesely 2000, S. 17) und dass die scheinbar klare Unterscheidung und Trennung zwischen ,männlich' und ,weiblich' selbst aus biologischer Perspektive nicht möglich ist. Helga Bilden schreibt dazu:

\begin{abstract}
„Die verschiedenen biologischen Kriterien für Geschlecht - anatomische, hormonale, gonadale, chromosomale - können nicht zu jedem Zeitpunkt ,weiblich' und ,männlich' klar voneinander unterscheiden und stimmen manchmal nicht mit einander überein. So gibt es zum Beispiel Personen mit weiblichen äußeren Genitalien und XY-Chromosomen" (Bilden 2001, S. 139).
\end{abstract}

Die herrschende Einordnung in nur zwei Geschlechter konnte somit auch aus biologischer Sichtweise in Frage gestellt werden. Die Kritik an Konzepten, die ,Geschlecht' als Bündel relativ statischer Identitäten, Eigenschaften und Verhaltensweisen definieren, führte zur Entwicklung (sozial-)konstruktivistischer Ansätze, die ,Geschlecht' und Geschlechterverhältnisse als Produkt andauernder sozialer Konstruktionsprozesse verstehen. Innerhalb dieses Paradigmas fokussiert das Konzept des doing gender auf soziale und individuelle Prozesse und Interaktionen. Das ,Geschlecht' wird dabei als etwas gedacht, das einem ständigen Reproduktionsprozess unterworfen ist. Das prozesshafte und dynamische eines solchen doing gender kann dabei auch ein reflektiertes undoing gender beinhalten (vgl. Nestvogel 2004, S. 157f.).

\title{
3.5 Konstruktion und Dekonstruktion von "Geschlecht‘
}

Seit Ende der 1980er, Anfang 1990er Jahre spielen konstruktivistische Ansätze in der angloamerikanischen und deutschsprachigen Debatte eine bedeutende Rolle. Mitunter ist sogar von einer konstruktivistischen 
Wende in den Sozialwissenschaften die Rede ${ }^{6}$ (vgl. Kubisch 2008, S. 17). Grob kann zwischen ,gemäßigten' und ,radikalen' Positionen des Konstruktivismus unterschieden werden, wobei erstere von einer empirisch erfahrbaren Außenwelt ausgehen, und letztere die Außenwelt als prinzipiell unzugänglich und das menschliche Gehirn als informationell geschlossen ansehen. So setzt beispielsweise die Ethnomethodologie bei der Frage an, wie das von Akteurinnen und Akteuren als objektive soziale Tatsachen Wahrgenomme, von ihnen selbst in ihren Handlungen produziert wird. Demgegenüber macht der kognitionstheoretische Konstruktivismus die Konstruktion der Wirklichkeit im Bewusstsein des Einzelnen zum Ausgangspunkt seiner Überlegungen (vgl. Kubisch 2008, S. 17). Gemeinsam ist den verschiedenen ,Spielarten des Konstruktivismus" (Knorr-Cetina 1989), dass sie die Naturgegebenheit und Objektivität von Kategorien wie ,Geschlecht' und Ethnizität in Frage stellen und statt dessen ihre soziale Konstruktion in den Mittelpunkt der Aufmerksamkeit rücken. In der Frauen- und Geschlechterforschung haben konstruktivistische Perspektiven einen ,gewissen Grad der Veralltäglichung erreicht" (vgl. Pühl et al. 2004, S. 12). Hier dominieren zwei Ansätze die Diskussion zur Konstruktion: Auf der einen Seite wird Konstruktion vor allem in den Sozialwissenschaften mit dem ethnomethodologisch orientierten Ansatz des doing gender von Candance West und Don Zimmermann (1987) in Verbindung gebracht (vgl. ebd., S. 12ff). Hier stehen die situativen, alltäglichen Konstruktionsprozesse, die ,Geschlecht' in der Interaktion hervorbringen, im Zentrum, wobei Mechanismen der Darstellung und der Zuschreibung im Fokus der Analyse stehen (vgl. Nestvogel 2004, S. 157f.). Auf der anderen Seite wird der dekonstruktivistisch-diskurstheoretische Ansatz Judith Butlers und die Aufösung der sex/gender-Dichotomie ${ }^{7}$ diskutiert. Im Fokus steht die Frage, wie Diskurse gender gestalten und hervorbringen.

6 Zum konstruktivistischen Paradigma zählen ethnomethodologische, wissenssoziologische oder diskurstheoretische und kognitionstheoretische Ansätze. Zudem wird auch die Kultursoziologie Bourdieus als konstruktivistisch bezeichnet. Schließlich interessiert sie sich für den Zusammenhang von Handeln und Strukturen sozialer Ungleichheit und erschafft mit dem Konzept des Habitus eine vermittelnde Instanz, mittels derer einerseits soziale Strukturen internalisiert und anderseits (re-)produziert werden (vgl. Knorr-Centina 1989; Gildemeister 2001; Kubisch 2004).

7 Vor allem im Anschluss an Butler (1990) wird inzwischen problematisiert, dass mit dieser Zweiteilung in den Begrifflichkeiten die Annahme einer natürlichen Grundlage der sozialen Konstruktion von ,Geschlecht' aufrechterhalten wird. Im Rahmen meiner Arbeit verwende ich in erster Linie den Begriff Geschlecht, der in einem nicht-essentialistischen Sinne zu verstehen ist. 
Die heterosexuelle Norm spielt hier eine wichtige Rolle. Auch wenn der (De-)Konstruktivismus die Unterscheidung der Kategorie ,Geschlecht" in biologisches Geschlecht sex und soziales Geschlecht gender kritisiert, war diese doch ein wichtiger Schritt, um zu zeigen, dass kein kausaler Zusammenhang zwischen biologischem und sozialem Geschlecht besteht. Geschlechterrollen müssen als historisch-gesellschaftliches Produkt begriffen werden (vgl. Maihofer 1994, S. 237) und nicht als Effekt ,natürlicher' Unterschiede (vgl. Gildemeister/Wetterer 1992, S. 205). Schließlich hat aber die Kritik an der sex/gender-Differenz die Entwicklung (sozial-)konstruktivistischer Ansätze inspiriert, welche ,Geschlecht' und Geschlechterverhältnisse als Produkt andauernder sozialer Konstruktionsprozesse versteht (vgl. Nestvogel 2004, S. 157f.).

\subsection{1 ,Geschlecht“ als soziale Konstruktion}

Die Überlegungen zu ,Geschlecht' als soziale Konstruktion basieren auf Forschungen zur Transsexualität. ${ }^{8}$ Die Perspektive der Transsexualität zeigt eine produktive Verfremdung des vormals Selbstverständlichen. Zudem werden die Nichtübereinstimmungen zwischen vergeschlechtlichten Körpereigenschaften und Geschlechtsidentität verdeutlicht. Dabei wird fragwürdig, ob körperliche Geschlechtlichkeit als ,natürliche' Basis des ,Geschlecht' gelten kann. Es zeigt, dass auch der Geschlechtskörper ein gesellschaftlich-kulturelles Phänomen ist und dass für die Geschlechtsidentität letztendlich das Gefühl bzw. die Gewissheit entscheidend ist, eine Frau oder ein Mann zu sein, auch wenn dies mit den vergeschlechtlichten Körpereigenschaften nicht übereinstimmt. Die Übereinstimmung von Geschlechtskörper und -identität und die Normalität dieser Herausbildung und Übereinstimmung erweist sich als hegemonialer Effekt des modernen bürgerlichen heterosexuellen Geschlechterdiskurses und als Zwang zur Identität (vgl. Maihofer 1994, S. 241). Dass Individuen ein ,Geschlecht' haben, und welches ,Geschlecht' sie haben, ist also nicht Ergebnis ,natürlicher', biologisch-anatomischer Gegebenheit, son-

8 Wie voraussetzungsvoll Frausein wirklich ist, wird durch die Transsexuellenforschung besonders deutlich. Durch die Studie „Agnes“ von Harold Garfinkel (1967) wird die Konstruiertheit individueller Geschlechtszugehörigkeit besonders sichtbar. Agnes muss jeden Tag aufs Neue die Aufgabe bestehen, Frau zu sein. Sie muss die verhaltens-, handlungs- und erlebnismäßige Kategorisierung immer wieder absolvieren, um als Frau akzeptiert zu werden (vgl. Micus-Loos 2004, S. 117). Als Frau muss Agnes ihr doing gender neu lernen; dadurch wird deutlich, dass jede einzelne Person für gender-Tun mitverantwortlich ist und bewusst mitbestimmen kann, obwohl doing gender gleichzeitig unvermeidbar ist und automatisch passiert. 
dern kann als Effekt interaktiver Leistungen angesehen werden. Dabei handelt es sich um Interaktionen, in denen einem Individuum Bedeutungen zugeschrieben werden und in denen ein Individuum sich anderen gegenüber als bestimmtes ,Geschlecht', Mann oder Frau, darstellt. Diese Zuschreibung von ,Geschlecht' setzt Alltagswissen voraus, demzufolge ein Individuum entweder eine Frau oder ein Mann sein muss. Diese Erwartungen und dieses Wissen führen dazu, dass jede Person unmittelbar als Frau oder Mann wahrgenommen wird (vgl. Maihofer 1994, S. 246). Das Verstehen sozialer Wirklichkeit basiert also nicht auf der Analyse des Individuums, sondern auf der kritischen Auseinandersetzung mit den Interaktionsprozessen (vgl. Dausien 1998, S. 260f.).

Dieser Ansatz des doing gender soll eine Möglichkeit bieten, ,Geschlecht' als soziale Konstruktion zu verstehen (vgl. Faulstich-Wieland 2004, S. 176). Candace West und Don Zimmerman (1987) gehen davon aus, dass Frauen und Männer das Tun (doing) von gender übernehmen, um sich als Teil der Gesellschaft zu identifizieren (vgl. Wesely 2000, S. 38). Doing gender umfasst individuelle Handlungssysteme und soziale Einrichtungen, die von Verhältnisbestimmungen strukturiert werden. Es beschreibt die wiederkehrende, routinierte und methodische Reproduktion von gender. Mit der Vorstellung, dass ,Geschlecht' bzw. Geschlechtszugehörigkeit ein Effekt vielschichtiger sozialer Prozesse ist und dass ,Geschlecht' lediglich gedacht wird, als etwas das man tut und nicht als etwas das man ist, nehmen VertreterInnen des doing gender-Konzepts eine grundlegend anti-essenzialistische Position ein. Sie gehen davon aus, dass das ,Geschlecht' omnirelevant ist, d. h. dass die Geschlechterdifferenzierung als Deutungsressource im Hintergrund praktisch aller sozialer Interaktionen wirksam ist (vgl. West/Zimmerman 1987, S. 136). Es sei deshalb auch unvermeidlich, gender herzustellen: „doing gender is unavoidable" (ebd., S. 137). Von zentraler Bedeutung für das Konzept des doing gender ist zudem die Annahme der gleichen Ursprünglichkeit von Differenzierung und Hierarchisierung, d. h. dass im Prozess der Herstellung von ,Geschlecht' gleichzeitig ein Verhältnis von Über- und Unterordnung produziert und damit die patriarchale Gesellschaftsstruktur erhalten wird. West und Zimmerman stellen in ihrem Ansatz einen Zusammenhang zwischen Interaktionen und Institutionen her und beziehen sich dabei auf den Institutionenbegriff von Erving Goffman. Wenn die Anordnung der Geschlechter mit Hilfe von Institutionen geregelt wird, sodass sie schließlich als ,natürlich' erscheint, spricht Goffman von der „,institutionelle Reflexivität“" (Goffmann 1994, S. 139). ${ }^{9}$

9 Das bekannteste von fünf Beispielen ist jenes öffentlicher Toiletten, die für Män- 
Auch dieser Ansatz bleibt nicht ohne Kritik. Ein wesentlicher Kritikpunkt an den Eklärungsmustern des doing gender betrifft deren Begrenzung auf die mikrosoziologische Ebene der Interaktion und die situationsbezogene Herstellung von Differenz. Sie vernachlässige die Stabilität der Kategorien, die diese über gesellschaftliche Institutionen und Strukturen erhalten, werfen beispielsweise Vertreterinnen der feministischen Gesellschaftstheorie, die ,Geschlecht' als Strukturkategorie konzipieren, den Vertreter_innen der Vorstellung von ,Geschlecht' als soziale Konstruktion, als Prozesskategorie, vor ${ }^{10}$ (vgl. Kubisch 2008, S. 19). Ein weiterer Kritikpunkt an der ethnomethodologischen Geschlechterforschung ist, dass diese - auf der Theorieebene - zwar überzeugend darlegt, wie doing gender funktioniert, nicht aber, in welcher Hinsicht sich das doing gender der Männer von dem der Frauen unterscheidet (vgl. Meuser 2007, S. 218). Eine andere Kritik, die sich auf eine grundlegende Ebene des doing gender-Konzepts bezieht, äußert Stefan Hirschauer. Er kritisiert eine Überfokussierung auf das Entdecken von Geschlechtsunterschieden (vgl. Hirschauer 2001, S. 213).

Ausgehend von der Omnipräsenz- bzw. Omnirelevanz-Annahme des doing gender-Konzepts widmet sich Hirschauer der Frage nach undoing gender und der Neutralisierung von ,Geschlecht' (vgl. Hirschauer 1994; 2001; 2012), indem er West und Zimmermans (1987) rhetorische Frage „Can we ever not do gender?" (West/Zimmerman 1987, S. 137) wörtlich nimmt. Er vermutet, dass diese ethnomethodologische Theorie von einer Omnipräsenz der Geschlechtsdifferenzierung ausgehe, da sie sich auf die Untersuchung von Transsexualität konzentriere. Er stellt fest, dass die Geschlechtszugehörigkeit für Transsexuelle, besonders in der Phase des Wechsels, „beinahe dauerthematisch“ sei und Geschlecht eine „thematische Obsession" darstelle (Hirschauer 1994, S. 676). Insofern wäre die Geschlechtsneutralität auch ein „blinden Fleck“ der wissenschaftlichen Thematisierung der Geschlechterdifferenz, da „Umstände, in denen das Geschlecht langweilig, nichtssagend, nebensächlich und uninteressant ist, sich systematisch der Fragestellung entziehen" (Hirschauer

ner und Frauen mit Blick auf eine vermeintlich biologische Differenz getrennt zur Verfügung gestellt werden, obwohl sie im privaten Raum, in der gemeinsamen Wohnung dieselben sanitären Anlagen teilen (vgl. Goffman 1994, S. 139).

,Geschlecht' als Strukturkategorie zu begreifen, bedeutet, ,Geschlecht' in seiner Verschränkung mit gesellschaftlichen Strukturen zu analysieren. Weiter wird davon ausgegangen, dass der Erhalt der kapitalistischen Gesellschaft auf die Reproduktion eines hierarchischen Verhältnisses der Geschlechter angewiesen ist (vgl. BeckerSchmidt/Knapp 2003, Gildemeister/Wetterer 1992, Wetterer 2002; Kubisch 2004). 
2001, S. 212). ${ }^{11}$ Statt von einem permanent fortlaufenden Konstruktionsprozess sei von einer "Diskontinuität der Geschlechtskonstruktion“ (Hirschauer 1994, S. 676) auszugehen. Hirschauer postuliert, empirische Forschung müsse zwar für die Geschlechterdifferenzierung sensibilisiert sein, aber genau beobachten, ob die Unterscheidung in der Praxis tatsächlich vollzogen werde. Sie müsse also für die Relevanzsetzung ebenso offen sein wie für Prozesse der Neutralisierung. So können Geschlechterdifferenzen nach der initialen Geschlechterzuordnung durch die Interaktions-Teilnehmer/innen im weiteren Verlauf einer Interaktion aktualisiert, d. h. fortgesetzt, aufrechterhalten oder in Gebrauch genommen werden, die Interagierenden könnten sie aber ebenso gut in den Hintergrund treten lassen (vgl. ebd., S. 677). Das Absehen von der Geschlechtszugehörigkeit bezeichnet Hirschauer als ,eine Art soziales Vergessen" (ebd., S. 678). Dabei handle es sich um eine konstruktive Leistung, um ein undoing gender (vgl. ebd.). Angelika Wetterer konkretisiert, dass das Konzept des undoing gender zwei unterschiedliche Formen beinhaltet, wie die Geschlechterdarstellung und -zuordnung nicht aktualisiert wird: Das „Ruhenlassen“ (ebd., S. 678) bezeichnet sie als einen Vorgang der „Nicht-Thematisierung" (Wetterer 2002, S. 149). Diesen unterscheidet sie von aktiven Strategien der Neutralisierung von ,Geschlecht":

„Im Unterschied zur Nicht-Thematisierung von Geschlecht, bei der die Hintergrunderwartung der Geschlechtsdarstellung latent bleibt, aber nicht thematisch wird, sind diese Neutralisierungsstrategien erkennbar auf die Normalitätserwartung der Geschlechtszuordnung bezogen und versuchen, ihr mehr oder weniger aktiv und mehr oder weniger absichtsvoll entgegenzuwirken" (ebd.).

Diese Differenzierung zwischen der Nicht-Thematisierung und der Neutralisierung von ,Geschlecht ${ }^{6}$ bzw. den Neutralisierungsstrategien ist insbesondere bei der Interpretation des empirischen Materials von Bedeutung. Selbiges gilt für die Präzisierung verschiedener Arten der Herstellung bzw. Aktualisierung oder Neutralisierung bzw. Nicht-Thematisierung in der Interaktion und in Institutionen. Hirschauer führt hierzu aus, dass in der Interaktion ,Geschlecht' als Mitgliedskategorie oder als Relationskategorie aufgebaut werden könne (vgl. 1994; 2001; 2012). Der Aufbau der Geschlechtszugehörigkeit zu einer Mitgliedskategorie erfolge darüber, dass sich die Beteiligten ,in ihrer Erkennbarkeit als Männer und Frauen adressieren und als Exemplar dieser Kategorie kenntlich machen"

11 Diese Arbeit entzieht sich dieser Kritik, da sie sowohl in der Fragestellung als auch im methodischen Vorgehen und in der Konzeption auf jene Personen fokussiert, für die ,Geschlecht' kein zentrales Thema und Interesse darstellt. 
(Hirschauer 2001, S. 218). Die Akteure/innen würden damit in ihrer Geschlechtszugehörigkeit aktiviert. Daneben könne mittels entsprechender Darstellungstechniken aber auch eine ,Selbstrekrutierung“ (ebd., S. 219) für die Geschlechtskategorien stattfinden (vgl. ebd., S. 219). Umgekehrt könnten entsprechende Darstellungsstile die Geschlechterdifferenz herunterspielen oder Evokationen blockieren.

„Schließlich können Adressierungen der Geschlechtszugehörigkeit auch der Konkurrenz anderer Mitgliedschaftskategorien erliegen. Eine solche Überlagerung durch andere Unterscheidungen kann durch die Teilnehmer aktiv unterstützt werden, indem sie Altersdifferenzen akzentuieren, Statusdifferenzen in den Vordergrund stellen usw." (Hirschauer 2012, S. 163).

,Geschlecht' als Relationskategorie aufzubauen bedeutet statt dessen, dass das Gleich- oder Verschiedensein der Interaktionsteilnehmer/innen deutlich gemacht wird. Aber auch hier gibt es die Möglichkeit, dass die Geschlechterrelation indifferent bleibt oder im Sinne des undoing gender neutralisiert wird. Dies kann z. B. in Form einer ,Spielverweigerung“" passieren oder Geschlechterdifferenz kann auch ,präventiv neutralisiert' werden, indem von vornherein Distanz gewahrt wird (vgl. ebd., S. 221). Die Teilnehmer/innen einer Interaktion können sich ferner unter Zuhilfenahme anderer Kategorien darauf verständigen, „dass die Geschlechterdifferenz für die Kennzeichnung der Interaktion ,gar nicht infrage kommt“" (ebd., S. 220). Im Sinne von Goffmans Konzept der ,institutionellen Reflexivität" (1994, S. 139) schaffen soziale Arrangements „Gelegenheiten für interaktive Geschlechtsdarstellungen, die ein Wissen von der Zweigeschlechtlichkeit bestätigen, das wiederum zur Legitimation der institutionellen Arrangements verwendet werden kann" (Hirschauer 1994, S. 680).

$\mathrm{Zu}$ den institutionellen Arrangements, welche die Geschlechterdifferenzierung unterstützen und stabilisieren, gehört beispielsweise die geschlechtliche Arbeitsteilung. Darüber hinaus führen Segregationsmaßnahmen, die Lokalitäten wie sanitäre Anlagen und Umkleidekabinen sexuieren, dazu, dass aus diesen Orte ,geschlechtlicher Besinnung“" werden (ebd., S. 687). Ähnlich wie im Bereich der Interaktionen zeigt Hirschauer auch für den Bereich der Institutionen auf, wie hier ,Geschlecht' neutralisiert werden kann. Auf institutioneller Ebene nennt er drei analytische Perspektiven, unter denen der Reproduktionszusammenhang offen für Geschlechtsneutralität ist: Erstens sei davon auszugehen, dass Institutionen nicht konkurrenzlos und auch nicht überhistorisch stabil seien. Für die Gegenwart könne festgestellt werden, dass es eine (wachsende) 
institutionelle Infrastruktur der Geschlechtsneutralität gebe. Zweitens hebt er hervor, dass vergeschlechtlichte Institutionen situativ aufrechterhalten und mit Leben gefüllt werden müssten. „Die soziale Struktur bleibt irrelevant, wenn sie nicht situiert wird" (Hirschauer 2001, S. 226). Drittens sei das Verhältnis struktureller Träger der Geschlechterdifferenz - wie beispielsweise Familie und Arbeitsmarkt - zueinander zu beachten, wenn es um Möglichkeiten der Neutralisierung gehe. Hier wären differenzverstärkende oder -minimierende Effekte denkbar.

Ortrun Brand kritisiert an Hirschauers Perspektive, dass er damit möglicherweise tatsächliche strukturelle Folgen von Geschlechterungleichheit übersehe. Diese dürften nicht vorschnell aufgrund des zunächst nicht sichtbaren sozialen Sinns als irrelevant kategorisiert werden. Vielmehr gelte es, die vielfachen Folgen erst sichtbar zu machen und diese systematisch zu erfassen. Insbesondere dürfe der Fokus auf die Praktiken der Neutralisierung nicht verschleiern, ob und wohin sich aktuell die soziale Bedeutung von ,Geschlecht' verschiebt (vgl. Brand 2013, S. 174). Zudem kritisiert Wetterer, dass bei Hirschauer die geschlechtshierarchische Komponente unberücksichtigt bleibe, wenn er in den Institutionen nur Gelegenheiten sieht, Interaktionen geschlechtlich zu polarisieren oder zu assoziieren (vgl. 2002, S. 125).

\subsection{2, Geschlecht' aus dekonstruktivistischer Perspektive}

Der dekonstruktivistisch-diskurstheoretische Ansatz stellt eine Möglichkeit dar, Differenzen zu überwinden, welche auf ,natürliche' Tatsachen Bezug nehmen. Aus dieser erkenntnistheoretischen Perspektive hinterfragt Judith Butler Begriffe wie Subjekt, Identität und sex als biologisches Geschlecht (vgl. Klinger 2008, S. 37). Der erkenntnistheoretische Ausgangspunkt von Judith Butler ist der dekonstruktivistischen Geschlechterforschung zuzuordnen. Ihre Theorie greift auf den Diskursbegriff von Michel Foucault (1983) zurück, wonach Subjekte hauptsächlich durch Machtdiskurse entstehen. Butlers Konzeption von Performativität setzt kein Subjekt voraus. Das heißt, es wird nicht davon ausgegangen, dass Subjekte gemeinsam mit anderen eine Welt konstruieren, sie werden vielmehr ihrerseits diskursiv konstruiert (vgl. Butler 1991, S. 16f.). Das Subjekt bildet sich, nach Butler, nicht als selbstbewusstes und schöpferisches Subjekt, sondern wird durch performative Wiederholung normativer Diskurse erst ermöglicht und durch Sprache hervorgebracht. Es ist in ein historisches Macht- und Diskursgeflecht verstrickt, das den Äußerun- 
gen erst Wirkung verleiht. Durch Macht kann das Subjekt erst existieren, jedoch ist die Handlungsmacht des Subjektes eingeschränkt (vgl. Klinger 2008, S. 36). Bei ihrem Ansatz geht Judith Butler auf die Konstruktion von ,Geschlecht', Geschlechterverhältnissen und Geschlechterordnung ein. Sie heftet ihren Blick auf die kulturellen Konstruktionen. Davon ausgehend, dass (Geschlechts-)Identität auf performative Aspekte der Sprache zurückgeführt werden kann, stellt sie die These auf, dass weder sex noch gender einen natürlichen Ursprung haben.

Gender bezeichnet demnach alle gesellschaftlichen Konstruktionen, die den Unterschied zwischen Frau und Mann ausmachen. Die Unterscheidung von zwei Geschlechtern ist für Judith Butler bereits das Produkt eines Vergeschlechtlichungsprozesses. Von Kategorien wie "Natur und ,biologisches Geschlecht" muss ihrer Ansicht nach Abschied genommen werden, da alles kulturell durchdrungen sei. In diesem Sinne ist auch sex etwas Kulturelles (vgl. ebd., S. 22f.). Gender definiert sie als Ergebnis von bereits vorher existierenden Normen und als ein Gebäude des Signifizierens. Signifizieren bedeutet, reglementierende Normen permanent zu wiederholen und zu bekräftigen, indem andere Regeln ausgeschlossen werden (vgl. Wesely 2000, S. 32).

Begriffe wie Subjekt, Identität und sex sind, nach Butler, ebenso wie die gesellschaftliche Aufteilung in öffentliche und private Bereiche, durch Diskurse entstanden, die sich im System der Sprache begründen. Innerhalb von Diskursen finden Bezeichnungen statt. Die Produktivität diskursiver und sprachlicher Macht ist das grundlegendste Konstruktionsprinzip von (Denk-)Wirklichkeiten. Diskurse umfassen alles: das Reale, Wahrnehmungs-, Handlungsweisen und abstrakte Begriffe. Dies geschieht prozesshaft und eine Bezeichnung muss entlang diskursiver Regeln in immer wiederkehrenden Wiederholungen von Neuem hergestellt werden. Judith Butler nennt dies performative acts (vgl. Butler 1991, S. 205). Der Körper (sex) ist demnach durch sprachliche Bezeichnungspraxis geprägt. Mittels kontinuierlicher diskursiver Wiederholungen wird das binäre System der Geschlechter aufrecht erhalten und die Wahrnehmung des Körpers als binär geschlechtlich verfestigt (vgl. MicusLoos 2004, S. 119). Auch ,männliche' oder , weibliche' Subjektivität entsteht durch performative acts, also durch sich wiederholende Aktionen oder Darstellungen. Machtverhältnisse, die in einem bereits vollendeten Sprechakt dargestellt werden, werden erst durch die Wiederholung der Bezeichnung gefestigt. Durch die Wiederholung performativer Diskurse kommt es zur Versteifung der durch die Sprechakte konstituierten materiellen Strukturen. Auf diese Weise werden Aussprüche wie „Es ist ein 
Junge“ oder „Es ist ein Mädchen“ zum sozialen Faktum, das einem so bezeichneten Körper nur ein ,Geschlecht“ zuordnet. Die Aussage „Es ist ein Mädchen" ist nicht nur eine Beschreibung oder Feststellung, sondern in ihrer Performativität eine determinierende wahrheitskonstruierende Formel ein weibliches ,Geschlecht` zu sein. Diese Anweisung, ein Mädchen zu sein, wird über verschiedene Zeitspannen hinweg kontinuierlich aufs Neue wiederholt. Für Butler bedeutet dies die Möglichkeit, die Normen in der Wiederholung nicht nur zu festigen, sondern auch zu verschieben (vgl. ebd., S. 119). Laut Butler geschieht die Bezeichnungspraxis, die Benennung, die Exklusion und die Wiederholung reglementierender Normen in einem Machtdiskurs. Hier beginnt bereits das Machtverhältnis, obwohl es keine/n konkrete/n Akteur/in gibt (vgl. Wesely 2000, S. 85).

Performativität kann die Möglichkeit der Subversion beinhalten, aber auch gesellschaftliche Sanktionen. Das zeichnet die Ambivalenz des Subjektes aus. Subjektivitäten, die nicht Produkt von Machtverhältnissen sind, werden verneint. Deshalb bleibt die Frage offen, woher Subjekte die Fähigkeiten und das Werkzeug erhalten, um Widerstand zu leisten und Veränderungen herbeizuführen (vgl. Micus-Loos 2004, S. 120). Die Fähigkeit der Selbstbestimmung, welche in den Erziehungs- und Bildungswissenschaften eine bedeutende Funktion einnimmt, wird durch das Subjektivitätsverständnis von Judith Butler in Frage gestellt. Laut Judith Butler wird die Persönlichkeit von Beginn an durch die Machtverhältnisse konstituiert und von ihnen infiziert, ohne begrenzt zu sein. Aufgrund dessen wird immer wieder die Frage gestellt, inwieweit Butlers Subjektverständnis für die pädagogische Arbeit relevant bzw. umsetzbar ist (vgl. Micus-Loos 2004, S. 121).

Gudrun-Axeli Knapp (2008) kritisiert an Judith Butlers dekonstruktivistischem Ansatz, dass sich dieser vorwiegend im geschichts- und empiriefreien Raum einer metatheoretischen Befragung begrifflicher Grundlagen bewege. Knapp stellt fest: „Derartige Reflexionen haben ihren eigenen Stellenwert - sie können jedoch eine historisch konkrete Bestimmung von Geschlechterregimes und kulturellen Symbolisierungen von Differenz, deren Widersprüchlichkeit und Tendenzgehalte nicht ersetzen" (ebd., S. 302). Auch Andrea Maihofer (1995) kritisiert an den Versuchen, ,Geschlecht', also sex und gender, als soziale Konstruktion zu begreifen, dass hierbei die körperlich-leibliche Ebene des Geschlechts nicht beachtet werde und dass die Realität des Geschlechts als eine historisch konkrete Existenzweise der Individuen nicht fassbar werde (vgl. Maihofer 1994, S. 236). Darüber hinaus habe das Verständnis, dass 
gesellschaftliche Phänomene vornehmlich Effekte sozialer Interaktionen sind, eine Reduktion der gesellschaftstheoretischen Perspektive zur Folge (vgl. ebd.) und fokussiere hauptsächlich darauf, wie ,Geschlecht' gemacht wird. Auch bei struktur- und machttheoretischen Ansätzen kritisiert Maihofer, dass die Konstruktion von Zweigeschlechtlichkeit im Mittelpunkt stehe, mit dem Ziel ihrer Dekonstruktion. Auch hier bleibe jedoch kein Platz für die Rekonstruktion des Geschlechts als eine historisch bestimmte gesellschaftlich-kulturelle Existenzweise (vgl. Maihofer 1994, S. 251). Mit gesellschaftlich-kultureller Existenzweise meint Maihofer die Art und Weise, wie in dieser Gesellschaft Individuen nicht nur zu Geschlechtern gemacht werden, sondern als ,gewordene' und als ,werdende' Geschlechter existieren (vgl. ebd., S. 251). Aus meiner Sicht spielt hier auch die Selbstkategorisierung der Individuen eine große Rolle. Auch in meiner empirischen Untersuchung war ich - in diesem Sinne - nicht nur mit einer körperlichen zweigeschlechtlichen Realität, sondern auch mit binären zweigeschlechtlichen Geschlechtsidentitäten konfroniert.

\section{6 ,Geschlecht' aus intersektionaler Perspektive}

Im Anschluss an die Diskussion um die (De-)Konstruktion wird in der Frauen- und Geschlechterforschung verstärkt die Frage nach ,Geschlecht" mit anderen Kategorien sozialer Differenzierung wie z. B. Ethnizität, ,Rasse', Sexualität, Klasse/Schicht, Nationalität, Alter, Lokalität (und damit jeweils einhergehende Normierungen, Marginalisierungen und Hierarchisierungen) in analytisch produktiver Weise zusammen gedacht. Dies wird zur Zeit vor allem unter den Termini Intersektionalität oder Interdependenz ${ }^{12}$ verhandelt (vgl. Dietze et al. 2007, S. 8). In anderen Kontexten, wie z. B. in den Erziehungs- und Bildungswissenschaften werden Begriffe wie Heterogenität oder Diversity verwendet (vgl. Dietze et al. 2007, S. 7). Im Gegensatz zu diesen Begriffsfeldern zeichnet sich die Intersektionalitätsforschung bzw. -debatte dadurch aus, dass sie sich ausschließlich auf Macht- und Herrschaftsverhältnisse konzentriert. Intersektionalität wird als ein wesentlicher und innovativer Analyseansatz anerkannt. Die Ursprünge des Paradigmas Intersektionalität sind

12 Näheres zur Diskussion rund um Intersektionalität und Interdependenz bietet das Internetportal „Intersektionalität: Forschungsplattform und Praxisforum für Intersektionalität und Interdependenzen": www.portal-intersektionalität.de [14.09.2012]. 
im Black Feminism und der Critical Race Theory (Crenshaw 1989; Chebout 2011) zu finden. Kimberley Crenshaw macht am Beispiel der spezifischen Subjektpositionen und Diskriminierungserfahrungen schwarzer Frauen deutlich, was sie unter Intersectionality versteht. Dabei bedient sie sich der Metapher einer Straßenkreuzung, bei der sich unterschiedliche Diskriminierungsformen überschneiden. Vor allem in den Gender Studies wird Intersektionalität mittlerweile für unterschiedliche Analysegegenstände produktiv gemacht (vgl. Walgenbach 2012a). Im deutschsprachigen Raum wird Intersektionalität im Allgemeinen als work in progress angesehen, Katharina Walgenbach bietet dennoch für einen ersten Zugang eine Definition:

„Unter Intersektionalität wird dabei verstanden, dass soziale Kategorien wie Gender, Ethnizität, Nation oder Klasse nicht isoliert voneinander konzeptualisiert werden können, sondern in ihren ,Verwobenheiten' oder, Überkreuzungen' (intersections) analysiert werden müssen. Additive Perspektiven sollen überwunden werden, indem der Fokus auf das gleichzeitige Zusammenwirken von sozialen Ungleichheiten gelegt wird. Es geht demnach nicht allein um die Berücksichtigung mehrerer sozialer Kategorien, sondern ebenfalls um die Analyse ihrer Wechselwirkungen" (Walgenbach 2012b, S. 81).

Somit sind Differenzkategorien wie z. B. Leistungsheterogenität oder Fachkompetenz als Diversity-Merkmal kein Gegenstand intersektionaler Analysen. „Das Forschungsfeld bzw. der gemeinsame Gegenstand von Intersektionalität sind Macht-, Herrschafts- und Normierungsverhältnisse, die soziale Strukturen, Praktiken und Identitäten reproduzieren" (Walgenbach 2012a). Walgenbach sieht es als problematisch, wenn Kimberlè Crenshaws Straßenkreuzungs-Metapher ${ }^{13}$ aus ihrem juristischen Entstehungskontext gelöst und in den Sozialwissenschaften für das Verständnis der, Überschneidungen' bzw. ,Überkreuzung' von Machtachsen bzw. sozialen Kategorien herangezogen wird. Für sie liegt das Problem darin, dass Machtverhältnisse als eindimensionale ,Linien' oder ,Relationen' konzeptualisiert werden (vgl. Walgenbach 2012a). Bei ihrer Kritik geht es ganz allgemein um das Verständnis von Machtverhältnissen bzw. sozialen Kategorien. Denn die Übertragbarkeit der Metapher der Straßenkreuzung auf die eigenen Erkenntnisinteressen der Sozialwissenschaften könnte suggerieren, dass die Kategorien gender und race vor (und auch nach) dem Zusammentreffen an der Kreuzung von einander

13 Weitere Kritikpunkte hierzu bei Walgenbach 2007 und 2012a. 
getrennt existierten. Das heißt, gender und race werden, mit Ausnahme der spezifischen Situation der Straßenkreuzung, immer noch als isolierte Kategorien gefasst (vgl. ebd.).

Im deutschsprachigen Raum offerieren Nina Degele und Gabriele Winker (2009) einen produktiven Beitrag zur Frage der Auswahl bzw. Gewichtungen von Kategorien. Ihr Ansatz leitet die Relevanz von Kategorien aus unterschiedlichen Analyseebenen ab. Die Autorinnen differenzieren in ihrer Mehrebenenanalyse zwischen drei Ebenen, die miteinander in Wechselwirkung stehen: Strukturebene, Repräsentationsebene und Identitätsebene (vgl. ebd., S. 18-62). Für die gesellschaftliche Strukturebene sei die Anzahl der relevanten Kategorien eingeschränkt. Nach Degele und Winker sind dies ,Geschlecht', Klasse, ,Rasse ${ }^{6}$ und Körper (Alter, körperliche Verfasstheit, Gesundheit und Attraktivität). Diese Kategorien werden als Strukturkategorien klassifiziert und ergeben sich für Degele und Winker deduktiv aus der Gesellschaftsanalyse eines modernen Kapitalismus. Hingegen muss gegenüber der Identitätsebene eine prinzipielle Offenheit angenommen werden. Unterschiedliche Differenzkategorien werden induktiv aus dem Forschungsprozess gewonnen. Gleiches gilt für die symbolische Repräsentationsebene, die sich auf Normen bzw. Ideologien bezieht. Nach Ansicht der Autorinnen grenzt der Ansatz der Mehrebenenanalyse die Beliebigkeit bei der Auswahl von Kategorien ein und ermöglicht gleichzeitig Vielfältigkeit in der Analyse (vgl. Degele/Winker 2009, S. 206; Walgenbach 2012a).

Auch wenn es den Autor innen in der Intersektionalitätsdebatte darum geht, isolierte bzw. eindimensionale Perspektiven auf Macht- und Herrschaftsverhältnisse zu überwinden, legen Termini wie, Überkreuzungen' oder ,Schnittpunkte' auf einer visuellen Ebene nach wie vor nahe, dass soziale Kategorien einen ,genuinen Kern' hätten, der sich mit weiteren Kategorien ,verkettet'. Die Idee eines ,genuinen Kerns' birgt die Gefahr in sich, soziale Kategorien durch die Annahme von Entitäten, die sich ,verbinden' oder , verquicken', eher zu stabilisieren als zu hinterfragen. Alternativ dazu hat Walgenbach deshalb gemeinsam mit Kolleginnen von der Humboldt Universität Berlin den Begriff interdependente Kategorien entwickelt (Dietze et al. 2007; Walgenbach 2000; Walgenbach 2007) und plädiert in ihrem Aufsatz ,Gender als interdependente Kategorie‘ für eine integrale Perspektive. Weiter schlägt sie vor, von interdependenten Kategorien auszugehen (vgl. ebd., S. 59), „da die Verbindung von ,inter' (zwischen) und ,Dependenz' (Abhängigkeit) deutlich macht, dass der Fokus des Begriffs auf der Konzeptualisierung wechselseitiger und nicht monodirektionaler Abhängigkeiten liegt" (Diet- 
ze et al. 2007, S. 19). Somit werden Beziehungen von Ungleichheit bzw. Marginalisierungen in den Vordergrund gerückt. Mit dem Begriff der Interdependenzen werden nicht mehr wechselseitige Interaktionen zwischen Kategorien gefasst (wie bei der „Intersektionalität" im Sinne Crenshaws 1989), sondern soziale Kategorien selbst werden als wechselseitig voneinander abhängig konzeptionalisiert. Aus diesem Grund wird von Walgenbach vorgeschlagen, „Gender"14 als interdependente Kategorie zu fassen. Diese Perspektive weist auch über die Gender Studies hinaus und kann allgemein für Forschungen zu Ungleichheit, Marginalisierung und Privilegierung produktiv angewendet werden (vgl. ebd., S. 9). „Durch die Verbindung der integralen Perspektive mit den Überlegungen zur strukturellen Dominanz sozialer Kategorien wird schließlich der Ansatz, "Gender als interdependente Kategorie" zu denken, innerhalb von Machtund Herrschaftsverhältnissen verortet" (Walgenbach 2007, S. 64). Dies gilt nicht allein für die Kategorie Gender, sondern auch für weitere soziale Kategorien wie soziales Milieu oder Ethnizität (vgl. Walgenbach 2012a).

Auch wenn sich die Intersektionalitätsforschung bzw. -debatte überaus breitgefächert und gehaltvoll gestaltet und ,sicherlich [...] kein vernünftiger Mensch grundsätzlich etwas ,gegen Intersektionalität' vorbringen" (Rendtorff 2012) will, werde ich in meiner Arbeit, weiterhin' zentral auf die Kategorie ,Geschlecht' setzen. Dies ist meiner Fragestellung geschuldet und zudem kommt ein solcher Zugang dem Bedürfnis nach Komplexitätsreduktion entgegen. Gleichwohl möchte ich betonen, die Bedeutung und die Wirkung der Kategorie ,Geschlecht" nicht als unterkomplex einzuschätzen, sondern zu berücksichtigen, „dass die Brisanz (und insofern auch die ,Wichtigkeit') von ,Geschlecht" nicht nur auf der Ebene von Verteilungen (von Macht und Einfluss usw.) zu suchen ist, sondern auf der Ebene der Bedeutungszuschreibungen" (ebd.).

Im folgenden Abschnitt wird der erweiternde Begriff der Existenzweise(n) dargestellt, der es erlaubt, ,Geschlecht' und Geschlechterdifferenzen als kulturelles, psychisches Bewusstseinsphänomen zu betrachten und zu begreifen. Gleichzeitig lenkt er die Aufmerksamkeit darauf, materiell-körperlich zu existieren. Zudem erlaubt er den Blick auf die

14 Walgenbach lehnt sich an den englischen Begriff „Gender" an, da dieser weniger mehrdeutig als der deutschsprachige Begriff Geschlecht sei. Mit der Großschreibung verweist sie auf die ,Eindeutschung، des englischen Begriffes. Mit der Begrifflichkeit „Gender" verweist sie auch darauf, dass es eine gegenderte Existenz gibt (vgl. Dietze et al. 2007, S. 15f.). Im Gegensatz zu Walgenbach verwende ich den deutschen Begriff Geschlecht. Dennoch werde ich während der Rezeption von Katharina Walgenbach ihre Schreibweise verwenden. 
jeweils eigene Logik und Dynamik, die beispielsweise der Geschlechtskörper gegenüber den psychischen Geschlechtsidentitäten oder kulturellen Geschlechtsnormen innerhalb eines Individuums haben mag. Maihofer verdeutlicht, dass der herrschende Geschlechterdiskurs ${ }^{15}$ nicht nur ein umfangreiches Set an ,männlich' oder ,weiblich` konnotierten Denkund Gefühlsweisen, Geschlechterrollen und -stereotype umfasst, sondern auch ein vielfältiges Repertoire an Körperpraxen und -gefühlen bis hin zu spezifischen Körperformen. So sind ,weibliche ${ }^{6}$ und ,männliche' Körper Ergebnis von disziplinierenden und formierenden Trainings und Stylings entlang geltender ,männlicher' und ,weiblicher' Gesten und als solche das Ergebnis eines langwierigen historischen Prozesses. Dies betrifft auch den scheinbar ,natürlichen' Geschlechtskörper. Einen ,männlichen' oder ,weiblichen' Körper zu ,haben' ist in dieser heterosexuellen Eindeutigkeit zweier Geschlechter keine einfache natürliche Gegebenheit (vgl. Maihofer 1995, S. 91). Zudem möchte ich hervorheben, dass der Begriff Existenzweisen m. E. ein Kontinuum an ,gewordenen', ,seienden' und ,sich verändernden' Geschlechtsidentitäten fasst.

\section{7 ,Geschlecht' als gesellschaftlich-kulturelle Existenzweise(n)}

Die Benennung von ,Geschlecht' als gesellschaftlich-kulturelle Existenzweise(n) soll eine begriffliche Balance zwischen Natur und Kultur, Körper und Geist darstellen. In der Vorstellung des Geschlechts als historisch entstandene Denk-, Gefühls- und Körperpraxen, eben als gesellschaftlichkulturelle Existenzweise(n), wird sowohl das Imaginäre der Realität des Geschlechts als auch die spezifische ,Materialität' und ,Realität' des Imaginären des Geschlechts erfasst. Damit verbunden ist die Auffassung, dass die gegenwärtige Art und Weise als ,Geschlecht' zu existieren, weder gesellschaftlich beliebig noch willkürlich veränderbar ist (vgl. Maihofer 1995, S. 108). Selbiges gilt für die Möglichkeiten individueller Kritik und Handlungsfähigkeit. Laut Maihofer liegt die Möglichkeit individueller Veränderung vor allem in der jeweils individuellen Einzigartigkeit und dem damit verbundenen breiten Spektrum an Möglichkeiten, auf gesell-

15 Diskurs ist eine komplexe Verbindung vielzähliger und spezifischer Denk-, Gefühlsund Handlungsweisen, Körperpraxen, Wissen(schaft)sformen, aber auch staatlicher und gesellschaftlich-kultureller Institutionen wie Gesellschafts- und Herrschaftsverhältnisse. Maihofer knüpft mit ihrem Diskursbegriff an Foucault an (vgl. Maihofer 1994, S. 256). 
schaftliche Gegebenheiten zu reagieren. Dies kann mal oppositionell, mal mit einem etwas weniger kritischen Blick auf die gegebene Gesellschaft passieren, erlaubt jedoch eine je individuelle Transformation geschlechtlicher Existenzweisen (vgl. ebd.) und versucht, eine ,identitäre Fixierung“" (vgl. Degele 2008, S. 43) hinter sich zu lassen.

„Ob individuelle Handlungen, Einsprüche, Widerstände etc. zu gesamtgesellschaftlichen Veränderungen führen, erweist sich aber meist erst im Nachhinein und hängt im Wesentlichen davon ab, ob sie Teil struktureller gesellschaftlicher Entwicklungsprozesse sind bzw. zu solchen werden oder in diese einmünden" (vgl. Maihofer 1995, S. 108).

Diese Überlegungen führen Maihofer zu der Forderung nach Anerkennung der (Geschlechter-)Differenz und vielfältigen Existenzweisen. Anerkennung der Differenz (positiv und nicht hierarchisierend) soll die Forderung nach Gleichheit nicht ersetzen, sondern die Anerkennung pluraler Lebensformen in den Fokus rücken. Dabei geht es um die Anerkennung grundlegender Differenzen, Anerkennung der kulturellen, ethnischen und klassenspezifischen Differenzen und Verschiedenheiten zwischen Individuen. Die Forderung nach Anerkennung der Geschlechterdifferenz, und m. E. der Geschlechterpluralität, stellt die Weiterentwicklung der Forderung nach Gleichheit bzw. Gleichberechtigung dar und tritt nicht an deren Stelle (vgl. ebd., S. 172). Maihofer beschreibt dies wie folgt:

„Sowohl die Anerkennung als gleichberechtigte Menschen an sich im Sinne der herkömmlichen Menschenrechte als auch die Anerkennung als gleichberechtigte Staatsbürgerinnen und -bürger im Sinne der herkömmlichen Staatsbürgerrechte bleiben notwendige Voraussetzungen. Die Forderung nach Anerkennung der Geschlechterdifferenz soll - gleichsam als nächster Schritt - die Gleichberechtigung der Frau nun auch als ,Frau' garantieren. Diese Forderung ist - wie sich historisch gezeigt hat - aber auch deshalb notwendig, weil nur dadurch für Frauen die Verwirklichung auch der anderen Ebenen wirklich garantiert scheint. Der nächste Schritt wäre dann die Anerkennung der/des Einzelnen auch in ihrer/seiner konkreten Individualität" (ebd., 172f.).

Der folgende Abschnitt soll deutlich machen, dass wir als Individuen von unterschiedlichen hegemonialen wie auch marginalen (Geschlechter-)Diskursen geprägt sind. ,Geschlecht' als gesellschaftlich-kulturelle Existenzweise(n) zu verstehen, ist ein Vorschlag, anhand der Differenzlinie ,Geschlecht` die Komplexität des Prozesse des Zusammenwirkens unter- 
schiedlicher Dimensionen zu erfassen, ohne jedoch von einer essentialistischen, binären Geschlechterdifferenz auszugehen oder diese zu reifizieren. Maihofer kommt dabei zu dem Schluss, dass wir alle individuelle Einzelne sind, die geprägt sind vom Zusammenwirken unterschiedlicher (hegemonialer) Diskurse zu Differenzlinien. Sie betont, dass es schlussendlich darum gehen müsse, diese Differenzen zwischen Individuen anzuerkennen und ausgehend von der Differenz die Gleichheit und Gleichberechtigung aller zu fordern und nicht deren Gleichmachung und Assimilation. Diese Forderung nach einer nicht-hierarchischen Anerkennung von ,Geschlecht', richtet sich nach meinem Verständnis auch an marginalisierte Existenzweisen von ,Geschlecht', da Maihofers Überlegungen darauf abzielen, neue ,gesellschaftliche Praxen zu finden, die eine Gesellschaft ermöglichen, in der man ohne Angst verschieden sein kann" (Maihofer 1995, S. 173 mit einem Zitat von Adorno 1969, S. 131). Dennoch orientiere ich mich bei den folgenden Ausführungen am hegemonialen westlichen Geschlechterdiskurs, da dieser maßgeblich die Thematisierung von ,Geschlecht' der für diese Untersuchung befragten Frauen und Männer beeinflusst und von ihnen reproduziert wird.

\subsubsection{Der hegemoniale westliche Geschlechterdiskurs}

Die Geschlechterdifferenz prägt den gegenwärtigen hegemonialen westlichen Geschlechterdiskurs und umfasst dementsprechend geschlechtsbezogene, je unterschiedliche Körperpraxen, -gefühle und -formen sowie ,männliche‘ und ,weibliche' Denk-, Gefühls-, und Verhaltensweisen, Identitäten, Normen, Rollen, Berufe etc., wobei im hegemonialen Geschlechterdiskurs der Mann bzw. ,Männlichkeit' der Maßstab ist (vgl. Maihofer 1995, S. 100ff). Hegemonial ist ein Diskurs, wenn er die herrschenden Normen, Werte und Verhaltensstandards einer Gesellschaft formuliert, aber auch dann, wenn in ihm das Denken, Fühlen und Handeln der Menschen sowohl normiert, zensiert und diszipliniert wie auch konstituiert wird (vgl. Maihofer 1994, S. 256). ,Geschlecht' als hegemonialen Diskurs und gesellschaftlich-kulturelle Existenzweise zu verstehen, bedeutet davon auszugehen, dass sich ,Geschlecht" in der Ausübung von verschiedenen, als ,weiblich' und ,männlich' konnotierten, Denk-, Gefühls- und Körperpraxen konstruiert und reproduziert. Einzelne Individuen werden aber nicht nur als Frauen und Männer identifiziert weil sie sich als solche in der Praxis inszenieren, sondern weil Verallgemeinerungen darüber existieren, wie Frau bzw. Mann oder die Rekonstruktion der hegemonialen Auffassung von ,Weiblichkeit' oder ,Männlichkeit' in der westlichen 
Gesellschaft zu sein haben. Allerdings treffen die Verallgemeinerungen nur in dem Maße zu, wie der hegemoniale Geschlechterdiskurs in der Gesellschaft real verbreitet bzw. im einzelnen Individuum präsent ist. Die Wirkung des herrschenden Geschlechterdiskurses ist nicht vornehmlich repressiver, sondern vor allem produktiver Art. Der moderne westliche Geschlechterdiskurs und seine Denk-, Gefühls- und Handlungsweisen dürfen nicht lediglich nur als hegemoniale Zuschreibungen verstanden werden (vgl. Maihofer 1995, S. 104). Er muss vielmehr

\begin{abstract}
„als Selbststilisierungen und -affirmationen der bürgerlichen Klasse und ihrer Lebensweise begriffen werden, und zwar im Sinne eines Selbstkonstituierungsprozesses sowohl des bürgerlichen Mannes (in Abgrenzung gegen die Männer anderer gesellschaftlicher Klassen und Schichten sowie zur Legitimierung seiner gesellschaftlichen Hegemonie) als auch der bürgerlichen Frau" (ebd.).
\end{abstract}

Die Geschichte der Frauen ist nicht vornehmlich als Geschichte der Unterdrückung oder des Widerstandes gegen patriarchale Zuschreibungen zu rekonstruieren, sondern ist Teil dieser. Erstgenanntes geschieht zwar nur allzu häufig, verstellt jedoch den Blick auf die hegemonialen schicht, klassen- und kulturspezifischen Differenzen sowie Macht- und Herrschaftsverhältnisse unter Frauen (vgl. ebd., S. 105). Diese Ausführungen machen deutlich, dass es in unserer Gesellschaft nicht nur hegemoniale, sondern auch marginale Diskurse und Formen von Existenzweisen gibt. Das heißt, Individuen sind von unterschiedlichen Diskursen durchzogen und in diesen verwoben. In unserer Gesellschaft existieren nicht nur der bürgerlich-hegemoniale Geschlechterdiskurs (mit seinen Modifikationen und Diversifikationen), sondern auch andere Geschlechterdiskurse in den einzelnen Individuen und in kleineren oder größeren gesellschaftlichen Gruppen. Ein historisch konkretes Individuum ist aufgrund seiner je eigenen Biografie (verschiedener Zugehörigkeit zu Klasse, Kultur, Ethnie) jeweils sehr komplex und für sich genommen eine einzigartige Verbindung unterschiedlicher, weiblich` wie ,männlich' konnotierter Denk-, Gefühls- und Körperpraxen (vgl. ebd.), die in dessen Lebenslauf zudem einer ständigen Veränderung unterliegen. Die Unterschiede zwischen den einzelnen Individuen bzw. zwischen den Geschlechtern können daher - je nach Kombination - sehr unterschiedlich sein, mal mehr, mal weniger umfassend und tiefgehend (vgl. ebd., S. 105f.). In der Regel wird ein Individuum jedoch schon aufgrund des sozialen Zwanges eine eindeutige - entweder , weibliche ${ }^{6}$ oder ,männliche ${ }^{6}$ - heterosexuelle Geschlechtsidentität entwickeln, und zwar in Übereinstimmung 
mit seinen vergeschlechtlichten Körpereigenschaften und entsprechend den Normen des hegemonialen bürgerlichen Geschlechterdiskurses. Diese ,Vereindeutigung" ist allerdings weder total noch endgültig. So ist die eindeutige und einzige Existenz von Individuen als ,männlich ${ }^{6}$ oder ,weiblich` zum Scheitern verurteilt, weil sie immer auch zugleich Angehörige einer spezifischen Klasse, Kultur oder ,Rasse' (ebenfalls historisch spezifische gesellschaftlich-kulturelle Existenzweisen) sind. Und letztlich sind sie immer auch unverwechselbare konkrete Einzelne. Es existieren immer Inkonsistenzen, Widersprüche, Ambivalenzen bzw. Möglichkeiten der Aktualisierung und Verstärkung anderer Aspekte der eigenen Person (vgl. ebd., S. 104ff). Maihofer meint, dass wir ,Geschlecht' nur begreifen, wenn wir einen Begriff entwickeln, der sowohl das Imaginäre dieser Existenzweise (also Geschlechtlichkeit, Subjektivität, Identität und Körperlichkeit als gesellschaftlich-kulturell produzierte Selbstverhältnisse) reflektiert, als auch die Realität dieser Existenzen als gelebte Denk-, Gefühls- und Körperpraxen (vgl. Maihofer 1994, S. 258f.).

Der Begriff der Existenzweise ist für diese Arbeit besonders interessant, da er sich nicht nur mit dem Gewordensein von ,Geschlecht" auseinandersetzt, sondern auch die Art und Weise im Blick hat, wie in dieser Gesellschaft Individuen, die sich als Frau oder Mann ,geworden' und ,seiend' empfinden, existieren, verändern, handeln und denken. Abschließend möchte ich nochmals hervorheben, dass ,Geschlecht' als gesellschaftlich-kulturelle Existenzweise(n) zu begreifen auch bedeutet, dass Existenzweisen nicht stabil und statisch, sondern flexibel und fluide sind. Somit ist mit dem Begriff der Existenzweisen die Anerkennung unterschiedlicher ,Geschlechter" und Geschlechtsidentitäten verbunden.

\subsection{Resümee}

$\mathrm{Zu}$ Beginn des Kapitels wurden moderne Geschlechterverhältnisse im Spannungsfeld rhetorischer Modernisierungsprozesse und struktureller Persistenz dargestellt, um im Folgenden auf ,Geschlecht` im Spiegel theoretischer und akademischer Debatten und Diskurse genauer einzugehen. Dies diente auch der Sensibilisierung für den empirischen Teil der Arbeit. In der Deskription der verschiedenen Ansätze wurde deutlich, dass es unterschiedliche „Spielarten der Konstruktion“ (Knorr-Centina 1989) und verschiedene forschungstheoretische Ausrichtungen auf den Forschungsgegenstand ,Geschlecht' gibt. Diese werden im Spannungsfeld von Geschlechtergleichheit und -differenz bis hin zur Aufhebung der Katego- 
rie ,Geschlecht' diskutiert. Aus den bisherigen Ausführungen ist deutlich geworden, dass es eine Fokussierung auf Geschlechtsidentität und -konstruktion gibt und dass bei Überlegungen zu ,Geschlecht' vornehmlich Effekte sozialer Interaktionen im Zentrum stehen. Diese Sichtweisen und Fragestellungen sind zwar wichtig und relevant, dennoch geht bei dieser Überfokussierung die Frage nach der Thematisierung und Relevanz von ,Geschlecht' bei Individuen, die sich zum jeweiligen Betrachtungszeitpunkt als Frau oder Mann geworden und seiend empfinden, ${ }^{16}$ und deren habitualisierten Handlungspraxis verloren.

Neben der Vernachlässigung der Ebene des Habitus und der habituellen Praxis mangelt es zudem an Ansätzen, die eine Verbindung zwischen der mikrosoziologischen Ebene von Identität und Interaktion und der makrosoziologischen Ebene sozialer Ungleichheit herstellen bzw. die Dichotomie zwischen diesen beiden Perspektiven zu überwinden vermögen. Die sozialen Strukturen, die einen Einfluss auf die Interaktion und Thematisierung haben und durch die Stabilität von Konstruktionen und Existenzweisen erklärt werden, können nicht erfasst werden. Dies gilt umso mehr, wenn es um den Zusammenhang zwischen der (De)Thematisierung von ,Geschlecht" und konjunktiver Erfahrungen innerhalb bestehender Strukturen geht. Es wird deutlich, dass sie sich lediglich auf die öffentliche bzw. definitorische Ebene der Wirklichkeitskonstruktion richten und die Bedeutungszuschreibung und das handlungsleitende Wissen und konjunktive Erfahrungen der einzelnen Individuen außen vor bleiben. Unter Berücksichtigung der aktuellen gesellschaftlichen Entwicklungen und einem ernstzunehmenden "gender scepticism“ (Knapp 2008, S. 297) oder der „Nicht-Thematisierung“" von Geschlechterdarstellungen (Wetterer 2002, S. 149), bedarf es neben den zahlreichen Spielarten der Konstruktion von ,Geschlecht' einer Analysefolie, die verstärkt die gesellschaftlichen Strukturen miteinbezieht und mit Thematisierungs- und Handlungspraxen der AkteurInnen verbindet.

Für diese Arbeit ist deshalb eine praxeologische Perspektive leitend, die im folgenden Kapitel ausgeführt wird. Es wird dargestellt, welche Rolle das Habitus-Feld-Konzept von Pierre Bourdieu für die empirische Untersuchung habitueller Praxis in Bezug auf ,Geschlecht' bei StudentInnen der Erziehungs- und Bildungswissenschaften spiel. Dabei beziehe ich mich auf die in der Tradition der Wissenssoziologie Karl Mannheims stehende dokumentarische Methode Ralf Bohnsacks (1989; 2008; 2010).

16 Auch wenn in dieser Arbeit sich als Männer und Frauen empfindende Individuen im Fokus stehen, möchte ich deutlich machen, dass Geschlechtsidentitäten nicht als etwas zeitlich Stabiles verstanden werden. 
Zudem schließe ich an dieses Kapitel Überlegungen an, die sich weniger mit der Konstruktion von ,Geschlecht' beschäftigen, sondern mehr mit der Frage, welche gesellschaftlichen (Verdeckungs-)Zusammenhänge und (rhetorische) Modernisierungsprozesse auf die (De-)Thematisierung von ,Geschlecht' einwirken. 


\section{Die Relevanz einer praxeologischen Perspektive}

Im vorherigen Kapitel wurden theoretische Konzepte zu Geschlechterkonstruktionen und -verständnisse vorgestellt. In der Kritik an ihnen wurde deutlich, dass sie auf die situative Herstellung der Differenz von ,Geschlecht' beschränkt bleiben und damit soziale Strukturen, welche einen Einfluss auf die Interaktion haben und die Stabilität der Konstruktionen erklären, nicht zu erfassen vermögen. Außerdem fokussieren die Konzepte auf eine theoretische und abstrakte Ebene weshalb Fragen der sozialen Identität dabei im Zentrum stehen. Der Habitus, die habituelle Praxis, bleibt jedoch unbeleuchtet. Statt dessen wird in diesen Betrachtungsweisen lediglich eine spezifische Form des Wissens erfasst, nämlich kommunikativ-generalisiertes Wissen, und rational orientiertes Handeln. Unberücksichtigt bleibt dabei das unmittelbar handlungsbezogene und handlungsleitende, d. h. das konjunktive Wissen (vgl. Bohnsack 2006, S. 280f.). Letzteres stellt ein milieuspezifisches Wissen dar, welcher in der gemeinsam gelebten Praxis zugleich in habitualisierter Weise zur Orientierung dient (vgl. Bohnsack 2003, S. 137). Im Rahmen dieses Kapitels soll nun ein dies berücksichtigender praxeologischer Ansatz vorgestellt werden, der an den genannten Punkten über Fragen zur Geschlechtsidentität und -konstruktion hinausreicht. Dieser ist von daher in theoretischer und methodologischer Weise geeignet für die Rekonstruktion handlungsleitender Orientierungen, Bewertungs- und Denkschemata von StudentInnen. Mit Hilfe dieses Zugangs wird nun die Grundlage für die empirische Untersuchung der habituellen Praxis in Bezug auf die (De-)Thematisierung von ,Geschlecht' und Geschlechterfragen bei StudentInnen der Erziehungs- und Bildungswissenschaften geschaffen.

Dazu wird im Folgenden zum einen das Habitus-Feld-Konzept von Pierre Bourdieu (1983; 1992; 1993; 1998; Kapitel 4.1) herangezogen. Dies dient der vorliegenden Arbeit als Folie, um Mechanismen und Funktionsweisen des wissenschaftlichen Feldes zu verdeutlichen und den (herkömmlichen) Dualismus von Individuum und ,Geschlecht` zu überwinden. Zum anderen wird im zweiten Abschnitt dieses Kapitels auf die in der Tradition der Wissenssoziologie Karl Mannheims stehende dokumentarische Methode Ralf Bohnsacks (1989; 2008; 2010; Kapitel 4.2) Bezug genommen. Die dokumentarische Methode bezieht sich bei wesentlichen 
Aspekten methodologischer Überlegungen auf das Habituskonzept Bourdieus und stellt eine Methode zur Habitusrekonstruktion bereit (vgl. Bohnsack et al. 2007; Meuser 2007; Kubisch 2008). Beide Ansätze verbindet somit nicht nur die Annahme, dass sich Individuen und die sie umgebende Welt in der sozialen Praxis gegenseitig herstellen und somit Homologien zwischen gesellschaftlichen Strukturen und habituellen Dispositionen bestehen (vgl. Bourdieu 2005, S. 260), sondern auch eine praxeologische Perspektive auf den Gegenstand. Wenn man nun die Art und Weise der (De-)Thematisierung von ,Geschlecht' und Geschlechterfragen als Werthaltungen, Weltanschauungen und Handlungsorientierungen versteht, wird deutlich, dass es neben individuellen Erfahrungen und Erlebnissen auch kollektive Erfahrungen und gesellschaftliche Strukturen und Diskurse gibt, die Vorstellungen und Praktiken leiten.

Der dritte Abschnitt des Kapitels (4.3) knüpft an diese Perspektive an und konzentriert sich zudem auf die gegenwärtig diagnostizierte Diskrepanz zwischen einer Gleichheitsrhetorik, die eine scheinbare Gleichberechtigung von Frauen und Männern fabriziert, und der sozialen Praxis, in der die Ungleichheit zwischen den Geschlechtern im Handeln und im handlungsleitenden Wissen weiterhin konstant bleibt (vgl. Rendtorff 2005; Wetterer 2006; Friebertshäuser 2012). In diesem Abschnitt stelle ich drei Analysefolien vor, deren gemeinsamer Fluchtpunkt die Fragen nach der (De-)Thematisierung von ,Geschlecht' und Geschlechterdarstellungen und dem Fokus auf das Handeln der Akteur_innen innerhalb gesellschaftlicher Rahmenbedingungen und Prozesse darstellt. Diese sind erstens Angelika Wetterers Gegenwartsanalyse der rhetorischen Modernisierung (2006)(vgl. Kapitel 4.3.1), zweitens die Theorie des Verdeckungszusammenhangs des Tübinger Instituts für frauenpolitische Sozialforschung e.V. (2000) (vgl. Kapitel 4.3.2) und drittens Angela McRobbies (2010) feministisch-politische Sichtweise (vgl. Kapitel 4.3.3).

Diese drei Analysefolien gehen in unterschiedlicher Weise davon aus, dass Denk-, Bewertungs- und Handlungsschemata von Individuen im Wechselverhältnis mit gesellschaftlichen Strukturen stehen. Doch erst die Kombination dieser drei Analysefolien ermöglicht es, den Blick auf die ,Bruchstellen` zwischen habitualisiertem Handeln (im Sinne von Geschlechterdifferenz) und rational orientiert Überzeugungen, Vorstellungen und Normen (in Sinne von Geschlechtergleichheit und Gleichberechtigung) zu richten. Der Grund für eine Zusammenführung dieser unterschiedlichen Perspektiven liegt darin, dass sie sich mit derselben Frage bzw. haben dieselbe Problemstellung als Ausgangslage. Im Zentrum der drei Ansätze steht außerdem dieselbe Problematik, die Individua- 
lisierung von gesellschaftlichen Problemlagen, die aufgrund von hierarchischen Geschlechterverhältnissen jenseits verbaler Beteuerungen von Gleichheit und Gleichberechtigung bestehen.

Ziel dieses Kapitels ist es, die Analysefolien zu erläutern und die verschiedenen Perspektiven, aus denen ich gewählte Problemstellungen dieser Arbeit betrachte, darzustellen. Durch die Verwendung verschiedener Perspektiven werden unterschiedliche Aspekte beleuchtet, was in einer umfassenden Rahmenanalyse sowie einer differenzierten Auswertung des empirischen Materials resultiert. Mein Untersuchungszugang ist somit nicht (nur) durch einen theoretischen Blickwinkel geprägt, sondern vielmehr von sich ergänzenden Überlegungen praxeologischer, methodologischer und geschlechterreflektierter Perspektiven.

\subsection{Das Habitus-Feld-Konzept von Pierre Bourdieu}

Die für das Alltagswissen typische Haltung, sich die Welt in Gestalt binären Oppositionen zu erklären, ist in den Sozial- und Geisteswissenschaften wie auch der Frauen- und Geschlechterforschung nicht unbekannt. Hier begegnen einem z. B. Gegenüberstellungen von Mikro- und Makrosoziologie oder von qualitativen und quantitativen Methoden. Die qualitativen Verfahren werden dabei vielfach der Mikro- und die quantitativen der Makrosoziologie zugeordnet. Auffallend ist, dass es sich bei den meisten solcher binärer Oppositionen jedoch nicht um ein Verhältnis komplementärer Gleichgewichtigkeiten handelt, sondern dass stets eine Seite die Relation dominiert bzw. höher gewertet wird. In dieser Asymmetrie finden sich die Mikrosoziologie und die qualitativen Verfahren gewissermaßen auf der, weniger relevanten' Seite wieder (vgl. Meuser 2007, S. 209).

Eine Forschungsperspektive bzw. ein Forschungsansatz, welcher die problematische Unterteilung in Mikro- und Makrosoziologie nicht vornimmt, ist die habitustheoretische Sozialstrukturanalyse Pierre Bourdieus (vgl. Engler 2010, S. 258). Seine Perspektive zeichnet sich dadurch aus, dass er die soziale Praxis von Akteurinnen und Akteuren zum Gegenstand macht; dabei rückt er die AkteurInnen als KonstrukteurInnen ihrer Realität ins Zentrum (ebd.). Bourdieus theoretische Konzepte sind von der Position geprägt, dass Wahrnehmungs- und Bewertungsschemata nicht losgelöst von ihrer sozialen Praxis erfasst werden können. Wissenschaftliche Aussagen, so sein Postulat, kommen ohne Bezug zum so- 
zialen Feld nicht aus. Damit will Bourdieu den herkömmlichen Dualismus von Individuum und Gesellschaft überwinden (vgl. Schlüter/FaulstichWieland 2009, S. 213). Die wissenschaftliche Reflexivität, die seine Arbeiten durchzieht, beinhaltet einen Bruch mit einem Denken in Dualismen (vgl. Engler 2010, S. 258). Das Habitus-Konzept, das Konzept von der symbolischen Gewalt, die Konstruktion des sozialen Raumes und die Vorstellung von sozialen Feldern sind Denkwerkzeuge, die von Bourdieu im Laufe seiner Forschungsarbeit entwickelt wurden, um die soziale Praxis mit ihrer eigenen, praktischen Logik und ihrem praktischen Sinn zu verstehen. Er liefert damit Denkinstrumentarien, die es ermöglichen, das Wirken und die Funktionsweise von Macht- und Herrschaftsverhältnissen in der sozialen Praxis offenzulegen (vgl. ebd., S. 257) und dem Problem der Vermittlung zwischen Selbst- und Weltverhältnissen zu begegnen (vgl. Rosenberg 2011, S. 70).

Vor dem Hintergrund, dass gesellschaftliche Herrschaftsbeziehungen eine enorme Stabilität aufweisen, richtet er seine Aufmerksamkeit auf Verhalten, Wahrnehmung und Denkweisen der Individuen. Bourdieu betont die ständige Wechselwirkung zwischen individuellem Handeln, symbolischer Ordnung und den sozialen Strukturen einer Gesellschaft. Demnach verweist die Analyse individuellen Handelns auf gesellschaftliche Strukturen und vice versa (vgl. Althoff/Bereswill/Riegraf 2001, S. 244). Im Kontext von Bourdieus Denken bedeutet dies, die subjektiven Sichtweisen, quasi die Ordnungsschemata, mit denen Individuen sich die Welt erklären als Verinnerlichung eines Habitus zu verstehen. Der Habitus wird dabei als Ausdruck eines sozialen und kulturellen Prozesses betrachtet, der immer Herrschaftsmechanismen unterliegt. Habitus meint somit nicht nur das Auftreten und Erscheinungsbild von Menschen, sondern beschreibt auch die Besonderheiten des persönlichen Verhaltensstils, die eben gerade nicht individuell, sondern gesellschaftlich geprägt sind (vgl. ebd.). Der Terminus umfasst im Weiteren auch die Strukturen des Denkens, Handelns und Sprechens.

Menschen leben in spezifischen Lebenswelten, die sich aus den objektiven Gegebenheiten und ihrer subjektiven Wahrnehmung (dem nicht hinterfragten Alltag) zusammensetzen. Verschiedene gesellschaftliche Klassen und soziale Gruppen haben jeweils eine eigene Kultur, die in Form von Geschmackspräferenzen und Lebensstilmustern ausgeprägt wird. Diese Kultur ist eng verbunden mit ihrer jeweiligen sozioökonomischen Lage und ihrem bildungskulturellen Kapital, und wird über den Habitus reproduziert (vgl. Bourdieu 1982, S. 8ff). Lebensbedingungen führen also zur Ausbildung spezifischer Lebensstile und werden durch diese 
ständig reproduziert, können aber auch modifiziert werden (vgl. Friebertshäuser 1992, S. 50). Der Habitus wird jedoch nicht wie die soziale Rolle, die als gesellschaftliche Zumutung verstanden werden kann, als ein dem Subjekt von außen angedachtes Bündel von Verhaltensregeln empfunden. Das Konzept der Rolle vermag nämlich die Kohärenz des Handelns einer Person nicht zu erklären, sondern bezeichnet immer nur einen Ausschnitt des gesamten Orientierungs- und Handlungssystems eines Akteurs/einer Akteurin (vgl. Krais/Gebauer 2010, S. 67f.). Der Habitus hingegen gilt als Bestandteil des lebenden Organismus, der entsprechend der Funktionsweise lebender Systeme arbeitet. Mit ihm wird also ein generierendes Prinzip, oder vielmehr ein Komplex von generierenden Prinzipien bezeichnet (vgl. Krais 2001, S. 248).

Im Gegensatz zu Sozialisationstheorien, die von einem wechselseitigen Einwirken von Gesellschaft und Individuum ausgehen, wird bei Bourdieu das Subjekt bereits von vornherein als radikal vergesellschaftlicht konstruiert (vgl. Schlüter/Faulstich-Wieland 2009, S. 212). Unter dieser Perspektive stellt sich also nicht die Frage, wie die Menschen zu gesellschaftlichen Wesen gemacht werden, sondern unter welchen Bedingungen ein Individuum ein bestimmter Teil einer Gesellschaft sein kann und eine spezielle Position einnehmen kann (vgl. Krais/Gebauer 2010, S. 61). Mit der Habitustheorie stellt Bourdieu des Weiteren einen unmittelbaren Zusammenhang zwischen Mikro- und Makroebene her. Er erklärt, wie Verhaltensweisen geordnet sein können, ohne dass ihnen eine Befolgung von Regeln zugrunde liegt (vgl. Bourdieu 1992a, S. 86).

Der Habitus fungiert als ,gesellschaftlicher Orientierungssinn“ (Bourdieu 1982, S. 728); durch ihn werden Wahrnehmungs-, Denk- und Beurteilungsschemata ausgeformt, mit denen Situationen, Handlungen und Gegenstände erkannt, interpretiert und bewertet werden. Dieses Konzept stellt somit ein Bindeglied zwischen Struktur und Handlung dar. Der Habitus bzw. die Habitusformen ${ }^{1}$ sind einerseits als eine Inkorporation sozialer Strukturen zu verstehen und andererseits die Erzeugungsgrundlage sozialer Praktiken (vgl. Kubisch 2008, S. 71). Damit ist es möglich, die komplexen Mechanismen zwischen vorfindlichen Strukturen und deren Reproduktion durch die Individuen als dialektischen Prozess zu begreifen (vgl. Friebertshäuser 1992, S. 50). Der Begriff des Habi-

1 Die deutschen Übersetzung des Plurals von Habitus mit Habitusformen wird von Beate Krais und Gunter Gebauer kritisiert. Sie schlagen vor, im Plural von Ha-

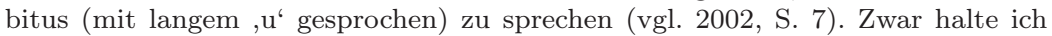
diese Kritik für sinnvoll, dennoch verwende ich, wenn es das Verständnis oder die Sinnhaftigkeit erschweren würde, den Plural der Habitusformen. 
tus, wie ihn Bourdieu verwendet, ist ein wissenschaftliches Konstrukt, welches das soziale Handeln der Subjekte erklärt und prognostizierbar machen soll. Er spricht im Zusammenhang von Habitus auch von einer „strukturierenden Struktur" oder einem „modus operandi“ (Bourdieu 1982, S. 281). Dies verweist auf die oben beschriebene Annahme, dass gleiche Existenzbedingungen oder Lebenslagen zur Erzeugung ähnlicher Habitusformen führen (vgl. Bourdieu 1993a, S. 98).

„In den Dispositionen des Habitus ist somit die gesamte Struktur des Systems der Existenzbedingungen angelegt, so wie diese sich in der Erfahrung einer besonderen sozialen Lage mit einer bestimmten Position innerhalb der Struktur niederschlägt" (Bourdieu 1982, S. 279).

Der Habitus ist somit strukturell bedingt, d. h. er ist abhängig von der Stellung, die ein/e Akteur/in innerhalb der Struktur gesellschaftlicher Relationen innehat. Für Bourdieu findet neben dem Habitus noch eine weitere Form von Objektivierung der Geschichte statt: die Institutionen. Dies erlaubt es:

„Institutionen zu bewohnen (habiter), sie sich praktisch anzueignen und sie damit in Funktion, am Leben, in Kraft zu halten, sie ständig dem Zustand des toten Buchstabens, der toten Sprache zu entreißen, den Sinn, der sich in ihnen niedergeschlagen hat, wieder aufleben zu lassen, wobei er ihnen allerdings die Korrekturen und Wandlungen aufzwingt, die Kehrseite und Voraussetzung dieser Reaktivierung. Besser noch, erst durch den Habitus findet die Institution ihre volle Erfüllung" (Bourdieu 1993a, S. 107).

Hier deutet sich die Verbindung des Habituskonzepts mit Bourdieus Konzepten des sozialen Raums, sozialer Felder und sozialer Klassen an. Von Habitus zu sprechen, hat demnach nur dann Sinn, wenn man ihn auf ein spezifisches Feld bezieht (vgl. Engler 2010, S. 262). Deshalb sollen im Folgenden die Mechanismen und Funktionsweisen des sozialen Feldes dargestellt werden, auf welches diese Studie fokussiert.

\subsubsection{Die Universität als konjunktiver Erfahrungsraum}

Bourdieu spricht vom sozialen Raum und den sozialen Feldern, wobei das Verhältnis zwischen beiden nicht immer eindeutig bestimmbar ist. Mit sozialem Raum meint er in der Regel die Struktur der ganzen Gesellschaft. Unter sozialen Feldern hingegen versteht er Ausdifferenzierungen 
wie beispielsweise das Feld der Kunst, der Politik oder der Wissenschaft ${ }^{2}$ (vgl. Kubisch 2008, S. 71). Wie das Habituskonzept ist auch das Konzept des sozialen Feldes ein offenes, das ausgehend von und für systematische, empirische Anwendungen entwickelt wurde, um zeitlich und räumlich bestimmbare Realitäten zu erforschen. Im Verständnis von Bourdieu bilden jedoch nicht Frauen und Männer als Einzelwesen den Ausgangspunkt von Untersuchungen, sondern Relationen als Realisierungen des historischen Handelns. Dieses relationale Denken kommt ohne einen Bezug auf soziale Felder nicht aus. Die Wahrnehmungs- und Bewertungsschemata der AkteurInnen verfügen über keine abstrakte und universelle Gültigkeit und sind immer verwoben mit der sozialen Praxis (vgl. Engler 2010, S. 258).

Der Begriff des Feldes dient außerdem zur Erinnerung, dass das eigentliche Objekt einer Untersuchung nicht das Individuum ist, auch wenn man ein Feld nur von den Individuen aus konstruieren kann. Aus dieser Perspektive ist ein soziales Feld zu verstehen als ein Netz oder eine Konfiguration von objektiven Relationen zwischen Positionen, die von AkteurInnen eingenommen werden. Diese sind verschieden, wobei gerade diese Heterogenität Bestandteil des Konzepts ist. Dabei werden soziale Felder als Kräftefelder imaginiert, deren Dynamik in den Beziehungen der AkteurInnen zueinander liegt. Dies gilt auch für die Hochschule in ihrer Rolle als Bildungsinstitution, die in dieser Arbeit als relativ autonomes Feld aufgefasst wird (vgl. Engler 2010, S. 262). Beate Krais und Gunter Gebauer haben fünf Aspekte hervorgehoben, die das soziale Feld auszeichnen:

(1) Es wird erstens als Kräftefeld gedacht, in dem es um einen spezifischen Einsatz geht und die AkteurInnen nach einer eigenen, feldspezifischen Logik handeln. Die Vorstellung des sozialen Feldes als ein Kräftefeld bedeutet nichts anderes, als dass sich z. B. eine Musikerin/ ein Musiker und eine Bankerin/ ein Banker in unterschiedlichen Feldern bewegen, und dass sie, wenn sie innerhalb dieser Felder eine Karriere anstreben, über diverse Vorzüge und Kompetenzen verfügen müssen. In beiden Fällen handelt es sich jedoch um ein ,Spiel', bei dem es den ,SpielerInnen', den sozialen AkteurInnen, um die Vorrangstellung geht. Ein soziales Feld ist ein nach einer eigenen Logik funktionierendes ,Spiel ${ }^{6}$ um Macht und Einfluss (vgl. Krais/Gebauer 2010, S. 56.).

2 Vor allem in der Frauen- und Geschlechterforschung wird das Feld-Konzept von einigen AutorInnen als theoretischer Zugang für empirische Forschung genutzt (vgl. Engler 2010, S. 262f.). Hierbei wird wiederholt die Hochschule als relativ autonomes Feld gefasst und AkteurInnen, die unterschiedliche Positionen einnehmen, ins Zentrum gerückt (z. B. Engler 1993; 2001; Hasenjürgen 1996; Schlüter 1999; Zimmermann 2000; Beaufays 2003). 
(2) Ein soziales Feld zeichnet weiterhin aus, dass es Personen gibt, die eine bestimmte Dimension gesellschaftlicher Praxis zu ihrem Beruf gemacht haben. Um beim Beispiel von der Bankerin/ dem Banker und der Musikerin/ dem Musiker zu bleiben, heißt das, dass das soziale Feld der Bankerin/ des Bankers immer die Finanzwelt ist, auch wenn sie/ er abends (zum Vergnügen) musiziert. Aufgrund ihrer/ seiner Freizeitbeschäftigung teilt sie/ er nicht das soziale Feld einer Berliner Philharmonikerin/ eines Berliner Philharmonikers, weil ihr/ sein Musik-Spiel „gewissermaßen außer Konkurrenz" läuft (ebd., S. 56).

(3) Drittens bedeutet das Konzept des sozialen Feldes die Vorstellung von einer inneren Homogenität des Systems. Da das Feld als Kräftefeld gedacht wird, ist von vornherein unterstellt, dass die ,Spieler_innen' in relevanten Merkmalen verschieden sind, ja diese Verschiedenheiten geradezu konstitutiv für das soziale Feld sind. An einer Auseinandersetzung um Macht und Einfluss in einem sozialen Feld kann sich nur beteiligen, wer im prinzipiellen Sinn als gleich anerkannt wird, doch sind die Gleichen wiederum keineswegs identisch. Es gibt sehr viele unterschiedliche AkteurInnen mit differenten Positionen, diese sind nichts anderes als die Verkörperung oder Personifizierung von Strukturen. Wird das wissenschaftliche Feld betrachtet, lassen sich Positionen bzw. Personen am Rande sowohl als auch im Zentrum des Feldes erkennen. Wie im sozialen Raum so gilt auch hier: Das Feld definiert einen Raum des Möglichen. Krais und Gebauer beschreiben die damit einhergehende Beweglichkeit des Feldes als: das Gewicht der einzelnen AkteurInnen bzw. Positionen durch ihre objektive Beziehung zu den anderen AkteurInnenen bzw. Positionen, ,es ist also, da es zu jedem gegebenen Zeitpunkt das Resultat vorangegangener Auseinandersetzungen ist, nicht ein für alle Mal gegeben, sondern verändert sich" (2010, S. 58).

(4) Ein viertes Charakteristikum des sozialen Feldes ist die Analogie zum Spiel. Jedes soziale Feld ist mit einer eigenen Logik ausgestattet, so wie auch jedes Spiel eigene Spielregeln besitzt. Allerdings ist das ,Spiel im sozialen Feld tiefer Ernst; das ist zwar rein metaphorisch zu verstehen, dennoch geht es auch um die soziale Existenz der AkteurInnen.

„Es ist ein ,Spiel“ um Macht und Einfluss, um die Durchsetzung der eigenen Sichtweisen im Horizont des in diesem Felde Möglichen; und jeder, der sich einlässt auf dieses ,Spiel', muss den Glauben an das Feld haben, die ,illusio', die Identifikation mit dem ,Spiel' die Bedingung dafür ist, dass man mitspielen kann" (Krais/Gebauer 2010, S. 58). 
Für das wissenschaftliche Feld bedeutet dies, dass wer Wissenschaft zu ihrem/seinen Beruf machen will, sich eine Position erkämpfen möchte, zunächst den Glauben haben muss, dass sich der Einsatz lohnt. Auch bei diesem Charakteristikum zeigt sich die Veränderbarkeit, denn die Metapher des Spiels verweist darauf, dass sich dieses nicht über ein fixiertes Gerüst von Positionen definiert und stagniert, sondern sich über die Praxis der AkteurInnen konstituiert. Die Konstellation der AkteurInnen verändert sich und somit auch ihr Gewicht im relationalen Gefüge, neue Konventionen bilden sich heraus und das ,Spiel' wird anders gespielt. So verschieden die Positionen bzw. die AkteurInnen auch sein können, sie haben den praktischen Glauben an das ,Spiel', die ,illusio“ gemeinsam(vgl. ebd., S. 58f.). Es gilt jedoch,

„wie bei den von Klassenlage und ,Geschlecht' geprägten Erfahrungen, die sich im Habitus einlagern, dass auch die mit dem Eintritt in ein spezifisches Feld und mit der Teilnahme an dem dort gespielten ,Spiel' entwickelten Aspekte des Habitus den Akteuren zur ,Natur' geworden sind. Dadurch bleibt unerkannt, wie die Logik des Feldes überhaupt funktioniert" (ebd., S. 60).

(5) Fünftens ist mit dem Konzept des sozialen Feldes die Vorstellung einer Entsprechung zwischen den verschiedenen Positionen der Akteure im sozialen Feld und den inhaltlichen Positionen verbunden. Bourdieu spricht hier von Homologien, einer Vorstellung, die vergleichbar ist mit der Entsprechung zwischen den Positionen im sozialen Raum und den verschiedenen Lebensstilen. Das heißt, dass z. B. die Wahl einer literarischen Gattung als Unterhaltungslektüre an die Stellung des Akteurs oder der Akteurin im Feld gebunden ist. So hängt die Geltung einer wissenschaftlichen Position, die Wertschätzung einer wissenschaftlichen Leistung, an der sozialen Anerkennung der Person, die diese Leistung im sozialen Feld Wissenschaft hervorgebracht hat. In diesem Sinne passiert die Durchsetzung wissenschaftlicher Denkstile, Theorien etc. nicht von alleine, sondern ist gebunden an die Aufgabe der jeweiligen Protagonistin, sich in ihrer scientific community durchzusetzen. Dazu ist nicht nur Fachwissen wichtig, sondern auch die dementsprechende Behauptung im sozialen Geschehen, mit der die Wissenschafts-(Re-)produktion untrennbar verbunden ist (vgl. ebd.).

Das Feld-Habitus-Konzept von Bourdieu mag strukturalistisch und deterministisch erscheinen, da, wie er es formuliert, die Struktur über den Habitus, den sie erzeugt hat, die Praxis regiert (vgl. Bourdieu 1993a, S. 102). Denn über den Habitus werden, soziale Strukturen von einzelnen Individuen inkorporiert, die dann in ihrem Handeln diese Struktu- 
ren wieder reproduzieren. Allerdings geschieht dies „nicht in den Gleisen eines mechanischen Determinismus, sondern über die Einschränkungen und Grenzen, die seinen Erfindungen von vornherein gesetzt sind" (ebd., 102f.). Bourdieu hebt somit hervor, dass der Habitus lediglich die Grenzen möglicher Praktiken aufzeigt, die aus unterschiedlichen Chancen resultieren und innerhalb derer das Handeln durchaus variiert werden kann. Die Formulierung der „geregelte(n) Improvisationen“ (Bourdieu 1976, S. 170 zit. n. Kubisch 2004, S. 73) umschreibt besonders treffend die Verbindung aus Determination und Freiheit im Begriff des Habitus. Bei den Akteuren und Akteurinnen entsteht der praktische Glaube an das Feld durch die Teilnahme am ,Spiel' ${ }^{6}$ Teilnahme am ,Spiel ${ }^{6}$ heißt, dass man unzählige Akte der Anerkennung erbringt, man in das ,Spiel ${ }^{6}$ investiert, sich anstrengt, alles richtig zu machen, indem man versucht, zu verstehen wie es funktioniert, erkennt, welche Positionen wichtig sind und welche expliziten und impliziten Regeln gelten etc. Soziale Felder sind komplexe ,Spiele und erfordern oftmals ein Durchlaufen langwieriger Prozesse des Lernens, welche häufig eingebunden sind in MeisterInnen-SchülerInnen-Verhältnisse. Dieses Eingebunden-Sein in die Praxis des ,Spiels ${ }^{6}$ ist ein zentraler Aspekt bei der Ausbildung des Habitus. Das Lernen durch Mitmachen, durch Ausprobieren und Einüben, aber auch durch körperliches Handeln, spielt eine zentrale Rolle, um als Mitglied des sozialen Feldes, des ,Spiels', anerkannt zu werden (vgl. Krais/Gebauer 2010, S. 62). Richtlinien dienen dabei der Orientierung in der sozialen Welt wie auch der Hervorbringung sozial angemessener Praktiken und bilden damit die Grundlage des sense pratique ${ }^{3}$ (vgl. Kubisch 2008, S. 72).

Strukturiert sind soziale Felder wie auch der soziale Raum entsprechend der Verteilung der Kapitalsorten, wobei in den verschiedenen Feldern eine je spezifische Zusammensetzung des Kapitals gefragt ist. Der Habitus und das soziale Feld sind Konzepte, die sich komplementär aufeinander beziehen und unterschiedliche Formen der Objektivierung von Geschichte in der sozialen Realität darstellen. In Verbindung der verschiedenen Bestandteile seiner Theorie bringt Bourdieu zum Ausdruck, dass soziale Strukturen durch Ungleichheit gekennzeichnet sind, dass also die Handlungsmöglichkeiten der Akteure unterschiedlich sind, was sich auch im Habitus niederschlägt (vgl. Kubisch 2008, S. 71). Ähnliche

Im Original lautet der Titel Bourdieus Buch „Sozialer Sinn“ (1993), ,Le sens pratique“ (1980). Diese Übersetzung von ,sense pratique“ mit ,sozialem Sinn“ kritisiert Michael Meuser als unzureichend, da die Dimension des praktischen Verstehens in der deutschen Übersetzung nur verkürzt vermittelt wird (vgl. 2007, S. 212). 
Existenz- oder Bildungsbedingungen, d. h. auch vergleichbare Verfügbarkeiten der verschiedenen Kapitalsorten, resultieren in ähnlichen Habitusformen. Sie bilden die Grundlage für die aufeinander abgestimmten Praktiken derjenigen, die derselben Gruppe oder Klasse zugerechnet werden. Bourdieu betont, dass aufeinander abgestimmte Praktiken ohne jede direkte Interaktion oder Abstimmung zwischen den Akteur_innen zustande kommen können. Die Art und Weise der Ausführung von Handlungen steht hierbei im Mittelpunkt. In verschiedenen sozialen Feldern sind unterschiedliche Dinge relevant, die wiederum bestimmte Praktiken und praktische Wahrnehmungs- und Bewertungsschemata erzeugen und somit eine spezifische Logik bewirken, welche das Besondere des Funktionierens eines Feldes jeweils ausmacht (vgl. Bourdieu 1982, S. 262).

Auch die Positionierung im sozialen Feld stellt eine wesentliche Grundlage für (De-)Thematisierung von ,Geschlecht' und Geschlechterfragen und die Ausbildung eines entsprechenden Habitus dar. Dies zeigt auf, wie ein Verständnis davon entwickelt werden kann, in welcher Form und nach welchen Spielregeln das Wechselverhältnis zwischen Individuum und Gesellschaft operiert. Demnach werden Werthaltungen, Weltanschauungen und Handlungsorientierungen erfasst, die in ähnlichen sozialen Lagerungen und gemeinsamen Erfahrungen gründen und die sich also auch auf die (De-)Thematisierung von ,Geschlecht' und Geschlechterfragen beziehen können.

Das soziale Feld der Bildungsinstitution stellt somit - nach dem Verständnis von Karl Mannheim (1980) und Ralf Bohnsack (2006) - für die befragten Studierenden einen konjunktiven Erfahrungsraum dar. Konjunktive Erfahrungsräume fußen auf gleichartigen bzw. ähnlichen Erfahrungen, die von den Mitgliedern dieser Erfahrungsräume geteilt werden und für diese daher selbstverständlich sind. Sie entstehen in verschiedenen Dimensionen: zum einen in konkreten Gruppen, wie peergroups von Jugendlichen oder Studierenden oder in Nachbarschaften; zum anderen gibt es aber auch in größeren organisationalen Kontexten wie beispielsweise Bundeswehr, Kirche, Parteien, Sportvereinen oder Bildungsinstitutionen wie der Universität Dimensionen konjunktiver Erfahrung. Diese Erfahrungen werden von allen, die sich darin bewegen, geteilt, ohne dass ein direkter Kontakt oder Austausch stattfindet. Und Gleiches gilt eben auch in Bezug auf noch größere soziale Entitäten wie das ,Geschlecht'. In solchen konjunktiven Erfahrungsräumen bilden sich kollektive Erfahrungsaufschichtungen, die sich verdichten und synthetisieren zu Herkunfts- und Bildungsmilieus, zu Geschlechts- und Generationszusammenhängen, zu auf Ethnizität oder organisationale Zugehörigkeit basie- 
renden Milieus. Konjunktive Erfahrungsräume treten niemals allein auf, sondern in vielfältigen Verflechtungszusammenhängen, weshalb jedes Individuum eine je spezifische biografische aufgeschichtete Mischung dieser Erfahrungsdimensionen in sich vereinigt. Deshalb ist bzw. erscheint ein Individuum für sich selbst und für andere unverwechselbar, verkörpert jedoch auch jeweils Typisches in sich (vgl. Schäffer 2012, S. 142).

\subsubsection{Gesellschaftliche Strukturen und Individuum im Wechselverhältnis}

Die Theorie Bourdieus und das Konzept sozialer Milieus zeigen auf, wie ein Verständnis davon entwickelt werden kann, dass Prozesse auf der Ebene von Identität und Interaktion notwendigerweise im Wechselverhältnis stehen und umgekehrt Gesellschaft nicht jenseits des Erlebens der Akteure anzusiedeln ist (vgl. Bohnsack 1998). Über den Begriff des sozialen Milieus bzw. der konjunktiven Erfahrung lassen sich demnach Werthaltungen, Weltanschauungen und Handlungsorientierungen erfassen, die auf ähnlichen sozialen Lagerungen und gemeinsamen Erfahrungen gegründet sind und die sich also auch auf ,Geschlecht' beziehen können. Pierre Bourdieu bezeichnet seinen Ansatz als ,strukturalistischen Konstruktivismus“ oder „konstruktivistischen Strukturalismus“ (1992, S. 135) und sieht es als sein wichtigstes Ziel an, den Gegensatz zwischen einer objektivistischen Position und einer subjektivistischen oder phänomenologischen Position zu überwinden:

„Mit dem Wort ,Strukturalismus' oder ,strukturalistisch' will ich sagen, dass es in der sozialen Welt selbst - und nicht bloß in den symbolischen Systemen, Sprache, Mythos usw. - objektive Strukturen gibt, die vom Bewusstsein und Willen der Handelnden unabhängig und in der Lage sind, deren Praktiken und Vorstellungen zu leiten und zu begrenzen. Mit dem Wort ,Konstruktivismus' ist gemeint, dass es eine soziale Genese gibt einerseits der Wahrnehmungs-, Denk- und Handlungsschemata, die für das konstitutiv sind, was ich Habitus nenne, andererseits der sozialen Strukturen und da nicht zuletzt jener Phänomene, die ich als Felder und als Gruppen bezeichne, insbesondere die herkömmlicherweise so genannten sozialen Klassen" (ebd.).

Die Wahrnehmungsschemata strukturieren die alltäglichen Wahrnehmungen; die Denkschemata umfassen Alltagstheorien, Klassifikationsmuster, implizite ethische Normen sowie ästhetische Maßsstäbe im Sinne des Geschmacks. Die Handlungsschemata im sozialen Feld beeinflussen 
die individuelle wie kollektive Praxis. Wenn man nun die Art und Weise der (De-)Thematisierung von ,Geschlecht' als Werthaltungen, Weltanschauungen und Handlungsorientierungen versteht, wird deutlich, dass es neben individuellen Erfahrungen und Erlebnissen auch kollektive Erfahrungen und gesellschaftliche Strukturen und Diskurse gibt, die Vorstellungen und Praktiken leiten. Bourdieu geht davon aus, dass eine ähnliche Positionierung innerhalb sozialer Felder damit einhergeht, dass ähnliche Wahrnehmungs-, Bewertungs- und Handlungsschemata im Sinne eines Habitus ausgebildet werden. Als Beweis führte Bourdieu beispielsweise Untersuchungen zu Nahrungsmittelkonsum, kulturellen Präferenzen und Wohnverhältnissen in Abhängigkeit von verschiedenen Berufen bzw. Klassen durch. Die Positionierung im sozialen Raum bildet die wesentliche Grundlage für die Ausbildung eines entsprechenden Habitus, der wiederum eine wichtige Erklärung für die verschiedenen Lebensstile bietet (vgl. Kubisch 2008, S. 63).

Hierfür stellt Bourdieus Klassenkonzept eine Erklärung dar. Dafür unterscheidet er zwischen vier verschiedenen Kapitalformen, anhand derer sich die Klassenposition bestimmen lässt: das ökonomische, das kulturelle, das soziale und schließlich das symbolische Kapital, wobei Letzteres im Vergleich zu den anderen drei Kapitalarten einen anderen Stellenwert hat (vgl. zum Folgenden Bourdieu 1983). Grundsätzlich versteht Bourdieu Kapital als ,akkumulierte Arbeit", die materialisiert oder verinnerlicht, d. h. ,inkorporiert" wird (ebd., S. 183).

Ökonomisches Kapital: Damit meint er die individuell wie auch kollektiv akkumulierbare Aneignung von materiellen Dingen: vor allem Geld, aber auch Güter, die unmittelbar in Geld konvertierbar sind.

Kulturelles Kapital: Kulturelles Kapital kann in drei verschiedenen Formen existieren: in inkorporiertem, objektiviertem und institutionalisiertem Zustand. Inkorporiertes Kulturkapital meint, dass Bildung angeeignet und verinnerlicht wird, d. h. in den Habitus einer Person übergeht. Während das inkorporierte Kapital damit an die jeweilige Person gebunden ist, lässt sich objektiviertes Kulturkapital in Form von Gemälden, Instrumenten und Ähnlichem ebenso gut übertragen wie ökonomisches Kapital. Nicht übertragbar ist allerdings die kulturelle Fähigkeit ein Gemälde zu genießen wozu es wiederum des inkorporierten Kulturkapitals bedarf. Akademische Titel, mit denen inkorporiertes Kapital anerkannt (vgl. Kubisch 2008, S. 61) wird, fasst Bourdieu als institutionalisiertes Kulturkapital zusammen. 
Soziales Kapital: Beim sozialen Kapital handelt es sich „um Ressourcen, die auf der Zugehörigkeit zu einer Gruppe beruhen" (Bourdieu 1983, S. 190f.). Er bezeichnet damit die Gesamtheit der aktuellen und potentiellen Ressourcen, die mit dem Besitz eines dauerhaften Netzes von mehr oder weniger institutionalisierten Beziehungen, gegenseitigen Kennens oder Anerkennens verbunden sind (vgl. ebd.). Entscheidend für das eigene soziale Kapital ist hierbei auch, in welchem Umfang die anderen, zu denen man soziale Beziehungen pflegt, über ökonomisches, kulturelles und symbolisches Kapital verfügen.

Symbolisches Kapital: Schließlich ist mit dem symbolischen Kapital jene Form gemeint, die die verschiedenen Arten von Kapital dann annehmen, wenn sie als legitim erkannt und anerkannt werden. Das symbolische Kapital unterscheidet sich insofern wesensmäßig von den anderen Kapitalarten, weil es nicht aus akkumulierter Arbeit besteht und nicht als Investitionsmittel zu verstehen ist. Es steht über den anderen Kapitalarten, weil es dazu dient, diese zu legitimieren (vgl. Kubisch 2008, S. 61).

Indem man nun Kapitalvolumen (Menge des insgesamt zur Verfügung stehenden Kapitals) und Kapitalzusammensetzung (Verhältnis der verschiedenen Kapitalsorten) auf zwei Achsen eines Koordinatensystems anordnet, erhält man ein Bild des Raums der sozialen Positionen, d. h. eines der beiden Aspekte des sozialen Raums. Hierbei werden auch soziale Laufbahnen berücksichtigt, d. h. Bewegungen einzelner Gruppen oder Klassen innerhalb des sozialen Raums im Zeitverlauf, die mit einer Zu- oder Abnahme des Kapitalvolumens oder einer Veränderung der Zusammensetzung der Kapitalarten verbunden sind. Die verschiedenen Positionen sind der Vorstellung Bourdieus zufolge aufeinander bezogen, d. h. relational. Innerhalb des sozialen Raums können entsprechend der so analysierten Struktur verschiedene Klassen sichtbar gemacht werden (vgl. Bourdieu 1998). Wichtig ist, dass Bourdieu die Klassen als konstruierte bzw. als theoretische Klassen bezeichnet und somit deutlich macht, dass es sich um ein wissenschaftliches Konstrukt handelt, das von tatsächlich mobilisierten Klassen zu differenzieren ist. Vom Raum der Positionen kann der Raum der Lebensstile unterschieden werden (vgl. Kubisch 2008, S. 62). Diese Überlegungen sind für meine Arbeit wichtig, da ich die These vertrete, dass die (De-)Thematisierung von ,Geschlecht in Abhängigkeit zum sozialen Raum und dem sozialen Feld des Studiums und gesellschaftlicher Diskurse steht. 


\subsubsection{Die Habitustheorie: Habituelle Praxis als stärkste Form des Handelns}

Mit dem Habituskonzept versucht Pierre Bourdieu, den Dualismus von objektivistischer oder rein subjektivistischer Perspektiven zu überwinden. Bezogen auf das Vermittlungsproblem von Selbst- und Weltverhältnissen geht es ihm darum, einerseits nicht von einem ausschließlich selbstbestimmten Subjekt auszugehen, andererseits trotzdem nicht eine vollständig gesellschaftliche Determination anzunehmen (vgl. Rosenberg 2011, S. 70). Formen des Habitus sind für Bourdieu, ,Systeme dauerhafter Dispositionen, strukturierte Strukturen, die geeignet sind, als strukturierende Strukturen zu wirken, mit anderen Worten: als Erzeugungs- und Strukturierungsprinzip von Praxisformen und Repräsentationen" (vgl. Bourdieu 1976, S. 165). Bourdieu verweist gelegentlich auf die Bedeutung der Sozialisation für die Herausbildung des Habitus und dass die Grundlagen der Habitusformen in der primären Sozialisation liegen. ${ }^{4}$ Er betont, dass der Habitus im Laufe der Sozialisation erworben wird. Als solcher ist dieser ein Produkt von individuellen und kollektiven Erfahrungen und fungiert als ein Generierungsprinzip für verschiedene Ordnungsschemata. Dabei ist der Habitus nicht als natürliche Kompetenz zu verstehen - im Sinne einer angeborenen Fähigkeit - sondern vielmehr gesellschaftlich, kulturell und historisch bedingt, also sozial produziert (vgl. Rosenberg 2011, S. 71).

Der Habitus bildet sich von frühster Kindheit an aus. In kontinuierlichen Lernprozessen prägen die Auseinandersetzung mit der Welt und in der Interaktion mit anderen den Rhythmus von Konstituierung und Modifikation des Habitus. Daher ist der Habitus dauerhaft, aber nicht gänzlich unveränderbar (vgl. Krais/Gebauer 2010, S. 61). Einerseits gewährleistet der Habitus die aktive Präsenz früherer Erfahrungen, andererseits operiert er unbewusst, da die Genese der habituellen Dispositionen vergessen wird (vgl. Kubisch 2008, S. 71). Durch Nachahmungen des Handelns anderer, d. h. also mimetisches Lernen (vgl. Krais/Gebauer 2010, S. 62), als praktisches, körperlich-sinnliches Tun in der Interaktion mit und durch die Wiederholung bestimmter Handlungen prägen sich Schemata ein und werden habitualisiert.

„Der Habitus funktioniert nicht mechanistisch, sondern nach dem Modell lebender Systeme. Auf Lernprozesse bezogen bedeutet dies, dass Gelerntes verarbeitet wird. Danach hat man sich den

4 Bourdieus Gegenstand sind jedoch die Sozialisationsergebnisse und nicht die -prozesse (vgl. Meuser 2006, S. 165). 
Vorgang der Aneignung des Sozialen - wie überhaupt Lernprozesse - nicht so vorzustellen, als sei das Individuum eine Art Schrank, in dessen Fächer und Schubladen nun soziale Ordnungen, Vorstellungen, Klassifikationsschemata, Wissensbestände und so weiter einsortiert würden, gerade so, wie sie in der sozialen Wirklichkeit außerhalb des Individuums vorkommen. Vielmehr wird die Fülle der einzelnen Erfahrungen, die Menschen auf Grund ihrer Tätigkeit in der Welt machen, zu einem komplexen Erfahrungswissen zusammengearbeitet und immer wieder transformiert" (Krais/Gebauer 2010, S. 63).

Erworben wird der Habitus in der und durch die Teilnahme an der Praxis. Dies geschieht weitgehend unabhängig von intentionalen pädagogischen Bemühungen und außerhalb der Institutionen des Bildungsund Erziehungssystems. Es ist ein praktisches, vorreflexives Lernen, in dem nicht (theoretische) Modelle, sondern die Handlungen der anderen nachgeahmt werden (vgl. Meuser 2006, S. 165). Auch Strukturübungen sind eine Form des vorreflexiven Lernens in Gestalt eines praktischen, körperlich-sinnlichen Tuns in der Interaktion mit anderen (vgl. ebd.). Der Begriff der Strukturübung fokussiert auf die aktiven Leistungen der Subjekte, gleichzeitig begreift er den Sozialisationsprozess als bestimmt durch die Strukturen, die angeeignet werden. Sozialisation bedeutet in diesem Verhältnis die Aneignung „einer spezifischen Position im sozialen Raum" (Krais/Gebauer 2010, S. 61). Im Sinne der Habitustheorie ist dies als eine aktive Leistung zu verstehen. Strukturübungen sind ein Modus unbewusster, vortheoretischer Aneignungen der sozialen Welt. Hier werden Struktur- und Ordnungsprinzipien erworben und inkorporiert. Mit den Strukturübungen entsteht qua Teilnahme ,der praktische Glaube an das Feld" (ebd., S. 62). Mit dieser Teilnahme erkennen die AkteurInnen das ,Spiel' selbst und dessen Regeln an und lernen eine Affinität zum ,Spiel'. In den Strukturübungen wird ein (feld-)spezifischer praktischer Sinn erworben (vgl. Meuser 2006, S. 174).

In modernen und differenzierten Gesellschaften treffen Individuen vermehrt auf Situationen, die sich von bereits erlebten unterscheiden. Sie müssen ihre Praktiken variieren, und diese neuen Erfahrungen modifizieren dann gegebenenfalls den Habitus. Das bedeutet, dass die Fülle der einzelnen Erfahrungen, die Menschen auf Grund ihrer Tätigkeit in der Welt machen, zu einem komplexen Erfahrungswissen zusammengefügt und dieses immer wieder transformiert wird (vgl. Krais/Gebauer 2010, S. 63). Der Habitus eröffnet einerseits in seiner Variationsbreite einen unendlich großen Möglichkeitsraum für die Generierung ver- 
schiedener Schemata, ist andererseits aber durch die gesellschaftlichen Strukturen stark eingegrenzt. Bei dem Eintritt in ein neues soziales Feld ist ein Habitus gefragt, der kongruent und lernfähig ist, sowie Aussicht auf Restrukturierungspotential bietet (vgl. ebd., S. 61f.). Diese komplexen und widersprüchlichen Strukturen der Gesellschaft bilden zugleich eine Art Sprengsatz im Habitus der Subjekte, die die Selbstverständlichkeit der Praxen immer wieder ein Stück in Frage stellen. Nimmt man allein die beiden grundlegenden Dimensionen sozialer Ungleichheit unserer Gesellschaft, Klasse und ,Geschlecht', dann wird deutlich, dass im Zusammenwirken dieser beiden Größenordnungen eine Fülle von Reibungspunkten und heterogenen Erfahrungen angelegt sind. So erlernen zwei Mädchen, einmal aus bürgerlicher Herkunft, einmal aus einer Arbeiterfamilie, unterschiedliche Sichtweisen und machen unterschiedliche Erfahrungen. Sie erleben unterschiedliche soziale Ordnungen. Die Selbstverständlichkeit der sozialen Ordnung, die Doxa, wird in konflikthaften Erfahrungen leicht brüchig (vgl. ebd., S. 72).

„Immer wieder werden Handlungsweisen und Interaktionen aus dem Fraglosen, Selbstverständlichen herausgerissen, werden $\mathrm{Zu}$ sammenhänge bewusst, gibt es Anstöße zum Nachdenken über das eigene Verhalten, über den eigenen Ort in der Welt und Ansätze zur Auflehnung oder der bewussten Auseinandersetzung mit der sozialen Ordnung, mit einem Wort, immer wieder erfahren sich die Individuen in ihrer Praxis als reflektierende, bewusst handelnde Subjekte“" (Krais/Gebauer 2010, S. 73).

Die Schwierigkeiten bei der Umsetzung von Gleichheit und Gleichberechtigung im Geschlechterverhältnis zeigt jedoch auch, dass wir aufgrund der durch unseren Habitus gegebenen Haltung zur Welt trotzdem in den sozialen Zusammenhang eingebunden bleiben. Gleichzeitig hat der Habitus eine Neigung, nach Bedingungen zu suchen, die denen seiner Erzeugung entsprechen, weil er für sie gut gerüstet ist (vgl. ebd.).

Bei einer gravierenden Änderung der sozialen Umstände zeigt sich der Habitus daher vorerst äußerst träge, es kommt lediglich zu einer geringen Passung von Struktur und Handeln (vgl. Kubisch 2008, S. 73). Bourdieu bezeichnet diese Trägheit des Habitus als „Hysteresis“ (Bourdieu 1993a, S. 116). Das heißt, der Habitus bleibt in gewohnten, überholten Erfahrungen und Existenzbedingungen verhaftet und erachtet die neuen Verhältnisse als nicht adäquat (vgl. Krais/Gebauer 2010, S. 21). Die Zähigkeit und die Trägheit einmal erworbener Sicht- und Handlungsweisen und eingeübter sozialer Praxen sowie Strategien werden besonders beim Wechsel in ein anderes soziales Feld bedeutsam. Denn gerade hier 
kann der Habitus Konflikte und Probleme dadurch produzieren, dass Dispositionen und Praxen hervorgebracht werden, die im neuen Feld als unerwünscht bzw. als sozial abweichende Verhaltensweisen gelten. $\mathrm{Zu}$ Veränderungen des Habitus kann somit auch der Wechsel zwischen sozialen Feldern, wie sie sich beispielsweise durch Bildungslaufbahnen ergeben, führen (vgl. Friebertshäuser 1992, S. 52). Auch Florian von Rosenberg sieht in der Relation zwischen Habitus und Feld eine Möglichkeit der Habitustransformation (vgl. 2011). Ein statisches, nicht auf die Probe gestelltes Anpassungsverhältnis zwischen Habitus und Feld erscheint hier unwahrscheinlich (vgl. Rieger-Ladich 2005, S. 289). Durch den Kontakt unterschiedlicher Erfahrungsräume und damit verschiedener Praxisformen und neuer Logiken im anderen Feld kann somit auch die Logik der Praxis innerhalb eines Habitus tangiert werden. Neben dem Anschluss an neue Felder besteht auch durch immer wieder reproduzierende Inkongruenzen zwischen Habitus und Feld die Möglichkeit einer Habitustransformation, aber auch die Transformation einer Feldstruktur (vgl. Rosenberg 2011, S. 81). Rosenberg konstatiert, „dass ein Habitus durch die Aspekte von Mehrdimensionalität, Iterabilität und Inkongruenz in seinem Bezug zu Feldern in dreifacher Weise als nicht abgeschlossen oder, besser, als per se nicht abschließbar gelten kann" (ebd.). Wie jedoch bereits zuvor bemerkt wurde, verändern sich bestehende Strukturen, wie auch Menschen, nur mit einer gewissen Trägheit, das bedeutet, dass sich der Habitus veränderten Bedingungen nur langsam anpasst. Gleichzeitig unterliegen auch bestehende Strukturen einem gewissen Trägheitseffekt, der Veränderungen nur langsam zulässt (vgl. Engler 2010, S. 262).

Bislang ist deutlich geworden, dass in den Habitus die Wahrnehmungsund Bewertungsschemata, die Prinzipien des Denkens, Fühlens und Handelns eingehen, die in einer Gesellschaft wirken. Anders formuliert: Wir bringen unsere je eigene und besondere Sichtweise der sozialen Welt hervor, aber wir tun dies nach Schemata, die wir nicht selbst erfunden haben, sondern die in uns und in der Welt enthalten sind (vgl. ebd., S. 260). Es bestehen also Homologien zwischen gesellschaftlichen Strukturen und habituellen Dispositionen (vgl. Bourdieu 2005, S. 260), d. h. Individuen und die sie umgebende Welt stellen sich in der sozialen Praxis gegenseitig her. Das bedeutet weiter, dass die Macht- und Herrschaftsverhältnisse den AkteurInnen nicht von außen oktroyiert werden, sondern dass die symbolische Ordnung der sozialen Welt in Form von Klassifikationssystemen in den Dingen, Institutionen, Gegenständen und u. a. in den Köpfen präsent ist. Dies betrifft auch das Klassifikationsschema ,Geschlecht', 
welches als weiblich und männlich, als binärer Gegensatz konstruiert ist (vgl. Engler 2010, S. 260). Bourdieu verwendet den Habitus als einen Operator, in den die zweigeschlechtliche Weltsicht eingeht und der zur zweigeschlechtlichen Ein- und Aufteilung der sozialen Welt führt. ${ }^{5} \mathrm{Er}$ bezeichnet ihn daher auch als ,vergeschlechtlichten und vergeschlechtlichenden Habitus“" (vgl. Bourdieu 1997a, S. 166).

,Geschlecht" wird dabei als eine Dimension des Sozialen verstanden, als eine Dimension der Hervorbringung sozialer Wirklichkeit durch die Ein- und Aufteilung der sozialen Welt, wie sie von AkteurInnen vorgenommen wird. Als vergeschlechtlichte, in den Habitus eingelagerte Sicht der Welt und als Dimension des Sozialen wie auch als Klassifikationsschema ist ,Geschlecht' Bestandteil der sozialen Ordnung und der von uns verwendeten Ordnungsschemata. Habitus sind ein Produkt der Geschichte und somit ein offenes Dispositionssystem, das ständig mit neuen Erfahrungen konfrontiert und von ihnen unentwegt beeinflusst wird. Dabei ist der Habitus nicht einfach gesellschaftlich bedingt, sondern er ist durch Mitspielen, durch Handeln in der sozialen Praxis erworben und wird in der sozialen Praxis auch verändert und umgebildet. Das bedeutet, dass gesellschaftliche Strukturen von den Individuen inkorporiert, also in den Körper ein- und festgeschrieben werden (vgl. Bourdieu 2005, S. 260).

,Geschlecht' stellt für Bourdieu eine ganz fundamentale Dimension des Habitus dar, die mit den sozialen Faktoren zusammenhängende soziale Eigenschaften modifiziert (vgl. Bourdieu 1997a, S. 222). In diesem Sinne ist ,Geschlecht' eine Dimension, die eine maßgebliche Rolle für die soziale Strukturierung einer Gesellschaft spielt. Bourdieu spricht auch davon, dass es ,männliche' und ,weibliche' Habitus gibt. Diese sind gegensätzlich, komplementär, relational in Abgrenzung zum anderen und hierarchisch strukturiert. Der Prozess, in dem ein geschlechtsbezogener Habitus erworben wird, kann beschrieben werden als eine ständige Prüfung von Handlungen, Signalen, Wahrnehmungen usw. an einem binären Code, bei der ständig die andere von zwei Alternativen des Seins verworfen und aus dem Bereich der eigenen Möglichkeiten ausgeschlossen wird (vgl. Krais 2001, S. 249). Den Erwerb der Habitusformen beschreibt Bourdieu in „Die männliche Herrschaft" als einen sehr anstrengenden Prozess für das Individuum, der auch konstitutiv mit der Körperlichkeit der Individuen verbunden ist: Habitus ist verkörperte Identität. Die

5 Hier ist kritisch anzumerken, dass er sich nur auf den hegemonialen westlichen Geschlechterdiskurs bezieht und dass marginalisierte Perspektiven abseits der Geschlechterdichotomie hier nicht mitgedacht werden. 
Habitus sind für Bourdieu inkorporierte soziale Gesetze und Einteilungsprinzipen (vgl. Bourdieu 2005, S. 91).

Das habituelle Handeln stellt für Bourdieu die am stärksten ausgeprägte Form des Handelns dar, daneben gibt es auch andere Formen wie beispielsweise rational orientiertes Handeln. Das Konzept des Habitus dient primär dazu, gesellschaftliche und klassenspezifische Praktiken zu erklären. Den Fokus dieser Analyse bilden daher die Regelmäßigkeiten in der Verbindung zwischen Struktur und Handeln (vgl. Kubisch 2008, S. 73). Aus Sicht von Krais und Gebauer ist mit der Konzeptionalisierung des Habitus auch ein Paradigmenwechsel im sozialwissenschaftlichen Denken verbunden, „nämlich die Abkehr von einer Vorstellung vom sozialen Handeln, die dieses als Resultat bewusster Entscheidungen bzw. als das Befolgen von Regeln begreift" (2002, S. 5). In der Berücksichtigung der dialektischen Beziehung zwischen objektiven Strukturen und dem Habitus (den strukturierten Dispositionen) sieht Bourdieu das Charakteristikum einer praxeologischen Perspektive (vgl. Bourdieu 1976, S. 147; Kubisch 2008, S. 74).

Die theoretischen Annahmen und die Forschungsperspektive der Denkfigur Pierre Bourdieus lassen sich sehr gut mit der dokumentarischen Methode verbinden, da sich diese bei wesentlichen Aspekten auf das Habituskonzept Bourdieus bezieht und auch Methoden zur Habitusrekonstruktion bereitstellt (vgl. Bohnsack et al. 2007; Meuser 2007; Kubisch 2008). Das heißt, sie fungiert als eine Art ,Übersetzerin' der ,theoretischen' Überlegungen von Bourdieu und operationalisiert diese für die qualitative empirische Forschung. Als Metatheorie dient ihr dazu die praxeologische Wissenssoziologie. Von zentraler Bedeutung ist hier die Unterscheidung zwischen kommunikativem und konjunktivem Wissen. Letzteres stellt ein milieuspezifisches Wissen dar, das in der gemeinsam gelebten Praxis zugleich in habitualisierter Weise zur Orientierung dient (vgl. Bohnsack 2003, S. 137). Im Gegensatz zu den vorgestellten Geschlechterforschungsansätzen, die sich lediglich auf die öffentliche bzw. definitorische Ebene der Wirklichkeitskonstruktion richten und auf kommunikativ-generalisiertes Wissen fokussieren, eröffnet die dokumentarische Methode einen Zugang zum konjunktiven Wissen und damit zur Handlungspraxis der AkteurInnen (vgl. Kubisch 2008, S. 70). Denn es ist von Bedeutung zwischen (Selbst-)Darstellungen, (Fremd)Konstruktionen und Fremdzuschreibungen auf der einen Seite und der habitualisierten Handlungspraxis der AkteurInnen auf der anderen Seite zu unterscheiden (vgl. ebd., S. 77). Diese von mir angewendete, methodologische, praxeologische Analysefolie und die damit einhergehen- 
de Unterscheidung von habituellem Handeln und rational orientiertem Handeln und die Möglichkeit der Rekonstruktion eines Bruchs zwischen diesen beiden Ebenen, spielt in Bezug auf die Frage nach Bewertungsund Handlungsschemata hinsichtlich ,Geschlecht` eine tragende Rolle.

\subsection{Die Dokumentarische Methode und praxeologische Wissenssoziologie}

Die dokumentarische Methode steht in der Tradition der Wissenssoziologie und basiert auf der Grundlage der Arbeiten von Karl Mannheim (1964a; 1964b; 1980), die Ralf Bohnsack für die empirische Forschung ausgearbeitet hat. Die dokumentarische Methode ist praxeologische Wissenssoziologie und vermag die Frage nach der handlungspraktischen Herstellung von Realität, also nach den habitualisierten Praktiken zu stellen, die auf dem handlungsleitenden und z. T. auf dem inkorporierten Erfahrungswissen der Akteure basieren (vgl. Bohnsack/Przyborski/Schäffer

2010, S. 11). Die Unterscheidung zwischen kommunikativem (oder immanentem) und konjunktivem (oder dokumentarischem) Sinngehalt ist hierbei zentral, da sie Zugang zu beiden Wissensebenen eröffnet (vgl. Kubisch 2005, S. 75; Bohnsack 2001a). Während es sich also bei dem kommunikativen Wissen um ein öffentliches und reflexiv zugängliches Wissen handelt, basiert das konjunktive Wissen auf der gemeinsam gelebten milieuspezifischen Praxis der Akteure. Mannheim bezeichnet das relevante Wissen als ein atheoretisches Wissen, welches im Gegensatz zum kommunikativ-generalisierten (oder theoretischen) Wissen steht. Das atheoretische Wissen wird vor allem in Form von Erzählungen und Beschreibungen vermittelt, bringt das handlungsleitende und z. T. inkorporierte Erfahrungswissen der Akteure zum Ausdruck und bezieht sich außerdem auf das zu Grunde liegende konjunktive Wissen (vgl. Bohnsack/Przyborski/Schäffer 2010, S. 12).

Mannheim spricht von „,konjunktiven Erfahrungsräumen“ (Bohnsack 2010, S. 211) dort, wo Menschen eine bestimmte Sozialisationsgeschichte und Handlungspraxis teilen. Diese ermöglicht ihnen, sich unmittelbar zu verstehen, während eine Verständigung über Milieugrenzen hinweg auf Interpretationen angewiesen ist, die auf kommunikativ-generalisiertes Wissen zurückgreifen (vgl. Bohnsack/Przyborski/Schäffer 2010, S. 12). Jede/r von uns ist Teilhabende/r vieler Erfahrungsräume. So können z. B. geschlechts-, bildungsmilieu- und generationstypische Erfahrungsräume voneinander unterschieden werden. Milieutypische Erfahrungs- 
räume ergeben sich im gemeinsamen Erleben von Wissensvermittlung in den je unterschiedlichen öffentlichen Institutionen und den entsprechenden biografischen Ablaufmustern (vgl. Przyborski/Wohlrab-Sahr 2009, S. 282f.). Das unmittelbare Verstehen innerhalb einer Gruppe wird möglich, indem sich gemeinsam geteiltes, atheoretisches Wissen in einem konjunktiven Erfahrungsraum konstituiert. „Diejenigen, die einander unmittelbar verstehen, teilen in jenen Bereichen, in denen dies der Fall ist, Gemeinsamkeiten eines Milieus oder eines konjunktiven Erfahrungsraums" (Bohnsack/Przyborski/Schäffer 2010, S. 12).

Der Milieubegriff, den Bohnsack im Zusammenhang mit der dokumentarischen Methode verwendet, verweist ebenso wie Mannheims Begriff des konjunktiven Erfahrungsraums insbesondere auf die Verschränkung von sozialer Lagerung und subjektiver Erfahrung, die von vornherein als kollektiv eingebunden gedacht wird (vgl. Bohnsack 1998, S. 120). Bei der dokumentarischen Methode wird davon ausgegangen, dass in Gruppendiskussionen kollektive Wissensbestände und kollektive Strukturen, die sich auf der Basis von existenziellen, erlebnisbezogenen Gemeinsamkeiten in konjunktiven Erfahrungsräumen gebildet haben, artikuliert werden (vgl. Przyborski/Wohlrab-Sahr 2009, S. 106). Karl Mannheim hat ein grundlagentheoretisches Konzept von Kollektivität entwickelt, in dem sich Kollektivität vom konkreten Individuum ebenso wie von der konkreten Gruppe ablöst. Die Gemeinsamkeiten der TeilnehmerInnen ergeben sich damit nicht mehr nur durch gemeinsam Erlebtes, wie der Handlungspraxis oder bestimmten Sozialisationserfahrungen. Statt dessen trennt Mannheim den Begriff der Zugehörigkeit, der mit dem direkten Zusammenleben in konkreten Gruppen verbunden ist, analytisch vom Begriff der Gruppe und fasst milieuspezifische Gemeinsamkeiten der Sozialisationsgeschichte als konjunktiven Erfahrungsraum zusammen (vgl. Bohnsack 2010, S. 211).

Dieser Erfahrungsraum verbindet diejenigen, die an den ihm immanenten Wissens- und Bedeutungsstrukturen teilhaben, z. B. bedingt durch Gemeinsamkeiten in der Ausbildung und/oder sozialräumlichen Gegebenheiten (vgl. ebd.). Dieses Prinzip funktioniert bei GesprächspartnerInnen, die sich kennen ebenso wie bei jenen, die sich (nur) durch eine (soziale) Einheit verbunden fühlen bzw. sind. Bei den gemeinsam artikulierten Rahmungen handelt es sich um ein Wissen, das gleichzeitig in der gelebten Praxis angeeignet wird und daher orientierendes Wissen ist (vgl. ebd., S. 105). Anders formuliert: die gemeinsame Erfahrung ist nicht auf das Kognitive zu reduzieren, sondern basiert auf dem gemeinsamen Handeln und Erleben der AkteurInnen. Diese gemeinsam 
erlebte und gelebte Handlungspraxis wird internalisiert und z. T. inkorporiert, „d. h. in das Wie, in den modus operandi der körperlichen und auch sprachlichen Praktiken eingeschrieben" (vgl. Bohnsack 2001, S. 331). Das inkorporierte konjunktive Wissen ist überwiegend atheoretisch, andere Bezeichnungen dafür wären das ,stillschweigende' oder implizite Wissen (vgl. Kubisch 2008, S. 73).

Durch die dokumentarische Methode kann ein scheinbar zusammenhangloser Diskursprozess einzelner Redebeiträge einer Gruppendiskussion ein gemeinsames, kollektives Sinnmuster bzw. Orientierungsdilemmata aufzeigen (vgl. Bohnsack 2010, S. 209). Wenn man die Wahl der Themen, der Struktur und der Art und Weise, wie der Gegenstand besprochen wird, analysiert, lassen sich zugrundeliegende Orientierungsmuster und -strukturen erkennen (vgl. Bohnsack 2008, S. 33). Diese kollektiven Orientierungen bzw. das kollektive Wissen lassen sich nur auf der Basis der wechselseitigen Bezugnahme der Teilnehmerinnen analysieren. Dazu muss eine gewisse Selbstläufigkeit der Diskussion gegeben sein: Die TeilnehmerInnen müssen zumindest phasenweise ohne Eingriffe des/der ForscherIn miteinander sprechen (können). Hinter der anzustrebenden Selbstläufigkeit der Gruppendiskussion steht folgende methodologische Überlegung: In der Dramaturgie, d. h. im Ablauf der Diskussion zeigt sich, ob und wo gemeinsame Erfahrungen der TeilnehmerInnen liegen (vgl. Bohnsack/Przyborski/Schäffer 2010, S. 11). Sie müssen diese erst im Verlauf der Diskussion herausfinden, um vom wechselseitigen Abtasten zum lebendigen Diskutieren zu kommen. Auf den Höhepunkten der Diskussion lassen sich kollektive Orientierungen finden (vgl. Przyborski/Wohlrab-Sahr 2009, S. 106). Die Analyse dieser kollektiven Orientierungen fokussiert auf dem der Handlungspraxis zugrunde liegenden modus operandi oder Habitus. Weil sie diese Verbindung zur Handlungspraxis eröffnet, wird die dokumentarische Methode als Methodologie einer praxeologischen Wissenssoziologie bezeichnet (vgl. Bohnsack 2001a, S. 331; vgl. Kubisch 2008, S. 77).

„[...] erst die Rekonstruktion dieses handlungspraktischen Erfahrungswissens ermöglicht es einzuschätzen, welche ,Wirklichkeitskonstruktionen' als inkorporierte derart in der habitualisierten Praxis verankert und in sie eingeschrieben sind, dass sie eine Kontinuität des vorreflexiven Routinehandelns, d. h. eine Existenzweise zu sichern oder auch zu erzwingen vermögen und somit strukturelle Bedeutung haben. Letztendlich ist jede soziale Kategorisierung, die geschlechtliche, als das ,doing gender', ebenso wie z. B. auch die Kategorisierung ,Jugend', das ,doing youth' sozusa- 
gen, in ihrer Doppelstruktur zu beleuchten: als Existenzweise wie zugleich als Darstellungs- und Verständigungsmodus" (vgl. Bohnsack 2001, S. 334).

Aus Sicht der dokumentarischen Methode gilt für den gegenstandsbezogenen empirischen Konstruktivismus - wie er sich zum Beispiel in der Gender- oder Migrationsforschung zeigt (und wie er in den ersten beiden Kapiteln meiner Arbeit zur Sprache kam) -, dass dieser sich lediglich auf die öffentliche bzw. definitorische Ebene der Wirklichkeitskonstruktion richtet. Es ist jedoch relevant, zwischen (Selbst-)Darstellungen, (Fremd-)Konstruktionen und Fremdzuschreibungen auf der einen Seite und der habitualisierten Handlungspraxis der Akteure auf der anderen Seite zu unterscheiden bzw. dem Unterschied zwischen der Ebene sozialer Identität und der Ebene des Habitus (auch empirisch) zu entsprechen (vgl. Kubisch 2008, S. 77; Bohnsack 2001a; Bohnsack/Nohl 2001; Nohl 2001; 2003). Es gilt somit anzumerken, dass der Konstruktivismus in den Varianten der Ethnomethodologie und der Sozialkonstruktivismus der gegenstandsbezogenen Empirie sich auf die Ebene des ,theoretischen Welt-Erkennens' richten und die ,existenzielle Ebene', d. h. die Ebene des ,Seienden' außen vor lassen (vgl. Bohnsack 2006, S. 10f). Ergo liegen die Möglichkeiten dieser Ansätze nicht darin, zwischen den beiden unterschiedlichen Ebenen des Wissens, dem kommunikativen und dem konjunktiven, zu unterscheiden. Im Vergleich dazu ist die dokumentarische Methode primär darauf gerichtet, einen Zugang zum konjunktiven Wissen als dem atheoretischen, je milieuspezifischen Orientierungswissen zu erschließen. Die dokumentarische Methode zielt also auf den modus operandi sozialer Prozesse (vgl. Kubisch 2008, S. 77).

Die bisherigen Ausführungen verdeutlichen, dass sich Bezüge zwischen der Habitustheorie Bourdieus und der dokumentarischen Methode herstellen lassen. Bourdieu, wie auch Mannheim, ging davon aus, dass sich die soziale Lage in der Weltanschauung, oder der Orientierung und dem Handeln von Individuen niederschlägt. Ebenso streben beide eine Verknüpfung der sogenannten mikro- und makrosoziologischen Perspektive bzw. die wissenssoziologische Auflösung dieser falschen Opposition an. In ihren Theorien stellen sie jeweils einen unmittelbaren Zusammenhang zwischen sozialen Strukturen und handelnden Subjekten her. Doch die Terminologie der sich die beiden bedienen, verrät die Differenzen. Während Mannheim den Begriff des konjunktiven Erfahrungsraums prägt, greift Bourdieu auf den Terminus des Habitus zurück. Bourdieu gibt mit dem Habituskonzept gewissermaßen die Sinndimension vor, auf die sich ein entsprechendes Bemühen zu richten hat. Die geeigneten me- 
thodischen Mittel lassen sich hingegen eher in der an die Mannheim'sche Wissenssoziologie anschließenden Methodologie der dokumentarischen Methode der Interpretation finden (vgl. Meuser 2007, S. 210). Aus der Perspektive der dokumentarischen Methode von Bohnsack stellt sich der Zusammenhang zwischen beiden Konzepten darin dar, dass eine geteilte konjunktive Erfahrung eine „habituelle Übereinstimmung“ begründet (Kubisch 2008, S. 78). Beide, Mannheim wie Bourdieu betonen darüber hinaus das atheoretische oder praktische Wissen, das Bourdieu als sense pratique versteht: „Praktisch ist der Sinn insofern, als das Verstehen nicht in einem Akt der bewussten Zuwendung auf die Situation geschieht, sondern integraler Teil des Handelns in der Situation ist" (Meuser 2007, S. 212).

Neben den beschriebenen Übereinstimmungen zwischen der Habitustheorie und der dokumentarischen Methode bestehen jedoch insbesondere in Hinblick auf die Methodologie deutliche Differenzen zwischen den beiden Ansätzen. ${ }^{6}$ Bohnsack hebt als eine Differenz zwischen der Habitustheorie Bourdieus und der dokumentarischen Methode hervor, dass bei ersterer eher Unterscheidungen, d. h. soziale Distinktion im Fokus stünde, während sich die dokumentarische Methode den in den ,konjunktiven Erfahrungen' fundierten habituellen Übereinstimmungen zuwende, den Habitus also im ,Medium der Konjunktion' analysiere (vgl. Kubisch 2008, S. 79). Mit Michael Meuser kann jedoch festgestellt werden, dass Konjunktion und Distinktion keine gegenläufigen und ausschließenden Tendenzen sind und sich beide mittels dokumentarischer Interpretation erfassen lassen (vgl. 2007, S. 218). Dies ist insbesondere für das Thema dieser Untersuchung wichtig, da das erhobene empirische Datenmaterial der Gruppendiskussionen vor allem bei der (De-)Thematisierung von ,Geschlecht' und Geschlechterfragen von Widersprüchen und Distinktionen geprägt ist. Aus Sicht von Bohnsack geht Bourdieu ,kausalgenetisch“ (2003, S. 68) vor, rekonstruiert also an dieser Stelle gerade nicht die milieuspezifischen Erlebnisschichtungen als Basis des Habitus. ${ }^{7}$ Die dokumentarische Methode berücksichtigt zwei zu differenzierende Ebenen des Wissens, indem sie im ersten Schritt der Analyse den wörtlichen,

6 Die Übereinstimmungen und Differenzen der Habitustheorie und der dokumentarischen Methode wird bei Meuser (2007) ausführlicher diskutiert.

7 Bourdieu entwickelte seinen Habitusbegriff auf der Basis umfassender empirischer Forschungen. Neben qualitativen Methoden wandte er häufig quantitative Verfahren an. Die ,Hauptbeweislast' (zumindest in den feinen Unterschieden) für die sozialstrukturanalytischen Diagnosen liegt hier bei den quantitativ erhobenen Daten. Aus diesem Grund wird das Vorgehen Bourdieus mitunter auch als „Habituskonstruktion" bezeichnet (Matthiesen 1989, zit. n. Meuser 2007, S. 214). 
thematischen Sinngehalt von Äußerungen erfasst (formulierende Interpretation) und im zweiten Schritt analysiert, wie die auf dieser Ebene identifizierten Themen bearbeitet werden (reflektierende Interpretation). Damit wird den Orientierungsrahmen derjenigen rekonstruiert, deren Äußerungen untersucht werden ${ }^{8}$ (vgl. Bohnsack 2008, S. 34, S. 134; vgl. Kapitel 5.4). Dieser bildet sich in der komparativen Analyse vor dem Hintergrund anderer Fälle heraus (vgl. ebd., S. 136). „Dieser Orientierungsrahmen (den wir auch Habitus nennen) ist der zentrale Gegenstand dokumentarischer Interpretation" (Bohnsack/Nentwig-Gesemann/Nohl 2007b, S. 15).

Zusammenfassend kann an dieser Stelle festgehalten werden, dass ein praxeologischer Ansatz die Polarisierung zwischen Mikro- und Makroebene zu überwinden vermag, indem er die Wirkung gesellschaftlicher Strukturen im Handeln der Akteure aufzeigt (vgl. Kubisch 2008, S. 79). Indem dieser Zugang nicht auf die Ebene der Alltagstheorien der Protagonisten sowie auf ihre Selbst-Interpretationen und SelbstDarstellungen, sondern auf die habitualisierte Handlungspraxis zielt, unterscheidet er sich insofern wesentlich von den (in den ersten beiden Kapiteln) vorgestellten Ansätzen der Geschlechterforschung und -theorie. Die dokumentarische Methode dient hier als eine notwendige Ergänzung, da damit die geregelten Improvisationen, d. h. die habitualisierten Wirklichkeitskonstruktionen, sichtbar werden, die Bestand über die jeweilige interaktive Situation hinaus haben (vgl. ebd., S. 80). Die dokumentarische Methode stellt die notwendigen Instrumente bereit, um den jeweiligen Orientierungsrahmen oder Habitus der Studierenden zu rekonstruieren, worauf ich im Kapitel 5.4 noch genauer eingehen werde. Auch der folgende, dritte Abschnitt dieses Kapitels knüpft an die bisher beschriebene praxeologische Perspektive an sowie an das Verständnis, dass Prozesse auf der Ebene von Identität und Interaktion notwendigerweise im Wechselverhältnis stehen und umgekehrt Gesellschaft nicht jenseits des Erlebens der Akteure/innen anzusiedeln ist. Im Weiteren geht es um die Konsequenzen des gewählten praxeologischen Standortes für die Beschäftigung mit dem Phänomen der (De-)Thematisierung von ,Geschlecht und Geschlechterfragen im Kontext gesellschaftlicher Modernisierungsprozesse. Dabei stehen gesellschaftliche Veränderungen und die Diskre-

8 Die rekonstruktive Methodologie der dokumentarischen Interpretation stellt eine Herausforderung an das Bourdieu'sche Verfahren der Habitusanalyse dar. Allerdings enthält die Bourdieusche Konzeptualisierung der Kategorie des Habitus ebenfalls eine Herausforderung an die rekonstruktive Methodologie. Diese ist insbesondere mit dem Gedanken der Inkorporierung gegeben (vgl. Meuser 2007, S. 221). 
panzen zwischen verbal beteuerter Gleichberechtigung von Frauen und Männern und deren praktischen Umsetzung in der sozialen Wirklichkeit und die Frage, wie diese die Art und Weise der (De-)Thematisierung von ,Geschlecht' und Geschlechterfragen beeinflussen, im Mittelpunkt der Betrachtung.

\subsection{Gesellschaftskritische und geschlechterreflektierende Analysefolien}

,Geschlecht' und Geschlechterverhältnisse in Bezug auf gesellschaftliche Ungleichheit in den Blick zu nehmen erscheint in der gegenwärtigen Zeit überholt (vgl. Rendtorff 2005, S. 34f.). Besonders in Anbetracht der hohen Anzahl von Studentinnen und Akademikerinnen ist es fast unglaublich, dass noch vor 100 Jahren darüber diskutiert wurde, ob man Frauen überhaupt zum Studium zulassen solle (vgl. Friebertshäuser 2012, S. 97). Mittlerweile scheint der Gleichberechtigung von Frauen und Männern nichts mehr im Weg zu stehen und feministische Einwände werden oftmals als überholt und unangemessen beurteilt (vgl. Rendtorff 2005, S. 34f.). Auch in der Frauen- und Geschlechterforschung hat in den letzten Jahren eine Diskussion darüber begonnen, ob „der Geschlechterdifferenz tatsächlich bzw. immer noch der Status einer Leitdifferenz zugesprochen werden kann" (Meuser 1999, S. 151; vgl. Wetterer 2002, S. 519-545). Die Positionen in dieser Diskussion sind kontrovers, und um die Diskussionslinien zu skizzieren, werden hier exemplarisch einige genannt: Auf der einen Seite ist von der „Auflösung der Geschlechterdifferenz" (Heintz 1993) die Rede; für die Gegenwart wird die ,De-Institutionalisierung، oder die ,De-Thematisierung' der Differenz konstatiert (vgl. Heintz/Nadai 1998; Nadai 1999; Pasero 1995); ,Geschlecht' gilt nicht länger als Ordnungs-, sondern als „Unordnungsfaktor" (Heintz/Nadai 1998) und undoing gender sei auf dem Vormarsch (vgl. Hirschauer 1994). Auf der anderen Seite meldet sich nicht weniger lautstark eine gegenteilige Einschätzung zu Wort: Hier gilt gender als eine der zentralen Basisinstitutionen auch gegenwärtiger Gesellschaften (vgl. Lorber 1999; Knapp 2008); steht die Persistenz und Veränderungsresistenz hierarchischer Geschlechtersegregationen im Berufsbereich im Zentrum der Analyse, scheint die geschlechtsbezogene Arbeitsteilung in Familie und Paarbeziehung kaum grundsätzlich verändert (vgl. Koppetsch und Burkart 1999; Maihofer/König 2004; vgl. Wetterer 2003, S. 287f.). Angelika Wetterer benennt den kleinsten gemeinsamen Nenner, auf den 
sich die ProtagonistInnen der einen und der anderen Position einigen könn(t)en:

„Der soziale Wandel, die Modernisierung des Geschlechterverhältnisses hat gegenwärtig einen Stand erreicht, der vor allem durch Widersprüche, Brüche und Ungleichzeitigkeiten gekennzeichnet ist: Brüche zwischen verschiedenen gesellschaftlichen Teilbereichen und - sobald man genau hinschaut - auch innerhalb einzelner Bereiche; Ungleichzeitigkeiten zwischen verschiedenen Regionen und sozialen Milieus; Widersprüche zwischen den verschiedenen Medien der Herstellung und Institutionalisierung geschlechtlicher Differenzierung und Hierarchisierung" (ebd., S. 288).

Diese gemeinsame Schnittstelle, wie Wetterer sie beschreibt, dient auch den im Folgenden vorgestellten Analysefolien - wie auch der hier durchgeführten Studie - als Ausgangspunkt. Die gemeinsame Problemstellung ist somit das gleichzeitige Nebeneinander einer , anscheinenden' Auflösung der Geschlechterdifferenz und die Persistenz traditioneller Rollenvorstellungen. Demnach lassen sich jenseits verbaler Beteuerungen von Gleichheit und Gleichberechtigung noch immer zahlreiche Diskrepanzen zwischen Anspruch und Wirklichkeit finden (vgl. Friebertshäuser 2012, S. 97$){ }^{9}$

Ziel der folgenden Ausführungen ist es nun, nicht ,eine' Perspektive vorzustellen, welche für meinen methodologischen und theoretischen $\mathrm{Zu}$ gang maßgeblich ist, sondern eine Triade von Blickwinkeln auf ,eine Problemstellung darzustellen, die als kumulativ, sich ergänzend und überlappend zu betrachten sind. Im ersten Schritt steht Angelika Wetterers These der ,rhetorische Modernisierung" im Mittelpunkt. Angelika Wetterer verweist hier auf die Gleichzeitigkeit von Modernisierungsprozessen und der Reformulierung von Geschlechterdifferenzen in ihrer strukturellen Persistenz. Ergänzung finden diese Ausführungen, im zweiten Schritt, in den Überlegungen zum ,Verdeckungszusammenhang“ des Tübinger Instituts für frauenpolitische Sozialforschung e.V. (2000). Hier konstatieren, Maria Bitzan, Heide Funk und Barbara Stauber, dass moderne Diskurse, z. B. zur Modernisierung von Weiblichkeitsbildern und Männlichkeitsbildern bzw. deren Thematisierungsformen, die realen strukturellen Probleme verdecken (vgl. Tübinger Institut für frauenpolitische Sozialforschung

9 Andrea Maihofer sieht in dieser Paradoxie des Nebeneinanders verschiedener Vorstellungen im Denken von den Geschlechtern (und zwar quer durch die gesellschaftlichen Schichten, wenn auch schichtspezifisch unterschiedlich gewichtet) ein Resultat von Inkonsistenz und widersprüchlichem Denken (vgl. Maihofer 2004, S. 39). 
e.V. 2000, S. 43). Eine Entöffentlichung geschlechtshierarchischer Widersprüche habe zur Etablierung einer Gleichberechtigungsnorm geführt (vgl. Bitzan 2000, S. 340; Kapitel 4.3.2). Im dritten Schritt werden die oben skizzierten Entwicklungen mittels der Ausführungen von Angela McRobbie (2010) in den Kontext feministischer Errungenschaften und neoliberalen Vereinnahmungspolitiken gesetzt. McRobbie führt das Konzept der Desartikulation ein, um zu verdeutlichen, wie die institutionellen Erfolge, die der Feminismus in den vergangenen 30 Jahren errungen hat, "gegenwärtig ausgehöhlt und unterminiert werden" (McRobbie 2010, S. 47). Mit Blick auf den Feminismus möchte sie zeigen, dass die Desartikulation zum machtstrategischen Kalkül eines neuen gender-Diskurses gehört. McRobbies These lautet, dass Frauen als Ersatz für einen als überholt geltenden Feminismus ein neuer Geschlechtervertrag angeboten wird, der sie (erfolgreich) als ,moderne Subjekte des 21. Jahrhunderts $^{6}$ anruft. Mit diesem neuen Geschlechtervertrag wird den Frauen das Angebot gemacht, öffentlich sichtbar zu werden, um am Arbeitsund Bildungsmarkt zu partizipieren und um an der Konsumkultur und Bürgergesellschaft teilnehmen zu können. Im Gegenzug findet eine Individualisierung und Entpolitisierung von Geschlechterungleichheit statt (vgl. McRobbie 2010, S. 87; Kapitel 4.3.3). Jede dieser Analysefolien beschäftigt sich auf ihre ganz spezielle Weise mit der Individualisierung von gesellschaftlichen Problemlagen, die aufgrund von hierarchischen Geschlechterverhältnissen bestehen.

In diesem dritten Abschnitt werden die eben genannten unterschiedlichen Perspektiven nun in Bezug auf dieselbe Frage bzw. Problemstellung beleuchtet. Zusammenfassend lässt sich sagen, dass die drei Ansätze in der gegenwärtigen (De-)Thematisierung von ,Geschlecht' und Geschlechterfragen die Gefahr einer Individualisierung und Entpolitisierung strukturell angelegter Problemlagen sehen und das Risiko, gesellschaftliche und vergeschlechtlichte Strukturen zu stabilisieren.

\subsubsection{Die rhetorische Modernisierung und die (De-) Thematisierung von Geschlechterdifferenz}

Angelika Wetterers These von der rhetorischen Modernisierung ist eine Gegenwartsanalyse, die den Umgang mit dem Nebeneinander von Gleichheit und Ungleichheit und die Diskrepanz zwischen den Überzeugungen und dem Handeln der Individuen beschreibt. ${ }^{10}$ Wetterers Ausgangspunkt

10 Aufgrund dessen sind ihre Überlegungen als Hintergrundfolie insbesondere für den empirischen Teil dieser Arbeit von Bedeutung. 
ist, dass das alltagsweltliche Differenzwissen, also was die Gesellschaftsmitglieder über den Unterschied der Geschlechter und die soziale Bedeutung der Geschlechterdifferenz, über die Geschlechterordnung und das Verhältnis der Geschlechter wissen, den Strukturen des Geschlechterverhältnisses und großen Teilen der sozialen Praxis ,vorausgeeilt‘ ist. Als Gründe für diese Entwicklung verweist sie auf die Gleichzeitigkeit von Modernisierungsprozessen, dem Wandel von Geschlechterverhältnissen und der Reformulierung von Geschlechterdifferenzierungen in ihrer strukturellen Persistenz. Durch diese parallelen Prozesse hat nach Wetterer ein ,neues Geschlechterwissen ${ }^{611}$ Einzug gehalten, das es erschwert, Geschlechterfragen überhaupt auf den Tisch zu bringen (vgl. Wetterer 2006, S. 5ff). Sie nimmt an, dass im Alltagswissen der Gesellschaftsmitglieder die Gleichstellung von Frau und Mann weitgehend anerkannt und vollzogen ist. Jedoch sind die sozialen Strukturen sowie das unbewusste Verhalten der Gesellschaftsmitglieder weitestgehend im ,alten Selbstverständnis ${ }^{12}$ der Geschlechterdifferenz verhaftet. Das bedeutet, dass sich kulturelle Deutungsmuster und Selbstkonzepte, Geschlechterdiskurse und mit ihnen der diskursfähige explizite und explizierbare Teil der Geschlechternormen merklich von den ,alten' Selbstverständlichkeiten entfernt haben weshalb geschlechtshierarchische Verteilungsasymmetrien der Legitimation bedürfen.

In diesem Fall spricht Angelika Wetterer von einer rhetorischen Modernisierung. Wetterer beschreibt dabei eine Modernisierung, „die sich im Diskurs und der Sprache, kaum jedoch in der Praxis zeigt" (Wetterer 2006, S. 12). Dieser Bruch ist auch bei den für diese Studie befragten Studierenden zu beobachten, wenn es um die Relevanz der Kategorie ,Geschlecht' für das erziehungswissenschaftliche Denken und pädagogische Handeln geht: die generelle Überzeugung lautet, dass der reflektierte

11 Genaueres zur Diskussion zu ,Geschlechterwissen' siehe auch Wetterer 2008; Dölling 2003 und 2005.

12 Hier stimmten das Alltagswissen (Männer und Frauen seien von Natur aus verschieden und deshalb für verschiedene Aufgaben bestimmt) und das Alltagshandeln (Frauen verhalten sich anders als Männer) überein (vgl. Wetterer 2003, S. 294). Die Effekte des Handelns konnten so das Alltagswissen validieren, welches wiederum das Alltagshandeln in den vorgegebenen Strukturen steuerte. Wetterer verdeutlicht das an einem Beispiel: Der Mann arbeitet Vollzeit, die Frau Teilzeit und in der Familie können sie sich so ihre vermeintlich unterschiedliche Natur gegenseitig „,vorexerzieren“ (ebd., S. 294). Die Erschaffung des Unterschieds und die Anerkennung des Unterschieds liegen dabei also eng beieinander. Dadurch herrschen relativ stabile Geschlechterverhältnisse, welche für die Gesellschaftsmitglieder eine Hilfestellung im Umgang mit sozialen Situationen darstellen (vgl. ebd., S. 295). 
Umgang mit ,Geschlecht' eine wichtige Rolle spielt, in der Praxis und im Handeln spiegeln sich diese Überzeugungen jedoch oftmals nicht wider. Angelika Wetterer begründet dies damit, dass sich auf der Ebene sozialer Strukturen und Institutionen, aber auch in den eingespielten Routinen des Alltagshandelns nach wie vor, wenngleich in modifizierter Form, die Sedimente und Spuren der ,alten' Verhältnisse finden lassen. Dies hat zur Folge, dass zeitgenössisches Differenzwissen und soziale Praxis nicht mehr bruchlos zusammenpassen. Eine ganz erhebliche Diskrepanz besteht insbesondere zwischen dem, was im Rahmen des alltagsweltlichen Differenzwissens thematisierbar ist, und dem, was nicht zur Sprache kommt aber u. a. in Gestalt latenter Geschlechternormen und institutionalisierter Strukturvorgaben weiterhin das soziale Handeln bestimmt (vgl. Wetterer 2003, S. 289f.). Die These der rhetorischen Modernisierung besagt, dass sich das Alltagswissen und das Alltagshandeln der Gesellschaftsmitglieder gegenwärtig im Widerspruch befinden und dieser Widerspruch von den Individuen mit der De-Thematisierung der Ungleichheit aufgelöst wird. Dabei wird vor allem die hierarchische Struktur der Geschlechterunterscheidung aus dem Wahrnehmungs- und Reflexionsrepertoire ausgeschlossen und damit unsichtbar. Für gesellschaftskritische Überlegungen bedeutsam und politisch brisant ist diese Diskrepanz vor allem deshalb, weil die widersprüchliche Beziehung zwischen Differenzwissen und differenzierenden Strukturen und Praktiken einen Verdeckungszusammenhang hervorbringt, der bestimmte Aspekte der sozialen Realität systematisch ausblendet (vgl. Wetterer 2003, S. 290). So schließst die rhetorische Modernisierung, die Modernisierung des diskursfähigen Differenzwissens, ,als ihre Kehrseite die De-Thematisierung der Ungleichheit zwischen den Geschlechtern ein" (ebd.), d. h. auf die Diskrepanz zwischen Handeln und Überzeugungen wird mit Schweigen über die noch immer bestehenden Ungleichheiten reagiert (vgl. ebd., S. 286). Es verändert sich also nicht nur das Reden über die Geschlechter, sondern auch das Schweigen und es ,verschiebt sich die Grenze zwischen dem, worüber sich sprechen, und dem, worüber sich nur Stillschweigen bewahren lässt" (ebd., S. 290).

Wetterer begreift die rhetorische Modernisierung mit ihrer Ausblendung der Ungleichheit als ,eine Antwort auf diese Problemkonstellation" (ebd., S. 296). Im Vergleich zu ,früher" hat sich das Alltagswissen vom ,zeitgenössische[n] Differenzwissen“" (ebd., S. 291) gewandelt und ist gegenwärtig von Egalität, Gleichberechtigung und Partnerschaftlichkeit bestimmt (vgl. ebd., S. 295). Sie verdeutlicht dies an Beispielen der Paarbeziehung und dem Lebenslauf: Betrachtet man den Lebenslauf junger 
Frauen, so kann man nach Wetterer von einer „Gleichheit der Ausgangslage" (ebd., S. 304) bezogen auf die Kompetenzentwicklung, die Lebenslage und das Selbstverständnis junger Frauen nach Beendigung ihrer Schullaufbahn im Vergleich zu jungen Männern sprechen. Die Institutionen des Bildungssystems stützen die „Idee der Gleichheit" (ebd.) und je länger der Aufenthalt im Bildungssystem anhält, desto stärker werden die jungen Frauen davon geprägt. Diese Annahme bestätigt sich auch in den für diese Studie durchgeführten Gruppendiskussionen mit Studierenden der Erziehungs- und Bildungswissenschaften. Zudem zeigt sich, dass die Studierenden von einer bereits erreichten Geschlechtergleichheit als Norm ausgehen und Benachteiligung nicht Teil dieser normativen Orientierung ist (vgl. Kapitel 6). Es wird allerdings auch deutlich, dass diese Modernisierung nur im Diskurs der Sprache stattfindet und auf der Ebene des handlungsleitenden Wissens nach wie vor die Geschlechterdifferenz und -hierarchie dominiert.

Wetterer knüpft bei ihren Überlegungen zu Paarbeziehungen an die Arbeit von Cornelia Koppetsch und Günter Burkart (1999) an. Auch sie haben eine Diskrepanz zwischen einer veränderten ,Gleichheitsrhetorik ${ }^{6}$ und konstant bleibenden alten Geschlechterpositionen in Paarbeziehungen aufgezeigt. Koppetsch und Burkart haben herausgearbeitet, dass beide Geschlechter zwar selbstverständlich die Gleichstellung der Geschlechter vertreten und die eigenen Paarbeziehungen als egalitär beschreiben. Bei genauer Betrachtung dessen, wie Paare sich tatsächlich verhalten, findet man hingegen nach wie vor Muster geschlechtsbezogener Arbeitsteilung vor. Viele Paare, die auf der Diskursebene die Gleichverteilung von Haus-, Erwerbs- und Erziehungsarbeit postulieren, praktizieren dennoch eine geschlechterungerechte Aufteilung, welche sie als Resultat freier Entscheidungen personalisieren (vgl. Koppetsch/Burkart 1999, S. 145ff). Man hat als Paar beispielsweise abgewägt, wer das höhere Einkommen erhält und danach entschieden, wer zuhause die Kinderbetreuung übernimmt. Diese Entscheidung wird dabei aber als individuell wahrgenommen. Sie hätte auch anders ausfallen können, wird beispielsweise argumentiert. Jedoch sind solche Entscheidungen auch das Ergebnis von Geschlechterungleichheit, da es in der Regel häufig immer noch Männer das höhere Einkommen haben. Dies führt dazu, dass Frauen in der Familienphase in Teilzeitarbeitsverhältnisse gehen, also in einem Lebensabschnitt, in dem wichtige Karriereschritte in unserer Gesellschaft erwartet werden (vgl. ebd.).

In Anlehnung an Koppetsch und Burkart beschreibt Wetterer die Mechanismen, auf die dabei zurückgegriffen wird, als die „Logik der Dis- 
kurse“ (Wetterer 2003, S. 298). Dabei wird die Ungleichheit als „Folge einer freien und bewusst getroffenen Wahl" (ebd., S. 298) verstanden, für welche die AkteureInnen selbst verantwortlich sind, indem sie strukturell angelegte Probleme personalisieren und individualisieren. In der alltäglichen Lebensführung scheitert oftmals die Idee der Gleichheit, weil es zu den neuen Vorstellungen von Gleichheit zum einen noch kein inkorporiertes Wissen gibt (vgl. ebd., S. 302) und zum anderen auch Institutionen den Lebenslauf von Frauen und Männern mitstrukturieren und auf je eigene Art und Weise die (strukturellen) Ungleichheiten verstärken können (vgl. ebd., S. 306). Wetterer sieht das Problem der rhetorischen Modernisierung darin, dass die Ungleichheiten mit der De-Thematisierung nicht aus der Welt zu schaffen sind, sondern sie vielmehr dadurch vor Kritik geschützt werden. Sie stellt fest, dass die theoretische Idee der Gleichheit und die scheinbare Wahlfreiheit zu alternativen Lebensformen neben herkömmlichen Geschlechterarrangements von einigen Gesellschaftsmitgliedern und -strukturen mit Beharrlichkeit verteidigt werden (vgl. ebd., S. 315) sind. Auch Koppetsch und Burkart sehen in der Idee der Gleichheit, eine „Quelle von Irrtümern und neuen Illusionen“, die „Ungleichheit nicht beseitigt, sondern in bestimmter Hinsicht sogar verschärft", weil sie sie der Thematisierbarkeit entzieht (Koppetsch/Burkart 1999, S. 193f).

Die paradoxe Situation, die wir heute vorfinden, ist dass auf der Ebene der Praxis soziale Ungleichheiten fortbestehen und auf der Ebene der Diskurse aber eine Gleichheitsrhetorik vorherrscht. Diese Divergenz macht es schwierig, Machtverhältnisse zwischen den Geschlechtern zu thematisieren.
„Das Verschwinden der Ungleichheit aus den Diskursen, die um Gleichberechtigung und Selbstverwirklichung kreisen, das Insis- tieren darauf, dass nicht normativ-geschlechtstypische Standards und tradierte Leitbilder, sondern die eigenen Entscheidungen das Handeln bestimmen, verdeckt wesentliche Aspekte der sozialen Wirklichkeit. Die Dethematisierung der Ungleichheit zwischen den Geschlechtern und zunehmend auch zwischen Frauen schafft die Ungleichheit nicht aus der Welt, sondern entzieht und schützt sie vor Kritik, und das ist nicht zuletzt in politischer Hinsicht prekär und problematisch" (vgl. Wetterer 2003, S. 314).

Die rhetorische Modernisierung ist Ausdruck einer Situation, in der sich vorerst nur das alltagsweltliche Differenzwissen von der Stelle bewegt hat. Die herkömmlichen Geschlechterarrangements haben ihr strukturbildendes Potenzial jedoch noch nicht verloren, sondern sind „ein Stück weit optional geworden" (ebd., S. 315). 


\subsubsection{Der Verdeckungszusammenhang und die Geschlechtergleichheit als unerreichte Norm}

Die Forscherinnen des Tübinger Instituts für frauenpolitische Sozialforschung e.V. (2000) Maria Bitzan, Heide Funk und Barbara Stauber, kommen zu einem ähnlichen Schluss wie Angelika Wetterer, wenn auch unter unterschiedlich gewichteten Vorzeichen und Wirkungszusammenhängen. Wie Wetterer diagnostizieren auch Bitzan, Funk und Stauber eine Diskrepanz zwischen Leitbild und Realität (Bitzan 2000, S. 343). Sie konstatieren, dass moderne Diskurse, wie z. B. die Modernisierung von Weiblichkeitsbildern und Männlichkeitsbildern bzw. deren Thematisierungsformen, die realen strukturellen Probleme verdecken (vgl. Tübinger Institut für frauenpolitische Sozialforschung e.V. 2000, S. 43). Demnach werden Diskriminierungen nicht mehr als struktureller Effekt wahrgenommen, sondern als individuelles Risiko oder persönliches Versagen. Hier kommt die Tatsache zum Tragen, dass in der Öffentlichkeit zunehmend davon ausgegangen wird, dass es keine Unterschiede mehr zwischen den Geschlechtern gibt, weil diese dem bestehenden Rechts- und Normengefüge widersprechen. Damit werden aber auch Ungleichheitserfahrungen tabuisiert (vgl. Bitzan 2002, S. 4). Als analytische Grundlage für ihre Überlegungen dient Bitzan, Funk und Stauber der ,Verdeckungszusammenhang“. Diese Analyseperspektive geht davon aus, dass in unterschiedlichen gesellschaftlichen Bereichen verdeckte geschlechtsbezogene und geschlechtshierarchische Strukturen wirken und dass Geschlechterhierarchie und Machtstrukturen noch immer wirksam sind (vgl. Tübinger Institut für frauenpolitische Sozialforschung e.V. 2000, S. 41f.).

Die Verdeckung von gesellschaftlichen, geschlechtlich formierten Herrschaftsverhältnissen, die durch eine Dichotomisierung der Geschlechter und den damit verbundenen Widersprüchen gestützt werden, geschieht dadurch, dass sie vor dem Hintergrund von Gleichheitspostulaten als solche nicht mehr benannt werden können und damit als Individualbelastungen definiert und auch erlebt werden. Dementsprechend wird es immer schwieriger, die dahinter stehenden, vielschichtigen, gesellschaftlichen Konfliktlinien zu thematisieren (vgl. Bitzan 2000, S. 343). Bitzan, Funk und Stauber sehen in der Personalisierung und Individualisierung von gesellschaftlichen Konflikten die „Entlastung einer staatlichen Verantwortung für die eigenen sozialen Verhältnisse - die Entlastung einer staatlichen Verantwortlichkeit durch Verdeckung von Verursachungszusammenhängen" (Bitzan 2000, S. 350). Die soziale Praxis sei demnach den Strukturen des Geschlechterverhältnisses nicht, voraus- 
geeilt', wie Wetterer betont, sondern das Resultat eines Verdeckungszusammenhangs, der sich als „Zusammenwirken sozialpolitischer Vorgaben/Regulierungen mit Ideologien der Machbarkeit und daraus resultierenden Selbst-Ansprüchen sowie mit konkreten Hierarchie- bzw. Machtkonstellationen" versteht (Bitzan/Daigler 2004, S. 208). Die Überlegungen des Tübinger Instituts für frauenpolitische Sozialforschung e.V. können somit als eine Erweiterung und Ergänzung von Wetterers Gegenwartsdiagnose gesehen werden, wenn es zu den Verursachungszusammenhängen kommt. Deutlich wird bei beiden Ansätzen, dass mit der (De-)Thematisierung von Ungleichheit aus den expliziten latente Geschlechternormen geworden sind, deren Problematisierung zunehmend schwieriger wird.

Als ein Ergebnis von Verdeckungszusammenhängen versteht das Tübinger Institut für frauenpolitische Sozialforschung, dass Diskriminierung nicht mehr als struktureller Effekt wahrgenommen wird, sondern als individuelles Risiko oder persönliches Versagen. Bitzan, Funk und Stauber konstatieren, dass in patriarchalen Gesellschaften Verdeckungen von geschlechtsbezogenen und geschlechtshierarchischen Strukturen permanent und überall stattfinden und Geschlechtergleichheit weiterhin eine Vorstellung und eine unerreichte Norm darstellt. Maria Bitzan, Heide Funk und Barbara Stauber gehen davon aus, dass in unterschiedlichen gesellschaftlichen Bereichen verdeckte geschlechtsbezogene und geschlechtshierarchische Strukturen wirken (vgl. Tübinger Institut für frauenpolitische Sozialforschung e.V. 2000, S. 41f.).

Die Überlegungen der Forscherinnen ermöglichen somit, in der Verbindung mit der rhetorischen Modernisierung das Ungleichheitstabu genauer in den Blick zu nehmen und die damit verbundenen Verdeckungs, und damit Machtmechanismen im Kontext der (De-)Thematisierung von ,Geschlecht' und Geschlechterfragen der vorliegenden Studie heraus zu arbeiten. Der Verdeckungszusammenhang ist ein Analysemodell, das gleichermaßen struktur- wie handlungstheoretisch angelegt ist: „Er ist Struktur, aber als Struktur wird er immer wieder durch das konkrete Handeln von konkreten Frauen und Männern reproduziert; er bedingt dieses Handeln genauso wie er durch dieses Handeln hervorgebracht wird" (ebd., S. 45). Verdeckungen werden demnach in Wechselwirkung zwischen den Gesellschaftsmitgliedern und gesellschaftlichen Strukturen geleistet. Sozialpolitische Definitions- und Umgangsweisen (denen auch Sozialpädagogik, Bildungs- und Soziale Arbeit zuzurechnen sind) tragen zur Verdeckung und Normalisierung bei (vgl. Bitzan 2000, S. 30). Maria Bitzan analysiert die Moderne als „Entöffentlichung geschlechtshierarchi- 
scher Widersprüche und Individualisierung gesellschaftlicher Konflikte, die ihre Lösung zu einer Privataufgabe machen. [...] Hinter individuellem Scheitern stehende gesellschaftliche Konflikte sind als solche nicht mehr erkennbar und somit auch nicht mehr politisierbar" (Bitzan 2000, S. 340). Dies bedeutet, dass typische, aus dem Geschlechterverhältnis resultierende Konflikte durch Erscheinungsformen der Moderne verdeckt und deshalb gerade nicht lösbar sind. Verdeckungen wirken als Vereindeutigungen, sie übergehen widersprüchliche, gesellschaftliche Anforderungen und Voraussetzungen und spielen die Folgen bzw. die Verantwortung für den Umgang mit diesen den Individuen zu (vgl. Tübinger Institut für frauenpolitische Sozialforschung e.V. 2000, S. 44). dies resultiert u. a. auch daraus, dass im gesellschaftlichen Kontext von Individualisierung der Druck zur Selbstbegründung und Selbstdarstellung auf eine ganz bestimmte Weise zugenommen hat und in der biografischen Bilanzierung nur noch das Erreichte zählt. Probleme, Benachteiligung und hinderliche gesellschaftliche Strukturen scheinen nicht mehr der Rede wert zu sein (vgl. ebd., S. 44).

Die Verdeckung von gesellschaftlichen, geschlechtlich formierten Herrschaftsverhältnissen ${ }^{13}$ und den damit verbunden Widersprüchen geschieht dadurch, dass sie als Individualbelastungen definiert und auch erlebt werden. Negative Gefühle wie Angst oder innere Konflikte, welche dem Selbstbild widersprechen, können somit schlechter empfunden und ausgedrückt werden. Wenn es beispielsweise verpönt ist, sich selbst als Opfer zu sehen, dann können entsprechende Erfahrungen auch schlechter verbalisiert werden. Dies führt, zugespitzt formuliert, zu einem kollektiven Realitätsverlust und eine ,Normaffinität‘. Maria Bitzan beschreibt dies wie folgt:

\begin{abstract}
„Normalität als Integration in die moderne Welt verlangt, von sich als Geschlechtswesen abzusehen, Bestätigungen da zu suchen, wo das angeblich Allgemeine verortet ist [...]. Sie bedeuten, von eigenen Erfahrungen abzusehen, sich an äußeren Bildern zu orientieren und darin, Verortung' zu suchen. Frauen und Mädchen lernen, sich nicht auf sich selbst, auf ihre Wahrnehmungen zu verlassen.
\end{abstract}

13 ,Geschlecht' ist eine Kategorie, die im Wechselverhältnis mit anderen Kategorien betrachtet werden muss (vgl. Kapitel 3.6). An dieser Stelle soll hervorgehoben werden, dass es neben den Geschlechterverhältnissen noch weitere Herrschaftsverhältnisse gibt, die diesen keinesfalls unterzuordnen sind. Allerdings ist der Geltungsbereich dieser Arbeit klar definiert, er fokussiert auf die Kategorie ,Geschlecht'. Erst die klare Definition des Gegenstandsbereichs entsteht die Möglichkeit, nach dem Zusammenspiel, etwa zwischen den Geschlechterverhältnissen und milieuspezifischen Unterschieden, zu fragen. 
Der Selbstbezug ist prekär. Damit ist auch die Bezugnahme auf andere Frauen oder Mädchen unsicher" (Bitzan 1997, S. 78).

Der Verdeckungszusammenhang stellt Lebensrealitäten, ihre strukturellen Bedingungen und deren Strategien in den Kontext der Geschlechterhierarchie und deren Wirkmechanismen. Der Verdeckungszusammenhang legt nahe, diese Gesellschaft als eine zu begreifen,

„die sich - nicht nur, aber auch - über die Aufrechterhaltung der Geschlechterhierarchie reproduziert und deren soziale Grundsäulen - die ökonomischen Prozesse, die Arbeitsmarktprozesse, die sozialen und kulturellen Prozesse etc. - durchherrscht sind von geschlechtshierarchischen Zuschreibungen, Zumutungen und Bewertungen. Letztere sind immer mit Verdeckungen verbunden: Im Sinne einer ,Mikrophysik der Macht' [...] durchdringen sie alle Ebenen der Realitätswahrnehmung. [. . . ] Sie zensieren Handlungsund Ausdrucksformen" (Tübinger Institut für frauenpolitische Sozialforschung e.V. 2000, S. 45).

Laut dem Tübinger Forschungsinstitut lassen sich Verdeckungsmechanismen in verschiedenen Bereichen aufzeigen und vielfältige Querverbindungen zwischen z. B. den Ebenen gesellschaftlicher Strukturen und kollektiver oder individueller Konstruktion von Realität entdecken (vgl. ebd., S. 41ff). Sie wirken im Bereich sozialpolitischer Normalitätsmuster (z. B. Familienorientierung), im Bereich gesellschaftlicher Symbolproduktion (z. B. Bilder von modernem Frausein) und sie schlagen sich nieder auf die eigenen biografischen Konstruktionen (z. B. als Individualisierung von Gelingen und Scheitern). Dies rechtfertige auch die Rede von einem ,Verdeckungszusammenhang' (vgl. ebd., S. 44f). Darüber hinaus stellt dies eine Erweiterung von Wetterers Überlegungen dar, die zwar Verdeckungen welche, im Zuge der rhetorischen Gleichheit geschehen berücksichtigen, diese aber nicht als zusammenhängende Verdeckungsmechanismen benennen, was erst die zur Beschreibung der Diskrepanz zwischen Leitbild und Realität bzw. zwischen Überzeugung und sozialer Praxis führt.

Diese nun ausführlich beschriebene widersprüchliche Gleichzeitigkeit von kulturellem Wandel und strukturellem Beharrungsvermögen ist als Hintergrundfolie meiner Überlegungen besonders wichtig. So stellt das Nicht-Reden über ,Geschlecht' bzw. die Nicht-Positionierung zu dem Thema eine Form des Verdeckens geschlechtlicher Differenzierungen und Hierarchien dar. Ausgehend davon, dass Verdeckungen permanent und überall stattfinden, ist auch nicht überraschend, dass diese gesellschaftlichen Verdeckungszusammenhänge auch in Bildungsinstitutionen und in 
Praxen der Lebensführung von Studierenden hineinwirken. Somit ist der Verdeckungszusammenhang, besonders mit Blick auf mein empirisches Material, eine wichtige Analysefolie. Abschließend wird nun neben den ersten beiden Erkenntnismodellen der rhetorischen Modernisierung und dem Verdeckungszusammenhang noch eine dritte und letzte Analysefolie vorgestellt.

Diese richtet ihren Blick auf die Ebene politischer Bedingungen und Mechanismen. Eine Antwort auf die Frage, warum es zu dieser aktuellen Situation von Verdeckungen und rhetorischen Modernisierungsprozessen kommt, bietet Angela McRobbie mit ihren Überlegungen zu einem neuen Geschlechtervertrag, welcher Frauen (und Männern) angeboten wird. Um diese darzustellen, greife ich im Folgenden auf McRobbies Ausführungen in ihrer Studie ,Top Girls - Feminismus und der Aufstieg des neoliberalen Geschlechterregimes“ (2010) zurück.

\subsubsection{Top Girls und ein neuer neoliberaler Geschlechtervertrag}

Angela McRobbie ${ }^{14}$ stellt in ihrer Studie „Top Girls - Feminismus und der Aufstieg des neoliberalen Geschlechterregimes" (2010) eine ähnliche Entwicklung fest wie zuvor bereits anhand Angelika Wetterers Ansatz und dem des Tübinger Instituts für frauenpolitische Sozialforschung dargestellt wurde. In ihrer Unstersuchung stellt sie fest, dass seit einigen Jahren in den westlichen Industrieländern ein bestimmtes modernes bzw. postmodernes Frauenbild dominiert. Dies ist geprägt von der jungen, ,weißen', gut ausgebildeten, karriereorientierten Frau, die ökonomisch und sexuell unabhängig ist und gegebenenfalls Beruf und Familie scheinbar problemlos vereinbart und dafür auch Angebote staatlicher Familienpolitik in Anspruch nimmt.

Diese Top Girls stehen für die Erfolge in der Gleichstellung der Geschlechter und dafür, dass feministische Interventionen und Kritik an (patriarchalen) Herrschaftsverhältnissen nicht mehr notwendig sind und ad acta gelegt werden können. Anders formuliert: Junge Frauen werden

14 Angela McRobbie wird häufig als Kultur- und Kommunikationswissenschafterin oder als Vertreterin der Cultural Studies rezipiert, dabei wird jedoch häufig übersehen, dass sich Angela McRobbie bereits 1982 gemeinsam mit Monika Savier im Rahmen des Sammelbandes „Autonomie aber wie! Mädchen Alltag Abenteuer“ Fragen zur Alltagswelt von Mädchen und jungen Fragen sowie zur Mädchenarbeit und Frauenforschung gestellt hat. Der Sammelband wird als Versuch deklariert, die „Tradition, die das Denken und Handeln trennt“ (McRobbie/Savier 1982, S. 7) zu verändern. 
heute als die neuen Leistungsträgerinnen des kapitalistischen und wohlfahrtsstaatlichen Umbaus präsentiert. Die Ursachen liegen für Angela McRobbie darin, dass insbesondere seit den 1990er Jahren wesentliche Ziele der Frauenbewegung in neoliberale Gesellschaftsentwürfe und Regierungsformen integriert und damit in einem veränderten gesellschaftspolitischen Kontext uminterpretiert wurden. Für das neoliberale Geschlechterregime ist zentral, dass das Verhältnis zwischen Öffentlichem und Privatem neu definiert wird und an die Stelle politischer Zuständigkeit zunehmend individuelle Verantwortung getreten ist.

Geschlechterverhältnisse werden dabei nicht mehr als strukturelle Hierarchien begriffen, sondern als private Arrangements - unter dem Titel der freien Wahl und der Freiheit des Individuums. Polit-ökonomische Rahmenbedingungen und der historische Kontext werden dabei ausgeblendet. Das ,Private - einst politisiert durch die neue Frauenbewegung wird im neoliberalen Geschlechterregime zunehmend entpolitisiert. Das neoliberale Geschlechterregime nimmt die Diskurse des Feminismus, inbesondere Vokabeln wie empowerment und ,Wahlfreiheit" scheinbar auf, doch werden diese in die eigene Logik eingespeist: Freiheit von patriarchaler Kontrolle, Selbstbestimmung oder Unabhängigkeit werden in der neoliberalen Wettbewerbslogik zu Flexibilität, Mobilität, individuelle Eigenverantwortung, Selbstausbeutung, Leistungsorientierung oder Abbau von wohlfahrtsstaatlicher Unterstützung.

Nach McRobbie besteht der neue Geschlechtervertrag, der jungen, gut ausgebildeten Frauen angeboten wird, in einem Verzicht auf feministische Inhalte. Im Gegenzug wird den jungen Frauen die Freiheit angeboten, an Bildung, Erwerbsarbeit, Karriere und Konsum zu partizipieren. Die Desartikulation feministischer Inhalte und Positionen scheint heute Grundlage dieses neuen Geschlechtervertrags zu sein. Die Erfolge des Feminismus werden zwar anerkannt - dies ist auch ein zentraler Unterschied zu traditionellen backlash-Debatten - doch der Feminismus wird als unzeitgemäß ausrangiert. Das heißt, dass der Feminismus heute zwar einerseits Teil des Alltagsverstandes geworden, zugleich wird er jedoch abgelehnt, denn „die Gleichberechtigung sei längst erreicht“ (ebd., S. 32). McRobbie spricht hier auch von einer „Politik der Desartikulation“ (2010, S. 47ff). Sie führt das Konzept der Desartikulation ein, „um verstehen zu können, wie die institutionellen Erfolge, die der Feminismus in den vergangenen 30 Jahren verzeichnen konnte, gegenwärtig ausgehöhlt und unterminiert werden" (ebd., S. 47). Dabei bezieht sie sich auf Stuart Hall und seine Ausführungen zur Politik der Artikulation (vgl. Hall 1989; 2003): 
„Mit Blick auf den Feminismus möchte ich zeigen, dass die Desartikulation zum machtstrategischen Kalkül eines neuen GenderDiskurses gehört, der darauf abzielt, die gruppenübergreifende politische Nutzbarmachung von intersektionalen Differenzkategorien und die Weitergabe feministischen Denkens von einer Frauengeneration zur nächsten zu unterbinden. Die Artikulationen werden entknüpft und aufgelöst, was zur Folge hat, dass eine neue feministische Politik immer weniger vorstellbar wird" (McRobbie 2010, S. 49).

Vielmehr bestärkt eine solche Logik Mechanismen der Individualisierung und Entpolitisierung ${ }^{15}$ sozialer Verhältnisse, die sie ebenfalls im Kontext einer globalen, neoliberalen Wirtschaftspolitik versteht, in der sich Anerkennung zunehmend an Kompetenz, Leistung sowie Erfolg bindet. McRobbies These lautet, dass Frauen als Ersatz für einen als überholt geltenden Feminismus von Medien, Populärkultur und staatlichen Einreichungen ein neuer neoliberaler Geschlechtervertrag angeboten wird, der sie (erfolgreich) als ,moderne Subjekte des 21. Jahrhunderts' anruft.

Mit der Frage, welche Auswirkungen diese Entwicklungen im Kontext feministischer Errungenschaften haben, setzt sich Angela McRobbie genauer auseinander. Gerade durch die Form der Anerkennung bzw. Inanspruchnahme, so McRobbie, würden etwaige Aufrufe zur Erneuerung des Feminismus bereits im Vorfeld diskreditiert. In ihrer Ursachenanalyse der Etablierung neuer Frauenbilder wendet sie sich insbesondere den Massenmedien bzw. der Populärkultur zu. Hierfür fokussiert sie vor allem auf popkulturelle Erzeugnisse wie Mode- und Frauenzeitschriften, zahlreiche Filme, Literatur und auf Frauen zugeschnittene Produkte. Diese macht sie als die entscheidenden Institutionen aus, die ein postmodernes Frauenbild entwerfen bzw. vermitteln und die Abwicklung des Feminismus betreiben, indem sie der Welt signalisieren, dass „feministische Ideen zentraler Bestandteil dessen sind, was heute Freiheit bedeutet" (ebd., S. 17). Sie sieht in der Integration (ehemals) feministischer Elemente in gesellschaftliche Institutionen eine neue, komplexere Variante von Antifeminismus, durch die das Entstehen einer neuen Frauenbewegung präventiv verhindert werden soll (vgl. ebd.).

15 Die Problematisierung der Individualisierung und Entpolitisierung verbindet Angela McRobbie mit den Überlegungen von Angelika Wetter und dem Tübinger Institut für frauenpolitische Sozialforschung. Alle drei Ansätze sehen hierbei die Gefahr, gesellschaftliche und vergeschlechtlichte Strukturen zu stabilisieren. 
„Was diese Praxen zu untermauern scheint, ist die Behauptung, junge Frauen hätten den Kampf um Gleichberechtigung mittlerweile gewonnen: Ihre Anerkennung als Subjekt gouvernementaler Aufmerksamkeit verdrängt jegliche Notwendigkeit einer feministischen Kritik [...]. Der Verzicht auf Kritik am Patriarchat ist eine der Bedingungen des neuen Geschlechtervertrags, der an jenen zentralen institutionellen Schauplätzen ausformuliert wird, an denen die Kategorie der jungen Frauen produziert wird" (McRobbie 2010, S. 91). ${ }^{16}$

Gleichzeitig sind die hohen Erfolgsquoten bei der Erlangung von Qualifikationen zum Maßstab der bisher erreichten Gleichberechtigung geworden. Es wird davon ausgegangen, dass Diskriminierung aufgrund von ,Geschlecht' kaum bis gar nicht vorkommt, sodass junge Männer heutzutage scheinbar eigentlich (Bildungs-)Verlierer sind und diskriminiert werden (vgl. McRobbie 2010, S. 113). Diese Diagnose kann auch auf die Studierenden meiner Untersuchung übertragen werden. In der Gruppe Holz sind sich die Studierenden darüber einig, dass es aufgrund des hohen Frauenanteils im Studium keine Notwendigkeit gebe, sich kritisch mit ,Geschlecht' auseinander zu setzen. Somit relativiert der hohe Frauenanteil die Notwendigkeit der kritischen Auseinandersetzung mit ,Geschlecht'.

McRobbie geht in ihren Ausführungen zum neuen Geschlechtervertrag mit Rückgriff auf Gilles Deleuzes Begriff der Luminosität (vgl. ebd., S. 88) ausführlich auf die Art und Weise ein, wie heute junge Frauen ,sichtbar' gemacht werden und Adressatinnen eines neuen Geschlechtervertrages sind. Mit dem Konzept der Luminosität zeigt McRobbie, wie die Macht, die Frauen kollektiv zu besitzen scheinen, vom Licht selbst geschaffen wird. Diese Luminositäten behaupten subtil eine postfeministische Gleichberechtigung, jedoch definieren und beschränken sie gleichzeitig ihre Bedingungen (vgl. ebd., S. 95). „Sie sind Lichtkegel, in denen junge Frauen gewissermaßen zum Leuchten gebracht werden und die zugleich das Terrain des unzweifelhaften und beruhigend Weiblichen abstecken" (ebd., S. 95).

McRobbie macht aktuell vier Bereiche aus in denen „Aufmerksamkeitsräume“ konstituiert werden: (1) „die postfeministische Maskerade“, (2) „die hoch gebildete, berufstätige Frau“, (3) „die phallische Frau“, (4)

16 Diese Überlegungen von McRobbie verbinden sich mit dem, was Susanne Maurer die „Verheißungen des Neoliberalismus“ (2006, S. 241) nennt, nämlich das Aufgreifen und die Funktionalisierung emanzipatorischer, feministischer Anliegen wie im Bereich der Bildung, der somit auf die Frage von Erwerbstätigkeit und Wettbewerbsfähigkeit reduziert wird (vgl. ebd.). 
„die globale Frau“ (ebd., S. 93). Zunächst verweist McRobbie auf den Mode- und Schönheitskomplex aus dem eine (1) ,postfeministische Maskerade" entsteht (ebd.). Die Autorin interpretiert die postfeministische Maskerade als „neue Form vergeschlechtlichter Machtverhältnisse“ (ebd., S. 100), die Patriarchat und männliche Herrschaft unter veränderten Bedingungen stabil hält und in der (weiterhin) eine klar differenzierte Seinsweise weiblicher Handlungsfähigkeit vorgeschrieben ist. Frauen werden so angesprochen, als seien sie durch die Gleichstellungsmaßnahmen z. B. im Bildungssektor bereits geschlechterreflektiert. „Ausgestattet mit diesem scheinbaren feministischen Rüstzeug werden sie nun nachdrücklich in die Unabhängigkeit und Eigenständigkeit gedrängt" (ebd., S. 94). Zu diesen gehören die genaue Selbstbeobachtung und -zensur und die Suche nach individuellen Lösungen. Diese weiblichen Individualisierungsprozesse fordern von jungen Frauen auch, sich selbst als wichtig wahrzunehmen. „In stressigen Phasen sollen sie sich in Therapie begeben und Beratungs- und Hilfsangebote wahrnehmen. Sie sind die intensiv gemanagten Subjekte der postfeministischen, gendersensiblen biopolitischen Praxen einer neuen Gouvernementalität" (ebd.). Die Maskerade erkennt den fiktiven Status des Weiblichen offen an, feiert diesen sogar, entwickelt aber gleichzeitig neue Strategien zur Durchsetzung der Geschlechterdifferenz (vgl. ebd., S. 100). Die postfeministische Maskerade zeichnet sich also dadurch aus, dass sie ,ironisch in das Repertoire der Weiblichkeit zurückführt“ (ebd., S. 101). Die neue Maskerade „verweist ständig auf ihre eigene Künstlichkeit" (ebd.), die übersteigerte Weiblichkeit, die den jungen Frauen durch die Konsumkultur angepriesen wird, erscheint nicht als Zwang, sondern als freiwillig gewählt und ist scheinbar desinteressiert an männlicher Anerkennung. Es ist „die maßregelnde Struktur des Mode- und Schönheitssystems, das als autoritäres Regime agiert" (ebd.) und den Verkennungseffekt erzeugt. Letztlich geht es mit der postfeministischen Maskerade darum, die entstandene „Rivalität mit den Männern in der Arbeitswelt [...] zu maskieren“ (ebd., S. 103) und die Konkurrenz, die sie nun darstellen, zu verbergen. Die postfeministische Maskerade operiert mit einer zweifachen Bewertung: Zum einen arbeitet ihre freiwillige Struktur daran, das immer noch aktive Patriarchat unsichtbar zu machen und zum anderen versichern Frauen durch die Erfüllung der Anforderungen des Mode- und Schönheitssystems ihren männlichen Kollegen, „dass sie trotzdem eine echte (also unterworfene) Frau bleib[en]" (ebd., S. 105) auch wenn sie in Konkurrenz mit ihnen steht (vgl. ebd.). ${ }^{17}$

17 Bereits 1966 hat Elisabeth Blochmann auf die Schwierigkeiten der Selbstdefiniti- 
Ihre Entsprechung findet die neue Maskerade in (2) der Sichtbarmachung „der hoch gebildeten, berufstätigen Frau“ (ebd., S. 108) - zum einen repräsentiert sie als kompetentes, sich beständig selbst optimierendes Subjekt den sozialen Wandel, zum anderen verbinden sich mit ihrer Sichtbarkeit neue soziale Grenzziehungen: Bildung, beruflicher Erfolg, Planung von Karriere und Familie werden zu Formen der Distanzierung und Abwertung von sozial Unterlegenen. Die Sichtbarkeit der hochgebildeten, berufstätigen Frau stellt für McRobbie den zweiten Aufmerksamkeitsraum dar. Staatliche Familienpolitik ermuntert Frauen nicht mehr, zu Hause zu bleiben, sondern verspricht Unterstützung bei der Vereinbarkeit von Beruf und Familie; sie „ersetzt gewissermaßen die Feministin“ (ebd., S. 118), indem sie Frauen bei der Bewältigung ihrer Doppelbelastung Hilfe anbietet und ,stabilisiert damit die heterosexuelle Matrix" (ebd., S. 118.). In der Konsequenz werden LeistungsversagerInnen, die nicht das erforderliche Maß an Motivation und Ehrgeiz haben, sich selbst zu verbessern, für ihren Mangel an Status und ihre sonstigen Fehler wesentlich entschiedener verurteilt als zuvor (vgl. ebd., S. 115).

Ein dritter Bereich, in dem Frauen als Adressatinnen eines neuen Geschlechtervertrages sichtbar gemacht werden, ist die Sexualität, Fertilität und Reproduktion. Die (3) „phallische Frau“ (ebd., S. 122) ist sexuell lustvoll aktiv, sorglos und genießt Sex als Freizeitspaß. Sie legt sich einen männlichen Habitus zu, trinkt exzessiv, pöbelt, raucht, prügelt sich und bleibt dabei weiterhin für Männer begehrenswert (vgl. ebd.). Solange sie sich nicht fortpflanzen, wird jungen Frauen damit das Recht darauf zugestanden, ,ihr Begehren scheinbar straffrei zu verfolgen“ (ebd., S. 124). Die ,phallische Frau“ scheint die Gleichberechtigung mit Männern erlangt zu haben, weil sie ihr männliches Gegenüber kopiert. Jugendliche Mutterschaft hingegen oder unzureichende Planung der Vereinbarkeit von Karriere und Familie werden - über Grenzen von Klassen und Ethnizität hinweg - negativ als Zeichen ,gescheiterter Weiblichkeit"(ebd.) bewertet . Zudem setzt das Sichtbarmachen der "phallischen Frau" in der Populärkultur stets „ein weißes weibliches Subjekt“ (ebd., S. 126) voraus. Außerdem beinhaltet diese Aneignung des Phallus keine Kritik männlicher Hegemonie (vgl. ebd., S. 122).

Die vierte Form von sichtbaren Frauen nennt McRobbie die (4) „globale Frau" (ebd., S. 126). Damit macht sie auf eine Form aufmerksam, mit der Frauen aus Drittweltländern repräsentiert werden. Diese Frauen

on und -darstellung von Frauen aufmerksam gemacht: „Die bloße Anpassung an den männlichen Maßstab oder die Übertreibung des rein Femininen sind aktuelle Gefahren" (S. 125). 
bedrohen ,den Westen nicht durch Migration und unkontrollierte Fruchtbarkeit" (ebd., S. 129), sondern bleiben wo sie sind. Ihr Begehren richtet sich allein auf Mode- und Schönheitsprodukte, „die zu der westlichen Weiblichkeit und Sexualität gehören" (ebd.).

Zusammenfassend kann gesagt werden, dass - nach McRobbie - heute jungen Frauen ein neuer Geschlechtervertrag angeboten wird, der sie dazu anhält, vor allem im Feld der Bildung und Berufstätigkeit aktiv zu werden und so ihre Teilhabe an der Produktion einer erfolgreichen Weiblichkeit, Sexualität und vielleicht auch Mutterschaft sicherzustellen. Zudem verlangt die Konsumsphäre von jungen Frauen, ihre sexuelle Intelligibilität in der Logik des heterosexuellen Begehrens durch massiven Konsum abzusichern. Die oben beschriebenen Aufmerksamkeitsräume und Luminositäten verdrängen die verschiedenen Formen von politischer Mobilisierung, die den Feminismus als soziale Bewegung geprägt haben, und setzten sich an ihre Stelle (vgl. ebd., S. 130). ${ }^{18}$ Zudem erzeugen die neuen Freiheiten, die jungen Frauen heute normativ zugestanden werden, mit ihren subtilen Zwängen und Regulierungen aufseiten der weiblichen Subjekte aber auch ,Unbehagen', das sich in ,postfeministischen Störungen' wie Bulimie, Anorexie, Selbstverletzungen usw. äußert. Junge Frauen werden in die Sphäre von Erwerb und Konsum eingebunden, ohne dass sie für Männer bedrohlich werden und die Arbeit(-steilung) in der Familie nicht in Frage gestellt stellt. Die These von Angela McRobbie, dass den jungen Frauen ein neuer Geschlechtervertrag angeboten wird, bietet eine interessante Interpretationsspur für das im Anschluss dargestellte empirische Material. Dieser neuer Geschlechtervertrag für junge Frauen und Männer leitet in Form von gesellschaftlichen Strukturen und Diskursen die individuellen wie auch kollektiven Erfahrungen und Erlebnisse, Vorstellungen und Praktiken, wirkt, als ein ,neuer ${ }^{6}$ konjunktiver Erfahrungsraum und beeinflusst so maßgeblich die (De-)Thematisierung von ,Geschlecht'. Bevor jedoch die eben angeführten Erkenntnismodelle in der empirische Analyse und Rekonstruktion Anwendung finden (Kapitel 6), werde ich das methodische Instrumentarium der dokumentarischen Methode nach Ralf Bohnsack und den Forschungsprozess explizieren (Kapitel 5).

Angesichts der breit angelegten Analysefolie erscheint es sinnvoll, genauer einzugrenzen, worin der Mehrwert dieser Perspektive liegt und was genau den Gegenstand der Untersuchung bilden soll. Das Potential

18 Aus der Perspektive von Bitzan, Funk und Stauber könnte die Fokussierung der Sichtbarmachung bestimmter Frauengruppen als ein Teil des Verdeckungszusammenhang gesehen werden. 
dieser unterschiedlichen Perspektiven auf ,eine' gesellschaftliche Entwicklung liegt insbesondere in der differenzierten Betrachtung des empirischqualitativen Datenmaterials das Gegenstand dieser Arbeit ist. Im Zentrum steht dabei die Frage, inwiefern die (De-)Thematisierung sowie die kollektiven Orientierungen bzw. Brüche zwischen Vorstellungen, Normen und Handeln von Prozessen der rhetorischen Modernisierung und gesellschaftlicher Verdeckungszusammenhänge geprägt sind. Zudem bietet die Vorstellung eines neuen Geschlechtervertrags eine interessante Interpretationsspur und könnte für einen ,neuen' gemeinsamen, konjunktiven Erfahrungsraum von jungen Frauen und Männern stehen.

Im Folgenden möchte ich in der Zusammenführung der vorangegangenen Ausführungen thesenartig skizzieren, wie eine praxeologisch fundierte, dokumentarische, studiumsbezogene und geschlechterreflektierende Forschung aussehen kann, was genau der Gegenstand der Untersuchung ist und wo meine rekonstruktive Forschung ansetzt. ${ }^{19} \mathrm{Im}$ Zentrum der Untersuchung steht die Rekonstruktion des Habitus bzw. der Habitusformen der Studierenden der Erziehungs- und Bildungswissenschaften zwei exemplarisch ausgewählter Universitäten. Es geht also um das handlungsbezogene und handlungsorientierende kollektive Wissen der Akteure_innen im Kontext ihres Studiums und den gesellschaftlichen, geschlechtlich formierten Denk- und Handlungsschemata im Rahmen eines modernisierten Geschlechterdiskurses. In ihrer praxeologischen Fundierung überwindet diese Untersuchung die Dichotomie von Mikround Makroebene, Kultur und Struktur. In der Rekonstruktion der handlungsleitenden Orientierungen bildet sie ab, wie sich soziale, gesellschaftliche und studiumsbezogene Strukturen im Handeln der AkteurInnen niederschlagen. Aufgrund des praxeologisch und dokumentarisch geprägten Zugangs der Untersuchung wird zwischen dem kommunikativen oder kommunikativ-generalisierten Wissen unterschieden. Das Studium der Erziehungs- und Bildungswissenschaften bildet, so die empirisch zu prüfende Annahme, einen konjunktiven Erfahrungsraum, innerhalb dessen die Studierenden im gemeinsamen Handeln kollektive Orientierungen ausbilden. Es ist von einer Überlagerung von studiums- und universitätsbezogenen Erfahrungsräumen bzw. Milieus auszugehen. Dabei lassen sich Orientierungen rekonstruieren, welche die Studierenden derselben Disziplin bei aller Differenz zwischen den Milieus miteinander teilen, die, so die These, auch maßgeblich mit gesellschaftlichen (rhetorischen) Moder-

19 Hier greife ich die Überlegungen von Sonja Kubisch auf, die sie in Bezug auf die dokumentarischen Organisationskulturforschung formuliert hat (vgl. 2008, S. 92$94)$. 
nisierungsprozessen und Verdeckungszusammenhängen verbunden sind. Die Orientierungen oder Habitusformen können unter differenten Blickwinkeln analysiert werden: In der Integrationsperspektive kann rekonstruiert werden, welche Orientierungen integrierend wirken, d. h. von der Mehrheit der Studierenden geteilt werden; in der Differenzperspektive kommen die verschiedenen (individuellen) Orientierungen verschiedener Milieus zur Geltung. Schließlich kann in einer Fragmentierungsperspektive auch deutlich werden, dass es keine (oder kaum) grundlegende gemeinsame Orientierungen unter den Studierenden gibt; im Sinne der dokumentarischen Methode würde man dann von Rahmeninkongruenzen sprechen (vgl. Kubisch 2008, S. 94). 


\section{Methodischer Zugang und der Forschungsprozess}

In den vorangegangenen Kapiteln wurde die Zielsetzung dieser Arbeit, die Thematisierung von ,Geschlecht' im Kontext des sozialen Feldes der Erziehungs- und Bildungswissenschaften und der Bildungsinstitution Universität zu erforschen, ausführlich hergeleitet bzw. ein Orientierungsrahmen erstellt. In einer praxeologischen Perspektive bedeutet dies, das kollektive, implizite und handlungsleitende Erfahrungswissen der Studierenden zu rekonstruieren, auf das sich ihre Wirklichkeitskonstruktionen gründen. Die empirische Bearbeitung des Forschungsthemas erfordert einen qualitativen bzw. rekonstruktiven Zugang.

Mein Ansatz basiert außerdem auf dem methodologischen Rahmen, der sich an den Prinzipien der rekonstruktiven Sozialforschung und des interpretativen Paradigmas orientiert (vgl. Kubisch 2008, S. 113). Beim handlungsleitenden Erfahrungswissen und bei der individuellen Reflexivität der Forschungssubjekte handelt es sich nicht nur um eine individuelle Sinn- und Wirklichkeitskonstruktion, sondern auch um die Konstruktion gesellschaftlichen Sinns. Anders formuliert geht es darum, die dialektische Verschränkung individueller und gesellschaftlicher Strukturierungsprozesse zu erfassen, um von den Individuen auf die Strukturen schließen zu können. Zudem ist davon auszugehen, dass Subjektivität und Individualität immer in die objektiven Strukturen des sozialen Raums eingebunden sind. Dies bedeutet, dass die Analyse von einzelnen Personen und Gruppen Auskunft über die Subjekt-Kontext-Relation gibt (vgl. Dausien 2009). Das implizite Wissen der Beforschten, um das es hier geht, eröffnet sich den Forschenden vor allem über Erzählungen und Beschreibungen der Beforschten. Um hierfür Raum und Möglichkeiten zu eröffnen, folgt die rekonstruktive Forschung den Prinzipien der Offenheit und der Kommunikation (vgl. Hoffmann-Riem 1980 und Kelle 1996). Für die Forschung bedeutet dies zweierlei: Zum einen sollte der/die Forschende eine Kommunikationsbeziehung mit den zu Erforschenden eingehen, in der das kommunikative Regelsystem der Forschungssubjekte gültig bleibt. Zum anderen muss der/die Forschende die theoretische Strukturierung des Forschungsgegenstandes zurückstellen, bis sich die Strukturierung des Forschungsgegenstandes durch die Forschungssubjekte herausgebildet hat (vgl. Kubisch 2008, S. 113). 
Neben der Erwägung der bereits jahrzehntelang bestehenden Diskussion um die Offenheit des Forschungsprozesses und der Forschenden und unter Berücksichtigung bereits etablierter Prinzipien wie ,Fremdheit' (vgl. Helfferich 2011) und native's point of view (vgl. Honer 2007) orientiere ich mich zudem am Konzept des beginner's mind ${ }^{1}$ (Suzuki 2006 [1970]) als Grundhaltung. ${ }^{2}$ Der beginner's mind zeichnet sich im Besonderen durch die Achtsamkeit gegenüber bestehenden und verdeckten Macht- und Herrschaftsstrukturen aus und dient zu deren Dechiffrierung mit dem Ziel, sie nicht zu reproduzieren. Dahinter steht zum einen die These, dass sichtbare Machtstrukturen besser verändert werden können, da nicht hinterfragt und bekämpft werden kann, was nicht sichtbar ist. Zum anderen basiert das Ziel transparenter Machtverhältnisse auf der Überlegung, dass Machtausübung auch besteht und wirksam ist, wenn diese latent vollzogen wird. Die Grundhaltung des beginner's mind ermöglicht somit die Reflexion von Hierarchie- und Machtverhältnissen zwischen Forscherin und Beforschten innerhalb des beforschten Feldes (vgl. Götsch/Klinger/Thiesen 2012, Abs. 47).

Besonders für das hier gewählte Forschungsfeld und den relevanten Gegenstand ist die Berücksichtigung und das Bewusstsein für implizite und explizite Hierarchien und Machtstrukturen bedeutsam. Eine disziplinäre Perspektive einzunehmen ist hierbei wichtig, da die Machtfelder und das Gefüge der Disziplinen eine starke Einflussgröße sind, wenn es um Thematisierungschancen und -dynamiken geht (vgl. Maurer 2008, S. 115). Neben den eigenen theoretischen Vorannahmen und Konzepten, deren alltagsweltlichen Voraussetzungen und politischen Konnotationen, sind auch die institutionellen (und disziplinären) Bedingungen der Proband_innen, aber auch die eigene Verflechtung darin, zu reflektieren. Deshalb wird an dieser Stelle bewusst und explizit auf meine

1 Genaueres über diese Grundhaltung, ihre Abgrenzung zu bereits bestehenden Prinzipien und eventuelle Überlappungen mit anderen Begriffen, werden bei Monika Götsch, Sabine Klinger und Andreas Thiesen 2012 expliziert.

2 Der beginner's mind ist ein Konzept des Zen Buddhismus und wird in der asiatischen Kampfkunst verwendet. Es beinhaltet bereits das Prinzip der Offenheit bzw. setzt dieses voraus. Dieser Begriff verweist darauf, von vorgefassten Meinungen und Denktraditionen und dogmatischen Denkschulen Abstand zu nehmen und ein Thema, einen Gegenstand oder einen Bereich aus der Position eines Anfängers/einer Anfängerin zu betrachten. Das heißt, sich temporär in den Zustand einer AnfängerIn zu versetzen die paternalistische Haltung des Besser-Wissens abzulegen. Die Vorstellung des leeren Blattes ist hier eine hilfreiche Metapher, ebenso wie die Vorstellung, dass eine Tasse geleert werden muss, bevor sie mit neuem Inhalt gefüllt werden kann, ganz nach dem Motto: „In the beginner's mind there are many possibilities, in the expert's mind there are few" (Suzuki 2006, S. 21; vgl. Götsch/Klinger/Thiesen 2012, Abs. 45). 
disziplinäre (also erziehungswissenschaftliche) Perspektive Bezug genommen (vgl. Maurer 2012, S. 134). Die Machtfelder und das Gefüge der Disziplinen sind durchaus eine starke Einflussgröße, wenn es um Thematisierungschancen und -dynamiken geht. Es ist davon auszugehen, dass Verdeckungen permanent und überall stattfinden, somit wirken gesellschaftliche Verdeckungszusammenhänge auch in die Praxen der Lebensführung von Studierenden und in Bildungsinstitutionen hinein (vgl. Kapitel 4.3.2; Tübinger Institut für frauenpolitische Sozialforschung e.V., S. 73).

Das Wissen über gesellschaftliche Verdeckungszusammenhänge wie auch über Machtfelder und Gefüge des Forschungsfeldes diente mir bei der Konzeption des methodischen Vorgehens und in der Auswertung als eine Hintergrundfolie, die Teil meiner reflexiven Haltung ist. Die Verdeckung von gesellschaftlichen, geschlechtlich formierten Herrschaftsverhältnissen, die durch eine Dichotomisierung der Geschlechter und den damit verbundenen Widersprüchen gestützt werden, geschieht dadurch, dass sie vor dem Hintergrund von Gleichheitspostulaten als solche nicht mehr benannt werden können und damit als Individualbelastungen definiert und auch erlebt werden. Die Haltung des beginner's mind ist für den Forschungsprozess sehr wichtig, da diese von mir verlangt, von meinen eigenen Erfahrungen und vorgefassten Meinungen vom Feld Abstand zu nehmen und es als ,Anfängerin' zu betrachten. Die Konsequenz besteht aber nicht darin, die eigenen Theorien und Vorannahmen gänzlich zu vergessen, sondern episodisch zur Seite zu stellen, um Platz für Neues zu schaffen (vgl. Götsch/Klinger/Thiesen 2012, Abs. 45) und die eigene theoretische Strukturierung des Forschungsgegenstandes zurückzustellen.

Um den beginner's mind nicht $\mathrm{zu}$ vorschnell wieder mit dem eigenen Fachwissen und -erfahrungen zu überfrachten, wurde gezielt die Diskussion mit fachfremden Personen gesucht und eine interdisziplinäre Austausch gepflegt. Somit waren heterogene ,Reflexionssettings' fester Bestandteil des Forschungs- und Interpretationsprozesses. Dem beginner's mind geht demzufolge das Prinzip der Offenheit voraus, außerdem sind die Prinzipien der ,wissenschaftlichen Reflexivität“3 (vgl. Bourdieu 1993b, S. 366) und der „Ko-Konstruktion“ (Dausien/Mecheril 2009) immanente und omnipräsente Bestandteile. In der Arbeit mit qualitativen Forschungsmethoden ist die Reflexion der verwendeten wissenschaftli-

3 Pierre Bourdieu interpretiert Reflexivität als eine in ihrer Bedeutsamkeit kaum zu überschätzende Bewegung, in der sich die Wissenschaft auf sich selbst zurückbezieht (vgl. Friebertshäuser/Rieger-Ladich/Wigger 2009, S. 11). 
chen Werkzeuge und Operationen sowie der darin eingelagerten Präsumptionen und Erwartungen ein bedeutendes Qualitätskriterium. Die Reflexion der eigenen Methoden und ihrer Anwendung wird zur wissenschaftstheoretischen Absicherung und in diesem Sinn wird sie nicht nur als nachträgliches Kriterium an die Forschung verstanden, sondern ist kontinuierlich und methodisch angeleitet im Forschungsprozess selbst verankert (vgl. Dausien 2007, Abs. 4).

„Die methodologische Begründung dafür findet sich in den Prinzipien des interpretativen Paradigmas: Wenn soziale Wirklichkeit immer schon interpretierte Wirklichkeit ist und die Deutungen in einer interaktiven Praxis konstruiert werden, so gilt dies auch für die Wissenschaft selbst. Ebenso wie sie die Methoden und Deutungspraxen der Alltagswelt kritisch analysiert, muss sie deshalb auch ihre eigenen Methoden und konstruktiven Praxen in den Blick nehmen (Postulat der Selbstanwendung) und - darüber hinaus - auch den Zusammenhang zwischen sozialweltlichen und wissenschaftlichen Bedeutungskonstruktionen reflektieren. Das Verhältnis von sozialer Wirklichkeit und wissenschaftlicher Erkenntnis ist nicht als einseitiges Abbildungsverhältnis zu denken, sondern eher als eine Praxis des ,Modellierens', in der die Forschung ihren Gegenstand aktiv formt" (ebd.).

Das bedeutet, der Forschungsprozess ist geprägt von einer systematischen Reflexion der eigenen interpretativen Praxis im Hinblick auf ihre Voraussetzungen. Dieses Verständnis der Interpretation wird von Bettina Dausien und Paul Mecheril (2009) als „Ko-Konstruktion“ bezeichnet. Die Idee der Ko-Konstruktion betrifft das Verhältnis zwischen einem empirischen Text oder Material und den dazu produzierten interpretativen und theoretischen Texten. Diese sind mit Alfred Schütz als „Konstruktionen zweiten Grades“ (1971, S. 6) zu verstehen. Der Begriff der KoKonstruktion geht allerdings über den Schütz'schen Ansatz hinaus. Die Ko-Konstruktion reflektiert stärker die wechselseitige Vermittlung und Dynamik zwischen dem empirischen Material und den im Forschungsprozess produzierten „Konstruktionen zweiten Grades.“4 Letztere sind

4 In dem Moment, in dem sich die sozial- und erziehungswissenschaftliche Forschung als Konstruktion zweiten Grades versteht, als Interpretation von Interpretationen, wird besonders die Reflexion auf die Voraussetzung der Auslegung der Interpretationen oder die kontingenten Bedingungen der Deutung der Interpretationen unumgänglich. In diesem Sinn ist eine reflexive Wendung auf die eigenen, nicht nur methodischen, sondern auch wissenschaftstheoretischen und womöglich auch erkenntnispolitischen Voraussetzungen eher in der qualitativen Forschung beheimatet, auch wenn sie nicht auf diesen Ort beschränkt sein muss (vgl. Mecheril/Rose 2012, S. 118). 
keine quasi-naturalistischen, objektiven oder linearen Rekonstruktionen, sondern unter gewissen Blickpunkten konstruierte Lesearten, die in der kommunikativen Interpretationspraxis herausgearbeitet, bzgl. ihrer Voraussetzungen reflektiert und an bestimmten Kriterien im Hinblick auf Plausibilität und Intersubjektivität begütet werden. Der Blick richtet sich also besonders auf die Relation zwischen den Konstruktionen im empirischen Material und den interpretativen Ko-Konstruktionen, die sich im Forschungsprozess wechselseitig strukturieren (vgl. Mecheril/Dausien 2009). Dies beinhaltet ein repetitives, die eigene Lesart des empirischen Materials hinterfragendes Reflektieren und sich der eigenen normativen Sichtweise bewusst zu sein, um diese nicht im Sinne der Deutungshoheit zu reifizieren.

Bereits mit der Wahl des methodischen Vorgehens wurde am Beginn des Forschungsprozesses die Grundlagen für die oben beschriebene Haltung gelegt. Die Entscheidung für die Verwendung eines offenen Verfahrens (z. B. Gruppendiskussionsverfahren) ermöglicht den Beforschten, ihr eigenes Relevanzsystem und ihr kommunikatives Regelsystem zu entfalten und die kollektiven und individuellen Orientierungen bei der Thematisierung von ,Geschlecht' und Geschlechterfragen eigenständig zu entfalten. Bereits dadurch können Unterschiede in den Interpretationsrahmen zwischen der Forscherin und den Erforschten sichtbar gemacht werden. Das Gruppendiskussionsverfahren, wie es auf der Basis der dokumentarischen Methode (vgl. Kapitel 5.2) entwickelt wurde, setzt die Prinzipien rekonstruktiver Forschung methodisch um.

Ziel der dokumentarischen Interpretation ist es, die kollektiven Rahmungen, die sich im Diskurs der Gruppendiskussion abbilden, zu rekonstruieren. Grundsätzlich können - je nach Erkenntnisinteresse - mit dieser Form der Datenerhebung und -auswertung jedoch sowohl individuelle und kollektive Orientierungen, die Gegensätzlichkeit zwischen institutionell erwünschten normativen Mustern einerseits und den individuellen und kollektiven Meinungen und Orientierungsmustern andererseits erfasst und für viele Anwendungsbereiche genutzt werden (vgl. Kutscher 2010, S. 190). Das Gruppendiskussionsverfahren stellt daher ein besonders geeignetes Erhebungsinstrument für meine empirische Studie dar und nimmt eine zentrale Stellung im Forschungsdesign ein. Im Rahmen meiner Forschung wurden insgesamt vier Gruppendiskussionen an zwei verschiedenen deutschen Universitäten mit 14 Studierenden der Erziehungs- und Bildungswissenschaften durchgeführt. Das erhobene empirische Material wird auf der Basis der dokumentarischen Methode ausgewertet. Im folgenden Kapitel werden entlang des Forschungs- 
prozesses die Erhebungs- wie auch Auswertungsmethoden genauer dargestellt. Um das forschungspraktische Vorgehen näher zu beschreiben, wird zunächst auf die Wahl des untersuchten Feldes, der untersuchten Universitäten (Kapitel 5.1), und auf das Gruppendiskussionsverfahren als zentrale Erhebungsmethode (Kapitel 5.2) eingegangen. Im nächsten Schritt steht die Datenerhebung und das empirische Material im Zentrum (Kapitel 5.3). Abschließend werden die Auswertungsschritte der dokumentarischen Methode expliziert (Kapitel 5.4). Da bereits zahlreiche Veröffentlichungen vorliegen, in denen ausführlich auf das Gruppendiskussionverfahren und die dokumentarische Methode eingegangen wird (vgl. z. B. Bohnsack 2003a, Bohnsack et al. 2001a, Bohnsack et al. 2006a, Loos/Schäffer 2001), konzentriere ich mich im Folgenden auf die für dieses Forschungsvorhaben relevanten Aspekte der Methoden.

\subsection{Zur Auswahl des Untersuchungsfeldes und den Universitäten ,Sonne“ und ,Mond"}

Bei der Auswahl des sozialen Feldes der Erziehungs- und Bildungswissenschaften als Forschungsfeld ging ich davon aus, dass gerade die Studierenden dieses Studiengangs eine besondere Sensibilität und Reflexivität im Umgang mit ,Geschlecht' aufweisen würden, da die Disziplin eng mit ihrem Gegenstand und den gesellschaftlichen Rahmenbedingungen verwoben ist und diese untrennbar mit den Vorstellungen über die Beschaffenheit des Subjekts und des Geschlechts verbunden sind. In der erziehungswissenschaftlichen Moderne hat sich, im Gegensatz zu anderen Disziplinen, eine lange Tradition für die pädagogische Thematisierung von ,Geschlecht' herausgebildet (vgl. Rendtorff/Moser 1999, S. 46f.).

Es wurden zahlreiche Ansätze in Hinblick auf einen Ausgleich von Benachteiligung entwickelt. Schließlich sind den Erziehungs- und Bildungswissenschaften neben der Frauen- und Geschlechterforschung eigene Forschungstraditionen zuzuordnen, die Diskurse zu Fragen von Normalität und Differenz, Inklusion und Exklusion, Fremdheit sowie Gerechtigkeit und Gleichberechtigung hervorgebracht haben (z. B. Migrationsforschung, Soziale Arbeit, Disability Studies). Zudem ist (mittlerweile) davon auszugehen, dass geschlechtersensible Inhalte Teil des Studiums sind. Diese Kriterien wurden bei der Auswahl der Universitäten der befragten Studierenden berücksichtigt. 
Neben diesen inhaltlichen Überlegungen stellt das Feld der Erziehungsund Bildungswissenschaften aufgrund seiner Größe einen interessanten Bereich dar. Zahlenmäßig gehören die Erziehungs- und Bildungswissenschaften inzwischen zu den großen Universitätsfächern. Dies gilt für die Zahl der Studierenden und der Absolventinnen und Absolventen ebenso wie für die Summe der Hochschulstandorte mit einem erziehungswissenschaftlichen Studienangebot und die Zahl der Professuren. Das gegenwärtige universitäre Erscheinungsbild ist nicht mehr ohne die Erziehungs- und Bildungswissenschaften zu denken und das Fach hat sich innerhalb der wissenschaftlichen Ausbildungslandschaft auf breiter Ebene etabliert (vgl. Otto/Rausenbach/Vogel 2002, S. 7f.). Im Wintersemester 2010/11 haben in Deutschland insgesamt 54.810 Studierende das Fach Erziehungs- und Bildungswissenschaften belegt, davon lag der Anteil der weiblichen Hauptfachstudierenden bei 77,6 \% (vgl. FaulstichWieland/Horstkemper 2012, S. 194). Somit zählen die Erziehungs- und Bildungswissenschaften neben der Psychologie und den Sprachfächern Germanistik und Anglistik zu den weit überproportional von Frauen gewählten Studienfächern (vgl. ebd., S. 195). Neben der Annahme, dass die Studierenden aufgrund der Inhalte im Studium eine gewisse Geschlechtersensibilität und einen reflektierten Umgang mit ,Geschlecht' hätten, stellt der hohe Frauenanteil im Studium einen weiteren Aspekt für die Wahl der Erziehungs- und Bildungswissenschaften als Untersuchungsfeld dar.

Im Zeitraum der Erhebung vollzog sich an den bundesdeutschen Universitäten und Hochschulen der Umstieg vom Diplom hin zum Bachelor/ Master-Modell. Von dieser Umstellung ist meine Untersuchung insofern betroffen, dass sich an der Universität ,Sonne` nur Bachelorstudierende und an der Universität ,Mond ${ }^{5}$ nur Diplomstudierende an der Untersuchung beteiligten. Diese Zusammenstellung wurde von mir nicht konstruiert, sondern hat sich aufgrund der Selbstmeldungen selbstläufig zusammengefügt. Diese unterschiedliche Zusammensetzung der Gruppen je nach Studienmodell hat Auswirkungen darauf, was schließlich anhand der Gruppendiskussionen rekonstruiert werden kann und ermöglicht die mehrdimensionale Erfassung verschiedener konjunktiver Erfahrungsräume. Aufgrund der Auswahl der Universitäten und Zusammenstellung der Gruppen nach Studienmodell können minimale und maximale Kontraste berücksichtigt werden.

5 In der Folge werden die gewählten Bezeichnungen der untersuchten Universitäten (Sonne und Mond) und der Gruppendiskussionen (Holz, Feuer, Metall, Wasser) nicht mehr hervorgehoben, sondern wie reale Namen verwendet. 
Die Homologien und Differenzen der Studienstandorte und -inhalte werde ich im Folgenden genauer erläutern. An beiden ausgewählten Universitäten haben die Studierenden inner- und außerhalb ihres Fachbereiches Möglichkeiten, sich mit dem Thema ,Geschlecht' und Geschlechterverhältnisse zu beschäftigen. An den Universitäten gibt es jeweils Zentren für die Erforschung der Geschlechterverhältnisse und für feministische Wissenschaft. Die Studierenden haben hier die Möglichkeit, ein Studienprogramm zu Frauenstudien/Gender Studies zu absolvieren. Beide Zentren bieten ein interdisziplinäres Lehrangebot, das in Kooperation mit zwei oder mehreren Lehrenden aus unterschiedlichen Disziplinen durchgeführt wird. Ziel ist es, Grundlagenwissen feministischer Theorie und Praxis zu vermitteln. Zudem werden Ringvorlesungen, Gastvorträge und Workshops angeboten. Studierende haben die Möglichkeit, eine Zusatzqualifikation bzw. ein Zertifikat in Frauenstudien/Gender Studies zu erwerben. Im Curriculum des Studienganges selbst haben die Bachelorwie auch die Diplomstudierenden Geschlechterfragen bzw. Themen aus Frauen- und Geschlechterforschung als Inhalt. Unter den Schlagworten „Geschlechterdifferenzen“, „Generationen- und Gender-Fragen“, „Geschlechterinszenierungen und Geschlechtersozialisation“ sowie „feministische Mädchenarbeit/-bildung und Frauenstudium" finden Studierende des Bachelorstudiums und des Diplomstudiums Geschlechterthemen im Vorlesungsverzeichnis ihres Studiengangs. Im Bachelorstudium der Universität Sonne wird ,Geschlecht' gemeinsam mit anderen Differenzkategorien bearbeitet und setzt sich aus Themen wie "soziale Lage“, „Migration" und „Behinderung" zusammen. An beiden Universitäten ist das Spektrum der Lehrveranstaltungen sehr breitgefächert, es umfasst Themen der historischen und international vergleichenden Pädagogik ebenso wie spezifische Themen der Sozialpädagogik, der empirischen Pädagogik, der Sonderpädagogik, der Erwachsenenbildung, der Schulpädagogik, der Didaktik sowie bildungspolitisch und -theoretisch relevante Themen.

An der Universität Sonne studierten im Wintersemester 2010/11, also am Beginn des Befragungszeitraums, 841 Personen am Bachelorstudiengang Erziehungs- und Bildungswissenschaften. $87 \%$ der Studierenden waren weiblich und $13 \%$ männlich. ${ }^{6}$ Der Bachelor Erziehungs- und Bildungswissenschaften gliedert sich insgesamt in sechs Module, in denen Grundlagenwissen über erziehungswissenschaftliche Theorien und Forschungsverfahren, über die Geschichte von Bildung und Erziehung sowie über pädagogisch-professionelles Handeln in verschiedenen Berufsfeldern bearbeitet werden. Weiter sieht der Bachelorstudiengang zwei Module als

6 Aus Gründen der Anonymisierung werden hier keine Quellenangaben gemacht. 
Wahlfächer vor: Im ersten Modul stehen Soziologie oder Psychologie zur Wahl und im zweiten Modul ist die Wahl des Faches freigestellt. Laut Studienplan wird das theoretische Studium zudem durch ein zweimonatiges Praktikum ergänzt. Der Studiengang hat eine Regelstudienzeit von sechs Semestern. An der Universität Sonne wurden die beiden Gruppendiskussionen Holz und Feuer geführt.

Am Institut für Erziehungs- und Bildungswissenschaften der Universität Mond waren im Wintersemester 2010/11 377 Studierende im Diplomstudium eingeschrieben. ${ }^{7}$ Davon waren $76 \%$ weiblich und $24 \%$ männlich. ${ }^{8}$ Auch hier betreuten die Lehrenden zum Zeitpunkt der Befragung sowohl den Bachelor- und Masterstudiengang der Erziehungsund Bildungswissenschaften sowie den auslaufende Diplomstudiengang Pädagogik. Das Praktikum im Grundstudium betrug mindestens 240 Stunden und im Hauptstudium acht Wochen bzw. 320 Stunden. Die Regelstudienzeit einschließlich Praktikum und Prüfungen beträgt neun Semester. Aufgrund der verschiedenen Regelstudienzeiten unterscheiden sich auch die Proband_innen der beiden Universitäten in ihrer bisherigen Studiendauer. Diese Differenz wird in der Auswertung des Datenmaterials Berücksichtigung finden. An der Universität Sonne wurden die beiden Gruppendiskussionen Metall und Wasser geführt.

\subsection{Gruppendiskussionsverfahren als zentrale Erhebungsmethode}

Um meine Forschungsschwerpunkte bestmöglich zu bearbeiten, habe ich das Gruppendiskussionsverfahren gewählt. Das Gruppendiskussionsverfahren ermöglicht, die Thematisierung von ,Geschlecht' bei Studierenden der Erziehungs- und Bildungswissenschaften zu untersuchen und unterschiedliche Zusammenhänge eines Phänomens zu beleuchten. Es können sowohl institutionell normative Muster wie auch individuelle und kollektive Meinungen und Orientierungsmuster beleuchtet werden. Die Erhebung in der Gruppe erlaubt es den TeilnehmerInnen, sich als Teil kollektiver Zusammenhänge und konjunktiver Erfahrungsräume zu artikulieren (vgl. Przyborski/Wohlrab-Sahr 2009, S. 106f.). In jeder Gruppe treffen Personen zusammen, die einige Erfahrungsräume gemeinsam

7 Auch hier werden aus Gründen der Anonymisierung keine Quellenangaben gemacht.

8 Im Bachelorstudiengang Erziehungs- und Bildungswissenschaft studierten zum Zeitpunkt der Erhebung 443 Personen. Knapp 20 \% waren männlich. 
haben und andere nicht. Als quasi kleinsten gemeinsamen Nenner teilen die Teilnehmer/innen der hier durchgeführten Gruppendiskussionen einen bildungsmilieutypischen Erfahrungsraum. Das heißt, die ,gemeinsam' erlebte Wissensvermittlung im Studiengang der Erziehungs- und Bildungswissenschaften und die Zugehörigkeit zu einer Universität bilden den konjunktiven Erfahrungsraum der Studierenden. Mittels Gruppendiskussionen erhält man verschiedene Perspektiven auf den untersuchten Gegenstand wodurch sich unterschiedliche Weltanschauungen rekonstruieren lassen. Trotzdem oder gerade deshalb werden in den Gruppendiskussionen normative Verständigungsstandards sichtbar. Das heißt, es werden Ritualisierungen sichtbar, die von Institutionen und strukturellen Mechanismen geprägt sind, aber auch jene, die aus der Peergruppe selbst kommen (vgl. Przyborski/Wohlrab-Sahr 2009, S. 105). Auch wenn in Gruppendiskussionen individuelle Meinungen formuliert werden und auch Bruchstücke biographischer Erzählungen vorkommen können, eignet sich das Verfahren nicht zur Bearbeitung von Fragen, bei denen nur die Individuen die zu untersuchende Einheit darstellen. Biografische Elemente oder individuelle Meinungen werden in Gruppendiskussionen oftmals stellvertretend für die ganze Gruppe, in Relation oder im Kontrast zu Erzählelementen anderer TeilnehmerInnen eingebracht. Meinungen, Normen und Werte tauchen nicht im Zusammenhang mit kohärentem individuellen Handeln auf, sondern in Kohärenz mit dem Geschehen der Diskussion. Aus diesem Grund kann in der Gruppendiskussion Individuelles nur begrenzt und nicht in seiner Eigengesetzlichkeit untersucht werden, sondern nur in Relation zum kollektiven Geschehen (vgl. Przyborski/Wohlrab-Sahr 2009, S. 106.). In Abgrenzung zu den kollektiven Orientierungsmustern bzw. wenn zu einem Thema keine kollektive Orientierung besteht, treten jedoch individuelle Orientierungsschemata und -rahmen in der Vordergrund.

Das Erkenntnisinteresse dieser Untersuchung liegt bei der Rekonstruktion kollektiv normativer Orientierungen und spezifischer Aspekte der Handlungspraxis und der Haltungen der Studierenden bei der Thematisierung von ,Geschlecht' und Geschlechterfragen. Durch die komparative Analyse der Gruppendiskussionen wird in dieser Untersuchung eine Verknüpfung von kollektiven und persönlichen Orientierungen in der konkreten Forschungspraxis geleistet. Die Gleichsetzung von ,Geschlecht' mit Frausein und Benachteiligung sowie die Individualisierung von (Diskriminierungs- und Benachteiligungs-)Erfahrungen im Zusammenhang mit der Kategorie ,Geschlecht' bzw. aufgrund der eigenen Geschlechtlichkeit werden vor dem Hintergrund von Gleichheitspostulaten 
negiert und sind ein Dokument für die Verdeckungs- und Neutralisierungstendenzen bei der (De-)Thematisierung von ,Geschlecht'.

Bevor ich jedoch genauer auf das empirische Material und die Auswertungsmethode eingehe, werde ich mich im Folgenden auf die Erhebungsprozesse und -verfahren eingehen. Dabei werde ich das Gruppendiskussionsverfahren auf Basis der dokumentarischen Methode, sowie die Durchführung und das Zustandekommen und die Zusammensetzung der Gruppen erläutern. Schließlich werden die Auswertungsschritte der dokumentarischen Methode expliziert (vgl. Kapitel 5.4).

\subsubsection{Gruppendiskussionsverfahren auf Basis der dokumentarischen Methode}

Die dokumentarische Methode wurde ursprünglich zur Interpretation von Gruppendiskussionen entwickelt (vgl. Bohnsack 1989; 2008; 2010). Mittlerweile wird sie für die Interpretation von Bildern, teilnehmenden Beobachtungen und Interviews herangezogen (vgl. Nohl 2009, S. 14). Ich orientiere mich jedoch beim Gruppendiskussionsverfahren daran, wie es ursprünglich im Zusammenhang mit der dokumentarischen Methode entwickelt wurde. Bei der dokumentarischen Methode setzt die erkenntnisleitende Differenz an der Unterscheidung zwischen implizitem, handlungspraktischem - in der Sprache von Mannheim ,atheoretischem bzw. ,konjunktivem Wissen' - und begrifflich explizitem, kommunikativem Wissen an. Das heißt, sie unterscheidet zwischen einerseits der im Erleben verankerten Herstellung von Wirklichkeit, dem handlungspraktischen Wissen und andererseits dem kommunikativ generalisierten Wissen, das uns in der Regel in begrifflich explizierter Form zur Verfügung steht (vgl. Przyborski/Wohlrab-Sahr 2009, S. 275).

Im deutschen Sprachraum stellen die empirischen Arbeiten des Frankfurter Instituts für Sozialforschung aus den 1950er Jahren den Ausgangspunkt des Gruppendiskussionsverfahrens dar. Werner Mangold (1960), als Vertreter der Frankfurter Schule, beschäftigte sich mit Gegenstand und Methode des Gruppendiskussionsverfahrens. Diese Beobachtung, wie wichtig in Gruppen die Beziehungen der einzelnen Gesprächsbeiträge zueinander sind, führte zu seinem Konzept der "Gruppenmeinung", das zum Schlüssel für die Analyse von Gruppendiskussionen wurde. Als Gegenstand der Erhebung sieht Mangold die Gruppenmeinung; sie ist das Produkt kollektiver Interaktionen (vgl. Przyborski/Wohlrab-Sahr 2009, S. 103). Der zu erforschende Gegenstand wird laut Mangold von der untersuchten Gruppe repräsentiert. Das bedeutet, dass sich die Grup- 
penmeinung bereits vor der Diskussionssituation in der Realität der Mitglieder, des betreffenden Kollektivs, ausgebildet hat. Das Kollektiv wird somit losgelöst von der konkreten Gruppe gefasst.

Hierbei geht es vor allem um gemeinsame Erfahrungen von Menschen, die ein gemeinsames Schicksal oder eine bestimmte soziale Lage teilen. Bohnsack spricht diesbezüglich von Milieus oder mit Bezug auf Mannheim von konjunktiven Erfahrungsräumen (vgl. Kubisch 2008, S. 117). Karl Mannheim hat ein grundlagentheoretisches Konzept von Kollektivität entwickelt, in dem sich Kollektivität vom konkreten Individuum ebenso wie von der konkreten Gruppe ablöst. Die Gemeinsamkeiten der TeilnehmerInnen ergeben sich damit nicht mehr nur durch gemeinsam Erlebtes wie der Handlungspraxis oder Sozialisationserfahrung. Mannheim trennt den Begriff der Zugehörigkeit, die mit dem direkten Zusammenleben in konkreten Gruppen verbunden ist, analytisch vom Begriff der Gruppe und erfasst milieuspezifische Gemeinsamkeiten der Sozialisationsgeschichte als konjunktiven Erfahrungsraum (vgl. Bohnsack 2010, S. 211). Dieser Erfahrungsraum verbindet diejenigen, die an den in ihm gegebenen Wissens- und Bedeutungsstrukturen teilhaben, z. B. bedingt durch Gemeinsamkeiten in der Ausbildung und/oder sozialräumlichen Gegebenheiten (vgl. ebd.). Das funktioniert bei GesprächspartnerInnen, die sich kennen und bei jenen, die sich (nur) durch eine (soziale) Einheit verbunden fühlen bzw. sind. Die Zusammensetzung der Gruppe und die Interaktionen werden als konstitutive Momente in die Methodologie des Verfahrens, der Erhebung und der Auswertung einbezogen. ${ }^{9}$ In den 1980er Jahren verknüpfte Ralf Bohnsack die Überlegungen zum Gruppendiskussionsverfahren von Werner Mangold und das Konzept der Kollektivität von Karl Mannheim und entwickelte die dokumentarische Auswertungsmethode. ${ }^{10}$

9 Die Kritik aus den 1970er Jahren, die Ergebnisse von Gruppendiskussionen seien weder reliabel noch valide, trifft jedoch nur zu, wenn der Gegenstand, auf den die Analyse abzielt, allein prozess- und damit situationsabhängig ist. Dann lassen sich die Ergebnisse nicht generalisieren und bleiben auf die aktuelle Situation beschränkt. Zudem ist die Methode des Gruppendiskussionsverfahrens umso genauer, je umfangreicher formale Strukturen oder Regeln alltagssprachlicher Diskursorganisation, also die natürlichen Standards, rekonstruiert werden können (vgl. Bohnsack/Przyborski/Schäffer S. 9, S. 104).

10 Die dokumentarische Methode hat in verschiedenen sozialwissenschaftlichen (interdisziplinären) Forschungsfeldern Anwendung gefunden. Neben der Gruppendiskussion zählen das Interview und die dokumentarische Methode zu beliebten Erhebungs- und Auswertungsverfahren der qualitativen Sozialforschung (vgl. Nohl 2009, S. 7). Die Interpretation von Interviews mittels der dokumentarischen Methode wurde von Arnd-Michael Nohl systematisch aufgearbeitet (vgl. ebd., S. 14). 
Im Forschungsprozess lassen sich auf Basis dieser methodologischen Überlegungen kollektive Orientierungen bzw. kollektives Wissen durch die wechselseitige Bezugnahme der Teilnehmerinnen analysieren. Dazu muss eine gewisse Selbstläufigkeit der Diskussion gegeben sein und die Teilnehmerinnen müssen zumindest phasenweise ohne Eingriffe der ForscherIn miteinander sprechen (können) (vgl. ebd., S. 106). Hinter der anzustrebenden Selbstläufigkeit der Gruppendiskussion liegt folgende methodologische Überlegung: In der Dramaturgie, d. h. im Ablauf der Diskussion, zeigt sich, ob und wo gemeinsame Erfahrungen der Teilnehmer und Teilnehmerinnen liegen (vgl. ebd.). Sie müssen diese quasi erst im Verlauf der Diskussion herausfinden, um vom gegenseitigen Abtasten zum lebendigen Diskutieren zu kommen. Hierfür ist es wichtig, den DiskussionsteilnehmerInnen ein Gespräch untereinander zu ermöglichen. In den Höhepunkten der Diskussion lassen sich somit auch kollektive Orientierungen finden. Zudem gibt die Diskursorganisation (also der Verlauf der Gruppendiskussion) Aufschluss darüber, ob und wie Studierende ,Geschlecht' thematisieren. Die Stärke der Gruppendiskussion als Methode spiegelt auch das zentrale Anliegen dieser Untersuchung wieder und liegt ,in der Möglichkeit der Rekonstruktion kollektiver Orientierungen, also des milieu- und kulturspezifischen Orientierungswissens, innerhalb und außerhalb von Organisationen und Institutionen" (Bohnsack/Przyborski/Schäffer 2010, S. 7).

\subsection{Datenerhebung und empirisches Material}

Bei dem vorliegenden empirischen Material handelt es sich um systematische Dokumente, d. h. sie wurden eigens zum Zweck der Analyse produziert. In der empirischen Untersuchung wurden insgesamt vier Gruppendiskussionen an zwei Universitäten durchgeführt. Es haben sich 14 Studierende (2 männlich, 12 weiblich; 7 Bachelor- und 7 Diplomstudierende) beteiligt. Die TeilnehmerInnen kannten sich zum Teil bereits aus dem Studium und befanden sich zum Zeitpunkt der Gruppendiskussion zwischen dem zweiten und dreizehnten Semester ihres Studiums. Das heißt, die ganze Bandbreite des Studienverlaufs ist vertreten.

Für die Auswahl der Personen oder Gruppen, die Gegenstand der Untersuchung werden sollen, wurden mehrere Wege genutzt, die auf Selbstmelder abzielten. Es wurde eine Einladung zur Gruppendiskussion als Aushang an den jeweiligen Instituten angebracht und über den Emailverteiler der Fachschaft versendet. Zudem wurden Studierende bei einer 
Lehrveranstaltungen zur Teilnahme an der Gruppendiskussion eingeladen. Die Einladung zur Gruppendiskussion erfolgte zum Thema „Erziehungswissenschaften studieren - Studieninteressen und -verläufe". Sie zielte neben dem Erfahrungsaustausch der Studierenden in Bezug auf ihr Studium und auf ihre Interessensgebiete und Schwerpunktthemen auch auf (un)wichtige Inhalte und Themen der Studierenden ab. Die Studierenden wurden dazu eingeladen, sich kritisch mit ihren Studiensituationen auseinanderzusetzen.

Bei diesem Vorgehen wurde den Forschungssubjekten das zu Grunde liegende Erkenntnisinteresse und die Forschungsfrage nicht gänzlich offenbart. Der Grund hierfür waren Bedenken, die sich aus den theoretischen Vorüberlegungen speisen. Da es heute als überholt und unangemessen erscheint, Geschlechterverhältnisse (als eine gesellschaftliche Ungleichheit) in den Blick zu nehmen (vgl. Rendtorff 2005, S. 34f.), habe ich befürchtet, dass eine komplette Offenlegung des wissenschaftlichen Interesses an der Thematisierung von ,Geschlecht' Abwehr und Skepsis bei den ProbandInnen auslösen könnte. Diese Überlegung erfolgte unter Berücksichtigung des „Verdeckungszusammenhangs“ (Tübinger Institut für frauenpolitische Sozialforschung e.V. 2000, S. 73) und der „Politik der Disartikulation“ (McRobbie 2012, S. 49; vgl. Kapitel 4.3.3). Unter dem Begriff Verdeckungszusammenhang machen Maria Bitzan und das Tübinger Institut für frauenpolitische Sozialforschung (1990) darauf aufmerksam, dass in der Öffentlichkeit zunehmend davon ausgegangen wird, dass es keine Unterschiede mehr zwischen den Geschlechter gibt, weil diese dem bestehen Rechts- und Normengefüge widersprechen. Angela McRobbie verweist in ihrer Studie „Top Girls. Feminismus und der Aufstieg des neoliberalen Geschlechterregimes" (2010) darauf, dass ,der Feminismus heute als , unsexy' dämonisiert wird. Die Erfolge des Feminismus werden zwar anerkannt, doch der Feminismus wird als unzeitgemäß ausrangiert (ebd., S. 49).

Eine weitere Vorüberlegung war, dass sich auf eine Einladung für eine Gruppendiskussion mit dem Fokus auf Geschlechterfragen nur Personen melden könnten, die bereits ein Interesse für die Thematik entwickelt haben. Die Frage, wie Studierende ,Geschlecht' thematisieren und von welchen Faktoren dies beeinflusst wird, sollte jedoch nicht nur unter Einbeziehung jener beantwortet werden, die die Kategorie ,Geschlecht' als relevant betrachten. Ziel war es, auch jene zu Wort kommen zu lassen, die sich nicht dafür interessieren bzw. die dem Thema ,Geschlecht' ambivalent oder wenig reflektiert gegenüber stehen. Dies ist mit der Ausschreibung auch gelungen. Es haben sich Personen mit unterschiedli- 
chem Interesse am Thema ,Geschlecht' und mit graduell verschiedenem theoretischen und atheoretischen Wissen zusammengefunden, was sich in den Gruppendiskussionen auch zeigt. Die Entscheidung für den oben beschriebenen, teils verdeckten Umgang mit dem Forschungsinteresse gegenüber den ProbandInnen ist eng verknüpft mit dem Erkenntnisinteresse, auch Widerstände, Desinteresse und zurückweisende Haltungen der Studierenden gegenüber Geschlechterthemen in den Blick zu bekommen.

In Hinblick auf die Zusammenstellung der zu untersuchenden Gruppen wurden von der Forscherin keine Konstruktionsleistungen erbracht, da jede Entscheidung einen Einfluss auf die Diskussion der Gruppe zum Thema ,Geschlecht' hätte bedeuten können. Somit folgt die Zusammensetzung der Gruppen dem Prinzip der „Realgruppen“ (Przyborski/Wohlrab-Sahr 2009, S. 113), da diese in der Regel über eine gemeinsame Erfahrungsbasis verfügen (vgl. ebd.). Zudem ist von Vorteil, dass das Erkenntnisinteresse nach der Thematisierung von ,Geschlecht' und Geschlechterfragen ein sehr weit gefasst ist und das Studium der Erziehungsund Bildungswissenschaften als ein Element der Gruppenbildung gesehen werden kann (vgl. ebd., S. 108). Eine künstliche Zusammenstellung der Gruppe hätte für die Entfaltung des eigenen Relevanzsystems problematisch sein können.

Carol Hagemann-White hat für die Adressierung von Frauen als eine homogene Gruppe deutlich herausgearbeitet, dass jede Forschung, die auf der Interaktion zwischen Forschenden und zu Erforschenden aufbaut, ihren Gegenstand stets mitkonstruiert. Sie stellt die Frage in den Raum, aufgrund welcher inhaltlichen Gemeinsamkeiten Personengruppen wie z. B. Frauen überhaupt als Gruppe gefasst werden können (vgl. Hagemann-White 1993, S. 71). Gleichzeitig haben es Forscher/innen in ihrer Praxis mit Menschen zu tun, die sich mehr oder weniger fraglos als Männer und Frauen erleben - ebenso wie auch die Forscher/innen in der Interaktion einem ,Geschlecht' zugeordnet werden (vgl. ebd.). Deshalb ist es insbesondere mit Blick auf die Kategorie ,Geschlecht" kaum möglich, als Forscher/in so genannte homogene Gruppen zu konstruieren, ohne Geschlechterkategorien damit zu reifizieren. Deshalb entschied ich mich, mit der Kontaktherstellung via Selbstmelder dem Prinzip der Offenheit bereits in der Zusammenstellung der Gruppen Rechnung zu tragen und es den Studierenden selbst zu überlassen, wer sich meldet und für welchen Termin sie sich Zeit nehmen wollten. Bei diesem Vorgehen haben sich an beiden Universitäten eine gemischtgeschlechtliche Gruppen und eine geschlechtshomogene (Frauen-)Gruppen zusammengefunden. 
Was die Studiendauer betrifft, sind die Gruppen durchmischt, dasselbe gilt jedoch nicht für den Studiengang, d. h. an der Universität Sonne haben sich nur Bachelorstudierende beteiligt und an der Universität Mond nur Diplomstudierende. Für die Analyse der Gruppendiskussionen ist dies von Bedeutung, da im Besonderen die Gruppe Holz von der Universität Sonne die Umstellung zum Bachelor/Master-Modell intensiv diskutierte. Bei den anderen Gruppendiskussionen stellte dies nicht eine derart zentrale Orientierung dar. Auch wenn ich die Auswahl meiner Probandinnen nicht im Sinne eines "theoretical sampling“ (Glaser/Strauss 1967, S. 45) getroffen habe, kann ich auch auf Basis der selbst zusammengefundenen Gruppen minimale und maximale Kontraste berücksichtigen. Der Ansatz der dokumentarischen Methode ermöglicht auch, zu rekonstruieren, welche konjunktiven Erfahrungen sich für die untersuchten Personen bzw. Gruppen selbst als relevant erweisen, ob sie sich also beispielsweise in einer Diskussion auf einen geschlechtsbezogenen oder bildungsmilieuspezifischen Erfahrungsraum beziehen und, wenn dies der Fall wäre, wie sie die tun (vgl. Kubisch 2008, S. 119). Somit kann die mehrdimensionale Erfassung verschiedener konjunktiver Erfahrungsräume bei der Auswertung berücksichtigt werden, ohne dies vorab gesteuert zu haben.

Meine hier dargelegte Entscheidung hinsichtlich der Zusammensetzung der Gruppen hat Auswirkungen darauf, was schließlich anhand der Gruppendiskussionen rekonstruiert werden kann. Mein Fokus solle im universitätsinternen und -übergreifenden Vergleich auf die kollektiven, normativen Orientierungen der Studierenden bezogen, auf das soziale Feld gerichtet sein. Zudem wird rekonstruiert werden, ob ein reflektierter Umgang mit ,Geschlecht' Teil des studentischen Habitus ist. Ich ging davon aus, dass es mir möglich sein würde, unterschiedliche konjunktive Erfahrungsräume in den einzelnen Gruppen zu rekonstruieren, jedoch würde dies nicht in eine sozio- oder sinngenetischen Typenbildung münden, sondern vielmehr in der Rekonstruktion von (De-)Thematisierungsmustern, wie ich in Kapitel 5.4.1 genauer erläutern werde. Davor wird jedoch die Durchführung der Gruppendiskussionen genauer erläutert.

\subsubsection{Durchführung der Gruppendiskussionen}

Insgesamt wurden vier Gruppendiskussionen an zwei Universitäten durchgeführt. Sie dauerten in der Regel zwischen zwei und drei Stunden und wurden je nach Möglichkeit in den Räumen der untersuchten Universi- 
tät oder in privaten Wohnräumen durchgeführt. Die Erhebungen fanden im Zeitraum von Oktober 2010 bis März 2011 statt. Die relativ großen Zeitabstände zwischen den Gruppendiskussionen spiegeln die Schwierigkeiten wieder, mit denen ich in Bezug auf die Datenerhebung konfrontiert war. Der Prozess der Datenerhebung hat sich deshalb als sehr schwierig erwiesen, da nur wenige Student_innen bereit waren, sich an einer Gruppendiskussion zu beteiligen. Zudem wurde die Terminfindung aufgrund der zeitlich knappen Ressourcen der Studierenden erschwert. Die Gruppendiskussionen wurden in der zweiten Person (Du-Form) geführt.

Im ersten Schritt wurden die TeilnehmerInnen über die Rolle der InterviewerIn, das allgemeine Erkenntnisinteresse und die Art und Weise der Erhebung informiert. Vor der Gruppendiskussion haben alle Beteiligten eine Interviewvereinbarung unterzeichnet, welche die Anonymisierung aller Daten garantiert und die Einwilligung zur Audioaufzeichnung und zur wissenschaftlichen Analyse der Daten beinhaltet. Für den Einstieg in die Diskussion wurde ein allgemeiner Stimulus zur Studiensituation gewählt. Danach folgte eine Frage zu interessanten oder langweiligen Themen im bisherigen Studium. Im Verlauf der Gruppendiskussion wurde auch nach Geschlechterinhalten im Studium und nach der Handhabung geschlechtergerechter Sprache gefragt. Abschließend wurde über die Zukunftspläne und Berufsziele der TeilnehmerInnen gesprochen. Die weitgefassten Themenkomplexe dienten demnach als Gesprächsanregungen, die antstelle starr Vorgaben lediglich einen Rahmen bieten sollten. (vgl. Friebertshäuser 1997, S. 375). ${ }^{11}$ Ziel dieses Vorgehens war es, die Thematisierung von ,Geschlecht" und Geschlechterinhalten im allgemeinen Kontext des Studiums zu besprechen. Bei Gruppendiskussionen ist es dabei von großer Bedeutung, dass sich die InterviewerIn zurücknimmt, damit ein selbstständiger und -läufiger Diskurs zustande kommen kann, und Themen von Gruppen aufgenommen und abgeschlossen werden können (vgl. Przyborski/Wohlrab-Sahr 2009, S. 112).

11 Nach eingehender Literaturrecherche wurden für die Zusammenstellung der Diskussionsstimuli in den Gruppendiskussionen zunächst möglichst viele Fragen hinsichtlich des Forschungsinteresses nach dem SPSS-Prinzip (vgl. Helfferich 2011) gesammelt. SPSS steht für sammeln, prüfen, sortieren und subsumieren von möglichen Interviewfragen. In einem zweiten Schritt wurden die gesammelten Fragen mit dem Ziel der Reduzierung und Strukturierung geprüft und im dritten Schritt in Themenkomplexe sortiert und abschließend subsumiert. Schließlich wurden Stimuli bzw. Fragen so formuliert, dass diese für die Studierenden verständlich sind und als Diskussionsanreize fungieren. Vor den Gruppendiskussionen wurden auch Probeinterviews geführt, um relevante Themen und Aspekte von Studierenden selbst zu erfassen, die von der Forscherin zuvor nicht in Betracht gezogen wurden, für die Gruppendiskussionen hingegen relevant sind. 

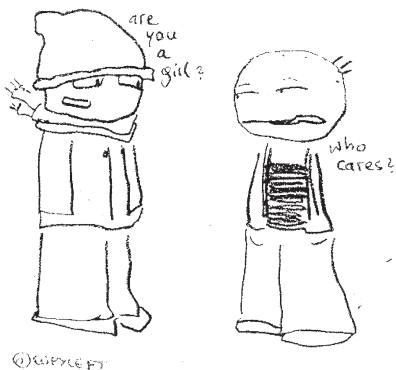

Abbildung 1: „Are you a girl?" „Who cares?"; Quelle: http://subjektstrucktur.blogsport.de/buch/ [24.10.2012]

Neben dieser Zurückhaltung in der Gruppendiskussion war der Umgang mit Begriffen zu ,Geschlecht', Geschlechterverhältnis(se), -ordnung(en), gender etc. bewusst sehr vage. Die vorsichtigen Frageformulierungen sollten verdeutlichen, dass man dem zu erforschenden Phänomen gegenüber in einer Fremdheitsrelation steht und außerdem bei den interviewten Personen Offenheit signalisieren (vgl. ebd.). Zugleich erhält die ,Gruppe' die Gelegenheit, das Thema in ihrer eigenen Sprache zu entfalten (vgl. Bohnsack 2008, S. 20.). Neben verbalen Diskussionsstimuli wurde den Gruppen auch eine visuelle Diskussionsanregung angeboten. Jeder Gruppe wurde eine Zeichnung vorgelegt, in der zwei Personen zu sehen sind und eine Person die andere fragt: „Are you a girl?", und diese antwortet: „Who cares?"12 Das Besondere an diesem Bild ist, dass keine der beiden Personen eindeutig als Mann oder Frau erkennbar ist beide androgyn wirken (siehe 1).

Die Befragten konnten ihre Kommunikation weitestgehend selbst strukturieren und selbst offen legen, wie sie die Fragestellung interpretieren. In Bezug auf die empirische Untersuchung verwendete ich ,Geschlecht' somit in einem sehr breitgefächerten Sinne, als heuristischen Suchbegriff, der dazu dient, Teilbereiche eines Verständnisses, einer Position oder habituellen Praxen, die damit in Verbindung gebracht werden, zu erfragen. Dahinter steckt das Ziel, neue Tatsachen, Zusammenhänge, Gedankengänge und Ideen in Bezug auf das handlungsorientierte Wissen hinsichtlich ,Geschlecht' zu entdecken. Einfacher formuliert: Wenn ich danach frage, wie und was Studierende über ,Geschlecht' und Geschlech-

12 Auf Deutsch: „Bist du ein Mädchen?“ Und die andere Person antwortet: „Wen kümmert's?" oder „Wenn interessiert's?" 
terfragen sprechen, dann interessiert mich alles, was die Studierenden diesbezüglich zu sagen oder eben auch nicht zu sagen haben. Angefangen bei Themen der Frauen- und Geschlechterforschung, Geschlechterfragen als Inhalt im Studium, der statistischen Repräsentation von Frauen und Männern im Studium, geschlechtergerechter Sprache, bis hin zu Schilderungen von Reflexions- und Sensibilisierungsprozessen zu ,Geschlecht', Erfahrungen in der pädagogischen Praxis, vergeschlechtlichte und vergeschlechtlichende Handlungs- und Denkschemata, Auseinandersetzung mit queer Identitäten, sowie Diskriminierung, Benachteiligung aber auch Bevorzugungen qua ,Geschlecht' ist alles relevant und wird in der Untersuchung genauer interpretiert. Diese Aufzählung mag an dieser Stelle vielleicht unsystematisch und durcheinander erscheinen, dennoch ist sie empirisch gefüllt und spiegelt meine Erfahrung aus dem Forschungsfeld und die (De-)Thematisierung von ,Geschlecht' bei Studierenden wider.

Die Besonderheit an Gruppendiskussionen ist, dass diese spontan und unangekündigt zu Assoziationen bezüglich Geschlechterfragen einladen. Das heißt, das Setting der Gruppendiskussion fordert die GruppendiskussionsteilnehmerInnen ${ }^{13}$ dazu auf, sich mit ,Geschlecht' und Geschlechterfragen auseinanderzusetzen bzw. geht davon aus, dass sich die GDT bereits mit ,Geschlecht' auseinander gesetzt haben. Diese Aufforderung wird von den Gruppen unterschiedlich aufgegriffen und bearbeitet. So wird die Diskussion der Gruppe Feuer zur Selbstreflexion und zur kritischen Reflexion der Kategorie ,Geschlecht' genutzt, wohingegen die Gruppe Holz die Diskussion nutzt, um theoretisch und abstrakt über ,Geschlecht' zu sprechen (siehe Kapitel 6).

Im Anschluss an die Diskussionen wurden die TeilnehmerInnen gebeten, einen kurzen Erhebungsbogen mit Fragen zu ihrer Person (z. B. Alter, Migrationshintergrund, Bildung der Eltern und ggf. der Geschwister) und Angaben zur Studiumsbiografie (siehe Anhang) auszufüllen. Das Ausfüllen des Kurzfragebogens wurde bewusst am Ende der Datenerhebungssituation platziert, um die klassische Frage-Antwort Situation und eine Atmosphäre des Abfragens zu vermeiden. Die auf diesem Wege ermittelten Informationen wurden jedoch nur vereinzelt genutzt und dienten eher der Kontextualisierung als der Rekonstruktion konjunktiver Erfahrungsräume. Mitunter kommentierten die Teilnehmer/innen beim Ausfüllen der Bögen einzelne Fragen, was ebenfalls Hinweise auf Relevanz und Thematisierung von ,Geschlecht' gab. So wurde in der Gruppe Feuer der Universität Sonne beispielsweise beim Ausfüllen bemerkt, dass

13 GruppendiskussionsteilnehmerInnen werden im Folgenden auch mit GDT abgekürzt. 
sie noch nie danach gefragt wurden, welches ,Geschlecht' sie in ihrem Ausweis angegeben hätten und dass diese Art der Frage eine Wahlmöglichkeit impliziert, die ihnen zuvor noch nie aufgefallen war.

Vor den Treffen wurde von der Forscherin ein Präskript verfasst. Dieses beinhaltet Notizen aus dem Vorfeld des Interviews. Es wurden Erwartungen an das konkrete Interview formuliert, aber auch die eigene Befindlichkeit festgehalten. Dies ist sinnvoll, um Informationen verfügbar zu machen, die sonst für die Forschungssupervision oder den Auswertungsprozess nicht zugänglich wären (vgl. Mey/Mruck 2007, S. 270.). Nach der Erhebungssituation wurde ein Postskript angefertigt. Es umfasst Auffälligkeiten und Befindlichkeiten, die sich auf die Erhebungssituation selbst und auf die nicht aufgezeichnete Zeit vor und nach der Befragung beziehen (vgl. ebd.). Diese Aufzeichnungen wurden in die Datenanalyse miteinbezogen.

Bei den Gruppendiskussionen, so meine Erfahrung mit der vorliegenden Untersuchung, zeigte sich immer wieder, dass die Studierenden in Bezug auf ,Geschlecht' zunächst Mühe hatten, nicht ,richtig', sozialerwünscht zu antworten, sondern ihre eigenen Statements und Erfahrungen zu diskutieren. Offen bleiben muss, welche interaktiven Effekte mein ,Geschlecht' und meine Rolle als erziehungswissenschaftliche Forscherin und Interviewerin bei den Studierenden ausgelöst hat.

\subsubsection{Transkription}

In der rekonstruktiven Sozialforschung wird die Audioaufnahme als Zeugnis der sozialen Welt durch Transkription zu einem abdruckbaren Dokument (vgl. Przyborski/Wohlrab-Sahr 2009, S. 162). In diesem Sinne wurden alle Diskussionen aufgezeichnet und vollständig transkribiert. Die Transkription der Gruppendiskussionen erfolgte direkt im Anschluss an die Durchführung. Die systematische Transkription trägt wesentlich zur intersubjektiven Überprüfbarkeit der Daten bei. Die Gesprächsinhalte wurden anonymisiert und authentisch wiedergegeben, d. h. Dialektsprache und Satzbaufehler wurden nicht behoben. Die Transkription erfolgte nach den Richtlinien des TiQ - Talk in Qualitative Social Research (vgl. Przyborski/Wohlrab-Sahr 2009, S. 166f; Bohnsack 2008, S. 235f.): Hauptwörter werden hiernach groß geschrieben und beim Neuansetzen eines/einer Sprecher/in wird das erste Wort mit Großbuchstaben begonnen. Nach Satzzeichen wird klein weitergeschrieben, wenn diese die Intonation anzeigen und nicht grammatikalisch gesetzt werden (vgl. Bohnsack 2007, S. 236). Um das Auffinden und Zitieren von Transkripti- 
onsstellen zu ermöglichen wird eine durchlaufende Zeilennummerierung verwendet (vgl. ebd.; genaueres siehe Anhang). Die transkribierten Daten wurden mit dem Instrumentarium der dokumentarischen Methode ausgewertet. Im weiteren Verlauf werde ich die Arbeitsschritte der dokumentarischen Methode zur Auswertung von Gruppendiskussionen genauer darstellen, um nochmals die methodische Brille, die auch meinen Blick auf den Forschungsgegenstand prägt, deutlich zu machen. Davor möchte ich jedoch noch das Reifizierungsproblem in der Geschlechterforschung ansprechen. Die empirische Untersuchung konfrontiert die Forschenden mit der Schwierigkeit, dass sie sich nie gänzlich von den Kategorien Frau/Mann, Homo-/Bi-/Heterosexualität distanzieren können, da die Heteronormativität als soziale Institution immer, eben auch im Forschungsprozess und bei den Forschenden selbst, wirksam ist (vgl. Götsch 2012, S. 96).

\subsubsection{Das Problem der Reifizierung der Kategorie 'Geschlecht' in der empirischen Forschung}

Für die empirische Forschung stellt sich die Herausforderung, wie Geschlechterverhältnisse und auf ,Geschlecht' bezogene Phänomene empirisch überzeugend untersucht werden können, ohne dadurch Geschlechterbinarität und Heteronormativität zu reifizieren. Zum einen besteht das Anliegen darin, ,Geschlecht' nicht als gegebene Kategorie vorauszusetzen, sondern als Differenzkonstruktion zu betrachten und zum anderen besteht die Gefahr, dass sich die Forschung selbst an diesen Konstruktionsprozessen beteiligt. Zudem stellt die Komplexität und Differenziertheit von Geschlechtertheorien die empirische Forschung vor eine Herausforderung (vgl. Dausien 2009, S. 159).

Die empirische Forschung steht somit vor einem methodologischen und methodischen Dilemma, zu dem es keine Lösung gibt, sondern nur die Strategie des reflexiven Umgangs mit dem Problem (vgl. ebd., S. 160). Regine Gildemeister schlägt vor, dieses grundlegende Problem in die Forschung einzubeziehen, indem „,man sich den eigenen Wissensbeständen gegenüber systematisch, dumm' stellt, oder [...] eine Perspektive der künstlichen Fremdheit einnimmt" (Gildemeister 2004, S. 33). Carol Hagemann-White (1993) plädiert dafür, die Perspektive der alltagsweltlichen Deutung der Geschlechterdifferenz beispielsweise in der Erhebungssituation zum einen bewusst einzunehmen und zum anderen in der Auswertungssituation durch Hinterfragen des scheinbar Selbstverständlichen außer Kraft zu setzen. 
„So wie wir keine Sprache untersuchen können, ohne die konsensuell alltäglich vorhandenen Bedeutungen der Worte zu wissen, so können wir den Umgang mit der Zweigeschlechtlichkeit in seinem prozessuellen Charakter nicht in der Forschung berücksichtigen, wenn die mit Geschlechtsbedeutung versehenen Elemente nicht identifiziert werden" (Hagemann-White 1993, S. 76).

Konkret wurden bei der Anonymisierung und der Interpretation des Datenmaterials bewusst möglichst geschlechtsneutrale Vornamen gewählt, um im Auswertungsprozess eine Haltung zu entwickeln, in der systematisch vermieden wird, bekannte Figuren und Stereotype unterschiedlicher Differenzkategorien zu reproduzieren und damit zu reifizieren. So soll vermieden werden, Männer und Frauen in essenzialisierender Weise miteinander zu vergleichen oder ,Geschlecht' als unhinterfragte Ressource der Forschung einzusetzen (vgl. Gildemeister 2007, S. 221). Die Verwendung von Unisex-Namen war hauptsächlich für den Auswertungsprozess dienlich sein, damit ,Geschlecht' nicht mehr zur (unbewussten) „Leitkategorie" (Degele 2004, S. 46) erhoben wird, sondern der Fokus bei der „Ver-/Entgeschlechtlichung“ (ebd.) von ,Geschlecht' liegt. Im Prozess der Auswertung, der u. a. in Interpretationsgruppen und Forschungswerkstätten $^{14}$ stattgefunden hat, hat dieses Vorgehen immer wieder Irritationen ausgelöst. Zu Beginn der Interpretationseinheiten erschienen die Fragen nach dem (biologischen), Geschlecht` der einzelnen GDT als wissenswert und relevant, weshalb erst ein Umgang mit dem Nicht-Wissen gefunden werden musste. Im Laufe der Interpretationseinheit(en) rückte die Frage nach der Geschlechteridentifikation schließlich in den Hintergrund und verlor an Relevanz, vielleicht auch deshalb, weil eine ,eindeutige ' Erkennung nur selten möglich war und die Zuordnungen und Zuschreibungen so vielfältig wie die Zahl der Mitglieder der Interpretationsgruppe waren. Schlussendlich war die Kategorisierung von Aussagen und Sprechweisen der GDT in Bezug auf ihr ,Geschlecht' nicht mehr von Belang.

In der Darstellung der Gruppendiskussionen habe ich nun diese Unisex-Namen beibehalten, jedoch nicht, um das ,Geschlecht' der SprecherInnen zu neutralisieren oder zu camouflieren, sondern um im besten Falle auch bei den LeserInnen und Rezipientinnen dieser Untersuchung Irritationen und vielleicht sogar ,Bewusstwerdungs- und Reflexionsprozesse' anzustoßen und ,Geschlecht' als binäre Kategorie zu

14 Hier soll darauf hingewiesen werden, dass die mit Bedacht vorgenommene Absicherung der Interpretation in den Interpretationsgruppen und Forschungswerkstätten ein Gütekriterium im Sinne von intersubjektiver Übereinstimmung darstellt. 
hinterfragen. Ziel ist es, nicht unreflektiert jene Konstruktionen von (Zwei-)Geschlechtlichkeit zu reproduzieren, die es zu kritisieren gilt. Für den Umgang mit Differenzkategorien in der qualitativen Forschung schlägt Regine Gildemeister (2007) vor, dass vermieden werden soll, Männer und Frauen in essenzialisierender Weise miteinander zu vergleichen oder ,Geschlecht' als fraglose Ressource der Forschung einzusetzen. Diesen Anspruch habe ich auch für andere dichotome Gegenüberstellungen wie z. B. ,bildungsnahe' vs. ,bildungsferne' Studierende angewendet. Eine Möglichkeit wäre es, ,Geschlecht' abwechselnd zu thematisieren und dann wieder gezielt auszublenden. Zudem wird empfohlen, Forschungsphasen zeitlich zu entzerren und in bestimmten Phasen der Auswertung das Material auf Hinweise wie Geschlechts- oder Ethnizitätszugehörigkeit etc. der Sprechenden im Text zu verschlüsseln. Dies hat zur Folge, dass Aussagen nicht als stereotype Formen klassifiziert werden können (vgl. ebd., S. 221; Götsch/Klinger/Thiesen 2012, Abs. 49).

\subsection{Auswertung auf Basis der dokumentarischen Methode}

Das dokumentarische Verfahren zielt nicht nur auf die Analyse textimmanenter Aussagen ab, sondern geht in weiteren methodisch-kontrollierten Schritten auch dem impliziten Gehalt der Aussagen beziehungsweise den grundlegenden Bedeutungszuschreibungen und Orientierungsmustern nach. Es versucht, die Oberflächensemantik des Gesagten systematisch zu transzendieren, indem es zunächst ihren wörtlichen thematischen Sinngehalt zusammenfassend formuliert, um dann die Frage zu stellen, wie (d. h. innerhalb welches Orientierungsrahmens) die auf dieser Ebene identifizierten Themen bearbeitet werden (vgl. Bohnsack/Przyborski/Schäffer 2010, S. 13). Hier spielt die Rekonstruktion der Diskursorganisation eine bedeutsame Rolle.

Bei der dokumentarischen Methode sind die Arbeitsschritte der formulierenden und der reflektierenden Interpretation, der Falldarstellung und der Typenbildung zu unterscheiden. Wesentliche Merkmale des Verfahrens sind ein sequenzielles und komparatives Vorgehen (vgl. Bohnsack 2008, S. 134ff). Die Basis für die einzelnen Interpretationsschritte und Darstellungen bilden die Transkripte der Gruppendiskussionen. 


\subsubsection{Schritte der Interpretation bei der Gruppendiskussion}

Das Interpretationsverfahren vollzieht sich in unterschiedlichen, jeweils aufeinander aufbauenden, Arbeitsschritten (vgl. Bohnsack 2008, S. 134). Im Folgenden werden die Interpretationsschritte der dokumentarischen Methode beschrieben. Nach diesen Schritten erfolgte auch die Auswertung des vorliegenden Datenmaterials.

\section{* Thematischer Verlauf, Auswahl von Passagen und Transkription}

Die ersten Schritte dienen dazu, sich in Form eines thematischen Verlaufs einen strukturierten Überblick über das konkrete Datenmaterial zu verschaffen. Dazu werden jeweils Abschnitte der Diskussion, die einen thematischen Zusammenhang bilden, in Form von Überschriften zusammengefasst. Diese dienen als Grundlage für die Auswahl jener Textabschnitte, die im Sinne der formulierenden und reflektierenden Interpretation ausgewertet werden (vgl. Przyborski/Wohlrab-Sahr 2009, S. 286). Der thematische Verlauf wird anhand der transkribierten Gruppendiskussion erstellt und erfolgt, indem die Themen bzw. thematische Wechsel sowie Anhaltspunkte für Interaktionsmerkmale festgehalten wird. Auf dieser Grundlage erfolgt die Auswahl werden die Passagen nach formalen und inhaltlichen Gesichtspunkten ausgewählt (vgl. ebd., S. 287). Zudem werden Passagen ausgewählt, die sich besonders durch interaktive und metaphorische Dichte auszeichnen (vgl. Bohnsack 2008, S. 134). In ihnen zeigt sich der Orientierungsrahmen bzw. Habitus einer Gruppe besonders deutlich; daher werden sie als Fokussierungsmetaphern bezeichnet. Zum anderen fiel die Wahl auf Passagen, die vor dem Hintergrund des Themas der Untersuchung von Relevanz sind. Im Rahmen meiner Studie habe ich die Passagen zu geschlechtergerechter Sprache sowie die Fokussierungsmetaphern aller Gruppen für die genauere formulierende und reflektierende Interpretation ausgewählt. Zusätzlich habe ich die Passagen analysiert, in denen Geschlechterdifferenzen und Geschlechtergleichheit, Benachteiligung und Diskriminierung thematisiert wurden.

\section{* Formulierende Interpretation}

In der formulierenden Interpretation geht es darum, zu den ausgewählten Passagen detaillierte formulierende Interpretationen zu erstellen; dazu werden wiederum einzelne Abschnitte der jeweiligen Passage in Form von Überschriften und Paraphrasierungen der Diskussionsinhalte feinge- 
gliedert. Dabei werden formulierende Zusammenfassungen erstellt, die innerhalb des Kommunikations- und Sinnhorizontes der befragten Person verbleiben. Zum Wahrheits- und Realitätsgehalt wird nicht Stellung genommen (vgl. Bohnsack 2008, S. 34, S. 134). In diesem zweiten Schritt geht es um eine zusammenfassende (Re-)Formulierung des allgemein verständlichen Sinngehalts der ausgewählten Passage. Der Inhalt wird mit dem Ziel paraphrasiert, die thematische Struktur und die Gliederung des Textes, die sich meist nicht unmittelbar erschließt, nachzuzeichnen (vgl. Przyborski/Wohlrab-Sahr 2009, S. 287). Er wird in Ober- und Unterthemen gegliedert, wobei ich vermerkt habe, ob das jeweilige Thema von den Befragten oder von der Befragenden initiiert wurde. Die explizierten Begriffe der Teilnehmenden der Gruppendiskussion wird also in diesem Analyseschritt zusammenfassend formuliert (vgl. Bohnsack 2003; 2008; Przyborski 2004).

\section{* Reflektierende Interpretation}

Der dritte Schritt zielt nun auf den Dokumentsinn, der in die Handlungspraxis eingelassen ist. Es handelt sich hierbei um die Explikation von Handlungsorientierungen und Habitusformen (vgl. ebd., S. 296). Hier steht im Mittelpunkt, wie bzw. in welchem Rahmen ein Thema in der jeweiligen Gruppe diskutiert und entfaltet wird. Dabei geht es nun um den dokumentarischen Sinngehalt. Der Übergang zur reflektierenden Interpretation besteht darin, dass der Rahmen thematisiert wird, innerhalb dessen etwas in einer bestimmten Weise exponiert wurde. Dieser Schritt zielt auf die Rekonstruktion und Explikation dieses Rahmens ab, also darauf, den Orientierungsrahmen oder Habitus einer Gruppe zu rekonstruieren (vgl. Bohnsack 2008, S. 135f.).

„In einem Sich-wechselseitigen-Steigern-und-Fördern, im diametralen Gegeneinander, in der kommentierenden Ergänzung oder auch in der systematischen Vereinnahmung der anderen finden jeweils andere Modi der Diskursorganisation und somit aber auch unterschiedliche - milieuspezifische - Formen fundamentaler Sozialität ihren Ausdruck, und es zeigt sich, ob den Beteiligten überhaupt ein ,Erfahrungsraum', ein Milieu gemeinsam ist oder nicht" (Bohnsack/Przyborski/Schäffer 2010, S. 8f.).

Dazu fokussiert die Analyse verschiedene Aspekte: Zunächst ist es hilfreich, positive und negative Gegenhorizonte sowie Enaktierungspotenziale in der Diskussion zu identifizieren (vgl. Przyborski/Wohlrab-Sahr 2009, S. 296). Bei der reflektierenden Interpretation ist die Rekonstruktion der Diskursorganisation zentral, also wie die Redebeiträge in for- 
maler Hinsicht aufeinander bezogen sind (vgl. Bohnsack 2008, S. 136). Beispielsweise macht es einen Unterschied, ob der Diskursverlauf durch ein argumentatives Gegeneinander nach dem Muster These-AntitheseSynthese organisiert ist (antithetische Diskursorganisation) oder ob in den Redebeiträgen Erzählungen und Beschreibungen ,nebeneinander' gestellt werden, in denen ein identisches Orientierungsmuster in Variationen immer wieder zum Ausdruck gebracht wird (parallelisierende Diskursorganisation) (vgl. Bohnsack/Schäffer 2007, S. 309). Bei der Diskursanalyse ist auch von Interesse, welche Orientierungen zur Sprache gebracht (Proposition), entfaltet (Elaboration) und geschlossen werden (Konklusion). ${ }^{15}$ Grundsätzlich wird zwischen inkludierenden und exkludierenden Modi der Diskursorganisation unterschieden, wobei letztere in Bezug zu den Rahmeninkongruenzen innerhalb der jeweiligen Gruppe stehen (vgl. Przyborski 2004, S. 95; Kubisch 2008, S. 125). In meiner Untersuchung zeigen sich in der Gruppe Holz deutliche Rahmeninkongruenzen wenn es um die Handlungspraxis von geschlechtergerechter Sprache geht. Hier kommt es unter den GDT zu Falschrahmungen und in diesem Zusammenhang weist die Diskursorganisation eine Reihe ritueller Konklusionen auf (genaueres siehe Kapitel 6.2). Es wird nicht abschließend zusammengefasst und konsensuell bewertet, sondern widersprechende Orientierungsgehalte werden nicht aufgelöst und Themen, die auf Rahmeninkongruenzen hinweisen, werden ausgeklammert oder als irrelevant abgetan (vgl. Przyborski 2004, S. 75). Bei der Analyse der Diskursorganisation liegt der Erkenntnisgewinn in der Rekonstruktion der Formen der Inszenierung des Kollektivs, wodurch diese für diese Untersuchung ein zentrales Moment der Auswertung darstellt. Deshalb sollen die Modi der Diskursorganisation im Folgenden genauer beschreiben werden.

\section{Inkludierende Modi}

Als inkludierende Modi bezeichnet Aglaia Przyborski jene Modi, in denen gemeinsame Orientierungen zum Ausdruck kommen. Hier unterscheidet sie parallele, antithetische (konkurrierende) und univoke Modi (vgl. ebd., S. 96). In der parallelen Diskursorganisation artikulieren sich gemeinsame Organisationen auf Basis gemeinsamer, im Sinne homologer Erfahrungen. Hier zeigt zunächst eine Aneinanderreihung, dass gewisse Darstellungen, die für eine fremde Beobachterin auf den ersten Blick manchmal wenig miteinander zu tun haben, für die Beteiligten jedoch

15 Das begriffliche Repertoire zur Diskursorganisation ist bei Aglaia Przyborski (2004) umfassend erläutert. 
die gleiche Sache betreffen. In der Interpretation erschließst sich dann dieselbe Orientierung in allen aufeinanderfolgenden Diskursbewegungen. Fasst man die dokumentarische Methode als eine Suche nach Homologien, die sich über Themen hinweg immer wieder finden, dann ist der parallele Modus letztlich der prototypische Modus dieser Methode (vgl. ebd., S. 97). Auch in der antithetischen Diskursorganisation kommen gemeinsame, homologe Orientierungen zum Ausdruck. Hier aber nicht in Form von einer immer wiederkehrenden Strukturidentität innerhalb der einzelnen Diskursbewegungen wie beim parallelen Modus, sondern durch Widerstreit und Verneinungen, ein oft konkurrierendes Gegeneinander, dessen gemeinsame Orientierungsgrundlage sich für den Interpreten/die Interpretin oft erst in der Synthese der widersprechenden Positionen als einander ergänzende Komponenten einer Orientierung, eines Habitus erschließst (vgl. ebd., S. 168).

Die Erfahrungen, die sich im univoken Modus inszenieren, sind homolog, also strukturidentisch, und sind dieselben identischen Erfahrungen. Dieser Modus findet sich systematisch in Diskursen auf der Grundlage derselben Erfahrungsbasis. Dabei ist wichtig zu unterscheiden, dass ein Vorfall, ein Ereignis oder auch eine historische Entwicklung von verschiedenen Beteiligten ganz unterschiedlich erfahren werden kann. Es genügt nicht, zur selben Zeit am selben Ort gewesen zu sein. Im univoken Modus inszeniert sich die Erfahrung derselben Begebenheit aus derselben Perspektive, die Erfahrung ist identisch und damit auch homolog strukturiert. Univoke Diskursbewegungen werden auch gemeinsam realisiert. Das Besondere an dieser gemeinsamen Realisierung ist das gleichzeitige Sprechen. Die ganze Gruppe spricht sozusagen unisono bzw. univok (vgl. ebd., S. 196).

\section{Exkludierende Modi}

Als exkludierende Modi bezeichnet Przyborski Modi, in welchen unterschiedliche, unvereinbare Orientierungen zum Ausdruck kommen. In diesen Formen diskursiver Praxis bringt das Kollektiv unterschiedliche, einander widersprechende Orientierungen hervor. Zur Analyse kommt also die Inszenierung bzw. die Artikulation unterschiedlicher Erfahrungsgrundlagen (vgl. ebd., S. 216). Exkludierende Modi werden von Przyborski als oppositionelle oder divergente Modi beschrieben. Exkludierende Modi lassen sich feststellen wenn z. B. eine Gruppe längere Zeit auf der Grundlage unterschiedlicher Erfahrungen spricht und dieser Bereich nicht gleich einem Themenwechsel zum Opfer fällt. Es lässt sich vorab nicht definieren, ob eine Gruppe einen exkludierenden Modus auf- 
weist. Wie die anderen Modi lässt sich auch der exkludierende Modus nicht mit einem oberflächlichen Blick auf das Material erkennen, sondern erst im Laufe der intensiven Interpretation (vgl. ebd.).

Eine oppositionelle Diskursorganisation findet sich in Gesprächen bzw. Gesprächsabschnitten, in welchen die Teilnehmer_innen über keine geteilten Erfahrungen verfügen. Hier kommt es zu Rahmeninkongruenzen, also zu offenen Erfahrungsunterschieden, die von den Teilnehmenden nicht in einen übergreifenden, kollektiv geteilten Rahmen überführt werden können. Hier münden widersprechende Orientierung(-skomponenten) nicht wie beim antithetischen Modus in eine Synthese, d. h. in eine Konklusion, in der Widersprüche als Teile einer Orientierung deutlich werden. Es kommt vielmehr zu sogenannten ,rituellen Konklusionen“ (ebd., S. 217), in welchen Themen - ohne dass auf sie eingegangen wird - abgeschlossen werden (vgl. ebd.). Das können beispielsweise Fragen nach einem neuen Thema sein, aber auch die Äußerung von Allgemeinplätzen wie ,jeder hat eine andere Meinung“" oder die Suspendierung eines Themas wie „darüber spricht man nicht" (ebd., S. 218). Typisches Merkmal des oppositionellen Modus ist ein offenes Gegeneinander von Orientierungen. Es kommt zu Themenabbrüchen oder zu Eskalationen an Stellen des Themenwechsels (vgl. ebd., S. 287).

In einem divergenten Diskurs beziehen sich die TeilnehmerInnen in Hinblick auf den Orientierungsgehalt nicht aufeinander, sondern reden vielmehr aneinander vorbei. Darin dokumentiert sich, dass die unterschiedlichen Orientierungen zu einem Thema für die in Frage stehenden Handlungsfelder bestehen bleiben (vgl. ebd., S. 72f.). Wie auch oppositionelle Modi finden sich divergente Modi der Diskursorganisation in Gesprächen bzw. Gesprächsabschnitten, in welchen die TeilnehmerInnen über keine geteilten Erfahrungen verfügen (vgl. ebd., S. 252). Im Unterschied zum oppositionellen Modus knüpfen die Diskursbewegungen aber aneinander an. So werden z. B. Sachverhaltsdarstellungen oft bestätigt, oder es werden Markierer (z. B. ,ja, aber") für die Bestätigung oder für eine lediglich leichte Differenzierung einer Proposition gesetzt. Im Anschluss daran werden aber einander widersprechende Orientierungsrahmen aufgeworfen. Beim divergenten Modus bleiben die Rahmeninkongruenzen eher verdeckt. Die einander widersprechenden Orientierungskomponenten münden, ebenso wie im oppositionellen Modus, nicht in eine Synthese, in der Widersprüche als Teil einer Orientierung deutlich werden, sondern in rituellen Konklusionen. Der divergente Modus der Diskursorganisation unterscheidet sich vom oppositionellen Modus dadurch, dass faktische Beschreibungen und Orientierungskomponenten 
immer wieder zur Stärkung einer widersprechenden Orientierung verwendet werden (vgl. ebd.). Typisches Merkmal des divergenten Modus ist die Vereinnahmung bzw. die „Falschrahmung“" (ebd., S. 287). Das Gegeneinander der Orientierungen bleibt bzw. wird verdeckt. Dabei werden entweder Orientierungskomponenten durch deren Ironisierung zur Artikulation einer widersprechenden Orientierung genutzt oder faktische Darstellungen befürwortet, um sie dann in eine widersprechende Orientierung einzubauen (z. B. Gruppe Holz, siehe Kapitel 6.2). Darin dokumentiert sich, dass die unterschiedlichen Orientierungen bestehen bleiben (vgl. ebd., S. 72f.). Neben der Rekonstruktion der Diskursorganisation(en) ist für die dokumentarische Methode charakteristisch, dass fallübergreifende und fallinterne Vergleiche schon früh in die Interpretation mit einbezogen werden. Sie spielen bereits in der reflektierenden Interpretation eine wichtige Rolle.

\section{* Komparative Analyse - Typenbildung bzw. die Bildung von (De-)Thematisierungsmustern}

Die komparative Analyse durchzieht letztlich den gesamten Interpretationsprozess. Ziel der Interpretation im Sinne der dokumentarischen Methode ist es, den Orientierungsrahmen der jeweiligen Gruppe, d. h. ihren Habitus zu rekonstruieren (vgl. Kubisch 2008, S. 126). Nachdem ein Orientierungsrahmen identifiziert wurde, wird dieser durch die fallinterne (fallexterne) komparative Analyse abstrahiert. Die komparative Analyse gewinnt bereits auf der Ebene der Fallanalyse Bedeutung: dort wo die Besonderheit des einzelnen Falles - innerhalb des übergreifenden Rahmens - strukturiert wird. In meiner Untersuchung habe ich zunächst fallübergreifende Vergleiche anhand von Passagen angestellt, in denen ,Geschlecht' (geschlechtergerechte Sprache, Geschlechterdifferenzen und -gleichheit, Benachteiligung und Diskriminierung usw.) zum Thema gemacht wurden. Im Zuge der parallel erfolgenden fallinternen Vergleiche wurden auf diese Weise Homologien in den Orientierungen der einzelnen Gruppen herausgearbeitet.

Die Beschreibung der jeweiligen Gruppendiskussionen hat primär die Aufgabe der vermittelnden Darstellung, Zusammenfassung und Verdichtung der Ergebnisse. Dabei werden zentrale Orientierungen und Rahmenkomponenten der jeweiligen Gruppe, die dramaturgische Entwicklung und die Diskursorganisation dargestellt und aufeinander bezogen.

„Eine Diskursbeschreibung wird umso runder und dichter, je umfassender es gelingt, die Beschreibung der Orientierungsmuster und Rahmenkomponenten (,Inhalt') einerseits und die Beschrei- 
bung von Dramaturgie und Diskursorganisation andererseits (,Form $\left.{ }^{6}\right)$ in einer Gesamtcharakteristik des Falles verschmelzen zu lassen, so z. B. indem sich zeigen lässt, wie sich die Gruppe die Artikulation dessen, was denn nun ihr eigentliches Anliegen und Problem zu einem Thema ist, Schritt für Schritt erarbeitet, erst allmählich zum Fokus vordringt, sich dabei dramaturgisch steigert (hinsichtlich interaktiver und metaphorischer Dichte) und gerade in dieser prozesshaften Bearbeitung unterschiedliche Schichten des Rahmens sichtbar werden lässt" (vgl. Bohnsack 2008, S. 140).

In meiner Studie habe ich zu insgesamt vier Gruppen, die zwei Universitäten zuzuordnen sind, ausführliche Beschreibungen angefertigt (vgl. Kapitel 6). Wie bereits angedeutet, hat die Zusammensetzung der von mir untersuchten Gruppen Auswirkungen auf die Typenbildung. So war es angesichts der Zusammenteilen des Samples nicht geplant, eine komparative Analyse zwischen so genannten homogenen Gruppen beispielsweise hinsichtlich des Geschlechts oder des Alters durchzuführen (vgl. Kubisch 2008, S. 128).

Mein Ziel der Ergebnisrekonstruktionen kann somit als sinngenetische Typenbildung verstanden werden, wobei ich nach sinnhaften, strukturierenden Mustern, die sich aus dem ,subjektiven' Sinn des Handelns und dem zugrunde liegenden ,objektiven' Sinn bzw. den sozialen Regeln ergeben, gesucht habe (vgl. Kelle/Kluge 2010). Für die konkrete Typenbildung habe ich mich somit an der Rekonstruktion der Habitusformen der jeweiligen Gruppen in Bezug auf die (De-)Thematisierung von ,Geschlecht' orientiert. Auf der Suche nach Thematisierungsmustern waren für mich die Fragen leitend, was die Studierenden über ,Geschlecht' erzählen, also inwiefern es für bestimmte Themen spezifische Muster gibt. Das Bilden von Mustern steht somit im Dienste der Verdichtung und Zuspitzung dessen, was sich in der Empirie findet. Das Muster vereint verschiedene aber zusammengehörige Elemente, die die jeweiligen Gruppen in wiederum übergreifender Weise vereinen. Im universitätsinternen und -übergreifenden Vergleich zwischen den Gruppen geht es darum, Ähnlichkeiten und Differenzen zu identifizieren.

Ziel ist es, im Vergleich der Universitäten die Homologien des sozialen Feldes und der gesellschaftlichen Strukturen der (De-)Thematisierung zu entwickeln und anhand der komparativen Analyse heraus zu arbeiten. Es lassen sich jedoch auch Muster herausarbeiten, die die Thematisierung von ,Geschlecht' auszeichnen. In diesem Sinne lassen sich in allen Gruppen Thematisierungsmuster rekonstruieren, die Heteronormativität und eine eindeutige und kohärente Geschlechterbinarität als Norm repro- 
duzieren. Das Ziel der empirischen Analyse steht somit im Zeichen der Herausarbeitung von Mustern der (De-)Thematisierung von ,Geschlecht und Geschlechterfragen. 



\section{Datenauswertung: ,,Geschlecht zwischen und auf den Zeilen ${ }^{16}$}

Im Rahmen des folgenden Kapitels sollen die Interpretationen und Rekonstruktionen des empirischen Materials dargestellt werden. Um die verschiedenen universitären Kontexte der Befragten nochmals in Erinnerung zu rufen, werden die Universitäten Sonne und Mond kurz darstellt (vgl. Kapitel 5.1). An der Universität Sonne haben sich nur Bachelorstudierende und an der Universität Mond nur Diplomstudierende an der Untersuchung beteiligt. An der Universität Sonne wurden Gruppendiskussionen mit der Gruppe Holz und Feuer geführt und an der Universität Mond wurden die Gruppen Metall und Wasser befragt. An beiden ausgewählten Universitäten haben die Studierenden inner- und außerhalb ihres Fachbereiches die Möglichkeit, Seminare zu Geschlechterfragen, oder „Genderseminare“, wie diese von den befragten Studierenden der Gruppe Wasser genannt werden, zu besuchen. An den Universitäten gibt es jeweils Zentren für die Erforschung von Geschlechterverhältnissen und für feministische Wissenschaft. Hier haben die Studierenden die Möglichkeit, an einem zertifizierten Studienprogramm teilzunehmen. Dies ist deshalb interessant, weil diese institutionalisierten Orte der Frauen- und Geschlechterforschung für die Studierenden als potentielle Räume für die Auseinandersetzung mit ,Geschlecht‘ (neben dem eigenen Studium) gesehen werden können. Zudem sind an der Universität Mond autonome Studierendengruppen aktiv, die allen Studentinnen die Möglichkeit bieten, sich über den eigenen Fachbereich hinaus zu engagieren.

Im folgenden Abschnitt wird als erstes herausgearbeitet, ,was ${ }^{6}$ die befragten Studierenden diskutierten, als sie in der Gruppendiskussion dazu aufgefordert waren, über ,Geschlecht' zu sprechen. Diese Darstellungen dienen dazu, die ,Was'-Ebene, also die besprochenen Themen der Gruppendiskussionen, für die Leserinnen und Leser zugänglich zu machen. Zudem bietet die gruppen- und universitätsübergreifende Explikation dieser deskriptiven Ebene den Einstieg in das empirische Material, analog zum Vorgehen der dokumentarischen Methode (vgl. Kapitel 6.1). Danach wird die Rekonstruktion habitueller Praxen und kollektiver Orientierungen bei der (De-)Thematisierung erläutert. Aufgrund

1 Hierbei handelt es sich um eine wörtliches Zitat von Susanne Maurer während des DoktorandInnenkolloquiums am 24.11.2013 in Marburg. 
der inneren Komplexität der Gruppen erfolgt diese Darstellung entlang der einzelnen Gruppendiskussionen und gruppiert nach der jeweiligen Universität. Es werden die jeweiligen Gruppen mit den für sie charakteristischen Orientierungen in Hinblick auf die (De-)Thematisierung von ,Geschlecht" und Geschlechterverhältnisse dargestellt. Hier geht es um fallinterne Homologien bzw. um den Habitus der einzelnen Gruppe (vgl. Kapitel 6.2, 6.3, 6.5, 6.6).

In der gruppenübergreifenden komparativen Analyse jener Gruppen, die derselben Universität zugeordnet sind, werden zudem im Kapitel 6.7 und 6.4 die gemeinsamen universitäts- und studiumsbezogenen konjunktiven Erfahrungen bei der (De-)Thematisierung zur Geltung gebracht.

Damit die Leser_innen den Gang der Rekonstruktion mitverfolgen können, sind Transkriptauszüge in die Falldarstellungen integriert.Alle Personen und Orte sind aufgrund der Anonymisierung umbenannt. Auch Angaben, die Hinweise auf Personen, die Universitäten oder Orte geben könnten, wurden verändert.

\subsection{Deskriptive Darstellung der Gruppendiskussionen: Themen und Inhalte}

In der folgenden deskriptiven Darstellung der Gruppendiskussionen werden vor allem Homologien bei den besprochenen Themen und Inhalten herausgearbeitet und dargestellt, wie das Thema ,Geschlecht" von den Gruppen unterschiedlich aufgegriffen und bearbeitet wurde. Ziel ist es, einen Über- und Einblick über das erhobene Datenmaterial zu darzustellen und vermitteln. Dies ist an dieser Stelle wichtig, da bei der späteren Rekonstruktion der (De-)Thematisierungsmuster nicht mehr auf alle besprochenen Themen eingegangen werden kann, sondern für die Fragestellung relevante Passagen, und somit vorab ausgewählte Themen, dargestellt werden.

Die deskriptive Darstellung der besprochenen Themen erfolgt gruppenund universitätsübergreifend, da eine getrennte Vorstellung der Gruppen zu Redundanzen führen würde. Die detaillierte Themenübersicht der jeweiligen Gruppe ist in Tabelle 1 und 2 (siehe S. 160) dargestellt. Die Aufzählung der Themen erfolgt in dieser Tabelle chronologisch und spiegelt den Themenverlauf der Gruppendiskussion wider. Bei den Themenaufzählung handelt es sich um bereits abstrahierte Beschreibungen der 
Forscherin; Begriffe, die aus den Gruppendiskussionen stammten werden als Direktzitate mit Anführungzeichen (, ") gekennzeichnet. In den Gruppen wurden auch die gleichen Themen, aber aus unterschiedlichen Perspektiven besprochen. So wird z. B bei der Frage nach der sexuellen Orientierung der Studierenden in der Gruppe Holz ausschließlich über die homosexuelle Orientierung männlicher Studierender spekuliert, in der Gruppe Feuer wird hingegen über die homosexuelle Orientierung von männlichen und weiblichen Studierenden gesprochen.

Im Anschluss an diese tabellarische Darstellung werden die Gemeinsamkeiten und Besonderheiten der Gruppendiskussionen hervorgehoben. In den Diskussionen wird nicht nur über ,Geschlecht' diskutiert, sondern auch andere Aspekte des Studiums besprochen. Dies ist ein Hinweis auf einen studiumsbezogenen konjunktiven Erfahrungsraum und auf Themen, die die Studierenden beschäftigen. So wird z. B. deutlich, dass in den Gruppen mit den Bachelorstudierenden (Holz und Feuer) intensiver über die Einführung des Bachelorstudiengangs diskutiert wurde als in den Gruppen mit den Diplomstudierenden (Metall und Wasser). Gruppen- wie auch universitätsübergreifend wurde die geringe gesellschaftliche Anerkennung und Wertschätzung gegenüber dem erziehungswissenschaftlichen Studium und der pädagogischen Arbeit diskutiert, was auf eine kollektive Erfahrung der Studierenden hinweist.

\subsubsection{Allgemeine Themen in den Gruppendiskussionen}

\section{„Das Bachelorkorsett"}

Die Studienplanumstellung vom Diplomstudium zum Bachelor- und Masterstudium ist ein Thema, das insbesondere die Gruppen Holz und Feuer beschäftigt, aber auch in der Gruppe Metall wird Kritik an der Umstellung auf den Bachelorstudiengang geübt. Dies kann damit zusammenhängen, dass in diesen Gruppen Bachelorstudierende befragt wurden und dass der Erhebungszeitraum in die Periode fällt, in der an beiden Universitäten die Bachelorstudiengänge eingeführt wurden.

Kritisiert werden das hohe Arbeitspensum, der hohe Leistungsdruck und die Einschränkung der individuellen Studiengestaltung. Die Studierenden sprechen in diesem Zusammenhang auch vom „Bachelorkorsett" (GD_Holz), das nicht nur die Studierenden, sondern auch die Lehrenden einschränkt. Die Bachelorstudierenden sehen sich im Vergleich zu den „Diplomern“ (GD_Holz)2 stark eingeschränkt. Auch die Diplomstu-

2 Bei den angegebenen Kurzzitate handelt es sich um Originalzitate aus den jeweiligen Gruppen. Für eine bessere Lesbarkeit werden Kurzzitate aus den Grup- 
Tabelle 1: Themenverläufe der jeweiligen Gruppendiskussion an der Universität Sonne

Gruppe Holz, Universität Sonne, Bachelorstudierende

$\Downarrow$ Kritik an den Studienbedingungen

$\Downarrow$ Kritik an der Einführung des Bachelorstudiengangs („Bachelorkorsett“)

$\Downarrow$ Androzentrische und androkratische Wissensvermittlung

$\Downarrow$ Bildungsgedanke: Selbstbildung vs. arbeitsmarktorientierte Bildung

$\Downarrow$ Lehrveranstaltungen zu Geschlechterfragen in den Erziehungs- und Bildungswissenschaften und in anderen Fächern

$\Downarrow$,Erziehungswissenschaft als typischer Frauenstudiengang“

$\Downarrow$,Reputationsproblem“ der Erziehungs- und Bildungswissenschaften \& Anerkennungsprobleme und Rechtfertigungsdruck aufgrund der Studienwahl

$\Downarrow$ Das generische Maskulinum als Norm der Sprech- und Schreibpraxis

$\Downarrow$ Die statistische Überrepräsentation weiblicher Studierender

$\Downarrow$ Die (homo)sexuelle Orientierung männlicher Studierender

$\Downarrow$ Vereinbarkeitsfragen von Familie und Karriere/Beruf

$\Downarrow$ Vor- und Nachteile der diffusen Arbeitsfelder der pädagogischen Praxis

$\Downarrow$ Die Rolle von ,Geschlecht' in der pädagogischen Praxis

Gruppe Feuer, Universität Sonne, Bachelorstudierende

$\Downarrow$ Kritik an den Studienbedingungen

$\Downarrow$ Kritik an der Einführung des Bachelorstudiengangs

$\Downarrow$ Die statistische Überrepräsentation weiblicher Studierender

$\Downarrow$ Lehrveranstaltungen zu Geschlechterfragen in den Erziehungs- und Bildungswissenschaften

$\Downarrow$ Anerkennungsprobleme und Rechtfertigungsdruck aufgrund der Studienwahl

$\Downarrow$ Die Relevanz der Thematisierung und Reflexion der Geschlechterdualität und -rollen

$\Downarrow$ Das generische Maskulinum als Norm der Sprech- und Schreibpraxis

$\Downarrow$, ,R]elativ geschlechtslos[e]“ Studierende

$\Downarrow$ Die (homo)sexuelle Orientierung von Studentinnen und Studenten

$\Downarrow$ Die (un),,eindeutig[e]" Geschlechterbinarität

$\Downarrow$ Die Rolle von ,Geschlecht' in der pädagogischen Praxis

$\Downarrow$ Geschlechterfragen als Teil des Curriculums in der Erziehungs- und Bildungswissenschaften

$\Downarrow$ Wunsch nach mehr Reflexion der eigenen vergeschlechtlichten und vergeschlechtlichenden Position im Studium 
Tabelle 2: Themenverläufe der jeweiligen Gruppendiskussion an der Universität Mond

Gruppe Metall, Universität Mond, Diplomstudierende

$\Downarrow$ Positives und Negatives im Studium

$\Downarrow$ Kritik an der Einführung des Bachelorstudiengangs

$\Downarrow$ Lehrveranstaltungen zu Geschlechterfragen in den Erziehungs- und Bildungswissenschaften

$\Downarrow$ Geschlechtergerechte Sprache als „gesellschaftskritische“ und „feministische" Sprache

$\Downarrow(\mathrm{Zu})$ starker ,weiblicher Fokus" bei der Thematisierung von ,Geschlecht"

$\Downarrow$ Distanzierung und Ablehnung von als extrem und überbetont wahrgenommenen Positionen zu Geschlechterfragen

$\Downarrow$ Anerkennungsprobleme und Rechtfertigungsdruck aufgrund der Studienwahl

$\Downarrow$ Die statistische Überrepräsentation weiblicher Studierender („Hühnerhaufen")

$\Downarrow$ Männliche Studierende, ,spannender" als Studentinnen?!

$\Downarrow$ Die Relevanz der Thematisierung und Reflexion der Geschlechterbinarität im Studium

$\Downarrow$ Möglichkeiten und Grenzen der Thematisierung von ,Geschlecht

$\Downarrow$ Geschlechterfragen als Teil des Curriculums in den Erziehungs- und Bildungswissenschaften

$\Downarrow$,Sensibilisierung“ für ,Geschlecht‘ als ein Teil der pädagogischen Bildung und der ,Selbstbildung"

Gruppe Wasser, Universität Mond, Diplomstudierende

$\Downarrow$ Positives und Negatives im Studium

$\Downarrow$ Lehrveranstaltungen zu Geschlechterfragen in den Erziehungs- und Bildungswissenschaften („Genderseminare“)

$\Downarrow$ Die statistische Unterrepräsentation männlicher Studierender: „weniger Männer studieren Pädagogik"

$\Downarrow$, ,[N]atürliche Haltung“" vs. reflexive Haltung gegenüber Geschlechterfragen

$\Downarrow$, ,[E]ine große Weiblichkeit" in den Erziehungs- und Bildungswissenschaften

$\Downarrow$ Distanzierung und Ablehnung von als extrem und überbetont wahrgenommenen Positionen zu Geschlechterfragen

$\Downarrow$ Frausein als Nachteil in der pädagogischen Praxis

$\Downarrow$ Anerkennung und Abwertung von „Weiblichkeit“

$\Downarrow$ Die Rolle von ,Geschlecht' in der pädagogischen Praxis

$\Downarrow$ Das generische Maskulinum als Norm der Sprech- und Schreibpraxis

$\Downarrow$ Möglichkeiten und Grenzen der Thematisierung von ,Geschlecht

$\Downarrow$ Reputationsproblem der Erziehungs- und Bildungswissenschaften und Anerkennungsprobleme und Rechtfertigungsdruck aufgrund der Studienwahl

$\Downarrow$ Geschlechterfragen als Teil des Curriculums in den Erziehungs- und Bildungswissenschaften 
dierenden der Gruppe Metall sehen Nachteile durch die Verschulung des Studiums aufgrund der Einführung des Bachelor- und Masterstudiums.

\section{Positives und Negatives im Studium}

Bachelor- wie Diplomstudierende üben Kritik am Studium, sie monieren über Studienbedingungen und -strukturen. Sie sind sich über langweilige und überlaufene Seminare einig und beanstanden die Qualität der Lehrveranstaltungen. Als positiv beurteilen die Gruppen Metall und Wasser die Wahlfreiheit im Studium, sie bemängeln jedoch die didaktische und methodische Aufbereitung der Seminarinhalte. Alle Gruppen stimmen darin überein, dass die Qualität einer Lehrveranstaltung sehr stark von den Dozierenden abhängt. In der Gruppe Metall wird auch die Motivation der Studierenden und ein mehrdimensionales Lernumfeld für das Gelingen eines Seminars hervorgehoben. In allen vier Gruppendiskussionen wird angemerkt, dass sie im Großen und Ganzen die Verbindung zwischen pädagogischer Praxis und erziehungswissenschaftlicher Theorie im Studium vermissen. Zudem wird von Schwierigkeiten bei der Vereinbarkeit von Studium und Berufstätigkeit berichtet. Auch hier deutet sich eine kollektive Erfahrung an.

\section{Bildung als Selbstbildung oder Systemanpassung?}

Die Frage nach dem Bildungsgedanken, der die Studierenden motiviert, wird in den Gruppen Holz und Metall diskutiert. In der Gruppe Holz wird das Spannungsfeld zwischen Bildung als Selbst- und Identitätsbildung (im Humboldt'schen Sinne) und der Bildung für den Arbeitsmarkt (der neoliberalen und kapitalistischen Regeln unterliegt) verhandelt. Dabei werden Selbstbildung und Systemanpassung einander gegenüber gestellt. In der Gruppe Metall wird die Selbst- und Identitätsbildung als Teil der Ausbildung und der Vermittlung pädagogischer Kompetenzen gesehen. Selbstbildung bildet hier nicht den Gegenpol zur Bildung für den Arbeitsmarkt, sondern stellt den Fluchtpunkt zwischen Persönlichkeitsentwicklung und pädagogischer Professionalität dar.

pendiskussionen im Fließtext mit Anführungszeichen hervorgehoben und zudem wird angegeben, aus welcher Gruppendiskussion das Zitat stammt. Die Zeilennummerierung aus den ursprünglichen Transkripten wird nur bei längeren Zitaten bzw. Passagen aus den Gruppendiskussionen angegeben, dadurch heben sich die längeren Passagen vom restlichen Fließtext und der Interpretation ab. Die Zitate aus den Transkripten entsprechen den TiQ-Regeln, das heißt u. a., dass die Interpunktion nicht syntaktischen Regeln, sondern der Intonation folgt (siehe dazu die Erklärungen im Anhang). 


\section{,Studierst Du jetzt Pädagogik?!‘ oder}

„das Reputationsproblem der Erziehungswissenschaften“" Ein Thema, das in allen Gruppendiskussionen, wenn auch in unterschiedlicher Intensität, debattiert wird, ist „das Reputationsproblem der Erziehungswissenschaft" (GD_Holz). Darunter wird die geringe gesellschaftliche Anerkennung und Wertschätzung gegenüber dem erziehungswissenschaftlichen Studium und der pädagogischen Arbeit verstanden. Die Studierenden sehen sich vielfach mit Vorurteilen und Stereotypen konfrontiert. Ihre kollektiven Erfahrungen sind, dass die Pädagogik nach wie vor oftmals mit der Lehrer_innenbildung gleichgesetzt wird und die Ausbildung zu PädagogInnen mit dem Berufsbild der Lehrerin/des Lehrers äquivalent ist. Zudem sehen sie sich mit der falschen Annahme konfrontiert, dass die Mehrzahl der AbsolventInnen des Faches entweder arbeitslos sei oder in fachfremde Tätigkeitsbereiche ausweiche. In den Gruppendiskussionen kommt die konjunktive Erfahrung zum Ausdruck, dass die Studierenden sowohl in ihrem Studium als auch in ihrer pädagogischen Praxis wenig Anerkennung und Wertschätzung erfahren. Das breite und diffuse Arbeitsfeld für PädagogInnen wird als Vor- und Nachteil wahrgenommen.

\subsubsection{Homologien und Besonderheiten bei der (De-)Thematisierung von ,Geschlecht"}

\section{Reden über ,Geschlecht': ein heteronom und autonom produziertes Thema}

Die Diskussionsimpulse der Gruppendiskussionsleiterin ${ }^{3}$ laden die TeilnehmerInnen dazu ein, über ,Geschlecht' zu diskutieren. Zudem regt die Diskussionsleitung in Form von immanenten oder exmanenten Nachfragen weitere Möglichkeiten über ,Geschlecht` und Geschlechterverhältnisse $\mathrm{zu}$ sprechen und produziert das Thema somit auch heteronom. In den beiden Gruppen Holz und Wasser wird unaufgefordert das Thema ,Geschlecht' von einer GDT ${ }^{4}$ platziert, nachdem die Interviewerin nach interessanten und langweiligen Themen gefragt hat. In der Gruppe Holz wird daraufhin von einer Teilnehmerin Kritik an den androzentrischen Inhalten einer Lehrveranstaltung geübt und bemängelt, dass geschlechterreflektierende Inhalte nicht als Querschnittsthema im Curriculum vertreten sind. Diese Kritik wird von ihren Kolleginnen nicht geteilt

3 Die Bezeichnungen Gruppendiskussionsleiterin und Interviewerin werden im Folgenden synonym verwendet.

4 Die Abkürzung GDT steht für GruppendiskussionsteilnehmerInnen. 
und bleibt als individuelle Orientierung im Raum stehen. In der Gruppe Wasser erzählt eine Teilnehmerin von ihrem Interesse für die Auseinandersetzung mit Geschlechterverhältnisse und -fragen und wie es dazu gekommen ist. Sie beschreibt: „,II]ch habe ein Seminar durch Zufall belegt was mit ,Geschlecht' und Erziehung war, [...] und dann fand ich das eigentlich irgendwie spannend [... ]". Sie erzählt weiter, dass sie seitdem zahlreiche Lehrveranstaltungen diesbezüglich besucht hat, die sie mehr oder weniger gut fand. Wie in der Gruppe Holz wird auch dieser autonom initiierte Redebeitrag zu ,Geschlecht' nicht als Diskussionsthema von der Gruppe aufgegriffen. Erst als die Gruppendiskussionsleiterin in Form von immanenten und exmanenten Nachfragen das Thema (erneut) anregt, wird es von der gesamten Gruppe aufgegriffen und diskutiert. Dies kann ein Indiz dafür sein, dass die Thematisierung und das Sprechen über ,Geschlecht' nicht Teil der studentischen Praxis sind. Indem die Studierenden in der Gruppendiskussion die Beitrage zu ,Geschlecht nicht aufgreifen - das ist im Besonderen in der Gruppe Holz der Fall werden Verdeckungszusammenhänge aufgrund konkurrierender konjunktiver Erfahrungsräume deutlich (vgl. Kapitel 6.2). Ein paar Beispiele für nicht-kommentierte Beträge sind: „Wenn mich einer Mädel nennt, dann werde ich auch aggressiv.“ (Mika, GD_Holz), oder: ,[I]ch meine, wir haben ja gerade gesagt, dass es so viele weibliche Studentinnen gibt in den Erziehungswissenschaften und von daher weiß ich nicht, ob das dann so das Thema ist." (Luan, GD_Holz). Auch wenn die Fragen nach ,Geschlecht" teilweise von der Gruppendiskussionsleiterin initiiert werden, haben die Studierenden selbstläufig diskutiert und die Themen, Inhalte und den Diskursverlauf selbst gesteuert.

\section{Lehrveranstaltungsangebot zu Geschlechterfragen}

In allen Gruppendiskussionen können Lehrveranstaltungen mit Geschlechterfragen als Inhalt genannt werden. Jedoch ist das Interesse und die Teilnahme an diesen Lehrveranstaltungen sehr unterschiedlich. Zur inhaltlichen Auseinandersetzung im Studium der Erziehungs- und Bildungswissenschaften wird von den Gruppen Feuer und Metall festgehalten, dass zwar über den Begriff gender gesprochen werde, jedoch ,viel zu wenig und viel zu selten" (GD_Feuer) im Zusammenhang mit der „persönlichen Realität“ (GD_Feuer) und den pädagogisch-praktischen Erfahrungen diskutiert und reflektiert wird. In der Gruppe Feuer wird zudem über das Potential der Universität und des Studiums diskutiert, einen Raum darzustelle, in dem eigene Vorstellungen reflektiert werden können. In der Gruppe Metall wird hingegen ein ,weiblich` dominier- 
ter Blick auf die Geschlechterfragen kritisiert. Neben den Möglichkeiten, sich innerhalb des eigenen Fachbereiches mit Geschlechterfragen und Geschlechterverhältnissen zu beschäftigen, werden auch andere Disziplinen wie Soziologie und Psychologie genannt. In den Gruppen Holz, Metall und Wasser werden in diesem Zusammenhang auch die Zentren für die Erforschung von Geschlechterverhältnissen und für feministische Wissenschaft angeführt, wobei der Unterschied in den Gruppen darin besteht, dass in den Gruppen Wasser und Metall im Gegensatz zur Gruppe Holz alle GDT die jeweiligen Genderzentren zumindest kennen oder Lehrveranstaltungen dort besucht haben. In der Gruppe Feuer findet das Zentrum keine Erwähnung.

\section{Distanzierung und Ablehnung von als extrem und überbetont wahrgenommenen Positionen zu Geschlechterfragen}

Charakteristisch für alle Gruppen - aber insbesondere für die Gruppen Metall und Wasser - ist, dass sich die GDT von „extremen“ Positionen und Maßnahmen sowie von einer Überfokussierung auf Geschlechterfragen distanzieren bzw. diese ablehnen. Es scheint eine Tendenz zu bestehen, sich von Thema ,Geschlecht' zu distanzieren bzw. eine ,moderate Position und Sichtweise zu vertreten. Als ,zu extrem wird in den Gruppen z. B. ,die geschlechtergerechte Sprache, ,die' Quotenregelung, ,der Feminismus und die Hervorhebung von ,Geschlecht' als wichtigste Ungleichheitskategorie thematisiert.

\section{Geschlechterdifferenz und sexuelle Orientierung}

Obgleich eine rhetorische Geschlechtergleichheit in den Gruppendiskussionen dominiert, wird von einer Differenz zwischen den Geschlechtern und einer Zweigeschlechtlichkeit ausgegangen. Jede Gruppe elaboriert, was Männer und Frauen unterscheide und welche gesellschaftlichen Erwartungen daran geknüpft seien. Hierin spiegelt sich die konjunktive Erfahrung der Studierenden wider wodurch Differenzen zwischen den Geschlechtern erneut tradiert werden. So wird z. B. zwischen männlichen und weiblichen Verhaltensweisen, Fähigkeiten, Äußerlichkeiten, Tätigkeiten etc. unterschieden. In der Gruppe Feuer wird z. B. der unterschiedliche Bewegungsdrang bei Mädchen und Jungen hervorgehoben; in der Gruppe Metall ist von verschiedenen Diskussionsstilen bei Studentinnen und Studenten die Rede; in der Gruppe Wasser wird ein unterschiedliches sexuelles Verlangen qua,Geschlecht' reproduziert und in der Gruppe Holz wird konstatiert, dass männliche Seminarteilnehmende kritischer seien. Auch wenn in der Gruppe Metall reflektiert wird, 
dass „Frau und Mann bestimmte Unterschiede haben, aber Individuen auch Unterschiede haben“, wird dennoch die Unterscheidung qua ,Geschlecht" hervorgehoben und reproduziert. Die Studierenden hinterfragen ihre eigene männliche oder weibliche Geschlechtsidentität nicht, hingegen werden queere Identitäten und Geschlechtsuneindeutigkeiten werden problematisiert und als verunsicherndes Moment in der alltäglichen Interaktion zur Sprache gebracht. Die eigene Heterosexualität ist eng mit einem toleranten und offenen Umgang mit Homosexualität verknüpft.

\section{Die statistische Überrepräsentation von Studentinnen und die vermutete Homosexualität von Studenten}

Eine weitere Homologie ist, dass die statistische Repräsentation von Frauen und Männern bei den Studierenden in jeder Gruppe zur Sprache kommt. Es wird die Überrepräsentation von Frauen hervorgehoben und das Studium der Erziehungs- und Bildungswissenschaften als ,typischer Frauenstudiengang“" bezeichnet (GD_Holz). Es wird auch ausgeführt, dass Männer nicht typischerweise dieses Studium wählen, sondern dass diese eine Ausnahme darstellen. Die Studenten werden in den Diskussionen (mit Ausnahme der in der Gruppe Metall) als von der männlichen (heterosexuellen) Norm abweichend konstruiert, indem die Vermutung diskutiert wird, dass die wenigen Männer im Studium „schwul“ (GD_Feuer) seien.

\section{Das generische Maskulinum und die geschlechtergerechte Sprache}

Eine andere Form der Repräsentation bzw. Sichtbarkeit von Männern und Frauen ist jene durch die Sprache. Diese Ebene wird in zwei Gruppen, Metall und Wasser, von den GDT initiativ angesprochen; bei den anderen beiden Gruppen wurde sie auf Nachfrage der Interviewerin thematisiert. In den Gruppen Metall und Wasser wird geschlechtergerechte Sprache als eine Form der Thematisierung von ,Geschlecht' an der Universität und im Studium dargestellt. Diese Form der Thematisierung wird nicht ausschließlich positiv bewertet, sondern auch als nicht notwendig oder übertrieben bezeichnet. Mit Ausnahme der Gruppe Metall dominiert die Verwendung des generischen Maskulinums; geschlechtergerechte Paarbildungen oder andere Formen geschlechtergerechter Sprache bilden die Ausnahme. 


\section{Die Relevanz der Kategorie ,Geschlecht' für die pädagogische Ausbildung und Praxis}

In den Gruppendiskussionen wird auch über die Bedeutungszuschreibung und über die Relevanz der Kategorie ,Geschlecht' für die pädagogische Ausbildung und Praxis gesprochen. Die Gruppen unterscheiden sich jedoch im Grad der Bedeutungszuschreibung und auch innerhalb der Gruppen gibt es starke Unterschiede und zahlreiche individuelle Orientierungen. In den Gruppen Feuer und Metall zeigt sich als kollektive Orientierung, dass ,Geschlecht' eine relevante Kategorie ist und deren Reflexion ein wichtiger Teil der Ausbildung im erziehungswissenschaftlichen Denken und pädagogischen Handeln sein sollte.

Auch wenn in der Gruppe Feuer die von der Interviewerin gestellte Frage nach ,Geschlecht‘ anfangs auf Irritation stößt („Also im ersten Moment habe ich gedacht, ja was was was soll diese Frage mit Geschlecht?" (GD_Feuer)), findet im Laufe der Gruppendiskussion eine intensive Auseinandersetzung und Reflexion über ,Geschlecht‘ und Zweigeschlechtlichkeit statt. Der hohe Grad an Reflexion und der starke Praxisbezug hebt die Gruppe Feuer stark von anderen Gruppen ab. Auch in den anderen Gruppen gibt es Passagen der Reflexion und der kritischen Auseinandersetzung mit der Kategorie Geschlecht, aber nicht in dem Ausmaß wie in Gruppe Feuer. Die Gruppe Feuer hat die Diskussion als Reflexionsraum genutzt und sich auch über persönliche Erfahrungen in der pädagogischen Praxis ausgetauscht. In den Gruppen Feuer, Metall und Wasser wird auch darüber gesprochen, warum das Thema ,Geschlecht' bei den KommilitonInnen Ablehnung oder Desinteresse auslöst.

\section{Keine Benachteiligung im Studium aufgrund von Geschlecht}

Eine weitere Gemeinsamkeit aller Gruppen ist die Hervorhebung, dass sich die Studentinnen und Studenten an der Universität aufgrund ihres Geschlechts nicht benachteiligt fühlen. Der hohe Frauenanteil dient in allen Gruppen als Argument dafür, dass es keine Benachteiligung im Studium gebe. Dies bedeutet aber nicht, dass die Einzelnen keine Diskriminierungserfahrung gemacht hätten. In den Gruppen wird Benachteiligung und Diskriminierung in anderen Bereichen, Kulturkreisen, Studiengängen usw. gesucht und gefunden. In Abgrenzung dazu wird jedoch über die Nachteile und Schwierigkeiten des Frauseins gesprochen wird. In der Gruppe Holz wird dies im Zusammenhang mit der pädagogischprofessionellen Rolle der Seminarleiterin und der Vereinbarkeit von Beruf und Familie exemplifiziert. In der Gruppe Wasser wird als Beispiel die schwierigere Position von Pädagoginnen in der Jugendarbeit, und hier 
im Speziellen in der Interaktion mit männlichen Jugendlichen, genannt. Problematisiert wird auch der Umgang mit der eigenen ,Weiblichkeit und der Wunsch, diese im äußerlichen Erscheinungsbild darzustellen und die möglichen gesellschaftlichen Konsequenzen, wie z. B. als Frau nicht ernstgenommen zu werden.

\section{Gleichsetzung von ,Geschlecht' mit Frausein}

Daran anschließend möchte ich hervorheben, dass in den Diskussionen, mit Ausnahme der Gruppe Feuer, ,Geschlecht" mit Frausein und Benachteiligung gleichgesetzt wird. Das heißt, dass beim Thema ,Geschlecht" hauptsächlich Bereiche, Anliegen und Aspekte angesprochen werden, die insbesondere für Frauen von Belang bzw. problematisch seien. Zugleich scheint die Frage nach ,Geschlecht' auch immer mit der Frage nach der Benachteiligung von Frauen verknüpft zu sein. Diese Gleichsetzung scheint die Thematisierung von ,Geschlecht' und Geschlechterfragen zu behindern, da die Studierenden vor der Hintergrundannahme diskutieren, dass feministische Interventionen und Kritik an (patriarchalen) Herrschaftsverhältnissen nicht mehr notwendig seien, da die Gleichstellung der Geschlechter bereits erreicht sei. Diese Orientierung der Studierenden am Gleichheitspostulat und an gesellschaftlichen Modernisierungsprozessen scheint eine Diskussion und eine Auseinandersetzung mit ,Geschlecht‘ und Geschlechterverhältnisse zu verhindern.

\subsection{Ein divergenter Diskurs bei der Gruppe Holz an der Universität Sonne}

In den folgenden Ausführungen wird der Fokus auf die einzelnen Gruppe gelegt. Die Darstellung konzentriert sich auf verschiedene Aspekte, die sich aus dem Material selbst, dem methodischen Zugang und der Fragestellung ergeben. Die Rekonstruktion der habituellen Praxen bei der (De-)Thematisierung von ,Geschlecht' und Geschlechterfragen sowie die identifizierten kollektiven und individuellen Orientierungen wie auch Mechanismen, die zur (De-)Thematisierung von ,Geschlecht' und Geschlechterverhältnissen führen, werden im Anschluss dargestellt. Die Darstellung erfolgt entlang der einzelnen Gruppendiskussionen, geordnet nach den jeweiligen Universitäten. Hier sollen die jeweiligen Gruppen mit den für sie charakteristischen Orientierungen zur Geltung kommen. Dabei geht es um fallinterne Homologien bzw. um die kollektiven Orientierungen der einzelnen Gruppen in Hinblick auf die (De-)Thematisierung 
von ,Geschlecht‘ und Geschlechterfragen. Die gruppenübergreifende und universitätsinterne komparative Analyse erfolgt jeweils nach der gesonderten Darstellung der Gruppen.

Die Diskussion der Gruppe Holz war die erste Gruppendiskussion, die an der Universität Sonne geführt wurde, insgesamt jedoch die zweite. Die Gruppendiskussion Holz fand ca. sechseinhalb Monate nach der Gruppendiskussion Metall an der Universität Mond statt. An der Diskussion nahmen sechs Studierende, fünf Frauen und ein Mann, teil. Alle waren zu diesem Zeitpunkt Studierende des Bachelorstudiengangs Erziehungsund Bildungswissenschaften an der Universität Sonne. Die TeilnehmerInnen waren zum Teil befreundet oder kannten sich von gemeinsam besuchten Lehrveranstaltungen. Die Gruppe zeichnet sich aufgrund ihrer Zusammensetzung hinsichtlich der Studiendauer aus. Während fünf Teilnehmer_innen im dritten und vierten Semester studierten, stand eine Studentin am Beginn des zweiten Semesters an der Universität Sonne. Bereits an dieser Stelle möchte ich hervorheben, dass sich diese Konstellation auf die Diskussion auswirkte, da Mika, die Studentin mit der geringsten Studiendauer, von ihren KommilitonInnen vor allem in ihrem Status als Studienanfängerin wahrgenommen wurde. ${ }^{5}$ Auch in Bezug auf ihr Alter ist diese Gruppe divers. Eine der Teilnehmerinnen war Anfang dreißig, eine Teilnehmerin war Mitte vierzig und die anderen TeilnehmerInnen waren zwischen Anfang und Mitte zwanzig. ${ }^{6}$ Dieser Altersunterschied lässt sich damit erklären, dass zwei Teilnehmerinnen ihr Studium als zweiten Bildungsweg gewählt haben. Drei Personen in der Gruppe Holz lebten zum Zeitpunkt der Diskussion in einer Lebenspartnerschaft und eine Teilnehmerin vermerkte Elternteil von vier Kindern zu sein. Keine der Teilnehmenden gab für sich einen Migrationshintergrund an. Die Interessensschwerpunkte im Studium waren bei den Teilnehmer_innen sehr breit gefächert und gingen von der Erwachsenenbildung über die Soziale Arbeit bis hin zur Sonderpädagogik. Auch das Ausmaß der Erfahrungen der TeilnehmerInnen in der pädagogischen Praxis war eher inhomogen. Das Zentrum für Gender Studies an ihrer Universität war nur einer Teilnehmerin (Mika) bekannt.

Bezeichnend für diese Gruppendiskussion ist, dass der Diskursverlauf sehr sprunghaft und zum Teil auch gegenläufig ist. Das heißt, die

5 Insbesondere bei den Passagen zur ,Geschlecht' und Geschlechterfragen wird dies deutlich.

6 Mika: w, 22 Jahre, 2. Semester; Luan: w, 45 Jahre, 3. Semester; Tomke: w, 24 Jahre, 3. Semester; Robyn: w, 32 Jahre, 3. Semester; Loris: w, 21 Jahre, 4. Semester; Luca: m, 24 Jahre, 4. Semester. 
Themen werden häufig gewechselt und angefangene Themen zu einem späteren Zeitpunkt wieder aufgegriffen und/oder (nicht) abgeschlossen. Die Studierenden reden aneinander vorbei, sie können manches Gesagte zum Teil nicht nachvollziehen oder einordnen. In der Gruppe Holz lassen sich hauptsächlich individuelle Orientierungen rekonstruieren oder Orientierungen, die nicht für die gesamte Gruppe, sondern nur für einen Teil zutreffen. Insbesondere Passagen, in denen es um die Relevanz der Kategorie ,Geschlecht' und/oder die (De-)Thematisierung von Geschlechterfragen und -verhältnisse geht, sind von einem oppositionellen und divergenten Diskurs ${ }^{7}$ geprägt. In der Gruppe scheint es kein geteiltes Interesse daran zu geben, über ,Geschlecht' und Geschlechterfragen zu diskutieren. Auch wenn das Thema - wie in der Gruppe Wasser - von einer Teilnehmerin in die Diskussion eingeführt wird, wird es von den GDT nicht aufgegriffen bzw. kommt es in dieser Passage zu eklatanten Verstehensproblemen. In der Gruppe Holz entwickelt sich keine selbst-reflexive Thematisierung von ,Geschlecht' und Geschlechterfragen, wie dies zum Teil in den anderen Gruppen der Fall ist, vielmehr zeigen sich unterschiedliche Facetten einer Thematisierungsverweigerung und dass es in dieser Gruppe keine etablierte Diskussionsform und -kultur zum Thema ,Geschlecht' zu geben scheint.

Im Folgenden sollen die zentralen Orientierungen und die Brüche bei der (De-)Thematisierung von ,Geschlecht' und Geschlechterfragen der Gruppe Holz anhand von Auszügen aus der Diskussion rekonstruiert werden. Bereits in der Anfangspassage der Gruppendiskussion zeigen sich erste Rahmeninkongruenzen.

\subsection{1 „Als Mann fällt man immer auf“}

Der Beginn der Diskussion ist insofern interessant, als hier bereits geschlechterbezogene Differenzen zur Sprache kommen. Darüber hinaus vermittelt dieser Auszug aus der Anfangspassage einen ersten Eindruck

7 Eine Opposition ist eine Orientierung, die nicht $\mathrm{zu}$ der vorangegangenen passt. Sind nicht auflösbare Widersprüche vorhanden, wird davon gesprochen, dass eine Gruppe - in diesem Zusammenhang - keinen gemeinsamen (Orientierungs-)Rahmen hat. In einem divergenten Diskurs beziehen sich die TeilnehmerInnen in Hinblick auf den Orientierungsgehalt nicht aufeinander, sondern reden vielmehr aneinander vorbei. Darin dokumentiert sich, dass die unterschiedlichen Orientierungen zu einem Thema für die in Frage stehenden Handlungsfelder bestehen bleiben (vgl. Przyborski 2004, S. 72f.). Beide Modi der Diskursorganisation finden sich in Gesprächen bzw. Gesprächsabschnitten, in welchen die TeilnehmerInnen über keine geteilten Erfahrungen verfügen (vgl. ebd., S. 252; Kapitel $5.4 .1)$. 
von der Diskursorganisation und den bestehenden Rahmeninkongruenzen. Im folgenden Auszug geht es um die Frage, ob sich die GDT im Laufe ihres Studiums der Erziehungs- und Bildungswissenschaften bereits kennengelernt haben. Bereits hier wird die Kategorie ,Geschlecht" als Unterscheidungs- und Erkennungsmerkmal eingeführt. Die Diskussion wird mit einer Feststellung und Frage der Interviewerin eröffnet. ${ }^{8}$

$1 \quad \mathrm{I}^{9}$ : $\quad$ Ihr studiert ja alle Erziehungswissenschaften, und jetzt

2

3

4

5

6 LUAN: ᄂ Mhm,

7 ROBYN: Wir kennen uns.

8 LORIS: (lacht)

9 LUCA: Ja.

10 MIKA:

11 ROBYN:

12

13

14 Seminare hatten.

so gut.

LUAN: $\quad$ Nee, genau.

LUCA: $\quad$ Mhm. kennen wir uns. einfach noch die Frage, ob ihr euch denn untereinander kennt und von wo denn.

TOMKE: Also ich kenne die beiden; weil wir schon gemeinsame

$\llcorner$ Als Mann fällt man immer auf.

$\llcorner$ Euch beide kenne ich nicht

MIKA: Als Mann fällt man immer auf; aber ich weiß auch nicht ja. Ich bin erst im ersten Semester; das kann sein dass es dann einfach noch nicht so viel Gelegenheit gab.

LUAN: Mhm, da man dann andere Sachen besucht.

MIKA: Ja; es gibt genug Studenten, ja.

LORIS: Wir sind im vierten und zusammen angefangen, ${ }^{\circ}$ daher ${ }^{\circ}$

LUCA: Mhm, vom Sehen her auch andere. $((\text { allgemeines Gelächter }))^{10}$

8 Bei der Transkription orientiere ich mich an den Richtlinien nach Talk in Qualitative Social Research (vgl. Przyborski/Wohlrab-Sahr 2009, S. 166f.; Bohnsack 2008, S. 235) (Siehe Anhang).

10 Die zitierten Passagen beginnen mit der jeweiligen Originalzeilennummerierung aus dem Transkript, im Verlauf der Passagen, z. B. aufgrund von gekennzeichneten Auslassungen, können diese jedoch davon abweichen. Zudem wurden die zitierten Passagen zur besseren Lesbarkeit leicht geglättet, d. h. für den Lesefluss störende Ähs, Ehms und Wortwiederholungen wurden entfernt. Der Sinngehalt der Aussagen wurde durch diese Überarbeitung nicht verändert. 
$\mathrm{Zu}$ Beginn wird von der Gruppendiskussionsleiterin der konjunktive Rahmen des Studiums Erziehungs- und Bildungswissenschaften abgesteckt und nach dem Bekanntschaftsgrad der Studierenden gefragt. Tomke greift die Frage als Erste auf und merkt an, dass sie zwei der Anwesenden (Luan und Robyn) kennt, da sie „gemeinsame Seminare hatten“. Luan bestätigt die Bekanntschaft mit einem „Mhm“. Als nächste bringt Robyn zum Ausdruck, dass sie Luca „kenne“. Indem Luca dies mit einem „Ja“" validiert, ist davon auszugehen, dass auch er Robyn bereits aus dem Studium kennt. Mika bezieht sich mit ihrem Beitrag auf Lucas ,Geschlecht‘ und kommentiert dies mit der Generalisierung: „Als Mann fällt man immer auf." Mit dieser Aussage macht Mika zwar nicht deutlich, ob sie Luca kennt oder ihn zuvor schon einmal gesehen hat, sondern betont, dass man als Mann im konjunktiven Erfahrungsraum des Studiums immer auffällt. Mika führt hiermit die Differenzierung nach Geschlecht ein, die jedoch von den anderen Studierenden nicht aufgegriffen wird. Gleichzeitig markiert Mika mit ihren Beitrag, dass Männer im Studium auffallen, da sie von der Norm abweichen, wobei die Norm hier unausgesprochen bleibt. Wenn wir hier für einen Moment die inhaltliche Ebene der Diskussion verlassen und auf die Gruppenzusammenstellung blicken, zeigt sich in Bezug auf die Geschlechterrepräsentation, dass fünf Frauen (exkl. der Interviewerin) und ein Mann in dieser Runde von Studierenden der Erziehungs- und Bildungswissenschaften sitzen. In diesem Verhältnis von Frauen und Männern spiegelt sich auch tendenziell die Geschlechterverteilung der eingeschriebenen Studierenden in den Erziehungs- und Bildungswissenschaften allgemein wieder. Dieses Studium wird weit überproportional von Frauen gewählt, mehr als drei Viertel aller Studierenden sind weiblich (vgl. Kapitel 2.2). Damit wird auch die Norm deutlich, auf die sich Mika bezieht und von der Luca abweicht.

Nachdem Robyn, Luan und Luca nochmals auf die Frage der Interviewerin zurückkommen und sich nicht auf Mikas Beitrag beziehen, wiederholt Mika nochmals ihre Feststellung: „Als Mann fällt man immer auf." In der Wiederholung dieser Aussage scheint eine Bekräftigung ihrer Proposition zu liegen. Wie bereits zuvor wird die Abweichung von einer unausgesprochenen Norm explizit. Dies verweist darauf, dass Mika von einem konjunktiven Wissen der Anwesenden ausgeht, dass keiner weiteren Explikation bedarf. Mika erklärt weiter, dass sie „erst im ersten Semester" studiere und es in dieser Zeit ,nicht so viele Gelegenheiten gab", anderen Studierenden und die GDT kennen zu lernen. Neben ,Geschlecht' wird hier auch die Studiendauer als Unterscheidungsmerkmal 
zwischen den Studierenden eingeführt. Luan, die im dritten Semester studiert, erklärt die wenigen Gelegenheiten damit, dass ,man dann andere Sachen besucht“. Mit „dann" scheint sich Luan auf die Studiendauer zu beziehen. Luan hat bereits drei Semester studiert und besucht deshalb andere Seminare als Mika. Mika schließst validierend an (,Ja“). Ihr Beitrag mündet jedoch auch in eine andere Erklärung: Sie sieht in der hohen Zahl von Studierenden („es gibt genug Studenten“) einen Grund dafür, warum sie die GDT nicht kennt. Abschließend greift Loris nochmals die Frage der Interviewerin auf und macht deutlich, dass sie und Luca sich seit dem Studiumsbeginn kennen und beide im vierten Semester sind. Luca fügt hinzu, dass er auch andere „,om Sehen her" kenne. Mehrere Teilnehmer/innen lachen daraufhin, was im Sinne einer Zwischenkonklusion gedeutet werden kann. Dieser Auszug deutet auf eine divergente Diskursbewegung hin, in denen die Rahmeninkongruenzen eher verdeckt bleiben und nicht direkt angesprochen werden. Zudem deutet sich bereits hier an, dass Mika die Differenzierung nach ,Geschlecht' thematisiert.

\subsubsection{Eklatante Verstehensprobleme und ein Habitus der (De-)Thematisierung von ,Geschlecht'}

Bei der Diskussion der Gruppe Holz ist auffällig, dass es nur wenige Passagen gibt, in denen sich die GDT auf ein Thema konzentrieren. Zudem ist diese Diskussion von schnellen und abrupten Themenwechseln geprägt. Dies kann als Hinweis dafür angesehen werden, dass es den Teilnehmer/innen der Gruppe nicht gelingt, sich auf einen konjunktiven Erfahrungsraum einzupendeln. Hierfür mag als Erklärung die Beteiligung von Personen unterschiedlicher Studiendauer herangezogen werden. Darüber hinaus bzw. in Verbindung damit werden jedoch auch Rahmeninkongruenzen in der Gruppe Holz deutlich, d. h. unterschiedliche und widersprüchliche Orientierungen, die nicht zu gemeinsamen verbunden werden können. Einen Hinweis hierauf geben rituelle Konklusionen, mit denen Themen abgeschlossen werden, ohne dass eine gemeinsam erarbeitete Orientierung damit verbunden wäre. Anhand der folgenden Passagen kann rekonstruiert werden, dass es in der Gruppe Holz bei der Thematisierung von ,Geschlecht' und Geschlechterfragen zu eklatanten Verstehensproblemen kommt und bei der Mehrzahl der GDT ein Habitus der Dethematisierung von ,Geschlecht' vorherrscht. 


\section{Androzentrismuskritik vs. fehlende Studienerfahrung}

Ein Beispiel für eklatante Verstehensprobleme ist der folgende Diskussionsausschnitt zum Thema „einseitige Art der Wissenschaft". Da diese Passage einen Referenzpunkt für die gesamte Diskussion darstellt und die Studierenden immer wieder auf sie zurückkommen, wird sie im Anschluss genauer betrachtet und ausführlich analysiert.

MIKA: Also mir fällt was ein was mich wirklich sehr überrascht hat, nämlich ja diese sehr einseitige Art der Wissenschaft; also da ranzugehen. Gut vielleicht liegt das auch jetzt einfach an an den Anfangsthemen, kann ich mir vorstellen, also zum Beispiel auch Anthropologie, also wenn man das wortwörtlich nehmen würde gibt es die Spezies Frau erst seit 1950, und vorher mal eher da ging es dann um die Bildung also Humboldt ist (dann) die Bildung von den Oberen, also den Söhnen der oberen Fünftausend, und ich war etwas überrascht dass die Veranstaltung, die Vorlesung dann eben Bildung von ja Idealismus heißt, „Bildung und Erziehung im Idealismus", und dann wird da nur die Humboldt'sche Bildungstheorie behandelt. Und dann da hab ich mich ja-, also irgendwas stimmt hier ganz und gar nicht; es ist natürlich hat alles seine Berechtigung, aber wie die Adeligen, in einer gewissen Zeit ihre Söhne ausgebildet haben würde ich jetzt nicht als Bildungs- und Erziehungstheorie für eine ganze Epoche oder Jahrhundert sehen.

ROBYN: Nee aber es geht ja gerade, also das ist damit verbunden, es sind ja drei Vorlesungen.

MIKA: Nee ich habe da noch ein zweites Seminar besucht. Und da ging es dann explizit um die Mädchenbildung; und dann, hatte ich mein gesamtes Bild über die gleiche Zeit, und da hab ich mich gefragt das kann ja n- also dass es das ist nämlich auch so eine Sache; gerade weil so wenig Zeit ist im Bachelor. Dann muss ich zwei Seminare besuchen, nur damit ich über eine Epoche wirklich Bescheid weiß, weil im einen wird nur Humboldt behandelt und 
im anderen, gut da geht es dann tatsächlich mal darum auch wie Bildung, Schulbildung, wie das überhaupt ausgesehen hat, also in diesem Mädchenbildungsseminar in dem

TOMKE: $\quad$ Ja aber ich glaube das liegt auch daran, dass die einfach einen Unterschied machen zwischen Überblicksseminaren, Einführungsseminaren wo du einen sehr breiten, gefächerten Überblick bekommst, wie (es zum Beispiel in der)

MIKA:

$\llcorner$ Ja das hatte ich ja gerade nicht, da ging es ja nur um die paar tausend von Humboldt.

TOMKE: Ja eben das meine ich ja; du hättest anscheinend lieber so einen Überblick gehabt,

MIKA:

$\llcorner$ Ja also von wegen Überblick.

Mika leitet ihre Proposition damit ein, dass sie diese als persönliche Einschätzung (,mir fällt was ein") eingrenzt. Sie elaboriert, was sie im Studium, ,wirklich [... ] sehr überrascht" habe. Sie beginnt hier eher wertneutral, denn ,überrascht" sein kann zunächst vieles bedeuten: Jemand kann positiv wie auch negativ von etwas/jemandem überrascht werden. Dieser eher neutrale Einstieg erfährt jedoch eine negative Wendung. Mika konkretisiert, dass sie von der ,sehr einseitige[n] Art der Wissenschaft" spricht. Mit dem Adjektiv „einseitig“ macht sie ihre individuelle Orientierung deutlich. Demnach wird nur eine Seite, ein spezieller Aspekt behandelt. Mika impliziert, dass ihr etwas fehlt - was ihr fehlt, spricht sie hier noch nicht an. Zu Beginn spricht Mika noch von einer sehr allgemeinen und abstrakten Ebene von „Wissenschaft". Eine mögliche Erklärung für ihre Wahrnehmung, dass Wissenschaft einseitig sei, sucht Mika bei den „Anfangsthemen“ des Studiums. Damit verweist sie auf ihren Status als Studienanfängerin. Die Einseitigkeit dieser Anfangsthemen macht Mika daran fest, dass es hier die „Spezies Frau erst seit 1950“ gebe. Mit dieser ironisch formulierten Äußerung (,Spezies Frau“), deutet Mika auch an, warum sie die „Wissenschaft" einseitig wahrnimmt. In der Verbindung des Begriffs ,Spezies“ und mit der Einführung der zeitlichen Dimension „1950" scheint Mika die wissenschaftliche Entdeckung der Frauen zum Ausdruck bringen zu wollen. Es ist zu vermuten, dass sich Mika in ihrer Kritik an der ,einseitigen Art" auf das Fehlen der historischen Thematisierung der „Spezies Frau" für die Zeit davor bezieht. 
Mika exemplifiziert ihre Kritik an einer Vorlesung, von der sie „überrascht" gewesen sei. Diese hatte den Titel „Bildung und Erziehung im Idealismus“, Inhalt sei jedoch „nur die Humboldt'sche Bildungstheorie“ gewesen und ,wie die Adeligen, in einer gewissen Zeit ihre Söhne ausgebildet haben". Wenn wir hier kurz den Schauplatz der Gruppendiskussion verlassen und in den Kontext des neuhumanistischen Bildungsideals mit ihrem Anspruch auf allgemeine Bildung wechseln, wird Mikas Kritik deutlicher. In den Schriften von Wilhelm von Humboldt wird zwar der Universalitätsanspruch der Bildungsidee formuliert, gleichzeitig jedoch dadurch unterlaufen, indem Humboldt Frauen auf spezifische Weise ausnimmt. Das Subjekt von Bildung bleibt damit weiterhin ein männliches Subjekt (vgl. Kapitel 2.3.1).

Neben der Androzentrismuskritik formuliert Mika hier auch eine Kritik am einseitigen Fokus der Vorlesung auf die „oberen Fünftausend“, auf die „Adeligen“. Somit scheint Mika neben der geschlechterreflektierten Perspektive auch eine milieusensible Perspektive der Vorlesung zu fehlen. Die Inhalte spiegeln für sie nicht die „Bildungsund Erziehungstheorie für eine ganze Epoche oder Jahrhundert" wieder. An diesem Beispiel veranschaulicht Mika ihre allgemeine Kritik an der „einseitigen Art der Wissenschaft" in dreifacher Weise: Sie kritisiert das Fehlen einer (1) geschlechter- und (2) milieusensiblen Perspektive und beanstandet, dass (3) wider eines allgemein formulierten Vorlesungstitels nur ein bestimmter Ausschnitt der Gesellschaft gezeigt wird. Diese Vorlesung bildet für Mika demnach einen negativen Horizont. Es ist zu vermuten, dass Mika ihre (singuläre) negative Erfahrung auf das gesamte Studium und die Erziehungs- und Bildungswissenschaften überträgt und deshalb von der ,sehr einseitige[n] Art der Wissenschaft" spricht und nicht nur von der Vorlesung. Mikas Kritik wird jedoch von den anderen Studierenden nicht geteilt bzw. ist für diese nicht nachvollziehbar.

Robyn bringt in ihrem Beitrag ihre oppositionelle Haltung zum Ausdruck. Sie beginnt mit der Verneinung (,Nee“), die sie mit einem Widerspruch anzeigenden „Aber" weiterführt. Sie greift Mikas Kritik am selektiven und eingegrenzten Inhalt der Lehrveranstaltung zwar auf, erklärt jedoch, dass damit „drei Vorlesungen“ verbunden sind. Mit „damit" bezieht sie sich auf die Veranstaltung „Bildung und Erziehung im Idealismus". Indem sie darauf verweist, dass damit „drei Vorlesungen“" verbunden seien, macht sie deutlich, dass der Inhalt dieser Vorlesung nicht in einer, sondern in drei Veranstaltungen behandelt werde. Damit relativiert sie Mikas Kritik, dass diese Vorlesung keine allgemeine und umfassende Perspektive vermittelt habe, da hierfür mehr als nur eine 
„Vorlesung“ nötig sei. In Bezug auf die (De-)Thematisierung von ,Geschlecht" ist interessant, dass sich Robyn (als auch die anderen GDT) nicht auf die Kritik an dem Fehlen der ,Spezies-Frau“ bezieht(en).

Mika schließt an Robyns Erwiderung an und erklärt, dass sie ein zweites Seminar - außerhalb dieser Reihe - besucht habe. Damit nimmt sie auf Robyns Einwand Bezug, dass mehr als eine Lehrveranstaltung besucht werden müsse, um ein umfassenden Blick über die „Bildung und Erziehung im Idealismus“ zu erhalten. In dem zweiten Seminar „ging es dann explizit um die Mädchenbildung", also um jene Inhalte, die Mika bei der Vorlesung vermisst hat. Mika wiederholt ihre Kritik an der Einseitigkeit der Inhalte, indem sie betont, zwei Veranstaltungen besuchen zu müssen, um „ein gesamtes Bild über die gleiche Zeit“ zu erhalten. In beiden Seminaren wurden je spezifische Aspekte einer „Epoche" behandelt: „Im einen wird nur Humboldt behandelt und im anderen, gut da geht es dann tatsächlich mal darum wie Bildung, Schulbildung, wie das überhaupt ausgesehen hat." Mika wird in ihrem Beitrag von Tomke unterbrochen, die mit den Worten ,,ja, aber" eine divergente Diskursbewegung ankündigt. Auch Tomke greift Mikas inhaltliche Kritik nicht auf, sondern versucht vielmehr eine Erklärung dafür zu finden, warum Mikas Erwartungen nicht erfüllt wurden. Tomke führt die Unterscheidung zwischen „Überblicksseminaren und Einführungsseminaren“ ein, die „die“ machen. Es ist zu vermuten, dass Tomke mit „die“ die Dozierenden meint. Das heißt, die Dozierenden würden die Unterschiede machen, somit erscheinen die Studierenden fremdbestimmt und davon abhängig, was die Dozierenden anbieten. Tomke führt die Unterscheidung weiter aus: Es gebe Seminare, „wo du einen sehr breiten, gefächerten Überblick bekommst". In Form einer Unterbrechung macht Mika nochmals klar, dass sie aber keinen Überblick, sondern einen sehr selektiven Einblick erhalten habe. Tomke schließt validierend an, indem sie ihre Vermutung zum Ausdruck bringt, Mika hätte ,lieber so einen Überblick gehabt". Daraufhin betont Mika erneut mit den Worten: ,ja also von wegen Überblick“ ihre Unzufriedenheit und ihre Kritik an der Vorlesung.

Bereits im Einstieg in die Passage zeigt sich, dass die Studierenden aneinander vorbei reden und zu keiner gemeinsamen Orientierung gelangen. Dies liegt, wie im Laufe der Gruppendiskussion noch deutlicher wird, daran, dass Mika von den anderen TeilnehmerInnen vor allem als Studienanfängerin gesehen wird, welche die feldspezifische Logik (noch) nicht inkorporiert habe und die ,Spielregeln" des Studiums nicht kenne (vgl. Kapitel 4.1.1). 
Vor diesem Hintergrund wird auch Mikas Kritik verstanden und die Diskussion nimmt phasenweise die Form eines Beratungsgespräches im Sinne einer StudienanfängerInnenberatung an. Die Perspektive von Mika wird abgewertet bzw. auf einer inhaltlichen Ebene nicht ernstgenommen, da die Studierenden mit ihrer längeren Erfahrung im Feld eine studiumsbezogene Vorrangstellung verknüpfen. Dieser Eindruck bestärkt sich im Laufe der Passage. Die TeilnehmerInnen scheinen über keine geteilten Erfahrungen zu verfügen und versuchen Mikas Kritik vor dem Hintergrund ihren eigenen Erfahrungen als StudienanfängerInnen zu lesen:

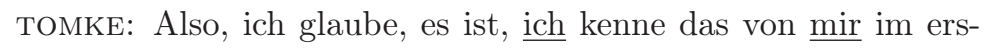
ten Semester man hat da irgendwie so ein bisschen den Anspruch, man glaubt man geht dahin, und jetzt kriegt man von jemanden der sich auskennt, wie niemand anderes, kriegt man das alles erzählt wie diese Zusammenhänge sind und wie sich das entwickelt hat.

ROBYN: Ja.

TOMKE: Und dann wird man so ein bisschen enttäuscht,

MIKA: Also ich denke [...] dass eben, dann muss da weniger vertieft werden in den Einführungsseminaren,

ROBYN: $\quad\llcorner$ Aber du hattest jetzt ein Semester; da kannst du doch nicht erwarten dass du so einen Gesamtüberblick schon hast; und er hat das in verschiedenen Modulen angeboten. Das, deswegen.
LUAN:
$\llcorner$ Das ist eine Reihe.

ROBYN: Es ist eine Reihe, die in verschiedenen Modulen angeboten wurde. Ich hatte das damals in Modul 3,[...] ich dachte auch so am Anfang, wir lernen so viel Zeug, aber irgendwie ich kann damit gar nichts anfangen, wie soll ich denn damit jemals arbeiten? Aber das wird im zwoten Semester wird das schon besser. [...]

MIKA: Also im zweiten Semester lerne ich denn die Wissenschaftlerinnen kennen, oder wie muss ich mir das vorstellen?

LUCA: $\quad$ D Du du ich glaube du darfst dir gar nicht so einen Kanon vorstellen, der systematisch abgehakt wird, 
ROBYN: ᄂ Nee.

LUCA: Sondern es gibt halt Gebiete wo du reinschaust und wo du nicht reinschaust.

ROBYN: Du kannst auch nicht alles lernen.

LUAN:

$\llcorner$ Zum Beispiel wenn sich was „Einführung in die Erziehungswissenschaften“ nennt, dann kann das entweder die Anthropologie sein, oder es kann der Idealismus sein, oder noch was ganz anderes; und je nachdem in welchem Semester du eingestiegen bist, hast du entweder das gehört oder das. Und das andere weißt du dann einfach nicht.

Tomke erzählt von ihren Erfahrungen im ersten Semester und schließt damit an Mikas Wunsch nach einem besseren „Überblick“ an. Tomke erzählt von ihren Ansprüchen als Studienanfängerin, und dass sich diese nicht mit ihren Erfahrungen im Studium gedeckt hätten und sie deshalb „enttäuscht" gewesen sei. Auch Robyn scheint diese Erfahrung gemacht zu haben, da sie validierend an Tomkes Beitrag anschließt (,ja“). Tomke und Robyn bringen hier erneut zum Ausdruck, dass sie Mikas Kritik und Unzufriedenheit mit ihrem Status als Studienanfängerin in Verbindung bringen („Aber du hattest jetzt ein Semester"), da dies Teil ihrer Erfahrung und ihrer Normalität war. Die Unzufriedenheit und die Kritik am Studium wird hier als temporäre studiumsbezogene konjunktive Erfahrung dargestellt, die Teil der unveränderlichen feldspezifischen Logik zu sein scheint, wobei der Inhalt der Kritik nebensächlich erscheint.

Dass die Teilnehmerinnen nicht vor demselben Hintergrund und demselben Relevanzsystem sprechen, spiegelt sich in Mikas Nachfrage wider. Wie bereits zuvor macht Mika auf das Fehlen geschlechterreflektierter Inhalte aufmerksam: „Also im zweiten Semester lerne ich dann die Wissenschaftlerinnen kennen, oder wie muss ich mir das vorstellen?" Es ist anzunehmen, dass sie sich mit den „Wissenschaftlerinnen“ auf Humboldt (siehe oben) bezieht und diese einen Gegenpol zu den männlich zentrierten Inhalten darstellen sollen. Erneut macht Mika auf eine Lücke aufmerksam, die aus ihrer Perspektive dazu beiträgt, dass die „Wissenschaft" einseitig sei.

Nun wiederholt sich dasselbe Diskursmuster wie oben: Die GDT gehen nicht auf Mikas Androzentrismuskritik ein, sondern folgen dem Muster der StudienanfängerInnenberatung, indem sie Mika die ,Spielregeln“ (erneut) erklären. Hinsichtlich der Kritik 
an der ,einseitigen Art der Wissenschaft" und der Androzentrismuskritik gelingt es den Teilnehmer/inne/n nicht, zu einer gemeinsamen Konklusion zu kommen. Schließlich wird die Passage (vorerst) von Luan mit einer rituellen Konklusion beendet und es wird deutlich, dass Mika mit ihrer Orientierung in Opposition zu der restlichen Gruppe steht. Auch wenn die Passage hier beendet erscheint, wird das Thema im Laufe der Gruppendiskussion immer wieder von Mika aufgeworfen bzw. darauf Bezug genommen.

Es gelingt den Teilnehmer/innen der Gruppe nicht, sich auf einen konjunktiven Erfahrungsraum und ein gemeinsames Thema einzupendeln. Als Erklärung für die eklatanten Verstehensprobleme unter den GDT kann die unterschiedliche Studiendauer der beteiligten Studierenden herangezogen werden. Darüber hinaus bzw. in Verbindung damit werden auch Rahmeninkongruenzen in der Gruppe Holz deutlich, d. h. unterschiedliche und widersprüchliche Orientierungen und Relevanzsysteme, die nicht zu gemeinsamen Orientierungen verbunden werden können. Auf der einen Seite bringt Mika ihre inhaltliche Kritik an einer Vorlesung zum Ausdruck, indem sie das Fehlen einer geschlechterreflektierten Perspektive betont und aufgrund dieser Erfahrung die Einseitigkeit der Wissenschaft problematisiert. Damit macht Mika auch deutlich, dass für sie die Thematisierung von ,Geschlecht' und Geschlechterfragen einen positiven Horizont darstellt und das Fehlen dieser Thematisierung den negativen Gegenhorizont. Auf der anderen Seite stehen Robyn, Luan und Tomke (später auch Luca), die Mikas Kritik als Unzufriedenheit einer Studienanfängerin interpretieren und diese vor dem Hintergrund ihrer konjunktiven Erfahrungen als fortgeschrittene Studierende diskutieren. Auch wenn alle Studierenden vor dem studiumsbezogenen konjunktiven Erfahrungsraum sprechen, zeigt sich, dass die Wahrnehmungs-, Denk- und Beurteilungsschemata der Studierenden maßgeblich von der Inkorporation sozialer Strukturen abhängen.

In dieser Passage zeigt sich zudem, dass es keine etablierte Diskussionsform und -kultur zum Thema ,Geschlecht' und Geschlechterfragen zu geben scheint. Somit lassen sich, wenn es um die Relevanz der Kategorie ,Geschlecht' (im Studium) geht, auch keine kollektiven Wissensbestände und habituelle Praxen rekonstruieren. Dies wird im weiteren Verlauf der Gruppendiskussion noch deutlicher. 


\section{Studiumsbezogene (De-)Thematisierung von ,Geschlecht‘ und Geschlechterfragen}

Um die Diskussion zur (De-)Thematisierung von ,Geschlecht' und Geschlechterfragen im studiumsbezogenen konjunktiven Erfahrungsraum nochmals anzuregen, fragt die Interviewerin nach ,konkreten Situationen“, an die sich die GDT erinnern können, in denen „Geschlecht eine Rolle gespielt hat". Mit dieser Frage ist die Präsumption verbunden, dass die GDT bereits Situationen erlebt haben, in denen, Geschlecht' relevant war, oder es Thema war. Zugleich setzt diese Frage voraus, dass sich die GDT bereits mit ,Geschlecht' auseinandergesetzt haben und fordert sie dazu auf, sich selbst damit ins Verhältnis zu setzten. Als Beispiel nennt die Interviewerin den Besuch von Lehrveranstaltungen, „wo es Thema war". Tomke greift die Frage als Erste auf:

846 TOMKE: Ich schreib gerade eine Hausarbeit über Gender; @aber

847

848

849

850

851

852

853

854

855

856

857

858

859

860

861

862

863

864 das wars@.

LUAN: Also in den Erziehungswissenschaften hab ich noch keine Veranstaltung gemacht. Ich hab in der Soziologie so was gemacht. Wobei ich dann da raus bin weil das nicht mehr ging, von der Zeit her, aber da wurde so was angeboten; „Einführung in die Geschlechterforschung“. Aber jetzt in der Erziehungswissenschaften ist mir das so konkret auch noch nicht begegnet, also glaube nicht.

ROBYN: Welches war das? Wo du dich so drüber aufgeregt hast?

LUAN:

$\llcorner$ Das war das in Soziologie.

TOMKE: Ich weiß aber das, also hab ich jetzt nur gelesen im Rahmen der Hausarbeit, dass an ganz vielen anderen Unis schon seit Jahren immer wieder Seminare zu geschlechtsgerechter Didaktik oder ja auch Gender im Schulunterricht oder so was gibt, aber das hab ich hier im Vorlesungsverzeichnis noch überhaupt nicht gefunden.

Tomke knüpft an die Orientierung der Interviewerin an, dass eine Auseinandersetzung mit ,Geschlecht' stattfindet und sagt, dass sie eine Hausarbeit über "Gender" schreibe. Der Begriff gender verweist hier auf eine Auseinandersetzung auf einer theoretischen Ebene. Auch das Schreiben einer Hausarbeit deutet auf eine universitäre Praxis hin, die auf eine 
theoretische Bearbeitung und Auseinandersetzung abzielt und keinen Hinweis auf die eigene Praxis oder das handlungsleitende Wissen gibt. Ihre Formulierung ,@aber das wars@“11 kann ein Hinweis dafür sein, dass sich Tomke außerhalb dieser universitären-theoretischen Ebene, also z. B. in der pädagogischen Praxis oder im Sinne einer Selbstreflexion, noch nicht mit ,Geschlecht' und Geschlechterfragen beschäftigt hat. Zudem relativiert Tomke mit diesem lachend gesprochenen Nachsatz ihre Auseinandersetzung und ihre Beschäftigung mit dem Thema. Luan nimmt nun auf das Beispiel der Interviewerin Bezug und berichtet, dass sie zwar eine Lehrveranstaltung besucht habe, aber nicht im Curriculum der Erziehungs- und Bildungswissenschaften, sondern in der Soziologie: ,[Da] wurde so was angeboten." Für den Abbruch des Seminars nennt Luan einen formalen Grund. Robyns Nachfrage, „Welches war das? Wo du dich so drüber aufgeregt hast?"“, macht deutlich, dass Robyn und Luan bereits über eine Lehrveranstaltung gesprochen haben, über die sich Luan ,aufgeregt" habe. Robyn möchte nun wissen, ob es sich dabei um diese Lehrveranstaltung handle. Dies lässt vermuten, dass es auch andere Gründe für den Abbruch dieser Veranstaltung gab, die Luan hier nicht nennen kann oder will. Luan bestätigt Robyn: „Das war das in der Soziologie." Dieser Austausch zwischen Robyn und Luan weist hier auf einen konjunktiven studentischen Erfahrungsraum hin, den die beiden teilen, und dass es eine Praxis gibt, sich über negative Erfahrungen auszutauschen, die jedoch im Rahmen der Gruppendiskussion keinen Platz haben. Worüber sich Luan empört hat, bleibt in der Gruppendiskussion unausgesprochen.

Tomke schließst validierend an Luans Proposition an. Anders als Luan bezieht sich Tomke allerdings nicht auf ihr eigenes Erfahrungswissen, sondern auf theoretisches Wissen, das sie sich ,im Rahmen der Hausarbeit" angeeignet hat. In dem Tomke beschreibt, dass es ,an ganz vielen Unis schon seit Jahren" ein Lehrveranstaltungsangebot zu ,Geschlecht" und Geschlechterfragen gebe, schwingt die implizite Kritik an ihrem Studienort mit. Robyn, Tomke und Luan scheinen hier die gemeinsame Erfahrung zu teilen, dass im Rahmen ihres Studiums der Erziehungs- und Bildungswissenschaften ,Geschlecht" und Geschlechterfragen nicht Teil des Lehrveranstaltungsangebots sind. Mika hat jedoch eine andere Erfahrung gemacht und erklärt, dass es hier eine "Genderforschungsgruppe“ gebe und sie ein Seminar besucht habe:

11 Wörter in @-Klammer illustriert, wenn ein Wort lachend gesprochen wird. 


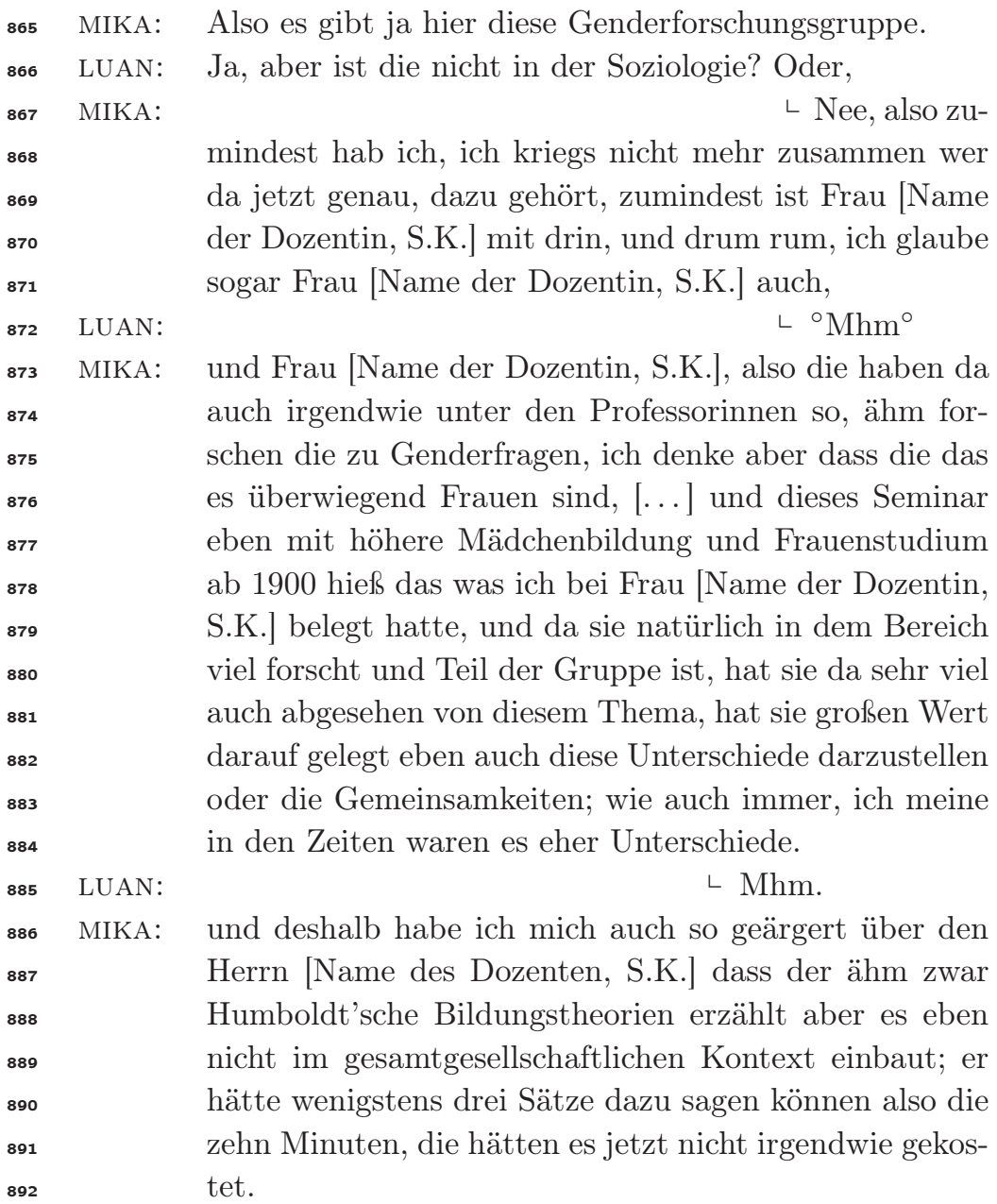

Mika hat im Gegensatz zu ihren Kommilitoninnen die Erfahrung gemacht, dass ,Geschlecht ${ }^{6}$ und Geschlechterfragen in ihrem Studiengang - wenn auch nicht in der Einführungsvorlesung, die sie oben kritisiert thematisiert wird: zum einen im Bereich der Forschung und zum anderen auch in der Lehre. Luans Nachfrage, ob es sich dabei nicht um einen anderen Fachbereich handelt, weist Mika zurück. Mika nennt einen Teil dieser „Genderforschungsgruppe“ beim Namen und dabei fällt auf, dass es „überwiegend Frauen" sind. Hier deutet sich bereits etwas an, das sich im Verlauf der Diskussion bestätigt: Die Thematisierung von ,Ge- 
schlecht ${ }^{6}$ und Geschlechterfragen im konjunktiven Erfahrungsraum des Studiums ist hauptsächlich von weiblichen Akteurinnen geprägt. Mit ihrem Wissen zu dieser "Genderforschungsgruppe“ und ihrem besuchten Seminar zur ,höheren Mädchenbildung und Frauenstudium ab 1900" belegt Mika ihr Expertinnenwissen und ihre Erfahrungen zur Thematisierung von ,Geschlecht' im Studiengang. Dieses Seminar stellt für Mika einen positiven Horizont dar. Nachdem Mikas Beitrag von Luan ratifiziert wird, kommt Mika nun auf das Seminar zur „Humboldt'schen Bildungstheorie“ und das Thema „einseitige Wissensvermittlung" zurück; beides stellt für sie einen negativen Horizont dar. Dieser Rückbezug ist ein weiterer Hinweis dafür, dass es sich bei der Gruppendiskussion um einen divergenten Diskurs handelt, und dass sich Mika zuvor nicht richtig verstanden fühlte und sich deshalb nochmals erklären möchte. Mika erklärt, dass sie sich über den Dozenten geärgert habe, da er den Inhalt seines Seminars nicht in den gesamtgesellschaftlichen Kontext eingebettet habe und eine geschlechterreflektierte Perspektive gänzlich ausgespart hat. Diese Passage wird durch einen Themenwechsel von Tomke beendet. Die Gruppe diskutiert zwar im Rahmen der „Genderfragen" weiter, jedoch wechselt der Fokus mit Tomkes Beitrag von einer inhaltlichen auf eine repräsentative Ebene:

TOMKE: Also ich glaube was in Genderfragen zumindest ungewöhnlich ist, ist das wir eine Dekanin haben. Also davon gibt es ja auch sehr wenige in den Positionen. Also man sagt ja je höher oder es ist so, dass je höher die äh der akademische Grad wird umso geringer wird der Frauenanteil dabei, und ja.

LUAN: $\quad$ L Also ich war bei der, der Frau [Name der Dekanin, S.K.] in einem Seminar und da kam unter anderem das Thema ,Geschlecht' drin vor; aber es dazu gehörten ja auch alle anderen Spektren ja? Von Behinderung bis was weiß ich was. Also von daher war es nur ein Aspekt und nicht ein Seminar zu diesem Thema.

MIKA: Aber ist es eigentlich nicht so dass ich meine gerade in der Erziehungswissenschaft, also zumindest in der Praxis ja also in der Arbeit jetzt auch mit Jugendlichen oder Kindern, da spielt ,Geschlecht' eine unglaublich große Rolle. [...] und eigentlich, wenn ich jetzt so drüber nachdenke, stimmt das schon dass es eigentlich re- 


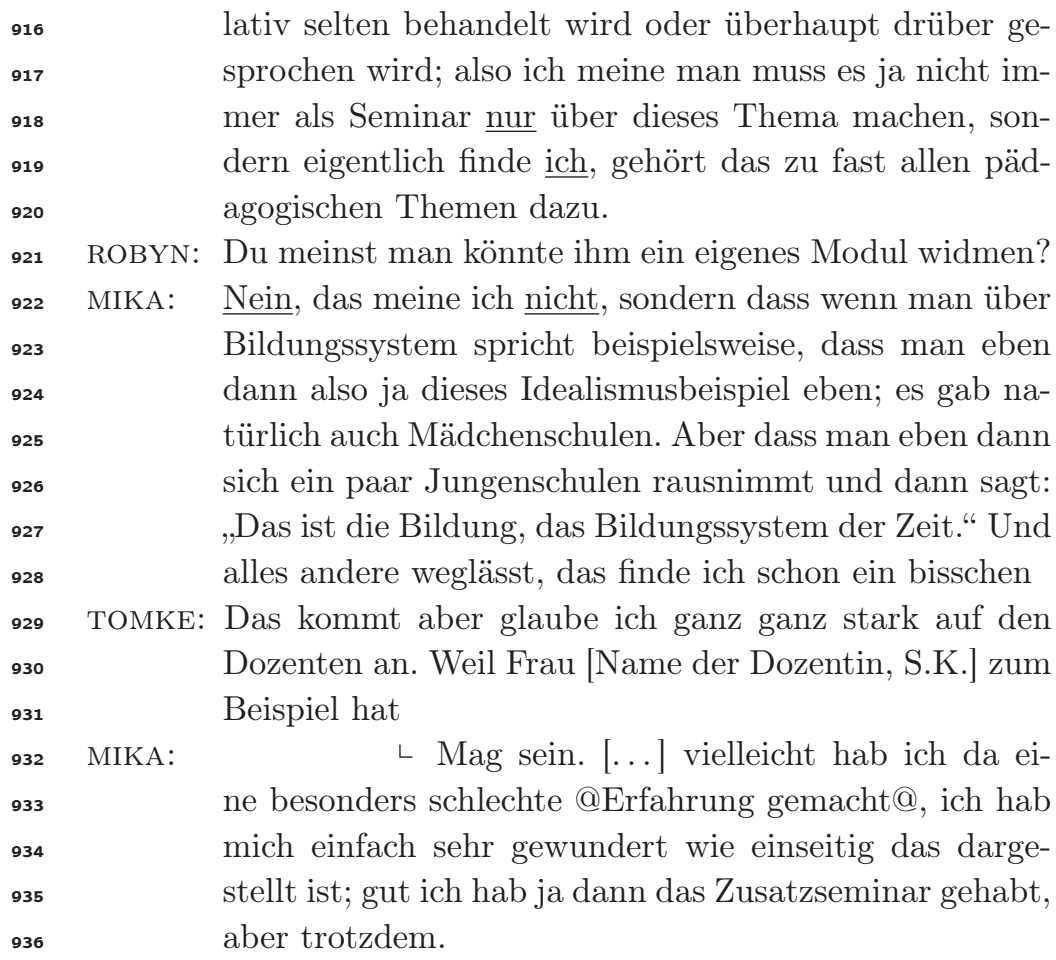

Tomke wirft hier eine neue Proposition auf, es geht nun nicht mehr um ,Geschlecht' in Bezug auf das Curriculum, sondern um die Verknüpfung von ,Geschlecht' (Frausein) mit Repräsentation und Führungsposition. Tomke elaboriert, dass es in Bezug auf „Genderfragen“ ungewöhnlich sei, dass sie „eine Dekanin haben“. „Genderfragen“ betrifft demnach auch die Repräsentation von Frauen in Führungspositionen. Mit dem Begriff „ungewöhnlich" markiert Tomke, dass eine Dekanin von der gewohnten Norm abweicht und eher selten der Fall ist. Tomkes Ausführungen sind erneut geprägt von theoretischem Wissen, sie elaboriert, dass ,,je höher die der akademische Grad wird umso geringer wird der Frauenanteil dabei". Auch bei diesem Beitrag wird der Begriff gender wieder als Expertinnenbegriff verwendet bzw. damit ein wissenschaftlicher Fachjargon bedient; dies weist darauf hin, dass gender zum geteilten Wortschatz der Studierenden gehörte, der ein Teil ihrer konjunktiven Erfahrung ist und einen wissenschaftlich-universitären Habitus repräsentiert. Luan berichtet nun, dass sie bei „der" Dekanin ein Seminar besucht habe, in dem ,unter anderem das Thema ,Geschlecht' drin 
vor" kam. Luan wechselt somit wieder zum Rahmen der Auseinandersetzung mit ,Geschlecht" in Bezug auf das Curriculum und elaboriert die bisherige Orientierung. Die Auseinandersetzung mit ,Geschlecht' muss nicht, wie bisher diskutiert, ein ganzes Seminar dominieren, sondern kann auch „nur ein[en] Aspekt“ sein. Mika eröffnet nun eine neue Orientierung und hebt die Relevanz von ,Geschlecht' für die pädagogische Praxis hervor: In der „Praxis [...] da spielt Geschlecht eine unglaublich große Rolle.“ In Bezug darauf generalisiert Mika, dass ,Geschlecht' „relativ selten behandelt wird oder überhaupt drüber gesprochen wird", und das obwohl es „fast [zu] allen pädagogischen Themen“ gehöre. Mika positioniert sich nun eindeutig und es wird deutlich, dass die Berücksichtigung von ,Geschlecht' und Geschlechterfragen im Studium für sie einen positiven Horizont darstellt. Robyn fragt nun nach, ob Mika dem Thema „ein eigenes Modul widmen" würde und bezieht sich wieder auf das Curriculum. Es zeigt sich aber auch, dass Robyn das Denken in universitären Strukturen und das Modulsystem bereits internalisiert hat.

Nachdem Mika die Frage verneint, exemplifiziert sie erneut am Seminar „Bildung und Erziehung im Idealismus“ ihre Proposition, dass ,Geschlecht' ein Querschnittsthema darstellen solle und kritisiert die androzentrische Perspektive im Seminar. Tomke grenzt Mikas Erfahrung ein, indem sie ihre persönliche Einschätzung nennt. Sie meint, dass es „ganz stark auf den Dozenten“ ankomme, ob eine geschlechterreflektierte Perspektive Teil des Seminars sei. Im Sinne einer Validierung ist Mikas „mag sein“ zu interpretieren, da sie Tomkes Orientierung zurückweist. Mit „mag sein“ signalisiert Mika zumindest eine Offenheit gegenüber Tomkes Proposition. Diese Vermutung wird damit bestätigt, dass Mika die Generalisierung ihrer negativen Erfahrung eingrenzt: „Vielleicht hab ich da eine besonders schlechte @Erfahrung gemacht@." Ihr Abschluss, „aber trotzdem", scheint zu betonten, dass sie dennoch an ihrer individuellen Orientierung festhält, dass eine geschlechterreflektierte Perspektive Teil eines jeden Seminars sein sollte, weil ,Geschlecht' in der Praxis eine "unglaublich große Rolle spielt".

In dieser Passage wird erneut sichtbar, dass es die Studierenden nicht schaffen, sich auf eine Orientierung einzupendeln und Bezüge zu ihren Beiträge herzustellen. Aber auch nach dieser Passage ist das Thema für Mika noch nicht beendet und sie greift es gegen Ende der Gruppendiskussion nochmals auf. Mika führt hier (erneut) die Orientierung ein, dass "Gendergeschichten“ auch in Vorlesungen allgemein einfließen sollten, um „einfach das Bewusstsein“ dafür zu schärfen. Für Mika scheinen, Geschlecht' und Geschlechterfragen kein 
abgetrenntes Spezialthema zu sein, sondern etwas, das in Zusammenhang mit anderen Themen stehen sollte.

\begin{tabular}{|c|c|c|}
\hline 78 & MIKA: & Ich denke diese Gendergeschichten, dass so was über- \\
\hline 79 & & haupt existiert, das darf man ruhig auch in Vorlesungen \\
\hline 80 & & allgemein einfach einfließen lassen, einfach als Bewusst- \\
\hline 31 & & sein dass das einfach das Bewusstsein, vorhanden ist, \\
\hline & & dass es da gewisse Unterschiede gibt auch gewisse Ge- \\
\hline 583 & & meinsamkeiten, gewisse Problematiken, und dass man \\
\hline 84 & & einfach ich meine es geht ja auch man braucht ja nicht \\
\hline 585 & & immer die Dinge so, separat voneinander betrachten, \\
\hline 86 & & dass die überhaupt nie zusammen kommen. Sondern \\
\hline 87 & & ich denke dafür sind wir auch an der Uni dass wir eben \\
\hline 88 & & Themen die natürlich am Anfang erstmal einzeln ver- \\
\hline 689 & & tieft werden, aber die dass wir die auch zusammenfüge \\
\hline 90 & : & {$[\ldots]$} \\
\hline 691 & LUCA: & Also vor zwei Jahren hat der [Name des Dozenten, S.K.] \\
\hline 92 & & noch eine Vorlesung die nur die KlassikerINNEN beha \\
\hline 693 & & delt hat. ${ }^{\circ}(\text { Pädagoginnen })^{\circ}$. \\
\hline 694 & ROBYN: & $\left\llcorner{ }^{\circ}(\mathrm{Ja})^{\circ}\right.$ \\
\hline 695 & LUCA: & Also ich vielleicht ist es einfach ein bisschen schwer auch \\
\hline 696 & & nach einem Semester zu beurteilen, \\
\hline 697 & ROBYN: & $\left\llcorner{ }^{\circ} \mathrm{Ja}^{\circ}\right.$ \\
\hline 1698 & LUCA: & was es für eine Bandbreite alles gibt, weil \\
\hline 699 & MIKA: & $\llcorner$ Ja, ich hoffe $\mathrm{i}$ \\
\hline 1700 & & irre mich (lacht) \\
\hline 01 & & ((allgemeines Gelächter)) \\
\hline 02 & LUCA: & ${ }^{\circ} \mathrm{Ja} .{ }^{\circ}$ \\
\hline
\end{tabular}

Nach der erneuten Kritik von Mika am Fehlen von „Gendergeschichten“ im Studium exemplifiziert Luca, dass es eine Vorlesung zu „KlassikerINNEN" gegeben hätte. Sein Beitrag stellt eine Opposition zu Mikas Erfahrung dar. Luca weist Mika auf die Grenzen ihrer Aussage hin. Nachdem Robyn Lucas Beitrag validiert, fährt Luca darin fort, den Geltungsbereich von Mikas Orientierung einzugrenzen und konstatiert, dass es Mika aufgrund ihrer kurzen Studienerfahrung ,vielleicht [...] einfach ein bisschen schwer" fällt, die Bandbreite des Lehrveranstaltungsangebots zu beurteilen. Damit grenzt er quasi Mikas Urteilskraft aufgrund ihrer erst kurzen Studiendauer ein. Damit wird betont, dass Mikas Sichtweise limi- 
tiert sei, was auch die Generalisierung ihrer Orientierung in Frage stellt. Die Passage endet mit einer (rituellen) Konklusion, indem Mika hier Luca und Robyn zustimmt, dass sie die „Bandbreite“ des Studienangebots nicht beurteilen könne. Mit der Formulierung ,ich hoffe ich irre mich“ lässt sie jedoch Zweifel daran offen, dass sie mit ihrer Androzentrismuskritik falsch liegt. Auch wird, indem Mika als Novizin im Feld gesehen wird, ihre Kritik auf einer inhaltlichen Ebene nicht wahrgenommen, sondern das Problem einer Studienanfängerin limitiert.

Auch diese Passage kann im Sinne der Diskursstruktur als oppositionell und divergent charakterisiert werden, demnach können auch hier keine kollektiven Orientierungen rekonstruiert werden. Darin wird deutlich, dass die Androzentrismuskritik keine kollektive Orientierung der Studierenden darstellt und die Studierenden in Bezug auf die Thematisierung von ,Geschlecht' auf keinen gemeinsamen Erfahrungsraum zurückgreifen können. In der Wahrnehmung ihrer Mitdiskutierenden konfligiert Mikas Kritik mit ihrem Status als Studienanfängerin. Als Studienanfängerin scheint Mika aus Sicht der anderen GDT über einen eingeschränkten studiumsbezogenen Erfahrungsraum zu verfügen. Mikas Kritik an der androzentrischen Wissenschaft und Wissensvermittlung wird von den anderen GDT, die über einen breiten studiumsbezogenen Erfahrungsraum verfügen, delegitimiert. Zudem wird deutlich, dass die Möglichkeiten über studiumsbezogene Erfahrungen in Bezug auf ,Geschlecht' zu sprechen - wie hier oftmals von Mika angeboten - von der Orientierung an der Dauer der studiumsbezogenen Erfahrungen überlagert wird. Hier bestätigt sich somit die These, dass es in dieser Gruppe keine etablierte Diskussionsform und -kultur zum Thema ,Geschlecht' gibt und sich diesbezüglich auch keine kollektiven Wissensbestände und habituelle Praxen rekonstruieren lassen. Bei den Passagen zur Relevanz von ,Geschlecht' und der Berücksichtigung dieser Kategorie zeigt sich vielmehr ein ,Habitus des nicht Verstehens" (Rieger-Ladich 2010, S. 92), der zu einer (De-)Thematisierung von ,Geschlecht' und Geschlechterfragen führt. Zudem zeigt sich, dass die Hintergrunderwartung der Geschlechtsdarstellung und -hierarchie ebenfalls nicht thematisiert und problematisiert wird. 


\subsection{3 „Erziehungswissenschaften: Ein typischer Frauenstudiengang"}

In der Gruppendiskussion gibt es aber auch Passagen, die von einem parallelen Diskurs geprägt sind, und sich daher neben individuellen auch kollektive Orientierungen rekonstruieren lassen, wie folgender Diskussionsausschnitt zeigt. Tomke beginnt mit der Proposition, dass es im Studium der Erziehungs- und Bildungswissenschaften einen „unglaublichen Frauenüberschuss" gibt:

937

938

939

940

941

942

943

944

945

946

947

948

949

950

951

952

953

954

955

956

957

958

959

960

961

962

963

964

TOMKE: Also was auf einer ganz anderen Ebene auffällig ist ist dass wir einen unglaublichen Frauenüberschuss haben.

MIKA:

$\llcorner$ Ja.

TOMKE: In der Erziehungswissenschaft. Also wenn da mehr als drei Männer in einem Seminar sitzen, ist das schon eine @ordentliche@ Quote.

LUAN: Findest du das auffällig?

TOMKE:

$\llcorner$ Ja.

MIKA:

ᄂ Ja.

ROBYN: Ich glaube das war aber schon also das ist halt so; oder? also bei den Lehrern findest du dann ja halt wieder mehr Männer.

TOMke: Genau. Das ist es ja. Also jetzt die Erziehungswissenschaftler, wenn man da mal guckt, ich meine aus dem Stegreif denke ich könnten wir alle mindestens drei vier fünf Professoren der Erziehungswissenschaften nennen, was verwundert, dass aber unter den Studenten, also in meinem Semester, mit mir angefangen da wüsste ich nicht wie viele Jungs da dabei waren. Da kenne ich mal vereinzelt welche aus höheren Semestern, aber auch nicht wirklich viele, also Erziehungswissenschaften,

MIKA:

$\llcorner$ Maximal fünf Prozent würde ich jetzt sagen aus dem Bauch. TOMKE:

$\llcorner$ Erzieh-

ungswissenschaften sind ja eindeutig eher so ein typischer Frauenstudiengang. Was eigentlich meiner Meinung nach nicht nicht unbedingt sein muss, denn es gibt genug Arbeitsfelder, der Erziehungswissenschaften wo 
Männer durchaus angebracht sind.

966 LUAN:

Tomkes Proposition, dass ,wir einen unglaublichen Frauenüberschuss haben", wird von Mika validiert, noch bevor sie fertig gesprochen hat. Tomke exemplifiziert: „Also wenn da mehr als drei Männer in einem Seminar sitzen, ist das schon eine @ordentliche@ Quote." Antithetisch fragt Luan nach, ob Tomke das wirklich ,auffällig" finde. Nicht nur Tomke, sondern auch Mika antworten auf diese Frage und bringen damit ihre gemeinsame Orientierung erneut zum Ausdruck. Robyn findet den Frauenüberschuss nicht auffällig, sondern konstatiert, dass dies „halt so" sei. Dass es einen Frauenüberschuss in den Erziehungs- und Bildungswissenschaften gibt, sei für sie nichts Auffälliges, im Lehramtsstudium ,findest du dann ja halt wieder mehr Männer". Auch wenn Robyn ihre Aussage als persönliche Einschätzung (,ich glaube") einklammert, hat sie einen sehr stark normierenden und festschreibenden Charakter. Tomke validiert Robyns Argument („Genau. das ist es ja."), mündet damit jedoch in eine andere Orientierung. Um das Auffällige an der Geschlechterverteilung hervorzuheben, betont Tomke das ungleiche Verhältnis von Professoren und den Studenten. Sie argumentiert, dass es gemessen an den wenigen Studenten „verwundert“, dass es viele Professoren gibt. Indem sie betont, dass ,wir alle" mehrere Professoren nennen könnten, generalisiert sie ihre persönliche Erfahrung und verleiht ihr damit mehr Gewicht. Mika bestätigt Tomke, dass es nur wenige Studenten gibt durch ihre persönliche Einschätzung und Erfahrung. Das Ungleichgewicht bei der Verteilung von Studentinnen und Studenten bringt Tomke zum Ausdruck, indem sie Erziehungs- und Bildungswissenschaften als ,typische[n] Frauenstudiengang" bezeichnet. Auch wenn Tomke im Weiteren erklärt, dass dies ihrer „Meinung nach nicht [... ] sein muss", reproduziert sie den Studiengang Erziehungs- und Bildungswissenschaften als ein weiblich dominiertes Fach. Luan schließst validierend an Tomkes Orientierung, dass es ,genug Arbeitsfelder" in den Erziehungs- und Bildungswissenschaften gebe, „wo Männer durchaus angebracht sind" und konkludiert, dass Männer in den pädagogischen Arbeitsfeldern auch „dringend benötigt werden“. Abschließend wird die von Tomke und Luan geteilte Orientierung deutlich, dass Männer einen legitimen und „dringend benötig[en]“ Teil unter PädagogInnen darstellen. Warum und in welchen Bereichen diese dringend benötigt werden, scheint Teil des kollektiven Wissensbestandes zu sein, da es hier nicht expliziert wird. Auch wenn sich die GDT am Be- 
ginn dieser Passage nicht einig sind, ob der Frauenüberschuss auffällig ist oder nicht, wird der Studiengang Erziehungs- und Bildungswissenschaften von allen GDT als ein weiblich dominiertes Fach reproduziert, indem männliche Studierenden als von der erziehungswissenschaftlichen Norm abweichend dargestellt werden.

\subsubsection{Die Orientierung am generischen Maskulinum und/oder an der geschlechtergerechten Sprache}

Die folgende Passage zur ,geschlechtergerechten Schreibpraxis in Hausarbeiten" 12 wurde von der Interviewerin initiiert. Sie ist von besonderer thematischer Relevanz, da sie die Basis für die im Anschluss durchgeführte komparative Analyse mit der Passage zur „Bedeutung und dem Umgang mit Begriffen und Kategorien im Allgemeinen" darstellt. Hinsichtlich der geschlechtergerechten Sprache kann in der Gruppe keine habituelle Praxis rekonstruiert werden; auch hier zeigen sich unterschiedliche individuelle Orientierungen.

1161 MIKA: Also bisher habe ich Schüler und dann großes I-n-n-e-n;

1162 geschrieben.

1163 LUAN: Genau; so mache ich das auch.

1164 TOMKE: Also ich muss ganz ehrlich sagen ich mache da nicht immer den Unterschied; ich mache oftmals eine Anmerkung, dass ich einfach durch den genormten Sprachgebrauch; der eben einfach der männlichen Norm entspricht, natürlich nur sag ,Ja dann werden die Schüler', ohne dann, ohne dass ich damit jetzt weibliche Schüler ausgrenzen möchte; sondern dass ich das einfach dass damit beide Gruppen benannt sind, wenn ich sie trennen möchte nenne ich sie weibliche Schüler und äh männliche Schüler, und ja; dass das letztlich oder oftmals auch einfach daran liegt, dass dass ich mir so Platz spare. Muss ich auch ganz ehrlich sagen also wenn ich immer ,Teilnehmer und Teilnehmerinnen' schreib,

LUAN:

$\left\llcorner{ }^{\circ}\right.$ Nee das ist blöd ${ }^{\circ}$.

1178 TOMKE: dass ich dann einfach also im Französischen ist es ja

12 Diese Passage ist in Form einer Beispielinterpretation nach der dokumentarischen Methode ausführlich im Anhang dargestellt. 


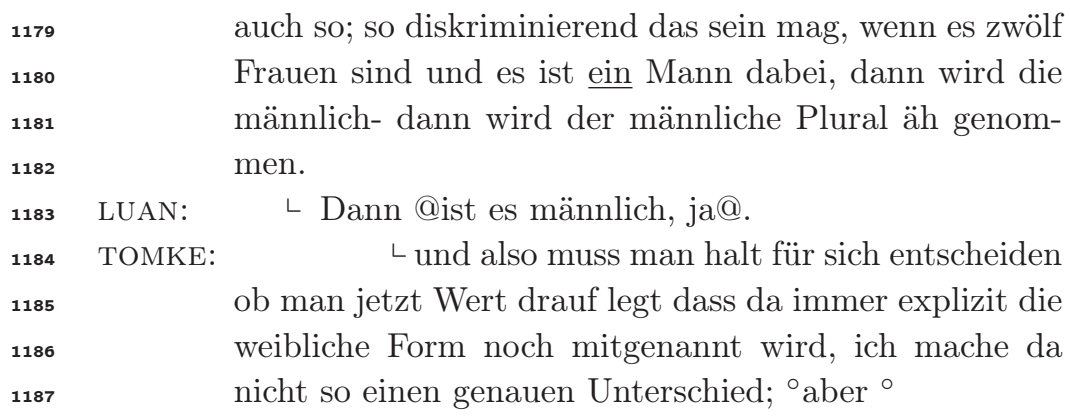

Mika und Luan geben als erste Auskunft über ihr eigene Handlungspraxis, sie kennzeichnen Frauen und Männer „,durch großes-I-n-n-e-n“ und verwenden somit die Form des Zusammenziehens mit dem Binnen-I. Das heißt, sie machen Frauen und Männer als Teile der Gesellschaft sichtbar und zudem werden beide Genusgruppen angesprochen. Tomkes Beitrag ist als Opposition zu verstehen, dabei orientiert sie sich am generischen Maskulinum. Tomke begründet ihre Orientierung „durch den genormten Sprachgebrauch, der eben einfach der männlichen Norm entspricht". Tomke führt fort, dass sie eine Anmerkung mache, dass damit beide Gruppen benannt seien, ohne dass sie damit ,weibliche Schüler ausgrenzen möchte". Das generische Maskulinum wird in diesem Beitrag positiv gerahmt und die geschlechtergerechte Sprache stellt den Gegenhorizont dar. Bevor Tomke ihren Satz zur Paarbildung fertig sprechen kann, wird sie von Luan mit einer Kommentierung unterbrochen. Luan lehnt diese Schreibweise ab: „Nee, das ist blöd.“ Damit validiert sie die von Tomke angekündigte negative Bewertung. Im Folgenden arbeitet Tomke ihre Orientierung weiter aus. Tomke erklärt, dass die Verwendung vollständiger Paarformen im Plural in den allgemeinen Sprachregeln nicht vorgesehen seien und Frauen beim generischen Maskulinum mitgemeint seien. Indem Tomke sich hier auf Grammatikregeln bezieht, ruft sie eine scheinbar neutrale, objektive und generalisierende Distanz an. Unter Rückbezug auf die vorherige Seite, wo Tomke sagt ,ohne dass ich damit jetzt weibliche Schüler ausgrenzen möchte", entsteht der Eindruck, dass Tomke das generische Maskulinum unter den Prämissen der Verallgemeinerung und der Inklusion verwendet. Tomke möchte niemanden ausgrenzen, sondern trifft ihre Entscheidung anscheinend aufgrund formaler und pragmatischer Kriterien. Luan bestätigt Tomkes Ausführungen zu den grammatikalischen Regeln auf einer Ebene des inhaltlichen Verständnisses. Die unterschiedlichen Orientierungen hinsichtlich der Schreibpraxis werden von Tomke mit einer rituellen Konklusion im Modus der 
Metarahmung verdeckt und individualisiert: „,[A]lso muss man halt für sich entscheiden, ob man jetzt Wert drauf legt dass da immer explizit die weibliche Form noch mitgenannt wird." Indem Tomke die „weibliche Form" hier als eine zusätzliche Nennung darstellt, bestätigt sie nochmals, dass sie sich am generischen Maskulinum orientiert.

Im Folgenden berichtet Luan, dass sie eine geschlechtsneutrale Pluralbildung verwende, wobei deutlich wird, dass sie sich dabei eigentlich auch am generischen Maskulinum orientiert.

\begin{tabular}{|c|c|c|}
\hline $\begin{array}{l}82 \\
83 \\
84 \\
85\end{array}$ & LUAN: & $\begin{array}{l}\llcorner\llcorner\text { Oder ich mach, } \\
\text { nehme einfach ein anderes Wort; so was wie ,der Rat- } \\
\text { suchende'. Der ist zwar dann auch männlich, aber für } \\
\text { mich nicht männlich }\end{array}$ \\
\hline & TOMKE: & $\llcorner$ Ja. \\
\hline & LUAN: & Ja oder ,der Klient` oder \\
\hline 38 & TOMKE: & $\llcorner$ Aber im Prinzip müsstest du dann \\
\hline & & auch (immer), der oder die \\
\hline & LUAN: & ᄂ Der Proband. \\
\hline & TOMKE: & ,der oder die Ratsuchende'; oder , der oder die Klientin'. \\
\hline 32 & LUAN: & \\
\hline & & das stimmt. Das ist mir zu viel Gedöns. \\
\hline & TOMKE: & $\begin{array}{l}\text { Eben. Also ich finde es auch im Lesefluss einfach manch- } \\
\text { mal behindernd, wenn Du immer wieder dieses Schräg- }\end{array}$ \\
\hline & & strich-N und dann noch in den Adjektiven Schrägstriche \\
\hline & & mit irgendwelchen Annexen hinten mit E und EN und \\
\hline & & was weiß ich, also \\
\hline & MIKA: & $\llcorner$ Dann kann man auch die weibliche Form neh- \\
\hline & & men. \\
\hline & LUAN: & Ich denke auch manchmal, also ich meine wir sind ja \\
\hline & & \\
\hline & & ge weibliche Wesen was erreicht haben, und gar nicht \\
\hline & & wir mehr so klar, es gibt diese Benachteiligung, aber \\
\hline & & ich finde so im Unialltag, habe ich da nicht das Gefühl \\
\hline & & ich müsse jetzt unbedingt, beweisen, dass Frauen und \\
\hline & & Männer; und Schülerinnen und Schüler oder so. [...] \\
\hline
\end{tabular}

Luan schließst an die Aussage von Tomke an und erklärt, dass sie ,einfach ein anderes Wort" verwende. Damit verweist sie zunächst auf eine alternative Umgangsweise. Als Beispiel führt sie „der Ratsuchende“ an. Mit 
dem Nachsatz: „Der ist zwar dann auch männlich, aber für mich nicht männlich besetzt", stellt Luan klar, dass dieses substantivierte Adjektiv, obwohl es maskulin ist, für sie ,nicht männlich besetzt" sei. Sie erklärt, dass dies für sie trotzdem einen verallgemeinernden und inkludierenden Charakter habe. Dies lässt erkennen, dass sich Luan wie auch Tomke an der männlichen Norm orientieren. Tomke unterbricht Luan hier und macht deutlich, dass wenn Luan explizit Männer und Frauen benennen wolle, sie ,im Prinzip [...] ,immer" den weiblichen und männlichen Artikel mitverwenden müsse. Luan validiert diese formalen Kriterien, sagt aber, dass sie sich in ihrer Praxis nicht daran orientiere, weil „das ist mir [Luan, S.K.] zu viel Gedöns". Auf einer theoretisch-formalen Ebene stimmt Luan hier Tomke zu, mündet jedoch in einen anderen Orientierungsgehalt, da sie dies nicht in die handlungspraktische Ebene umsetzt. Eine Interpretation ist, dass der Begriff „Gedöns" ein Problem bei der Differenzierung von Männer und Frauen in der Schreibpraxis markiert, das Luan nicht wichtig genug ist, um deshalb auf einer handlungspraktischen Ebene einen Mehraufwand in Kauf zu nehmen. Das Befolgen der Regeln für eine geschlechtergerechte Sprache ist Luan zu viel "Gedöns“. Mit „eben“ stimmt Tomke Luan zu und verstärkt gleichzeitig Luans Feststellung, dass die Verwendung der geschlechtergerechten Sprache zu viel "Gedöns" sei. Als Interpretin hat man den Eindruck, dass sich Tomke nun von Luan verstanden fühlt. Mit „eben“ im Sinne von „wir meinen doch dasselbe" fasst Tomke das Vorausgegangene bestätigend zusammen. Es entsteht der Eindruck, dass sie dieselbe Orientierung teilen. Bei genauerer Betrachtung erkennt man, dass diese Synthese nur rituell ist und nicht wirklich zwei Themen zusammenführt, sondern Tomkes Orientierung bestärkt. Jetzt geht es nicht mehr nur um zu viel „Gedöns“ beim Schreiben von Texten, sondern auch beim Lesen. Tomke elaboriert, dass sie sich durch geschlechtergerechte Sprache mit „den Adjektiven Schrägstriche mit irgendwelchen Annexen hinten" behindert fühle und deshalb ablehne.

Mika schließt das Argument von Tomke (weil geschlechtergerechte Sprache zu viel „Gedöns“ sei, folgt eine Orientierung an der männlichen Norm) auf und merkt an, dass nicht nur die Orientierung an der männlichen Norm möglich sei, sondern es auch die Möglichkeit gebe, sich an der weibliche Form zu orientieren: „Dann kann man auch die weibliche Form nehmen." Damit eröffnet sie eine neue Orientierung: Neben der geschlechtergerechten Sprache und dem generischen Maskulinum gebe es auch noch die Möglichkeit, sich am generischen Femininum zu orientieren und die Männer mit zu meinen. Diese Proposition steht in Opposi- 
tion zur männlichen Norm. Mikas Opposition, die weibliche Form als Norm zu verwenden, wird von den Mitdiskutierenden nicht aufgegriffen, und bleibt undiskutiert im Raum stehen. Die DeThematisierung und die Verdeckung von ,Geschlecht' und Geschlechterverhältnisse scheint Teil ihrer habituellen Praxis zu sein.

Luan hebt ihre Orientierung hervor, dass Frauen ,heute ${ }^{6}$,in der privilegierten Situation“ seien und „dass für uns schon einige weibliche Wesen was erreicht haben". Hier erkennt Luan den Erfolg früherer Frauengenerationen an, jedoch ist zu vermuten, dass sie heute keine Notwendigkeit mehr darin sieht, auf die Sichtbarmachung von Frauen zu pochen, da ,heute' zumindest im Studium, alles besser und egalitärer erscheint. Der Vergleich von ,früher' und ,heute' deutet auf die Vorstellung modernisierter Geschlechterverhältnisse und -gleichstellung hin. Zudem wird in Luans Orientierung deutlich, dass sie davon ausgeht, dass feministische Interventionen und Kritik an (patriarchalen) Herrschaftsverhältnissen nicht mehr notwendig seien und ad acta gelegt werden können (vgl. Kapitel 4.3.3). Im „Unialltag“ habe Luan ,nicht das Gefühl, ich [Luan, S.K.] müsse jetzt unbedingt beweisen, dass Frauen und Männer und Schülerinnen und Schüler, oder so" benachteiligt werden. In dieser Logik müssen Frauen im universitären Alltag durch die Schreibweise nicht hervorgehoben werden, weil hier keine Benachteiligung bestehe. Das heißt, die Unterscheidung von Männer und Frauen sei hier nicht nötig und die männliche Norm als Verallgemeinerung angebracht. In dieser Gruppendiskussion wird eine Argumentationsfigur verwendet, die auch in den anderen Gruppendiskussionen (Metall, Feuer) zum Vorschein kommt. Tomke und Luan betonen, dass sie das generische Maskulinum als ihre selbst gewählte Schreibpraxis favorisieren und sie damit niemanden ausschließen wollen. Dahinter steht zum einen die Annahme, das geschlechtergerechte Sprache verwendet wird, um Frauen nicht zu diskriminieren, die Repräsentation von Vielfalt und das Ziel, für starre Geschlechterrollen zu sensibilisieren, bleibt hierbei ganz und gar unbedacht. Es deutet sich folgende Orientierung an: Wenn Frauen nicht benachteiligt sind, ist die männliche Norm in ihrer Verallgemeinerung legitim. In Bezug auf ,Geschlecht' und Geschlechterfragen findet eine Neutralisierung und Verdeckung von sozialen Ungleichheiten und deren Wirkmechanismen statt. Der von Angela McRobbies angenommene neue Geschlechtervertrag scheint hier als konjunktiver Erfahrungsraum wirksam zu sein. Im weiteren Verlauf der Passage erklärt Loris auf Nachfrage der Interviewerin, dass sie sich in ihrer Schreibpraxis an der geschlechtergerechten 
Sprache orientiere und sowohl die weibliche als auch die männliche Form verwende. Dass diese Praxis keine kollektive Orientierung darstellt, zeigt sich an den Reaktionen der anderen GDT.

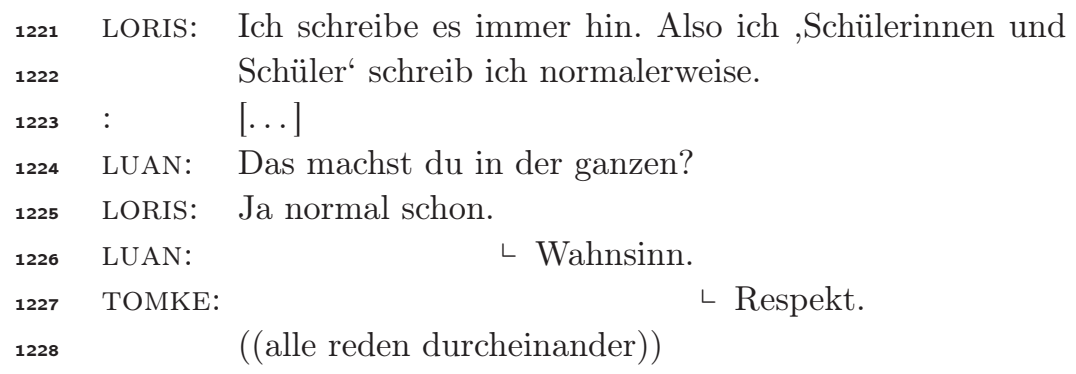

Luan möchte nun wissen, ob Loris „Schülerinnen und Schüler“ in der ganzen Arbeit schreibt. Nachdem Loris diese Frage bejaht, kommentieren Luan und Tomke dies mit „Wahnsinn“ und „Respekt". Dies hört sich zunächst wie eine Anerkennung an, ist aber eher als eine ironische bzw. überzogene Anmerkungen zu interpretieren. Loris macht etwas, das zuvor als ,zu viel Gedöns“ und zu viel Aufwand diskutiert wurde. Luan und Tomke grenzen sich davon ab, indem sie es als etwas Besonderes, Außergewöhnliches hervorheben. Es wird erneut erkennbar, dass die Orientierung an Sprache keine kollektive Orientierung der Gruppe darstellt, sondern eine individuelle Orientierung einzelner Studierender. Auch im folgenden Auszug, in dem Loris eine neue Form der geschlechtergerechten Sprache einführt, wird deutlich, dass ein reflektierender Umgang mit Geschlechterfragen nicht Teil des erziehungswissenschaftlichen-studentischen Habitus der Gruppe $\mathrm{Holz}$ ist.

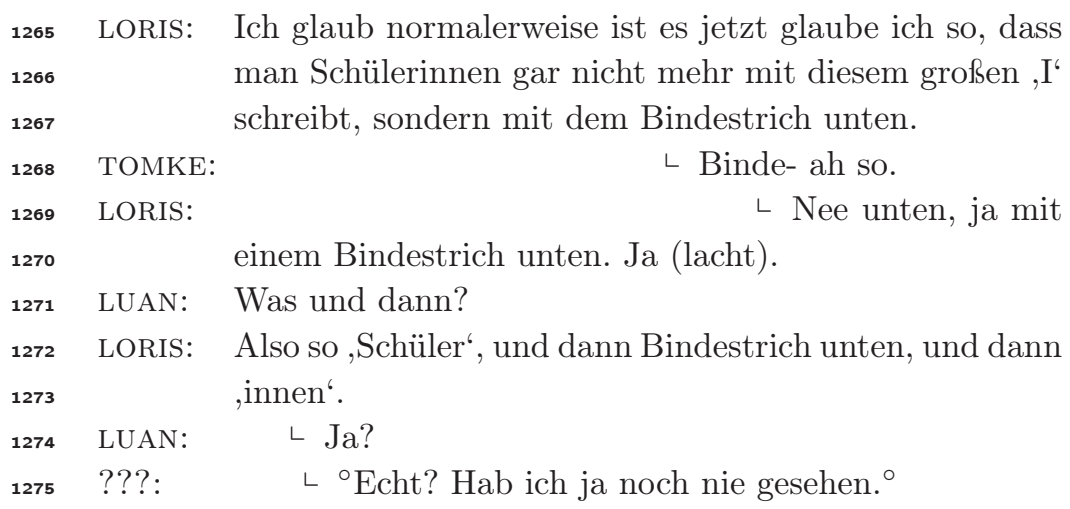




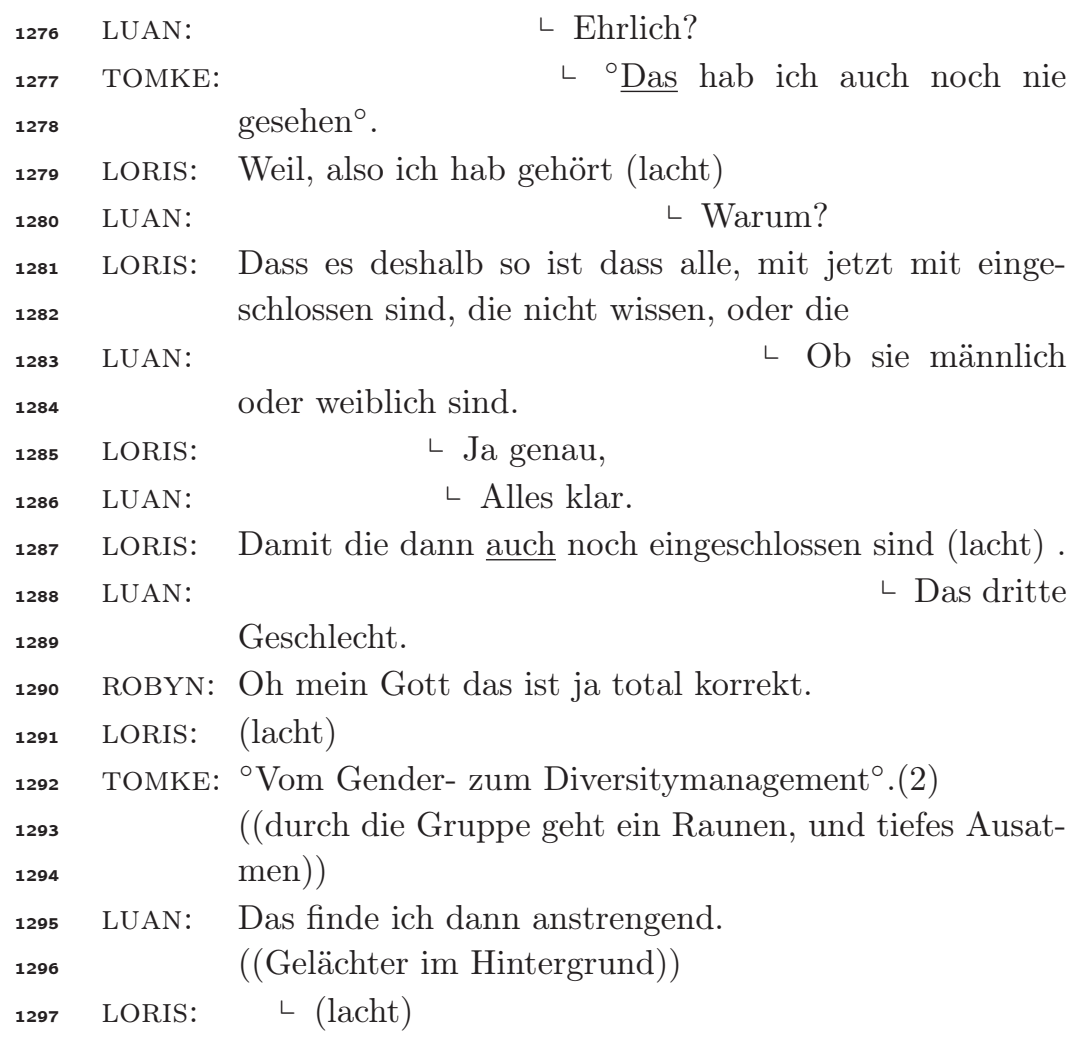

Loris nennt hier in Bezug auf die geschlechtergerechte Sprache eine neue Norm: „Ich glaub, normalerweise ist es jetzt, glaube ich, so, dass man Schülerinnen gar nicht mehr mit diesem großen I schreibt, sondern mit dem Bindestrich unten." Loris verweist hier auf den gender gap, der keine Handlungspraxis von Loris beschreibt, sondern hier ein theoretisches Wissen über eine ,neue ${ }^{6}$ Norm darstellt. Es folgt eine Reihe von ungläubigen Nachfragen und Kommentierungen von verschieden GDT: „Ja?“; „Echt? Hab ich ja noch nie gesehen“; „Ehrlich?“. Es wird deutlich, dass Loris als Einzige diese Schreibweise kennt. Luan fragt nach, ,warum" das so gemacht werde. Loris erläutert, dass damit ,alle [...] eingeschlossen sind, die nicht wissen, oder die". Hier ringt Loris um Worte und Luan spricht für sie weiter: „Ob sie männlich oder weiblich sind.“ Es wirkt wie aus einer Stimme gesprochen, denn Loris ratifiziert Luans Fertigstellung und Luan gibt ihrerseits an, dass sie verstanden habe. Luan elaboriert noch weiter: „Damit die dann auch noch eingeschlossen sind 
(lacht)." Demnach gibt es nicht nur Männer und Frauen, die ein- oder ausgeschlossen werden, sondern, auch „das dritte Geschlecht". Die vage und zaghaft wirkende Aussage ,also ich habe gehört" verleiht der Aussage den Charakter eines Gerüchts und wirkt wie Halbwissen, zudem erscheint ihr Erklärungsversuch aufgrund ihres Lachens etwas unsicher bzw. nimmt diesem die Ernsthaftigkeit. Loris bringt durch ihren Beitrag einen neuen Aspekt in die Diskussion ein, nämlich, dass es mehr als zwei Geschlechter und Geschlechtsidentitäten gebe, und auch diese durch die Sprache ein- bzw. ausgeschlossen werden können. Dies stellt für die GDT jedoch kein geteiltes Wissen und somit auch keine kollektive Orientierung der Gruppe dar, wie sich an den Reaktionen von Robyn, Tomke und Luan erkennen lässt.

In Robyns Beitrag („Oh mein Gott, das ist ja total korrekt") steckt eine implizite Ablehnung. „Das“ kann sich zum einen auf die Schreibweise „Bindestrich-Unten“ beziehen, aber zum anderen auch auf die Auseinandersetzung mit einem dritten Geschlecht. Die Ablehnung dieser Orientierung wird auch durch ein „Raunen“, das durch die Gruppe geht, hörbar. Abschließend konkludiert Luan: „Das finde ich dann anstrengend.“ Dies ist auch ein Hinweis dafür, dass die kritische Auseinandersetzung mit der Zweigeschlechtlichkeit und der Möglichkeit weiterer Geschlechter ,zu anstrengend' sei und somit einen negativen Horizont darstellt. Es lässt sich rekonstruieren, dass die Gruppe die Orientierung am Bindestrich-Unten ablehnt.

Interessant ist an dieser Stelle, dass „das dritte Geschlecht“" bzw. die Information, dass es Personen gibt, die nicht wissen „ob sie männlich oder weiblich sind"6, nicht diskutiert wird. Eine (kritische) Auseinandersetzung mit der geschlechtlichen Norm wird nicht aufgegriffen. Das Thema „dritte[s] Geschlecht" und queere Identitäten knüpft in der Gruppe Holz an keinen konjunktiven Erfahrungsraum an. Es wird eine Verbindung zu konjunktiven Erfahrungen der Gruppe in Hinblick auf die eigene Geschlechtlichkeit und Geschlechtsidentität deutlich. Zudem wird in der Passage bestätigt, dass die Studierenden in Bezug auf ihre Sprachpraxis und hinsichtlich der Relevanz von geschlechtergerechter Sprache keine kollektive Orientierung teilen. Dass diese unkritische und unreflektierte Praxis Teil der (De-)Thematisierung von ,Geschlecht' und Geschlechterfragen ist und in Kontrast zu anderen Themenbereichen und Differenzkategorien steht, zeigt sich an folgender Passage zur ,Relevanz und Reflexion von Begriffen und Bezeichnungen im Studium". 


\section{Die Relevanz von Begriffsdiskussionen im Studium}

Die direkt daran anschließende Passage beginnt mit einer Proposition von Robyn und mündet in eine Debatte zur Frage nach der Relevanz einer Reflexion und Diskussion über Begriffe und Bezeichnungen im Rahmen des Studiums. Im Gegensatz zu oben zeigt sich dabei ein studiumsbezogener konjunktiver Erfahrungsraum der Studierenden.

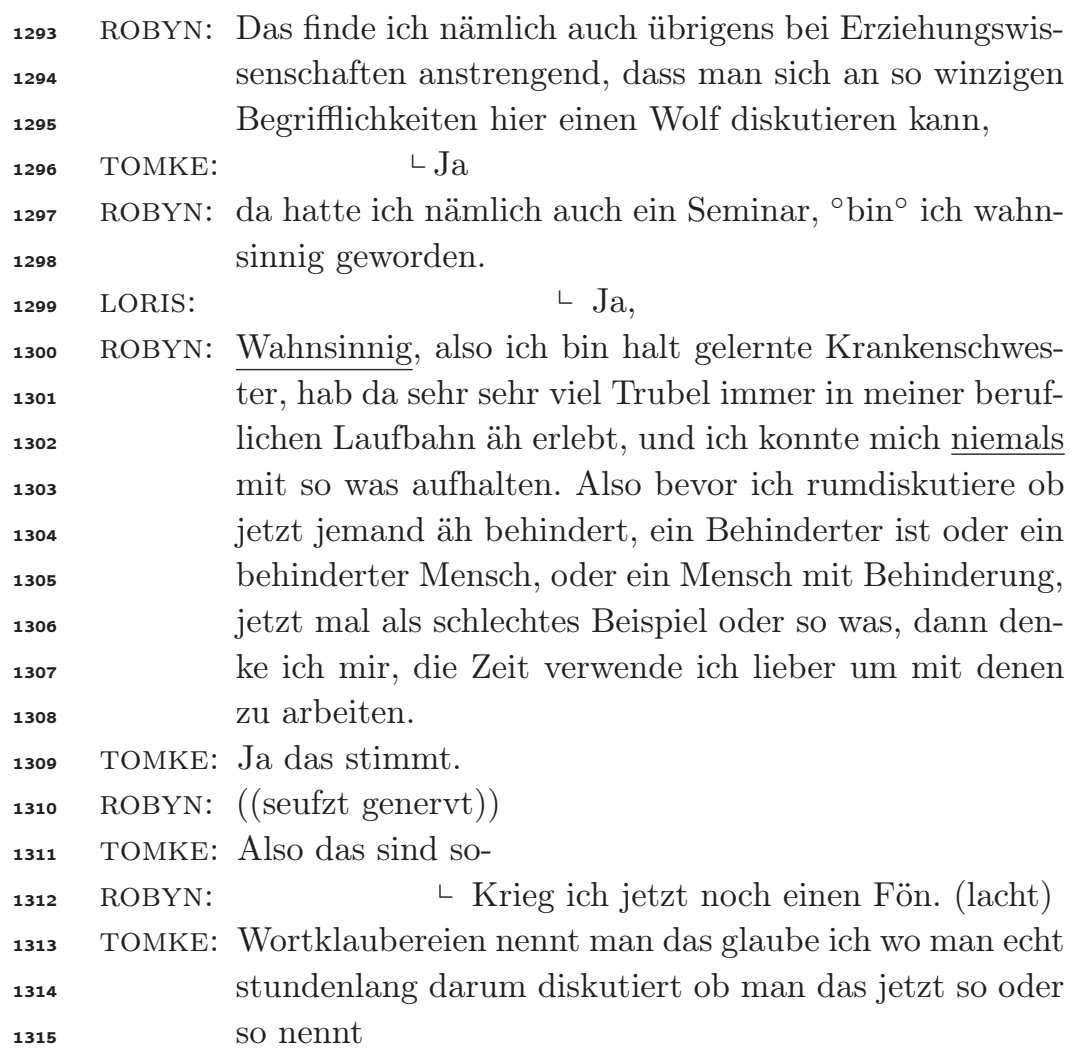

Mit ihrer Proposition greift Robyn die Facette der Anstrengungen heraus und bezieht sich implizit auf die vorherige Passage zur geschlechtergerechten Schreibpraxis. Sie findet es „bei Erziehungswissenschaften anstrengend, dass man sich an so winzigen Begrifflichkeiten hier einen Wolf diskutieren kann." Damit kommt es zu einer dreifachen Ablehnung. Erstens kommt es hiermit zu einer negativen Bewertung der vorherigen Diskussion, zweitens zur Abwertung eines Teils des Studiums und drit- 
tens wird der geschlechtergerechten Sprache und ihren Schreibweisen die Relevanz entzogen. Indem Robyn die Erziehungs- und Bildungswissenschaften hier so hervorhebt, macht sie deutlich, dass es auch noch andere Räume und Orte gebe, wo dies nicht so wichtig sei. Tomke bestätigt die aufgeworfene Orientierung („Ja“). Robyn exemplifiziert ihre Erfahrung und macht deutlich, dass sie die Notwendigkeit dieser Diskussion nicht nachempfinden könnte. Diese Diskussionen bilden für sie einen negativen Horizont. Robyns Beschreibung wird durch Loris mit einem "Ja“ validiert. Aufgrund ihrer Tätigkeit als Krankenschwester, habe sich Robyn ,niemals mit so was aufhalten“ können und habe bislang nie die Zeit gehabt, über Begrifflichkeiten zu diskutieren; so etwas habe sie nur aufgehalten. Es hätte wichtigere Dinge zu tun gegeben als darüber zu diskutieren, wie man eine bestimmte Gruppe von Personen bezeichnen solle. Um ihre Orientierung besser verständlich zu machen, erklärt sie diese an der Bezeichnung „Mensch mit Behinderung“. Sie elaboriert: „Also bevor ich rumdiskutiere", wie diese Gruppe von Personen zu adressieren sei, „,denke ich mir, die Zeit verwende ich lieber um mit denen zu arbeiten“. Hier wird deutlich, dass die Arbeit mit den Menschen für Robyn den positiven Horizont und die Diskussion darüber, wie diese zu bezeichnen und zu adressieren seien, den negativen Horizont darstellen. Validierend schließst Tomke an diese Orientierung an und wertet diese Diskussion als „Wortklaubereien"13 ab.

Der folgende Teil der Diskussion kann, auch wenn der Beitrag von Mika zunächst wie eine Opposition wirkt, im Sinne der Diskursstruktur als antithetisch charakterisiert werden, denn er mündet in eine Synthese der Orientierungen.

$\begin{array}{lll}{ }_{\mathbf{1 3 1 4}} & \text { MIKA: } & \llcorner\text { Das ist natürlich dann der pädagogische Akt die } \\ { }_{\mathbf{1 3 1 5}} & \text { Studenten erstmal dahin zu bekommen; die Menschen } \\ { }_{\mathbf{1 3 1 6}} & \text { so zu nehmen wie sie sind. Und dann muss man erstmal } \\ { }_{\mathbf{1 3 1 7}} & \text { diskutieren welche Arten es überhaupt gibt; vielleicht } \\ { }_{\mathbf{1 3 1 8}} & \text { ist das der Sinn dahinter, was dann manche nicht ver- } \\ { }_{\mathbf{1 3 1 9}} & \text { stehen die da schon weiter @sind@. } \\ { }_{\mathbf{1 3 2 0}} & \text { ROBYN: } \text { Ich denke ja dann immer man ist vielleicht falsch im } \\ { }_{\mathbf{1 3 2 1}} & \text { Pädagogikstudium wenn man die Menschen nicht so } \\ { }_{\mathbf{1 3 2 2}} & \text { nehmen kann wie sie sind. }\end{array}$

13 „Wortklauberei“ ist eine abwertende alltagssprachliche Beschreibung für eine pedantische oder enge Auslegung von Worten oder von etwas Gesagtem bzw. Geschriebenem. 
1323 MIKA: Ja, aber es sind offenbar auch welche dabei hier, das

1324

1325

1326

1327

1328

1329

1330

1331

1332

1333

1334

1335

1336

1337

1338

1339

1340

1341

1342

1343

1344

1345

1346

1347

1348

1349

1350

1351

1352

1353

1354

1355

1356

1357

1358 glaube ich schon.

ROBYN: $\quad\llcorner$ Ja, nee aber ich muss sagen das Seminar hat mir trotzdem geholfen, weil es ja tatsächlich Sachen gibt die disku- also die muss man halt diskutieren, weil halt einfach wirklich was dahintersteckt.

LORIS: Ich glaube es geht halt bei diesen Bezeichnungen generell um die Bezeichnung an sich, dass überhaupt Menschen, Bezeichnungen bekommen überhaupt das ich glaube das ist einfach dann auch diese Kritik daran und deswegen finde ich es schon wichtig dass man über diese Begriffe spricht. Weil der behinderte Mensch, ist ein Mensch, und nicht, die Behinderung sollte nicht im Vordergrund stehen, deswegen finde ich diese Diskussion um die Begriffe schon sehr wichtig.

TOMKE: Ja das stimmt. Auch so das Bewusste, Bewusstsein schaffen.

LORIS: $\quad$ Es geht einfach diese, warum sagt man es überhaupt? Das finde ich (weil), warum, es ist doch einfach ein Mensch.

TOMKE: $\quad L$ Ja ich glaube da ist man sich manchmal echt nicht bewusst, was mit Begriffen, teilweise mitschwingt.

LORIS:

$\llcorner$ Ja ich glaub, das: finde ich total. ja

TOMKE: Und das Bewusstmachen, also diese Diskussionen sind glaube ich

MIKA: ᄂ Wenn mich einer ,Mädel` nennt dann werde ich auch aggressiv.

(Tomke und Mika reden gleichzeitig)

TOMKE: $\llcorner$ Die Diskussion ist glaube ich einfach auch dazu da um das Bewusstsein zu schaffen

LORIS:

$\left\llcorner{ }^{\circ} \mathrm{Ja}^{\circ}\right.$.

TOMKE: wieso diese Begriffe bestehen und was eigentlich ja in dem Begriff mitschwingt ohne dass wir uns dessen bewusst sind. (2) Von daher sind die sind die nicht überflüssig, aber trotzdem manchmal sehr strapaziös @für 
Mika versteht diese Begriffsdiskussionen als „pädagogischen Akt", der versucht, „die Studenten erstmal dahin zu bekommen, die Menschen so zu nehmen wie sie sind“. Menschen zu nehmen wie sie sind, ist laut dieser Aussage keine ,Naturgegebenheit', sondern ein Zustand, den „Studenten" erlernen können, indem sie über Bezeichnungen von Menschen diskutieren. Für Mika sind diese Diskussionen nicht unnötig, sondern ein pädagogischer Akt und Teil der Ausbildung. Antithetisch meint Robyn, dass dies bereits Teil des Habitus von Studierenden sein sollte und wer diese Voraussetzung nicht erfülle, sei ,vielleicht falsch im Pädagogikstudium" und jene, die „Menschen so nehmen können wie sie sind“ seien richtig. Mika stimmt Robyn zunächst zu, glaubt aber, dass die Realität anders aussieht und es nicht nur diese, richtigen' Student innen hier gebe, ,ansonsten gäbe es diese Diskussionen nicht“. Im Sinne einer Synthese elaboriert Robyn nun, dass ihr das oben angesprochene Seminar „trotzdem geholfen" habe, weil „es tatsächlich Sachen gibt, die muss man halt diskutieren". Hier unterscheidet Robyn zwischen Themen, die man „tatsächlich diskutieren muss", also wichtigen Themen, und unwichtigen Themen (dieser Teil bleibt unausgesprochen). Diese wichtigen Themen unterscheiden sich von den unwichtigen, ,weil halt einfach wirklich was dahintersteckt" und somit nicht (nur) theoretisch, sondern auch praktisch von Bedeutung seien. Loris greift die Fragen nach der Relevanz von Themen auf und arbeitet diesbezüglich die Frage nach der Diskussion zu „Bezeichnungen generell“ genauer aus. Für Loris sind die Diskussionen über die Bezeichnungen ,schon wichtig“" und „dass man über diese Begriffe spricht“. Dabei ginge es um die „Kritik daran“, dass Menschen überhaupt Bezeichnungen bekommen. Als Beispiel greift sie das zuvor bereits angeführte Beispiel um den „behinderten Mensch“ auf und rahmt dieses nun positiv. Für Loris ist ein behinderter Mensch „ein Mensch“. Das sollte auch in der Sprache ,im Vordergrund stehen“ und nicht „die Behinderung“. Um das zum Ausdruck zu bringen, findet Loris die „Diskussion um die Begriffe schon sehr wichtig“. Wie auch Mika zuvor wertet hier auch Loris die „Diskussion um die Begriffe“ auf. Validierend schließt Tomke an und erweitert die Sinnhaftigkeit dieser Diskussionen, da diese dazu da seien, um „Bewusstsein [zu] schaffen“. Tomke elaboriert weiter, dass ,man sich manchmal echt nicht bewusst ist, was mit Begriffen, teilweise mitschwingt". Damit macht sie nochmals deutlich, wie wichtig es sei, über Begriffe zu diskutieren, weil im Sprachgebrauch die Komple- 
xität und die Mehrdeutigkeit von Begriffen oftmals verloren gehe bzw. diese nicht bedacht werde. Loris bestätigt diese Orientierung: „Ja, finde ich total, ja." In Bezug auf ein pädagogisches Subjekt scheinen diese Begriffsdiskussionen einen positiven Horizont darzustellen.

Mika nennt nun ein Beispiel aus ihrer eigenen Erfahrung, wo Personen nicht bewusst sei, was in Begriffen ,mitschwingt": „Wenn mich einer Mädel nennt, dann werde ich auch aggressiv." Für Mika schwingt bei der Bezeichnung „Mädel“ etwas mit, das anderen nicht bewusst sei und sie ,aggressiv" mache. Mika versucht hier ohne Erfolg, den Geltungsbereich der Orientierung auszuweiten, da das von ihr eingebrachte Thema der Fremd-Bezeichnung ,Mädel“14 auch hier von den übrigen Teilnehmer/inne/n nicht aufgegriffen wird. Ein möglicher Grund kann darin liegen, dass diese keine ähnlichen Erfahrungen gemacht haben. Einen anderen und wahrscheinlicheren Grund sehe ich darin, dass die übrigen GDT keinen bewussten Umgang mit ,Geschlecht' haben. Unbeeindruckt von Mikas ,Zwischenruf' elaboriert Tomke ihre Orientierung weiter („die Diskussion, ist glaube ich, einfach auch dazu da, um das Bewusstsein zu schaffen"), erneut von Loris validiert wird. Im Sinne einer Konklusion fasst Tomke abschließend auf einer abstrakten und wenig konkreten Ebene zusammen, dass diese Diskussionen um Begriffe wichtig seien, aber trotzdem „sehr strapaziös @für die Nerven@“ sein können.

Im Gegensatz zur Passage zur geschlechtergerechten Sprache kommt es hier zu einer Synthese und zu einer geteilten Orientierung, dass Diskussionen hinsichtlich verwendeter Begriffe im studiumsbezogenen konjunktiven Erfahrungsraum wichtig sind, um Bewusstsein darüber zu schaffen, was mit den Begriffen ,mitschwingt". Jedoch kann dies nicht auf alle Differenzkategorien im gleichen Maß übertragen werden. Ein Vergleich der Passagen zur "geschlechtergerechten Sprache" und „den Begriffsdiskussionen" zeigt, dass es in beiden um eine sprachliche Sensibilität geht und darum, wie durch Sprache und Begriffe Bewusstsein geschaffen werden kann. Im Gegensatz zum Abschnitt zu den „Diskussionen in Bezug auf Begriffe" zeigt sich im Abschnitt zur ,geschlechtergerechten Sprache" kein gemeinsames Interesse an der Thematisierung und Reflexion von ,Geschlecht". Zudem wird im Vergleich deutlich, dass es keine geteilten normativen Vorstellungen hinsichtlich geschlechtergerechter Sprache gibt und keinen studiumsbezo-

14 „Mädel“ ist eine umgangssprachliche Verkleinerungsform bzw. eine Verniedlichung von Frau oder Mädchen. Damit kann eine Abwertung von Frausein und Weiblichkeit verbunden sein. 
genen konjunktiven Erfahrungsraum. Im Gegensatz zu „,Behinderung" scheint die Differenzkategorie ,Geschlecht" kein oder zumindest ein wenig besprochenes Gesprächsthema darzustellen. Die Sensibilisierung für ,Geschlecht' scheint, anders als für Behinderung, nicht thematisierbar zu sein. Während Mika mit ihren Versuchen, auf Geschlechterfragen aufmerksam zu machen, kein Gehör findet, sind sich die Studierenden darüber einig, dass Sensibilisierungen und Bewusstwerdungsprozesse durch Begriffsdiskussionen sehr wichtig seien. Auf einer abstrakten Ebene scheinen Begriffsdiskussionen für die Studierenden eine Norm und Orientierung darzustellen, in ihrer handlungsleitenden Praxis spiegelt sich dies jedoch nicht wider, wie an den Passagen zur ,geschlechtergerechten Sprache“ und der „einseitigen Art der Wissenschaft" deutlich wurde. Der studiumsbezogene konjunktive Erfahrungsraum scheint sich hier mit dem neuen Geschlechtervertrag als konjunktiver Erfahrungsraum zu überlappen, da hier der Eindruck vermittelt wird, dass (junge) Frauen den Kampf um Gleichberechtigung mittlerweile gewonnen hätten.

\subsubsection{Ein Student in „der Pädagogik“: „Ist der schwul?“}

Die sexuelle Orientierung von Studenten der Erziehungs- und Bildungswissenschaften wird, mit Ausnahme der Gruppe Metall, auch in den anderen Gruppen thematisiert. ${ }^{15}$ In dieser Gruppendiskussion wird das Thema bezugnehmend auf die visuelle Diskussionsanregung - einem Bild, in dem zwei Personen zu sehen sind und eine die andere fragt: „Are you a girl?“, worauf diese antwortet: „Who cares?“( (siehe Kapitel 1) - aufgenommen:

1378 LUAN: Also [...], ich meine wir haben ja gerade gesagt, dass

1379 es so viele, weibliche Studentinnen gibt in den Erzie-

1380

1381

1382 hungswissenschaften, und von daher weiß ich nicht ob das dann so das Thema ist.

1383

TOMKE: Also mir ist aufgefallen dass nicht von mir, aber ich wurde von vielen gefragt, wenn irgendwo zwischen 20

1384 Mädchen ein Junge saß, ,ist der schwul'?

1385 MIKA: $\quad \ddot{\mathrm{Ah}} \mathrm{hm} \mathrm{hm} \mathrm{hm}$

15 Diese Diskussion wird aber nicht immer von der gleichen Frage ausgelöst, was darauf hinweist, dass sie unabhängig vom Diskussionsstimulus den kollektiven Wissensbestand widerspiegelt bzw. einen kollektiven Orientierungsrahmen der Studierenden darstellt (vgl. Przyborski/Wohlrab-Sahr 2009). 


\begin{tabular}{lll}
$\mathbf{1 3 8 6}$ & LUAN: & \multicolumn{1}{c}{$\llcorner$ Nein. } \\
$\mathbf{1 3 8}^{\mathbf{3 8 7}}$ & TOMKE: & Ja. Das ist mindestens schon drei oder vier Mal passiert; \\
$\mathbf{1 3 8 8}$ & & wo ich sagte, pff haha \\
$\mathbf{1 3 8 9}$ & MIKA: & Ja was denkst Du denn, natürlich ist der schwul! \\
$\mathbf{1 3 9 0}$ & TOMKE: & Also bei einem hat man es wirklich gesehen, aber das \\
$\mathbf{1 3 9 1}$ & & ist ja auch vollkommen egal. Ich meine selbst wenn er \\
$\mathbf{1 3 9 2}$ & & es ist, @ich wusste nicht was mit dieser@ Frage jetzt \\
$\mathbf{1 3 9 3}$ & & weiter verfolgt werden sollte. \\
$\mathbf{1 3 9 4}$ & ROBYN: & $\llcorner$ Ah das ist halt menschlich
\end{tabular}

Auf die Einladung der Forscherin, von Situationen zu erzählen, in denen sich die Studierenden auch schon einmal gedacht haben: „Wen interessiert's, wen kümmert's", opponiert Luan zunächst gegen die Orientierung der Forscherin, dass ,Geschlecht' im Studium der Erziehungs- und Bildungswissenschaften ,so das Thema ist". Als Begründungsfigur nennt sie den hohen Frauenanteil bei den Studierenden, der zuvor von der gesamten Gruppe („wir") bestätigt wurde. Dahinter steckt die Orientierung, dass wenn Frauen in einem Bereich nicht die Minderheit darstellen, es keine Notwendigkeit gebe ,Geschlecht" und Geschlechterfragen zu thematisieren. Hier wird die Verknüpfung deutlich, dass Luan für die Notwendigkeit der Thematisierung von ,Geschlecht' die Unterrepräsentanz/Benachteiligung von Frauen voraussetzt. Somit wird deutlich, dass Luan ,Geschlecht' mit Frausein und Geschlechterfragen mit Frauenfragen verbindet. Die Legitimation für eine Thematisierung von ,Geschlecht' scheint in der Benachteiligung und Diskriminierung von Frauen zu liegen. Hier wiederholt sich eine Argumentationsfigur, die bereits bei der Relevanz von geschlechtergerechter Sprache zum Tragen kam. Wenn Frauen nicht (mehr) benachteiligt sind, scheint es keinen Grund für die Verwendung einer geschlechtergerechten Sprache zu geben. Da Frauen im erziehungswissenschaftlichen Studium nicht unterrepräsentiert sind, scheint es für die Studierenden auch keine Benachteiligung an der Universität zu geben. Auch wenn punktuell immer wieder betont wird, dass außerhalb des Studiums und in anderen Studiengängen Benachteiligungen existieren, scheinen die Studierenden der Erziehungs- und Bildungswissenschaften nicht davon betroffen zu sein. Der Frauenüberschuss dürfte die Notwendigkeit und den Bedarf der Student_innen, über ,Geschlecht' zu sprechen, aushebeln. Hier wird auch deutlich, dass aufgrund rhetorischer Modernisierungsprozesse und hinter den modernen Diskursen strukturelle Probleme verdeckt werden. 
Auch Tomke greift in ihrem antithetischen Beitrag auf den weiblich dominierten konjunktiven Erfahrungsraum des Studiums zurück, ihre Proposition geht jedoch in eine konträre Richtung. Tomke hat die Erfahrung gemacht, dass ,Geschlecht' ein Thema im Studium sei. Gerade weil das Studium eine weibliche Domäne darstellt, wird über die homosexuelle Orientierung der Studenten spekuliert: Wenn ,irgendwo zwischen 20 Mädchen ein Junge saß" wird ,von vielen gefragt": „[I]st der schwul?" Mika und Luan machen ungläubige und verwunderte Bemerkungen, woraufhin Tomke betont, dass ihr das schon mehrmals passiert sei. Mika antwortet in ihrem Beitrag auf die Frage „[I]st der schwul?“ mit: „Ja was denkst du denn, natürlich ist der schwul!“ Ob es sich hierbei um eine ironische Kommentierung oder um eine tatsächliche Bestätigung und Generalisierung der Frage handelt, kann hier nicht mit Sicherheit gesagt werden. Tomke validiert Mikas Aussage zwar, schränkt jedoch die Generalisierung dieser Annahme ein, indem sie von „einem“ Studenten spricht, bei dem es ,augenscheinlich' gewesen sei, dass er schwul ist. Woran man die sexuelle Orientierung einer Person erkennt, wird jedoch nicht erläutert. Dies deutet auf einen kollektiven Wissensbestand hin. Tomke betont jedoch, dass es ihr nicht darum gehe, ob die Studenten wirklich homosexuell seien (,das ist ja auch vollkommen egal“), sondern sie nicht wisse „was mit dieser Frage jetzt weiter verfolgt werden sollte“. Studenten der Erziehungs- und Bildungswissenschaften werden zum einen als von der heterosexuellen Geschlechternorm abweichend konstruiert und zum anderen wird die Feminisierung, die ,Verweiblichung' des Studiums zementiert, da nichtheterosexuelle Männer oftmals mit ,nicht-männlich' ${ }^{6}$ und dem geschlechterbinären Denken folgend mit ,Verweiblichung' konnotiert sind (Hirschauer 1996).

Robyn schliefst mit einer rituellen Konklusion im Modus einer Metarahmung an. Sie konstatiert, dass die Frage nach der sexuellen Orientierung „halt menschlich“ sei. Mit der Metarahmung (,das ist halt menschlich") nimmt sie eine starke Normierung vor und sagt, dass dieses Interesse an der sexuellen Orientierung anderer ein Teil des Menschen sei, der unveränderbar und vielleicht sogar etwas Natürliches sei. Mit diesem Satz schwingt aber auch so etwas wie Resignation mit: Es sei unveränderbar und somit könne und müsse man dagegen auch nichts tun. Damit hat diese Aussage auch einen entlastenden Charakter. Implizit gibt sie zu verstehen, dass dieses Interesse an der sexuellen Orientierung einer Person legitimer Teil des menschlichen Verhaltens sei und zugleich kommt es damit zu einer Generalisierung und Normalisierung dieses In- 
teresses bzw. Verhaltens. Diese Äußerung bezeichne ich als ,rhetorisches Schachmatt". Es zeigt sich in der Gruppendiskussion immer wieder und scheint charakteristisch für Robyn. Damit werden (scheinbar) eindeutige und unumstößliche Feststellungen und Wahrheiten formuliert, die signalisieren, dass es nichts mehr zu diskutieren gebe. Mit einem ,rhetorischen Schachmatt' ist die Diskussion meist rituell beendet. Rhetorisch ist dieses Schachmatt deshalb, weil damit keine Synthese von unterschiedlichen Thesen verbunden ist, sondern das Thema nur ,oberflächlich', aber nicht inhaltlich zum Ende kommt. Dass es sich hierbei um keine kollektive Orientierung handelt, bringen Mika und Tomke zum Ausdruck:

\begin{tabular}{|c|c|c|}
\hline & MIKA: & $\llcorner$ Aber das ist ein gesell- \\
\hline & & \\
\hline & TOMKE: & $\begin{array}{l}\text { Aber es ist eine Zuschreibung. Also sobald jemand sagt } \\
\text {.Ich will Friseur werden', sagst du. Äh:: ja'. Obwohl das }\end{array}$ \\
\hline & & $\begin{array}{l}\text {, Ich will Friseur werden, sagst du, An:: ja. Ubwonl das } \\
\text { früher ein ganz normaler Männerberuf war, und so ist es }\end{array}$ \\
\hline & & oder sehe ich die Tendenz zumindest mittlerweile auch \\
\hline & & in sozialen Berufen, und ganz stark auch in der Pädago- \\
\hline & & gik. [...] Also \\
\hline & & oder ich merke dass Zuschreibungen übe \\
\hline & & gang stattfinden. $\mathrm{V}$ \\
\hline & & \\
\hline & : & {$[\ldots]$} \\
\hline & LUAN: & $\begin{array}{l}\text { Also ich finde es lächerlich, wenn ich einen Mann in } \\
\text { der Erziehungswissenschaften sehe, zu sagen gleich zu } \\
\text { fragen ,ist der schwul', das würde mir nie in den Sinn } \\
\text { kommen. }\end{array}$ \\
\hline & MIKA: & $\begin{array}{l}\llcorner\text { Vielleicht sollte man die Herrn Professor mal } \\
\text { fragen ,Sind Sie schwul oder warum unterrichten Sie } \\
\text { hier?' Ja also es ist irgendwie komisch. Was kann man } \\
\text { dagegen machen? }\end{array}$ \\
\hline & LUAN: & $\llcorner$ Seltsam. Nix. \\
\hline
\end{tabular}

Mika widerspricht Robyn und konstatiert, dass das Interesse an der sexuellen Orientierung nicht menschlich im Sinne von ,natürlich gewachsen', sondern „ein gesellschaftliches Ding“ sei. Das Interesse an der sexuellen Orientierung sei somit nichts ,Natürliches", sondern gesellschaftlich (historisch) gewachsen und konstruiert. Auch Tomke schließst sich dem Widerspruch an: Für sie handelt es sich dabei um eine "Zuschreibung“. 
An ihrer Exemplifizierung wird deutlich, dass sie damit nicht das Interesse an der sexuellen Orientierung meint, sondern die ,Zuschreibung einer homosexuellen Orientierung aufgrund spezifischer Rahmenbedingungen wie z. B. der Berufs- oder Studienwahl. Als Vergleichsfolie nennt sie den Beruf „Friseur“. Hier problematisiert sie den kausal erscheinenden Zusammenhang zwischen Berufswahl in einem aktuell weiblich dominierten bzw. konnotierten Bereich und die Abweichung von der hegemonialen Männlichkeit. Tomke kritisiert die Zuschreibung, dass Männer, die in einem Beruf tätig sind oder ein Studium wählen, in dem jeweils überwiegend Frauen zu finden sind, homosexuell seien. In dieser Zuschreibungspraxis schwingt zugleich eine Abwertung der Männer wie auch der von Frauen dominierten Bereiche mit.

Auch Luan lehnt diese Zuschreibung ab: „Also ich finde es lächerlich, wenn ich einen Mann in der Erziehungswissenschaften sehe, zu sagen, gleich zu fragen ,ist der schwul', das würde mir nie in den Sinn kommen." Mika führt die Frage quasi ad absurdum, indem sie diese rhetorisch an die „Herrn Professor[en]" richtet (,sind Sie schwul oder warum unterrichten Sie hier?"). Damit karikiert sie die zuvor beschriebene Kausalität zwischen der Berufs- bzw. Studienwahl von Männern und ihrer sexuellen Orientierung. Sie schließst mit der Konklusion, dass es ,irgendwie komisch“ sei und stellt die Frage: „Was kann man dagegen machen?" Damit signalisiert sie, dass sie diesbezüglich Handlungs- und Veränderungsbedarf sieht. Luan bestätigt zwar nochmals, dass sie diese Zuschreibungspraxis ,seltsam“ findet, auf Mikas Frage weiß sie jedoch auch keinen Rat. Ihre einsilbige Antwort („nix") wirkt hier etwas resigniert. Mit Mikas Frage wird ein Themenwechsel eingeleitet. Auch wenn in dieser Passage, die Frage nach der homosexuellen Orientierung von Studenten von Teilen der Gruppe abgelehnt wird, werden männliche Studierende als von der heterosexuellen Geschlechternorm abweichend konstruiert und zudem wird die Feminisierung der Erziehungs- und Bildungswissenschaften reproduziert. Es wird damit das Stereotyp verfestigt, dass Männer dieses Studium nicht typischerweise wählen, und wenn sie es tun, sie von der heterosexuellen Geschlechternorm abweichen.

\subsection{6 (De-)Thematisierung von Benachteiligungserfahrungen}

Auch wenn die Studierenden in Bezug auf die geschlechtergerechte Sprache betont haben, dass sie sich nicht benachteiligt fühlen, bedeutet das 
nicht, dass sie noch keine Benachteiligungs- oder Diskriminierungserfahrungen gemacht haben, sondern dass diese nicht thematisierbar sind und somit verdeckt werden, wie folgende Passage zeigt:

2176 MIKA: Die Frauen, die tatsächlich ihre Kinder mit in die Vor-

$2177 \quad$ lesung bringen, die kriegen nicht wirklich viel mit.

2178 TOMKE: Och das kommt auf das Kind an

2179 MIKA: Ja genau @und das Alter von dem Kind@. Also

2180 TOMKE: $\llcorner$ (Ich glaub schon)

2181 LUAN: Aber die Studentinnen haben heute immerhin die Mög-

${ }_{2182}$ lichkeit die haben hier ja so eine Art Krippe.

2183 TOMKE: Ja.

${ }_{2184}$ LUAN: Ich hatte das nicht. Ich hab meine, mein erstes Kind da-

2185 mals mitgenommen zu einem Philosophieprofessor, um

2186 da eine Prüfung abzulegen, die hab ich dann auch ab-

$2187 \quad$ gelegt, und danach hat er mich dann angesprochen und

$2188 \quad$ gemeint, ob ich denn nicht lieber zuhause bleiben wolle.

2189 LORIS: (lacht entsetzt)

2190 LUAN: Und da war ich dann noch so jung, dass ich zwar noch

$2191 \quad$ ein bisschen weiter studiert hab aber nicht mehr viel.

${ }_{2192}$ Und dann hab ich es halt abgebrochen.

2193 LUCA: Hm.

2194 LUAN: Und alle meine Kinder bekommen, und jetzt

${ }_{2196}^{2195}$ TOMKE: $\quad\llcorner$ Wie viele hast $\mathrm{Du}$

2197 LUAN: Vier. (lacht)

$2198 \quad$ ((allgemeines Gelächter $))$

2199 TOMKE: ((pfeift))

2200 LUAN: ${ }^{\circ} \mathrm{Naja}^{\circ}$.

2201

Mika knüpft an das zuvor besprochene Thema Vereinbarkeitsfragen von Beruf und Familie (hier ohne Auszug aus dem Transkript) an und verlagert dieses Thema auf das Studium: „Die Frauen, die tatsächlich ihre Kinder mit in die Vorlesung bringen, die kriegen nicht wirklich viel mit." Damit beschreibt Mika eine Situation, die nicht der Regel entspricht, sondern eine Ausnahme darstellt. Gemeinsam mit Tomke kommt sie zu dem Schluss, dass es vom Alter des Kindes abhänge, ob die Frau etwas von der Vorlesung „mitkriegt.“ Zudem wird auch in dieser Passage 
ein traditionelles Rollenbild reproduziert: Kinderbetreuung ist Aufgabe der Mutter. Männer werden hier von Betreuungsaufgaben der Kinder ausgenommen. Auch Luan knüpft an diese Orientierung an und hebt hervor, dass ",die Studentinnen" heutzutage ,,ja so eine Art Krippe" hätten, d. h. sie hätten Unterstützung bei der Kinderbetreuung. Interessant ist, dass hier die Kinderkrippe als entlastende Institution genannt wird und dass weitere Elternteile keine Erwähnung finden. Zuvor (hier ohne Auszug aus dem Transkript) wurde darüber gesprochen, dass die Kindererziehung heutzutage halbe/halbe organisiert sein sollte und nicht zu Lasten der Frauen gehen sollte. Dieser Aspekt der Inklusion der Väter in die Betreuungs- und Erziehungsaufgaben wird hier nicht umgesetzt, hat somit kein Enaktierungspotential und stellt damit auch keinen Orientierungsrahmen dar. Bei der Orientierung an einer egalitären Partnerschaft handelt es sich um ein Denkschema und eine Wertvorstellung, die sich in der habituellen Praxis der Studierenden nicht widerspiegelt und somit nicht als Orientierungsmuster dient. Zudem wird hier erneut im Vergleich von ,früher' und ,heute' die Gegenwart als positiver Horizont dargestellt und dadurch die Norm der Gleichberechtigung der Geschlechter reproduziert.

Der negative Horizont ,früher' wird von Luan exemplifiziert. Sie erzählt davon, dass sie, als sie Studentin und junge Mutter war, keine institutionelle Unterstützung erhalten habe. Luan verdeutlicht, dass sie ,damals ${ }^{6}$ ihr erstes Kind zu einer Prüfung mitgenommen habe, die sie bei einem Philosophieprofessor abgelegt habe, der sie fragte, ob sie „denn nicht lieber zuhause bleiben wolle". Damit erzählt Luan, dass ihr damals vermittelt worden sei, dass Kinder an der Uni, in Prüfungssituationen, nicht angebracht seien und Mütter besser ,zuhause" bleiben sollten. Luan wurde hier implizit nahegelegt, ihr Studium zu beenden und sich stattdessen um ihr Kind zu kümmern. Dabei wird die Orientierung an einer Entweder-oder-Entscheidung deutlich, die ,früher' als negativen Horizont erzeugt. Luan erzählt, dass sie „Zwar noch ein bisschen weiter studiert" habe, aber dann das Studium „halt abgebrochen" habe. Auf diese Erzählung folgt ein Hörsignal $\left(, \mathrm{Hm}^{“}\right)$ von Luca, das auch eine gewisse Sprachlosigkeit zum Ausdruck bringt. Luan erzählt weiter, dass sie sich schlussendlich gegen das Studium entschieden habe und in der Folge ,alle meine [ihre, S.K.] Kinder bekommen“ habe. Mit „und jetzt" macht sie deutlich, dass sie ihr Studium wieder aufgenommen hat, allerdings wird sie in ihren Ausführungen von Tomke unterbrochen, die sie danach fragt, wie viele Kinder Luan denn hat. Luan antwortet, dass sie vier Kinder hat und lacht dabei laut auf. Auch die anderen Studierenden lachen mit und 
es erfolgt ein Pfeifton von Tomke, der als Zeichen der Überraschung oder Bewunderung interpretiert werden kann. Luan beendet die Situation mit einem leisen „Naja“.

Hier ist auffällig, dass es zu keiner weiteren Nachfrage oder einem Zeichen der Empörung hinsichtlich der erzählten Erfahrung kommt. Indem Luans Erzählung unkommentiert im Raum stehen bleibt, zeigt sich erneut, dass die Studierenden über keinen konjunktiven Erfahrungsraum verfügen, um über Diskrimierungs- und Benachteiligungserfahrungen zu sprechen. Die Orientierung der Studierenden am Gleichheitspostulat und an gesellschaftlichen Modernisierungsprozessen scheint eine weitere Diskussion zu verhindern. Indem das Thema von der Studierenden nicht aufgegriffen wird, findet eine De-Thematisierung von Benachteiligung aufgrund von stereotypen Geschlechterrollen und -hierarchien statt. Wie bereits oben dargestellt, deutet auch hier alles darauf hin, dass die (De-)Thematisierung und die Verdeckung von ,Geschlecht ${ }^{6}$ und Geschlechterverhältnissen Teil der studentischen-habituellen Praxis ist und der studiumsbezogene konjunktive Erfahrungsraum vom konjunktiven Erfahrungsraum des neuen Geschlechtervertrags umspannt wird.

\subsubsection{Zusammenfassung der Gruppe Holz}

Die Diskussion der Gruppe Holz ist durch Rahmeninkongruenzen geprägt. Bereits der Beginn der Diskussion gibt deutliche Hinweise darauf, dass es unterschiedliche Orientierungen hinsichtlich der Relevanz von ,Geschlecht' und Geschlechterfragen gibt. Den Teilnehmer/inne/n gelingt es kaum, sich auf gemeinsame Themen einzulassen. Widersprüchliche Orientierungen treten in verschiedenen Passagen zutage; bevor es jedoch zu offenen Widersprüchen kommen kann, werden die kritischen Themen mit rituellen Konklusionen beendet. Insbesondere in Hinblick auf die Androzentrismuskritik und das Fehlen geschlechterreflektierter Inhalte in Lehrveranstaltungen konnten gegensätzliche Orientierungen rekonstruiert werden. Das Bewusstsein und das Interesse, sich mit Fragen zu ,Geschlecht' und Geschlechterverhältnissen auseinanderzusetzen, ist bei den befragten StudentInnen sehr divers. Das Spektrum reicht von offenem Interesse bis zur Ablehnung. Auch wenn es kein gemeinsames Interesse und kein gemeinsames Relevanzsystem gibt, über ,Geschlecht und Geschlechterfragen zu diskutieren, zeigt sich, dass die gemeinsame Sprache die sie dennoch finden, eine theoretisch-akademische Sprache, 
ist und somit vom studiumsbezogenen konjunktiven Erfahrungsraum geprägt ist. Die gemeinsame Sprache ist eine akademische, die sich an theoretischem Wissen und curricularen Fragen orientiert sowie vom generischen Maskulinum geprägt ist.

In den Passagen zur Relevanz von ,Geschlecht' und der Berücksichtigung dieser Kategorie im Curriculum zeigen sich in der Gruppe Holz eklatante Verstehensprobleme und ein „Habitus des nicht Verstehens“ (Rieger-Ladich 2010, S. 92). Die De-Thematisierung und die Verdeckung von ,Geschlecht' und Geschlechterfragen scheint somit Teil der studentisch-habituellen Praxis zu sein. Die Probleme unter den Studierenden sind ein Hinweis dafür, dass es keine gemeinsame Orientierung zu Geschlechterfragen gibt und Studierende keine etablierte Diskussionsform und -kultur zum Thema, Geschlecht' haben.

Wie in den anderen Gruppen wird auch hier der Studiengang Erziehungs- und Bildungswissenschaften als ein weiblich dominiertes Fach reproduziert, in dem männliche Studierende als von der erziehungswissenschaftlichen und der hegemonialen (heterosexuellen) Norm abweichend dargestellt werden. In der Gruppe Holz lässt sich außerdem zeigen, dass es sich bei der Orientierung an einer egalitären Partnerschaft um ein Denkschema und eine Wertvorstellung handelt, die sich jedoch nicht in der habituellen Praxis der Studierenden widerspiegelt. So wird z. B. das Geschlechterstereotyp reproduziert, dass Kinderbetreuung die Aufgabe der Mutter sei und Väter dafür nicht zuständig seien. Zudem wird auch die eigene heterosexuelle, vergeschlechtlichte und vergeschlechtlichende Position nicht hinterfragt.

Die in der Gruppe Holz vorgenommene Gleichsetzung von ,Geschlecht' mit Frausein und Geschlechterfragen mit ,Frauenfragen' und Benachteiligung scheint die Thematisierung von ,Geschlecht' und Geschlechtverhältnissen zu behindern, da sich die Studierenden am Gleichheitspostulat und an gesellschaftlichen Modernisierungsprozessen orientieren und nicht (mehr) als benachteiligt wahrgenommen werden wollen. Das von einer Teilnehmerin eingebrachte Thema ,geschlechterbezogener Benachteiligungs- und Diskriminierungserfahrungen" wird von den Studierenden nicht aufgegriffen und es kommt zu einer (De-)Thematisierung und Individualisierung dieser Erfahrungen. Dies deutet darauf hin, dass die (De-)Thematisierung und die Verdeckung von ,Geschlecht' und Geschlechterfragen Teil der studentisch-habituellen Praxis sind. Zudem zeigt sich die Argumentationsfigur, dass bei Nicht-Überrepräsentierteit der Frauen im erziehungswissenschaftlichen Studium, es für die Studierenden auch keine Benachteiligung an der Universität zu geben scheint. 
Damit wird die rhetorische Norm der Gleichstellung der Geschlechter reproduziert und Geschlechterungleichheiten werden verdeckt. In Bezug auf ,Geschlecht' und Geschlechterfragen finden eine Neutralisierung und Verdeckung von sozialen Ungleichheiten und deren Wirkmechanismen statt.

In der Gruppe Holz scheint sich der studiumsbezogene konjunktive Erfahrungsraum mit dem neuen Geschlechtervertrag als konjunktiver Erfahrungsraum zu überlappen bzw. in einem konkurrierenden Verhältnis zu stehen. Zudem wird die Orientierung deutlich, dass feministische Interventionen und Kritik an (patriarchalen) Herrschaftsverhältnissen für die Studierenden nicht mehr notwendig sind und ad acta gelegt werden können, da die Gleichstellung der Geschlechter, zumindest auf einer rhetorischen Ebene, als erreicht erscheint. Ein reflektierender Umgang mit ,Geschlecht' scheint in dieser Gruppe nicht Teil des erziehungswissenschaftlich-studentischen Habitus zu sein.

\subsection{Die (selbst)reflektierende Gruppe Feuer an der Universität Sonne}

Zwei Monate nach der Gruppendiskussion Holz wurde mit der Gruppe Feuer die zweite Gruppendiskussion mit Studierenden an der Universität Sonne geführt. An dieser Gruppe wollten sich ursprünglich vier Personen beteiligen. Zum vereinbarten Termin sind jedoch zwei Studierende nicht erschienen. An der Gruppe Feuer haben sich somit nur zwei Studentinnen des Bachelorstudiengangs Erziehungs- und Bildungswissenschaften beteiligt. Für eine der Studentinnen ist dies das Zweitstudium, im Hauptstudium studiert sie Grundschullehramt und steht hier kurz vor der Examensarbeit. Beide Frauen leben in einer Partnerschaft oder haben familiäre Betreuungs- oder Pflegearbeiten. Weiters haben beide keinen Migrationshintergrund angegeben. Die zwei Frauen sind Anfang zwanzig bzw. Anfang dreißig. ${ }^{16}$ Alex wie auch Nikita haben Lehrveranstaltungen zu ,Geschlecht' und Geschlechterfragen im Laufe ihres Studiums besucht. Das Zentrum für Gender Studies an ihrer Universität ist ihnen allerdings nicht bekannt.

In dieser Gruppe wird das Thema ,Geschlecht" von der Interviewerin eingeführt, damit handelt es sich um ein Interesse der Forscherin und um einen Diskurs, der heteronom produziert wurde. Trotz den anfänglichen

16 Alex: w, 32 Jahre, 4. Semester; Nikita: w, 22 Jahre, 5. Semester. 
Bekundung, dass ,Geschlecht' und Geschlechterfragen für die GDT keine Rolle spielen und nicht von Interesse seien, entwickelt sich ein autonomer Diskurs, der sich durch eine direkte und selbst-reflexive Thematisierung ${ }^{17}$ von ,Geschlecht' auszeichnet. Es kommt in der Diskussion nicht nur zu einer allgemeinen, theoretischen Thematisierung, sondern es wird auch das persönliche Selbst und die pädagogisch-praktische Erfahrung involviert. Das hohe Maß an Reflexion und Selbstbezug hebt die Gruppe Feuer von den anderen Gruppen ab. Neben dem Studium verbindet die beide Frauen ihre facettenreichen Erfahrungen in der pädagogischen Praxis, der schulischen und außerschulischen Jugendarbeit. Alex belegt (nur) im Zweitstudium den Bachelorstudiengang Erziehungs- und Bildungswissenschaften und im Hauptstudium Grundschullehramt. Dennoch wird in der Gruppendiskussion deutlich, dass Alex und Nikita über genügend gemeinsame Erfahrungsräume inner- und außerhalb des Studiums verfügen.

In dieser Zweier-Konstellation werden, im Gegensatz zur Gruppe Holz, zahlreiche Beispiele erzählt. Nikita und Alex führen einen inkludierenden Diskurs in Form eines parallelen Modus. Sie artikulieren gemeinsame Orientierungen auf der Basis gemeinsamer (im Sinne homologer) Erfahrungen auch explizit auf der, Was'-Ebene. Dies wird in der anschließenden Darstellung und Auswertung der Gruppendiskussion deutlich. In dieser Zweier-Konstellation verläuft die Diskussion sehr harmonisch und wenig konflikthaft. Nachdem sich die GDT darüber einig waren, über ,Geschlecht' und Geschlechterfragen zu sprechen, muss das Thema nicht mehr legitimiert werden bzw. mit anderen Themen konkurrieren (wie z. B. in der Gruppe Holz). In dieser Diskussion werden ,Geschlecht' und Geschlechterfragen nicht mit Frausein und Frauenproblemen gleichgesetzt; dies scheint die Diskussion zu befruchten. Die Ebene, auf der gesprochen wird, ist eine pädagogisch-professionelle, auf der die Selbst-Reflexion von Geschlechterrollen und -stereotypen einen selbstverständlichen Teil einnimmt. Die GDT, Alex und Nikita, haben die Diskussion im Sinne eines Reflexionsraums genutzt, in welchem sie über das Thema, Geschlecht' reflektieren und ihre Erfahrungen besprechen können. Bei der anschließenden Darstellung und Auswertung dieser Gruppendiskussion wird aufgrund der Zweier-Konstellation stärker auf die inhaltliche Ebene als auf die Diskursorganisation fokussiert.

17 Im Vergleich dazu spricht Wolfram Fischer-Rosenthal (1995) in Bezug auf die Auseinandersetzung und Aufarbeitung der deutschen Vergangenheit des Nationalsozialismus auch von verschiedenen Thematisierungsweisen, die ebenfalls heteronom produziert wurden, wobei es jedoch zu keiner selbst-reflexiven Aufarbeitung kam. 


\subsection{1 „Was soll diese Frage mit Geschlecht?“}

Der erste Diskussionsimpuls zu ,Geschlecht" wird in der Gruppe Feuer von der Interviewerin initiiert, indem sie die GDT fragt, ob ,Geschlecht ein Thema sei, das sie interessiere. Auf diese Frage reagieren Alex und Nikita eher zurückweisend: ${ }^{18}$

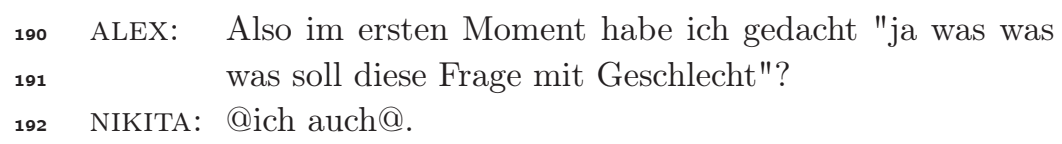

Beide bringen als erste Reaktion zum Ausdruck, dass sie von der „Frage mit Geschlecht" irritiert sind und keinen Bezug dazu haben. Damit grenzen sie sich im ersten Schritt von dem Thema ab. Im zweiten Schritt findet jedoch durch Alex eine Annäherung zum Thema ,Geschlecht' statt. Sie erklärt, dass "Geschlecht" in ihrem Studium „eine große Rolle spielt". $\mathrm{Zu}$ diesem Schluss kommt sie zum einen aufgrund der statistischen ungleichen Geschlechterverteilung bei den Studierenden ihrer Studienfächer „Pädagogik und Grundschullehramt“. Diese Fächer studieren „fast nur Frauen“" und „ein paar Jungs". Zum anderen spielt ,Geschlecht" auch bei den „Gerüchten“ über die sexuelle Orientierung der Studenten eine Rolle: „[U]nd dann gibt es immer so Gerüchte, naja, wer das macht der muss ja schwul sein oder irgendwie, nicht ganz richtig." Nikita validiert die Erfahrung, dass auch in ihrem Studiengang Frauen dominieren und dass „man ganz oft hört", dass die wenigen Männer im Studium „schwul" seien. Beide GDT verfügen über das Erfahrungswissen, dass das Studium der Erziehungs- und Bildungswissenschaften eine „Frauendomäne ${ }^{66}$ ist.

Ihre Erfahrung ist, dass Männer nicht typischerweise dieses Studium wählen und dass diese eine Ausnahme darstellen. Das erziehungswissenschaftliche bzw. pädagogische Studium lässt sich somit auch in dieser Gruppendiskussion als ein „Frauenstudium“ rekonstruieren. Verstärkt wird dies, indem über die sexuelle Orientierung der wenigen Studenten spekuliert wird. Auch wenn es sich bei den Gerüchten nicht um bestätigtes Wissen handelt, handelt es sich um verallgemeinernde Aussagen, welche die Studenten als von der heterosexuellen Geschlechternorm und damit auch von einer gesellschaftlich hegemonialen Männlichkeit abweichend konstruieren und die Feminisierung des Studiums der Erziehungs-

18 Die zitierten Passagen beginnen mit der jeweiligen Originalzeilennummerierung, und weichen im Verlauf der Passagen, z. B. aufgrund von gekennzeichneten Auslassungen, davon ab. 
und Bildungswissenschaften zementieren. Wenn man den Rahmen der Gruppendiskussion kurz verlässt, dann kann man feststellen, dass Homosexualität erst in der Neuzeit und mit der aufkommenden Unterscheidung zweier sich ausschließender, gegensätzlicher Geschlechter zu einem exklusiven, z. T. bis heute pathologisierten und kriminalisierten Phänomen gemacht wurde. Zudem wurde Homosexualität mit einer Verweiblichung von Männern gleichgesetzt (Hirschauer 1996).

In dieser Passage wird deutlich, dass sich die GDT im ersten Moment vom Thema, Geschlecht' distanzieren und den Anschein erwecken, als ob Geschlechterfragen für sie nicht von Interesse seien. Sie signalisieren, dass ,Geschlecht' für sie kein Thema sei und relativieren auf einer rhetorischen Ebene die Bedeutung dieser Kategorie. Nach dieser Situation der vorläufigen rhetorischen Zurückweisung werden ,Geschlecht' und Geschlechterfragen jedoch zu einem Thema, das nicht mehr legitimiert werden muss und wird somit thematisierbar. Im Verlauf der Gruppendiskussion wird das Interesse an dem Thema deutlicher und es entwickelt sich ein Gespräch, in dem die GDT ,Geschlecht' sowohl in Bezug auf ihre persönliche Realität als auch auf ihre pädagogische Praxis reflektieren. ,Geschlecht' wird von einem von außen herangetragenen Diskussionsthema zu einem Thema, das auch das Interesse der GDT widerspiegelt und zu dem sie sich selbst verhalten. Im Laufe der Gruppendiskussion, ca. 1640 Zeilen im Transkript bzw. zwei Stunden später, kommen Nikita und Alex zu dem Schluss, dass es doch „ein spannendes Thema“ sei und es einer theoretischen wie auch persönlichen Auseinandersetzung bedürfe. Letzteres stellt für sie eine Lücke im Studium dar.

1827 ALEX: Ja, das [Geschlecht(erdifferenzen), S.K.] spielt schon eine Rolle. [...]

NIKITA: ${ }^{\circ}$ Das ist schon ein spannendes Thema $^{\circ}$ (lacht)

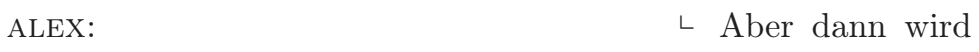
das hier in der Uni doch sehr auf irgendwelche, Forschungsarbeiten und Thesen bezogen, und also als Genderbegriff, oder was auch immer, diskutiert, aber viel zu wenig und viel zu selten an unserer ganz persönlichen Realität hier; ja das kommt jetzt, finde ich, hier so gerade so raus.

NIKITA: $\quad$ Mhm 
In Bezug auf die Thematisierung von ,Geschlecht' und Geschlechterfragen an der Universität bzw. in ihrem Studium konstatieren die GDT, dass das Thema häufig auf „Forschungsarbeiten und Thesen“ bezogen oder ,als Genderbegriff" besprochen werde. Als positiver Gegenhorizont zu dieser an Forschung und Wissenschaft orientierten Thematisierung wird die Einbeziehung der „ganz persönlichen Realität" genannt. Dies finde „viel zu wenig und viel zu selten“ statt. Die GDT befürworten demnach eine Thematisierung und Auseinandersetzung mit ,Geschlecht ${ }^{6}$ und Geschlechterfragen, die auch die individuelle und persönliche Lebensrealität inkludiert und nicht alleine auf eine theoretisch-abstrakte Ebene fokussiert.

\subsubsection{Möglichkeiten und Grenzen der Auseinandersetzung und Thematisierung}

Anhand der Diskussion der Gruppe Feuer lassen sich die Möglichkeiten und die Grenzen rekonstruieren, die das Studium für eine Thematisierung und Auseinandersetzung mit ,Geschlecht' und Geschlechterfragen bietet. Nikita wie auch Alex beschreiben ihr Studium als einen Ort, in dem sie sich erstmals bewusst mit ,Geschlecht' und Geschlechterfragen auseinandergesetzt hätten: Nikita exemplifiziert dies am besuchten „Sexualpädagogik-Seminar“: Hier sei „ganz bewusst thematisiert" und kritisch infrage gestellt worden, „warum wir eigentlich von Anfang an unterscheiden, irgendwie zwischen Jungen und Mädchen". Sie erzählt weiter, dass die Differenzierung zwischen Männern und Frauen und ,Geschlecht' als Ordnungskategorie kritisch hinterfragt worden sei. Nikita resümiert: ,[D]as Seminar fand ich auch sehr interessant", weil sie sich über diese Inhalte „,vorher überhaupt noch nie Gedanken gemacht“ und sich „,noch nie so damit auseinandergesetzt" habe. Allerdings konstatiert Nikita an einer anderen Stelle der Gruppendiskussion, dass ,Geschlecht kein Querschnittsthema im Studium darstelle, sondern ein Spezialthema sei, das in speziellen Seminaren behandelt werde:

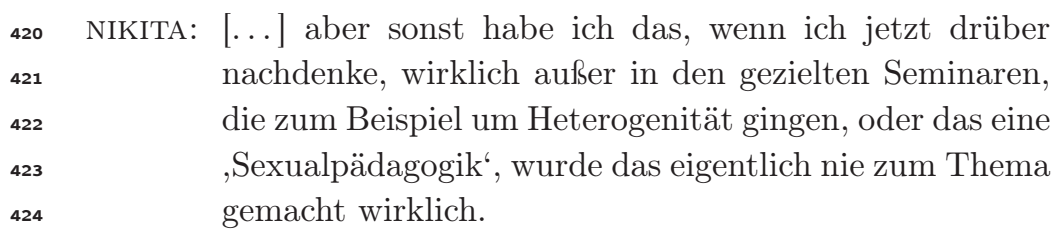

Auch Alex hat sich im Rahmen ihres Studiums mit ,Geschlecht' und Geschlechterfragen beschäftigt und beschreibt, dass ihr die Thematisie- 
rung von ,Geschlecht“ „unter dem Begriff doing gender von Anfang an begegnet" sei. Damit verweist sie auf einen theoretischen Analyseansatz, den sie im Laufe ihres Studiums kennengelernt habe und der sie dafür sensibilisiert habe, ,Geschlecht" als soziale Kategorie zu verstehen, die in Interaktionen (re-)produziert werde. Alex resümiert, dass dies auch ihre „Perspektive“ auf die Interaktionen der AkteurInnen im Unterricht geschärft habe und darauf, „welche unterschiedlichen Erwartungen" sie an Jungen und Mädchen habe, „bewusst oder unbewusst“.

In Bezug auf ihre pädagogische Praxis ist es Alex wie auch Nikita wichtig, geschlechterreflektiert zu handeln:

1582 NIKITA: Mhm, na ich denke, generell in der pädagogischen Ar1583 beit, wenn man eben schon so geprägt ist mit den Ge${ }_{1584}$ schlechterrollen und so weiter, dass man das einfach im1585 mer für sich selbst, reflektieren sollte. Habe ich jetzt so gehandelt, weil das eine Frau ist, oder hätte ich vielleicht doch anders handeln sollen, und nicht nur so weil es eine Frau ist? [...] Ja. Ich denke, man macht sich wirklich erst wenig Gedanken bis man dazu angeregt wird; [...] also das müsste vielleicht doch mehr thematisiert werden. Also hier in der Uni, um eben die Grundlagen zu haben, um damit reflektiert umzugehen.

1593

ALEX: Mhm, also ich denke auch, dass das eine Rolle spielt, egal in welcher Altersstufe man sich gerade bewegt beruflich. Weil man ja selber immer eine Perspektive hat, und die Menschen, mit denen man zu tun hat, die ja auch haben.

1598 NIKITA: Mhm.

Eine selbst-reflexive Haltung gegenüber Geschlechterrollen und -stereotype stellt für Nikita und Alex einen wichtigen Aspekt ,in der pädagogischen Arbeit" dar. Diese geschlechterreflektierende Haltung schildert Nikita als eine Haltung, die (von außen) angeregt werden müsse. Deshalb sollten ,Geschlecht' und Geschlechterfragen in ihrem Studium „mehr thematisiert werden“. Das ,Geschlecht” spiele für Alex unabhängig vom Alter der pädagogischen Subjekte bzw. der pädagogischen Zielgruppe eine Rolle, nicht nur in Bezug auf ihre eigenen Geschlechterstereotype, sondern auch auf jene der anderen. Sowohl Nikita als auch Alex beschreiben ihr Studium als einen Ort, der die Möglichkeit biete, 
sich mit ,Geschlecht' und Geschlechterfragen auseinanderzusetzen. Es lassen sich aber auch die Grenzen und Lücken rekonstruieren. Die GDT resümieren, dass ,Geschlecht‘ und Geschlechterfragen im Studium „mehr thematisiert werden" müssen, um eine geschlechterreflektierende Haltung anzuregen. Zudem wird deutlich, dass ,Geschlecht' kein Querschnittsthema im Studium darstellt, sondern sich Studierenden ohne Anregung wenig mit ,Geschlecht' und Geschlechterfragen auseinandersetzen. Dies hat den Nachteil, dass sich letztlich nur ein Teil der Studierenden damit beschäftigt.

Eine weitere Lücke in der Thematisierung wird von Alex eingeführt. Sie hebt hervor, dass es an der Universität auch "Übergriffe gibt", diese aber nicht ,thematisiert" werden:

427 ALEX: Also mir fällt gerade was ein, dass es eher nicht thematisiert wird. Also mir ist das in all den Jahren nicht begegnet, dass einmal thematisiert wurde, ob es Übergriffe gibt. [...] oder hier gibt es Hilfe oder hier gibt es ein Angebot, sich da irgendwie zu melden oder irgendwas, gar nichts. [...] Und ich hatte auch mal ein Erlebnis selber, [...]. Also Übergriffe innerhalb der Universität; dass es so was gibt, das mag ja also relativ harmlos sein, aber dass es das gibt, und dass es auch eine Rolle spielt, dieses Geschlechtliche; scheint also bei uns in unserem Verhalten als Studenten so zu sein, dass wir relativ geschlechtslos durch die Uni laufen.

NIKITA:

ᄂ Ja.

ALEX: Es soll keinen Unterschied machen, ob das jetzt eine Studentin oder ein Student ist, der das mit dem Professor oder der Professorin bespricht, oder Studenten, die miteinander arbeiten, habe ich auch nie erlebt, dass es da irgendeine Unterscheidung gab, ob wer jetzt männlich weiblich ist und welche Rolle das spielt wenn man zum Beispiel gemeinsam irgendein Projekt zu machen hat oder so.

NIKITA:

$\llcorner$ Ja.

ALEX: Dann ist man geschlechtslos eigentlich. [...] Also es scheint zumindest, dass man es sein soll; also ich weiß auch, dass ich das für mich so empfunden habe, wenn 
ich es jetzt reflektiere.

NIKITA: Mhm, ich glaube auch, die Rolle der Studierenden hat ja jeder, und egal @welchen Geschlechts@.

ALEX: Ja, "die Studierenden", die haben dann irgendwie keins mehr; auch sprachlich.

NIKITA:

$\llcorner$ Ja genau.

In dieser Passage resümiert Alex, auch aufgrund ihrer eigenen Erfahrung, dass es an der Universität Übergriffe gebe. Damit versucht sie zu verdeutlichen, dass das „Geschlechtliche“ „eine Rolle spielt", auch wenn dies im allgemeinen, ,geschlechtslosen" Verhalten der Studierenden nicht den Anschein habe. Indem Alex die Übergriffe mit,Geschlecht' in Verbindung bringt, ist anzunehmen, dass sie hier von sexuellen Übergriffen spricht. Das ,geschlechtslose“ Verhalten der Studierenden scheint eine Norm, eine Orientierung zu sein, mit der Alex nicht übereinstimmt. Interessant ist, dass genau an der Stelle, an der Alex über die Geschlechtslosigkeit der AkteurInnen an der Universität spricht, sie nicht wie im Rest der Diskussion das generische Maskulinum verwendet, sondern das ,Geschlecht ${ }^{\text {* }}$ hervorhebt und von ,Studentin“ und „Student", „Professor" und „Professorin" spricht. Die Geschlechtslosigkeit wirkt wie eine Verhaltensregel, gegen die Alex opponiert. Auch die Formulierung, dass man „eigentlich“ „geschlechtslos" sei, signalisiert, dass Alex diese Norm infrage stellt bzw. relativiert. Alex reflektiert, dass sie die Geschlechtslosigkeit als ,Schein“ empfunden habe, als etwas, das so ,sein soll"; das bedeute aber nicht, dass es so sei. Letzteres hat Alex am Beispiel der Übergriffe deutlich gemacht.

In dieser Passage lässt sich zeigen, dass Geschlechtslosigkeit eine ,universitäre' Orientierung darstellt, in der zum Ausdruck kommen soll, dass ,Geschlecht' an der Universität keine Rolle spielt. Beide GDT nehmen das geschlechtslose Verhalten zwar als Orientierung wahr, wobei deutlich wird, dass Alex diese Norm kritisch(er) betrachtet. In ihren Ausführungen wird deutlich, dass der normative Anspruch an der Universität, geschlechtslos zu sein und zu agieren, zu einer DeThematisierung von sexuellen Übergriffen an der Universität führt bzw. diese verdeckt. 


\subsubsection{Gleichberechtigung von vs. Differenzierung nach Geschlecht}

In dieser Gruppendiskussion lässt sich rekonstruieren, dass sich die GDT zum einen an der Gleichheit und Gleichberechtigung von Männern und Frauen als Norm orientieren. Zum anderen wird deutlich, dass ,Geschlecht' auf der Handlungsebene weiterhin eine Differenzierungskategorie darstellt. ,Geschlecht' fungiert demnach im handlungsleitenden Wissen nach wie vor als Ordnungs- und Strukturmerkmal, dies wird jedoch durch die Gleichheitsnorm im kommunikativ-generalisierten Wissen verdeckt. Die Geschlechtergleichheit ist ein Motiv, um die Wirksamkeit der Kategorie ,Geschlecht' zu neutralisieren und zu verdecken. Dies wird im Folgenden anhand der Passagen zur geschlechtergerechten Sprache und zum Generationenwechsel sowie in der Kontrastierung mit der Passage zur „Physiotherapeuten-Ausbildung“ deutlich.

\section{Geschlechtergerechte Sprache und Generationenwechsel}

Wie auch in den Gruppen Holz und Wasser wird hier deutlich, dass sich die Studierenden von der geschlechtergerechten Sprache distanzieren und sich bei Hausarbeiten an den formalen Erwartungen und Normen der Dozierenden orientieren. Nikita und Alex teilen die Orientierung, dass Studierende geschlechtergerechte Sprache in Hausarbeiten nur aufgrund von äußeren Zwängen und Bedingungen verwenden und bestätigen damit das generische Maskulinum als normative Orientierung.

NIKITA: Es gibt Dozenten, die zum Beispiel darauf bestehen, dass man in einer Hausarbeit unterscheidet. Oder beziehungsweise beides nennt. Und das ist so ein bisschen was, gegen@das ich mich wehre@, weil ich sage, die deutsche Sprache ist halt eine Sprache, schon immer, die sich irgendwie auf das männliche Geschlecht fokussiert, und dann heißt es halt, "der Pädagoge", erstmal wenn ich allgemein davon rede und nicht, „die der Pädagoge Schrägstrich -in“ oder wie auch immer, und ich mich selbst nicht damit angegriffen fühle einfach; wenn da steht "Pädagoge". Das ist mir egal. [...] Aber wie gesagt ich wehre mich da ein bisschen dagegen; also ich finde es einfach überflüssig. 
Nikita distanziert sich von der Forderung durch Dozierende, „dass man in einer Hausarbeit unterscheidet. Oder beziehungsweise beides [Frauen und Männer] nennt.“ Für sie sei diese Schreibweise „einfach überflüssig“, da die „deutsche Sprache, schon immer [...] auf das männliche Geschlecht fokussiert" gewesen sei. Wenn ein Wort „allgemein" verwendet werde, ,dann heißst es halt, der Pädagoge, [...] und nicht ,die der Pädagoge Schrägstrich -in" oder wie auch immer". Nikita erläutert, dass das generische Maskulinum in ihrer Orientierung niemanden ausschließe, sondern einen inkludierenden Charakter habe und durch seine unkomplizierte Schreibweise besteche. Zudem argumentiert sie, dass sie sich ,selbst nicht damit angegriffen fühle [...] wenn da steht, ,Pädagoge““. Nikita betont, dass sie sich durch das generische Maskulinum in ihrem Frausein nicht angegriffen fühle. Damit bestätigt und reproduziert sie auf einer rhetorischen Ebene das kommunikativ-generalisierende Wissen, dass Frauen nicht (mehr) benachteiligt seien und deshalb keine besondere Nennung oder Behandlung brauchen. Dadurch stellt sie gleichzeitig die Notwendigkeit geschlechtergerechter Sprache infrage. Die Gleichheit und Gleichberechtigung von Männern und Frauen scheint durch die Orientierung am generischen Maskulinum bestätigt bzw. dadurch Gültigkeit zu erlangen.

Nachdem Alex erläutert, dass sie sich mit Fragen zur geschlechtergerechten Schreibweise bislang nicht beschäftigt habe, fragt die Interviewerin nochmals nach den Reaktionen der Studierenden, wenn Professorinnen und Professoren auf die geschlechtergerechte Sprache pochten.

ALEX: Also ich vermute das auch, also dass da von uns, also in unserem Alter niemand das für wirklich so bedeutend hält; also es gibt sicherlich welche, aber dass es eher die die Minderheit, ist die sich da so, wie du es sagst dafür einsetzen wird und=sagen=würde. Es ist wichtig, sondern es kommt uns eher so vor wie: "Naja gut, das ist jetzt so eine formale Erwartung, und wenn es halt sein muss, erfüllen wir das", oder es ist uns eigentlich klar, warum das ist, aber im Prinzip ist uns auch schon klar, das haben wir schon hinter uns, das Thema. Müssen wir das jetzt noch mal diskutieren und noch mal feststellen, dass in dem Beruf man mit solchen und solchen Geschlechtsteilen arbeiten kann; ja? Also es ist so (wäre) schon was Banales wo man denkt: "Das nervt jetzt 
langsam mal". So. Also so kommt es mir jetzt gerade vor.

NIKITA: Ja ich hab immer das Gefühl, dass es von denen, die das so fordern, im Prinzip auch so ein Punkt @Emanzipation ist@, dass (noch) die Frau genannt werden muss, unbedingt die weibliche Form, aber wie gesagt, für mich spielt das irgendwie keine Rolle, weil ich mich einfach damit selbst nicht angegriffen @fühle wenn das da so steht@; also das ist mir, das ist unsere Sprache, und das finde ich, muss man nicht ändern, und das stört nur wenn man das die ganze Zeit dann immer wieder in Doppelform schreibt, ja?

ALEX: Also mir würde das auch so gehen, wenn ich in so einer Arbeit irgendwas von Therapeuten und Pädagogen lese, dass mir aus meiner Alltagserfahrung klar ist, da gibt es männliche und weibliche, [...], Das gibt es bei Ärzten, das gibt es eigentlich in allen Berufen. Natürlich mit den Tendenzen, dass eben (sagen wir mal) in manchen Berufen doch die mehr die einen oder mehr die anderen sind, aber es steht ja letztlich doch allen offen.

Alex validiert die Orientierung, dass geschlechtergerechte Sprache nicht wichtig sei und grenzt ihren Geltungsbereich auf eine bestimmte Altersgruppe bzw. Generation ein. Mit dem Verweis auf das „Alter" wird implizit auf einen Generationenwechsel hingedeutet. Die Ausführungen zur Sprache sind in einen vergleichenden Bedeutungsrahmen eingebettet, der die ,heute' erreichte Gleichberechtigung (,das haben wir schon hinter uns das Thema") und der ,früheren' Benachteiligung von Frauen nebeneinander stellt. Für eine ,jüngere' Generation, zu der sich auch die GDT zählen, sei die geschlechtergerechte Sprache nicht relevant, nur die „Minderheit" setze sich dafür ein. Die Gleichberechtigung und „Emanzipation" gelte - zumindest in der Arbeitswelt - als erreicht, und deshalb erscheint die kontinuierliche Forderung danach als „banal" und als etwas, das ,.jetzt langsam mal nervt". Die Studentinnen machen deutlich, dass sie sich bereits emanzipiert und gleichberechtigt fühlen. Und mit der Argumentationsfigur, sich vom generischen Maskulinum angesprochen zu fühlen, validieren sie die Emanzipation und Gleichberechtigung von Frauen. Es kommt zu einer verbaler Beteuerungen von Gleichheit und 
Gleichberechtigung. Indem die GDT das generische Maskulinum als ,richtige und normale' Sprache darstellen, betonen sie, dass die geschlechtergerechte Sprache eine formale Anforderung sei, die es in einem bestimmten universitär-akademischen Rahmen zu erfüllten gilt.

Die Logik dieser Passage lässt sich demnach so zusammenfassen: Für Alex und Nikita ist die Emanzipation und Gleichberechtigung heute bereits erreicht, deshalb sei dieses Thema für die jüngere (Frauen-)Generation abgeschlossen. Die erreichte Gleichberechtigung identifizieren Nikita und Alex in der für beide Geschlechter offenen Arbeitswelt, diese „steht ja letztlich doch allen offen". Demnach stünden Männern und Frauen alle Wege offen, es kommt zu einer Individualisierung von Gelingen und Scheitern. Probleme, Benachteiligung und hinderliche gesellschaftliche Strukturen scheinen nicht der Rede wert. Indem sich Alex und Nikita von der geschlechtergerechten Sprache distanzieren und das generische Maskulinum verteidigen, bringen sie ihre eigene Gleichberechtigung und Emanzipation zum Ausdruck. Damit wird die Norm der Gleichstellung der Geschlechter reproduziert und die Relevanz von ,Geschlecht ${ }^{6}$ und Geschlechterfragen rhetorisch entdramatisiert.

Dieser vergleichende Bedeutungsrahmen von ,früher' und , heute' lässt sich auch in einer späteren Passage in der Diskussion rekonstruieren. In dieser Passage fragt die Interviewerin die GDT, ob es für sie etwas Besonderes sei, wenn eine Frau die Position der Dekanin innehabe. Damit führt sie die Orientierung ein, dass Frauen in Leitungspositionen eine Besonderheit darstellen. Diese Orientierung wird von den GDT nicht geteilt:

\begin{tabular}{|c|c|}
\hline & $\begin{array}{l}\text { gibt ja auch Personen die das aus ihrer Kindheit und } \\
\text { Jugend und vielleicht auch lange im Erwachsenenalter } \\
\text { noch ganz anders kennen; und wir kennen das schon so, } \\
\text { es gibt Männer und Frauen, die können alles Mögliche } \\
\text { sein. }\end{array}$ \\
\hline NIKITA: & $\begin{array}{l}\text { Ich meine, seitdem ich studiere haben wir eine Dekanin; } \\
\text { ich weiß nicht wie lange sie das schon ist, also es ist für } \\
\text { mich halt, ich kenne es ja auch nicht anders jetzt, ja? }\end{array}$ \\
\hline ALEX: & $\begin{array}{l}\text { Ja, ich meine, ich würde mich auch nicht mehr darüber } \\
\text { wundern, dass es eine Bundeskanzlerin gibt, ja? }\end{array}$ \\
\hline NIKITA: & $\llcorner$ Ja. \\
\hline
\end{tabular}


1793 ALEX: Also ich kann mir vorstellen, dass das für andere bedeutender und besonderer ist als es mir vorkommen würde.

1795 NIKITA: $\left\llcorner{ }^{\circ} \mathrm{Mhm}^{\circ}\right.$,

Auch in dieser Passage wird im Vergleichshorizont von ,früher' und ,heute' zum einen auf die Modernisierung von Geschlechterverhältnissen und -gleichstellung, und zum anderen auf einen Generationenwechsel verwiesen. Mit ,heute wird impliziert, dass Frauen und Männer ,alles Mögliche sein" könnten, beiden Gruppen stünden ,heute' alle Wege offen. ,Früher" sei dies nicht der Fall gewesen, damals hätten das die Menschen in ,ihrer Kindheit und Jugend und vielleicht auch lange im Erwachsenenalter" anders kennen gelernt und deshalb seien Frauen in Führungspositionen auch ,bedeutender und besonderer" gewesen. Alex verweist hiermit auf die ältere Generation, für die Frauen in Führungspositionen eine Besonderheit darstell(t)en. Zu dieser Generation zählen sich Nikita und Alex nicht, das wurde auch oben deutlich; für sie löst eine „Dekanin“ oder „Bundeskanzlerin“ keine Verwunderung aus: Es sei „normal“. An dieser Stelle möchte ich auch auf die Wortwahl von Nikita und Alex verweisen: Sie sprechen davon, dass sie, wie auch die ,ältere' Generation, die Geschlechterverhältnisse und -rollen auf eine bestimmte Weise „kennen“. „Kennen“ wird im Sinne von ,etwas wissen“ und „mit etwas bekannt sein" verwendet und verweist hier eher auf ein kommunikativgeneralisiertes Wissen als auf die Erfahrungsebene. Die Generationen sind mit unterschiedlichen Geschlechternormen bekannt, die ,früher' von der Geschlechterungleichheit und die ,heute' von Geschlechtergleichheit und modernisierten Geschlechterverhältnisse geprägt sind.

\section{„Physiotherapeuten-Ausbildung6"}

Auch wenn sich rekonstruieren lässt, dass sich die Studierenden an der Geschlechtergleichheit als Norm orientieren, ist ,Geschlecht' weiterhin eine Kategorie, nach der sie differenzieren. Das heißt, ,Geschlecht' stellt nach wie vor ein Ordnungs- und Strukturmerkmal dar, auch wenn dies durch die Gleichheitsnorm verdeckt wird. Nikita exemplifiziert dies im Folgenden am Gespräch, das sie mit einer Freundin geführt habe.

1809 NIKITA: [...] also ich habe, mir fällt gerade ein, gerade gestern, 1810 meine beste Freundin hat jetzt seit ersten November angefangen mit einer Physiotherapeuten-Ausbildung, und dann habe ich gestern mit ihr telefoniert und habe sie dann gefragt, ja, habe ich sie automatisch auch ge- 
fragt. Sind da mehr mehr Frauen oder Männer?". Und dann meinte sie: "Ja, mehr Frauen", dann war ich total: „Echt? Ich hätte jetzt echt gedacht dass das mehr Männer machen, Physiotherapie; ja? Das war für mich irgendwie ganz klar, aber scheint wohl in der Ausbildung jetzt erst, also bei ihr in der Ausbildung erstmal nicht so zu sein; ja?

ALEX:

$\left\llcorner^{\circ} \mathrm{Gibt}^{\circ}\right.$. Hm;

NIKITA: Ich hätte die auch fragen können: "Wie viele machen denn die Ausbildung. "; ich hätte ja die Unterscheidung gar nicht erst treffen müssen, aber ich habe es gemacht, ja? Also wenn ich jetzt drüber nachdenke.

ALEX: Ja.

Nikita erzählt, dass sie ihre Freundin ,automatisch“ nach der Geschlechterverteilung bei der „Physiotherapeuten-Ausbildung“" gefragt habe: „Sind da mehr mehr Frauen oder Männer?" Nikitas handlungsleitendes Wissen ist demnach ganz ,automatisch" von der Differenzierung zwischen Frauen und Männern geprägt. Die Geschlechterdifferenz wird hier von Nikita als Leitdifferenz eingeführt. In der Gruppendiskussion reflektiert Nikita, dass sie eigentlich nicht nach der Geschlechterverteilung hätte fragen müssen und damit die Unterscheidung zwischen Männern und Frauen nicht hätte treffen müssen, sondern auch ganz allgemein nach der Teilnehmerinnenzahl hätte fragen können. Ihr wird nachträglich bewusst, dass sie automatisch die Unterscheidung zwischen Männern und Frauen treffe und auch bestimmte (Berufs-)Bilder mit dem, Geschlecht' verknüpfe. Damit ist die Frage oder die Feststellung der Geschlechterverteilung nicht nur eine Frage oder Feststellung der statistischen Verteilung. Es findet dabei auch eine Verknüpfung mit den jeweiligen Geschlechterbildern und -rollen statt - dies wird auch in der Passage zur statistischen Geschlechterverteilung in den Erziehungs- und Bildungswissenschaften deutlich.

Wie bereits gezeigt wurde, gehen die GDT von einer heteronormativen Gleichheitsnorm und einem toleranten, offenen und modernisierten Geschlechterwissen (das ich als heteronormatives Wissen verstehe) aus. Die Geschlechter werden in dem Sinne als gleich gesehen, als sie dieselben Möglichkeiten haben. Männer und Frauen könnten die gleichen Ämter bekleiden und beide könnten, wenn sie nur wollten, alles erreichen und jeden Weg für sich wählen. Diese Gleichheitsnorm verdeckt, dass die Dif- 
ferenzierung nach ,Geschlecht' im handlungsleitenden Wissen nach wie vor eine Rolle spielt. Geschlechtergleichheit ist ein Motiv, um die Wirksamkeit der Kategorie ,Geschlecht' und Geschlechterhierarchien $\mathrm{zu}$ neutralisieren und $\mathrm{zu}$ verdecken.

\subsubsection{Homosexualität: „Ich habe überhaupt gar kein Problem damit."}

In der Diskussion drücken Alex und Nikita eine (vermeintliche) Toleranz gegenüber Transpersonen und Homosexualität aus. In der folgenden Passage wird zudem die Rolle der Universität in bzw. das universitäre Umfeld als Ort/Raum der Auseinandersetzung mit der eigenen Heteronormativität sichtbar. Die Toleranz gegenüber Homosexualität ist nicht nur Teil einer heterosexuellen, sondern auch einer universitären/pädagogischen Norm. Nikita und Alex betonen, dass sie noch nie ein Problem mit Homosexualität gehabt hätten, aber trotzdem wäre es ,auf jeden Fall was Neues, mit dem [sie] konfrontiert wurde[n], hier in der Uni“.

693

694

695

696

697

698

699

700

701

702

703

704

705

706

707

708

709

710

711

712

NIKITA: Ich muss auch ganz ehrlich sagen, auch hier in der Uni, also ich habe bevor ich angefangen hab zu studieren, in meinem Freundeskreis niemanden gehabt, oder zumindest niemand von dem ich es weiß, dass er homosexuell ist; oder sie homosexuell ist. Und hier, seitdem ich studiere, habe ich einige kennengelernt die homosexuell sind; und das war für mich, ich habe überhaupt gar kein Problem damit, noch nie gehabt, aber das war für mich trotzdem was Neues. [...] aber es ist auf jeden Fall was Neues, mit dem ich konfrontiert wurde hier in der Uni.

ALEX: Also mir ging das ganz ähnlich. Jetzt wo du es sagst wird mir das auch klar, dass es eigentlich erst jetzt so in der Studienzeit, so naja greifbar geworden ist sage ich mal. Also indem ich auch einen Kommilitonen hier kennengelernt habe, [...] Und dann war das auch interessant, weil er sich dann auch ja, also naja auch also auch sehr offen damit umgegangen ist.

NIKITA: Finde ich aber ganz viele hier an der Uni.

ALEX:

$\llcorner\mathrm{Ja}$.

NIKITA: Also ich habe, ich habe noch keinen kennengelernt, der 


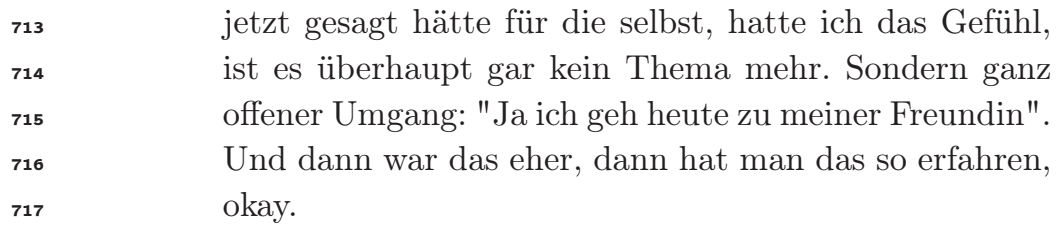

Für Nikita und Alex stellt das Studium einen Ort dar, an dem sie erstmals mit Homosexualität als ,reale' sexuelle Orientierung konfrontiert wurden. Davor war Homosexualität zwar Teil ihres common sense, aber nicht Teil ihrer erlebten Erfahrung. Dies habe sich durch das Studium und die dort geknüpften Kontakte geändert. Beide betonen den „offenen“ Umgang mit Homosexualität an der Universität und stellen diesen Umgang als positiven Horizont dar. Homosexualität werde hier als nichts Besonderes hervorgehoben, sondern es herrsche ein ,ganz offener Umgang“. Die Normalität der Homosexualität wird hier explizit betont, während die Normalität der Heterosexualität als selbstverständlich erscheint. Zugleich wird damit Heterosexualität, im Gegensatz zur Homosexualität und Transsexualität, implizit als „,normal“, selbstverständlich und nicht erklärungsbedürftig konzipiert.

Im Gegensatz zur Universität als toleranter und offener Raum, wird die Schule als Arbeitsfeld zum negativen Gegenhorizont. Hier ist nicht so ein offener Umgang mit der eigenen Homosexualität, wie an der Universität möglich:

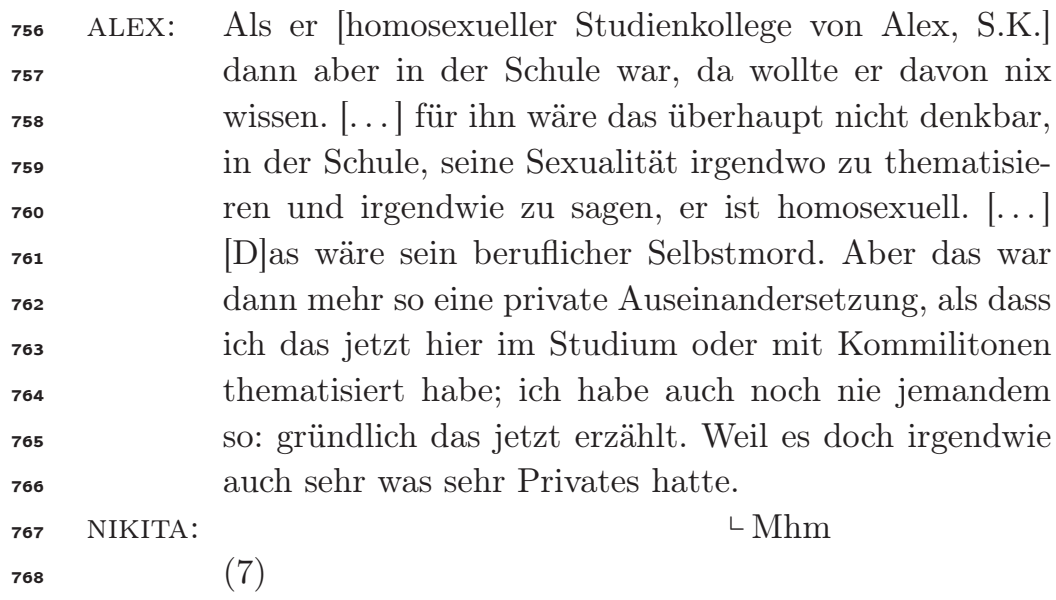

In Abgrenzung zum Arbeitsfeld der Schule wird die besondere Stellung der Universität deutlich. Was an der Universität positiv erscheint, kann 
im Arbeitsfeld zum „,beruflichen Selbstmord“ führen, hier gelten andere Bewertungs- und Handlungsschemata. Damit wird der Geltungsbereich des toleranten und offenen Umgangs mit Homosexualität auf die Universität eingeschränkt. Zudem werden die Probleme, die das Abweichen von der heterosexuellen Norm betrffen, als Privatsache und individuelles Problem positioniert, indem diese als etwas ,sehr Privates" dargestellt werden. Negative Erfahrungen und Probleme werden zur Privatsache erklärt und dadurch die realen strukturellen Probleme sowie Ungleichheitsstrukturen verdeckt. Auf der einen Seite wird in dieser Passage der tolerante und offene Umgang mit Homosexualität an der Universität hervorgehoben und zum anderen wird deutlich, dass diese Orientierung nicht in allen gesellschaftlichen Bereichen Gültigkeit hat. Durch die Individualisierung und Verdeckung der Probleme bleiben zum einen die Brüche zwischen rhetorischer Modernisierung, dem ,neuen, modernisierten' Geschlechterwissen und der nach wie vor herrschenden heterosexuellen Geschlechternorm verdeckt, und gleichzeitig wird das kommunikativ-generalisierende Wissen zur Gleichheit und Gleichstellung weiter tradiert. In Abgrenzung zum Arbeitsfeld der Schule wird in dieser Passage deutlich, dass die Toleranz und der offene Umgang mit Homosexualität Teil einer universitären/pädagogischen Orientierung ist. Außerhalb des universitären, erziehungswissenschaftlichen Feldes wird Homosexualität nach wie vor tabuisiert.

\subsubsection{Die binäre Geschlechternorm und das, tolerante und modernisierte‘ Geschlechterwissen}

Wie bereits deutlich wurde, werden die Geschlechterbinarität und die damit verbundenen Geschlechterverhältnisse implizit wie auch explizit als Norm formuliert. In der folgenden Passage wird die binäre Geschlechternorm deutlich, indem Transsexualität von den GDT als Gegenhorizont herangezogen wird. Hier wird auch der Anspruch einer toleranten Haltung gegenüber Transsexualität formuliert, jedoch zeigt sich gleichzeitig, dass dieser Anspruch eine Vorstellung bleibt und nicht Teil des handlungsleitenden Wissens ist.

NIKITA: Ich denke gerade mit diesen Zwischengeschlechtlichen, da sind wir einfach schon selbst so geprägt. Also ich weiß, ich bin letztens durch die Uni hier gelaufen, und da ist mir jemand entgegengekommen, der hatte eine 
blonde Perücke auf, aber war wohl eigentlich geboren ein Mann, ja? Und da bin ich auch mit noch zwei anderen, und natürlich, wir haben uns alle umgedreht und haben uns gefragt, war das jetzt eine Frau oder ein Mann, ja? Und waren total, also ich glaube, man ist da selbst so, dass man ohne dass man es eigentlich möchte, dann doch hinguckt, ja? Und sich irgendwie fragt, und das einfach nicht als normal irgendwie ansehen kann, ja? Also dass wir kein Problem damit haben, klar, aber es ist trotzdem so, dass man geprägt ist und das dann vielleicht doch hinterfragen sollte, warum das so ist, ja?

ALEX: Ja, ich würde das, ich würde dir das wirklich bestätigen; also es ist vielleicht sogar so eine so eine Diskrepanz, die man dann selber wahrnimmt, dass man so sagen wir mal wie so eine Stimme im Kopf hat, die einem sagt: „Ja eigentlich musst du jetzt tolerant sein, und eigentlich musst du ja wissen, dass es das alles gibt, und das ist alles völlig okay; das ist alles völlig normal", und gleichzeitig merkst du aber, dass es sofort, also noch bevor du irgendwie wirklich denken kannst, irgendwelche Reaktionen in dir gibt, auf Dinge, die du da siehst, ja?

NIKITA: $\llcorner$ Ja.

ALEX:

$\llcorner$ Eben so was.

NIKITA:

$\llcorner$ Ja.

ALEX: Mit Perücke, oder auch andere Sachen; also diese Reaktion auf Geschlecht, und auf diese Darstellung oder auch diese Erwartungen, die ich habe und und wie die sich bestätigen oder nicht ganz schnell passiert, ja? Und dann irgendwie vielleicht oben so ein kleiner Zensor sitzt @der@ dann sagt:

NIKITA:

ᄂ Ja.

ALEX: ,So soll man aber nicht." 
Nikita erzählt von einer Begegnung mit einer trans-/queergender ${ }^{19}$ Person. „[O]hne dass man es eigentlich möchte“ und auch wenn man ,kein Problem damit ha[t]", errege Transgeschlechtlichkeit Aufmerksamkeit. In ihrer Reaktion auf diese queerperson und dem Impuls, eine eindeutige und kohärente Geschlechtlichkeit zu identifizieren, macht sie deutlich, dass dies nicht der Norm entspreche und es ,nicht als normal irgendwie an[ge]sehen" werde. Auf einer reflexiven Ebene gibt Nikita jedoch zu bedenken, dass man ,vielleicht doch hinterfragen sollte, warum das so ist". Auch Alex bestätigt diese Reaktion und diese Überlegungen und arbeitet sie weiter aus. Alex betont die Diskrepanz zwischen dem, wie man tatsächlich reagiert, „,[man] dann doch hinguckt", und wie man reagieren sollte: „[E]igentlich musst du jetzt tolerant sein."

Alex und Nikita beschreiben hier den Bruch zwischen dem common sense („das ist alles völlig normal“) und dem handlungsleitenden Wissen (,,bevor du irgendwie wirklich denken kannst, [merkst du, dass es] irgendwelche Reaktionen in dir gibt, auf Dinge, die du da siehst"). Letzteres ist nach wie vor von der binären und kohärenten Geschlechtlichkeit als Orientierungsmuster bestimmt. Dass diese Toleranz und Offenheit nicht Teil der Handlungsebene ist, bringt Alex sehr bildlich zum Ausdruck. Sie spricht von einem ,kleine[n] Zensor“" im Kopf, der darauf hinweise, wenn man nicht so handle, wie man sollte. Der Anspruch einer toleranten Haltung gegenüber Transsexualität scheint eine Selbst- wie auch Fremdzuschreibung zu sein, die Teil eines ,neuen, modernisierten' Geschlechterwissens zu sein scheint und im Widerspruch zur handlungsleitenden Geschlechternorm steht. Zugleich wird durch den „kleine[n] Zensor“" auch der normative Druck sichtbar, gemäß des ,neuen, modernisierten' Geschlechterwissens zu handeln bzw. dieses rhetorisch zu reproduzieren. Eine tolerante Haltung gegenüber Transsexualität ist Teil des ,modernisierten' rhetorischen Geschlechterwissens, welches die Persistenz der eindeutig binären Geschlechternorm verdeckt. Das Geschlechterwissen ist zwar weiterhin von heteronormativem Wissen geprägt, erweist sich jedoch in dem Sinne als modernisiert, als es von einer offenen und toleranten Haltung gegenüber Homosexualität und Transsexualität gekennzeichnet ist. Zu dem ist es von der

19 Im Weiteren werden die Begriffe Transgender, Transperson(en) oder queergender, queerperson(en) verwendet - auch wenn die GDT nicht diese Bezeichnungen benutzen - da damit ,alle möglichen Formen der Trans-, Mehr- oder Zwischengeschlechtlichkeit" (Funk 2002, S. 391) Beachtung finden. Zudem wird damit der optionale Widerstand von Transgender gegenüber der Binarität von ,Geschlecht bzw. einer kohärenten, lebenslang stabilen Geschlechtsidentität angesprochen (vgl. Götsch 2012, S. 130). 
Vorstellung bestimmt, dass Frauen und Männer in ihrem Maß an Selbstbestimmung, Autonomie und Gleichheit teilhaben und gleichberechtigt sind. Dies sind jedoch nur rhetorische Bekundungen und Lippenbekenntnisse, da in der sozialen Praxis und im handlungsleitenden Wissen weiterhin die Ungleichheit und Differenz zwischen den Geschlechtern konstant bleibt.

Die Universität als soziales Feld der Toleranz und Offenheit repräsentiert jedoch nicht die gesamtgesellschaftlichen Denk- und Handlungsschemata. Nikita elaboriert in der folgenden Erzählung, dass in anderen Orten und Feldern (visuelle) geschlechtliche Eindeutigkeit und Kohärenz gefordert wird. Sie erzählt von ihren Erfahrungen als LeichtathletikTrainerin und beschreibt die sozialen Zwänge zur vermeintlich offensichtlichen geschlechtlichen Kohärenz, als eindeutig weiblich oder männlich zu gelten. In dieser Passage wird deutlich, wie ,Geschlecht' in alltäglichen sozialen Interaktionen mit dem körperlichen Auftreten einer Person sichtbar wird bzw. als eindeutig sichtbar verlangt wird. Damit wird bereits angedeutet, dass Geschlechterwissen auch ein körperliches und inkorporiertes Wissen ist.

${ }_{944}$ NIKITA: Und ja, also ich gebe ja Leichtathletik-Training, und an

945

946

947

948

949

950

951

952

953

954

955

956

957

958

959

960

961

962

963 Wettkämpfen, da sind halt meistens schon die gleichen Kinder, weil das ja ein Kreis ist, und da gibt es auch zwei Jungen, in der Altersklasse sieben Jahre und acht Jahre oder so, und die haben beide lange Haare, lange blonde Haare. [...] Und die werden bei jedem Wettkampf von den Erwachsenen gefragt, ob die vielleicht in der falschen Gruppe sind. Ich finde das immer ganz schlimm. [...] [A]ber denke mir dann auf der anderen Seite auch, vielleicht wäre das für sie einfacher, auch wenn es sehr schlimm ist, dass es so ein Thema wird und so gefragt wird, wenn die Eltern nicht einfach irgendwie doch die Haare kurz schneiden würden, weil ich das Gefühl habe, die werden damit bestimmt ja auch belastet. Weil die das permanent gefragt werden, und die Erwachsenen sagen dann zu denen,

ALEX:

$\llcorner$ Sind die es? Sind die belastet? Also sagen die dann, öh das passiert ja andauernd"? Oder ist das für die so: "Haha, wieder jemanden ausgetrickst"? 

selbst noch nie so wirklich mitbekommen; [...] aber ich krieg das mit, bei jedem Wettkampf wird darüber geredet, und es ist ja eigentlich schlimm, die wissen ja wohl, wo sie stehen und dass sie Jungs sind, aber das ist im Prinzip genau die gleiche Frage, um die es da immer geht, also jedesmal.

Im Gegensatz zur Universität wird im sportlichen Wettkampf von den Teilnehmer_innen geschlechtliche Konformität im Sinne einer eindeutig erkennbaren Geschlechtszugehörigkeit gefordert. Nikita berichtet davon, dass ,zwei Jungen, in der Altersklasse sieben Jahre und acht Jahre“ aufgrund ihrer Frisur (,beide [haben] lange Haare; lange blonde Haare“) bei Wettkämpfen von den Erwachsenen immer wieder gefragt werden, „ob die vielleicht in der falschen Gruppe sind“. Das Reglement der Geschlechtertrennung im Wettkampf scheint hier im Sinne der ,institutionellen Reflexivität" (Goffmann 1994, S. 139) die Anordnung der Geschlechter zu bestimmen, in Übereinstimmung mit den gesellschaftlich konstruierten und zugeschriebenen weiblichen und männlichen Geschlechtsmerkmalen. Durch die Intervention der Erwachsenen wird deutlich, dass diese Jungen in der Außenwahrnehmung für die Erwachsenen nicht eindeutig männlich erscheinen, dies aber von ihnen verlangt wird. An diesem Beispiel wird die normative Orientierung am Zwei-Geschlechter-Modell und dessen Ausschließlichkeit deutlich. Zudem zeigt sich der soziale Zwang und Druck, eindeutig männlich oder weiblich zu erscheinen. Nikita erzählt, dass diese Situation für sie selbst ,immer ganz schlimm“ sei und sie auch annehme, dass dies die betroffenen Jungs „belastet“. Als nicht eindeutig männlich oder weiblich identifizierbar zu sein, stellt für Nikita einen negativen Horizont im Vergleich zur unausgesprochenen und selbstverständlichen Geschlechterdifferenzierung dar. In der Anpassung an die Geschlechternorm, in diesem Fall an die männliche Norm, sieht Niktita eine mögliche Lösung dieser für sie problembehafteten Situation. Die Logik dahinter ist eine Verdeckung von Problemen, die aufgrund von nach wie vor bestehenden binären Geschlechternormen, entstehen. Indem Nikita auf die Anpassung der beiden Jungs an die herrschende Geschlechternorm und die Forderung nach eindeutig erkennbarer Geschlechtszugehörigkeit fokussiert, blendet sie eine Veränderung der Forderung der Erwachsenen aus. Somit bleiben „die“ Erwachsenen als pädagogische Subjekte der Veränderung eine Leerstelle und somit jene, denen Definitionsmacht und die Legitimität zur Erhaltung der Norm zukommt. Nikita 
hinterfragt die Forderung nach Anpassung nicht, und in dieser Reaktion wird deutlich, wie selbstverständlich diese Norm auch für Nikita selbst und Teil ihres inkorporierten Wissen ist. Ihr Geschlechterwissen folgt demnach weiterhin den binären Prinzipien, die nach Eindeutigkeit verlangen und durch die es gleichzeitig hergestellt wird.

Als positiven Gegenhorizont nennt Alex einen spielerischen Umgang mit den Geschlechternormen von Seiten der betroffenen Jungen. Sie elaboriert die These, dass sich die Jungen nicht belastet fühlen könnten, sondern Irritationen gezielt auslösen und Spaß daran haben könnten, die Erwachsenen ,ausgetrickst" zu haben. Auch wenn Nikita die „Reaktion von den [Jungen] selbst noch nie so wirklich mitbekommen" habe, greift sie diese alternative Lesart von Alex für sich nicht auf, sondern betont die Persistenz, mit der die Jungen auf ihr Abweichen von der männlichen Geschlechternorm hingewiesen werden. Dadurch wird auf die gesellschaftlich verlangte Stabilität und Persistenz der binären und kohärenten Geschlechternorm verwiesen. Geschlechtrollen und -normen werden somit nicht als reflektierbares, sondern als quasi ,naturgegebenes' Faktum verhandelt.

\subsubsection{Zusammenfassung der Gruppe Feuer}

Die Diskussion der Gruppe Feuer zeichnet sich durch ein hohes Maß an Reflexion und Selbstbezug aus. Zudem findet ein Austausch in Bezug auf die pädagogisch-praktische Erfahrung statt, der in diesem Ausmaß in keiner anderen Gruppe zu sehe ist. Die Ebene, auf der gesprochen wird, ist eine pädagogisch-professionelle, auf der die Selbst-Reflexion von Geschlechterrollen und -stereotype einen selbstverständlichen Teil einnimmt. Ein Austausch über theoretisches Geschlechterwissen (Bücherwissen) findet in dieser Gruppe im Gegensatz zu den Gruppen Holz und Metall kaum statt. Bei der Thematisierung von ,Geschlecht' orientieren sich die GDT vor allem an ihrer pädagogischen Praxis und ihrem Studium. Somit kann neben dem Studium auch die pädagogische Praxis als konjunktiver Erfahrungsraum rekonstruiert werden.

Anfangs distanzieren sich die Studierenden noch vom Thema, Geschlecht' und bringen zum Ausdruck, dass sie mit Geschlechterfragen nichts anzufangen wissen. Nach dieser rhetorischen Zurückweisung wird im Verlauf der Diskussion jedoch ihr Interesse an dem Thema deutlich und es entwickelt sich eine Diskussion, in der sie ,Geschlecht' in Bezug auf ihre persönliche Realität wie auch auf ihre pädagogische Praxis reflektieren. Das Thema wird thematisierbar/kommunizierbar, weil sie im 
Sinne von Selbstreflexion über die eigene Erfahrung sprechen. Darüber kommen sie zur Relevanz des Themas; sie kommen schließlich zu dem Schluss, dass es (doch) ein spannendes Thema ist, über das es viel zu reden gibt. In dieser Gruppendiskussion wird deutlich, dass in einem Raum, in dem kritische Reflexion Platz hat, ,Geschlecht' und Geschlechterfragen thematisiert werden.

Das Studium skizzieren Nikita und Alex als einen Ort, der für sie die Möglichkeit bot, sich erstmals mit ,Geschlecht‘ und Geschlechterfragen auseinanderzusetzen. Es wird jedoch auch deutlich, dass ,Geschlecht' kein Querschnittsthema im Studium darstellt, sondern ein Spezialthema ist, das in speziellen Seminaren behandelt wird. Zudem resümieren die GDT, dass ,Geschlecht' und Geschlechterfragen im Studium „mehr thematisiert werden“ müssten, um eine geschlechterreflektierende Haltung anzuregen. Auch in dieser Gruppendiskussion wird das Studium der Erziehungs- und Bildungswissenschaften als eine „Frauendomäne" rekonstruiert. Damit kommt jedoch nicht nur die ungleiche Geschlechterverteilung im Studium zum Ausdruck, sondern die Feminisierung einen ganzen Feldes. Verstärkt wird dies durch verallgemeinernde Aussagen, welche beispielsweise die Studenten als von der heterosexuellen Geschlechternorm abweichend konstruieren. Zusammenfassend kann auch gesagt werden, dass die binären Geschlechterverhältnisse implizit wie auch explizit als Norm formuliert werden. Im sozialen Feld des Studiums wird eine starke Toleranz und Offenheit gegenüber Homosexualität und Transsexualität formuliert, jedoch zeigt sich gleichzeitig, dass dies ein Anspruch ist, der nicht Teil des handlungsleitenden Wissens ist. Damit wird der Geltungsbereich des toleranten und offenen Umgangs mit Homosexualität auf die Universität eingeschränkt. In Abgrenzung zum Arbeitsfeld der Schule wird die besondere Stellung des Studiums deutlich. Die Toleranz gegenüber Homosexualität und Transsexualität lässt sich als Teil einer heterosexuellen und pädagogisch-universitären Orientierung rekonstruieren.

Die GDT gehen von einer Gleichheitsnorm und einem toleranten, offenen und modernisierten Geschlechterwissen aus. Die Geschlechter werden in dem Sinne als gleich gesehen, weil sie vermeintlich über dieselben Möglichkeiten verfügen. Es wird vorausgesetzt, dass Männer und Frauen den gleichen Zugang zum Arbeitsmarkt hätten und beide Geschlechter könnten, wenn sie (nur) wollten, alles erreichen und jeden Weg für sich wählen. Diese Gleichheitsnorm verdeckt, dass die Differenzierung nach ,Geschlecht' im handlungsleitenden Wissen nach wie vor eine Rolle spielt. Das heißt, ,Geschlecht' stellt nach wie vor ein Ordnungs- und Struktur- 
merkmal dar, auch wenn dieses durch die Gleichheitsnorm verdeckt wird. Die rhetorische Beteuerung und Inszenierung der Geschlechtergleichheit ist somit ein Motiv, um die Wirksamkeit der Kategorie ,Geschlecht' zu neutralisieren und zu verdecken. Auch wenn auf der Handlungsebene die herkömmlichen Differenzen und Hierarchien reproduziert werden, sind sie nicht mehr thematisierbar. So lässt sich mit dieser Gruppendiskussion Angelika Wetterers These bestätigen, „dass zeitgenössisches Differenzwissen und soziale Praxis nicht mehr bruchlos zusammenpassen" (Wetterer 2003, S. 290). (Implizite) Vorstellungen bzw. das inkorporierte Wissen von Geschlechterdifferenz und -hierarchie treffen dabei auf explizit gemachte Vorstellungen von Egalität und Individualität.

\subsection{Komparative Analyse: Gruppenübergreifende Orientierung an der Universität Sonne}

In der komparativen Analyse, d. h. im Vergleich zwischen den Orientierungen der Gruppe Holz und der Gruppe Feuer, den beiden Gruppen aus der Universität Sonne, zeigen sich hinsichtlich der (De-)Thematisierung von ,Geschlecht' und Geschlechterfragen Gemeinsamkeiten wie auch Unterschiede. Im Vergleich fällt zunächst auf, dass in beiden Gruppen der weiblich dominierte konjunktive studiumsbezogene Erfahrungsraum eine große Rolle spielt. In der Gruppe Feuer bildet auch die Erfahrungen der Studierenden in der pädagogischen Praxis einen konjunktiven Erfahrungsraum. In der Gruppe Holz wird der studiumsbezogene konjunktive Erfahrungsraum deutlicher als in der Gruppe Feuer vom konjunktiven Erfahrungsraum des neuen Geschlechtervertrags, wie ihn Angela McRobbie diagnostiziert, überlagert. Demnach seien feministische Interventionen und Kritik an (patriarchalen) Herrschaftsverhältnissen nicht mehr notwendig und die Geschlechterverhältnisse werden nicht mehr als strukturelle Hierarchien begriffen, sondern als private Arrangements (vgl. McRobbie 2010). In der Gruppe Holz lassen sich somit unterschiedliche Orientierungen hinsichtlich der Relevanz von ,Geschlecht' und Geschlechterfragen rekonstruieren, den TeilnehmerInnen gelingt es kaum, sich auf gemeinsame Themen einzupendeln bzw. eine gemeinsame Position zu finden. Daher sinds im Gegensatz zur Gruppe Feuer ,Geschlecht und Geschlechterfragen in der Gruppe Holz kein Thema, das auf kollektive Wissensbestände oder ein kollektives Reflexionsinteresse stößt. 
Während in der Gruppe Feuer ein inkludierender Diskurs in Form eines parallelen Modus geführt wird, ist die Diskussion der Gruppe Holz von einem divergenten Diskurs und durch Rahmeninkongruenzen geprägt. Auch wenn in der Gruppe Holz eine Teilnehmerin immer wieder versucht, ,Geschlecht' und Geschlechterfragen bzw. die Kritik an einer androzentrischen Wissensvermittlung zum Thema zu machen, kommt es zu keiner gemeinsamen Bearbeitung des Themas, da zum einen das Bewusstsein und das Interesse, sich mit Fragen zu ,Geschlecht' und Geschlechterverhältnisse auseinandersetzen, bei den befragten StudentInnen sehr divers ist; zum anderen kommt es zu einer Falschrahmung, die verdeutlicht, dass die (De-)Thematisierung und die Verdeckung von ,Geschlecht' und Geschlechterfragen Teil der studentisch-habituellen Praxis der GDT der Gruppe Holz sind.

In der Gruppe Holz wird im Gegensatz zur Gruppe Feuer deutlich, dass die Studierenden über keine etablierte Diskussionsform und -kultur zum Thema ,Geschlecht' verfügen. Im Gegensatz dazu wird in der Gruppe Feuer ,Geschlecht' nach einer vorläufigen rhetorischen Zurückweisung im Verlauf der Diskussion das Interesse an dem Thema deutlich und es entwickelt sich eine Diskussion, in der ,Geschlecht' und Geschlechterfragen in Bezug auf die persönliche Realität der GDT wie auch auf ihre pädagogische Praxis reflektiert werden. Die Gruppe Feuer kommt schließlich zu dem Schluss, dass es (doch) ein spannendes Thema sei, über das es viel zu reden gebe. In dieser Gruppendiskussion wird deutlich, dass in einem Raum, in dem kritische Reflexion Platz hat, ,Geschlecht thematisiert wird. In der Gruppe Feuer wird eine Thematisierung und Auseinandersetzung mit ,Geschlecht' und Geschlechterfragen befürwortet, die die individuelle, persönliche Lebensrealität reflektiert und eine theoretisch-abstrakte Ebene inkludiert.

Gemeinsam ist den beiden Gruppen, dass die binären Geschlechterverhältnisse implizit wie auch explizit als Norm formuliert werden und die GDT ihre eigene heterosexuelle vergeschlechtlichte und vergeschlechtlichende Position nicht hinterfragen. In der Gruppe Holz wie auch in der Gruppe Feuer wird das Studium der Erziehungs- und Bildungswissenschaften als eine „Frauendomäne“ und als ein feminisiertes Feld rekonstruiert. Verstärkt wird dies durch verallgemeinernde Aussagen hinsichtlich einer möglichen homosexuellen Orientierung von männlichen Studierenden. Bei der Thematisierung der sexuellen Orientierung von Studenten der Erziehungs- und Bildungswissenschaften wird in der Gruppe Feuer die Universität als toleranter und offener Raum dargestellt, während die Gruppe Holz durch Spekulationen über die sexuelle Orientie- 
rung der Studenten diese als von der heterosexuellen Geschlechternorm abweichend konstruiert.

In der Gruppe Holz wird als ungewöhnlich charakterisiert, dass der Fachbereich Erziehungs- und Bildungswissenschaften an der Universität Sonne von einer Dekanin geleitet wird; Leitungs- bzw. Führungspositionen werden dabei implizit als typisches Arbeitsfeld von Männern angesehen. In unterschiedlicher Weise reproduzieren beide Gruppen traditionelle Rollenbilder und charakterisieren bestimmte Bereiche als weiblich oder männlich. In der Gruppe Holz wird beispielsweise die Kinderbetreuung als weibliche/mütterliche Aufgabe diagnostiziert und Männer werden von Kinderbetreuungsaufgaben ausgenommen.

Die Orientierung an der Gleichstellungsnorm der Geschlechter wird in beiden Gruppen reproduziert und die Relevanz von ,Geschlecht' und Geschlechterfragen (punktuell) rhetorisch entdramatisiert. Diese Gleichheitsnorm verdeckt, dass die Differenzierung nach ,Geschlecht' im handlungsleitenden Wissen nach wie vor eine Rolle spielt. Zudem ist die Geschlechtergleichheit ein Motiv, um die Wirksamkeit der Kategorie ,Geschlecht' zu neutralisieren und zu verdecken. Im Vergleich der beiden Gruppen zeigt sich, dass in der Gruppe Feuer ,Geschlecht' und Geschlechterfragen nicht mit Frausein und ,Frauenproblemen' gleichgesetzt werden; dies scheint die Diskussion anzuregen, wohingegen die Gleichsetzung von ,Geschlecht' mit Frausein und Geschlechterfragen mit ,Frauenfragen' die Diskussion der Gruppe Holz zu behindern scheint. Vor dem Hintergrund der Annahme fast aller TeilnehmerInnen der Gruppe Holz, dass feministische Interventionen und Kritik an (patriarchalen) Herrschaftsverhältnissen nicht mehr notwendig sei und ad acta gelegt werden können, da die Gleichstellung der Geschlechter bereits erreicht sei, ist dies besonders interessant und legt den neuen Geschlechtervertrag als konjunktiven Erfahrungsraum nahe. Dieser konjunktive Erfahrungsraum und die Orientierung der Studierenden am Gleichheitspostulat und an gesellschaftlichen Modernisierungsprozessen scheint eine Diskussion und eine Auseinandersetzung mit ,Geschlecht' und Geschlechterfragen in der Gruppe Holz zu verhindern.

Auch hinsichtlich geschlechterbezogener Benachteiligungs- und Diskriminierungserfahrungen verfügt die Gruppe Holz über keine kollektive Diskussionskultur. Das von einer Teilnehmerin eingebrachte Thema wird von den Studierenden nicht aufgegriffen und es kommt zu einer De-Thematisierung von geschlechterbezogener Benachteiligung. Dies deutet darauf hin, dass die De-Thematisierung und die Verdeckung von ,Geschlecht' und Geschlechterfragen Teil der studentischen-habituellen 
Praxis ist. Zudem zeigt sich in der Gruppe Holz die Argumentationsfigur, dass wenn Frauen im erziehungswissenschaftlichen Studium nicht unterrepräsentiert sind, es für die Studierenden auch keine Benachteiligung an der Universität zu geben scheint. In Bezug auf ,Geschlecht und Geschlechterfragen findet eine Neutralisierung und Verdeckung von sozialen Ungleichheiten und deren Wirkmechanismen statt. In der Gruppe Feuer kommt es ebenfalls zu einer Beteuerungen von Gleichheit und Gleichberechtigung, jedoch hier in Bezug auf die Orientierung am generischen Maskulinum. Die Ablehnung geschlechtergerechter Sprache wird mit der Annahme begründet, dass Emanzipation und Gleichberechtigung von Frauen bereits erreicht sei.

\subsection{Die ,Ja, aber'-Mentalität der Gruppe Metall an der Universität Mond}

An der Universität Mond wurde die erste Gruppendiskussion durchgeführt. Diese Gruppe wird im Folgenden Metall genannt. Diese Diskussion erfolgte ca. fünf Monate vor der zweiten Gruppendiskussion mit der Gruppe Holz an der Universität Sonne. Die Gruppe Metall besteht aus drei Studierenden des Diplomstudiengangs Erziehungs- und Bildungswissenschaften. Sie befinden sich zwischen dem neunten und dreizehnten Studiensemester und sie sind somit eher am Ende ihres Studiums. Zwei der beteiligten Personen sind weiblich, eine männlich. ${ }^{20}$ Alle drei TeilnehmerInnen sind Mitte zwanzig und kinderlos und niemand von ihnen gibt für sich einen Migrationshintergrund an. Eine Teilnehmerin lebt in einer festen Partnerschaft. Die Personen kennen sich vom Studium, da sie zum Teil dieselben Seminare besucht haben. Die Gruppe Metall stellt somit eine homogene Gruppe dar. Dies zeigt sich auch in den gewählten Schwerpunkten im Studium: Hier haben alle drei Erwachsenenbildung und außerschulische Jugendarbeit gewählt. Alle drei GruppendiskussionsteilnehmerInnen haben in ihrem Haupt- oder Nebenfach Seminare zu ,Geschlecht‘ und Geschlechterfragen besucht. Zudem haben zwei der TeilnehmerInnen das zertifizierte Studienprogramm des Zentrums für Gender Studies an der Universität Mond abgeschlossen. In dieser Gruppendiskussion diskutieren drei Studierende, die ,Geschlecht' als ein relevantes Thema ansehen und sich bereits im Laufe ihres Studiums damit auseinandergesetzt haben.

20 Neele: w, 24 Jahre, 9. Semester; Michi: w, 26 Jahre, 11. Semester; Jona: m, 27 Jahre, 13. Semester. 
Es soll voraus geschickt werden, dass die Diskussion trotz, oder gerade wegen dieser Erfahrung der GDT zum Teil recht diffus, verwirrend und schwer durchschaubar wirkt und vor allem die Thematisierung von ,Geschlecht" und Geschlechterfragen im universitären Kontext verhandelt wird. Insbesondere erschweren die sprunghaften Themenwechsel und die überkomplexe Darstellung von ,Geschlecht' und Geschlechterfragen die Interpretation. Aufgrund dessen erfolgt die Rekonstruktion der Orientierung der Gruppe Metall nicht ausschließlich dem Verlauf der Gruppendiskussion folgend, sondern auch nach themenbezogenen Kriterien. ,Geschlecht' wird in dieser Gruppendiskussion als ein janusköpfiges Thema besprochen, es erscheint den GDT als ,interessant" und „schwierig“" zugleich. In Bezug auf die Relevanz von ,Geschlecht' zeigt sich eine ,Ja, aber'-Mentalität und eine Kritikaffinität gegenüber der studiumsbezogenen Thematisierung von ,Geschlecht'. Sie befürworten das Thema zwar, betonen aber die negativen Aspekte der Thematisierung. In der Diskussion wird deutlich, wie schwierig es für die GDT ist, eine gemeinsame Position zu finden; sie ringen förmlich mit dem Thema und im Laufe der Diskussion wird die Thematisierung von ,Geschlecht' und Geschlechterverhältnissen immer heterogener und komplexer. Die Thematisierung von ,Geschlecht' lässt sich in dieser Gruppe als eine Suchbewegung nach Positionen, Lösungen und kollektiven Orientierungen charakterisieren. Im Folgenden sollen die zentralen Orientierungen der Gruppe Metall anhand von Auszügen aus der Diskussion rekonstruiert werden, zudem soll es darum gehen, Zusammenhänge zwischen der Themenwahl und den konjunktiven Erfahrungsräumen der Teilnehmer_innen herauszuarbeiten.

\subsubsection{Kritik an der studiumsbezogenen Thematisierung von ,Geschlecht"}

Auch in dieser Gruppe wurde die Diskussion zum Thema ,Geschlecht von der Gruppendiskussionsleiterin initiiert. Somit handelt es sich zuerst um ein Interesse der Forscherin und um einen Diskurs, der heteronom produziert wurde. Dieser wurde von den GDT augegriffen und es entsteht ein autonomer Diskurs zu ,Geschlecht' und Geschlechterfragen unter den GDT. Im Gegensatz zur Gruppe Holz wird deutlich, dass die Thematisierung von ,Geschlecht' und Geschlechterfragen eine Dimension der studiumsbezogenen konjunktiven Erfahrung im sozialen Feld der Bildungsinstitution bildet, sie stellt für die GDT eine gemeinsame Erfahrung und eine kollektive Orientierung dar. Darüber hinaus vermittelt 
dieser Einstieg bereits einen ersten Eindruck von der Diskursorganisation. In dieser Gruppendiskussion wird vor allem die Thematisierung von ,Geschlecht' und Geschlechterfragen im universitären Kontext kritisch hinterfragt. Im folgenden Abschnitt wird diese ,Ja, aber'- Mentalität und die Kritikaffinität der Studierenden deutlich.

\section{Kritik an einer einseitigen und weiblich dominierten Thematisierung von ,Geschlecht"}

Jona berichtet als erster von seinen Erfahrungen und nennt Seminare, in denen es ,auch um gender" ging. Er betont seine Erfahrungen und seine Kenntnisse und bringt zudem seine Kritik an der Verhandlung des Themas ,gender“ im Studium zum Ausdruck.

301

302

303

304

305

306

307

308

309

310

311

312

313

314

315

316

317

318

319

320

321

322

323

324

JONA:@Ja:a@; also es gab explizite Seminare in der Pädagogik in den Veranstaltungskalender sozusagen es gab Seminare zu Mädchenbildung, zu geschlechterdifferenzierter, Pädagogik, zu Sexualpädagogik, wo es dann auch um gender ging; da hab ich viel besucht, [...] also bei den männlichen Kollegen des Fachbereichs fand nichts dazu statt, obwohl sich jetzt z. B. Herr [Name des Professors, S.K.] mit Jungenarbeit beschäftigt hat, oder auch mi:it männlicher Sozialisation, aber es gab in meiner Zeit hier kein Seminar zum Beispiel dazu, was ich sehr schade finde, [...] und dann war ich immer in Veranstaltungen, wo es um weibliche Sozialisation ging wo die weiblichen Kolleginnen sehr viel Wert darauf legten, wobei der Bereich Jungen für mich zu ausgeklammert wurde, wo ich mir dachte, nee, das kann jetzt auch nicht sein, das ist mir wieder zu einseitig, (3) also eher dann in expliziten

MICHI:

$\left\llcorner{ }^{\circ} \mathrm{mhm}^{\circ}\right.$

JONA: Lehrveranstaltungen und gerade halt vor allem natürlich von [Name von zwei Professorinnen, S.K.] würde ich sagen, als die zwei Hauptvertreterinnen und

MICHI:

$\left\llcorner{ }^{\circ} \mathrm{Mhm}^{\circ}\right.$

JONA: dann [Name einer Professorin, S.K.] mit Mädchenbildern, aber auch Medienzeugs

MICHI:

$\llcorner\mathrm{mhm}, \mathrm{ja}$ 


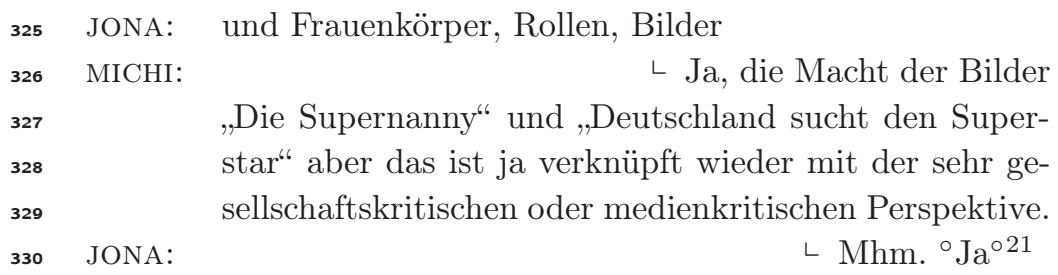

Jona beginnt hier damit, ,Seminare in der Pädagogik“ aufzuzählen, in denen ,Geschlecht' und Geschlechterfragen „explizit" thematisiert wurden. Indem Jona hier von ,gender" spricht und erklärt, dass er viele Seminare besucht habe, markiert er sein Expertenwissen und seine Auseinandersetzung auf einer theoretischen Ebene. Als negativen Aspekt bei der Thematisierung von „,gender“ beschreibt er die Abwesenheit von „männlichen" Professoren und männer-/jungenspezifischen Inhalten. Jona hebt hervor, dass das Thema in der Lehre nicht von den „männlichen Kollegen des Fachbereichs" abgedeckt werde, obwohl z. B. ,Jungenarbeit" auch der Schwerpunkt eines Professors sei. In der Lehre werden Seminare zu Geschlechterfragen anscheinend nur von Frauen angeboten. Es hat den Anschein, als wäre die Thematisierung von, Geschlecht' eine ,Frauen-Sache'. Jona bedauert dies und findet es „einseitig“, wenn „der Bereich Jungen [... zu ausgeklammert“ werde und in einer Lehrveranstaltungen nur „die weibliche Sozialisation" im Zentrum stehe. Er beschreibt die Thematisierung als einseitig und kritisiert die auf weibliche Lebenslagen fokussierten Inhalte weiblicher Dozentinnen. Es ist anzunehmen, dass Jona das Fehlen männlicher Perspektiven im Studium generell bemängelt. Michi stimmt ihm in seiner Kritik zu, woraufhin Jona wiederum zwei weibliche Professorinnen als „Hauptvertreterinnen“ für Geschlechterfragen hervor hebt, um die weibliche Dominanz nochmals zu bestätigen. Michi und Jona teilen die Erfahrung, dass ,Geschlecht' und Geschlechterfragen im Studium der Erziehungs- und Bildungswissenschaften ,weiblich' dominiert seien Frauen sprechen über weibliche Sozialisation, Mädchenbilder, Frauenkörper, -rollen und -bilder - Männer bzw. auf männliche Lebenslagen ausgerichtete Inhalte bilden eine Leerstelle. Als positiven Aspekt hebt Michi jedoch hervor, dass in den Seminaren ,Geschlecht" und Geschlechterfragen zumindest mit einer ,gesellschaftskritischen oder medienkritischen Perspektive" verknüpft sind. Bereits hier deutet sich der gesellschaftskritische und hinterfragende Impetus bei der Themati-

21 Die zitierten Passagen beginnen mit der jeweiligen Originalzeilennummerierung, und weichen im Verlauf der Passagen, z. B. aufgrund von gekennzeichneten Auslassungen davon ab. 
sierung von ,Geschlecht' als positiver Horizont an. Auch Neele bestätigt zu einem späteren Zeitpunkt in der Gruppendiskussion die Einschätzung einer weibliche Dominanz: ,[A]lso manchmal liegt mir, wie du schon gesagt hast, der Fokus zu sehr auf dem Weiblichen." In Bezug auf die Thematisierung von ,Geschlecht" im Curriculum lässt sich rekonstruieren, dass diese als, „einseitig“6 wahrgenommen wird. Damit beziehen sich die GDT auf einen, weiblichen' Fokus auf Seiten der Lehrenden und der Lehrveranstaltungsinhalte und auf die marginale Stellung männlicher Lebenswelten und Akteure. Als positiver Horizont wird von Michi die gesellschaftskritische und medienkritische Perspektive in Verbindung mit den Geschlechterfragen dargestellt. Gleichwohl die Studierenden unterschiedliche Erfahrungen damit gemacht haben, ob ,Geschlecht' und Geschlechterfragen im Studium nun als Querschnittsthema oder als Spezialthema behandelt werden, ist ihnen die Erfahrung gemeinsam, dass die Thematisierung von ,Geschlecht' , weiblich' ist und vor allem von Frauen über Frauen und Mädchen gesprochen wird.

\section{Kritik an einer populärwissenschaftlichen und undifferenzierten Thematisierung von ,Geschlecht"}

In der folgenden Passage wird die Kritik an der Thematisierung von ,Geschlecht' und Geschlechterfragen innerhalb der Universität Mond anhand eines Beispiels konkretisiert und erweitert. Jona weitet die Kritik an der Thematisierung von ,Geschlecht' aus und exemplifiziert diese anhand eines besuchten „Vortrag[s] bei den Psychologen“. Hier geht es vor allem um die Kritik an der undifferenzierten Vermittlung und der naturalisierenden Darstellung von Geschlechterdifferenzen.

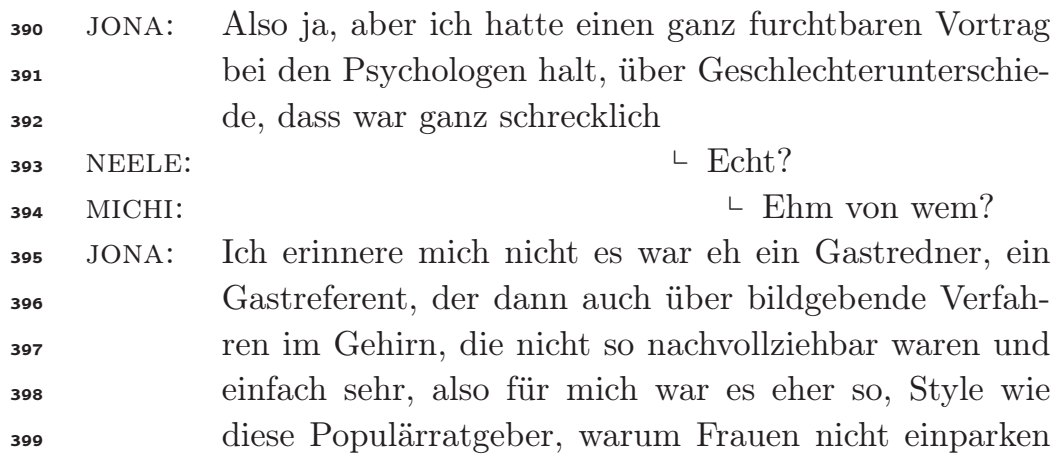




\begin{tabular}{|c|c|c|}
\hline 400 & & können, jetzt aber \\
\hline 401 & MICHI: & $\llcorner$ (stöhnt) Ja; \\
\hline 402 & NEELE: & ட (stöhnt) Ach, ja; \\
\hline 403 & JONA: & aber vielleicht auch weil ich auch geprägt bin von der \\
\hline 404 & & Soziologie hier und \\
\hline 405 & NEELE: & $\llcorner$ Mhm, ja, ja \\
\hline 406 & JONA: & ich finde so was von vornherein abstoßend, wenn man \\
\hline 407 & & überhaupt so ran geht, über biologische Determinanten- \\
\hline 408 & & aber ja, das fand ich jetzt einfach nicht so differenziert. \\
\hline 409 & MICHI: & $\left\llcorner{ }^{\circ} \mathrm{Mhm}^{\circ}\right.$ \\
\hline
\end{tabular}

Jona berichtet von einen „Vortrag“" eines Psychologen über „Geschlechterunterschiede". Bei der Passage werden gleich mehrere negative Horizonte bei der Thematisierung von ,Geschlecht' zum Ausdruck gebracht: Zum einen wird die Naturalisierung von Geschlechterunterschieden kritisiert und zum anderen wird die Psychologie als negativer Horizont darstellt. Indem Jona von einem ,furchtbaren Vortrag“" und einer ,ganz schrecklichen" Erfahrung spricht, verleiht er seiner Kritik quasi doppeltes Gewicht. Als negativ beschreibt Jona die unwissenschaftliche und undifferenzierte Darstellung des Themas. Geschlechterfragen seien in diesem Vortrag populärwissenschaftlich (,so wie diese Populärratgeber") behandelt und Geschlechterunterschiede tradiert sowie naturalisiert worden. Auch wenn Michi und Neele nicht bei diesem Vortrag waren, scheinen sie zu wissen, wovon Jona spricht und validieren ihrerseits die Ablehnung einer undifferenzierten und biologistischen Thematisierung von Geschlecht. Diese stellt für die GDT einen negativen Horizont dar und bildet zugleich eine kollektive Orientierung. Als positiven Gegenhorizont nennt Jona die Soziologie, dies wird auch von Neele validiert. Es kann an dieser Stelle vermutet werden, dass sich Jona und Michi in der Folge auch auf die sozial-(de-)konstruktivistischen Ansätze, in der die Tradition der Frauen- und Geschlechterforschung der Soziologie steht, beziehen. Damit validieren sie, dass die Thematisierung von ,Geschlecht' eine (relevante) studiumsbezogene, konjunktive Erfahrung darstellt.

\section{Kritik an einer möglichen Überbetonung von ,Geschlecht‘}

Neele bestätigt mit folgendem Beitrag, dass die Thematisierung eine studiumsbezogene, konjunktive Erfahrung ist. Mit dem Wort ,aber" kündigt 
Neele ihren Widerspruch gegen die bisherige implizite Annahme an, dass die Thematisierung von ,Geschlecht' omnirelevant sei:

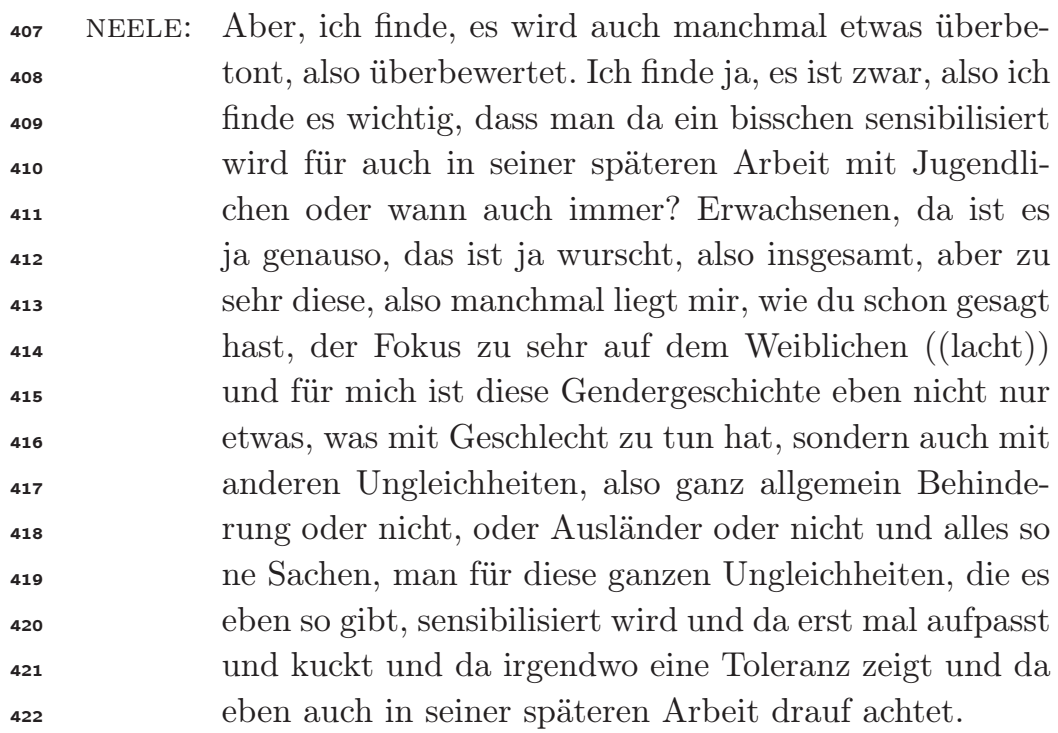

Neele betrachtet die Thematisierung von ,Geschlecht' und Geschlechterfragen als „manchmal etwas überbetont, also überbewertet“. Sie wechselt in ihrem Beitrag nun von einer konkreten, beispielhaften auf eine abstraktere Ebene. Für Neele wird ,Geschlecht' demnach zu stark betont. Sie schränkt den Geltungsbereich ihrer Aussage jedoch ein, indem sie von „manchmal" spricht und ihre Aussage als persönlich (,finde ich“) einklammert. Das lässt eine Distanzierung von Neele zum Thema vermuten, aber nicht auf eine generelle Ablehnung schließen. Diese Vermutung bestätigt sich, indem sie sagt, dass „man da ein bisschen sensibilisiert wird“ für die spätere pädagogische Arbeit. Die Kritik an einer Omnirelevanz des Themas wird darin deutlich, da sie von „ein bisschen“ Sensibilisierung spricht.

Für Neele sei zwar wichtig, dass eine Sensibilisierung für die Kategorie ,Geschlecht' stattfinde, sie schränkt diese jedoch auf ein minimales und nicht zu intensives Maß („bisschen“) ein. Zudem liege für sie „der Fokus sehr auf dem Weiblichen". Damit nimmt sie auf Jona Kritik an einer weiblichen Dominanz bei der Thematisierung Bezug. Die Sensibilisierung für ,Geschlecht' sei für die „spätere Arbeit" wichtig. Anders als Jona hebt sie jedoch nicht die marginalisierte männliche Position hervor, 
sondern betont den Zusammenhang dieser "Gendergeschichte“ mit „anderen Ungleichheiten“. Mit dem Stichwort „Gendergeschichte“ markiert sie ihre Kritik an dem Thema, da hier eine implizite Abwertung mitschwingt. Für Neele scheint ,Geschlecht' eine gesellschaftliche Differenzund Ungleichheitskategorie unter vielen zu sein, so wie „Behinderung“" oder Migration. Neben ,Geschlecht' gebe es noch andere Ungleichheiten, die für die pädagogische Arbeit wichtig seien und für die man ,sensibilisiert" werden sollte. Damit betont Neele auch die Relevanz anderer gesellschaftlicher Ungleichheiten für die „spätere Arbeit“. Dafür sollte man laut Neele auch ,sensibilisiert" sein und „Toleranz" zeigen.

An dieser Stelle ist anzunehmen, dass Neele ihre Befürchtung zum Ausdruck bringt, dass durch eine Fokussierung auf die Ungleichheitskategorie ,Geschlecht' andere Differenzlinien nivelliert werden bzw. unberücksichtigt bleiben. Da Neele ihre Befürchtung hier nicht durch ein Beispiel darstellt, scheint es sich hier wohl eher um ein Vorurteil bzw. eine Vorstellung als um eine konkrete Erfahrung zu handeln. Mit ihrem Beitrag bestätigt sie jedoch die Kritik an der Thematisierung von ,Geschlecht' als habituelle Praxis. Somit kann die Kritik an der Art der Thematisierung von ,Geschlecht' und Geschlechterfragen im universitären Feld als ein wesentliches Element des Habitus der Gruppe in Hinblick auf die Thematisierung von ,Geschlecht ${ }^{6}$ charakterisiert werden. Diese lässt sich anhand der folgenden Passagen verdichten.

\section{Kritik am als ausgrenzend und diskriminierend wahrgenommenen Diskussionsklima}

Die oben eingeführte Kritik von Jonas an einer unwissenschaftlichen und wenig differenzierten Darstellung von Geschlechterbinarität betrifft nicht nur inhaltliche Gesichtspunkte der Thematisierung, sondern auch die Diskussionskultur und das -klima im Studium bzw. im universitären Kontext. Im folgenden Auszug der Gruppendiskussion bestätigt Michi in ihrem Beitrag auch Neeles Proposition, dass das Thema manchmal überbewertet und überbetont werde. Damit validiert sie die Orientierung, dass die Thematisierung von ,Geschlecht' eine konjunktive Erfahrung darstellt.

Jedoch wird auch hier deutlich, dass bestimmte Formen der Thematisierung bzw. ein ausgrenzendes Diskussionsklima einen negativen Horizont der Thematisierung darstellen. 
MICHI: Was ich zum Beispiel schlimm finde ist dieses Diskussionsklima [...] ja also so dass man das Gefühl hat, es gibt, ne Gruppe von Leuten die da so extrem sind und je mehr das in die Extreme geht, egal wobei, finde ich das immer wahnsinnig anstrengend und wieder ausgrenzend, weil es ist dann irgendwie, wenn ich das Gefühl habe, so ja, wenn man sich äußern würde in die Richtung. Ich möchte aber vielleicht einfach gerne Hausfrau sein und Kinder haben, und dann irgendwie als Loser oder unemanzipiert dargestellt wird.

NEELE: Mhm:m [...]

мICHI: Auf der Uni bin ich generell für das Geschlechterthema sehr sensibilisiert worden, auch über die Sprache, dass ich darauf achte und das vor anderen auch vertrete, aber jetzt zu sagen, nur weil man auf die Sprache achtet, dann diskriminiert man weniger oder auch das Gefühl, dass, wenn man sehr auf diese feministische Sache achtet, dann diskriminiert man vielleicht an anderer Stelle, also das ist-, oder darüber hab ich mich einfach mit diesen Themen generell mehr auseinander gesetzt: Je mehr man irgendwie versucht, eine Position extrem einzunehmen, desto mehr gerät man selber in Gefahr, wieder intoleranter zu werden und das gehört zu den Themen auch.

NEELE: Mhm

Michi beginnt ihren Beitrag damit, von ihrem persönlichen Empfinden zu sprechen. Sie erlebt ein „Diskussionsklima“ als „,schlimm“, wenn „,man das Gefühl hat" es gebe eine „Gruppe von Leuten“ , die „so extrem“ sei. Michi geht es hier um das Gefühl der Ausgrenzung, das sie vermutlich schon erlebt hat. Michi möchte Personen nicht als „Loser oder unemanzipiert" betrachtet, weil sich diese einer traditionell Frauenrolle orientiert. Damit elaboriert Michi, dass sie sich gegenüber einem diskriminierenden und ausgrenzenden Diskussionsklima außerhalb von Lehrveranstaltungen abgrenzt. Sie erläutert, dass eine extreme Ansicht/Position für sie dann bestehe, wenn dadurch wieder Ausgrenzungen stattfinden, andere Positionen delegitimiert und Personen abgewertet werden. ,Extreme Positionen werden von Michi generell negativ bewertet, egal zu welchem 
Thema. Für Michi stellt eine ,extreme Position einen negativen Horizont dar, weil dadurch wieder Ausgrenzungen stattfinde. Diese Orientierung wird auch von Neele validiert.

Auch wenn Michi ,auf der Uni generell für das Geschlechterthema sehr sensibilisiert" worden sei und „auf die Sprache achtet" sowie ihre Position auch vor anderen vertrete, schließt sie aus, dass sie selbst ein ausgrenzendes Diskussionsklima schaffe. Im Gegensatz zu der „Gruppe von Leuten" habe sich Michi ,einfach mit diesen Themen generell mehr auseinander gesetzt". Mit "diesem Thema" bezieht sich Michi darauf, dass eine geschlechtergerechte Sprache und eine Sensibilisierung für das Geschlechterthema nicht davor bewahre, andere Personen zu diskriminieren. Michi warnt davor, „eine Position extrem einzunehmen“, da sie damit die Gefahr verbindet, ,intoleranter zu werden“. Anders als die von Michi angeführten ,extremen' Positionen stellt die geschlechtergerechte Sprache für sie einen positiven Horizont dar. Gleichzeitig wird in diesem Beitrag deutlich, dass das universitäre Feld für Michi ein Ort ist, der die Möglichkeit bietet, sich mit ,Geschlecht' und Geschlechterfragen auseinanderzusetzen und sie auch dazu angeregt hat. Im Beitrag von Michi zeigt sich eine eigentümliche Melange, die sich auf der einen Seite an der Relevanz der Thematisierung von ,Geschlecht' orientiert und zum anderen an der Abgrenzung von einer ,zu extremen" Thematisierung von ,Geschlecht'. Hier lässt sich die Orientierung rekonstruieren, dass die Thematisierung von ,Geschlecht' nur dann legitim ist, wenn es dabei zu keiner Diskriminierung oder Ausgrenzung kommt.

In Bezug auf ,Geschlecht` zeigt sich in der Gruppendiskussion eine ,Ja, aber'-Mentalität, die ich auch mit dem Begriff der Kritikaffinität fasse. ,Geschlecht' wird zwar als ein wichtiges Thema darstellt, allerdings wird die Relevanz limitiert und ihr Geltungsbereich eingeschränkt.

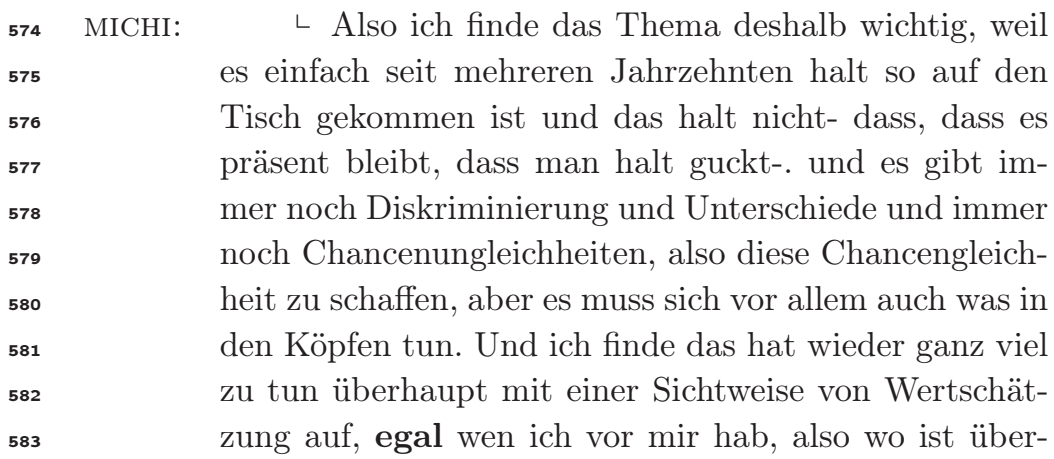




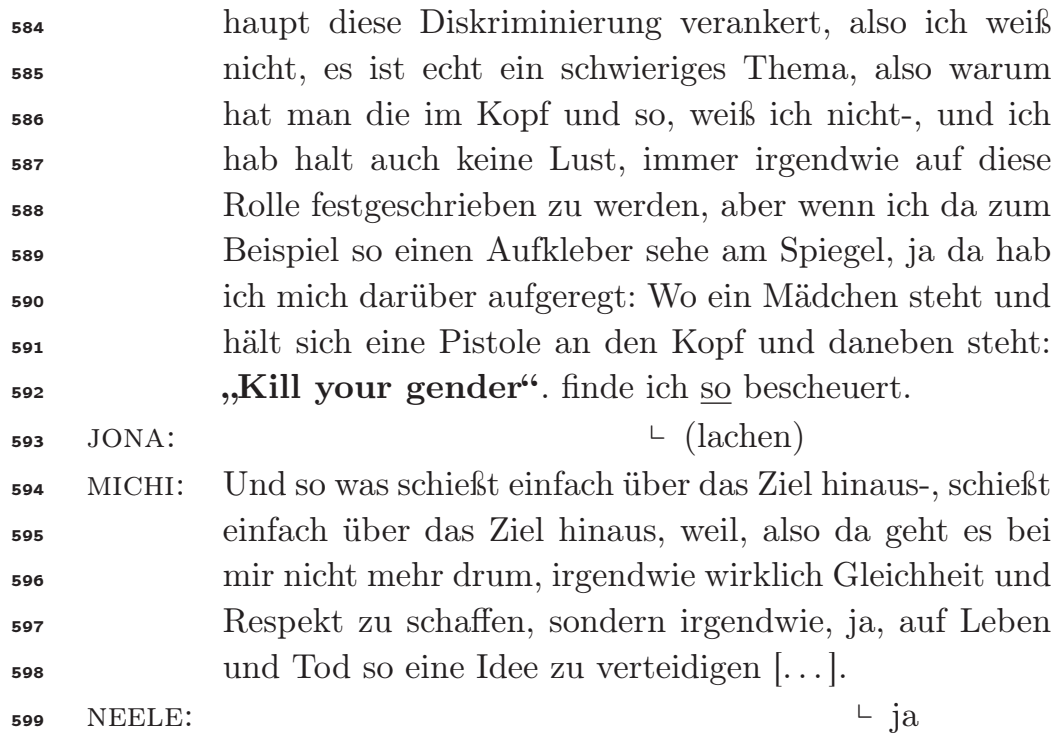

Michi beginnt ihren Beitrag damit, die Relevanz des „Geschlechterthema[s]" zu betonen. Für sie sei „das Thema" wichtig, weil es noch immer Diskriminierung, Unterschiede und Chancenungleichheiten zwischen Männern und Frauen gebe und das, obwohl „das Thema“ bereits seit „mehreren Jahrzehnten“ präsent sei. Die Schaffung von Chancengleichheit wird hier als positiver Gegenhorizont zur Persistenz von Diskriminierung und Differenzierung eingeführt. In Bezug darauf wie Chancengleichheit geschaffen werden kann, konstatiert Michi: ,[A]ber es muss sich vor allem auch was in den Köpfen tun." Michi geht davon aus, dass Chancengleichheit in erster Linie dadurch erreicht werden könne, indem sich das, „was in den Köpfen“ der Subjekte stecke, verändere. Gesellschaftliche Strukturen bleiben ungenannt. Hier zeigt sich die Tendenz einer Individualisierung struktureller Probleme (vgl. Wetterer 2003; Tübinger Institut für frauenpolitische Sozialforschung e.V. 2000). Mit ,in den Köpfen“ bezieht sich Michi vermutlich auf individuelle Handlungs- und Denkschemata und verweist auf das Veränderungspotential durch die Individuen selbst. Eine ,Sichtweise von Wertschätzung" wird von Michi als positiver Gegenhorizont zum Handlungsund Denkschema der Diskriminierung und Differenzierung dargestellt. Im wertschätzenden Umgang miteinander („,egal wen ich vor mir hab“) sieht Michi eine Möglichkeit der Veränderung und eine normative Orientierung. Allerdings konstatiert sie, dass es „echt ein schwieriges Thema“ 
sei, den Ursachen von Diskriminierung und den Rollenzuschreibungen auf den Grund zu gehen. Auch wenn sie sich an einem wertschätzenden Umgang orientiert, ist ihr Erleben davon charakterisiert, dass sie ,immer irgendwie auf diese Rolle festgeschrieben“ werde und dazu „keine Lust" habe. Hier ist davon auszugehen, dass Michi von geschlechtsbezogenen Rollenzuschreibungen spricht und es ablehnt, kontinuierlich darauf festgeschrieben zu werden. Michis Vorstellung und Wunsch nach Chancengleichheit/Geschlechtergleichheit stimmt nicht mit ihren Erfahrungen überein. Um das Ziel der "Gleichheit"/Gleichbehandlung zu erreichen, ist Michi ,aber“ nicht jedes Mittel, nicht jede Aktion recht.

Als negatives Beispiel nennt sie eine Initiative des Frauen- und Lesben-Referats an der Universität Mond: Es wurden Aufkleber an verschiedenen Orten angebracht, die zur Thematisierung und Überwindung längst überholter Stereotype und Geschlechterrollen beitragen und für neue Rollenbilder in der Gesellschaft sensibilisieren sollen. Michi beschreibt einen Aufkleber, auf dem ein Mädchen zu sehen ist, dass sich eine Pistole an den Kopf hält. Dieses Bild ist von der Aufschrift begleitet: „Kill your gender“ (siehe Bild: 2). Auch wenn sie nicht auf geschlechterstereotype Rollen festgeschrieben werden will, geht für Michi dieser Aufkleber zu weit und sie lehnt diese Initiative ab. Indem Michi diesen negativen Horizont der Thematisierung von Stereotypen, und Geschlechterrollen beschreibt, macht sie deutlich, dass bei der Thematisierung und Veränderung von Geschlechterstereoptypen und -rollen „Wertschätzung", „Gleichheit und Respekt" eine Orientierung darstellen. Diese Orientierung sieht Michi durch diese Initiative nicht gewährleistet, vielmehr sieht sie darin die Orientierung an einer kämpferischen Verteidigung einer „Idee“ „auf Leben und Tod“. Diese (radikale) Kampfhaltung stellt für sie einen negativen Gegenhorizont zu einem wertschätzenden und respektvollen Umgang miteinander dar. In den bisherigen Passagen wurde der Begriff gender an sich, aber auch in seinen Variationen wie z. B. „gendergerecht" und „Gendergeschichten“ von den GDT verwendet. Demnach scheint der Begriff gender zum geteilten Wortschatz der Studierenden und der Universität zu gehören und ist Teil ihrer konjunktiven Erfahrung.

Im weiteren Verlauf der Diskussion wird das Thema der negativen Erfahrungen mit und die Kritik an einer falschen Thematisierung von ,Geschlecht' erneut von Jona eingebracht. Hier wird jedoch die Kritik an einem diskriminierenden und ausgrenzenden Verhalten am Beispiel eines Seminars ,im Bereich interkulturelle Pädagogik“" explizit. Die Passage soll im Folgenden dargestellt und knapp zusammengefasst werden, 


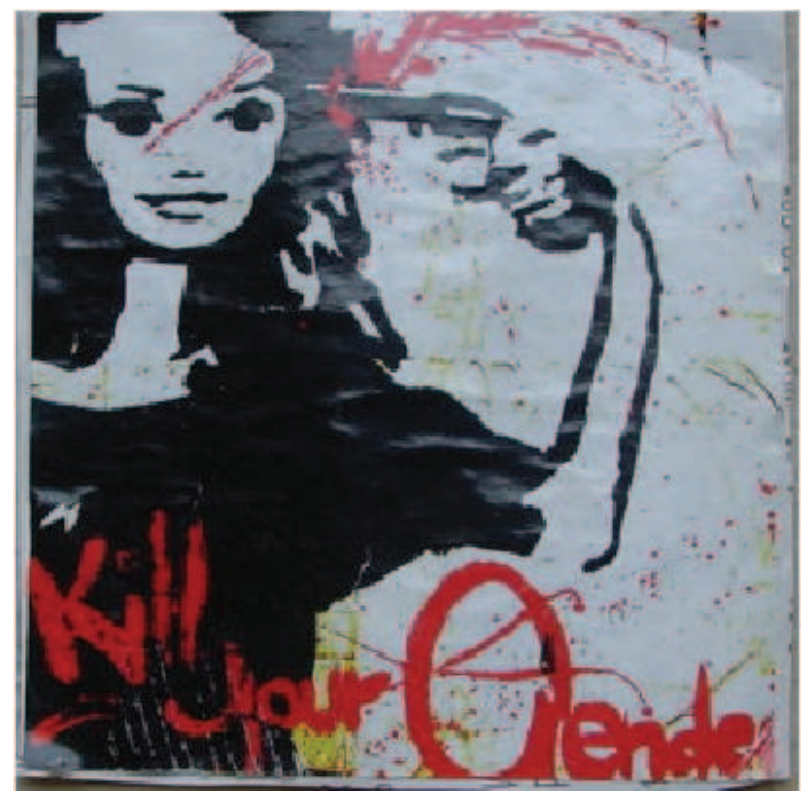

Abbildung 2: Kill your gender; Quelle: Street Art, Ber$\operatorname{lin}$ 2006, http://plusnine.twoday.net/stories/1889601/ [12.08.2012]

da sich hier die rekonstruierten Orientierungen der Gruppe Metall in Hinblick auf die normativen Erwartungen an die Thematisierung von ,Geschlecht' und Geschlechterfragen verdichten.

906 JONA: Ich erinnere mich jetzt grad an ein ganz schreckliches Seminar im Bereich interkulturelle Pädagogik bei Frau [Name der Dozentin, S.K.] [...] weil da extrem undifferenziert sowohl von den Teilnehmern als auch von ihr über die Unterdrückung der Frau im Islam geredet wurde $[\ldots]$, also ich bin dann auch wieder raus, also nach drei Sitzungen, weil ich das ganz furchtbar fand, weil dann, weil so da, weil da Kämpfe statt gefunden haben zwischen der Seminarleitung und einzelnen Muslima mit Kopftuch, die im Seminar saßen und die dann im Endeffekt rausgeschmissen wurden, weil sie 


\begin{tabular}{|c|c|c|}
\hline 917 & MICHI: & $\llcorner$ Wie die \\
\hline 918 & & rausgeschmissen wurden? \\
\hline 919 & JONA: & $\llcorner$ weil sie das nicht wissenschaftlich \\
\hline 920 & & betrachten konnten. Aber die Seminarleitung selbst halt \\
\hline 921 & & auch nicht. Also es wurde sehr emotional und unsach- \\
\hline 922 & & lich, wirklich, also ganz komisch, wirklich geschrien und \\
\hline 923 & & diskutiert, aber auf 'ne sehr auf 'ne sehr undialogische \\
\hline 924 & & Weise, so dass viele das Seminar verlassen haben und \\
\hline 925 & & sie wörtlich auch mehrmals gesagt hat. Dann könnt ihr \\
\hline 926 & & den Raum verlassen, also zu diesen drei Muslima, die \\
\hline 927 & & halt \\
\hline 928 & MICHI: & $\llcorner$ Krass \\
\hline 929 & NEELE: & $\llcorner$ Krass \\
\hline 930 & MICHI: & $\llcorner$ Ey das find ich- \\
\hline 931 & JONA: & $\begin{array}{l}\llcorner\text { das war ganz, das fand ich ganz, } \\
\text { dass ist natürlich unmöglich. }\end{array}$ \\
\hline 933 & MICHI: & Das- das, find ich diskriminierend. \\
\hline 934 & JONA: & $\mathbf{J a}$ \\
\hline
\end{tabular}

Jona erzählt von seiner Erfahrung aus einem ,schreckliche[n] Seminar im Bereich interkulturelle Pädagogik". Die ,sehr emotional und unsachlich" geführte Diskussion wird als negativ bewertet. Wie bereits bei Michis eingebrachtem Beispiel ,Kill your gender“" bildet eine aggressive, kämpferische und emotionale Diskussionskultur bei der Thematisierung von ,Geschlecht' einen negativen Horizont. Jona beschreibt vor allem die konflikthafte, aggressive und unwissenschaftliche Diskussion in dem Seminar sowie generalisierende Aussagen ,über die Unterdrückung der Frau im Islam" als negative Erfahrung und negative Diskussionskultur. In dem Seminar wurde nicht wertschätzend und respektvoll diskutiert (was die normative Orientierung der GDT darstellt), sondern da haben „Kämpfe statt gefunden [... ] zwischen der Seminarleitung und einzelnen Muslima mit Kopftuch“. Es ist anzunehmen, dass es in diesem Seminar zu einer Konfrontation zwischen Muslima und Nicht-Muslima kam. Jona beschreibt den Konflikt so, dass sich die Frauen mit Kopftuch gegen den Vorwurf gewehrt hätten, dass sie aufgrund ihres Glaubens unterdrückt seien (hier ohne transkribierte Passage). Diese Studentinnen haben sich gegen die Generalisierung von Seiten der Dozentin gewehrt. In diesem Seminar hat Jona erlebt, wie ein eurozentrisch-feministischer Diskurs Frauen mit anderem ethnischen und religiösen Hintergrund ausschließt. 
Dieser Ausschluss fand im Seminar nicht nur durch Zuschreibungen und einer ausgrenzenden Diskussion statt, sondern wurde auch faktisch vollzogen, indem die Seminarleitung die „Muslima“ „im Endeffekt rausgeschmissen" habe. Die Ablehnung dieses Verhaltens der Seminarleitung bringt Jona auch auf der Handlungsebene zum Ausdruck, indem er, wie viele andere, das Seminar verlassen hat. Damit wird die Abwertung und Ablehnung dieses Verhaltens als kollektive Orientierung deutlich. Auch die GDT bewerten das von Jona beschriebene „Diskussionsklima“ als „diskriminierend“ und bringen damit ihre Ablehnung zum Ausdruck. Auch wenn die GDT nicht dasselbe Seminare besucht haben, teilen sie ähnliche Erfahrungen und die kollektive Orientierung, dass sie in Bezug auf ,Geschlecht" und Geschlechterfragen ein aggressives, undifferenziertes, naturalisierendes und diskriminierendes „Diskussionsklima“ ablehnen. Hier lässt sich die Orientierung rekonstruieren, dass die Thematisierung von ,Geschlecht' nur dann legitim ist, wenn es dabei zu keiner Diskriminierung oder Ausgrenzung kommt.

Der Beitrag von Jona wird in der Gruppe jedoch nicht dazu genutzt, ihre (normative) Orientierung gegenüber KommilitonInnen mit Kopftuch zu benennen; das eingebrachte Thema zur ,interkulturellen Pädagogik" dient den GDT, um die ausgrenzende und diskriminierende Form der Thematisierung von ,Geschlecht' zu benennen. Das Thema Religion/Glaube oder der Umgang mit KommilitonInnen mit islamischem Glauben oder Migrationshintergrund knüpft in der Gruppe Metall an keinen konjunktiven Erfahrungsraum an, ebenso wenig wird eine Verbindung zu konjunktiven Erfahrungen der Gruppe in Hinblick auf den eigenen Glauben und die eigene Herkunft deutlich.

Anhand dieser Passagen lässt sich rekonstruieren, dass die Thematisierung von ,Geschlecht' und Geschlechterfragen für die befragten Studierenden im sozialen Feld der Bildungsinstitution eine Dimension konjunktiver Erfahrung darstellt. Gemeinsam haben die GDT, dass sie alle Kritik daran formulieren, wie ihnen das Thema ,Geschlecht' bislang an der Universität begegnet ist. Die Studierenden orientieren sich an der Vorstellung, dass eine Thematisierung von und eine Sensibilisierung für ,Geschlecht' relevant ist, jedoch haben sie alle eine andere Vorstellung davon, wie diese aussehen soll. Auf Basis ihrer je spezifischen Erfahrung kritisieren sie zwar nicht die Thematisierung von ,Geschlecht' und Geschlechterfragen grundsätzlich, sondern zeigen die negativen Aspekte und Horizonte von Thematisierungsweisen auf. Sie bringen zum Aus- 
druck, dass es bestimmte Formen der Thematisierung gibt, die sie als ,extrem" wahrnehmen und aus ihrer Sicht, „über das Ziel hinaus schieß[en]". Das Thema ,Geschlecht' ist für die Studierenden zwar wichtig, aber auch „schwierig“, weil es andere Personen ausgrenzen kann. Diese Kritikaffinität und ,Ja, aber'-Mentalität der Studierenden ist für die Gruppe Metall zentral. Die Kritikaffinität bezüglich der Thematisierungsweisen von ,Geschlecht" und Geschlechterfragen im universitären Feld lässt sich als ein wesentliches Element des Habitus der Gruppe in Hinblick auf die Thematisierung von ,Geschlecht" charakterisieren. Bestimmte Formen der Thematisierung bzw. ein ausgrenzendes Diskussionsklima sowie eine Naturalisierung von Geschlechterdifferenz stellen einen negativen Horizont der Thematisierung dar. Die Thematisierung von ,Geschlecht' ist nur dann legitim, wenn es dabei zu keiner Diskriminierung oder Ausgrenzung kommt. Einen positiven Horizont stellen gesellschaftskritische Momente dar und die Orientierung der Thematisierung von ,Geschlecht“ an „Wertschätzung“, „Gleichheit und Respekt". Die Geschlechtersensibilität und -reflexion, sowie die Fähigkeit wissenschaftlich zu argumentieren sind für die Studierenden von großer Bedeutung.

\subsubsection{Orientierung an einer ,kritische[n] und feministische[n]", "gendergerechte[n] Sprache“"}

In Bezug auf die Auseinandersetzung mit ,Geschlecht' und Geschlechterfragen werden auch geschlechtergerechte Sprache im Studium und das als gesellschaftskritisch wahrgenommene Studienumfeld als Anregung zur Thematisierung und Reflexion genannt. Die Gruppe Metall ist somit die einzige, in der die geschlechtergerechte Sprache einen positiven Horizont darstellt, an dem sich die GDT orientieren. In der folgenden Passage wird deutlich, dass eine Thematisierung nicht nur durch Seminarinhalte erfolgen kann, sondern auch vom Wahrnehmungs- und Bewertungsschema des sozialen Feldes der Bildungsinstitution angeregt wird.

332

333

334

335

336

337

338

Michi: [...] ich hab während meinem Studiums, wobei ich jetzt eben nicht genau weiß, ob das dann an den Erziehungswissenschaften jetzt hauptsächlich liegt ode::r-, wird schon auch daran gelegen haben, also, dass ich auf die Sprache sehr geachtet habe nach und nach und das ist sicher auch etwas was [Name der Universität, S.K.] generell prägt oder die Geisteswissenschaften vielleicht auf 
340 JONA:

341 MICHI:

342

343

344

345

346

347

348

349

350

351

352

eine kritische Sprache ${ }^{\circ}$ oder feministische Sprache ${ }^{\circ}$

ᄂ@Gendergerecht@

$\llcorner$ ja genau, gendergerechte Sprache. Und dass es unterschiedliche Dozentinnen und Dozenten ((lacht)) gibt, die da sehr drauf achten, also jetzt nicht alle; andere wieder nicht so, aber zum Beispiel Frau [Name der Dozentin, S.K.] ja sehr stark und dass man es ja auch in der Literatur findet-, jetzt auch nicht gerade in

JONA: $\quad$ L ${ }^{\circ} \mathrm{Mhm}^{\circ}$

MICHI: jeder, aber so in ganz viel wissenschaftlicher Literatur, dass es immer irgendwie Erwähnung findet, wie mit den Geschlechterformen bei der Schreibweise umgegangen wird und so.

Michi beschreibt, dass sie im Laufe des Studiums immer mehr auf „die Sprache" geachtet hätte, und stellt die ,gendergerechte Sprache" als einen positiven Bezugspunkt und als Handlungsorientierung im sozialen Feld der Bildungsinstitution dar. Damit verbindet sie auch eine „kritisch“ und „feministisch“ orientierte Perspektive. Indem Jona Michi auf die richtige Bezeichnung hinweist, unterstreicht Jona erneut seinen Expertenstatus und macht deutlich, dass er diesbezüglich ähnliche Erfahrungen teilt. Michi erklärt nun weiter, dass sie im Studium von den „Dozentinnen und Dozenten" wie auch von "wissenschaftlicher Literatur" für eine geschlechtergerechte Sprache sensibilisiert worden sei. Auch wenn sie hier von „Dozentinnen und Dozenten“ spricht, scheint die geschlechtergerechte Sprache in der universitären Praxis eine weibliche Stimme zu haben, da sie als Beispiel eine Dozentin anführt und männliche Vortragende unerwähnt bleiben. Auch hier bestätigt sich die konjunktive Erfahrung, dass die Thematisierung von ,Geschlecht' im universitären Kontext vor allem von weiblichen Akteurinnen bestimmt ist. Zudem lässt sich rekonstruieren, dass die geschlechtergerechte Sprache Teil der konjunktiven Erfahrung der Studierenden der Gruppe Metall und Teil ihrer habituellen Praxis ist.

\subsection{3 „Ja, sind wir jetzt benachteiligt oder nicht“}

Wie auch in den anderen Gruppendiskussionen wird in der Gruppe Metall die Orientierung geteilt, dass Frauen im Studium nicht benachteiligt 
sind. Dies wird in der folgenden Passage nochmals verdichtet. Das Thema „Benachteiligung von Frauen im Studium“ wird von Neele angesprochen. Ihren Beitrag führt sie ein, indem sie erzählt, dass „,dieses Thema“ (damit bezieht sie sich auf die zuvor angesprochene "Gendergeschichte") auch außerhalb von Seminaren „unter den Kommilitonen“ besprochen werde.

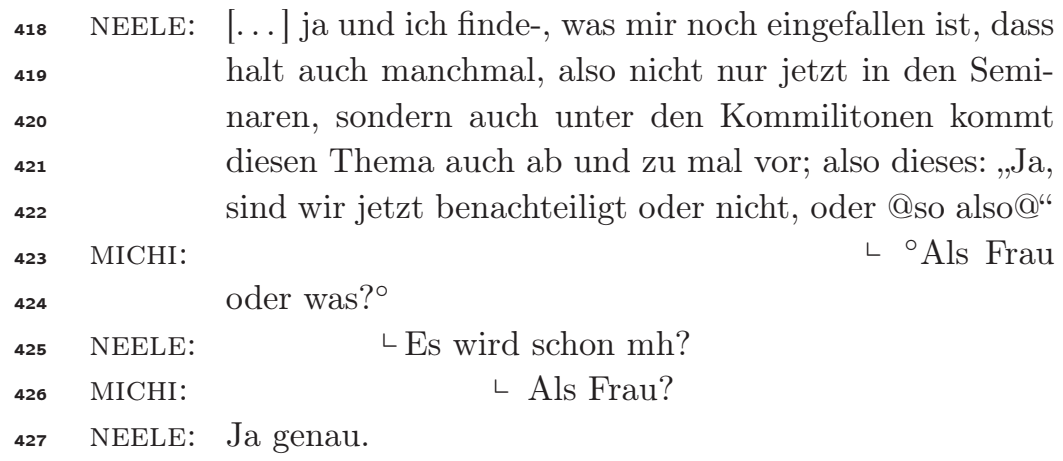

Neele elaboriert hier ihre Erfahrung, dass die "Gendergeschichte" nicht nur in Seminaren, sondern auch unter den Kommiliton_innen ,ab und zu mal" besprochen werde. Hier ginge es dann um die Frage: „[S]ind wir jetzt benachteiligt oder nicht?". Die Formulierung von Neele (,sind wir") lässt darauf schließen, dass Neele hier von ihrer eigenen Geschlechtlichkeit ausgeht und von sich und ihren Kolleginnen spricht. $\mathrm{Zu}$ diesem Zeitpunkt ist dies aber nicht eindeutig klar, da sie zuvor von ihren „Kommilitonen“ spricht. Erst durch die Bejahung von Michis Nachfrage („Als Frau oder was?") bestätigt sich diese Annahme. An Neeles Beitrag ist interessant, dass es in erster Linie um die Benachteiligung von Frauen geht. Hier zeigt sich die Verknüpfung von "Gendergeschichten" mit Frauen und Benachteiligung. Das verweist darauf, das Geschlechterfragen mit Ungleichbehandlung von Frauen verbunden wird. Dies ist ein erstes Indiz dafür, dass ,Geschlecht' und Geschlechterfragen von den Studierenden mit Frausein und Benachteiligung von Frauen gleichgesetzt wird. Die Frage nach Benachteiligung knüpft in der Gruppe Metall an einen konjunktiven Erfahrungsraum an. Jona möchten nun die Antwort auf diese Frage wissen:
426 JONA:
$\llcorner$ Und was ist die Antwort?
427 MICHI:
$\left\llcorner{ }^{\circ}((\text { lacht }))^{\circ}\right.$
428 NEELE:
(3) ja, es gibt eben irgendwie nicht wirklich eine Ant- 
wort, also es ist schon so ein Streitthema irgendwie, es hat jeder andere Ansichten was denn Frausein überhaupt bedeutet, da fängt es ja schon an

MICHI:

$\left\llcorner{ }^{\circ} \mathrm{Ja}^{\circ}\right.$

NEELE: also:o ich finde das ist auch so eine individuelle Geschichte, also (3) ja, wo soll ich da jetzt anfangen, also wenn ich, also es gibt eben die sagen: „Ja ich will nur arbeiten und Karriere oder ich will beides, ich will es vereinbaren." Oder die sagen: „Nö ich will Hausfrau und Mutter" und dann fängt die Diskussion an @so ungefähr@so;

Auf die Nachfrage von Jona antwortet Neele, dass es „nicht wirklich eine Antwort" gebe und es ein "Streitthema“" unter den KommilitonInnen sei. Das Problem beginne nämlich schon dabei, dass es keine kollektive Orientierung dazu gibt, ,was denn Frausein" bedeute. Michi validiert die Orientierung, dass es vielfältige Vorstellungen von „Frausein" gebe. Die Thematisierung von Benachteiligung ist Teil des konjunktiven studiumsbezogenen Erfahrungsraums und geprägt von der Orientierung, dass Benachteiligung in erster Linie Frauen betrifft. Es scheint jedoch keine kollektive Orientierung, sondern unterschiedliche individuelle Orientierungen in Bezug auf Frausein zu geben. Neeles persönliche Einschätzung ist, dass es sich bei der Frage, ,was denn Frausein überhaupt bedeutet", um eine ,individuelle Geschichte" handle. Sie belegt dies an den unterschiedlichen Bedürfnissen und Lebensentwürfen von Frauen. Indem sie die drei Optionen ,Karrie$\mathrm{re}^{6}$, ,Hausfrau/Mutter und Karriere' und ,Hausfrau/Mutter formuliert, macht sie auf die angeblich querliegenden unterschiedlichen Entscheidungen zur Lebensgestaltung von Frauen aufmerksam. Gesellschaftliche Probleme und Strukturen werden von ihr nicht angesprochen, sondern individualisiert. Zugleich reproduziert Neele eine geschlechterstereotype Rollenverteilung: Familienbezogene Betreuung und Hausarbeit ist ,Frauensache' und die Frage nach Vereinbarkeit von Beruf und Familie ist eine ,Frauenfrage'. Michi nimmt zu einem späteren Zeitpunkt in der Gruppendiskussion auf Neeles Beitrag Bezug. Dabei distanziert sie sich von Neeles Orientierung, dass zuerst darüber diskutieren werden müsse, was eine Frau ausmache, bevor man über Fragen der Benachteiligung sprechen könne: 


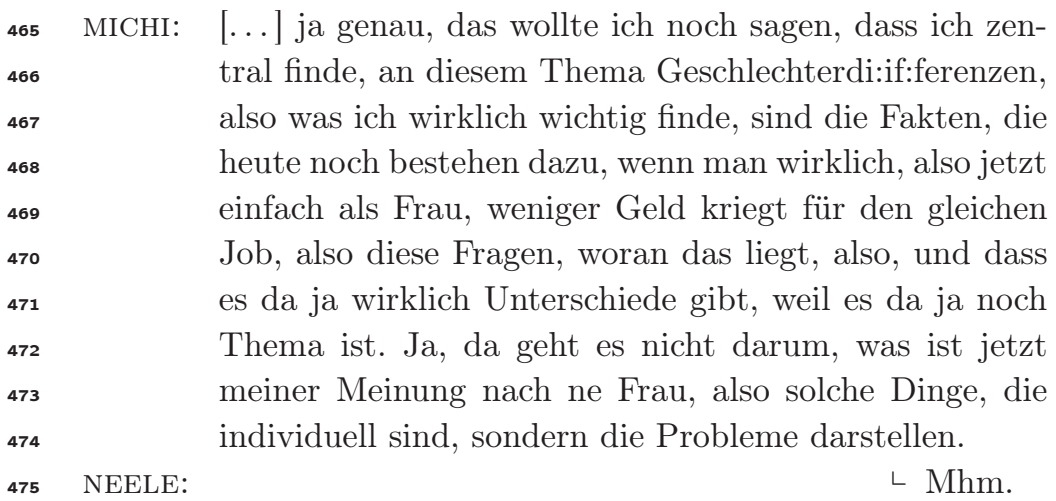

Interessant ist, dass Michi in Verbindung mit Ungleichbehandlung von Frauen und Männern von "Geschlechterdifferenzen“ spricht. Es ist anzunehmen, dass sie nicht biologische Geschlechterdifferenzen meint, sondern jene, die sich in einer Ungleichbehandlung qua ,Geschlecht" manifestieren. Ihrer persönlichen Meinung nach sei es „wirklich wichtig“, nach wie vor bestehende faktische Ungleichbehandlungen zu diskutieren und zu beseitigen. Als aktuelles Beispiel nennt Michi den gender pay gap. Michi verweist auf strukturelle Probleme, die aufgrund der gesellschaftlich konstruierten Differenzierung zwischen den Geschlechtern entstehen. Gleichzeitig wird an einer früheren Stelle angemerkt, dass Chancengleichheit, laut Michi, erreicht werden könne, wenn „was in den Köpfen“ verändert werde. Bei „diesem Thema Geschlechterdifferenzen“" stehen für Michi strukturelle und gesellschaftliche Probleme im Zentrum. Die Fragen nach verschiedenen und ,individuell[en]" Weiblichkeitskonzepten und Existenzweisen (,also solche Dinge") erfahren eine indirekte Abwertung. Neele ratifiziert Michi auf einer Ebene des inhaltlichen Verständnisses mit einem sogenannten Hörsignal („mhm“). Somit wird nicht deutlich, ob sie Michi zustimmt oder nicht. Bei diesen Beiträgen zum Thema „Benachteiligung“ lässt sich die kollektive Orientierung rekonstruieren, was Frausein Ungleichbehandlung bedeutet. Michi validiert die Orientierung, dass es vielfältige Vorstellungen und Verständnisweisen von „Frausein" gebe und eine ,individuelle Geschichte" sei.

\section{Keine Benachteiligung von Frauen oder Männern im Studium}

Für Neele scheint das Thema Benachteiligung noch nicht beendet zu sein. Auch wenn, oder vielleicht auch gerade weil die Studierenden davon aus- 
gehen, dass Benachteiligung in erster Linie Frauen betrifft, fragt Neele nach, ob sich Michi „als Frau benachteiligt" fühle. Mit dem Nachsatz „als Studentin“ grenzt Neele den Geltungsbereich der Frage ein und beschränkt ihn auf den studiumsbezogenen konjunktiven Erfahrungsraum.

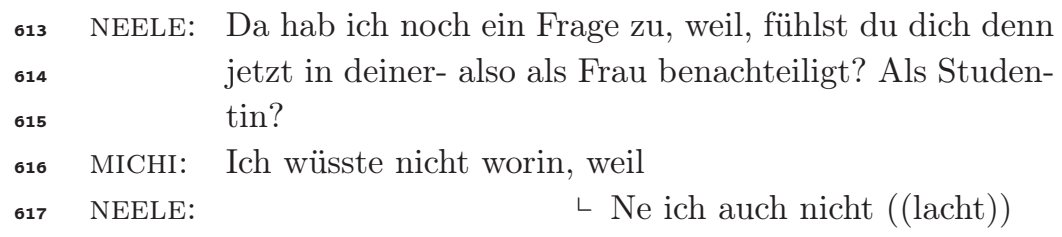

Michi verneint diese Frage indirekt und betont, sie „wüsste nicht worin“ sie sich benachteiligt fühlen sollte. Indem sie damit antwortet, dass sie nicht wisse, in welcher Sache und unter welchem Umstand sie sich benachteiligt fühlen könnte, weist sie Benachteiligung als erlebte Erfahrung von sich. Es kann an dieser Stelle vermutet werden, dass sie annimmt, dass dies auch auf andere Studentinnen zutrifft. Mit ,weil“ kündigt sie eine Erklärung ihres Beitrags an, wird jedoch von Neele unterbrochen. Neele schließt validierend an die Bemerkungen von Michi an, auch sie fühle sich nicht benachteiligt. Geschlechtsbezogene Benachteiligungen existieren demnach in ihrer Wahrnehmung und ihrem Erleben nicht (vgl. Hark 2008).

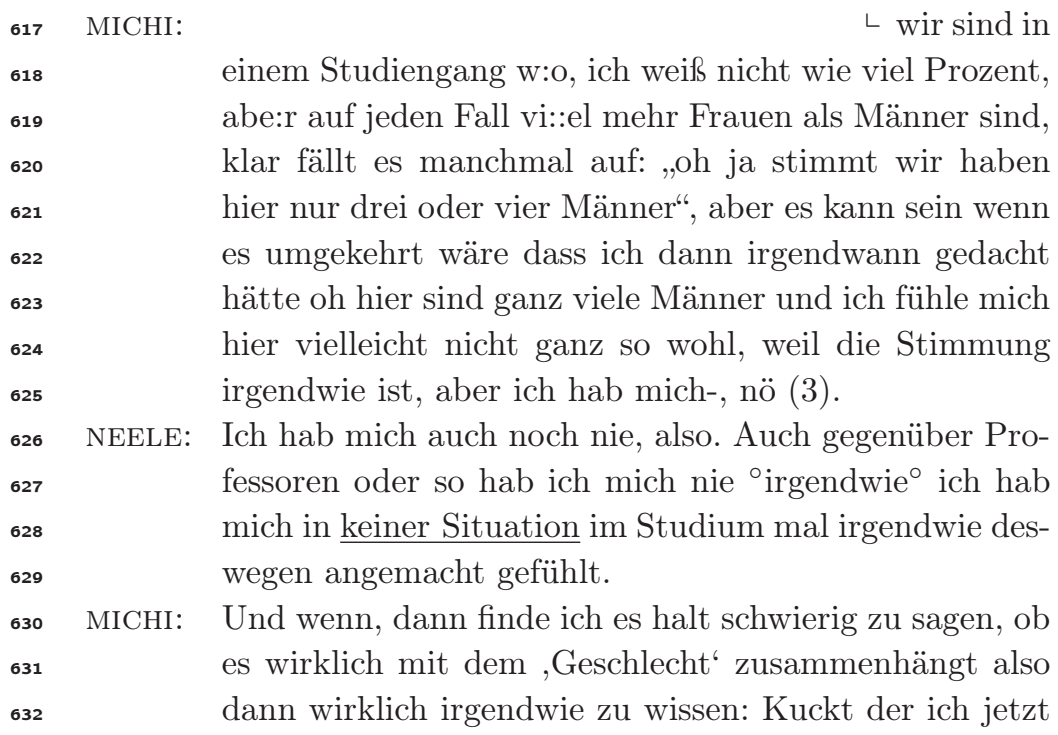


so- oder redet der jetzt so mit mir weil er genervt ist und einen schlechten Tag hat und ich Student/Studentin bin? Oder macht der mich so an weil ich ne Frau bin. Und dann vielleicht nicht mal bewusst weil unbewusst haben wir alle irgendwelche (hustet) Schubladen in uns, die wir nie ganz ausschalten können

NEELE:

ᄂ Ja, richtig.

\section{(4)}

NEELE: Das geht auch gar nicht; glaub ich

JONA:

ᄂ Nö

NEELE:

$\llcorner\mathrm{Ne}$

MICHI:

$\left\llcorner{ }^{\circ} \mathrm{Ne}\right.$, man kann

es halt nur immer wieder beobachten und ja. ${ }^{\circ}$

Michi knüpft in der nächsten Wortmeldung an ihre angekündigte Begründung an. Sie begründet ihre Orientierung und ihr Erleben, keine geschlechtsbezogene Benachteiligung erfahren zu haben, mit dem gemeinsamen studiumsbezogenen konjunktiven Erfahrungsraum. Hier hebt sie die ungleiche Geschlechterverteilung (,vi::el mehr Frauen als Männer") im Studiengang hervor. Michi scheint sich aufgrund der weiblichen Dominanz unter den Studierenden nicht benachteiligt zu fühlen. Michi schließt aber nicht aus, dass sie sich bei männlicher Dominanz „nicht ganz so wohl“ gefühlt hätte, weil die „Stimmung“ anders gewesen wäre. Damit bestätigt sie die Orientierung, dass es im Studiengang Erziehungs- und Bildungswissenschaften aufgrund der weiblichen Dominanz keine geschlechtsbezogene Benachteiligung von Frauen gebe. Hierbei wird von Michi auch das Stereotyp des erziehungswissenschaftlichen Studiengangs als „Frauenstudiengang“ reproduziert. Abschließend wiederholt Michi einsilbig (,nö“), dass sie sich nicht benachteiligt fühle. Michi elaboriert die Orientierung auf die professorale Ebene. Auch von „Professoren" habe sie sich „nie [...] in keiner Situation im Studium mal irgendwie deswegen angemacht gefühlt".

In den bisherigen Beiträgen wird Benachteiligung mit Formulierungen wie ,ich fühle mich", ,ich habe mich", ,finde ich" als eine individuelle/subjektive Empfindungen inszeniert. Geschlechtsbezogene Benachteiligung hat hier nicht mehr viel mit faktischer Benachteiligung zu tun, sondern mit einem subjektiven Empfinden. Diese Einschätzung wird von Michi weiter tradiert. Sie erklärt, auch wenn sich jemand aufgrund des Geschlechts benachteiligt fühle, sei es ,schwierig zu 
sagen“ bzw. zu beurteilen, „ob es wirklich mit dem Geschlecht zusammenhängt". Als hypothetische Beispiele führt Michi Gespräche und Blickkontakt an; sie scheint hier eine Verknüpfung zwischen geschlechtsbezogener Interaktion und Benachteiligung zu sehen. Michi elaboriert weiter, dass es schwer zu ergründen sei, warum man komisch/anders behandelt werde und ob es einen unmittelbaren, das heißt, einen direkten oder indirekten Zusammenhang mit dem ,Geschlecht” („Frausein“) gebe. Im Beitrag von Michi wird das Verständnis von Benachteiligung als ungleiche Behandlung deutlich, sowie die Orientierung, dass es schwierig sei, geschlechtsbezogene Benachteiligung/Behandlung als solche zu identifizieren und nicht als eine subjektive Empfindung fehl zu interpretieren. Verstärkt wird dies durch die kollektive Orientierung, Benachteiligung passiere „nicht mal bewusst", sondern könne auch „unbewusst" geschehen, weil „wir alle irgendwelche Schubladen in uns" hätten, „die wir nicht ganz ausschalten können". Neele und Jona validieren diese Orientierung. Die drei sind sich darin einig, dass ,wir alle“ in Kategorien (,Schubladen") denken und diese unser Handeln und Verhalten willkürlich steuern. Damit wird das bewusste Verhalten und Handeln eingeschränkt, „man [kann] es halt nur immer wieder beobachten" und in der Situation nicht aktiv verändernd eingreifen. Mit dieser Begründungsfigur werden intentionale geschlechterbezogene Benachteiligung und Behandlung quasi nivelliert und eine ungleiche Behandlung normalisiert. In Sinne von Maria Bitzan zeigt sich hier ein ,prekärer Selbstbezug“ (Bitzan 1997, S. 78): Wenn es keinen eindeutigen und stichhaltigen Beweis dafür gibt, dass ein bestimmtes Verhalten oder eine Ungleichbehandlung mit dem ,Geschlecht' zu tun hat, werden die eigene Wahrnehmung und Empfindungen in diese Richtung relativiert/nicht ernst genommen. Verbunden mit der oben rekonstruierten Orientierung, dass es keine geschlechtsbezogene Benachteiligung im Studium gibt, wird deutlich, dass die Studentinnen von sich als Geschlechtswesen absehen und Bestätigungen da suchen, wo das angeblich Allgemeine und Normale verortet ist.

In Bezug auf geschlechtsbezogene Benachteiligung möchten nun die weiblichen GDT von Jona, dem Angehörigen der männlichen Minderheit, wissen, ob er sich „benachteiligt gefühlt" hätte. Diese Passage zum Thema Benachteiligung ist von einer hohen interaktiven Dichte gekennzeichnet.

641 NEELE: Hast du dich ${ }^{\circ}$ benachteiligt gefühlt? ${ }^{\circ}$

642 MICHI: 'Ja genau, das hab ich mir auch grad gedacht ${ }^{\circ}$ 
NEELE: Nein ((lacht)), ich fühl mich wohl in dem Hühnerhaufen ((lacht))

MICHI: $\quad\llcorner$ ((lacht)

JONA: Mir war ehrlich gesagt manchmal langweilig, dass so wenig Jungs da waren, also ich hätts spannender gefunden, wenn mehr

MICHI:

ᄂ Langweilig? Ach ja.

NEELE:

$\llcorner$ Spannender?

JONA: Männer in meinen Studium gewesen wären, auf jeden Fall. Aber ich hab mich nicht benachteiligt gefühlt

NEELE:

$\llcorner\mathrm{Mhm}$

MICHI: Warum hättest du es spannender gefunden?

JONA: weil (2) ich die, die da waren, sehr interessant fand

MICHI: Die Männer, oder wie?

JONA: Ja genau; viele denen ich in den Seminaren begegnet bin, fand ich interessanter als eine Masse

NEELE:

$\llcorner$ (stöhnt)

JONA: an StudentInnen denen ich auch begegnet bin.

MICHI:

$\left\llcorner{ }^{\circ}\right.$ Was? ${ }^{\circ}$

NEELE:

$\llcorner$ da muss-

MICHI: $\llcorner$ Also du

meinst jetzt, spannender als viele der Frauen,

JONA: Als ein großer Teil der weib- meiner weiblichen Kommilitoninnen

Wie bereits am Anfang des aktuellen Abschnitts (Zeile 613) stellt auch hier wieder Neele die Frage nach Benachteiligung. Nachdem zuvor von der Benachteiligung von Frauen gesprochen wird, wird nun nach der Benachteiligung von Männern im Studium gefragt. Hierfür richtet sich das Interesse von Neele und Michi nun auf Jonas Erfahrungen. Dahinter steht vermutlich die Annahme, dass Männer und Frauen unterschiedliche Erfahrungen machen. In Bezug auf Benachteiligung lässt sich in der Gruppe Metall ein geschlechtsbezogener konjunktiver Erfahrungsraum rekonstruieren.

Bevor Jona antworten kann, schlägt Neele eine Antwortmöglichkeit vor. Diese Wortmeldung möchte ich im Folgenden genauer betrachten, da sie die Diskursorganisation der Passage repräsentiert. Neeles Proposition ist, dass sich Jona nicht benachteiligt fühlen, sondern sich ,in dem Hüh- 
nerhaufen“ wohlfühlen würde. „Hühnerhaufen“ ist eine umgangssprachliche Beschreibung einer Gruppe von Frauen; zudem wird „Hühnerhaufen“ auch oftmals verwendet, um eine planlose und konfuse Gruppe zu adressieren. Mit dieser Bezeichnung ist eine tendenziell abwertende Haltung verbunden. Damit betont sie die weibliche Dominanz im Studium. Indem sie die weibliche Dominanz karikiert, entzieht sie der Gruppe von Studentinnen (implizit) die Ernsthaftigkeit und die Seriosität. Die große Anzahl von Studentinnen wird hier (implizit) abgewertet. Wenn wir kurz in dieser Metapher des Hühnerhaufens verbleiben, wird Jona (implizit) als der Hahn in der Gruppe von Hühnern (Studentinnen) angesprochen, wobei der Hahn im „Hühnerhaufen“ meist als Mittelpunkt erscheint. Mit dieser Metapher suggeriert Neele eine positive Erfahrung von Jona. Im Rahmen des Studiums erfährt er aus dieser Sichtweise in seiner Rolle als Mann eine Besonderung und Aufwertung. Den Gegenpol dieser Aufwertung stellen die (vielen) Studentinnen dar. Jona konstatiert, dass ihm „ehrlich gesagt manchmal langweilig" gewesen sei. Jona hätte es spannender gefunden, wenn mehr Männer im Studium gewesen wären. Er verneint, dass er sich benachteiligt gefühlt hätte, nennt jedoch das Gefühl der Langeweile, weil ,so wenig Jungs da waren“, als negative Erfahrung. Wie bereits zuvor Neele, stellt auch Jona Männer im Studium der Erziehungsund Bildungswissenschaften als etwas Besonders dar. Er rekonstruiert ein

Männerbild, das im Vergleich zum Frauenbild spannender und interessanter und besonders erscheint. Michi und Neele sind von dieser Äußerung überrascht („Langweilig? Ach ja."; „Spannender?“), damit haben sie wohl nicht gerechnet. Auf die Nachfrage von Michi: „Warum hättest du es spannender gefunden?“, wenn mehr Männer im Studium gewesen wären, antwortet Jona, dass er „die [Männer], die da waren, sehr interessant fand". Auf ein erneutes Nachfragen von Michi wiederholt er, „dass Männer interessanter und spannender waren, als die Masse an Studentinnen, als viele Frauen“. Durch Michis (ungläubige) Nachfrage wird ihre Verwundung und eventuell auch eine oppositionelle Haltung deutlich. Sie teilt Jonas Orientierung, dass Männer im Studium interessanter und spannender seien als die weiblichen Kommilitoninnen, nicht. In einer späteren Passage, die hier aus Platzgründen nicht dargestellt werden kann, erklärt Michi, dass sie eine konträre Erfahrung gemacht habe und mit zahlreichen Kommilitoninnen spannende und kritische Auseinandersetzungen geführt hätte.

NEELE: Aber da muss ich auch mal was sagen. Dass ich in die 
seminare; das waren meistens Männer, die das gemacht haben. Das fällt mir jetzt im Nachhinein mal so auf, dass ich lieber-

JONA: $\quad$ L Also die Lehrenden meinst du jetzt?

NEELE: Genau, dass ich lieber in Lehrveranstaltungen gegangen bin, wo Männer die Dozenten waren.

JONA:

ᄂ@ah ha@

NEELE: Warum weiß ich nicht; aber bei Frauen war ich glaube ich weniger im Verhältnis.

NEELE: Weiß nicht warum das so is', aber (4) vielleicht hab ich sowas fachlich Kompetentes gesucht oder so.

JONA: Okay, und hast du es dann gefunden?

NEELE: Ja;

JONA: Und woanders nicht, oder?

NEELE: Mh?

JONA: Und woanders nicht?

NEELE: Bei den Frauen war es mir manchmal zuviel (2) Bla, ich weiß nicht? [...]

NEELE: Ich war vielleicht in meinem ganzen Studium in (2) drei Veranstaltungen die Frauen gemacht haben

MICHI:

ᄂ Echt? Krass!

NEELE: Ja. Das fällt mir jetzt mal so auf. ${ }^{\circ}$

MICHI: Also bei mir waren so mit die, die ich gemacht hab, eigentlich bei Frauen. (2)

JONA: Die kritischsten? Im Sinne von

MICHI:

$\llcorner$ hinterfragen, sich eigene Gedanken machen:n; [...] Ich bin generell auch eher in Seminare lieber gegangen mit Frauen; weil die, weil ich die Professorinnen oft lieber mochte als di:ie Männer.

Neele reflektiert hier, dass sie die meisten Seminare bei „Männern“ gemacht und ,lieber" an Lehrveranstaltungen von „Dozenten" teilgenommen habe. Jonas Beitrag „@ah ha@“ veranlasst Neele, über den Grund ihrer Präferenz nachzudenken bzw. sich zu rechtfertigen. Sie spekuliert: „[V]ielleicht hab ich sowas fachlich Kompetentes gesucht oder so." Gefunden habe sie das bei ,den' Männern und nicht bei ,den' Frauen. Auch hier werden, die' Frauen (implizit) abgewertet und Männer erfahren eine fachliche Anerkennung. In Neeles Orientierung werden Dozenten im Ge- 
gensatz zu Dozentinnen mit fachlicher Kompetenz gleichgesetzt. Neeles Kritik an Dozentinnen bleibt unkonkret und diffus („Bla“). Die Dozentinnen bleiben hier das undefinierte und uninteressante Andere, Gegenüber. In Neeles Orientierung scheinen Männer qua,Geschlecht' fachlich kompetent zu sein. Als Gegenhorizont erzählt Michi von ihrer Erfahrung, dass sie „die kritischsten Seminare“ im Sinne von „hinterfragen [und] sich eigene Gedanken machen:n" bei Frauen besucht habe. Mit ihrem Beitrag macht Michi zum einen deutlich, dass die Orientierung von Neele und Jona (Männer seien spannender und kompetenter), keine kollektive Orientierung darstellt und sie eine andere Erfahrung gemacht habe und somit eine konträre Position vertritt. Zum anderen verweist sie darauf, dass die Vermittlung von und Anregung zum (gesellschafts-)kritischen und hinterfragenden Denken im Studium für sie eine positive Qualifikation darstellt.

Die Studierenden sprechen im Anschluss daran darüber, welche Dozentinnen und Dozenten sie am Institut besser fänden, kommen jedoch zu keiner gemeinsamen Bewertung (hier ohne transkribierte Passage). Im univoken Diskursmodus beenden sie diese Passage, indem sie die Präferenz für Dozentinnen und Dozenten zu einer individuellen Entscheidung erklären.

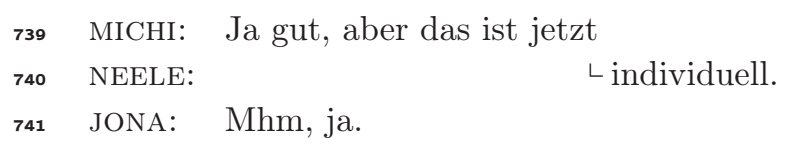

Durch den oben dargestellten Abschnitt lässt sich rekonstruieren, dass Geschlechterzuschreibungen, Essentialisierungen sowie Differenzierungen nach ,Geschlecht' Teil der habituellen Praxis und eine kollektive Orientierung der Studierenden darstellt. Der Geschlechterdifferenz kann in der Gruppe Metall der Status einer Leitdifferenz zugesprochen werden. Die Handlungsschemata sind von der Geschlechterdifferenz bestimmt, wohingegen sich die rational orientierten Überzeugungen, Vorstellungen und Normen auf Geschlechtergleichheit und Gleichberechtigung zu richten scheinen. Probleme und Konflikte werden nur oberflächlich besprochen, durch die Betonung von Individualität und Individualisierung wird die Differenzierung nach ,Geschlecht ${ }^{6}$ tabuisiert und verdeckt.

In dieser Gruppendiskussion wird ,Geschlecht' als ein janusköpfiges Thema besprochen. Zum einen ist ,Geschlecht' für die Studierenden ein interessantes und (über)komplexes Thema und zum anderen ist die Diskussion davon geprägt, wie schwierig es für die Studierenden sei, diesbe- 
züglich eine ,eigene' Position zu entwickeln. Das wird auch in den Passagen zur Kritik an der universitären Thematisierung von ,Geschlecht" und Geschlechterfragen erkennbar. Immer wieder kommt es zur Vermischung von vortheoretischem Geschlechterwissen, dem modernisierten Geschlechterdiskurs und dem theoretischen-reflexiven Geschlechterwissen aus dem studiumsbezogenen konjunktiven Erfahrungsraum.

Diese Melange aus (a),Alltagswissen' oder vortheoretischem Wissen, das sich (implizit) an Vorstellungen bzw. an inkorporiertem Wissen von Geschlechterdifferenz und -hierarchie orientiert, (b) einer (explizit) gemachten Vorstellung von Toleranz, Egalität und Individualität und (c) dem Wissen über theoretische Konzepte zu Geschlechterkonstruktionen stiftet bei den Studierenden Verwirrung und erzeugt eine Überkomplexität des Themas, die es für die GDT schwierig macht, eine eigene wie auch eine gemeinsame Position dazu zu finden.

\subsection{4 ,Geschlecht': ,[I]ch find's extrem wichtig“" aber es ist ,schwierig irgendwie 'ne Position zu entwickeln“"}

Neele habe sich bereits in ihrem Grundstudium mit „diese[m] Genderaspekt" beschäftigt und ihr „Vordiplom dann auch so ein bisschen darauf ausgerichtet". Sie habe auch ein „Genderzertifikat" ${ }^{\text {22 }}$ erworben, indem sie „bestimmte Seminare“ am Zentrum für Gender Studies an der Universität Mond besucht habe. Trotz dieser sehr umfangreichen und umfassenden theoretischen Beschäftigung mit ,Geschlecht' und Geschlechterfragen beschreibt Neele in der Gruppendiskussion, wie schwierig es für sie sei, diesbezüglich Antworten und eine Position zu finden.

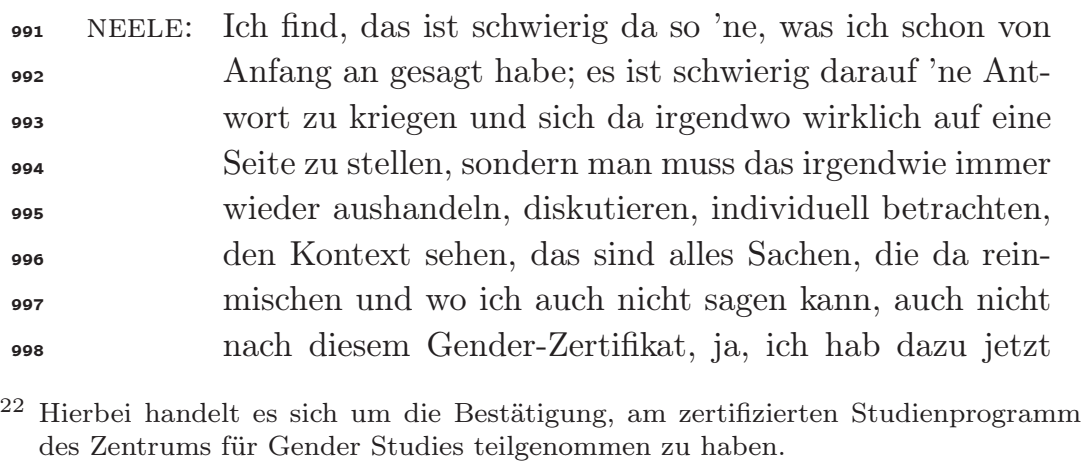




\begin{tabular}{|c|c|c|}
\hline 999 & & 'ne Position \\
\hline 1000 & JONA: & $\left\llcorner{ }^{\circ}(\text { lachen })^{\circ}\right.$ \\
\hline $\begin{array}{l}1001 \\
1002\end{array}$ & NEELE: & $\begin{array}{l}\text { ich weiß wie das läuft. Nee, das ist völliger Quatsch. } \\
\text { Also- }\end{array}$ \\
\hline $\begin{array}{l}1003 \\
1004 \\
1005 \\
1006 \\
1007 \\
1008\end{array}$ & JONA: & $\begin{array}{l}\qquad \text { Aber 'ne Position kann man ja trotzdem haben, } \\
\text { auch wenn man nicht weiß, wie alles läuft. Also ich finds } \\
\text { extrem wichtig, irgendwie 'ne Position zu entwickeln, } \\
\text { halt im Laufe der Zeit, auch wenn du das selbst oder } \\
\text { wir oder ich nicht, jetzt, mich permanent unterdrückt } \\
\text { oder ausgegrenzt oder so was fühle. }\end{array}$ \\
\hline 1009 & NEELE: & ᄂ @.Ja@ \\
\hline
\end{tabular}

Neele spricht hier von ihrer persönlichen Schwierigkeit, ,eine' Antwort auf Geschlechterfragen zu ,kriegen und sich da irgendwo wirklich auf eine Seite zu stellen". Mit dieser Beschreibung wird Neeles Annahme sichtbar, dass es eine singuläre/einfache Antwort auf Geschlechterfragen gebe und ihre Hoffnung/Erwartung, dass ihr diese Antwort, vielleicht auch im Sinne von Rezeptwissen, vermittelt werden würde. Neele hat den Eindruck, sich auf „eine Seite“ stellen zu müssen, sich positionieren zu müssen. Auch wenn die Seiten hier ungenannt bleiben, ist anzunehmen, dass Neele auf die Frage nach der Relevanz und der Legitimität von Geschlechterfragen anspielt und darauf, ob diese Geschlechterfragen nun wichtig seien oder nicht.

Gleichwohl ihr Wissen zu ,Geschlecht' und Geschlechterfragen universitär zertifiziert ist, konstatiert sie, dass sie nicht wisse, „wie das läuft“. Neeles Orientierung ist, dass sich jemand sehr intensiv mit ,Geschlecht" und Geschlechterfragen auseinandersetzen müsse, um eine Position entwickeln zu können. Für Neele scheint es jedoch aufgrund der von ihr wahrgenommenen Überkomplexität des Themas nicht möglich zu sein, eine generelle Position einzunehmen. Es lässt sich auch vermuten, dass sich dahinter eine Form der Distanzierung verbirgt, die nicht offen ausgesprochen werden kann, sondern in Form einer divergenten Position ihren Ausdruck findet. Jona opponiert gegen Neeles Orientierung. Er beginnt seinen Beitrag mit ,aber" und leitet damit seinen Widerspruch ein. Jona finde es „extrem wichtig“, „,'ne Position“ zu entwickeln, ,auch wenn man nicht weiß, wie alles läuft". Er bezieht sich hier nicht darauf, ,eine' Antwort zu finden, sondern eine Position zu haben, auch wenn nicht alle Aspekte einer Sache/eines Problems ausgeleuchtet sind. Jona 
spart jedoch aus, wie diese Position entwickelt werden kann; der Prozess (,halt im Laufe der Zeit", „irgendwie“) bleibt im Dunkeln. Für Jona ist es wichtig, eine Position zu haben, ,auch wenn [sich jemand] jetzt nicht permanent unterdrückt oder ausgegrenzt" fühle. Nicht das Ausmaß an theoretischem Wissen oder die eigene Betroffenheit sind für Jonas auschlaggebend, um eine Position zu vertreten, die Geschlechterfragen als wichtig zu erachten, sondern er orientiert sich daran, dass nach wie vor (andere) Personen ausgegrenzt und unterdrückt werden. Für Neele scheint Jonas Darstellung plausibel zu klingen, da sie dieser zustimmt („Ja“). Es lässt sich allerdings abschließend nicht klären, ob es sich hier um eine Ratifikation handelt, also um eine Bestätigung auf einer Ebene des inhaltlichen Verständnisses, oder um eine Validierung der aufgeworfenen Orientierungen.

\subsubsection{Die Orientierung an der Geschlechterdifferenz: „Du musst ja wissen, wen du suchst"}

Geschlechterbinarität und Geschlechterdifferenz lassen sich auch in der Gruppe Metall als normative Orientierung rekonstruieren. Es werden aber auch individuelle Ansichten deutlich, wenn es um die Stabilität einer eindeutigen, äußerlichen Geschlechterdifferenz geht. In der folgenden Passage wird das Thema Geschlechteruneindeutigkeit von Jona eingebracht und anschließend von Neele und Michi differenziert.

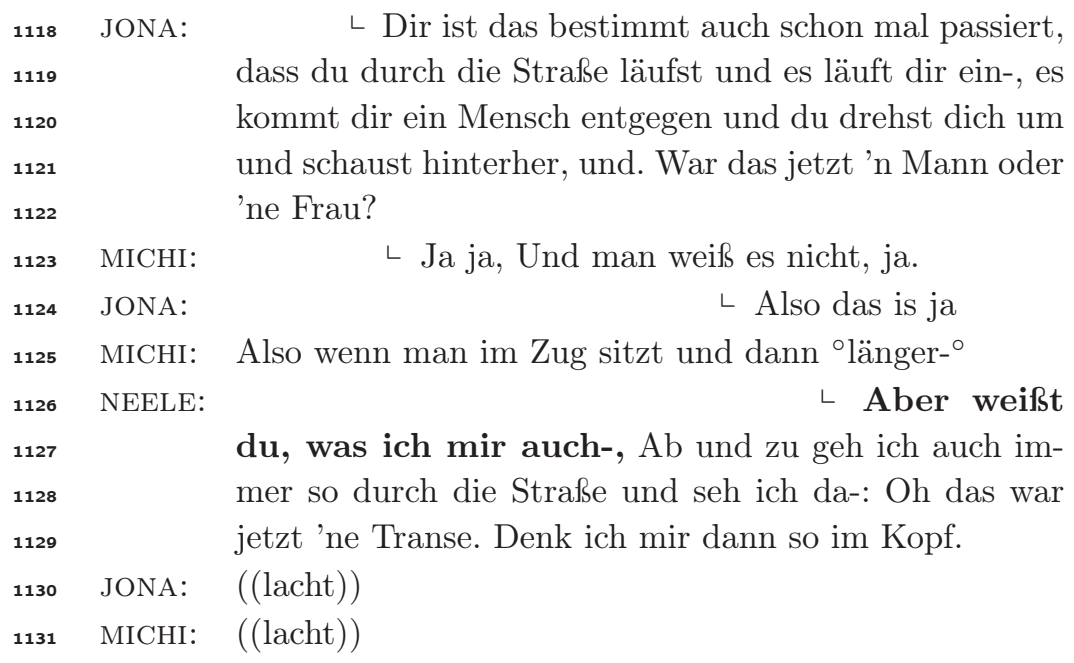


1132 NEELE: Ja @das ist echt@ passiert, wirklich. [...]

1133 MICHI: Ja, das is ja auch oft, vom Gesicht alleine her, das hat

1134

1135

1136

1137

1138

1139

1140

1141

1142

1143

1144

1145

1146

1147

1148

1149

1150

1151

1152

1153

1154

1155

1156

1157

1158 auch natürlich immer viel mit der Aufmachung zu tun. Also ich mein eigentlich freu ich mich auch, dass ich nicht irgendwie, wenn ich rumlaufe, weiß- ich nicht, ich finde das ist schon auch schön zu wissen, dass man irgendwie 'n bisschen was Weibliches hat und ausstrahlt oder was Männliches, umgekehrt halt, also ich glaub dieses, es würde mir fehlen wenn ich diese Differenzierung überhaupt nicht-

JONA: $\quad\llcorner$ Ja, aber natürlich, weil das alle von dir erwarten

MICHI: Nein, ich meinte jetzt, wenn ich diese Differenzierung überhaupt nicht hätte, wenn ich rumlaufen würde, wenn alle Leute, also wenn man's nie wüsste, ist das 'n Mann oder 'ne Frau, das wär doch ganz komisch, oder nicht?

NEELE:

$\llcorner$ Mhm

MICHI: also wenn ich durch die Stadt laufe?

NEELE:

$\llcorner$ Du musst ja wis-

sen, wen du suchst ((lacht))

MICHI: Ja, ja so ungefähr, genau. Mhm((lacht)) ja, ist doch so.

NEELE: Such ich jetzt 'ne Freundin oder 'n Freund?

MICHI: Ich mein, es ist doch so, dass dieses Thema, also ich mein, dieses Thema ist einfach immer immer immer immer immer präsent.

NEELE: $\llcorner$ Ja.

Jonas Beitrag beginnt damit, seine individuelle Erfahrung als konjunktive Erfahrung einzuführen („Dir ist das bestimmt auch schon mal passiert"). Er geht also davon aus, dass auch andere die Erfahrung gemacht hätten, einer Person begegnet zu sein, bei der nicht eindeutig erkennbar gewesen sei, ob, sie ,'n Mann oder 'ne Frau" sei. Michi schließt validierend an und wiederholt das für sie Essentielle des Beitrags in eigenen Worten: ,[M]an weiß es nicht." In dieser Passage lässt sich rekonstruieren, dass die (visuelle) geschlechtliche Eindeutigkeit sowie die Geschlechterdualität eine kollektive Orientierung und Norm darstellt. Michi beginnt mit einer Elaboration, wird jedoch von Neele unterbrochen, die 
das Thema der Geschlechtsuneindeutigkeit auf Transsexualität („Transe") ausweitet. Ob sich Neele mit dem Begriff „Transe" auf Transsexualität, Transgender, Transvestitismus oder Cross-Dressing bezieht, bleibt offen. Durch den Beitrag von Michi wird auch deutlich, dass Personen, die von einer eindeutigen und kohärenten Geschlechtlichkeit abweichen, als nicht der Norm entsprechend wahrgenommen werden und deshalb auffallen. Umgekehrt würde wohl nicht erzählt werden, dass man einen Mann oder eine Frau auf der Straße gesehen habe.

Nachdem Michi und Jona über ihre Erfahrung lachen, betont Neele die Echtheit ihrer Aussage. Michi signalisiert, dass sie ihr glaube und erklärt, dass die Ein- bzw. Uneindeutigkeit von ,Geschlecht' „,immer viel mit der Aufmachung“" zu tun habe. Michi versucht abschließend, pointiert zum Ausdruck zu bringen, dass ihr diese (äußerliche) Differenzierung zwischen Männern und Frauen fehlen würde, wenn es diese nicht mehr gäbe. Interessant ist hier, dass Michi quasi vom Einzelfall auf ein generelles und sich ausbreitendes Phänomen schließst. Die eindeutige und kohärente Geschlechterbinarität ist für Michi eine normative Orientierung, die sie nicht verändert sehen will. Jona sieht dies anscheinend anders und weist Michi darauf hin, dass ihr die Differenzen nur deshalb fehlen würden, ,weil das alle von [ihr] erwarten." Damit verweist er auf die gesellschaftlichen Mechanismen und Denkschemata, die auf Individuen einwirken. Michi weist Jonas Widerspruch zurück („nein“) und wiederholt ihre Orientierung in Form einer Suggestivfrage: „[W]enn man's nie wüsste, ist das 'n Mann oder 'ne Frau, das wär doch ganz komisch, oder nicht?" Michi äußert sich hier ablehnend bzw. abwertend (,,komisch $^{6}$ ) gegenüber der Vorstellung, dass Männer und Frauen in ihrer äußerlichen Erscheinung nicht unterscheidbar wären. Neele bestätigt Michis Orientierung an der Geschlechterbinarität. Sie greift das von Michi Gesagte auf und transferiert es in ihren eigenen Erfahrungsrahmen. Für sie ist die Differenzierung zwischen den Geschlechtern wichtig, weil „du musst ja wissen, wen du suchst": ,'ne Freundin oder 'n Freund". Damit bringt Neele, wenn auch mehr implizit als explizit, ihre Orientierung an Paarbeziehungen und Partnerschaft zur Sprache, die sie anscheinend mit der Geschlechterbinarität verbindet. Michi validiert Neeles Orientierung zuerst etwas vage (,ja, so ungefähr") dann aber deutlicher (,ja, ist doch so"). Diese Passage wird von den beiden beendet, indem der Geltungsbereich einer eindeutigen Geschlechterbinarität in Verbindung mit Partnerschaft ausgedehnt und als omnipräsent und -relevant erklärt wird: ,[D]ieses Thema ist ein- 
fach immer immer immer immer immer präsent." Da sich Jona nach seiner Intervention nicht mehr zu Wort meldet, ist anzunehmen, dass er die Orientierung von Michi und Neele nicht teilt. Auch wenn sich Jona, wie am Anfang der Passage deutlich wird, an einer geschlechtlichen Eindeutigkeit sowie die Geschlechterdualität orientiert, stellt die Vorstellung einer äußerlichen Geschlechtergleichheit für ihn möglicherweise keinen negativen Horizont dar.

\subsubsection{Die Thematisierung von ,Geschlecht': „Die Frage ist: Wie und mit welchem Ziel?"}

In der Gruppen Metall stehen alle GDT am Ende ihres Studiums und haben im Laufe ihrer Ausbildung studiumsbezogene konjunktive Erfahrungen in Bezug auf die Thematisierung von ,Geschlecht' und Geschlechterfragen gemacht. Auf Basis dieser Erfahrungen diskutieren Michi, Jona und Neele auf Nachfrage der Interviewerin auch darüber, was diese Thematisierung im Studium (nicht) leisten könne und was ihnen bei der studiumsbezogenen Thematisierung von ,Geschlecht' und Geschlechterfragen wichtig sei. Zusammenfassend lässt sich sagen, dass die Studierenden diesbezüglich sehr individuelle Orientierungsschemata äußern. Es lassen sich aber auch kollektive Orientierungsschemata rekonstruieren.

1269

1270

1271

1272

1273

1274

1275

1276

1277

1278

1279

1280

1281

1282

1283

1284

1285

MICHI: Die Frage ist: Wie und mit welchem Ziel? Also wofür und wie eben, also, weil ich es halt wichtig fände, wenn es so wäre, dass es dann eben auch differenziert passiert und eben nicht wo sicher immer schnell die Gefahr ist, dass es dann polarisiert oder pauschalisiert wird und dadurch eigentlich wieder mehr getrennt wird als vereint wird, also wenn, dann muss es wirklich differenziert und immer wieder von anderen Ecken beleuchtet werden und viel eigenes kritisches Denken möglich sein. [...

NEELE: ${ }^{\circ} \mathrm{Also}^{\circ}$, also ich fände, dass es eine Que:erverbindung zu den eigentlichen Fächern geben sollte. Also nicht als eigenständiges Fach. Das finde ich irgendwie nicht so ansprechend. Sondern, dass es in einzelnen Seminaren immer als Punkt oder als Themenpunkt oder beiläufig mitlaufen sollte oder mal aufgegriffen wird.

JONA: Ich find aber auch, dass, man also dass- [...]ich weiß 
nicht wer das gesagt hat, mit sozialer Ungleichheit, und also dass das halt einfach einen ganz klaren Stellenwert haben muss in der Beschäftigung mit sozialer Ungleichheit das finde ich irgendwie klar, und neben anderen Formen von Diskriminierung und Ausgrenzung von Andersheit oder der Konstruktion von Gruppen und gleichzeitig finde ich es aber total interessant, das wär für mich was, was mitlaufen muss irgendwie bei dieser Beschäftigung wenn es um Gesellschaft, wenn's um Identitätsbildung oder um Bildungssystem geht, also das ist für mich klar, es muss, es muss, es muss Geschlecht thematisiert werden, auf 'ne Weise. Aber ich find's total wichtig oder eigentlich auch wenn's so Raum gäbe, um irgendwie eigene Identität zu reflektieren [...] also was bedeutet das für dich, für deine Rolle als Pädagogin in der pädagogischen Praxis, unabhängig davon, ob du selbst diskriminiert bist oder nicht;

NEELE: Mhm

JONA: Also allein, was transportierst du selber mit raus, mit zu den Menschen, mit denen du zu tun hast; so das find ich super wichtig. Und das passiert halt nicht in 'nem Seminar wenn einmal in der Sitzung über Geschlecht geredet wird

NEELE: ᄂ $\mathrm{Ja}$

JONA: sondern, dann also da-, viele haben da ja auch so 'ne Scheu, sich zu äußern, und dann gibt es halt diese Kampfwesen, die dann da so aggressiv auftreten und alles andere auch irgendwie unterdrücken, also im Endeffekt das jetzt alles gegendert sein muss. Also ich fänd's spannend also, sowohl es mir wichtig ist, dass es halt 'n Querschnittsthema ist, aber auch, dass es so explizite Räume gäbe, also auch Seminare oder Blockseminare oder was auch immer;

NEELE: $\llcorner\mathrm{Ja}$

JONA: was hängt da alles mit zusammen, was spielt da rein? NEELE: Das find ich auch sehr gut, ja, Identitätsbildung (4) 
Bereits durch die Diskursorganisation wird die Aneinanderreihung individueller Positionen sichtbar. Michi beginnt mit der Proposition, dass eine ,differenziert|e]" Auseinandersetzung mit ,Geschlecht" wichtig sei, in der auch „viel eigenes kritisches Denken“ möglich sei. Eine Thematisierung, die „polarisiert" oder ,pauschalisiert" und wieder neue Trennlinien ziehe bzw. andere Personen ausgrenze, lehne sie ab. Eine Thematisierung von ,Geschlecht' sei nur dann sinnvoll, wenn es sich um eine differenzierte Auseinandersetzung handle. Wie bereits beim Thema „geschlechtergerechte Sprache" bringt Michi ihre Orientierung an ein gesellschaftskritisches Denken zum Ausdruck. Dem gegenüber steht der negative Horizont einer pauschalisierenden und ausgrenzenden Thematisierung von ,Geschlecht', welche die Differenzierungen nicht auföst, sondern verstärkt. Michi spricht hier von einer inhaltlich-didaktischen Ebene.

Neele schließst mit ihrem Beitrag nicht an, sondern formuliert eine neue Proposition, in der sie auf die curriculare Ebene fokussiert. Neele findet die Idee eines eigenständigen Fachs bzw. Schwerpunktes zu ,Geschlecht' „nicht so ansprechend“. ,Geschlecht” sieht sie als ein Querschnittsthema, das in einzelnen Seminaren ,beiläufig mitlaufen sollte oder mal aufgegriffen wird". Eine Fokussierung auf ,Geschlecht" und Geschlechterfragen stellt für sie einen negativen Horizont dar. Neele wiederholt hier ihre Orientierung, die sie bereits zum Thema „Kritik an einer möglichen Überbetonung von ,Geschlecht“" geäußert hat. Michis Frage nach der inhaltlich-didaktischen Vermittlung ist für Neele nicht relevant.

Jona schließt mit einer Antithese an und bezieht sich dabei auf einen anderen Beitrag zu ,Geschlecht' und Ungleichheit, der bereits zu einem früheren Zeitpunkt in der Gruppendiskussion aufkam. Jona konstatiert, dass ,Geschlecht" „in der Beschäftigung mit sozialer Ungleichheit" einen „Stellenwert haben muss". Anders als Michi und Neele spricht er sich eindeutig für die studiumsbezogene Thematisierung von ,Geschlecht" und Geschlechterfragen aus. Jona nennt zudem noch andere Formen von Diskriminierung, Ausgrenzung, Andersheit, Konstruktion von Gruppen, Gesellschaft und Identität sowie Fragen zur (Identitäts-)Bildung als geschlechterrelevante Themen. Für ihn stellt eine fokussierte Thematisierung von ,Geschlecht" und Geschlechterfragen einen positiven Horizont dar: „[F] ̈̈r mich [ist] klar, es muss, es muss, es muss ,Geschlecht" thematisiert werden, auf "ne Weise." Zudem finde er es wichtig, wenn es einen „Raum gäbe, um irgendwie eigene Identität zu reflektieren“ und darüber nachzudenken, was ,Geschlecht' „für deine Rolle als Pädagogin in der pädagogischen Praxis" bedeute. Neben der Implementierung von theoretischen Ansätzen zu Geschlechterfragen und -konstruktion plädiert 
Jona für die Selbstreflexion und ihre Relevanz für pädagogische Professionalität in einer vergeschlechtlichten und vergeschlechtlichenden Gesellschaft. Reflexion stellt für ihn einen wichtigen Teil der Ausbildung im erziehungswissenschaftlichen Denken und pädagogischen Handeln dar. Jona macht hier die Vielschichtigkeit und die Komplexität des Themas deutlich. Wie bereits an einem anderen Punkt der Gruppendiskussion verdeutlicht er hier nochmals, dass diese Orientierung „,unabhängig davon [ist], ob du selbst diskriminiert bist oder nicht". In seinem Beitrag macht er zwei Ebenen der Auseinandersetzung deutlich: die pädagogischprofessionelle und die persönliche Ebene. Antithetisch gibt Jona zu bedenken, dass dies nicht geleistet werden könne, wenn ,Geschlecht', wie von Neele dargestellt, in einem Seminar nur in einer Sitzung besprochen werde. Neele validiert zwar („Ja“), dass das von Jona Gesagte nicht von einem Seminar oder einer Sitzung geleistet werden könne, es kann aber nicht gesagt werden, ob sie der Orientierung von Jona generell zustimmt. Jona fährt darin fort, für eine Thematisierung hinderliche Aspekte zu benennen. Zum einen gebe es viele Studierende, die eine „Scheu“ hätten „sich zu äußern“, und zum anderen gebe es diese „Kampfwesen“, die „aggressiv auftreten und alles andere auch irgendwie unterdrücken“. Hier lässt sich eine Überschneidung mit Michis Orientierung, die ein ausgrenzendes Verhalten ablehnt, feststellen. Auch mit Neeles Orientierung scheint Jona eine Überlappung zu sehen. Wie Neele sieht auch er ,Geschlecht' als ein Querschnittsthema. Ihm sei wichtig, „dass es halt 'n Querschnittsthema ist". Allerdings mündet er in einen anderen Orientierungsrahmen. Er greift Neeles Argument zwar auf und bestärkt es, verwendet es dann jedoch für seinen Orientierungsrahmen. Neben einem Querschnittsthema sieht Jona ,Geschlecht' als ein Thema, das auch in „explizite[n] Räume[n]“, ,also auch [in] Seminare[n]" reflektiert und thematisiert werden sollte. Abschließend validiert Neele die Relevanz von ,Geschlecht' für die eigene Identitätsbildung.

In dieser Passage wird deutlich, dass die Studierenden hinsichtlich der Frage, wie und ob ,Geschlecht' und Geschlechterfragen ins Curriculum des Studiengangs implementiert werden sollen, kein gemeinsames Orientierungsschema teilen, auch wenn sich die individuellen Ansichten punktuell überschneiden. 


\section{„Identitätsbildung“" und „Persönlichkeitsbildung“: geschlechterreflektierendes Verhalten als Orientierungsschema}

Bereits in der oben dargestellten Passage wurde eine Überschneidung der individuellen Orientierungen deutlich. Im weiteren Verlauf der Diskussion kristallisiert sich ein kollektives Orientierungsschema im Sinne eines kleinsten gemeinsamen Nenners heraus. Diese Passage soll im Folgenden, wenn auch vergleichsweise knapp zusammengefasst, wiedergegeben werden, da sich hier die rekonstruierten Orientierungen der Gruppe Metall in Hinblick auf normative Erwartungen an die studiumsbezogene Thematisierung von ,Geschlecht' und Geschlechterfragen verdichten:

1368

1369

1370

1371

1372

1373

1374

1375

1376

1377

1378

1379

1380

1381

1382

1383

1384

1385

1386

1387

1388

1389

1390

1391

1392

MICHI: Also ich stoß mich so=n bisschen daran, wenn ich merke, dass es halt zu sehr, das- oder dass für mich zu sehr dieses Thema fokussiert wird und andere Sachen 'n bisschen rausfallen; und ich glaube, was ich wichtig fände, wenn das im Studium immer mit dabei ist, wobei ich halt auch finde, dass es das ja auch schon ist, dadurch dass es auch sprachkritisch beachtet wird. [...] Ich würd mir aber wünschen, dass es nicht- dass es nicht so:o dominant ist, dass es irgendwie alles überschattet oder dass es so scheint, als ob das Thema wichtiger ist als alle anderen; weil ich finde, also, diese Frage auch mit der Identitätsbildung- da hab ich gedacht, ja:a? ich finds auch wichtig, zum Beispiel irgendwie nochmal zu gucken, hab- hab ich vielleicht wirklich Unsicherheiten weil ich 'ne Frau bin? Also auch seine eigene Rolle sein eigenes Rollenverständnis zu reflektieren; und zu gucken was man selber eigentlich auch für Männer-, Frauenbilder hat und von seiner eigenen Rolle, würde ich mich, also hab ich-, oder hab ich Probleme damit, mich gegenüber Männern zu positionieren, oder was auch immer?

JONA:

$\llcorner\operatorname{Mhm}[\ldots]$

NEELE: Ja ja.

JONA: [..] also so das Ausbilden, also im Endeffekt die Selbstbildung in Richtung Aushalten-können von Unklarheiten auch; und von schwierigen Situationen sozusagen, 
1412

1413

1414

1415

1416

ja? Und natürlich das Selbstreflexive, aber ganz wichtig eben weg, also neben dem Rationalen so was, so 'ne Toleranz auszubilden, so 'ne gefühlte, emotionale Fähigkeit mit Unklarheiten umzugehen.

MICHI:

NEELE:

JONA:

MICHI:

???:

NEELE: $\llcorner$ Mhm, ja

$\llcorner\mathrm{ja}$,

[... ] Im Endeffekt dadurch aber auch Identitätsbildung und Selbstbildung, Persönlichkeitsentwicklung, Reifung so was wir hier auch die ganze Zeit betreiben in dem wir dauernd nur rumdiskutieren und uns gegenseitig annerven, auch aber im Endeffekt ja auch so Perspektivwechsel einüben und Empathie ausprobieren und so dieses Diskurshafte, und das finde ich, ist ein Ding, also dieses Persönlichkeitsding voranbringen und gleichzeitig aber irgendwie, dass man fachlich argumentieren kann auch, und dadurch auch irgendwelche dummen Argumente relativieren kann, wenn das den anderen halt interessiert ((lacht)). Also aufgrund irgendwelcher Studien, zum Beispiel

$\llcorner\mathrm{Ja}$

$[\ldots]$

Also was ich auch sehr schön fand; dieses Aushalten von Unsicherheiten. Mhm. Das find ich auch total-, das trifft es so, ${ }^{\circ}$ find ich $\operatorname{auch}^{\circ}(3)$, fand ich gut ((lacht))

Michi distanziert sich von Jonas Orientierung und macht deutlich, dass es sie störe, wenn das, ,Thema zu sehr fokussiert wird" und deshalb andere Themen nicht mehr berücksichtigt werden (,andere Sachen 'n bisschen rausfallen“). Ihre persönliche Einschätzung ist, ,dass es das ja auch schon ist, dadurch dass es auch sprachkritisch beachtet wird". Eine Überfokussierung und Dominanz des Themas, also wenn es ,so scheint, als ob das Thema wichtiger ist als alle anderen", stellt für sie einen negativen Horizont dar. Als positiven Gegenhorizont greift Michi den bereits in der vorherigen Passage verwendeten Begriff der ,Identitätsbildung“ auf. Damit verbindet sie eine selbstreflexive Haltung (,sein eigenes Rollenverständnis zu reflektieren ${ }^{6}$ ) und ein geschlechterreflektierendes Verhalten (,zu gucken was man selber eigentlich auch für Männer-, Frauenbilder hat"). Jona und Neele schließen validierend an und Jona 
buchstabiert die gemeinsame Orientierung genauer aus. Er hebt hervor, dass es bei der Thematisierung von ,Geschlecht“ auch darum ginge, „Unklarheiten" und „schwierige Situationen“ auszuhalten. Jona ginge es dabei nicht (nur) um das „Rationale“, also eine theoretische Ebene, sondern um die ,Selbstreflexion“ und den Erwerb der „emotionale[n] Fähigkeit, mit Unklarheiten umzugehen“" Bestätigt von Michi und Neele fasst Jona das Gesagte mit den Begriffen „Identitätsbildung und Selbstbildung, Persönlichkeitsentwicklung, Reifung" zusammen. Das Studium biete für Jona auch, dass, indem „diskutiert" werde, „Perspektivwechsel" und somit auch „Empathie“ eingeübt werde. Abschließend erklärt Jona, ihm sei wichtig, dass „man fachlich argumentieren kann“, um unangebrachten Argumenten etwas entgegenzusetzen. Jona orientiert sich an einer Mischung aus Selbstreflexion und theoretischer, fachlicher Kompetenz. Neele stimmt Jona darin zu, dass das "Aushalten von Unsicherheiten“ auch für sie wichtig sei.

In der Gruppe Metall lässt sich in Bezug auf die studiumsbezogene Thematisierung von ,Geschlecht', Identitätsbildung, Selbstreflexion und eine geschlechterreflektierende Haltung sowie die ,emotionale Fähigkeit, mit Unklarheiten umzugehen“" als gemeinsamer positiver Horizont rekonstruieren.

\subsubsection{Zusammenfassung der Gruppe Metall}

Die Thematisierung von ,Geschlecht' und Geschlechterfragen verläuft bei der Gruppe Metall sehr sprunghaft und ist von verschiedenen Diskursmodi geprägt. Es kommt sowohl zu parallelen Modi (z. B. beim Thema Benachteiligung von Frauen im studiumsbezogenen konjunktiven Raum), aber auch zu divergenten (z. B. bei der Kritik an der studiumsbezogenen Thematisierung von ,Geschlecht') und zu oppositionellen Diskursorganisationen (z. B. bei der Passage ,Geschlecht': „[I]ch find's extrem wichtig", aber es ist ,irgendwie "ne Position zu entwickeln"). In dieser Gruppe wurde ,Geschlecht' viel diskutiert und war in selbstverständliches Thema, das nicht legitimiert werden musste. Es lassen sich zahlreiche individuelle aber auch kollektive Orientierungen rekonstruieren. Im Facettenreichtum der Diskussion zeigt sich auch die Vielfältigkeit und die Variationsbreite, mit welcher der Begriff ,Geschlecht' Gebrauch findet. ,Geschlecht' wird in der Gruppe Metall stellvertretend für ein inhaltliches Thema im Studium im Zusammenhang mit Geschlechtsidentität, Sexualität, ,biologischem' und sozialem, Geschlecht' sowie für eine Form der Existenzweise verwendet. Geschlechterfragen werden hauptsächlich 
mit Ungleichheit, Differenz und Benachteiligung in Verbindung gebracht. ,Geschlecht' und Geschlechterfragen werden von den Studierenden mit der Benachteiligung von Frau gleichgesetzt und quasi auch vorausgesetzt. Die eigene (eindeutige) Geschlechtlichkeit, die heterosexuelle Geschlechternorm sowie die Geschlechterdifferenz wird nicht in Frage gestellt.

In der Gruppe Metall lässt sich das soziale Feld der Bildungsinstitution Universität als studiumsbezogener konjunktiver Erfahrungsraum rekonstruieren, der geprägt ist von Möglichkeiten, sich mit Geschlechterfragen auseinanderzusetzen. Gender gehört zum geteilten Wortschatz der Studierenden und ist Teil ihrer konjunktiven Erfahrung. Auch die geschlechtergerechte Sprache ist Teil der konjunktiven Erfahrung der Studierenden der Gruppe Metall und Teil ihrer habituellen Praxis. In Bezug auf die Thematisierung von ,Geschlecht' im Curriculum lässt sich rekonstruieren, dass diese als einseitig wahrgenommen wird. Damit beziehen sie sich auf einen, weiblichen' Fokus auf Seiten der Lehrenden und der Lehrveranstaltungsinhalte und auf die marginale Stellung männlicher Lebenswelten und Akteure. Auch in der Gruppe Metall wird das Stereotyp des erziehungswissenschaftlichen Studiengangs als ,Frauenstudiengang" reproduziert. Die große Anzahl von Studentinnen wird in der Gruppendiskussion (implizit) abgewertet und die Studenten stellen aufgrund ihrer geringen Anzahlen eine Besonderheit dar und erfahren explizit eine Aufwertung.

Bei den Studierenden der Gruppe Metall wird eine Theoretisierung von Geschlechterfragen markant, die sich durch die studiumsbezogenen Erfahrungen mit der Thematisierung von ,Geschlecht' erklären lässt. Es wird aber auch deutlich, dass dieses theoretische Geschlechterwissen nicht Teil des habituellen Handelns ist und sich die Studierenden der Gruppe Metall diesbezüglich an vortheoretischem Geschlechterwissen orientieren. Es zeigt sich, dass die Geschlechterzuschreibungen, Essentialisierungen sowie Differenzierungen nach ,Geschlecht' Teil der habituellen Praxis und eine kollektive Orientierung der Studierenden darstellen. Der Geschlechterdifferenz kann in der Gruppe Metall der Status einer Leitdifferenz zugesprochen werden. Die Handlungsschemata sind von der Geschlechterdifferenz bestimmt, wohingegen sich die rational orientierten Überzeugungen, Vorstellungen und Normen auf Geschlechtergleichheit und Gleichberechtigung zu richten scheinen. Personen, die einer eindeutigen und kohärenten Geschlechtlichkeit nicht entsprechen, werden als von der Norm abweichend wahrgenommen und thematisiert.

Die Thematisierung von Benachteiligung ist Teil des konjunktiven studiumsbezogenen Erfahrungsraums und geprägt von der Orientierung, 
dass Benachteiligung in erster Linie Frauen betrifft. Allerdings betonten die StudentInnen, keine geschlechtsbezogene Benachteiligung erfahren zu haben; dies scheint mit dem gemeinsamen studiumsbezogenen konjunktiven Erfahrungsraum in Zusammenhang zu stehen, da sich die Studentinnen aufgrund der weibliche Dominanz im Studiengang nicht benachteiligt fühlen. Im Studiengang Erziehungs- und Bildungswissenschaften scheint es aufgrund der hohen Anzahl von Studentinnen, so zumindest die Argumentationsfigur der Studierenden, keine geschlechtsbezogene Benachteiligung von Frauen (und Männern) zu geben. Geschlechtsbezogene Benachteiligung wird in der Gruppe Metall als individuelle/subjektive Empfindung inszeniert und somit als intentionale geschlechterbezogene Benachteiligung und Behandlung nivelliert und quasi als individuelles Problem normalisiert.

Zentral ist für die Gruppe Metall ihre Kritikaffinität und die ,Ja, aber'-Mentalität in Bezug auf die studiumsbezogenen konjunktiven Erfahrungen bei der Thematisierung von ,Geschlecht' und Geschlechterfragen. Die Kritikaffinität lässt sich als ein wesentliches Element des Habitus der Gruppe in Hinblick auf die Thematisierung von ,Geschlecht ${ }^{\text {* }}$ charakterisieren. Die Studierenden orientieren sich an der Vorstellung, dass eine Thematisierung von und eine Sensibilisierung für ,Geschlecht relevant seien, jedoch haben sie individuelle Vorstellungen davon, wie diese aussehen sollen. Gemein ist ihnen die Kritik an der studiumsbezogenen Thematisierung von ,Geschlecht' und Geschlechterfragen. Hier sind die Erwartungen seitens der Studierenden in gewisser Weise paradox: Das Thema ,Geschlecht' ist für die Studierenden zwar wichtig, weil es Chancengleichheit schaffe, aber auch ,schwierig“, weil im Zuge der Diskussionen dazu auch andere Personengruppen ausgegrenzt werden könnten. Die Geschlechtersensibilität und -reflexion sowie die Fähigkeit wissenschaftlich zu argumentieren, sind für die Studierenden von großer Bedeutung. Weitere wichtige Aspekte der Thematisierung von ,Geschlecht' stellen gesellschaftskritische Momente dar und die Orientierung an Wertschätzung, Gleichheit und Respekt. Der gesellschaftskritische und hinterfragende Impetus bei der Thematisierung von ,Geschlecht' stellt somit einen positiven Horizont dar. Bestimmte Formen der Thematisierung bzw. ein ausgrenzendes Diskussionsklima sowie eine Naturalisierung von Geschlechterdifferenz bilden dagegen einen negativen Horizont. Die Thematisierung von ,Geschlecht' ist nur dann legitim, wenn es dabei zu keiner Diskriminierung oder Ausgrenzung kommt. Auch eine mögliche Überfokussierung und Dominanz des Themas stellt für diese Gruppe einen negativen Horizont dar. 
In Bezug auf die Frage, was die Thematisierung von ,Geschlecht' und Geschlechterfragen im Studium (nicht) leisten kann und was ihnen bei der studiumsbezogenen Thematisierung von ,Geschlecht' und Geschlechterfragen wichtig sei, lässt sich zusammenfassend sagen, dass die Studierenden diesbezüglich sehr individuelle Orientierungsschemata äußern. Gemeinsam ist den Studierenden, dass sie davon ausgehen, dass die Thematisierung von ,Geschlecht' für die Identitätsbildung, Selbstreflexion und eine geschlechterreflektierende Haltung wichtig sei und dazu beitrage, die „emotionale Fähigkeit mit Unklarheiten umzugehen“ ausbilden zu können. Zudem sehen sie eine Relevanz sowohl für die pädagogischprofessionelle als auch die persönliche Ebene. Einer Implementierung des Themas ins Curriculum des Studiengangs stehen die GDT grundsätzlich positiv gegenüber, jedoch dominieren bei der Frage nach dem ,Wie ${ }^{6}$ individuelle Orientierungsschemata.

\subsection{Ein „,naturgegebener“ Zugang bei der Gruppe Wasser an der Universität Mond}

Die zweite Gruppendiskussion an der Universität Mond wurde sieben Monate nach der Gruppe Metall geführt und wird in der Folge als Gruppe Wasser bezeichnet. Diese Gruppendiskussion besteht aus drei Studierenden des Diplomstudiengangs Erziehungs- und Bildungswissenschaften. Alle drei beteiligten Personen sind weiblich, verpartnerschaftlicht und ohne Betreuungs- und Pflegeaufgaben. Ein Migrationshintergrund ist auch bei diesen Studierenden nicht gegeben. Die Studentinnen sind zwischen Anfang und Mitte Zwanzig. Sie stehen am Ende ihres Studium und sind im achten und dreizehnten Semester. ${ }^{23}$ Die drei Frauen kenn einander vom Studium, Ike und Juno verbindet eine Freundschaft. Die Studienschwerpunkte der GDT dieser geschlechtshomogenen Gruppe fassen Erwachsenenbildung, außerschulische Jugendarbeit und Sonderpädagogik. Zudem hat Ulli das zertifizierte Studienprogramm des Zentrums für Gender Studies an ihrer Universität absolviert. Sie ist die einzige in dieser Gruppe, die sich im Rahmen des universitären Lehrveranstaltungsangebots mit ,Geschlecht' und Geschlechterfragen beschäftigt hat. Im Gegensatz zur Gruppe Holz und Feuer ist den GDT das Lehrveranstaltungsangebot zur Frauen- und Geschlechterforschung inner- und außerhalb des erziehungswissenschaftlichen Studiengangs bekannt.

23 Ulli: w, 23 Jahre, 8. Semester; Ike: w, 26 Jahre, 13. Semester; Juno: w, 26 Jahre, 13. Semester. 


\subsubsection{Eine „,natürliche Haltung“" zum Thema vs. Reflexion von ,Geschlecht"}

Auch in dieser Gruppendiskussion ist die Thematisierung von ,Geschlecht" und Geschlechterfragen heteronom produziert, also von außen initiiert, dennoch kommt es auch hier zu autonomen und selbstläufigen Diskursen. In diesen wird deutlich, dass die Studierenden sich häufig auf ihr eigenes ,Geschlecht ' und auf ihre ,selbstverständliche' Heterosexualität beziehen. Neben impliziten heteronormativen Annahmen lassen sich auch eine explizite heterosexuelle und binäre Geschlechterordnung rekonstruieren. In Bezug auf die Thematisierung von ,Geschlecht' und Geschlechterfragen wird der Facettenreichtum der Auseinandersetzung bzw. der Beschäftigung der GDT mit Geschlechterfragen deutlich.

Als erste beschreibt Ulli, warum sie begonnen habe, sich mit ,Geschlecht' und Geschlechterfragen zu beschäftigen. Sie bezieht sich dabei auf die Frage der Forscherin, welche Themen für die GDT im Studium interessant bzw. langweilig wären und nennt ,Geschlecht' als ein spannendes Thema: ${ }^{24}$

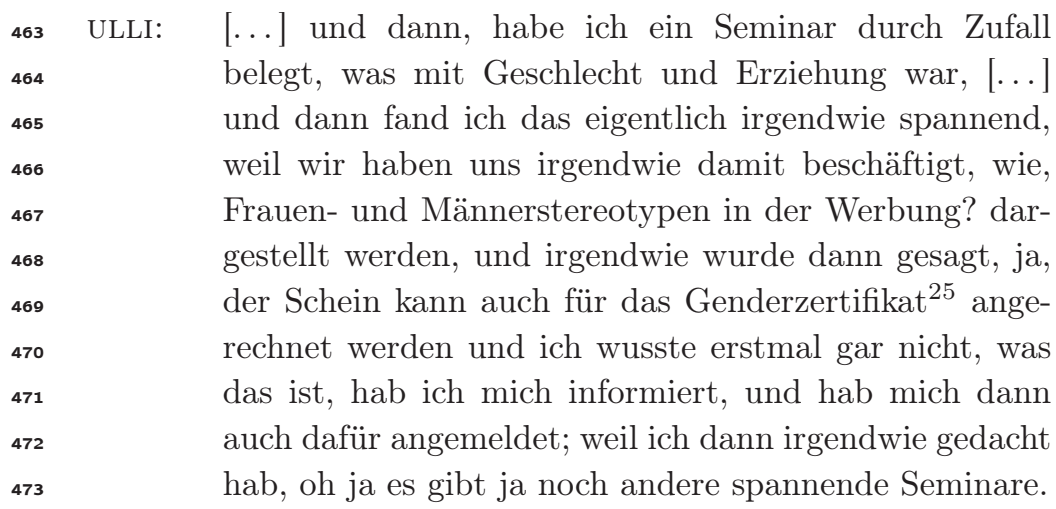

Hier wird deutlich, dass ihr Interesse an Geschlechterfrage im Studium durch ihre Teilnahme an einer Lehrveranstaltung zu diesem Thema geweckt wurde und dass das Lehrveranstaltungsangebot inner- und außerhalb ihres Fachbereichs, Ulli zu einer weiteren Auseinandersetzung angeregt hat. Für Ulli stellt das Studium demnach einen wichtigen Raum

24 Die zitierten Passagen beginnen mit der jeweiligen Originalzeilennummerierung, und weichen im Verlauf der Passagen z. B. aufgrund von gekennzeichneten Auslassungen davon ab.

25 Hierbei handelt es sich um die Bestätigung, am zertifizierten Studienprogramm des Zentrums für Gender Studies teilgenommen zu haben. 
für die Auseinandersetzung mit ,Geschlecht' dar. Sie spricht zwar davon, „durch Zufall", also unbeabsichtigt ein Seminar zu Geschlechterfragen in den Erziehungs- und Bildungswissenschaften belegt zu haben, hat sich dann aber aktiv und bewusst für eine weitere Auseinandersetzung da mit entschieden. ${ }^{26}$ Sie beschreibt weiter, dass sie nicht alle Seminare und Vorlesungen gut gefunden hat, dennoch hat dies ihr Interesse und ihre Neugierde an dem Thema nicht getrübt. In dieser Gruppendiskussion wird das Thema ,Geschlecht' zwar als erstes von Ulli genannt, jedoch nehmen die anderen GDT nicht darauf Bezug, sondern sprechen über andere (für sie relevante Themen) weiter. Erst als ,Geschlecht' von außen (von der Forscherin) nochmals initiiert wird, nehmen alle GDT darauf Bezug und skizzieren ihren Zugang zum Thema. Die Thematisierung von ,Geschlecht' und Geschlechterfragen ist für diese Gruppe keine Dimension des studiumsbezogenen konjunktiven Erfahrungsraums.

\begin{tabular}{|c|c|c|}
\hline \multirow{2}{*}{\multicolumn{2}{|c|}{545 IKE: }} & \multirow[b]{2}{*}[\ldots]{ ich habe mich nicht großartig damit beschäftigt, } \\
\hline & & \\
\hline & & witzigerweise, weil ich nämlich eigentlich relativ poli- \\
\hline 547 & & tisch interessiert bin, und das eigentlich schon ein The- \\
\hline 548 & & ma ist, dass vor allem die Dozentinnen, die ich gut fin- \\
\hline 549 & & de an der Uni, machen, aber ich hab eben einfach da- \\
\hline 550 & & durch, dass ich immer andere Sachen gemacht habe, \\
\hline 551 & & keinen direkten Zugang bekommen, finde es auch nicht \\
\hline 552 & & so schlimm, weil ich so eine relativ, denke ich natürlich \\
\hline 553 & & Haltung dazu schon habe, einfach durch meine Mutte \\
\hline 554 & & die durch die Frauenbewegung da einiges mitbekomm \\
\hline 555 & & hat, ich hab da viel gelesen Richtung de Beauvoir vi \\
\hline 556 & & leicht, ja. So. Von daher finde ich es jetzt nicht so: tr \\
\hline 557 & & gisch; also es ist jetzt kein Thema, bei dem ich den \\
\hline & & ch, dass ich es nie eingehender studiert hab \\
\hline
\end{tabular}

Ike bezieht sich zumindest am Beginn ihrer Wortmeldung auf ihr Studium und beschreibt, dass sie sich im Rahmen dessen ,nicht großartig“" mit ,Geschlecht' und Geschlechterfragen beschäftigt und keinen Zugang zum Thema gefunden habe. In ihren Ausführung wird deutlich, dass Ike aber auch keinen Zugang zum Thema gesucht hat. Aufgrund der „natürlichen Haltung", die sie sich selbst unterstellt, sei eine vertiefende Auseinandersetzung für sie nicht nötig gewesen. Dies stellt für Ike jedoch kein

26 Auf ähnliche Weise beschreibt Neele aus der Gruppe Metall ihren Zugang zu Geschlechterfragen als passiv und als einen Prozess, in den sie so „reingerutscht" sei (GD_Metall). 
Versäumnis dar, da sie sich bereits aufgrund ihrer „Mutter, die durch die Frauenbewegung da einiges mitbekommen hat" außerhalb ihrer universitären Ausbildung mit Geschlechterfragen auseinandergesetzt habe. Ike verweist hier auf das Selbststudium im familiären Raum und auf den Generationenaustausch zwischen Mutter und Tochter als einen weiteren Ort der Thematisierung. Indem Ike davon ausgeht, dass sie ,schon eine“ „natürliche Haltung dazu“ habe, impliziert sie, dass es für sie im Rahmen ihres Studiums diesbezüglich nichts mehr zu lernen, erfahren und reflektieren gebe. Ike finde es dennoch seltsam, dass sie sich im Rahmen ihres Studiums nicht damit beschäftigt habe, da zwei Aspekte dafür sprechen würden: Zum einem scheinen insbesondere jene „Dozentinnen“, die Ike gut heißt, Lehrveranstaltungen oder Ähnliches zu diesem Thema zu „machen“ und zum anderen beschreibt sie sich selbst als „relativ politisch interessiert" und antizipiert damit, dass Geschlechterfragen politische Fragen seien bzw. ,Geschlecht' ein politisches Thema sei. Ike steht einer Thematisierung von ,Geschlecht' nicht abwertend oder negativ gegenüber, sondern beschreibt eher, dass sie keine Notwendigkeit für sich sehe, ,Geschlecht' bzw. Geschlechterfragen „eingehender“" zu studieren. Hier lässt sich die Annahme entwerfen, dass eine ,natürliche Haltung" eine Art Legitimationsfigur zu sein scheint, die für Ike eine (theoretische) Auseinandersetzung im universitären Kontext unnötig macht. Offen bleibt jedoch, was Ike unter einer ,natürlichen Haltung" versteht und wie sich diese äußert. Zudem lässt sich aufgrund der folgenden Wortmeldung bereits hier eine These formulieren, die im Verlauf der Auswertung noch bestätigt wird: Geschlechterfragen werden in dieser Gruppendiskussion als ,Frauenfragen verstanden und die Thematisierung (im Studium, aber auch außerhalb) wird von Frauen übernommen. In Bezug auf ,einen gleichberechtigten Bildungszugang" erläutert Ike: „[N]atürlich habe ich das im Hinterkopf, ich bin eine Frau, und mir ist es wichtig." Ike verdeutlicht, dass sie das Thema qua ihres Geschlechts, ihres Frauseins, im Blick habe, auch wenn es nicht ihr Hauptinteresse sei.

Auch Juno geht (in einer anderen Stelle der Diskussion) davon aus, dass sie einen ,naturgegebenen Zugang" zum Thema habe und impliziert wie auch Ike, dass ihr intuitiver Zugang zumindest nicht offensichtlich mit einem Bildungsprozess bzw. einer aktiven und bewussten Auseinandersetzung mit dem Thema zusammenhänge, sondern mit ihrem Frausein. Juno elaboriert: ,[A]lso es ist eigentlich auch etwas, das ich automatisch habe, in meinem Verständnis, und da war nie irgendwie ein Bedürfnis danach, das zu vertiefen." Juno macht zudem deut- 
lich, dass das Thema zwar im Laufe des Studiums präsent, aber für sie nicht relevant und interessant gewesen sei: ,[F]ür mich war Emanzipation jetzt nie ein großes Thema." Auch hier wird nicht ausgeführt, was einen „naturgegebenen Zugang" ausmacht, es hat aber den Anschein, als würde dieser auch hier als Legitimationsfigur für ein Desinteresse und eine Nicht-Thematisierung dienen. Juno und Ike teilen die Orientierung an einem ,natürlichen Zugang'، zu ,Geschlecht' und Geschlechterfragen, der unabhängig von Bildungsprozessen besteht und qua Frausein existiert.

Im Gegensatz dazu beschreibt Ulli ihren Zugang am Thema als gewachsen und ihre Auseinandersetzung damit als nicht abgeschlossen:

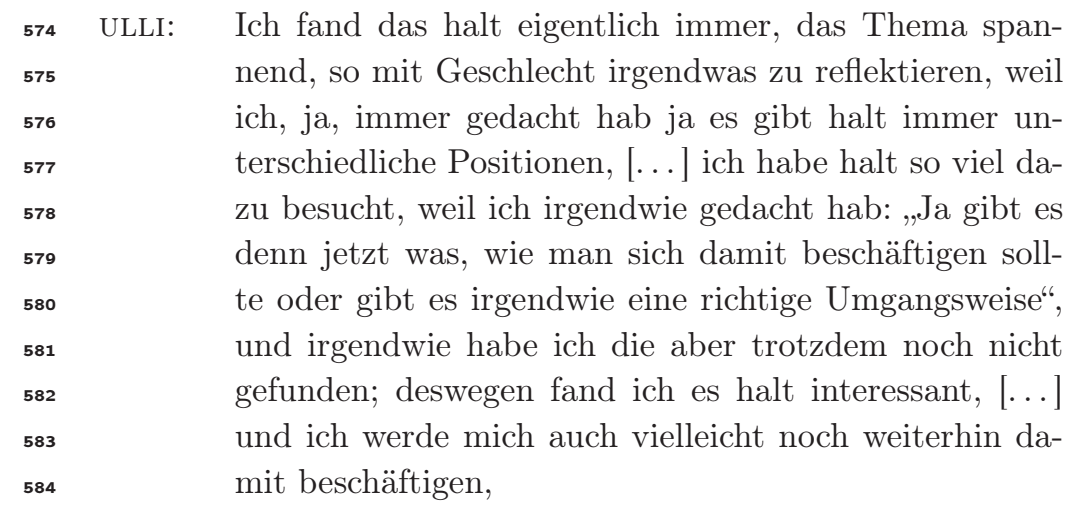

Ulli fand es „eigentlich immer spannend“ mit „Geschlecht irgendwas zu reflektieren" und führt weiter aus, dass sie sich gerne mit dem Thema auseinandersetze, um Lösungen für „Probleme“ und eine „richtige Umgangsweise" mit dem Thema zu finden. Damit führt Ulli eine neue Proposition ein Ulli resümiert jedoch, dass sie diese ,richtige Umgangsweise" trotz vieler Seminare „noch nicht gefunden" habe. Diese Suche nach einem Umgang, einer Haltung findet Ulli ,interessant" und stellt für sie den positiven Horizont dar. Im Gegensatz zu Ike und Juno geht Ulli nicht davon aus, aufgrund ihres Frauseins bereits über eine ,natürlich' angelegte Haltung zu verfügen. Für Ulli stellt die studiumsbezogene Thematisierung von ,Geschlecht' und Geschlechterfragen einen positiven Horizont dar, auch wenn sie negative Erfahrungen gemacht hat. Sie beschreibt, dass die Diskussionen in den Seminaren immer wieder auf das Gleiche hinauslaufen und die Schuld bei der „Politik“ gesucht werde. Ulli stimmt mit dieser Diagnose nicht überein, sondern gibt zu bedenken, dass es die Lösung vielleicht gar nicht gebe, weil „eigentlich weiß man 
gar nicht so richtig, wie man jetzt mit dem Thema umgehen soll" und „vielleicht weiß einfach keiner, was richtig und was falsch ist". Für Ulli stellen die unterschiedlichen Zugänge und Debatten auf der Suche nach Lösungen für das ,Geschlechterproblem' eine gewisse Faszination dar, die dazu motiviert, sich auch zukünftig mit Geschlechterfragen zu beschäftigen.

In der Passage werden die unterschiedlichen Arten der Auseinandersetzung bzw. der Beschäftigung der GDT mit Geschlechterfragen deutlich. Ullis Beschäftigung ist im Gegensatz zu Ikes und Junos universitär und theoretisch geprägt. Zudem betont Ulli, dass sie noch keinen Umgang mit ,Geschlecht" und Geschlechterfragen gefunden habe und sie daran zweifle, dass es eine, richtige' Form der Beschäftigung damit gebe. Für Ulli stellt die studiumsbezogene Thematisierung von ,Geschlecht' und Geschlechterfragen einen positiven Horizont dar. Ike und Juno heben hingegen ihren „natürlichen“, „,naturgegebenen“ Umgang hervor, der keiner universitären Auseinandersetzung bedürfe, sondern dass qua Frausein eine (scheinbare) Sensibilisierung für das Thema und eine Selbst-Reflexion gegeben sei. Damit zeigt sich bei ihrer Argumentation ein Wirkmechanismus für ein Desinteresse und eine (De-)Thematisierung von Geschlecht.

Die theoretische bzw. curriculumsbezogene Thematisierung von ,Geschlecht ${ }^{6}$ und Geschlechterfragen stellt somit keine Dimension des studiumsbezogenen konjunktiven Erfahrungsraums dar. Für Ulli stellt die studiums- und curriculumsbezogene Thematisierung einen positiven, und für Ike und Juno einen negativen Horizont dar. Gemein ist den Studierenden die Gleichsetzung von Geschlechterfragen mit ,Frauenfragen und -problemen'. Diese scheinen jedoch nicht thematisierbar zu sein, da sie im Widerspruch zur rhetorischen Modernisierung und ihrer Vorstellung von Gleichheit, Gleichberechtigung und Egalität von Männern und Frauen stehen.

\subsubsection{Die statistische Geschlechterverteilung bei den Studierenden und den ProfessorInnen}

Auf die Frage der Gruppendiskussionsleiterin, ob sich die GDT an Situationen im Studium erinnern können, in denen ,Geschlecht thematisiert wurde und auf welche Art, beschreibt Ulli eine Situation am Anfang des Studiums, in der auf die relativ hohe Zahl von Studenten im Studiengang hingewiesen wurde: 


\begin{tabular}{|c|c|}
\hline ULLI: & Also ich kann halt direkt, muss ich direkt ans Anfang \\
\hline & des Studiums denken, dass schon eigentlich in der Eröff- \\
\hline & nung des Studiengangs direkt gesagt wurde: „Oh, hier \\
\hline & sind ja 20 Männer, das ist ja schon viel"; und damit fing \\
\hline & es eigentlich ging schon direkt die Eröffnung los, was \\
\hline & ha- also dass dann die Männer bei uns gesagt haben: \\
\hline & „Ja stimmt eigentlich, was machen wir jetzt hier einen \\
\hline & erziehungswissenschaftlichen Studiengang", und die das \\
\hline & Gefühl hatten, sich immer wieder behaupten zu müssen; \\
\hline & immer wieder darstellen, warum sie das jetzt mach \\
\hline & und das gut finden. (Dass die dann irgendwie) sagt, ja, \\
\hline & sie haben einen besonderen Bezug zur Theaterpädag \\
\hline & gik, oder sie wollen, ich weiß auch nicht später mal Do- \\
\hline & zent werden, oder Professor, oder also und für Frauen \\
\hline & war das sofort klar; warum sie Erziehungswissenschaf- \\
\hline & ten studieren. Also sie mussten jetzt nicht sich darstel- \\
\hline & len und sagen: „Ich studiere Erziehungswissenschaften \\
\hline & weil“, sondern die haben gesagt: „Ja, ich studiere das \\
\hline & halt", und Punkt. Und bei Männern hatte ich immer \\
\hline & das Gefühl, kam direkt, „ich erziehe- ich studiere das \\
\hline & weil", also und ja, damit war das eigentlich schon direkt \\
\hline & Thema, für sich und dass dann auch in anderen Semina- \\
\hline & ren immer geguckt wurde, ja, welche Studiengänge sind \\
\hline & jetzt besonders männerdominiert, wie zum Beispiel In- \\
\hline & formatik, warum sind da keine Frauen, und wie schafft \\
\hline & man es, Frauen da rein zu kriegen; wo man andererseits \\
\hline & jetzt nicht fragt, wie würde man Männer in die Erzie- \\
\hline & hungswissenschaften bekommen; also zumindest ist $\mathrm{m}$ \\
\hline & das nicht so aufgefallen. \\
\hline
\end{tabular}

In dieser Erzählung wird auf die statistische Geschlechterverteilung bei den Studierenden Bezug genommen und deutlich, dass Erziehungsund Bildungswissenschaften als ein weiblich dominiertes Fach reproduziert werden. Männer (männliche Studierende) weichen von der erziehungswissenschaftlichen Norm ab. Ulli erzählt, dass für die Studentinnen wie auch für die Studenten selbst bereits zu Beginn des Studiums die Aufmerksamkeit dafür geschaffen worden sei, dass Männer eine marginale Position einnehmen und diese Rechtfertigung bedürfe. Ulli hat 
den Eindruck, dass die Studenten „das Gefühl hatten, sich immer wieder behaupten zu müssen" und ,immer wieder darstellen, warum sie das jetzt machen und das gut finden". Die Studenten scheinen ihre Studienwahl mit einem besonderen Ziel oder einem speziellen Interesse rechtfertigen zu müssen. Im Gegensatz dazu sei bei den Frauen sofort klar, „warum sie Erziehungswissenschaften" studieren. Sie müssen ihre Studienwahl nicht erklären oder begründen, sie stellen die unhinterfragte geschlechtliche Norm im Studium der Erziehungs- und Bildungswissenschaften dar. Die Frage nach der statistischen Geschlechterverteilung wurde auch auf andere Studiengänge übertragen, hier werde jedoch der Fokus umgekehrt und gefragt, wie man mehr Frauen für ,männerdominierte“ Studiengänge interessieren könnte. In den Erziehungs- und Bildungswissenschaften hat Ulli nicht die Erfahrung gemacht, dass danach gefragt worden sei, wie man mehr Männer für den Studiengang motivieren könne. Die Thematisierung der ungleichen Geschlechterverteilung endet innerhalb der Erziehungs- und Bildungswissenschaften bei deren Feststellung und Reifizierung.

Juno nimmt auf die statistische Geschlechterverteilung Bezug, verschiebt diese jedoch auf die Ebene der ProfessorInnen:

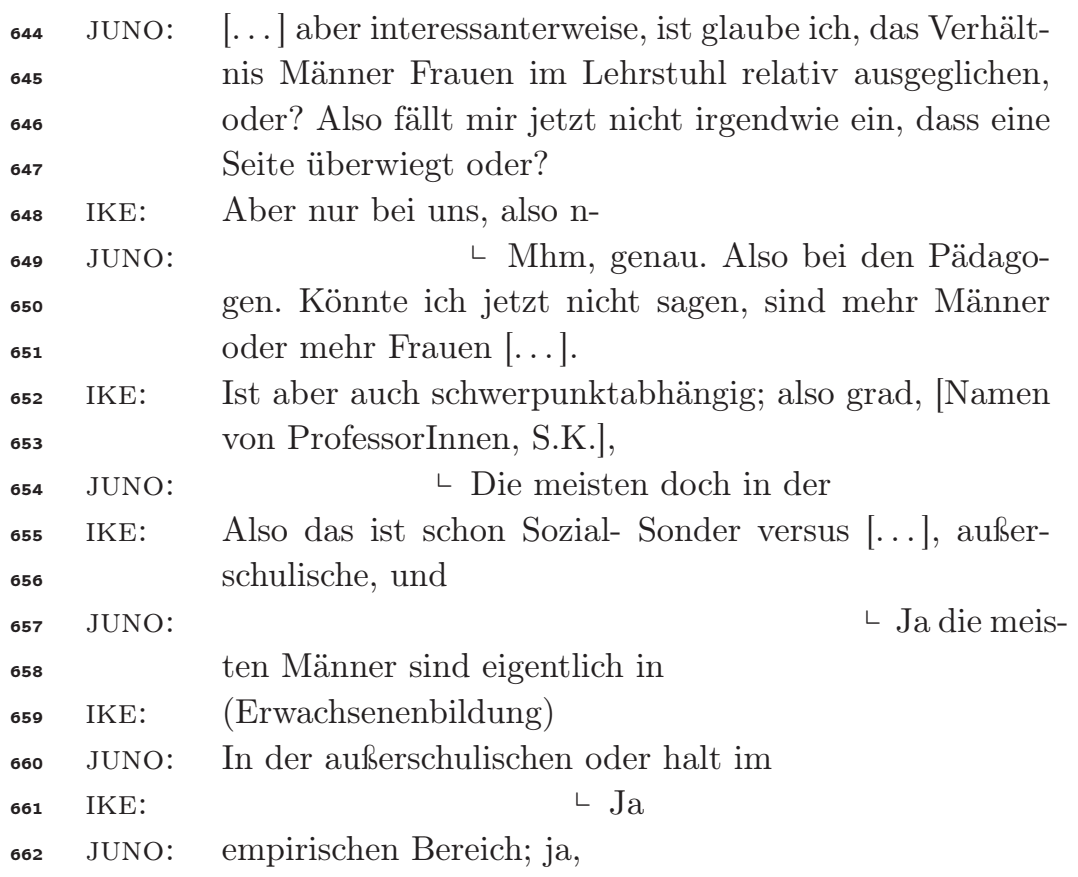




$$
\begin{array}{llr}
\mathbf{6 6 3} & \text { IKE: } & \multicolumn{2}{c}{\text { Ja. }} \\
\mathbf{6 6 4} & \text { JUNO: } & \text { Genau. Also ich glaube in der Sozial- und Sonderpäd- } \\
\mathbf{6 6 5} & & \text { agogik } \\
\mathbf{6 6 6} & \text { IKE: } & \llcorner\text { Lehrstühle glaube ich. } \\
\mathbf{6 6 7} & \text { JUNO: } & \text { Haben wir keinen männlichen Dozenten. }
\end{array}
$$

Juno und Ike arbeiten hier gemeinsam heraus, dass das statistische Geschlechterverhältnis bei den ProfessorInnen in der „Pädagogik" „relativ ausgeglichen" sei; dies scheint für sie einen positiven Horizont im Gegenzug zum statistischen ungleichen Geschlechterverhältnis bei den Studierenden zu bilden. Die konstatierte Geschlechtersegregation entlang der jeweiligen Schwerpunkte - sie kommen zu dem Schluss, dass es in der Sozial- und Sonderpädagogik mehr Professorinnen gebe und in der außerschulischen Jugendarbeit, Erwachsenenbildung und im empirischen Bereich mehr Professoren - bleibt unkommentiert im Raum stehen. Durch den oben dargestellten Abschnitt lassen sich die Geschlechterdifferenz und die Geschlechterdualität in der Gruppe Wasser als Leitdifferenz rekonstruieren.

Auf Nachfrage von Ulli geben Ike und Juno an, dass in ihrem Jahrgang mit ca. einem Drittel ,relativ viele Männer" das Studium begonnen hätten, dennoch seien die Studentinnen in der Mehrheit. Somit bestätigen sie die statistische Dominanz von Studentinnen im Studium. Aufgrund der interaktiven Dichte wird in dieser Passage deutlich, dass die statistische Geschlechterverteilung eine selbstverständliche Thematisierung von ,Geschlecht ${ }^{6}$ zu sein scheint. Wie bereits Ulli zuvor reproduzieren nun auch Ike und Juno das Stereotyp vom erziehungswissenschaftlichen Studiengang als ,Frauenstudiengang6, in dem Männer als von der Norm abweichend dargestellt werden.

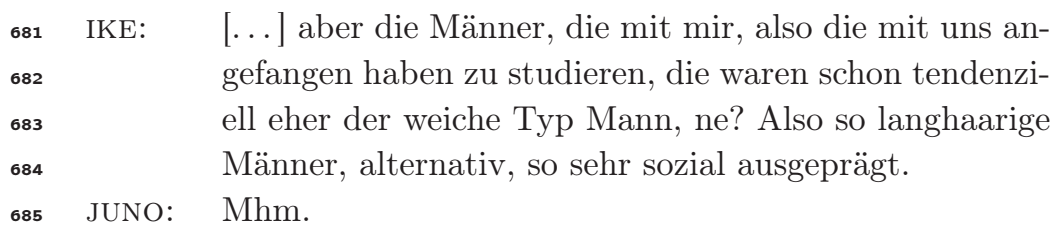


Mit dem Wort ,aber“ kündigt Ike einen Widerspruch zu dem davor Gesagten an und bringt gleichzeitig zum Ausdruck, dass sie sich auf das Vorherige, in diesem Fall auf ihre „relativ vielen Männer" als Kommilitonen, bezieht. Diese wären, laut Ike, schon „eher der weiche Typ Mann“. Diesen „Typ“ macht sie am Erscheinungsbild fest. Mit „alternativ" verweist Ike auf eine ,alternative" Subkultur, die sich z. B. im Kleidungsstil kenntlich macht. Zudem hebt sie die ausgeprägte soziale Kompetenz dieses ,Männertyps' hervor. Diese stellt eventuell auch eine Qualifikation für das Studium dar. Auch wenn in ihrem Studiengang „relativ viele Männer" seien, handle es sich dabei eher um den „weichen Typ Mann“. Mit dieser Hervorhebung wirkt es so, als würden ihre Kommilitonen von einer männlichen Norm abweichen. Dieser Orientierungsgehalt wird von Ike bestätigt. Die Gruppe Wasser teilt die konjunktive Erfahrung, dass es mehr Studentinnen gibt und dass die Studenten von der Norm abweichen: zum einen von der weiblich geprägten erziehungswissenschaftlichen und pädagogischen Norm und zum anderen von der hegemonialen Männlichkeitsnorm.

\subsubsection{Die Thematisierung von ,Geschlecht' wird als Überbetonung wahrgenommen}

In der Gruppendiskussion wird auch darüber gesprochen, dass die Thematisierung von ,Geschlecht' überbetont werden könne und es zum Teil auch eine ,zu starke ${ }^{6}$ und ,zu persönliche Involviertheit' mit dem Thema gebe. Für Juno stellt dies einen negativen Horizont dar. Juno führt dies am Beispiel einer (ehemaligen) „Genderfreundin“ aus:

$\begin{array}{ll}{ }_{741} \text { JUNO: } & \text { Also ich weiß noch, dass eine Freundin, also auch aus } \\ \text { 742 } & \text { dieser Anfangsclique vom Studium, sich auf einmal ganz } \\ & \text { stark in Richtung Gender orientiert hat, das hab ich } \\ \mathbf{7 4 4} & \text { früher gar nicht so mitbekommen, aber die war halt } \\ \mathbf{7 4 5} & \text { auf einmal so vereinnahmt in diesem Thema, dass es } \\ \mathbf{7 4 6} & \text { halt wirklich schwierig war, mit ihr da drüber zu reden } \\ \mathbf{7 4 7} & \text { weil sie so sehr wütend dann wurde; weil sie sich sofort } \\ \mathbf{7 4 8} & \text { irgendwie persönlich da angegriffen gefühlt hat, sobald } \\ \mathbf{7 4 9} & \text { man irgendwie was in Frage gestellt hat, was die Rolle } \\ \mathbf{7 5 0} & \text { der Frau oder die Rechte der Frauen anging. }\end{array}$

Hier eröffnet Juno ihre Orientierung, dass man sich auch ,zu stark“" mit gender auseinandersetzen können. Diesen negativen Horizont der The- 
matisierung zeigt sie am Beispiel einer ehemaligen Freundin auf. Juno resümiert, dass es mit dieser ,wirklich schwierig“" gewesen sei, über das Thema zu sprechen, ,weil sie so sehr wütend“ gewesen sei und sich schnell „persönlich angegriffen gefühlt hat", wenn sie den Eindruck hatte, „die Rechte der Frauen" würden in Frage gestellt werden. Juno beschreibt ihre Freundin als ,zu sehr' und ,zu persönlich' vom Geschlechterthema vereinnahmt. Zudem deutet sich hier an, dass das Thema "Gender" inhaltlich mit ,Frauenfragen' und ,Frauenproblemen' gefüllt sei. Junos Kritik an einer ,zu starken' Orientierung ,in Richtung Gender" grenzt sich von einer theoretischen Auseinandersetzung/Beschäftigung ab und fokussiert auf eine ,zu starke' persönliche Identifizierung mit ,Frauenfragen'. Als negativ nimmt sie die Thematisierung wahr, wenn sie auf einer zu „persönlich“ involvierten Ebene stattfinde. Indem Juno hinzufügt, dass die Gespräche mit ihrer „Genderfreundin“ manchmal „auch sehr interessant" gewesen seien, signalisiert sie, dass sie die Thematisierung von ,Geschlecht' und Geschlechterfragen nicht per se ablehnt. Den positiven Horizont der Thematisierung beschreibt sie als Horizonterweiterung (,„über den Tellerrand zu gucken“). Ähnlich stellt auch für Ike die Nicht-Thematisierung von ,Geschlecht' einen positiven Horizont dar. Sie erzählt (in einer hier nicht zitierten Stelle der Diskussion) von einer „Lesbe“, die im Gegensatz zu Junos „Genderfreundin“ nicht „die ganze Zeit auf solchen Themen rumgeritten ist". In der Formulierung „,rumgeritten“" kommt eine abwertende Haltung gegenüber der Thematisierung von ,Geschlecht' zum Ausdruck. Juno wie auch Ike teilen die Orientierung an der Nicht-Thematisierung von ,Geschlecht' und Geschlechterfragen. Was Juno an der Thematisierung von ,Geschlecht' ablehnt, elaboriert sie wie folgt:

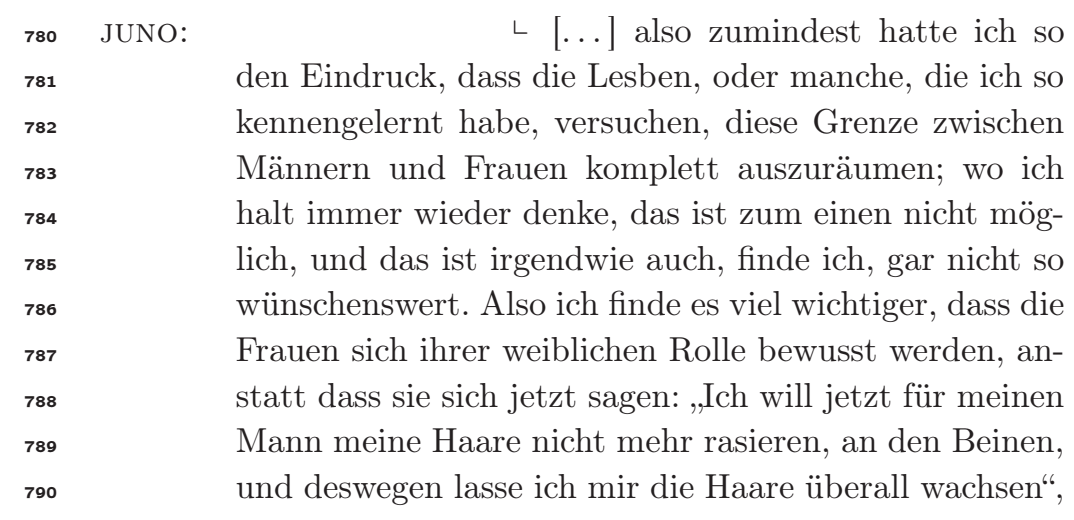


damit man sieht, dass ich die diese Grenze halt aufheben will; dass ich mir nichts sagen lasse. Aber ich finde, an solchen Grundsätzen kann man es gar nicht festmachen; und das ist irgendwie auch ein kultureller Hintergrund wo Frauen herkommen, und weiß nicht ich habe es, das heißt natürlich, ich will jetzt nicht über das über das Beine-Enthaaren reden, aber irgendwie vielleicht @so was@ so was Stures, was man sich dann so überlegt, um den Männern zu zeigen, dass ich nicht ihr Lakai bin, rasiere ich mir die Haare jetzt nicht, anstatt frei zu sagen: "Ich bin meine eigene Göttin und ich rasiere mich" oder so was. ((lacht)) Es ist, führt jetzt gerade so ein bisschen über Körperhygiene rum, aber solche Sachen irgendwie, ja? Also sind es halt so, (2) ehm so Kampfappelle irgendwie; die man da bei gender, glaube ich, gebraucht, denen man sich dann halt so anpasst. $[\ldots]$

Juno führt ihren Eindruck aus, das manche „Lesben“, die sie kennengelernt hätte, die „Grenze zwischen Männern und Frauen komplett auszuräumen“ möchten. Juno lehnt dies ab, weil sie denkt, dass dies ,zum einen nicht möglich" und zum anderen diese Entwicklung aus ihrer Perspektive nicht „wünschenswert" sei. Juno möchte diese Differenzen nicht aufösen, sondern setzt dem entgegen, dass „Frauen sich ihrer weiblichen Rolle bewusst werden" sollen. Was sie hier mit ,weiblicher Rolle" meint, wird nicht deutlich, es zeigt sich allerdings, dass Juno die Geschlechterdifferenz als ,naturgegeben' und unveränderlich ansieht und von einer komplementären Geschlechterbinarität ausgeht.

Am Beispiel der Debatte um die Körperhaarentfernung bei Frauen macht Juno deutlich, dass es ihr darum geht, dass sich Frauen nicht an Männern orientieren, sondern von ihren eigenen Bedürfnissen ausgehen sollen, anstatt mittels „Kampfappelle“ in den Widerstand zu gehen. Juno dient das Motiv der „Körperhygiene“ jedoch vielmehr dazu, ihre Ablehnung gegenüber der „Kampfappelle“ „die man da bei Gender [...] gebraucht" zum Ausdruck zu bringen. Juno macht deutlich, dass sie eine habitualisierte kämpferische Haltung und Gesprächsführung ablehnt. Diese Ausführungen zur Überbetonung der Thematisierung von ,Geschlecht' durch ,zu starke, persönliche' Involviertheit und einer kämpferischen Haltung kann auch als negativer Gegenhorizont zur oben darge- 
stellten ,natürlichen Haltung“" fungieren. Sie reproduziert damit das Stereotyp und den Mythos einer ,maskulinen Kampfemanze'. In dem Beitrag von Juno wird die implizite normative Orientierung vor Augen geführt, dass eine, wirklich' emanzipierte Frau selten ,Kampfargumente ${ }^{6}$ verwendet, sondern versucht, sich (gegen Männer und ihre verbliebenen Vorurteile) ohne Kampf durchzusetzen vermag.

\section{Der „Nervfaktor“ ,Geschlecht“}

Ulli meldet sich nun zu Wort und zeigt sich davon überrascht, dass Juno und Ike mit ihren Kommilitoninnen ,Geschlecht' und Geschlechterfragen diskutieren. Ulli hätte nämlich die Erfahrung gemacht, dass sich ihre Kommiliton_innen nicht für Geschlechterfragen interessieren, da sie das Thema langweilig fänden und als obsolet betrachten. Hier zeigt sich, dass die Gruppe Wasser in Bezug auf die Thematisierung von ,Geschlecht $^{6}$ und Geschlechterfragen mit KommilitonInnen über keine konjunktiven Erfahrungen verfügen.

\begin{tabular}{|c|c|}
\hline ULLI: & Also find ich ganz interessant, dass ihr so irgendwie \\
\hline 832 & Kommilitonen habt, die das interessiert oder so, also \\
\hline & weil ich habe halt gerade mal nachgedacht, und mir ist \\
\hline & es halt immer so ergangen, dass irgendwie, wenn ic \\
\hline & jetzt zu Kommilitonen gesagt hab, „Ja, ich das: Gen- \\
\hline & derseminar dödeldö besucht", wie auch immer das jetz \\
\hline & hieß, dann wurde immer gesagt: „Wieso das denn? Is ja \\
\hline & voll langweilig! Das kennt man doch alles." Und dar \\
\hline & immer so: „Ja und was kennt man denn jetzt wirklich?“, \\
\hline & also weil es wurde dann immer abgetan ja, ach man weiß \\
\hline & doch, dass es Ungleichheiten gibt und dass das irgen \\
\hline & wie behoben werden muss, und ich dann immer (gesą \\
\hline & habe), „Ja und wie?“, und dann wurde halt immer g \\
\hline & sagt: „Ja, ach, keine Ahnung, ist doch langweilig, u \\
\hline & will ich auch nicht wissen, und keine Ahnung, ich hab \\
\hline & keine Lust, mit denen zu diskutieren," und so, und des- \\
\hline & wegen also ich hab irgendwie ganz andere Erfahrung \\
\hline & gemacht; und fand das dann immer halt recht schad \\
\hline & dass viele Erziehungswissenschaftler sich gar nicht \\
\hline & irklich für das Thema interessieren, obwohl es eig \\
\hline
\end{tabular}


lich für mich, also ich finde es wichtig. Auch wenn das jetzt nicht in meiner Arbeit später vorkommen wird, ich finde es generell wichtig.

Ihre KommilitonInnen können es nicht nachvollziehen wenn Ulli ein "Genderseminar" besuche, da sie der Meinung seien, dort nichts Neues mehr zu erfahren. Ulli beschreibt das Desinteresse und die Langeweile, die das Thema bei ihren Kommilitonen auslöse und dass die Thematisierung oberflächlich bliebe: „,[M]an weiß doch, dass es Ungleichheiten gibt und dass das irgendwie behoben werden muss." Hier wird deutlich, dass das Wissen über Geschlechterungleichheit und -differenz Teil des ,Alltagswissens ${ }^{6}$ oder des ,vortheoretischen Wissens ${ }^{6}$ der Studierenden ist, jedoch besteht kein Interesse, sich mit Geschlechterfragen in Seminaren (theoretisch-reflexiv) auseinanderzusetzen. Sie hätten „,keine Lust, mit denen zu diskutieren“. Es bleibt zwar unbestimmt, mit wem sie nicht diskutieren möchten, es ist jedoch anzunehmen, dass es sich hierbei um Personen handelt, die sich für Geschlechterfragen engagieren. Mit dem demonstrativen Desinteresse scheinen sie einer Diskussion entgehen zu wollen.

Für Ulli ist diese Haltung nicht nachvollziehbar, da aus ihrer Sicht die Geschlechterfragen für die Erziehungs- und Bildungswissenschaften ,generell" ein wichtiges Thema darstellen und damit einen positiven Horizont markieren. Die Beschäftigung mit ,Geschlecht' und Geschlechterfragen verkörpert für sie einen wichtigen Teil der Ausbildung im erziehungswissenschaftlichen Denken und pädagogischen Handeln dar. Ike versucht nun, Ulli zu erklären, warum die KommilitonInnen keine Lust hätten zu diskutieren und warum sie glaube, dass das „Thema Gender" „schnell ein großer Nervfaktor" werde. Ike erklärt, dass es häufig mit „hetzenden Emanzen" und „zu persönlichen" Diskussionen assoziiert und deshalb „der wissenschaftliche Aspekt" auch oftmals übersehen werde. Letzteres stellt für Ulli und Ike einen positiven Horizont dar, wohingegen die persönlichen Diskussionen bei Ike einen negativen Horizont bieten. Ike elaboriert weiter, dass eine weibliche ,Opferrhetorik' und eine polemische Diskussionsweise zu Ablehnung führe. Frauen wollen nicht mehr, dass ihre Probleme primär auf ihr Frausein zurückgeführt werden.

In dieser Aussage wird ein Beispiel dafür deutlich, dass es mittlerweile verpönt ist, sich selbst als Opfer zu sehen, womit einhergeht, dass entsprechende Erfahrungen schwieriger verbalisierbar werden. Dies führt, zugespitzt formuliert, zu einem kollektiven Realitätsverlust und einer ,Normaffinität‘ (vgl. Bitzan 1997, S. 78). 


$\begin{array}{ll}{ }_{868} \text { IKE: } & \text { Und ich denke dann, dass die wahrscheinlich denken es } \\ & \text { geht dann in solchen Seminaren stark um ,wir armen } \\ { }_{870} & \text { Frauen, wir sind unterbezahlt, wir werden ausgenutzt, } \\ { }_{871} & \text { wir sind nur Sexsymbole“, und so. Also dass diese gan- } \\ { }_{872} & \text { zen Klischees teilweise, oder was natürlich auch teilwei- } \\ { }_{873} & \text { se Probleme sind, da sehr polemisch diskutiert werden } \\ { }_{874} & \text { und nicht mehr so viel mit der Wissenschaft zu tun ha- } \\ { }_{875} & \text { ben, und dass man deshalb von vorne herein eigentlich } \\ { }_{876} & \text { nicht so viel damit zu tun haben möchte. } \\ { }_{877} & \text { ULLI: }\end{array}$

Hierbei handelt es sich um implizite und explizite Vorstellungen bzw. um Alltagswissen darüber, wie ,Geschlecht' und Geschlechterfragen diskutiert werden; die Möglichkeit einer produktiven, theoretischen und wissenschaftlichen Debatte wird verdeckt bzw. ist nicht vorstellbar. Auch wenn Ike hier quasi für andere Kommiliton_innen spricht, wird darin auch ihre eigene Position deutlich, dass Klischees und Vorurteile - in diesen Seminaren werde angriffslustig, überspitzt und unsachlich diskutiert bzw. die Frauen inszenierten sich als Opfer davon abhalten, "Genderseminare" zu besuchen. Der Effekt dieser Klischees ist, dass man von dem Thema bereits "genervt" ist, bevor man sich damit auseinandergesetzt hat. Auch hier wird eine Legitimationsfigur der Nicht-Thematisierung von ,Geschlecht' deutlich. Zudem wird das Stereotyp reproduziert, dass in „Genderseminare[n]“ Frauen als Opfer darstellt werden und unwissenschaftlich diskutiert werde. Ulli bestätigt Ike auf einer Ebene des inhaltlichen Verständnisses. Es wird damit nicht markiert, ob diese Orientierung geteilt wird oder nicht.

Da Ulli als Einzige auf ihr Erfahrungswissen aus besuchten Seminaren zu ,Geschlecht' und Geschlechterfragen zurückgreifen kann, erklärt sie, dass man diese Klischees nicht generalisieren könne und grenzt sich von diesen ab. ${ }^{27}$ Auch wenn Ulli bereits Seminare besucht hat, die ihr nicht gefallen habe, hat sie noch nie erlebt, dass die Diskussionen ,zu persönlich' wären oder sich die Frauen als Opfer positioniert hätten. Ihrer Erfahrung nach treffen die von Ike dargestellten Vorurteile nicht zu, ganz im Gegenteil. Sie erklärt, dass vor allem theoretische Fragen im Zentrum stünden, z. B. ,welche Theorien passen denn in unsere Gesellschaft und kann man sie irgendwie verändern". Diese seien es auch, wofür sich Ulli

27 Da es sich hier um einen relativ langen Beitrag handelt, der aus Platzgründen nicht als Transkript-Ausschnitt angeführt werden kann, wird Ullis Wortmeldung im Sinne einer Pharaphrasierung wiedergegeben; selbes gilt für Ikes Antwort. 
interessiert: „[I]ch find das halt spannend.“ Damit grenzt sie die Gültigkeit der oben dargestellten Vorurteile ein. Für Ulli ist die ablehnende und desinteressierte Haltung ihrer KommilitonInnen jedoch noch nicht geklärt und sie wiederholt, dass sie es schwer nachvollziehen könne, dass Personen das Thema überhaupt nicht spannend finden, sondern ,sofort sagen nö, ach ich kenn alles, ich weiß alles". Mit Ullis Nachfrage bzw. Verständnisfrage wird erneut deutlich, dass die Studentinnen nicht auf dieselben Erfahrungen zurückgreifen. Damit zeigt sich auch, dass Ulli sich nicht an den herrschenden Vorurteilen und Klischees gegenüber der Thematisierung von ,Geschlecht' orientiert, sondern auf ihr gegensätzliches Erfahrungswissen zurückgreift.

Ike versucht nun, diese Gegenposition genauer zu beleuchten und erklärt, warum sich Leute abgrenzen würden. Ike erklärt, dass sie nicht davon ausgehe, „dass Kommilitonen, die so eine Aversion gegen die Genderthemen haben" sich vorstellen können, dass ,sehr theoretisch diskutiert werden kann“, sondern dass in deren Vorstellung „Hetzthematiken“ dominieren. Ike glaubt, dass deshalb eine ,negative Meinung gleich“ gegenüber dem „Thema Gender" herrsche. Mit „gleich“ verweist sie vermutlich darauf, dass das Thema bereits im Vorhinein in seiner Gesamtheit abgelehnt werde und eine Auseinandersetzung und eine Beschäftigung dadurch nicht angestrebt werden. Ike geht von der Annahme aus, dass Personen, die sich nicht für Geschlechterfragen interessieren, durch die herrschenden Vorurteile und Klischees abgeschreckt werden.

Juno reflektiert, dass auch sie von diesen „ganzen Klischees“ beeinflusst sei und diese auch reproduziere:

JUNO: Also ich merke es ja bei mir selbst auch, dass das so ein bisschen vorherrscht, dass ich jetzt auch gerade so diese ganzen Klischees so rausgehauen hab, und jetzt auch so denke, ja, vielleicht sollte ich mich da wirklich noch mal ein bisschen in so ein Seminar setzen, oder mich damit mehr auseinandersetzen, aber man kommt ja dann, wenn man nicht jetzt in der, in so einer Diskussion oder mit jemandem, den das größer beschäftigt, sich unterhält gar nicht darüber groß dazu, sich darüber Gedanken zu machen, wenn man jetzt nicht andere Sachen, oder oft andere Sachen hat, die einen jetzt mehr interessieren; was vielleicht auch schade ist, dann hät- 
Juno überlegt, ob es für sie nicht doch sinnvoll wäre, sich „damit mehr auseinanderzusetzen". Sie meint, dass sie diese Klischees vielleicht nicht hätte, wenn sie ein „Genderseminar" besucht hätte und dass sie es schwierig fände, sich über ,Geschlecht' Gedanken zu machen, wenn man niemanden habe, der/die sich mit Geschlechterfragen schon ,größer beschäftigt" hätte.

Das Studium wird von den Teilnehmerinnen der Gruppe Wasser als ein Ort gezeichnet, in dem Geschlechterfragen in Seminaren besprochen werden können, jedoch würden Klischees und Vorurteile darüber, dass dabei Frauen ,nur' als Opfer darstellt und Diskussionen dort ,zu' aggressiv geführt werden, Studierende davon abhalten, diese Seminare zu besuchen. Es wird so dargestellt, als würde das Thema bereits abgelehnt werden, bevor eine Auseinandersetzung und eine Beschäftigung damit stattgefunden hat. Diese Orientierung wird vor allem von Ike und Juno dargestellt; Ulli hat diesbezüglich andere Erfahrungen gemacht.

\subsubsection{Geschlechterdualität als „Strukturmerkmal“}

An der folgenden Passage lässt sich rekonstruieren, dass eine Differenzierung nach ,Geschlecht' Teil der habituellen Praxis und eine kollektiven Orientierung der Studierenden darstellt. Die Geschlechterdifferenz und -dualität hat in der Gruppe Wasser den Status einer Leitdifferenz. Die Unterscheidung zwischen Männern und Frauen wird auch in der folgenden Wortmeldung von Juno als Orientierung bestätigt. Sie betont die Schwierigkeit ,wenn sich zwei Menschen auf so einer ungeschlechtlichen Ebene begegnen" und arbeitet anschließend die strukturierende Funktion der Geschlechterdualität in Interaktionen heraus.

1083 JUNO: Es ist schwierig wenn sich zwei Menschen auf so einer ungeschlechtlichen Ebene begegnen; also wenn ich eine Freundin oder generell eine Frau treffe, dann habe ich einen gewissen Umgang, irgendwie. Dann spreche ich über bestimmte Themen, oder ich frage irgendwie was ich denke, das könnte sie jetzt interessieren, das müssen ja jetzt nicht irgendwie, keine Ahnung, Blumen und Klamotten sein, aber man hat ja einen bestimmten Ha- 


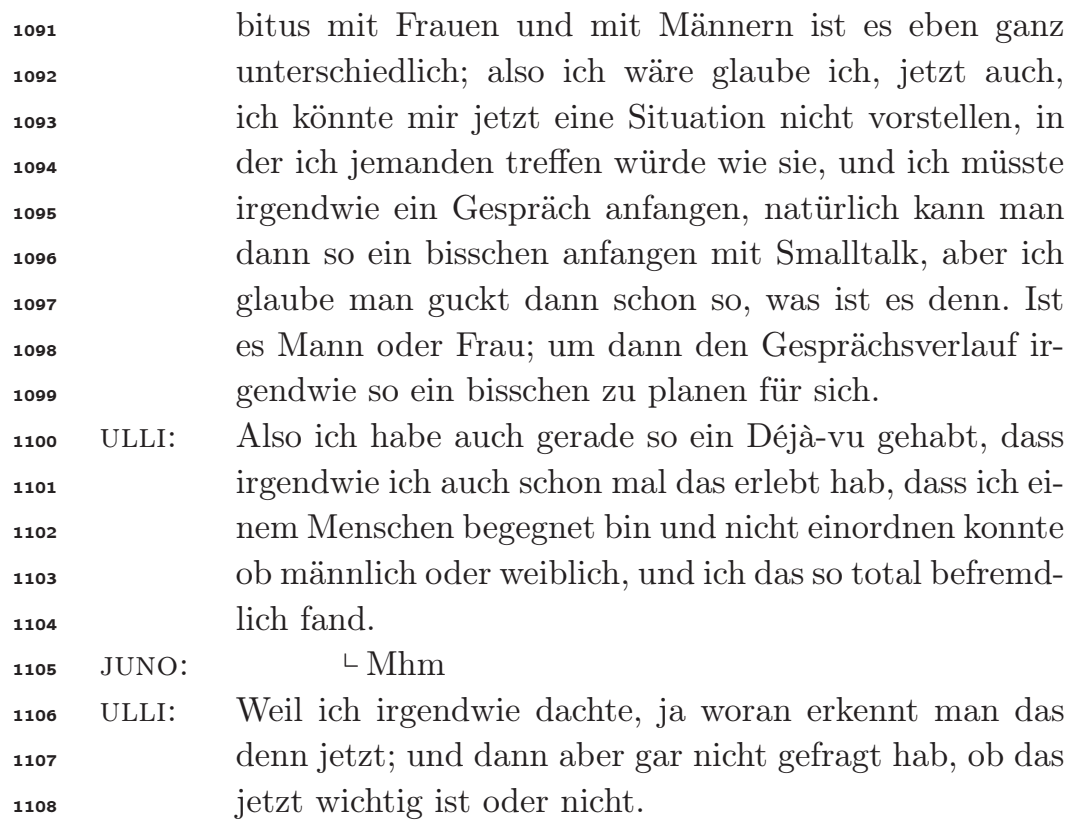

Juno beschreibt, dass man ,einen bestimmten Habitus mit Frauen und Männern" habe und einen geschlechtsbezogenen Habitus vom Gegenüber erwarte. Sie elaboriert das verunsichernde Moment und die Orientierungslosigkeit, die ein ,geschlechtsuneindeutiges' Gegenüber auslöse. Damit beschreibt sie unhinterfragt ihr vergeschlechtlichendes Verhalten und dass sie zwischen Männern und Frauen unterscheidet. Ulli bestätigt das „,befremdlich[e]“ Gefühl, wenn man „einem Menschen“ begegnet, der/die nicht eindeutig einem ,Geschlecht" zugeordnet werden könne. Diese Erfahrung beschreibt Ulli als „total befremdlich“. Sie reflektiert jedoch selbstkritisch, dass sie sich in dieser Situation in erster Linie gefragt habe, woran man ,Geschlecht' erkennen könne, anstatt sich die Frage zu stellen, ob die Eindeutigkeit von ,Geschlecht' für die Situation relevant sei. Es hat den Anschein, als würde Ulli hier ihren Fokus auf ,Geschlecht' hinterfragen und kritisch betrachten. Auch hier ist es wieder Ulli, die über ein alternatives Orientierungsschema nachdenkt. Auch in der Gruppe Feuer wird darüber gesprochen, dass die GDT gegenüber queere Personen kein Handlungsrepertoir besitzen und nicht wissen, wie sie reagieren sollen. In der Gruppe Feuer wird zudem der Bruch zwischen Orientierungsschema und -rahmen angesprochen. Die GDT wissen, dass sie einen ganz normalen Umgang mit queere Per- 
sonen haben soll(t)en, auf ihrer Handlungsebene spiegelt sich das jedoch nicht wider.

In der folgenden Passage verdichten sich erneut die Vorstellungen von einer kohärenten und eindeutigen Geschlechtlichkeit als Teil einer habituellen Praxis.

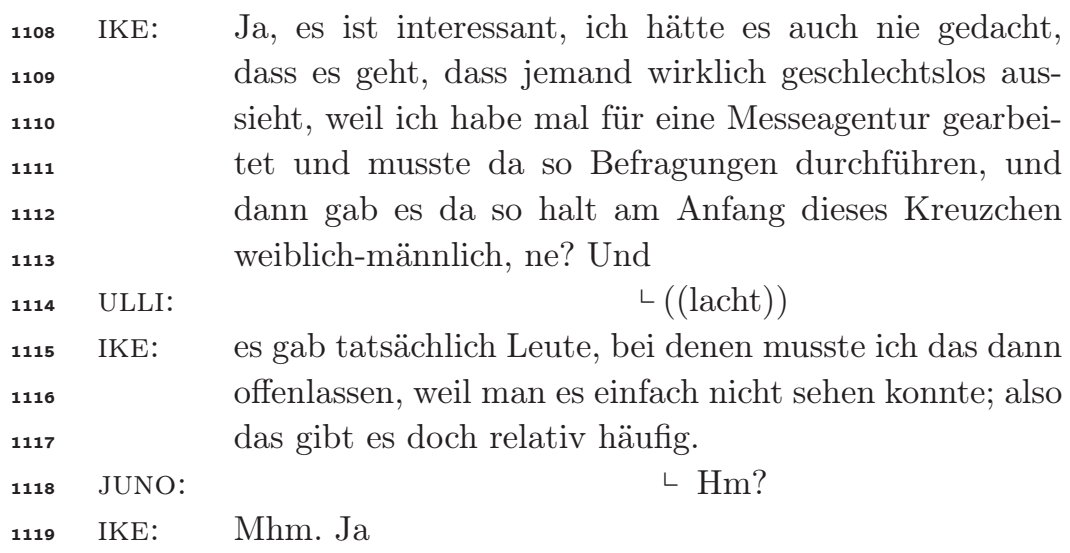

Ike beginnt damit, von einer vergangenen Orientierung („hätte es auch nie gedacht, dass es geht, dass jemand wirklich geschlechtslos aussieht") $\mathrm{zu}$ sprechen und schließt gleichzeitig an das Thema der vorherigen Diskussion zwischen Juno und Ulli an. Mit dem Wort „hätte“ kündigt sie an, eine differente Erfahrung gemacht zu haben. Sie erzählt nun auch von einer Situation, in der sie Personen nicht eindeutig als ,weiblich oder ,männlich‘ identifizieren konnte (,es gab tatsächlich Leute bei denen musste ich das dann offenlassen“). In ihrer Begründung (,weil man es einfach nicht sehen konnte") wird ihre Orientierung an der Geschlechterbinarität deutlich. Abschließend konstatiert sie, dass es „relativ häufig“ vorkomme, Personen zu begegnen, bei denen man nicht eindeutig erkennen könne, ob es eine Frau oder ein Mann sei. Interessant ist, dass Ike die Möglichkeit der Mehr- oder Zwischengeschlechtlichkeit nicht in den Sinn kommt. Auch beim anschließenden Diskurs zwischen Ike und Juno verdichtet sich die Orientierung an der (eindeutigen) Geschlechterbinarität. Die Passage soll nur kurz zusammengefasst werden.

Für Juno ist es nur schwer vorstellbar, dass Personen nicht entlang der dualen Geschlechterkategorien einordenbar sind:

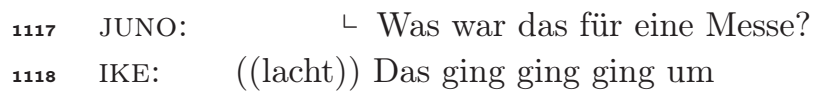




\begin{tabular}{|c|c|c|}
\hline 1119 & JUNO: & $\llcorner$ Wenn du da gleich so \\
\hline 1120 & & viele getroffen hast. \\
\hline 1121 & IKE: & $\llcorner$ um Forschung; also Marktforschung \\
\hline 1122 & & einfach. \\
\hline 1123 & JUNO: & ட Mhm? \\
\hline 1124 & IKE: & Ja. Interesse für irgendwelche Produkte. \\
\hline 1125 & JUNO: & Das heißt, die hatten mitunter so Hosenanzüge oder so \\
\hline 1126 & & was an, wo man es nicht, also oft kann man es ja dann \\
\hline 1127 & & schon so an der Kleidung oder so was. \\
\hline 1128 & IKE: & Nee, nee, nee; ich musste, ich musste, ich musste quasi \\
\hline 1129 & & Kunden befragen; also die waren nicht ${ }^{\circ}$ formal angezogen ${ }^{\circ}$ \\
\hline 1130 & JUNO: & $\llcorner$ Du hast \\
\hline 1131 & & die aber gesehen, oder? Oder war das so am Telefon \\
\hline 1132 & IKE: & $\left\llcorner\right.$ Jaja, ich hab die ${ }^{\circ}$ gesehen $^{\circ}$. \\
\hline 1133 & JUNO: & ${ }^{\circ} \mathrm{Okay},{ }^{\circ} \mathrm{mhm}$ \\
\hline 1134 & IKE: & $\llcorner$ Mhm. \\
\hline
\end{tabular}

Juno versucht hier anhand verschiedener Konstruktionen Ursachen dafür zu finden, warum Ike das ,Geschlecht' der befragten Personen nicht erkennen könne. Es fällt ihr sichtlich schwer zu glauben, dass man das ,Geschlecht' einer Person nicht eindeutig erkennen kann. Frauen und Männer sind nach Junos Orientierung eindeutig unterscheidbar und wenn dies nicht der Fall ist, dann muss dies einen speziellen Grund haben.

\subsubsection{Thematisierung von ,Geschlecht' ist weiblich}

In der Gruppendiskussion lässt sich rekonstruieren, dass die Thematisierung von ,Geschlecht' und Geschlechterfragen im konjunktiven Erfahrungsraum des Studiums weiblich geprägt ist, d. h. diese Inhalte von Frauen angesprochen und thematisiert werden.

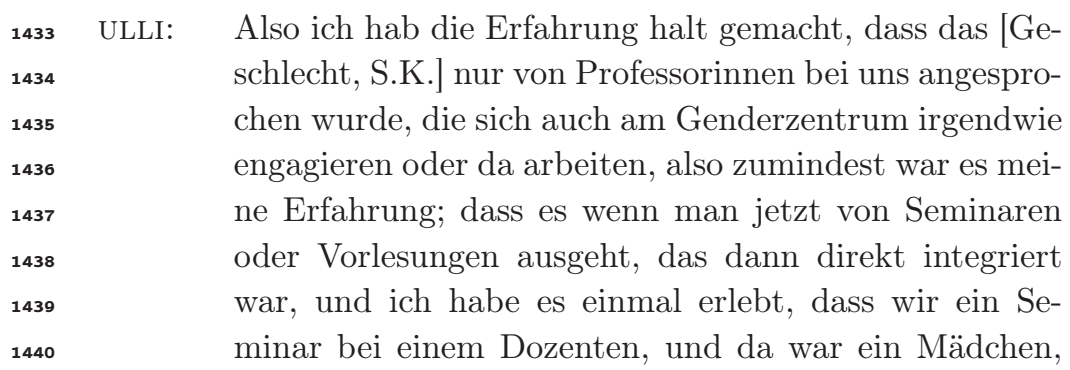


und die hat sich beschwert, die hat gesagt, das kann sie so nicht akzeptieren, sie möchte bitte mindestens eine Sitzung wo es auch um Geschlecht geht; und da war er völlig schockiert und hat gesagt, ja aber das hat er jetzt nicht so vorgesehen, und er kennt sich damit jetzt auch nicht aus, und dann hat sie gesagt, das ist egal, sie kennt sich aus, sie würde das Referat auch übernehmen. Und ja; und dann wurde das halt auf den Seminarplan für ein anderes Thema ersetzt, und das fand ich halt dann schon ziemlich beeindruckend, weil ich wäre jetzt nicht auf die Idee gekommen, das so wirklich so demonstrativ zu sagen, so „das muss da jetzt mit rein und das gehört dazu" und so, aber das fand ich gut dass sie das gemacht hat, und ja.

IKE: Ja also, die die Professorinnen die kann ich bestätigen, also dass die das ansprechen, also klar, nei also gerade Sozial- und Sonderpädagogik, [...].

IKE: Ich habe auch, also ich wüsste nicht, dass das in den Seminaren thematisiert wurde, was aber bei mir auch einfach bei der Auswahl der Seminare liegen kann. Also in der Erwachsenenbildung einfach schon mal viel weniger, würde ich behaupten, Programmplanung; da macht man sowas. Das ist kein, kein, kein großes Thema, oder Methoden, also in der Erwachsenenbildung; da ist das nicht so (2) aktuell, oder?

ULLI: Ja also ich weiß nicht, also ich weiß halt, dass eine Dozentin bei uns sich auch mit der $\mathrm{VHS}^{28}$ viel beschäftigt, und die halt auch erzählt dass das halt ein Problem ist, [...] und sie macht jetzt gerade eine Dissertation zu der Geschlechterkultur in der VHS, also und sie hat da ganz viel mit dem Thema auch angesprochen.

IKE: $\quad$ Mhm, ja das kann sein; [...] so und ach ja, im Beratung Mediation; auf jeden Fall. Also gerade Mediation,

Das Akronym „VHS“ steht für Volkshochschule. Eine Volkshochschule ist eine gemeinnützige Einrichtung zur Erwachsenen- und Weiterbildung. 
wenn es darum geht, dass quasi jeder einen Ansprechpartner haben, soll, also man überlegt zwei Mediatoren $\mathrm{zu}$ haben beispielsweise um einen weiblichen und einen männlichen Ansprechpartner zu haben, solche Dinge.

1479 (9)

Ulli bringt ihre Proposition ein, dass ,Geschlecht " und Geschlechterfragen nur von Professorinnen angesprochen werden. Die Thematisierung sei in den ,Seminaren oder Vorlesungen [...] direkt integriert". Ulli elaboriert, dass sich diese Professorinnen auch am "Genderzentrum" und somit auch außerhalb ihrer Disziplin engagieren. Damit betont sie die intensive Beschäftigung der Professorinnen mit ,Geschlecht'. Auch in ihrem zweiten Beispiel exemplifiziert Ulli die Proposition, dass die studiumsbezogene Thematisierung von ,Geschlecht' und Geschlechterfragen weiblich sei. Als singuläres Ereignis beschreibt Ulli, wie eine Studentin in einem Seminar den Dozenten auf das Fehlen von Geschlechterthematiken hingewiesen und diese auch eingefordert hätte. „[D]a war er [der Seminarleiter, S.K.] völlig schockiert und hat gesagt, ja aber das hat er jetzt nicht so vorgesehen, und er kennt sich damit jetzt auch nicht aus." Durch das Nicht-Wissen des Dozenten wird die Thematisierung als weiblich bestärkt. Indem Ulli auch die Ausnahme beschreibt, dass das Thema von einer Studentin angesprochen wurde, bestätigt sie die Regel, dass ,Geschlechterfragen' von Frauen angesprochen und thematisiert werden. Ulli erzählt, dass das Thema dann doch noch in den Seminarplan aufgenommen worden sei, weil sich die Studentin dazu bereit erklärt hatte, die Seminareinheit zu diesem Thema zu gestalten. Dass die Studentin das Thema „,demonstrativ" eingefordert und gesagt hätte, „das muss da jetzt mit rein und das gehört dazu", stellt für Ulli einen positiven Horizont dar. Dieses Verhalten fände sie ,ziemlich beeindruckend“, damit kommt zum Ausdruck, dass Geschlechterfragen auch für Ulli ein relevantes Thema darstellen. Ike schließt validierend an und bestätigt die Erfahrung, dass „Professorinnen“ ,Geschlecht" und Geschlechterfragen ansprechen und damit thematisieren würden. In Bezug auf den studiumsbezogenen Erfahrungsraum hebt Ike die ,Sozial- und Sonderpädagogik“ als Raum der Thematisierung hervor. Ike macht die Thematisierung nicht nur an den Professorinnen fest, sondern auch an Schwerpunkten, für die das Thema besonders relevant zu sein scheint.

Erst nach einer zehn Sekunden langen Pause führt Ike ihren Beitrag weiter und bringt ihre oppositionelle Erfahrung hinsichtlich eines anderen Aspekts zum Ausdruck. Im Gegensatz zu Ulli habe Ike nicht 
die Erfahrung gemacht, dass ,Geschlecht“ „in den Seminaren thematisiert wurde". Diese unterschiedliche Erfahrung erklärt sich Ike mit ihrer „Auswahl der Seminare" und darin, dass ,Geschlecht" und Geschlechterfragen im Gegensatz zur Sozial- und Sonderpädagogik in ihrem Studienschwerpunkt der Erwachsenenbildung „kein großes Thema“ darstellen. Ike generalisiert ihre Erfahrung und konstatiert, dass ,Geschlecht' in der Erwachsenenbildung ,nicht so aktuell“" sei. Anithetisch grenzt Ulli diese Generalisierung ein, indem sie von einer Dozentin in diesem Bereich erzählt, die sich intensiv mit Geschlechterfragen in der Erwachsenenbildung beschäftigt. Ulli formuliert hier die Antithese zu der Annahme, dass Geschlechterfragen in der Erwachsenenbildung nicht relevant seien und bestärkt gleichzeitig ihre These, dass ,Geschlecht' von Frauen thematisiert werde. Es kommt zu einer (rituellen) Konklusion der beiden Thesen, indem nun auch Ike bestätigt, dass ,Geschlecht' unter bestimmten Geschichtspunkten und in bestimmten Kontexten auch in der Erwachsenenbildung eine Rolle spielen können. Als Beispiele nennt sie die Bereiche Beratung und Mediation. In dieser Passage wird zum einen deutlich, dass die Thematisierung von ,Geschlecht ${ }^{6}$ und Geschlechterfragen im konjunktiven Erfahrungsraum des Studiums weiblich geprägt ist und dass die curriculums- bzw. seminarbezogene Thematisierung keine konjunktive Erfahrung darstellt.

In der Gruppendiskussion wird deutlich, dass die Thematisierung von ,Geschlecht' im universitären Studium weiblich ist und somit als eine ,Frauensache‘ erscheint. Ähnliches gilt auch für die Erfahrung der GDT im außeruniversitären Raum, in ihren privaten Beziehungen, wie folgende Passage zeigt:

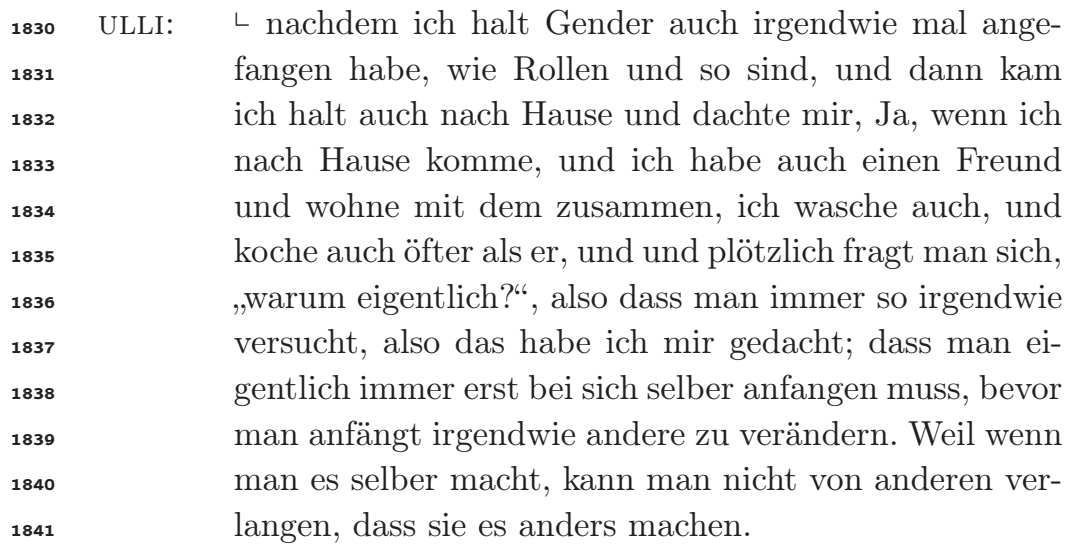




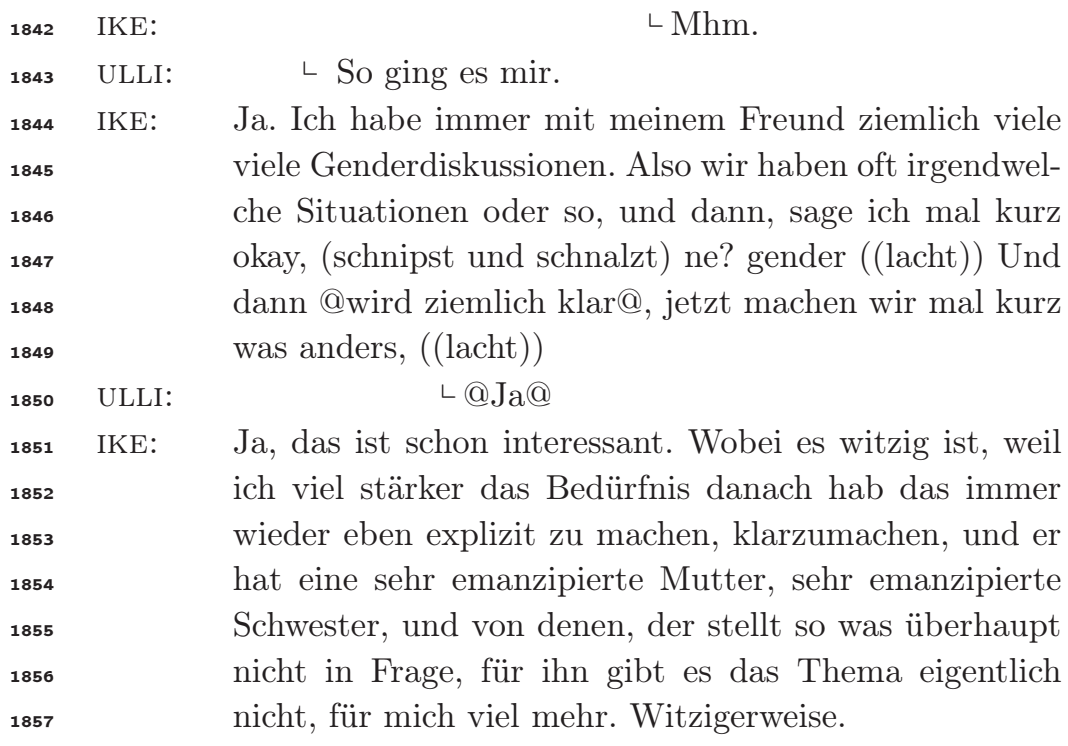

Ulli beginnt damit, darzustellen, dass sie aufgrund ihrer Auseinandersetzung mit Geschlechterfragen begonnen habe, ihr eigenes Verhalten zu reflektieren und zu verändern. Durch ihrer Auseinandersetzung mit den Geschlechterrollen habe sie auch ihr eigenes Leben danach kritisch befragt und habe sich die Frage gestellt, warum sie eigentlich im gemeinsamen Haushalt mit ihrem Partner häufiger Tätigkeiten übernimmt, die aus einem traditionellen Blickpunkt Frauen zugeschrieben sind. Hier wird eine Sensibilisierung für und eine Reflexion des eigenen geschlechterbezogenen Verhaltens erkennbar, sowie der Wunsch nach Veränderung, auch um ein Vorbild für andere zu sein. Ike validiert diese Erfahrung. Sie scheint zumindest ähnliche Erfahrungen gemacht zu haben. Sie erzählt, dass sie mit ihrem Freund sehr viele „Genderdiskussionen“ führe und versuche wie auch Ulli keine Geschlechterstereotype zu reproduzieren und ihr Verhalten aktiv zu verändern. Ike reflektiert weiter, dass ihr auffalle, dass sie ,viel stärker [als ihr Freund, S.K.] das Bedürfnis hat", geschlechtsbezogenes Verhalten und Rollen anzusprechen. Die Thematisierung von ,Geschlecht' scheint somit auch in Ikes Privatleben, weiblich zu sein. Sie mache die Erfahrung, dass ihr Freund, obwohl er eine ,sehr emanzipierte Mutter, sehr emanzipierte Schwester" habe, „so was überhaupt nicht in Frage" stelle. Hier wird auch Ikes Erwartung deutlich, dass emanzipierte Frauen im familiären Umfeld auch Männer sensibilisieren und emanzipieren. Allerdings mache sie die Erfahrung, dass ,es das 
Thema“ für ihn, im Gegensatz zu ihr, ,eigentlich nicht" gebe. An diesem Beispiel wird sichtbar, wie explizit gemachte Vorstellungen von Egalität und Partner_innenschaft und (implizite) Vorstellungen bzw. das inkorporierte Wissen von Geschlechterdifferenz und -hierarchie aufeinander treffen und somit Brüche entstehen.

Anhand dieser Gruppendiskussionsauszüge wird deutlich, dass die GDT in ihrem studiumsbezogenen konjunktiven Erfahrungsraum wie auch in ihrem Privatleben die gemeinsame Erfahrung machen, dass ,Geschlecht" und Geschlechterfragen von Frauen thematisiert werden und demnach auf dieser Ebene die aktiven ProtagonistInnen und AkteurInnen weiblich sind. Geschlechterfragen scheinen somit zu ,Frauenfragen', also zu von Frauen gestellten Fragen zu werden. Frauen scheinen die Expertinnen zu sein und die Thematisierung durch Männer bildet eine Leerstelle.

\subsubsection{Frausein wird „direkt negativ assoziiert“}

Für die Gruppe Wasser ist charakteristisch, dass Frausein explizit negativ besetzt ist und die GDT immer wieder die Erfahrung machen, dass sie aufgrund ihres Geschlechts anders (als Männer) behandelt werden. Gleichzeitig lässt sich eine heteronormative Geschlechtsdualität rekonstruieren. Dabei beziehen sich die Studierenden häufig explizit auf ihr eigenes ,Geschlecht' und eher implizit auf ihre ,selbstverständliche' Heterosexualität. Bezugnehmend auf die visuelle Diskussionsanregung (siehe 1) beschreibt Ike den Bruch zwischen ihrer eigenen Idealvorstellung, dass ,Geschlecht' bzw. Frausein keine Rolle in ihrem privat- und Berufsleben spielen soll und ihren Erfahrungen, dass es doch eine Rolle spielt.

Ike erklärt einführend: ,[A]lso ich finde es interessant, das Bild, weil es so ein bisschen meine Situation widerspiegelt." Sie betont, dass es für sie in ihrer Arbeit sehr wichtig sei, nicht zu sehr ,weiblich zu sein", sich nicht ,zu weiblich“, zu „präsentieren“. Auch wenn sie versuche, ihre Geschlechtszugehörigkeit zu nivellieren, mache sie die Erfahrung, dass dieses Thema eben doch eine Rolle spiele und „dass gecared wird, dass man sich darum kümmert" (hier ohne transkriptive Passage). Auch in ihrem Privatleben versuche Ike, ihr ,Geschlecht ",nicht so wichtig werden zu lassen".

${ }_{1027}$ IKE: [...] Dass es [das Geschlecht, S.K.] nicht egal ist, obwohl ich mich eher identifizieren würde, quasi, mit die- 
ser Person, die sagt, „Who cares?“, weil ich nämlich immer versucht habe, eher das eben nicht so wichtig werden zu lassen, gerade in weil ich zum Beispiel einen großen männlichen Freundeskreis habe und ich da immer versucht habe, ein bisschen, also nicht so sehr als Frau aufzutreten, ja, damit ich da nicht irgendwelche Fantasien wecke.

JUNO: Mhm, wobei mir dazu jetzt auffällt, also so ohne Kritik und einfach nur so spontane, eine spontane Eingebung ist, wenn ich mich quasi an Männern orientiere, dass ich Männern in einer gewissen Art und Weise begegne, passe ich mich ihnen ja auch eigentlich wieder an anstatt ihnen zu zeigen, wie sie mich behandeln sollten.

Auch Ike würde gerne sagen: „Wen, kümmert oder interessiert es?“, ob sie eine Frau ist. Dennoch versucht sie nicht nur im Berufsleben, sondern auch im Freundeskreis, ihre, Weiblichkeit', ihr Frausein zu neutralisieren, um keine „Fantasien“ bei ihren männlichen Freunden zu wecken. Mit dem Wort „Fantasien“ könnte Ike sexuelle Fantasien meinen, die sie als Frau bei ihren männlichen Freunden wecken könnte. Hier verweist sie auf die Objektivierung und Sexualisierung von Frauen. Es wird ihre Orientierung an einer heterosexuellen Norm deutlich. Interessant ist, dass Ike den umgekehrten Fall, dass ihre männlichen Freunde bei ihr oder anderen Freundinnen sexuelle Fantasien auslösen könnten, nicht thematisiert. Juno kritisiert an Ikes Beitrag die Orientierung an Männern und daran, dass man sich ihnen anpasse. Die Anpassung von Frauen an Männer bzw. auch die Neutralisierung der ,Weiblichkeit' stellt für Juno einen negativen Horizont dar. Bereits in der Passage zu „Desinteresse und Aversion gegenüber Geschlechterfragen" wurde deutlich, dass Juno eine Orientierung an und Fokussierung auf Männer ablehnt. Juno distanziert sich allerdings nicht von der Orientierung an einer heterosexuellen Normativität. Bei Ulli löst dieses Bild eine andere Reaktion aus, und sie bringt ihre eigene Proposition ein.

1068 ULLI: [...] und dieses „Who cares", da habe ich gerade so gedacht, das könnte so ein, so ein Wunschbild der Zukunft sein, dass es nicht mehr so wichtig ist, ob man männlich oder weiblich ist, [...] obwohl man ja eigentlich gar nicht will, dass es keine Männer und Frauen gibt, ei- 
gentlich will man ja nur die Diskriminierung nicht mehr. Und eigentlich müsste man versuchen, dass also wie diese Frage gestellt wird, dass es halt eben nur neutral ist; und dann eben nicht dieses Negative oder Positive immer mitschwingt. Ne, dieses „du bist ein Mädchen das ist schlecht", oder „du bist ein Mädchen, das ist gut", oder „du bist ein Junge, das ist gut", und „du bist ein Junge, das ist schlecht".

Ulli assoziiert mit dem Bild eine Kennenlernsituation, weil man sich hier die Frage stelle, ob das Gegenüber „,männlich oder weiblich“ sei und damit, dass man versuche, das Gegenüber in die Kategorien ,männlich` und ,weiblich ${ }^{6}$ einzuordnen. Für sie habe ,Geschlecht' „eine Strukturierungsfunktion". Im Weiteren äußert Ulli auch ihre Eindrücke zur bisherigen Diskussion (hier ohne transkriptive Passage): Sie spricht nun über das bisherige Gespräch in der Gruppendiskussion und konstatiert, dass die Frage: „Bist du ein Mädchen?“ „in dieser Diskussion [...] direkt negativ assoziiert" werde und dass Mädchen- bzw. Frausein negativ besetzt sei. Ulli findet das „ein bisschen befremdlich“ und grenzt sich somit von Ikes Orientierung ab. Als Gegenhorizont zur bisherigen Diskussion initiiert Ulli die alternative Lesart, dass die Frage ,ja auch ganz neutral" sein oder sogar eine ,positive Frage sein" könnte. Diese alternative Lesart hat jedoch einen theoretischen Charakter und wird an keinem Beispiel exemplifiziert. Sie verbleibt auf einer kommunikativen Ebene und wird nicht als handlungsleitendes Wissen bestätigt. Für Ulli stellt „dieses Who cares?" auch ein „Wunschbild der Zukunft" dar, in dem Männer und Frauen nicht mehr unterschiedlich (hierarchisch) bewertet werden. Das bedeutet für Ulli aber nicht, ,dass es keine Männer und Frauen gibt", sondern dass die „Diskriminierung“" und Bewertung aufgrund von ,Geschlecht' der Vergangenheit angehören solle. Ullis Betrag wird von den anderen GDT nicht aufgegriffen, sondern bleibt ein unkommentierter Einschub.

Hingegen scheint die Diskussion um die ,Neutralisierung v von ,Weiblichkeit' (siehe oben) noch nicht beendet zu sein. Ike greift das Thema nochmals auf und korrigiert Junos ,falsche' Rezeption ihrer Aussage und erklärt, dass sie nicht wie Männer behandelt werden wolle:

1134 IKE: Ja also ich, um jetzt noch mal an das anzuknüpfen, Juno was du gesagt hast, ich finde nicht, dass dieses 
Männern, sondern man macht sich neutral. So.

JUNO: Mhm.

IKE: Aber ich habe die Erfahrung gemacht, dass es einfach nicht geht. Also ich habe das oft versucht, und es funktioniert nicht. Also ich weiß jetzt nicht ob es bei anderen funktioniert als bei mir, ich bin halt einfach auch relativ typisch weiblich so von meiner ganzen Erscheinung her, ja, kann ich jetzt auch nicht ändern, hätte ich gerne geändert, damit es einfach praktischer ist, aber dann muss man damit einfach umgehen. Musste ich damit umgehen; also dieses „Who cares", das ist eben eine Wunschvorstellung, Ulli, wie du das gerade gesagt hast, quasi, aber ist mir nicht begegnet, gerade im kulturellen Unterschied überhaupt nicht.

JUNO: Mhm

ULLI: Darf ich fragen, wo du jetzt gerade gesagt hast, du hättest es gerne gehabt, dass es nicht so wäre, dass du weiblich aussiehst, ist das halt eben weil, du das Gefühl hast, dass weibliche Personen eher negativ behandelt werden?

IKE:

$\llcorner$ Genau.

ULLI: Ja; also war es eher so, dass wenn das jetzt eigentlich neutral gewesen wäre, ob man männlich oder weiblich ist, dann wäre das nicht so wichtig gewesen.

IKE:

$\llcorner$ Dann wäre es

nicht wichtig, genau,

ULLI:

$\llcorner$ Genau.

IKE: Also in beispielsweise in Finnland, ist es angenehmer als in Deutschland, eine Frau zu sein, also da ist man ist man neutraler, wird man neutraler wahrgenommen, war meine, war meine Wahrnehmung, auch das was ich gelesen hab, in aber in asiatischen Ländern ist es einfach eine Katastrophe; ${ }^{\circ}$ so. ${ }^{\circ}$ Und auch in der Jugendarbeit in Deutschland ist das superschwierig, weiblich zu sein. Also da muss man sich erstmal gegen die ganzen Vorurteile und gegen die ganzen ,kommst du nicht heute 
Nacht doch noch mit mir ins Bett" erstmal durchsetzen; ne? So, also es ist ganz schwierig, da so eine weibliche Rolle zu haben die sich bestimmt aber abgrenzt gegen solche Dinge [...]. Also da wäre es einfach einfacher @wenn man keine@ keine Frau wäre, ja.

Ike kritisiert, dass Frauen als sexualisierte Wesen wahrgenommen werden und reagiert darauf, indem sie versucht, ihre, Weiblichkeit', ihr Frausein zu neutralisieren.

Hier wird erneut der Bruch zwischen Ikes Orientierungsschema und -rahmen deutlich: In ihrer (idealen) Vorstellung sollte ,Geschlecht' bzw. ,Weiblichkeit" keine Rolle spielen und es sollte nicht wichtig sein, ob man ,männlich' oder , weiblich' ist/aussieht. Ike hat jedoch die Erfahrung gemacht, dass diese Vorstellung nicht zutreffe und ihre ,Weiblichkeit' (oftmals) relevant gemacht werde. Im Austausch mit Ulli wird deutlich, dass Ike das Gefühl hat, ,,dass weibliche Personen eher negativ behandelt werden" und dass sie ihre ,weibliche" Körperlichkeit als Nachteil empfindet. In Ikes Erfahrungen und Erzählungen dominieren die negativen und erschwerenden ,Auswirkungen/Faktoren' von Frausein. Ihre Erfahrungen differenziert sie jedoch im Ländervergleich. Als einzigen positiven Erfahrungshorizont nennt Ike ihre Erlebnisse in Finnland, wo es angenehmer sei, „eine Frau zu sein, also da ist man neutraler, man wird neutraler wahrgenommen". Im Gegensatz zu Deutschland sei Ike in Finnland „neutraler" behandelt und wahrgenommen worden. Als negativen Erfahrungshorizont nennt sie ,asiatische Länder“, dort ,ist es einfach eine Katastrophe". Als Beispiel nennt sie in der Diskussion ihre Erfahrungen in Sri Lanka. Dort sei es für sie negativ gewesen, als Frau gesehen und behandelt zu werden. Ein weiterer Ort, an dem es für Ike „superschwierig [ist], weiblich zu sein", sei die Jugendarbeit in Deutschland. Auch hier müsse man gegen Sexismen kämpfen und sich gegen Vorurteile durchsetzten: „Also es ist ganz schwierig, da so eine weibliche Rolle zu haben, die sich bestimmt aber abgrenzt gegen solche Dinge." Die asiatischen Länder und die Jugendarbeit in Deutschland stellen für Ike Orte und Räume dar, in denen es sehr ,schwierig ist eine weibliche Rolle" zu haben. ,Weiblich $^{6}$ zu sein, bedeutet für Ike, mit Vorurteilen und Sexismen konfrontiert zu werden und stellt daher ein Erschwernis dar. Durch die Zustimmung von Ulli und Juno wird deutlich, dass sie diese Orientierung mit Ike teilen. Die normative Vorstellung 
von Gleichheit und Gleichberechtigung passt mit der sozialen Praxis der GDT nicht zusammen. Dieser Bruch ist jedoch nicht thematisierbar, sondern wird als Individualbelastung definiert und erlebt.

Das eingebrachte Thema zu Ländervergleich und kulturellen Unterschieden knüpft in der Gruppe Wasser an keinen konjunktiven Erfahrungsraum an; ebensowenig wird eine Verbindung zu konjunktiven Erfahrungen der Gruppe in Hinblick auf die eigene Herkunft und eigene Kultur deutlich.

In dieser Passage wird erneut deutlich, dass die GDT von einer Geschlechterdifferenz ausgehen und dass die Geschlechter in einer unterschiedlichen Weise bewertet werden. Sie heben die Probleme, die mit Frausein verbunden sind, hervor. Auch wenn Ulli eine positive Lesart hinsichtlich einer Neutralisierung vorschlägt, setzt sich eine problemorientierte Thematisierung von Frausein und ,Weiblichkeit' durch. Auch in dieser Passage wird deutlich, dass die GDT Geschlechterfragen mit ,Frauenfragen‘ und ,Frauenproblemen' gleichsetzen. Zudem wird deutlich, dass die GDT nicht aufgrund ihres Frauseins als ein Opfer oder als benachteiligt erscheinen wollen - auch wenn das ihrer Erfahrung entspricht. Diese Erfahrung wird jedoch negiert bzw. es findet ein Absehen davon statt.

Verlässt man hier den Rahmen der Gruppendiskussion und tritt in den theoretischen Bezugsrahmen ein, so lässt sich dies mit dem ,prekären Selbstbezug“" (Bitzan 1997, S. 78) erläutern: Dieser hebt hervor, dass die Frauen von sich selbst und von ihren Erfahrungen absehen, um sich in einer normativen Welt verhalten zu können. Negative Gefühle wie Angst oder innere Konflikte, welche dem Selbstbild widersprechen, können somit schlechter wahrgenommen und ausgedrückt werden. Wenn es beispielsweise verpönt ist, sich selbst als Opfer zu sehen, dann können entsprechende Erfahrungen auch schwieriger verbalisiert werden. Dies führt, zugespitzt formuliert, zu einem kollektiven Realitätsverlust und einer ,Normaffinität'. Maria Bitzan beschreibt, dass die moderne Welt für eine Integration die Anpassung an die Normalität verlange, das bedeutet, von sich als Geschlechtswesen abzusehen und Bestätigungen dort zu suchen, wo das angeblich Allgemeine verortet ist. Es umfasst, von eigenen Erfahrungen abzusehen, sich an äußeren Bildern zu orientieren und darin ,Verortung" zu suchen (vgl. Bitzan 1997, S. 78). Dies wird auch in der am Ende dargestellten Passage zum ,generischen Maskulinum als Norm der Sprech- und Schreibpraxis" deutlich. 


\subsubsection{Das generische Maskulinum als Norm der Sprech- und Schreibpraxis}

Die Gruppe Wasser orientiert sich in ihrer Sprechpraxis am generischen Maskulinum und betont, dass die geschlechtergerechte Sprache für sie kein „großes Thema“ darstelle. In Bezug auf die Schreibpraxis (z. B. in Hausarbeiten oder in Beiträgen für Zeitschriften) teilen die Studierenden keine gemeinsame Orientierung und betonen, dass die Entscheidung für oder gegen das generische Maskulinum nicht immer der persönlichen Präferenz entspreche, sondern von (äußeren) Normen abhängig sei und es viele verschiedene Schreibweisen und unterschiedliche Normen gebe, an denen man sich orientieren müsse (hier ohne transkribierte Passage). Ike exemplifiziert, dass die Verwendung geschlechtergerechter Sprache in Hausarbeiten auch von der Orientierung der Dozierenden abhängig sei und nicht immer die persönliche Orientierung widerspiegle:

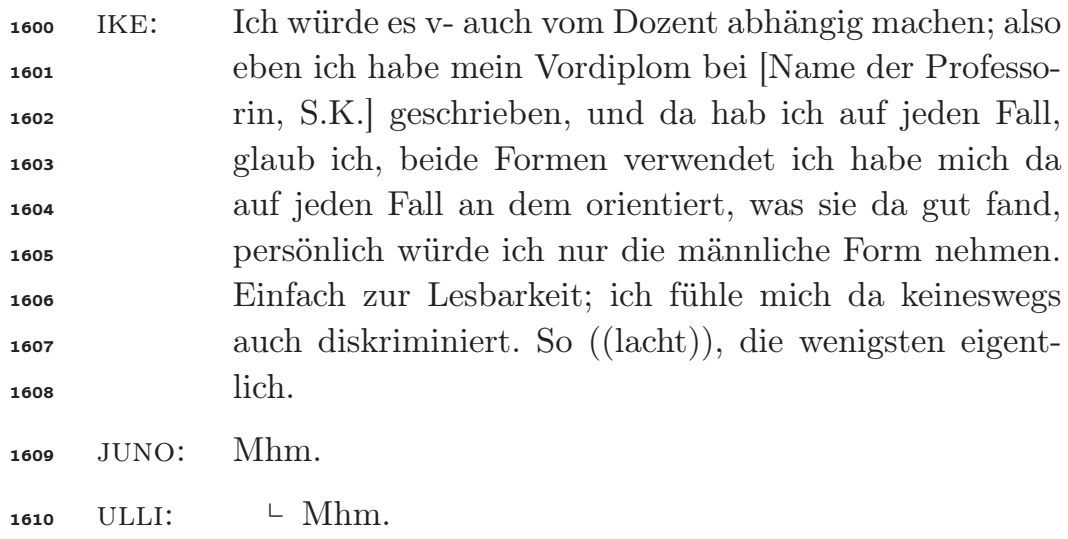

In dieser Gruppendiskussion wird eine Argumentationsfigur verwendet, die auch in den anderen Gruppendiskussionen (Holz, Feuer) in Erscheinung tritt. Ike betont, dass sie aus Gründen der Lesbarkeit das generische Maskulinum in ihrer selbstgewählten Schreibpraxis favorisiere und sich davon ,keineswegs diskriminiert" fühle. Nicht nur sie, sondern in ihrer Wahrnehmung würden sich „die wenigsten“" aufgrund des generischen Maskulinums diskriminiert fühlen. Somit generalisiert Ike ihre Annahme und weitet den Geltungscharakter ihrer Aussage aus. Diese Aussage wird von Juno und Ulli quasi simultan validiert. Dahinter steht zum einen die Annahme, dass geschlechtergerechte Sprache verwendet werde, um Frauen nicht zu diskriminieren. Die Repräsentation von Vielfalt 
und das Ziel, für starre Geschlechterrollen zu sensibilisieren, bleibt hierbei ganz und gar unbedacht. Zum anderen passt die Beteuerung, sich nicht diskriminiert zu fühlen, zu den rhetorischen Gleichheitsbekundungen und der Vorstellung (!), dass ,Geschlecht' keine Rolle spiele. Speziell vor dem Hintergrund, dass Frausein und ,Weiblichkeit' in dieser Gruppendiskussion negativ gerahmt werden, wirkt diese Argumentationsfigur hier paradox und spiegelt den Bruch zwischen dem kommunikativ-generalisierten Wissen und dem Erfahrungswissen wider.

\subsubsection{Zusammenfassung der Gruppe Wasser}

Zusammenfassend kann gesagt werden, dass in der Gruppe Wasser ein inkludierender Diskurs geführt wird, der sowohl in einer parallelen als auch antithetischen Diskursorganisation Ausdruck findet (vgl. Kapitel 5.4.1). In der Gruppe kommen homologe Orientierungen zum Ausdruck, die sich zum Teil im Gegeneinander (antithetisch) entwickelt haben (z. B. Frausein wird ,negativ assoziiert"), aber auch durch eine (parallele) Aneinanderreihung von Darstellungen (z. B. Geschlechterdualität als ,Strukturmerkmal").

Auch wenn die Thematisierung von ,Geschlecht' und Geschlechterfragen heteronom produziert wird, kommt es zu keiner Thematisierungsverweigerung, sondern diese wird affirmativ aufgegriffen. Alle drei GDT sind dazu bereit, über Geschlechterfragen zu sprechen und dabei wird deutlich, dass das Studium und das universitäre Umfeld hinsichtlich dieses Themas als konjunktiver Erfahrungsraum fungiert. Zudem wird deutlich, dass Ulli, Ike und Juno ihr Frausein verbindet und dies implizit auf ihre eigene Geschlechtlichkeit und auf ihre ,selbstverständliche' Heterosexualität beziehen. Dies ist hier, im Vergleich zu geschlechtshomogenen Gruppe Feuer stärker der Fall. Neben den impliziten heteronormativen Annahmen lassen sich auch eine explizite hierarchische Geschlechterordnung und -dualität rekonstruieren. Die eigene heterosexuelle und , weibliche‘ Geschlechtsidentität wird von Ulli, Ike und Juno nicht infrage gestellt. In der Gruppendiskussion wird immer wieder deutlich, dass Frausein für die GDT tendenziell negativ besetzt ist und sie immer wieder die Erfahrung machen, dass sie aufgrund ihres Geschlechts anders (als Männer) behandelt werden. Sie heben die Probleme, die mit Frausein verbunden sind, hervor. Geschlechterfragen scheinen von den GDT mit ,Frauenfragen' und ,Frauenproblemen' gleichgesetzt zu werden. Bei der Thematisierung von ,Geschlecht' kann die negative Asso- 
ziation mit Frausein als homologes Muster rekonstruiert werden. Daher kann Frausein als negativer Gegenhorizont als wesentliches Element des Habitus der Gruppe Wasser in Hinblick auf die Thematisierung von ,Geschlecht' charakterisiert werden.

In der Gruppendiskussion zeigt sich auch der Bruch zwischen Orientierungsschemata und -rahmen. In der (idealen) Vorstellung bzw. dem Alltagswissen der GDT sollte ,Geschlecht' und ,Weiblichkeit' keine Rolle spielen, jedoch machen die Studentinnen die Erfahrung, dass diese Vorstellung nicht zutrifft und ihre ,Weiblichkeit' im Alltag relevant gemacht wird. Zusammenfassend kann festgestellt werden, dass die GDT in ihrem konjunktiven Erfahrungsraum wie auch in ihrem Privatleben die gemeinsame Erfahrung machen, dass Geschlecht- und Geschlechterfragen von Frauen thematisiert werden und demnach auf Ebene der aktiven ProtagonistInnen und AkteurInnen weiblich sind. Frauen scheinen hinsichtlich Geschlechterfragen die Expertinnen zu sein und die Thematisierung durch Männer bildet eine Leerstelle. Das Studium der Erziehungs- und Bildungswissenschaften selbst wird als ein weiblich dominiertes Fach hervorgehoben, in welchem Männer (männliche Studierende) weichen von der (erziehungswissenschaftlichen) Norm ab weichen.

In Bezug auf die Thematisierung von ,Geschlecht' und Geschlechterfragen werden damit die unterschiedlichen Arten der Auseinandersetzung bzw. Beschäftigung der GDT deutlich. Diese Gruppendiskussion ist durchmischt von theoretisch-abstrakten Vorstellungen und persönlichen Erfahrungen. Ullis Interesse an Geschlechterfragen wurde im Studium durch ihre Teilnahme an einer Lehrveranstaltung geweckt und sie hat sich als Einzige in der Gruppe im Rahmen ihres Studium auch mit theoretischen Debatten zu ,Geschlecht' auseinandergesetzt. Im Gegensatz dazu sprechen Ike und Juno davon, dass sie einen „naturgegebenen Zugang" zum Thema hätten, der eine (theoretisch-akademische) Auseinandersetzung im universitären Kontext unnötig mache. Qua Geschlecht, qua Frausein scheinen sie aus ihrer Sicht über eine geschlechterreflektierende Perspektive zu verfügen, die sich jedoch im empirischen Material in keiner Hinsicht rekonstruieren lässt. Dieser „naturgegebene Zugang“ erscheint als eine Art Legitimationsfigur, um ein Desinteresse und eine De-Thematisierung von ,Geschlecht` zu begründen. Diese unterschiedlichen Haltungen haben auch Einfluss auf die Frage nach der Relevanz von ,Geschlecht' für erziehungswissenschaftliches Denken und Handeln. So stellen für Ulli, im Gegensatz zu Ike und Juno, ,Geschlecht' und Geschlechterfragen ein wichtiges Thema für die Erziehungs- und Bildungswissenschaften dar. 
Auch in dieser Gruppendiskussion orientieren sich die GDT in ihrer Sprech- und Schreibpraxis am generischen Maskulinum. Sie betonen, dass sie sich vom generischen Maskulinum nicht diskriminiert fühlen; dahinter scheint sich die rhetorische Bekundung zu verbergen, dass ,Geschlecht" nicht relevant ist. Vor dem Hintergrund, dass das Frausein und ,Weiblichkeit' in dieser Gruppendiskussion negativ gerahmt werden, und die GDT die Erfahrung machen, dass ,Geschlecht' im Alltag eine Rolle spielt, wirkt diese Bekundung eher wie eine rhetorische Entdramatisierung von ,Geschlecht' und Geschlechterfragen.

Eine Überbetonung der Thematisierung von ,Geschlecht' durch ,zu starke' persönliche Involviertheit und durch eine habitualisierte kämpferische Haltung wird von einem Teil der GDT abgelehnt, sie grenzen sich dadurch von einer theoretischen Auseinandersetzung/Beschäftigung ab. In der Diskussion wird deutlich, dass ersteres auch dazu führt, dass ein Desinteresse und eine Aversion gegenüber Geschlechterfragen (unter den Studierenden) existieren und dass diese Einstellung die Meinung der Kommiliton_innen spiegelt. Das Studium wird als ein Ort besprochen, in dem Geschlechterfragen in Seminaren diskutiert werden können, und in dem zu einer Auseinandersetzung mit ,Geschlecht' angeregt wird, jedoch halten Klischees und Vorurteile darüber, dass Frauen als Opfer darstellt werden und Diskussionen dort sehr persönlich und aggressiv geführt werden, Studierende davon ab, diese Seminare zu besuchen. Für zwei GDT der Gruppe Wasser konnte ein geschlechtsbezogener konjunktiver Erfahrungsraum rekonstruiert werden. Das von einer Teilnehmerin eingebrachte Thema des Ländervergleichs und der kulturellen Unterschiede knüpft in der Gruppe Wasser an keinen konjunktiven Erfahrungsraum an.

\subsection{Komparative Analyse: gruppenübergreifende Orientierungen bei der Universität Mond}

Die vorgestellten Gruppen Metall und Wasser verbindet neben dem studiumsbezogenen auch der universitätsbezogene konjunktive Erfahrungsraum. Wie bereits ausgeführt, haben die Studierenden gemeinsam, dass sie zum Zeitpunkt der Gruppendiskussion am Ende ihres Diplomstudiums der Erziehungs- und Bildungswissenschaften stehen. In ihrer Zusammensetzung unterscheiden sich die Gruppen darin, dass die Gruppe Metall eine geschlechtsheterogen, und die Gruppe Wasser eine geschlechts- 
homogen ist. In der geschlechtshomogenen Gruppe Wasser wird deutlich, dass das Frausein der Teilnehmerinnen einen wichtigen Referenzpunkt darstellt und das Geschlecht der GDT einen konjunktiven Erfahrungsraum bildet. Zudem skizzieren beide Gruppen das Studium als einen Ort, der die Möglichkeit bietet, sich mit ,Geschlecht' und Geschlechterfragen auseinanderzusetzen, weiters ist den Studierenden der Universität Mond das Zentrum für Gender Studies an ihrer Universität bekannt.

In Bezug auf die Thematisierung von ,Geschlecht' und Geschlechterfragen wird in der Gruppe Wasser deutlich, dass zwischen unterschiedlichen Dimensionen der studiumsbezogenen konjunktiven Erfahrungen differenziert werden muss. Auch wenn die Studierenden studiumsbezogene Erfahrungen teilen, stellt eine theoretische bzw. curriculumsbezogene Thematisierung von ,Geschlecht' und Geschlechterfragen keine gemeinsame Erfahrung dar. Das ergibt sich daraus, dass nicht alle Teilnehmerinnen Seminare zu diesem Thema besucht haben. Es kann somit rekonstruiert werden, dass in der Gruppe Wasser verschiedene studiumsbezogene Erfahrungsräume existieren, innerhalb derer die Thematisierung von ,Geschlecht' und Geschlechterfragen unterschiedlich bewertet wird. In der Gruppe Wasser wird die studiumsbezogene bzw. die theoretische und curriculumsbezogene Thematisierung von einer Studentin positiv bewertet, dies stellt bei den beiden anderen Teilnehmerinnen jedoch einen negativen Horizont dar. Diese Differenzierung ist bei der Gruppe Metall nicht der Fall. Hier verfügen die Studierenden über einen konjunktiven Erfahrungsraum, der eine studiumsbezogene bzw. die theoretische und curriculumsbezogene Thematisierung von ,Geschlecht "und Geschlechterfragen umfasst. Die Gruppen haben gemeinsam, dass ausgiebig über negative Horizonte der Thematisierung von ,Geschlecht' und Geschlechterfragen diskutiert wird und sie ein als übertrieben und kämpferisch wahrgenommenes Diskussionsklima ablehnen. Beide Gruppen reproduzieren somit in unterschiedlicher Weise Stereotype von ,hetzenden Emanzen“ (GD_Wasser) und ,aggressiven Kampfwesen“ (GD_Metall), und dass ,Geschlecht" eine „Hetzthematik“ (GD_Wasser) ,auf Leben und Tod" sei bzw. immer wieder als solche von den Studierenden wahrgenommen wird. In beiden Gruppen wird rekonstruiert, dass sich die Mehrheit der KommilitonInnen an den Klischees, den Vorurteilen und den negativen Erfahrungen bei der studiumsbezogenen Thematisierung von ,Geschlecht' orientieren. In der Gruppe Metall lässt sich eine Kritikaffinität in Hinblick auf die studiumsbezogene Thematisierung von ,Geschlecht als ein wesentliches Element des Habitus der Gruppe charakterisieren. Dennoch lässt sich in der Gruppe Metall im Gegensatz zur Gruppe Was- 
ser eine gemeinsame (Ideal-)Vorstellung in Bezug auf die Thematisierung von ,Geschlecht' und Geschlechterfragen rekonstruieren, die sich an „Wertschätzung“, „Gleichheit" und „Respekt" orientieren soll. In der Kritikaffinität, die zwar in beiden Gruppen unterschiedlich ausfällt, lässt sich trotzdem ein gemeinsames Muster der Thematisierung identifizieren, das Stereotype reproduziert und eine positive Bewertung und Bezugnahme auf eine produktive Thematisierung von ,Geschlecht' und Geschlechterfragen verhindert und verdeckt.

In beiden Gruppen lässt sich außerdem rekonstruieren, dass die Differenzierung nach ,Geschlecht' Teil der habituellen Praxis ist und eine kollektive Orientierung der Studierenden darstellt. Die Geschlechterdifferenz und die Geschlechterbinarität haben in den Gruppen Metall und Wasser den Status einer Leitdifferenz. Die eigene (eindeutige) Geschlechtlichkeit, die heterosexuelle Geschlechternorm sowie die Geschlechterdifferenz werden nicht infrage gestellt. Geschlechterfragen werden hauptsächlich mit Ungleichheit, Differenz und Benachteiligung in Verbindung gebracht. In unterschiedlicher Weise reproduzieren die beiden Gruppen traditionelle Rollenbilder und charakterisieren bestimmte Bereiche als ,weiblich' oder ,männlich'. So wird von der Gruppe Metall wie auch von der Gruppe Wasser das Studium der Erziehungs- und Bildungswissenschaften implizit und explizit als typisches Studium und Arbeitsfeld für Frauen angesehen. In beiden Gruppen spielt die statistische Geschlechterverteilung unter den Studierenden eine wichtige Rolle, hierbei scheint es sich um eine studiumsbezogene konjunktive Erfahrung zu handeln. Männer werden in diesem Feld als von der Norm abweichend wahrgenommen und dargestellt. Sie divergieren zum einen von der weiblich geprägten erziehungswissenschaftlichen und pädagogischen Norm und zum anderen von der hegemonialen (heterosexuellen) Männlichkeitsnorm. Das bedeutet aber nicht, dass Männer in den Gruppendiskussionen eine negative Bewertung erfahren. In der Gruppe Metall werden Studenten aufgrund ihrer geringen Anzahl aufgewertet und besondert. Auch in der Gruppe Wasser werden Männer aufgewertet, jedoch auf anderer Weise. Hier kann die negative Assoziation mit Frausein als ein wesentliches Element des Habitus in Hinblick auf die Thematisierung von ,Geschlecht' charakterisiert werden. Einen positiven, aber unausgesprochenen und demnach selbstverständlichen Gegenhorizont bildet die positive Assoziation mit Mannsein.

Zudem fällt auf, dass die Thematisierung von ,Geschlecht' und Geschlechterfragen in den studiums- und universitätsbezogenen Erfahrungsräumen als , weiblich' rekonstruiert wird. Das heißt, in beiden Grup- 
pen der Universität Mond werden Frauen (Dozentinnen und Studentinnen) als die aktiven Protagonistinnen und Akteurinnen beschrieben. Es lässt sich rekonstruieren, dass Geschlechterfragen als ,Frauenfragen', von Frauen gestellt und thematisiert werden. Frauen scheinen die Expertinnen zu sein und die Thematisierung durch Männer bildet eine Leerstelle. Im Vergleich der beiden Gruppen fällt auf, dass der „Fokus auf dem Weiblichen" (GD_Metall) in der Gruppe Metall negativ bewertet und als einseitig wahrgenommen wird. Damit bezieht sich die Gruppe auf einen ,weiblichen' Fokus auf Seiten der Lehrenden, der Lehrveranstaltungsinhalte und auf die marginale Stellung männlicher Lebenswelten und Akteure. Vor dem Hintergrund, dass die Geschlechterfragen mit ,Frauenfragen' und ,-problemen' gleichgesetzt werden, die Männer nur partiell bzw. nicht tangieren, scheint diese weibliche Dominanz innerhalb der studiums- und universitätsbezogenen Erfahrungsräume besonders problematisch.

Geschlechtsbezogene Benachteiligung wird in der Gruppe Metall als individuelle/subjektive Empfindung inszeniert, weshalb intentionale geschlechterbezogene Benachteiligung und Behandlung nivelliert und quasi als individuelles Problem ,normalisiert' wird. Dies scheint mit dem gemeinsamen studiums- und universitätsbezogenen konjunktiven Erfahrungsraum im Zusammenhang zu stehen, da angenommen wird, dass die Studentinnen (und Studenten) aufgrund der weiblichen Dominanz im Studiengang nicht benachteiligt werden. In der Individualisierung und in der Verschiebung von realen strukturellen Problemen auf andere Bereiche und Personen zeigt sich ein weiteres Muster, das zu einer (De-)Thematisierung führt und zur Etablierung einer Gleichberechtigungsnorm, auch wenn dies nicht den Erfahrungen der Studierenden entspricht. Hier scheint eine Art Third Person Effekt(vgl. Huck/Brosius 2007, S. 335) wirksam zu werden: Demnach sind andere Personen in anderen Bereichen, Institutionen und Strukturen, in denen die Studierenden nicht selbst situiert sind, von (benachteiligenden Wirkungen) der Kategorie ,Geschlecht' stärker betroffen (vgl. ebd.). In der Gruppe Wasser kommt geschlechtsbezogene Benachteiligung implizit zum Ausdruck, indem Frausein für die GDT tendenziell negativ besetzt ist und sie immer wieder die Erfahrung machen, dass sie aufgrund ihres Geschlechts anders (als Männer) behandelt werden. Dem folgend heben sie Probleme, die mit Frausein verbunden sind, hervor. Erfahrungen geschlechtsbezogener Benachteiligung finden in der Gruppe Wasser ihren Ausdruck in der negativen Orientierung am Frausein. 
In den Gruppen Metall und Wasser gehört der Begriff gender zum geteilten Wortschatz der Studierenden, ist Teil ihrer konjunktiven Erfahrung und wird hauptsächlich in Verbindung mit einer theoretischen Auseinandersetzung und theoretischem Wissen genutzt. Was sich bereits in der Gruppe Wasser dargestellt hat, zeigt sich auch in der komparativen Analyse mit der Gruppe Metall. In Bezug auf die Thematisierung von ,Geschlecht' und Geschlechterfragen kann zwischen einem theoretischabstrakten Zugang und einem, von den Diskutierenden als „naturgegeben" bezeichneten Zugang unterschieden werden. Der theoretischabstrakte Zugang zeichnet sich durch eine theoretisch-akademische Auseinandersetzung im universitären Kontext aus und ist von einer curriculumsbezogenen Thematisierung geprägt. Der als „naturgegeben“ beschriebene Zugang sei qua Frausein ein ,intuitiver' Zugang und hängt nicht offensichtlich mit einem formalen Bildungsprozess bzw. mit einer aktiven und bewussten Auseinandersetzung mit dem Thema zusammen. Dieser „naturgegebene Zugang" erscheint als eine Art Legitimationsfigur, um ein Desinteresse und eine (De-)Thematisierung von ,Geschlecht und Geschlechterfragen zu begründen. Diese unterschiedlichen Haltungen haben auch Einfluss auf die Frage nach der Relevanz von ,Geschlecht ${ }^{\text {* }}$ für erziehungswissenschaftliches Denken und Handeln und einen reflektierenden Umgang mit ,Geschlecht'. So sind die Geschlechtersensibilität und -reflexion sowie die Fähigkeit, wissenschaftlich zu argumentieren, für die Studierenden der Gruppe Metall von großer Bedeutung. Zudem werden alleine in der Gruppe Metall individuelle Orientierungsschemata in Bezug auf die Frage, was die Thematisierung von ,Geschlecht ${ }^{6}$ und Geschlechterfragen im Studium (nicht) leisten kann und was ihnen bei der studiumsbezogenen Thematisierung von ,Geschlecht' und Geschlechterfragen wichtig ist, diskutiert. Auch wenn die Gruppe Metall diesbezüglich über keine kollektive Orientierung verfügt, wird deutlich, dass sie einer Implementierung den Themas im Curriculum des Studiengangs grundsätzlich positiv gegenüber steht.

In der komparativen Analyse der beiden Gruppen der Universität Mond zeigen sich in Bezug auf die geschlechtergerechte Sprache unterschiedliche Orientierungen, die auf differenten Erfahrungen fußen. In der Gruppe Metall wird die geschlechtergerechte Sprache als positiver Horizont dargestellt, da die Studierenden damit die konjunktive Erfahrung einer gesellschaftskritischen Perspektive verbinden. Die geschlechtergerechte Sprache ist somit eine kollektive Orientierung und Teil der habituellen Praxis. Die Mehrzahl der Gruppe Wasser hingegen orientiert sich am generischen Maskulinum, aber auch an Normen, die von außen, 
z. B. von Dozentinnen, verlangt werden. Anders als in der Gruppe Metall wird hier betont, dass die geschlechtergerechte Sprache kein ,großes Thema" (GD_Wasser) sei. In der Gruppe Wasser wird die Notwendigkeit einer geschlechtergerechten Sprache mit einer benachteiligten und diskriminierten Position von Frauen verbunden. Jedoch wird hervorgehoben, dass sich die GDT vom generischen Maskulinum nicht diskriminiert fühlen. Hinter dieser Argumentationsfigur verbirgt sich auf einer rhetorischen Ebene das Orientierungsschema, dass ,Geschlecht' keine Rolle spiele. Vor dem Hintergrund, dass die Studierenden der Gruppe Wasser Frausein und ,Weiblichkeit" negativ assoziieren und in dieser Hinsicht bereits als negativ wahrgenommene Erfahrungen gemacht haben, wird hier der Bruch zwischen einer rhetorischen Geschlechtergleichheit und Egalität einerseits, und einer erfahrenen Geschlechterungleichheit und Differenz andererseits, deutlich. In diesem Bruch lässt sich ein Muster der (De-)Thematisierung von ,Geschlecht' identifizieren. Dieser scheint jedoch nicht thematisierbar zu sein, da er im Widerspruch zur dominanten gesellschaftlichen Norm von Gleichheit, Gleichberechtigung und Egalität von Männern und Frauen steht. 


\section{Ergebnisdiskussion: Spielarten der (De-)Thematisierung von ,Geschlecht' und Geschlechterfragen}

In diesem die Arbeit abschließenden Kapitel sollen nun die zentralen Ergebnisse der empirischen Analysen vor dem Hintergrund des zu Beginn der Arbeit entfalteten theoretischen Rahmens und Analysefolien zusammengefasst werden. Hierzu ist es sinnvoll, sich zunächst die Zielsetzungen der Arbeit nochmals zu vergegenwärtigen. Der Fokus der Analysen liegt auf der habituellen Konstruktion bei der (De-)Thematisierung von ,Geschlecht' und Geschlechterfragen. Diese Frage wurde auf das soziale Feld der Erziehungs- und Bildungswissenschaften zugespitzt, da - so zumindest die Annahme - die Auseinandersetzung und die Beschäftigung mit ,Geschlecht' und Geschlechterfragen Teil der studentisch-habituellen Praxen sei. Dem universitären und pädagogisch-praktischen Feld der Erziehungs- und Bildungswissenschaften kommt eine besondere Verantwortung für die Tradierung der hierarchischen (Geschlechter-)Differenz zu, da es heutzutage für Studierende üblich/möglich ist, an Seminaren mit geschlechtersensiblen bzw. geschlechterreflektierten Inhalten teilzunehmen. Allerdings ist weitgehend unerforscht, wie und in welcher Weise Studierende ,Geschlecht" und Geschlechterfragen thematisieren und in welcher Weise sie dabei in ihren Wahrnehmungen, Denk- und Handlungsmustern, in ihrer Kommunikation von den Erfahrungen in der erziehungswissenschaftlichen Fachkultur und -umwelt wie auch von gesellschaftlichen Modernisierungsdiskursen geprägt sind. Deshalb wurden über die situativen Konstruktionsleistungen der Akteur_innen hinausgehend nach Mustern gesucht, die für die untersuchte Gruppe jeweils charakteristisch in Hinblick auf die (De-)Thematisierung von ,Geschlecht' und Geschlechterfragen sind. Es wurden ausführliche Falldarstellungen angefertigt, die die einzelnen Gruppen in den Fokus genommen und deren zentrale Orientierungen rekonstruiert haben, welche als Habitus bezeichnet werden können (vgl. Kapitel 6).

Die Ergebnisse sind damit auf einer Ebene anzusiedeln, die die Dichotomie von Struktur und Handlung, Mikro- und Makroebene überwindet, welche für viele Ansätze, die sich mit sozialer Differenz und ,Geschlecht ${ }^{*}$ 
und Geschlechterfragen auseinandersetzen, charakteristisch ist (vgl. Kubisch 2008, S. 311).

Die Ergebnisdiskussion beginnt mit einer Zusammenfassung der rekonstruierten habituellen (De-)Thematisierungsmuster der jeweiligen Gruppen (vgl. Kapitel 7.1), diese ist als sinngenetische Typologie zu verstehen. In der universitätsinternen und -übergreifenden komparativen Analyse lassen sich Ähnlichkeiten und Differenzen identifiziert, die im Kapitel 7.2 und 7.3 zusammengefasst werden. Im Kapitel 7.4 wird genauer herausgearbeitet, dass es neben studiumsbezogenen Erfahrungen und Erlebnissen und individuellen Erfahrungen auch gesellschaftliche Strukturen und Diskurse gibt, die Vorstellungen und Praktiken der AkteurInnen leiten. Hier wird deutlich, was sich im (vermeintlich) selbstverständlichen Umgang mit der Kategorie ,Geschlecht` versteckt. Unter der Überschrift „Verdeckungszusammenhänge und Wirkmechanismen“ (Kapitel 7.4.2) werden Diskursmodi zusammengefasst, die eine Thematisierung erschweren und/oder zu einer (De-)Thematisierung führen. Die Arbeit endet mit einem Resümee und einem Ausblick, die sich der Notwendigkeit von geschlechterreflektierenden studiumsbezogenen Erfahrungsräumen und Lehrkonzepten im Rahmen des erziehungswissenschaftlichen Studiums widmen (Kapitel 7.5). Dies betrifft die Wissensvermittlung ebenso wie Reflexions- und Austauschmöglichkeiten.

\subsection{Habituelle Konstruktionen bei der (De-)Thematisierung von ,Geschlecht"}

Im Rahmen der Gruppendarstellungen wurden anhand verschiedener Passagen die wesentlichen handlungsleitenden Orientierungen der einzelnen Gruppen rekonstruiert (vgl. Kapitel 6). Ein zentrales Ergebnis der Arbeit, hinsichtlich der Thematisierung von ,Geschlecht' und Geschlechterfragen ist die stattfindende Neutralisierung und Verdeckung von sozialen Ungleichheiten und deren Wirkmechanismen. Des Weiteren kann es auch im Rahmen einer Thematisierung zu einer Dethematisierung von ,Geschlecht' und Geschlechterfragen kommen. Zudem zeigt sich, dass eine geschlechterreflektierende Haltung und geschlechterreflektiertes Handeln nicht notwendigerweise Teil des erziehungswissenschaftlichstudentischen Habitus sind. Neben dem studiumsbezogenen konjunktiven Erfahrungsraum ist die (De-)Thematisierung von ,Geschlecht' und Geschlechterfragen geprägt von rhetorischen Modernisierungsprozessen und Verdeckungszusammenhängen. Die rekonstruierten Habitusformen 
der interviewten Gruppen Holz, Feuer, Metall und Wasser sollen nun noch einmal im Überblick dargestellt werden.

\subsubsection{Ein Habitus des Nicht-Verstehens}

In der Gruppe Holz, deren sechs Teilnehmerinnen Bachelorstudierende des Fachbereichs Erziehungs- und Bildungswissenschaften an der Universität Sonne sind, steht die Thematisierung von ,Geschlecht' und Geschlechterfragen im Zeichen eklatanter Verständigungsprobleme; der Gruppe Holz gelingt es nicht, sich auf das Thema einzupendeln. Gerade darin ist das für die Gruppe typische Muster zu sehen, welches auf Rahmeninkongruenzen und fehlenden studiumsbezogenen konjunktiven Erfahrungen basiert. Da sich der Großteil der Gruppe Holz am Gleichheitspostulat und an gesellschaftlichen Modernisierungsprozessen orientiert und auf keine studiumsbezogenen Erfahrungen hinsichtlich der Thematisierung von ,Geschlecht" verfügt, scheint eine Diskussion verhindert bzw. brüchig zu werden. Die Beteiligung von Personen mit einer unterschiedlichen Studiendauer mag als eine Erklärung hierfür herangezogen werden.

Darüber hinaus bzw. in Verbindung damit zeichnen sich unterschiedliche und widersprüchliche Orientierungen ab, die nicht zu gemeinsamen Ansichten hinsichtlich der Relevanz von ,Geschlecht' und Geschlechterfragen verbunden werden können. Es zeigt sich, dass es keine etablierte und konjunktive Diskussionsform und -kultur zum Thema ,Geschlecht in der Gruppe Holz gibt und sich daher diesbezüglich auch keine kollektiven Wissensbestände und habituellen Praxen rekonstruieren lassen. Auch wenn es aufgrund des fehlenden studiumsbezogenen konjunktiven Erfahrungsraums problematisch ist, von einem Habitus zu sprechen, charakterisiere ich diese Gruppe hinsichtlich der Thematisierung von ,Geschlecht' und Geschlechterfragen dennoch mit dem Habitus des NichtVerstehens und der Dethematisierung von ,Geschlecht', da sich dieser nicht nur auf den studiumsbezogenen konjunktiven Erfahrungsraum bezieht, sondern auch auf einen neuen Geschlechtervertrag (vgl. Kapitel 4.3.3), der bei den Studierenden als konjunktiver Erfahrungsraum wirksam ist.

In der Gruppe Holz deutet vieles darauf hin, dass die Dethematisierung und die Verdeckung von ,Geschlecht' und Geschlechterfragen Teil der studentisch-habituellen Praxis sind und der studiumsbezogene konjunktive Erfahrungsraum vom konjunktiven Erfahrungsraum des neuen Geschlechtervertrags überlagert wird. 


\subsubsection{Der Habitus der rhetorischen Distanzierung}

In der Gruppe Feuer, die sich aus zwei Bachelorstudentinnen der Erziehungs- und Bildungswissenschaften der Universität Sonne zusammensetzt, ist die Thematisierung von ,Geschlecht" und Geschlechterfragen geprägt von der Reflexion pädagogisch-praktischer Erfahrungen, die ich als Erweiterung des studiumsbezogenen konjunktiven Erfahrungsraums verstehe. Diese Reflexion ist jedoch erst nach einer Distanzierung von der Bedeutungszuschreibung und Relevanz von ,Geschlecht' und Geschlechterfragen von Seiten der Student_innen möglich. Nach dieser rhetorischen Distanzierung und Zurückweisung von Geschlechterfragen gelang eine Thematisierung auf einer Ebene der Reflexion.

Es ist anzunehmen, dass diese aufgrund des offenen Diskussionsraums des Gruppendiskussuionssettings begünstigt wurde. In der Gruppe Feuer zeigt sich somit, dass in einem Raum, in dem kritische Reflexion Platz hat, ,Geschlecht' thematisiert werden kann. Hinsichtlich des Studiums lautet das Fazit der Akteurinnen, dass ,Geschlecht' und Geschlechterfragen verstärkt thematisiert werden sollten, um eine geschlechterreflektierende Haltung anzuregen, welche für die pädagogische Praxis aus Sicht der Gruppe Feuer von Bedeutung sei. An der Gruppe Feuer ist interessant, dass es trotz des hohen reflexiven Gehalts der Diskussion zur Reproduktion eines binären Geschlechterverständnisses kommt und sie sich an der Geschlechtergleichheit als Norm orientiert. Die GDT gehen also von einer Gleichheitsnorm und einem toleranten, offenen und modernisierten Geschlechterwissen aus. In der Gruppe Feuer zeigt sich, dass die Kategorie ,Geschlecht' nach wie vor ein Ordnungs- und Strukturmerkmal darstellt, auch wenn dieses tendenziell durch die Gleichheitsnorm verdeckt wird.

Die rhetorische Beteuerung und Inszenierung der Geschlechtergleichheit ist somit ein Motiv, um die Wirksamkeit der Kategorie ,Geschlecht $\mathrm{zu}$ neutralisieren und zu verdecken. So lässt sich mit dieser Gruppendiskussion Angelika Wetterers These bestätigen, ,dass zeitgenössisches Differenzwissen und soziale Praxis nicht mehr bruchlos zusammenpassen" (Wetterer 2003, S. 290). (Implizite) Vorstellungen bzw. das inkorporierte Wissen von Geschlechterdifferenz und -hierarchie treffen dabei auf explizit gemachte Vorstellungen von Egalität und Individualität. 


\subsubsection{Der kritikaffine Habitus und eine ,Ja, aber'-Mentalität}

Die Gruppe Metall, deren drei TeilnehmerInnen an der Universität Mond Erziehungs- und Bildungswissenschaften im Diplomstudiengang studieren, zeichnet sich bei der Thematisierung von ,Geschlecht' durch ihre Kritikaffinität aus. In der Gruppe Metall lässt sich eine selbstverständliche und habitualisierte Thematisierung von ,Geschlecht' rekonstruieren, jedoch ist diese nicht von einem reflektierenden Umgang mit der Kategorie ,Geschlecht' geprägt, sondern von einer inhaltlichen Kritik an der Thematisierung von ,Geschlecht" und Geschlechterfragen, vor allem im Kontext des bildungs- und universitätsbezogenen Milieus.

In ihrem studiumsbezogenen Alltag erleben die TeilnehmerInnen eine Thematisierung von ,Geschlecht' und Geschlechterfragen, die nicht ihrer Vorstellung entspricht. Sie orientieren sich daran, dass eine Thematisierung von und eine Sensibilisierung für ,Geschlecht' im Zentrum stehen sollte, sie haben jedoch alle eine unterschiedliche Vorstellung davon, wie dies konkret umgesetzt werden soll. Gemein ist ihnen aber, und dass macht den Habitus der Gruppe aus, die Kritik an der studiumsbezogenen Thematisierung von ,Geschlecht' und Geschlechterfragen: Sie kritisieren erstens das als ausgrenzend und diskriminierend wahrgenommene Diskussionsklima, wenn es um, Geschlecht ' und Geschlechterfragen geht, zweitens empfinden sie die Thematisierung von Geschlechterfragen als undifferenziert und unterkomplex und drittens bemängeln sie eine Naturalisierung der Geschlechterdifferenzen. Viertens problematisieren sie eine einseitige und an Frauen orientierte Thematisierung sowie eine mögliche Überfokussierung des Themas. Auch hier zeigen sich Spuren des konjunktiven Erfahrungsraums des neuen Geschlechtervertrags, da feministische Interventionen und eine Kritik an (patriarchalen) Herrschaftsverhältnissen nicht mehr notwendig erscheinen.

Die Thematisierung von ,Geschlecht" und Geschlechterfragen scheint für die Gruppe Metall nur dann legitim, wenn es dabei zu keiner Diskriminierung oder Ausgrenzung kommt. Die Erwartungen seitens der Studierenden sind in gewisser Weise paradox: Das Thema ,Geschlecht" ist für sie zwar relevant, weil es Chancengleichheit schaffe, aber auch problematisch, weil im Zuge der Diskussionen potentiell auch andere Personengruppen ausgegrenzt werden könnten. Der gesellschaftskritische und hinterfragende Impetus bei der Thematisierung von ,Geschlecht' stellt in der Gruppe Metall ein wichtiges Denk- und Bewertungsschema dar. 


\subsubsection{Der Habitus des prekären Selbstbezugs}

Hinsichtlich der negativen Bezugnahme und der Problematisierung von Frausein kann in der Gruppe Wasser, an der sich drei Diplomstudentinnen des Fachbereichs Erziehungs- und Bildungswissenschaften an der Universität Mond beteiligt haben, von einem Habitus bei der Thematisierung von ,Geschlecht' und Geschlechterfragen gesprochen werden. Auf einer kommunikativ generalisierenden Ebene findet jedoch ein Negieren bzw. ein Absehen von negativen Erfahrungen aufgrund des eigenen Frauseins statt. Demnach kann in der Gruppe Metall von einem Habitus des ,prekären Selbstbezug[s]" (Bitzan 1997, S. 78) gesprochen werden. Dieser hebt hervor, dass die Frauen von sich selbst und von ihren Erfahrungen absehen, um sich in einer normativen Welt verhalten zu können. Negative Gefühle wie Angst oder innere Konflikte, welche dem Selbstbild und den gesellschaftlichen Denkschemata widersprechen, werden durch die Rezeption des rhetorisch modernisierten Geschlechterwissens verdeckt.

Die Thematisierung von ,Geschlecht" und Geschlechterfragen steht in der Gruppe Wasser zudem zwischen einer ,natürlichen' Haltung und einer bildungsbezogenen reflexiven Haltung gegenüber Geschlechterfragen. Zwei Teilnehmerinnen teilen die Orientierung an einem ,natürlichen ${ }^{6}$ Zugang zu ,Geschlecht' und Geschlechterfragen, der unabhängig von Bildungsprozessen bestehe und qua Frausein gegeben sei. Diese Orientierung wird jedoch von der dritten Teilnehmerin nicht geteilt; ihr zufolge könne eine reflektierende Haltung im Kontext des Studiums prozesshaft entwickelt werden. Diesbezüglich können die Teilnehmerinnen auf keine curriculums- bzw. seminarbezogenen konjunktiven Erfahrung zurückgreifen und wie in der Gruppe Holz scheint für einen Teil der Gruppe Wasser der neue Geschlechtervertrag als konjunktiver Erfahrungsraum $\mathrm{zu}$ fungieren.

\subsection{Universitätsbezogene (De-)Thematisierung von ,Geschlecht“}

Über die Habitusformen hinausgehend hat die Arbeit nach Antworten auf die Frage gesucht, inwiefern es Aspekte in der (De-)Thematisierung von ,Geschlecht' und Geschlechterfragen gibt, die für die Studierenden der Erziehungs- und Bildungswissenschaften einer Universität im Vergleich zu denen einer anderen typisch sind und in diesem Sinne als uni- 
versitätsbezogene konjunktive Erfahrungen bezeichnet werden können. $\mathrm{Zu}$ Beginn der Interpretationen habe ich mir häufig die Frage gestellte, warum in den einzelnen Gruppen die (De-)Thematisierung von ,Geschlecht" und Geschlechterfragen auf unterschiedliche Weise diskutiert wurde, es in einigen Gruppen als selbstverständliches Thema aufgegriffen wurde und in anderen Abwehr und Distanz auslöste. Im Laufe der mittels der dokumentarischen Methode durchgeführten Fallrekonstruktionen und -vergleiche wurden jedoch in zunehmendem Maße Zusammenhänge zwischen der (De-)Thematisierung von ,Geschlecht' und Geschlechterfragen und den universitäts- und studiumsbezogenen konjunktiven Erfahrungsräumen der jeweiligen Gruppen deutlich.

Es zeigt sich, dass das Studium der Erziehungs- und Bildungswissenschaften einen konjunktiven Erfahrungsraum bildet, der jedoch auch vom universitätsbezogenen konjunktiven Erfahrungsraum überlagert wird. Zudem wird deutlich, dass die Studierenden derselben Disziplin bei aller Differenz zwischen den universitären Standorten einen konjunktiven Erfahrungsraum miteinander teilen, der maßgeblich mit den gesellschaftlichen (rhetorischen) Modernisierungsprozessen und Verdeckungszusammenhängen verbunden ist. Zusammenfassend kann der neue Geschlechtervertrag als grundlegender konjunktiver Erfahrungsraum bezeichnet werden, auf dessen Basis die (De-)Thematisierung von ,Geschlecht' und Geschlechterfragen angesiedelt ist. Diese unterschiedlichen und zum Teil überlappenden oder konkurrierenden konjunktiven Erfahrungsräume sollen im Folgenden dargestellt werden.

\subsubsection{Der konjunktive Erfahrungsraum der Bachelorstudierenden der Universität Sonne}

Wenn man die Gruppen Holz und Feuer der Universität Sonne miteinander vergleicht, wird auf einer allgemeinen Ebene deutlich, dass bei den Gruppen der Universität Sonne zum Zeitpunkt der Untersuchung die Kritik am neu eingeführten Bachelor- und Masterstudiengang eine kollektive Orientierung darstellt. Auch wenn diese in der Gruppe Holz einen größeren Raum einnimmt als in der Gruppe Feuer, stellt die gemeinsame Betroffenheit der Bachelorstudierenden ein verbindendes Element und kollektiv besprochenes Thema dar. Dies steht im Gegensatz zur Thematisierung von ,Geschlecht' und Geschlechterfragen, diesbezüglich scheint es keine gemeinsame Diskussionskultur und kein affirmatives Diskussionsklima zu geben. Es fällt auf, dass beide Gruppen trotz der unterschiedlichen Positionen bestimmte (De-)Thematisierungsmuster und 
Orientierungen miteinander teilen, die auf universitätsbezogene Aspekte verweisen. Es wird deutlich, dass der konjunktive Erfahrungsraum der Universität Sonne nahelegt, das Thema ,Geschlecht' zunächst abzulehnen, und dass es keinen Inhalt darstellt, der selbstverständlich diskutiert wird. Dies wird in beiden Gruppen, wenn auch in unterschiedlicher Intensität, deutlich. Den TeilnehmerInnen der Gruppe Holz gelingt es kaum, sich auf eine gemeinsame Ebene bei der Thematisierung von ,Geschlecht und Geschlechterfragen einzupendeln.

Auch in der Gruppe Feuer kommt es erst nach einer anfänglichen $\mathrm{Zu}$ rückweisung des Themas zu einer Auseinandersetzung mit ,Geschlecht und Geschlechterfragen, vor allem in Bezug auf ihre pädagogische Praxis und ihr Studium. Das Thema wird thematisierbar/kommunizierbar, weil die Studierenden im Sinne einer Selbstreflexion über die eigene Erfahrung sprechen und darüber zur Relevanz des Themas kommen. In dieser Gruppendiskussion wird deutlich, dass in einem Raum, in dem kritische Reflexion Platz hat, ,Geschlecht' thematisiert wird. Dieser Raum wird durch die Gruppendiskussion bereitgestellt und scheint im Bachelorstudiengang der Erziehungs- und Bildungswissenschaften der Universität Sonne zu fehlen. Zu diesem Schluss kommt auch die Gruppe Feuer, indem sie resümiert, dass ,Geschlecht' und Geschlechterfragen im Studium „mehr thematisiert werden“ müssten, um eine geschlechterreflektierende Haltung anzuregen.

In beiden Gruppen spielt der weiblich dominierte konjunktive studiumsbezogene Erfahrungsraum eine große Rolle bei der (De-)Thematisierung von ,Geschlecht‘ und Geschlechterfragen. In der Gruppe Holz wie auch in der Gruppe Feuer wird das Studium der Erziehungs- und Bildungswissenschaften als eine „Frauendomäne" und als ein feminisiertes Feld rekonstruiert und pädagogische bzw. erziehungswissenschaftliche Tätigkeiten als, weibliche' Bereiche reproduziert. Verstärkt wird dies durch verallgemeinernde Aussagen und Spekulationen hinsichtlich einer möglichen homosexuellen Orientierung männlicher Studierender. Der hohe Frauenanteil bei den Studierenden dient beiden Gruppen als Argumentationsfigur, dass Frauen nicht benachteiligt seien und ,Geschlecht" sowie Geschlechterfragen keine Rolle spielten. Demnach ist die kollektive Orientierung folgende: Wenn Frauen im erziehungswissenschaftlichen Studium nicht unterrepräsentiert sind, scheint es für die Studierenden auch keine Benachteiligung an der Universität zu geben. In Bezug auf ,Geschlecht‘ und Geschlechterfragen findet so eine Neutralisierung und Verdeckung von sozialen Ungleichheiten und deren Wirkmechanismen statt, die mit der hohen Zahl an weiblichen Studierenden legitimiert 
werden. In unterschiedlicher Weise reproduzieren beide Gruppen traditionelle Rollenbilder und charakterisieren bestimmte Bereiche als weiblich oder männlich. Gemeinsam ist den beiden Gruppen, dass die binären Geschlechterverhältnisse implizit wie auch explizit als Norm formuliert werden und die Studierenden ihre eigene heterosexuelle vergeschlechtlichte und vergeschlechtlichende Position nicht hinterfragen.

Die Orientierung an der Gleichstellungsnorm der Geschlechter wird in beiden Gruppen reproduziert und verdeckt, dass die Differenzierung nach ,Geschlecht' im handlungsleitenden Wissen nach wie vor eine Rolle spielt. Zudem ist die Geschlechtergleichheit ein Motiv, um die Wirksamkeit der Kategorie ,Geschlecht' zu neutralisieren und zu verdecken. Der studiums- und universitätsbezogene Erfahrungsraum der Universität Sonne dient den Studierenden demnach nicht als Raum und Möglichkeit ihre eigene heterosexuelle vergeschlechtlichte und vergeschlechtlichende Position zu hinterfragen und zu reflektieren. Auch eine geschlechtergerechte Sprache ist nicht Teil der habituellen Sprechpraxis der Studierenden an der Universität Sonne. Die Verwendung des generischen Maskulinums wird durch die Annahme begründet, dass Emanzipation und Gleichberechtigung von Frauen bereits erreicht sei und es deshalb nicht mehr notwendig sei, Frauen in der Sprache hervorzuheben bzw. durch eine geschlechtergerechte Sprache der Benachteiligung von Frauen entgegenwirken zu müssen. Interessant ist auch, dass den Studierenden der Universität Sonne, mit einer Ausnahme, das Zentrum für Gender Studies an ihrer Universität nicht bekannt ist.

Es ist nun deutlich geworden, dass ein reflektierter Umgang mit ,Geschlecht' und Geschlechterfragen bei den Teilnehmer_innen nicht Teil des erziehungswissenschaftlich-studentischen Habitus ist und somit auch nicht Teil des universitäts- und studiumsbezogenen konjunktiven Erfahrungsraums der Universität Sonne. Gleiches gilt für die Relevanz und die Bedeutung der Kategorie ,Geschlecht' für das erziehungswissenschaftliche Denken und Handeln. Der studiums- und universitätsbezogene Erfahrungsraum der Universität Sonne legt eine (De-)Thematisierung von ,Geschlecht' und Geschlechterfragen nahe. Verstärkt wird die (De-)Thematisierung vom konjunktiven Erfahrungsraum des neuen Geschlechtervertrags, wie ihn Angela McRobbie diagnostiziert (siehe Kapitel 7.4). 


\subsubsection{Der konjunktive Erfahrungsraum der Diplomstudierenden der Universität Mond}

Bei den beiden Gruppen der Universität Mond fällt (speziell im Vergleich zu den Gruppen der Universität Sonne) auf, dass die Einführung des Bachelor- und Masterstudiengangs nur am Rande thematisiert wird und keine kollektive Erfahrung darstellt. Dies liegt wohl daran, dass die befragten Diplomstudierenden von der Studiengangsumstellung nicht unmittelbar betroffen sind. Anders als an der Universität Sonne können die Studierenden bei der Thematisierung von ,Geschlecht' und Geschlechterfragen auf den studiums- und universitätsbezogenen konjunktiven Erfahrungsraum zurückgreifen, was sich besonders in der Gruppe Metall zeigt. Dies legt nahe, dass es an der Universität Mond eine gemeinsame und habitualisierte Diskussionskultur gibt und Geschlechterfragen ein Diskussionsthema sind, das nicht (wie an der Universität Sonne) legitimiert werden muss. Das Thema ,Geschlecht' scheint inner- und außerhalb von Lehrveranstaltungen präsent zu sein. Zum einen wird dies daran deutlich, dass in beiden Gruppen Professorinnen genannt werden, die sich in ihrer Lehre und Forschung mit ,Geschlecht' und Geschlechterfragen beschäftigen. Zum anderen sind auch Studierende, die sich nicht im Rahmen einer Lehrveranstaltung mit ,Geschlecht' und Geschlechterfragen auseinandergesetzt haben, zumindest im ersten Impuls, bereit, über ,Geschlecht' und Geschlechterfragen zu diskutieren und über studiumsbezogene konjunktive Erfahrungen verfügen bzw. diese auch nennen. Somit lässt sich in beiden Gruppen das soziale Feld der Universität Mond als konjunktiver Erfahrungsraum rekonstruieren, der geprägt ist von Möglichkeiten, sich mit Geschlechterfragen auseinanderzusetzen. Allerdings bedeutet dies nicht, dass die Studierenden eine geschlechterreflektierte Haltungen inkorporiert haben.

In Bezug auf die Relevanz und Bedeutung von ,Geschlecht' und Geschlechterfragen zeigt sich bei den Studierenden der Universität Mond eine ,Ja, aber'-Mentalität. ,Geschlecht' wird zwar als ein wichtiges Thema darstellt, allerdings wird die Relevanz limitiert und ihr Geltungsbereich eingeschränkt. Die Kritik an der studiumsbezogenen Thematisierung von ,Geschlecht ${ }^{6}$ und Geschlechterfragen ist eine wesentliche Gemeinsamkeit der Gruppen Metall und Wasser. In beiden Gruppen werden bestimmte Formen der Thematisierung bzw. eine als ausgrenzende und überbetrieben wahrgenommene Diskussion abgelehnt. Zudem werden in beiden Gruppen antifeministische Stereotype hinsichtlich ,hetzende[r] Emanzen" (GD_Wasser) und ,aggressive[r] Kampfwesen“" (GD_Metall) ge- 
äußert. In der Kritik, die zwar in beiden Gruppen unterschiedlich ausfällt, lässt sich ein gemeinsames Muster der (De-)Thematisierung identifizieren, das antifeministische Stereotype reproduziert und eine positive Bewertung und Bezugnahme auf eine produktive Thematisierung von ,Geschlecht' und Geschlechterfragen verhindert und verdeckt. Hier wird deutlich, dass der konjunktive Erfahrungsraum der Studierenden von Diskussionen und Positionen geprägt ist, welche die Studierenden als zu extrem und übertrieben wahrnehmen, da sie davon ausgehen, dass keine Geschlechterungleichheit und -benachteiligung mehr besteht.

Im Vergleich der Gruppen fällt auf, dass die Studierenden die Erfahrung gemeinsam haben, dass die Thematisierung von ,Geschlecht' , weiblich' ist und vor allem von Frauen über Frauen und Mädchen gesprochen wird; dies wird als einseitige Thematisierung wahrgenommen. Das heißt, in beiden Gruppen der Universität Mond werden Frauen (Dozentinnen und Studentinnen) als die aktiven Protagonistinnen und Akteurinnen in der Thematisierung von ,Geschlecht' beschrieben. Frauen scheinen die Expertinnen zu sein, und die Thematisierung durch Männer und auf männliche Lebenslagen ausgerichtete Inhalte werden von den Studierenden als Leerstelle diagnostiziert. Der weiblich dominierte konjunktive studiumsbezogene Erfahrungsraum spielt bei der (De-)Thematisierung von ,Geschlecht' und Geschlechterfragen in beiden Gruppen eine große Rolle. So wird von der Gruppe Metall wie auch von der Gruppe Wasser das Studium der Erziehungs- und Bildungswissenschaften implizit und explizit als typisches Studium und Arbeitsfeld für Frauen angesehen und als weiblich dominiertes Feld reproduziert. Zudem wird die statistische Geschlechterverteilung unter den Studierenden diskutiert und Studenten als von der weiblich geprägten erziehungswissenschaftlichen und pädagogischen Norm abweichend konstruiert. Das bedeutet aber nicht, dass Männer in den Gruppendiskussionen eine negative Bewertung erfahren. In der Gruppe Metall erfahren Studenten aufgrund ihrer geringen Anzahl eine Aufwertung und Besonderung. In der Gruppe Wasser zeigt sich eine negative Assoziation mit Frausein als ein wesentliches Element. Bei den Studierenden der Universität Mond kommt es zu einer positiven Assoziation mit Mannsein und damit zu einer impliziten wie auch expliziten Aufwertung. Es ist zu vermuten, dass dies nicht nur vom studiumsbezogenen konjunktiven Erfahrungsraum geprägt ist, sondern mit gesellschaftlichen geschlechterbezogenen Zuschreibungen in Verbindung steht.

Auch an der Universität Mond wird von den befragten Studierenden die (eigene) eindeutige Geschlechtlichkeit, die heterosexuelle Geschlech- 
ternorm sowie die Geschlechterdifferenz nicht infrage gestellt. In beiden Gruppen lässt sich rekonstruieren, dass die Differenzierung nach ,Geschlecht' Teil der habituellen Praxis und eine kollektive Orientierung der Studierenden darstellt und somit Teil ihres konjunktiven Erfahrungsraums ist. Gemein ist den Studierenden zudem die Gleichsetzung von Geschlechterfragen mit ,Frauenfragen' und ,-problemen'. Geschlechterfragen werden hauptsächlich mit Ungleichheit, Differenz und Benachteiligung in Verbindung gebracht. Auch wenn die befragten Studierenden der Universität Mond über keine kollektive Orientierung hinsichtlich der Relevanz und Bedeutungszuschreibung der Thematisierung von ,Geschlecht' und Geschlechterfragen teilen, wird deutlich, dass sie einer Implementierung des Themas ins Curriculum des Studiengangs grundsätzlich positiv gegenüber stehen.

Hinsichtlich einer geschlechtergerechten Sprechpraxis unterscheiden sich die Orientierungen der Gruppen Metall und Wasser. Während in der Gruppe Metall in Formen der geschlechtergerechten Sprache diskutiert wird, orientieren sich die Studierenden der Gruppe Wasser am generischen Maskulinum. Hierin spiegelt sich die Diversität des studiumsbezogenen konjunktiven Erfahrungsraums selbst wieder. In der Gruppe Wasser wird, wie in den Gruppen der Universität Sonne, die Notwendigkeit einer geschlechtergerechten Sprache mit einer benachteiligten und diskriminierten Position von Frauen verbunden. Es wird jedoch von den weiblichen Studierenden hervorgehoben, dass sie sich vom generischen Maskulinum nicht diskriminiert fühlen. Hinter dieser Argumentationsfigur verbirgt sich auf einer rhetorischen Ebene das Orientierungsschema der Geschlechtergleichheit, und dass die Geschlechterdifferenz keine soziale Ungleichheit auslöse. Auch hier werden rhetorische Modernisierungsdiskurse und Verdeckungszusammenhänge deutlich.

Zusammenfassend kann gesagt werden, dass die Studierenden der Universität Mond zwar über einen konjunktiven Erfahrungsraum und eine Diskussionskultur verfügen, wenn es um ,Geschlecht' und Geschlechterfragen geht. Jedoch legt der studiums- und universitätsbezogene Erfahrungsraum der Universität Mond eine Thematisierung von ,Geschlecht" und Geschlechterfragen nahe, die weniger von einem geschlechterreflektierten Umgang geprägt ist als vielmehr von einer ,Ja, aber'Mentalität, welche die studiumsbezogene Thematisierung von ,Geschlecht ${ }^{\text {* }}$ und Geschlechterfragen selbst zum Zentrum der Kritik macht und nicht die binären Geschlechternormen oder (patriarchalen) Herrschaftsverhältnisse. Wie bereits an der Universität Sonne zeigt sich auch an der Universität Mond, dass die Studierenden davon ausgehen, dass feministische In- 
terventionen und Kritik an der soziale Ungleichheit (re-)produzierenden Geschlechterdifferenz nicht mehr notwendig sei.

\subsection{Universitätsübergreifende und studiumsbezogene (De-) Thematisierung von ,Geschlecht“}

Im Laufe der Auswertung hat sich nicht nur die Frage ergeben, welche Art der (De-)Thematisierung der studiumsbezogene konjunktive Erfahrungsraum nahe legt, sondern auch, welche Themen von den Studierenden bevorzugt werden. Im Verlauf der komparativen Analyse wurde deutlich, dass das Studium zu einer theoretischen und abstrakten Auseinandersetzung mit ,Geschlecht" und Geschlechterfragen anregt. Die eigene Erfahrungs- und Erlebenswelt der Studierenden wird dabei aber nur wenig angesprochen und eine selbstreflexive Auseinandersetzung mit bzw. die Frage nach der Relevanz und Bedeutung der Kategorie ,Geschlecht" nimmt wenig Raum ein und ist von individuellen Orientierungen bestimmt.

Nahe liegende Themen sind für die Studierenden Lehrveranstaltungen mit Geschlechterinhalten und somit ein curriculumsbezogener Austausch. Auch die statistische Verteilung von Männern und Frauen bei den Studierenden und Professor_innen und hier vor allem der hohe Frauenanteil bei den Studierenden sowie Spekulationen hinsichtlich der sexuellen Orientierung von Studenten sind aufkommende Themen. In allen Gruppen spielt der weiblich dominierte konjunktive studiumsbezogene Erfahrungsraum eine große Rolle bei der (De-)Thematisierung von ,Geschlecht' und Geschlechterfragen. Die Diskussionen zu diesen Bereichen werden nicht immer von den gleichen Fragen ausgelöst, was darauf hinweist, dass sie unabhängig von den gestellten Fragen zu studiumsbezogenen konjunktiven Erfahrungen gehören. Die bereits angesprochene theoretische und abstrakte Auseinandersetzung mit ,Geschlecht" und Geschlechterfragen findet ihren Ausdruck auch in der Verwendung des Begriffs gender, der zum geteilten Wortschatz der Studierenden gehört und hauptsächlich in Verbindung mit einer theoretischen Auseinandersetzung und theoretischem Wissen genutzt wird.

Im Gegensatz zu den Bachelorstudierenden der Universität Sonne ist den befragten Diplomstudierenden der Universität Mond das Zentrum für Gender Studies an ihrer Universität bekannt. Die gemeinsame Er- 
fahrung der Studierenden der Universität Mond hinsichtlich der studiumsbezogenen Thematisierung von ,Geschlecht' und Geschlechterfragen kann an der längeren Studiendauer der Diplomstudierenden liegen. Allerdings muss hier einschränkend gesagt werden, dass die ,Studienanfängerin` an der Universität Sonne, anders als ihre bereits länger studierenden Kommilitoninnen, über die institutionellen Verankerung von Geschlechterfragen in ihrem Studiengang wie auch im Zentrum für Gender Studies Bescheid weiß.

Insgesamt werden in den Diskussionen affirmative wie auch ablehnende Muster bei der (De-)Thematisierung von ,Geschlecht' und Geschlechterfragen sichtbar. Hierin spiegelt sich die Diversität des studiumsbezogenen konjunktiven Erfahrungsraums selbst wider, der unterschiedliche Spielarten der (De-)Thematisierung von ,Geschlecht' und Geschlechterfragen zulässt und wenig konsistent erscheint. Was die Gruppen trotz aller Unterschiede in den Habitusformen miteinander teilen, ist, dass die (De-)Thematisierung von ,Geschlecht' und Geschlechterfragen von Verdeckungszusammenhängen, rhetorischen Modernisierungsprozessen und dem neuen Geschlechtervertrag geprägt ist, und dass ein Bruch zwischen dem kommunikativ-generalisierten Geschlechterwissen und dem handlungsleitenden Geschlechterwissen besteht.

\subsection{Der neue Geschlechtervertrag und rhetorische Modernisierungsprozesse als konjunktiver Erfahrungsraum}

Bei der Rekonstruktion der Thematisierung von ,Geschlecht' wird deutlich, dass es neben studiumsbezogenen Erfahrungen und Erlebnissen sowie individuellen Erfahrungen auch gesellschaftliche Strukturen und Diskurse gibt, die Vorstellungen und Praktiken leiten. In der Analyse wird fassbar, dass sich die Studierenden zur rhetorischen Modernisierung und zum neuen Geschlechtervertrag verhalten müssen und diese somit einen relevanten gesellschaftsbezogenen konjunktiven Erfahrungsraum darstellen. Dadurch werden die verschiedenen Formen von politischer Diskussion und Mobilisierung, welche den Feminismus als soziale Bewegung geprägt haben, verdrängt (vgl. McRobbie 2010, S. 130). Im Laufe der Untersuchung zeigt sich insbesondere bei der Rekonstruktion des Geschlechterwissens und -verständnisses der Student_innen die Wirksamkeit der rhetorischen Modernisierung und des neuen Geschlech- 
tervertrags. Daher wird im Folgenden ein gruppen- und universitätsübergreifendes Geschlechterverständnis und -wissen rekonstruiert, welches die (De-)Thematisierung von ,Geschlecht' und Geschlechterfragen maßgeblich beeinflusst bzw. eine Thematisierung verhindert.

\subsubsection{Die Rekonstruktion eines binären Geschlechterwissens und -verständnisses}

Das rekonstruierte Geschlechterverständnis und -wissen ist zum einen von der Persistenz einer eindeutig binären Geschlechternorm geprägt, zum anderen zeigen sich Brüche, indem modernisierte Vorstellungen und Phänomene auf der Ebene des kommunikativ-generalisierenden Wissens betont werden. Das heißt, bei dieser Modernisierung handelt es sich um eine rhetorische (vgl. Wetterer 2003): Obgleich das Geschlechterwissen und -verständnis von den Vorstellungen geprägt sind, dass ,Geschlecht in modernen Gesellschaften pluralisiert, individuell und eigenverantwortlich gestaltbar sowie frei wählbar sei, orientieren sich die Studierenden ebenso an der ,naturhaften' Geschlechterdifferenz und reproduzieren diese. Das Geschlechterwissen und -verständnis ist von dem Denkschema bestimmt, dass Frauen und Männer im gleichen Maß an Selbstbestimmung, Autonomie und Gleichheit teilhaben und gleichberechtigt sind. Dies sind jedoch nur rhetorische Bekundungen, da in der sozialen Praxis und im handlungsleitenden Wissen weiterhin die Ungleichheit und Differenz zwischen den Geschlechtern konstant bleibt. Wenn die AkteurInnen also immer wieder die Gleichheit der Geschlechter rhetorisch betonen, ist Gleichheit im Sinne der Gleichstellung von Differentem zu verstehen, dessen Differenz dadurch aufrechterhalten wird bzw. werden soll. ${ }^{1}$

In den Gruppendiskussionen werden von den jeweiligen Akteur_innen die eigene männliche oder weibliche Geschlechtlichkeit, Geschlechtsidentität und Heterosexualität nicht infrage gestellt. Die Vorstellung einer kohärenten und eindeutigen Geschlechterbinarität lassen sich in allen Gruppen als normative Orientierung rekonstruieren und sind somit Teil der habituellen Praxis. Das kollektive Geschlechterwissen folgt demnach weiterhin den binären Prinzipien, die nach Eindeutigkeit verlangen und durch die es gleichzeitig hergestellt wird. Queere Identitäten

1 Zu einem ähnlichen Schluss kommt auch Monika Götsch in ihrer derzeit noch unveröffentlichten Dissertation an der Albert-Ludwigs-Universität Freiburg im Breisgau: ,,,n' Kerl mit sechzehn Jahren, der noch nie 'nem Weib an die Wäsche gegangen ist, gibt's ja eigentlich kaum noch." Sozialisation heteronormativen Wissens" (2012). 
und Geschlechtsuneindeutigkeiten werden als verunsicherndes Moment zur Sprache gebracht. Personen, die einer eindeutigen und kohärenten Geschlechtlichkeit nicht entsprechend werden als Ausnahmen und Besonderheiten wahrgenommen und als nicht der Geschlechternorm entsprechen, thematisiert. Gleichzeitig wird jedoch eine tolerante Haltung gegenüber Transsexualität betont. Während die Normalität und Alltäglichkeit von Homosexualität in der heutigen modernisierten Gesellschaft explizit betont werden muss, wird die Normalität von Heterosexualität implizit gewusst und ist Teil der kollektiven Orientierung. Diese Toleranz gegenüber Transsexualität und Homosexualität kann als ein Teil des ,modernisierten' rhetorischen Geschlechterwissens identifiziert werden. Das heißt, auch wenn die rhetorische Ebene von einer offenen und toleranten Haltung gegenüber Homosexualität und Transsexualität gekennzeichnet ist, ist die habituelle Praxis weiterhin vom heteronormativen Geschlechterwissen geprägt.

Auch das in dieser Arbeit vorgestellte Geschlechterverständnis von Andrea Maihofer, ,Geschlecht' als Existenzweise (vgl. Kapitel 3.7) zu fassen, kann nicht als handlungsleitendes Wissen bei den befragten Studierenden rekonstruiert werden. Das Konzept ,Geschlecht' als gesellschaftlich-kulturelle Existenzweise(n) zu begreifen, würde bedeuten, dass ,Geschlecht' nicht stabil und statisch, sondern flexibel und fluide ist. Aufgrund der praxeologischen Perspektive dieser Arbeit lässt sich in allen Gruppen eine eindeutige, stabile und kohärente Geschlechterbinarität als Norm rekonstruieren. Somit wird ersichtlich, dass die theoretischen Überlegungen von Maihofer nicht Teil einer habituellen Praxis oder eines , alltäglichen' Geschlechterverständnisses der befragten Studierenden sind. Bei der Rekonstruktion der Thematisierung von ,Geschlecht' wird deutlich, dass zwischen Geschlechterwissen und Geschlechterhandeln unterschieden werden muss.

\subsubsection{Verdeckungszusammenhänge und Wirkmechanismen}

Im gruppen- und universitätsübergreifenden Vergleich wird deutlich, dass nicht nur zwischen einer Neutralisierung und „Nicht-Thematisierung“ (Wetterer 2002, S. 149) von ,Geschlecht' gesprochen werden kann, sondern dass auch bei einer Thematisierung von ,Geschlecht' und Geschlechterfragen Dynamiken wirken, die zu einer (De-)Thematisierung führen. Das heißt, die grundsätzliche Annahme bzw. die idealtypische Vorstellung, dass die Thematisierung von ,Geschlecht' und Geschlechterfragen, 
aber auch die Auseinandersetzung mit dem objektivierten Thema ,Geschlecht", unwillkürlich zu einem reflektierten Umgang mit ,Geschlecht" führt, kann in dieser Arbeit nicht bestätigt werden. Die in dieser Arbeit herausgearbeitete Orientierung der Studierenden am neuen Geschlechtervertrag und an gesellschaftlichen Modernisierungsprozessen scheint eine Diskussion und eine Auseinandersetzung mit ,Geschlecht' und Geschlechterfragen zu verhindern. Zudem sind weiterhin Verdeckungszusammenhänge wirksam, die zu einer Individualisierung von Problemen führen und eine Thematisierung von ,Geschlecht' und Geschlechterfragen verhindern. Auch wenn in den Gruppen punktuell das eigene Erleben verhandelt wird, reicht dies nicht aus, um eine kritische Reflexion anzustoßen. Deshalb sollen im Folgenden die Wirkmechanismen zusammengefasst werden, die eine Thematisierung erschweren und/oder zu einer Dethematisierung führen. Hierfür wird auf die Analysen aller vier Gruppen zurückgegriffen.

\section{Rhetorische Geschlechtergleichheit oder das Gleichheitspostulat}

Die Orientierung der Studierenden am Gleichheitspostulat und an gesellschaftlichen Modernisierungsprozessen verhindert eine Diskussion und eine Auseinandersetzung mit ,Geschlecht' und Geschlechterfragen. Dies kann auch als Grund dafür gesehen werden, warum die Bedeutungszuschreibung von ,Geschlecht' und Geschlechterfragen bei den Akteuren und Akteurinnen an Relevanz und Legitimation verliert. Die Verdeckung von gesellschaftlichen geschlechtlich formierten Herrschaftsverhältnissen und den damit verbundenen Widersprüchen geschieht dadurch, dass sie als Individualbelastungen definiert und auch erlebt werden. Wie bereits Irene Dölling 2003 konstatierst, ist auch hier festzustellen, dass die Verbindung von Individualisierung mit Gleichberechtigung eine sehr spezifische Bedeutung einnimmt: Der Rekurs auf Gleichberechtigung funktioniert als Regulativ des Redens, das die thematisierbaren von den nicht oder nur auf Umwegen thematisierbaren Beständen des alltagsweltlichen Geschlechterwissen trennt. Dies wird wiederrum führt zu einer (De)Thematisierung geschlechtlicher Differenzierungen und Hierarchien. Daher gelten gleichstellungspolitischer Maßnahmen als überflüssig, weil Geschlecht ohnedies keine Rolle mehr spielt (vgl. Dölling 2003, S. 153-158). So werden Brüche zwischen der rhetorischen Geschlechtergleichheit und Egalität und einer erfahrenen Geschlechterungleichheit und Differenz individualisiert, da diese im Widerspruch zur dominanten gesellschaftlichen Norm von Gleichheit, Gleichberechtigung und Egalität von Män- 
nern und Frauen stehen. Aufgrund des Widerspruchs zwischen der Logik des Diskurses und der Logik der Praxis werden entwickeln Studierdende Bewältigungsstrategien. Zentrale Bewältigungsstrategien sind das Absehen vom eigenen Erleben (siehe prekärer Selbstbezug) und die Inkorporierung der (Det-)thematisierung von Geschlechterfragen. Dies resultiert darin, dass die fortbestehenden Ungleichheiten zwischen den Geschlechtern nicht als Geschlechterungleichheit thematisiert werden (vgl. Wetterer 2013, S. 256).

\section{Gleichsetzung von ,Geschlecht' mit Frausein und Benachteiligung}

Bei der Thematisierung von ,Geschlecht' und Geschlechterfragen kommen vor allem ,die ${ }^{6}$ Frauen in den Blick bzw. werden in den Blick genommen. Das bedeutet, dass beim Thema Geschlecht hauptsächlich Bereiche, Anliegen und Aspekte angesprochen werden, die insbesondere für Frauen von Belang bzw. problematisch seien. Damit findet gleichzeitig eine Delegation der Verantwortung an Frauen statt. Gerade die Gleichsetzung von ,Geschlecht' mit Frausein und Benachteiligung scheint die Thematisierung von Geschlecht und Geschlechterfragen zu behindern, da Geschlecht scheinbar keine Rolle mehr spielt (vgl. ebd., S. 258) und Selbstbestimmung und Gleichberechtigung zu einem common sense geworden sind (vgl. ebd., S. 246).

Die befragten Studierenden diskutieren vor der Hintergrundannahme, dass die Gleichstellung der Geschlechter bereits erreicht sei und deshalb (feministische) Interventionen und Kritik an (patriarchalen) Herrschaftsverhältnissen nicht mehr notwendig seien. Für Cornelia Koppetsch und Günter Burkhart (1999) ist vor allem die "Idee der Gleichheit" (...) „zu einer Quelle von Irrtümern und neuen Illusionen“ (1999, S. 193f.) geworden. Damit wird die Ungleichheit nicht beseitig, sondern entzieht sich der Thematisierbarkeit und wird somit ,in bestimmter Hinsicht sogar verschärft" (ebd.). Desweiteren wird in einer Gruppendiskussion die Annahme deutlich, dass qua Frausein ein ,intuitiver' Zugang zum Thema ,Geschlecht' bestehe, welcher nicht von einem Bildungsprozess bzw. von einer aktiven und bewussten Auseinandersetzung mit dem Thema bestimmt sei. Dieser „,naturgegebene Zugang“ übernimmt in der Diskussion die Rolle einer Legitimationsfigur, um ein Desinteresse und eine Dethematisierung von ,Geschlecht' und Geschlechterfragen (als Frau) zu begründen. 


\section{Third Person Effekt}

In der Individualisierung und in der Verschiebung von realen strukturellen Problemen auf andere Bereiche und Personen zeigt sich ein weiteres Muster, das zu einer (De-)Thematisierung führt und zur Etablierung einer Gleichberechtigungsnorm, auch wenn sich diese nicht mit den Erfahrungen der Studierenden deckt. Hier scheint eine Art Third Person Effekt $^{2}$ (vgl. Huck/Brosius 2007, S. 335) wirksam zu werden: Demnach sind andere Personen in anderen Bereichen, Institutionen und Strukturen, in denen die Studierenden nicht selbst situiert sind, von (benachteiligenden Wirkungen) der Kategorie ,Geschlecht' stärker betroffen als sie selbst (vgl. ebd.).

\section{Der Vergleich von ,früher' und ,heute}

Mit dem Vergleich der aktuellen Situation und ,früher' versuchen die Studentinnen deutlich zu machen, dass sich Frauen heute emanzipiert und gleichberechtigt fühlen und Selbstbestimmung, Autonomie und Gleichberechtigung zentrale Bestandteile des Alltagswissens junger Frauen sind. In Abgrenzung zu früher wird betont, dass heutzutage Frauen und Männern alle Wege offen stünden. Auch mit dieser Argumentationsfigur kommt es zwar zu einer Thematisierung von ,Geschlecht' und Geschlechterfragen, der historische Vergleich impliziert jedoch, dass diese heute nicht mehr notwendig sei und somit kommt es schlussendlich zu einer (De-)Thematisierung.

\section{Prekärer Selbstbezug}

In der Untersuchung zeigt sich ein Absehen von der eigenen Geschlechtlichkeit und von strukturellen Problemen. Negative Erfahrungen werden negiert und negative Gefühle wie Angst oder innere Konflikte, welche dem Selbstbild widersprechen, werden nicht (mehr) artikuliert bzw. können schlechter empfunden und ausgedrückt werden. Es scheint verpönt zu sein, sich selbst bzw. andere Frauen als Opfer zu sehen, und dementsprechende Erfahrungen werden individualisiert. Dies führt, zugespitzt formuliert, zu einem kollektiven Realitätsverlust und einer Normaffinität (vgl. Bitzan 1997, S. 78). Es wird von den eigenen Erfahrungen abgesehen und es findet eine Orientierung an den abstrakten äußeren Bildern der Gleichheitsnorm statt. Damit ist auch die Bezugnahme auf andere

2 Ursprünglich beschreibt dieser Effekt ein verzerrtes Wahrnehmungsphänomen der Wirkungsweisen von Massenmedien. Er beschreibt die Tendenz vieler Menschen, zu glauben, dass die Massenmedien andere stärker beeinflussen als sie selbst (vgl. Huck/Brosius 2007, S. 335). 
Frauen oder Mädchen von Unsicherheit geprägt. Hier wird deutlich, dass sich das private Leben und die persönlichen Erfahrungen nicht in Diskursen der öffentlichen Sphäre übersetzen lassen.

\section{Überkomplexität des Themas}

Des Weiteren stiftet folgende Aspekte bei den Studierenden Verwirrung: (a) ,Alltagswissen' oder vortheoretischem Wissen, dass sich (implizit) an Vorstellungen bzw. an inkorporiertem Wissen von Geschlechterdifferenz und -hierarchie orientiert, (b) einer (explizit) gemachten Vorstellungen von Toleranz, Egalität und Individualität und (c) dem Wissen über theoretische Konzepte zu Geschlechterkonstruktionen, die eine kritische Analyse von gesellschaftlichen Geschlechterverhältnissen und strukturellen Problemen schwierig macht.

\section{Das feminisierte Feld der Erziehungs- und Bildungswissenschaften}

Ein anderes Argument für die (De-)Thematisierung von ,Geschlecht ‘ stellt hohe Frauenanteil bei den Studierenden des Studienganges Erziehungsund Bildungswissenschaften dar. Die Studierenden gehen davon aus, dass es aufgrund der weiblichen Dominanz keine Benachteiligung im Studium gebe, obgleich Einzelne sehr wohl Diskriminierungserfahrung gemacht haben. Die Vorstellung, dass es keine Benachteiligung von Frauen aufgrund der statischen Überrepräsentanz gibt, führt in Folge dazu, dass nicht mehr nach der Relevanz der Kategorie ,Geschlecht' für erziehungswissenschaftliches Denken und Handeln gefragt wird. Zudem erscheint die geschlechtergerechte Sprache aufgrund des feminisierten Feldes als obsolet. In dieser Logik müssen Frauen im universitären Alltag durch die Schreibweise nicht hervorgehoben werden, weil hier keine Benachteiligung bestehe. Die Argumentationsfigur lautet: Sobald die anteilsmäßige Verteilung von Frauen und Männer gleich oder angenähert ist, erscheint das generische Maskulinum in seiner Verallgemeinerung legitim, mittels derer niemanden ausgeschlossen werden.

\section{Ausgrenzung anderer marginalisierter und benachteiligter Gruppen}

Abschließend möchte ich hervorheben, dass auch die potentielle Gefahr, andere marginalisierte und benachteiligte Gruppen aufgrund der Fokussierung auf die Ungleichheitskategorie ,Geschlecht' auszugrenzen oder zu benachteiligen, die Thematisierung von ,Geschlecht' und Geschlechterfragen einschränken kann. In diesem Sinne kann auch die Distanzierung 
bzw. die Ablehnung von „extremen“ Positionen und Maßnahmen sowie von einer Überfokussierung auf Geschlechterfragen verstanden werden. Es scheint eine Tendenz zu bestehen, sich vom Thema, Geschlecht' zu distanzieren bzw. eine moderate Position und Sichtweise vertreten zu wollen. Als ,zu extrem' wird in den Gruppen z. B. ,die' geschlechtergerechte Sprache, ,die' Quotenregelung, ,der ${ }^{6}$ Feminismus und die Hervorhebung von ,Geschlecht' als wichtigste Ungleichheitskategorie thematisiert.

\subsection{Resümee und Ausblick: Plädoyer für einen geschlechterreflexiven Erfahrungsraum}

Die vorliegende Arbeit kommt zu dem Ergebnis, dass die Studierenden der Erziehungs- und Bildungswissenschaften bei der (De-)Thematisierung von ,Geschlecht' nicht nur vom studiumsbezogenen konjunktiven Erfahrungsraum geprägt sind, sondern auch von gesellschaftlichen rhetorischen Modernisierungsprozessen und einem neuen Geschlechtervertrag. Es wird deutlich, dass sich diese konjunktiven Erfahrungsräume zum Teil überlappen, aber auch in Konkurrenz zueinander stehen. Der gemeinsame konjunktive Erfahrungsraum und die sozialen Strukturen haben einen großen Einfluss auf die (De-)Thematisierung von ,Geschlecht und Geschlechterfragen. Die dargestellten Ergebnisse zeigen, dass die Relevanz und Bedeutungszuschreibung der Kategorie ,Geschlecht' keine subjektiv individuelle und partikuläre Wahl ist, sondern vom sozialen Feld und von gesellschaftlichen Diskursen beeinflusst wird (vgl. Klinger 2011). Es bleibt festzuhalten, dass ein reflektierter und reflektierender Umgang mit ,Geschlecht' nicht Teil der studentisch-habituellen Praxis ist und die eigene vergeschlechtlichte und vergeschlechtlichende Position von den Studierenden nicht hinterfragt wird.

Dieses ernüchternde Ergebnis veranlasst mich dazu, dafür zu plädieren, dass das kritische und ermächtigende Potential von Bildungsprozessen im Studium besser genutzt werden sollte und geschlechterreflektierte Lehrkonzepte verstärkt werden müssen, um den Studierenden ihr eigenes Verhalten und Verhältnis zu gesellschaftlichen und pädagogischen Kontexten stärker zu vergegenwärtigen. So vertrete ich die Einschätzung, dass Bildung und Bildungsprozesse gerade in Zeiten neoliberaler Vereinnahmung und Verkürzungen zentrale Instrumente für die künftige Entwicklung der Gesellschaft und ihrer Mitglieder sein können, gerade 
weil Inhalt und Methode der Erziehung und Bildung eng mit Macht- und Geschlechterverhältnissen einer Gesellschaft zusammenhängen.

Vor diesem Hintergrund möchte ich eine geschlechterreflektierende Haltung im Hinblick auf ihre Relevanz für pädagogische Professionalität in einer vergeschlechtlichten und vergeschlechtlichenden Gesellschaft betonen. Die Reflexivität ist für das erziehungswissenschaftliche Denken und pädagogische Handeln besonders wichtig und sollte eine zu vermittelnde Schlüsselkompetenz in der Ausbildung darstellen (vgl. Combe/Kolbe 2004, S. 835). In Bezug auf Erfahrungen mit Geschlechterkonstruktionen hat die Reflexion eine doppelte Funktion: Es ist eine Sache, über gemachte Erfahrungen nachzudenken und eine andere, sich selbst zu diesen Erfahrungen in ein Verhältnis zu setzen. Bettina Dausien unterscheidet in diesem Sinne zwischen Selbst- und einfacher Reflexion (vgl. Dausien 2006, S. 36). Reflexion verlangt von den Individuen, sich sorgfältig und eingehend mit ihrem Handeln und dessen Effekten auseinander zu setzen. Paradox wirkt dabei, dass die tradierten Geschlechterrollen und -differenzen zunächst sichtbar und bewusst gemacht, also betont werden müssen, um überwunden werden zu können (vgl. Hanschitz/Schmidt/Schwarz 2009, S. 117). Dazu gehört auch die Überprüfung der Bedingungen des eigenen Denkens, was Sabine Hark „reflexive Produktivität" nennt (vgl. Dietze 2008, S. 39). Die Reflexion ${ }^{3}$ der Kategorie ,Geschlecht' bedeutet aber auch, die (eigene) hegemoniale Position innerhalb gesellschaftlicher Diskurse zu erkennen und die jeweilige Positionierung im Feld zu begreifen. Die Hervorhebung der Relevanz von einer kontinuierlichen Thematisierung von ,Geschlecht' und Geschlechterverhältnissen intendiert somit auch eine (hegemoniale) Selbstreflexion. ${ }^{4}$

Einen reflexiven Umgang mit, Geschlecht' verstehe ich demnach als Teil einer pädagogisch-professionellen Haltung, der sich durch ein geschlechterkritisches, hinter- und befragendes Verhältnis gegenüber ,Normalitäten' und ,Selbstverständlichkeiten' auszeichnet. Mit Hilfe einer reflexiven Haltung sollen ,Normalitäten' in kritischer Perspektive erschlos-

3 Eine selbstreflexive Haltung ermöglicht, dass ,blinde Flecken' der Wahrnehmung bewusst gemacht werden, gänzlich beseitigt werden können diese jedoch nicht (vgl. Siebert 2002, S. 25). Toleranz gegenüber Andersdenkenden und Verantwortung für die selbstständigen Entscheidungen und Denkweisen der eigenen Person werden durch die konstruktivistische Erkenntnistheorie impliziert (vgl. Siebert 2002, S. 38).

4 Der Begriff der hegemonialen (Selbst-)Kritik wurde vor allem von Gabriele Dietze im Diskurs um die Critical Whiteness Theory und den Kritischen Okzidentalismus geprägt (Dietze 2006; 2008). 
sen, thematisiert und problematisiert werden (vgl. Mecheril/Rose 2012, S. 117). Diese Kompetenz ist auch für die pädagogische Praxis relevant, da diese nicht durch ein Rezeptwissen bewältigt werden kann, das lediglich im Studium einzuverleiben wäre. Die pädagogische Wirklichkeit ist nicht etwas Stabiles und Konstantes, auf das sich Studierende sozusagen technisch vorbereiten können. Das pädagogische Feld ist etwas Fließendes, in kontinuierlicher Veränderung Begriffenes und auch die erfahrensten Pädagog_innen und Erziehungs- und BildungswissenschafterInnen tun gut daran, diese Tatsache ernst zu nehmen und zu vermitteln. Der Bewusstwerdungsprozess und die Reflexion der eigenen vergeschlechtlichten und vergesellschaftlichten Position ist eine wichtige Voraussetzung für erziehungswissenschaftliche Professionalität in der Wissenschaft wie auch der Praxis, um geschlechterreflektierende Perspektiven in das Konzept einer kritischen, differenzreflexiven Pädagogik einzufügen und für eine Praxis des demokratischen Umgangs mit Differenzen und Identitäten Sorge zu tragen. Ziel wäre es, einerseits von einem unreflektierten Umgang mit ,Geschlecht' zu einem reflektierten Umgang mit ,Geschlecht' zu gelangen und andererseits, im Anschluss daran eine Entdramatisierung von ,Geschlecht` zu ermöglichen (vgl. Faulstich-Wieland 2005, S. 2). Das heißt, ,Geschlecht' auf einer Metaebene kontinuierlich zu reflektieren, auch wenn es bewusst nicht (mehr) explizit thematisiert wird.

Wie in der vorliegenden Studie deutlich wird, müssen für einen reflektierten und reflektierenden Umgang mit ,Geschlecht' auf institutioneller Ebene aber erst Voraussetzungen, Räume und Möglichkeiten geschaffen werden. Zum Beispiel braucht es in der universitären pädagogischerziehungswissenschaftlichen Ausbildung zunehmend Räume, um einen solchen reflexiven Umgang mit ,Geschlecht', emanzipatorische Abstraktionen und gesellschaftskritische und geschlechterkritische Perspektiven und Praxen zu ermöglichen. Denn indem einzelne Konkretheiten und Erfahrungen auf das (abstrakte) Allgemeine bezogen werden können, lässt sich ein Stück Aufklärung und reflexiver Raum gewinnen. Hierfür sind vor allem Seminarkonzepte notwendig, welche in der Auseinandersetzung und Bearbeitung von ,Geschlecht' und Geschlechterfragen die Triade bestehend aus theoretischer und pädagogisch-praktischer Ebene sowie eine knritischen (biografischen Selbst-)Reflexion berücksichtigen. Somit sollte auch verstärkt das Erkenntniswissen aus der Geschlechterforschung ${ }^{5}$ (in

5 Es besteht meist Unklarheit über die Weite des geschlechtertheoretischen Feldes und der mangelnde Einblick in die unterschiedlichen, parallel geführten, akademischen Diskurse der Frauen- und Geschlechterforschung zieht oftmals Irritationen nach sich (vgl. Hanschitz/Schmidt/Schwarz 2009, S. 120). 
den Erziehungs- und Bildungswissenschaften) in die Inhalte des Studiums transferiert werden. Weiter scheint eine intensivere Rezeption (erziehungswissenschaftlicher) Geschlechterforschung im allgemeinen erziehungswissenschaftlichen Diskurs nötig. Die Vermittlung der Wirkungsmechanismen und -zusammenhänge der Kategorie ,Geschlecht' sollte in der erziehungswissenschaftlichen Ausbildung verstärkt werden.

Die Frage danach, wie sich die Erziehungs- und Bildungswissenschaften zu den hier rekonstruierten Verdeckungszusammenhängen verhalten soll, bedarf einer intensiven Diskussion, die, als eine Konsequenz dieser Studien, bei einem geschlechterreflektierten Verständnis von Lerninhalten und Orten ansetzen sollte. Vor dem Hintergrund der Ergebnisse dieser Studie stellt sich zudem die Frage nach der Relevanz eines kritischen Bildungsbegriffes hinsichtlich der Wissensvermittlung im Kontext neoliberal vereinnahmter Bildungsprozesse und -diskurse. Hier gilt es eine kritische Perspektive bzw. die Relevanz von (politisch) positionierten Erziehungs- und Bildungswissenschaften in Bezug auf Bildungspolitik und -diskurse zu unterstreichen und an ihr sozialkritisches Moment zu erinnern.

In weiterer Folge zeigen sich hier Anknüpfungsmöglichkeiten für fortführende Forschungsvorhaben. So steht weiterhin die Frage im Raum, wie sich die (De-)Thematisierung von ,Geschlecht' und Geschlechterfragen bei Pädagog_innen und Erziehungswissenschaftler_innen gestaltet, die ihr Studium bereits abgeschlossen haben. Zudem wäre ein Vergleich der Ergebnisse dieser Studie mit denen anderer Studiengänge, die z. B. statistisch nicht von Frauen dominiert sind, interessant, um den Einfluss des weiblich dominierten studiumsbezogenen konjunktiven Erfahrungsraums besser einordnen zu können. Eine neue Frage wirft das Verhältnis zwischen dem studiumsbezogenen und dem gesellschaftsbezogenen Erfahrungsraum der rhetorischen Modernisierung und des neuen Geschlechtervertrags auf, das mit dieser Studie nicht umfassend beantwortet werden kann. Zudem bleibt offen, in welcher Weise der neue Geschlechtervertrag auch junge Männer tangiert. Es wäre auch zu fragen, wie die (De-)Thematisierung von ,Geschlecht ${ }^{6}$ und Geschlechterfragen im Sinne der Intersektionalität (vgl. Winker/Degele 2009; Walgenbach 2007) von Ungleichheitskategorien (Rassismen, Klassizismen, Sexismen, usw.) und deren Wechselwirkungen bedingt werden bzw. wie sie diese Ungleichheitsverhältnissse re-produzieren. In diesem Sinne wäre eine Untersuchung interessant, an der mehrere Personen mit Migrationshintergrund oder mit queere Identitäten teilnehmen würden. So kann meine Arbeit nicht nur als Instrument der Abklärung einzelner Mechanismern 
der (De-)Thematisierung von ,Geschlecht' und Geschlechterfragen in den Erziehungs- und Bildungswissenschaften, sondern auch als Ausgangspunkt für daran ansetzdnde interessante Forschungsfragen dienen. 



\section{Literaturverzeichnis}

Acker, Joan (1990): Hierarchies, Jobs, Bodies: A Theory of Gendered Organizations. In: Sage Publications, Inc. (Hrsg.): Gender and Society. Jahrgang 4, Heft 2, S. 139-158.

Adorno, Theodor W. (1969): Minima Moralia. Frankfurt am Main: Suhrkamp Verlag.

Adorno, Theodor W./Horkheimer, Max (1971). Dialektik der Aufklärung. Frankfurt am Main: Fischer Verlag.

Althoff, Martina/Bereswill, Mechthild/Riegraf, Birgit (2001): Klasse, Milieu und Geschlecht: Das Konzept des Habitus im Kontext feministischer Fragestellungen. In: Althoff, Martina/Bereswill, Mechthild/Riegraf, Birgit (Hrsg.): Feministische Methodologien und Methoden: Tradition, Konzepte, Erörterungen. Opladen: Leske \& Budrich Verlag, S. 243-245.

Andresen, Sabine/Casale, Rita/Moser, Vera/Prengel, Annedore/Rendtorff, Barbara (2005): Editorial. Geschlechterforschung in der Kritik. In: Casale, Rita/Rendtorff, Barbara/Andresen, Sabine/Moser, Vera/Prengel, Annedore (Hrsg.): Jahrbuch Frauen- und Geschlechterforschung. Geschlechterforschung in der Kritik. Opladen und Bloomfield Hills: Verlag Barbara Budrich, S. 9-17.

Andresen, Sünne/Koreuber, Mechthild/Lüdke, Dorothea (Hrsg.) (2009): Gender und Diversity: Albtraum oder Traumpaar? Interdisziplinärer Dialog zur „Modernisierung” von Geschlechter- und Gleichstellungspolitik. Wiesbaden: VS Verlag für Sozialwissenschaften.

Beaufays, Sandra (2003): Wie werden Wissenschaftler gemacht? Der Glaube und das Geschlecht des Feldes Wissenschaft. Dissertation. Darmstadt: Technische Universität.

Becker-Schmidt, Regina (2003): Frauenforschung, Geschlechterforschung, Geschlechterverhältnisforschung. In: Becker-Schmidt, Regina/Knapp, GudrunAxeli (Hrsg.): Feministische Theorien zur Einführung. 3. Auflage, Hamburg: Junius Verlag, S. 14-62.

Becker-Schmidt, Regina/Knapp, Gudrun-Axeli (Hrsg.) (2003): Feministische Theorien zur Einführung. 3. Auflage, Hamburg: Junius Verlag.

Beauvoir, Simone de (2003): Das Andere Geschlecht. Sitte und Sexus der Frau. 3. Auflage, Reinbek bei Hamburg: Rowohlt Verlag.

Bilden, Helga (1991): Geschlechtsspezifische Sozialisation. In: Hurrelmann, Klaus/Ulich, Dieter (Hrsg.): Neues Handbuch der Sozialisationsforschung. Weinheim \& Basel: Beltz Verlag, S. 270-301. 
Bitzan, Maria (1997): Der geschlechterdifferenzierende Blick Zur Arbeit mit dem weiblichen Gemeinwesen. In: Widersprüche. Zeitschrift für sozialistische Politik in Bildungs-, Gesundheits- und Sozialbereich. Jahrgang 17, Heft 65, S. 77-91.

Bitzan, Maria (2000): Konflikt und Eigensinn. In: Neue Praxis. Jahrgang 30, Heft 4, S. 335-346.

Bitzan, Maria (2002): Sozialpolitische Ver- und Entdeckungen. Geschlechterkonflikte und Soziale Arbeit. In: Widersprüche. Zeitschrift für sozialistische Politik in Bildungs-, Gesundheits- und Sozialbereich. Jahrgang 22, Heft 84, S. 27-42.

URL: www.widersprueche-zeitschrift.de/artickel993.html

[Zugriff:04.07.2011]

Bitzan, Maria/Daigler, Claudia (2004): Eigensinn und Einmischung: Einführung in Grundlagen und Perspektiven parteilicher Mädchenarbeit. Weinheim: Juventa Verlag.

Blochmann, Elisabeth (1966): Das ,Frauenzimmer' und die ,Gelehrsamkeit'. Eine Studie über die Anfänge des Mädchenschulwesens in Deutschland. Heidelberg: Verlag Quelle \& Meyer.

Bohnsack, Ralf (1989): Generation, Milieu und Geschlecht. Ergebnisse aus Gruppendiskussionen mit Jugendlichen. Opladen: Leske \& Budrich Verlag.

Bohnsack, Ralf (1998): Milieu als konjunktiver Erfahrungsraum. Eine dynamische Konzeption von Milieu in empirischer Analyse. In: Matthiesen, Ulf (Hrsg.): Die Räume der Milieus. Neue Tendenzen in der sozial- und raumwissenschaftlichen Milieuforschung, in der Stadt- und Raumplanung. Berlin: Edition Sigma, S. 119-131.

Bohnsack, Ralf (2001): Dokumentarische Methode. Theorie und Praxis wissenssoziologischer Interpretation. In: Hug, Theo (Hrsg.): Wie kommt Wissenschaft zu Wissen? Einführung in die Methodologie der Sozial- und Kulturwissenschaften. Baltmannsweiler: Schneider Verlag Hohengehren, S. 326345 .

Bohnsack, Ralf (2003): Praxeologische Wissenssoziologie. In: Bohnsack, Ralf/Marotzki, Winfried/Meuser, Michael (Hrsg.): Hauptbegriffe Qualitativer Sozialforschung. Ein Wörterbuch. Opladen: Leske \& Budrich Verlag, S. 137-138.

Bohnsack, Ralf (2006): Mannheims Wissenssoziologie als Methode. In: Tänzler, Dirk/Knoblauch, Hubert/Soeffner, Hans-Georg (Hrsg.): Neue Perspektiven der Wissenssoziologie. Konstanz: UVK Verlag, S. 271-291.

Bohnsack, Ralf (2007): Typenbildung, Generalisierung und komparative Analyse. Grundprinzipien der dokumentarischen Methode. In: Bohnsack, Ralf/Nentwig-Gesemann, Iris/Nohl, Arnd-Michael (Hrsg.): Die dokumenta- 
rische Methode und ihre Forschungspraxis. Grundlagen qualitativer Sozialforschung. Wiesbaden: VS Verlag für Sozialwissenschaften, S. 225-254.

Bohnsack, Ralf (2008): Rekonstruktive Sozialforschung. Eine Einführung in qualitative Methoden. 7. Auflage, Opladen \& Farmington Hills: Verlag Barbara Budrich.

Bohnsack, Ralf (2010): Gruppendiskussionsverfahren und dokumentarische Methode. In: Friebertshäuser, Barbara/Langer, Antje/Prengel, Annedore (Hrsg.): Handbuch Qualitative Forschungsmethoden in der Erziehungswissenschaft. 2. Auflage, Weinheim \& München: Juventa Verlag, S. 205-218.

Bohnsack, Ralf/Nentwig-Gesemann, Iris/Nohl, Arnd-Michael (Hrsg.) (2007a): Die dokumentarische Methode und ihre Forschungspraxis. Grundlagen qualitativer Sozialforschung. Wiesbaden: VS Verlag für Sozialwissenschaften.

Bohnsack, Ralf/Nentwig-Gesemann, Iris/Nohl, Arnd-Michael (2007b): Einleitung: Die dokumentarische Methode und ihre Forschungspraxis. In: Bohnsack, Ralf/Nentwig-Gesemann, Iris/Nohl, Arnd-Michael (Hrsg.): Die dokumentarische Methode und ihre Forschungspraxis. Grundlagen qualitativer Sozialforschung. Wiesbaden: VS Verlag für Sozialwissenschaften, S. 9-28.

Bohnsack, Ralf/Schäffer, Burkhard (2007): Exemplarische Textinterpretation: Diskursorganisation und dokumentarische Methode. In: Bohnsack, Ralf/Nentwig-Gesemann, Iris/Nohl, Arnd-Michael (Hrsg.): Die dokumentarische Methode und ihre Forschungspraxis. Grundlagen qualitativer Sozialforschung. Wiesbaden: VS Verlag für Sozialwissenschaften, S. 309-323.

Bohnsack, Ralf/Nohl, Arnd-Michael (2007): Exemplarische Textinterpretation: Die Sequenzanalyse der dokumentarischen Methode. In: Bohnsack, Ralf/Nentwig-Gesemann, Iris/Nohl, Arnd-Michael (Hrsg.): Die dokumentarische Methode und ihre Forschungspraxis. Grundlagen qualitativer Sozialforschung. Wiesbaden: VS Verlag für Sozialwissenschaften, S. 303-307.

Bohnsack, Ralf/Przyborski, Aglaja/Schäffer, Burckhard (2010): Einleitung. Gruppendiskussion als Methode rekonstruktiver Sozialforschung. In: Bohnsack, Ralf/Przyborski, Aglaja/Schäffer, Burckhard (Hrsg.): Das Gruppendiskussionsverfahren in der Forschungspraxis. Opladen \& Farmington Hills: Verlag Barbara Budrich, S. 7-22.

Borst, Eva (2003): Anerkennung der Anderen und das Problem des Unterschieds. Perspektiven einer kritischen Theorie der Bildung. Baltmannsweiler: Schneider Verlag Hohengehren.

Bourdieu, Pierre (1976): Entwurf einer Theorie der Praxis. Frankfurt am Main: Suhrkamp Verlag.

Bourdieu, Pierre (1982): Die feinen Unterschiede. Kritik der gesellschaftlichen Urteilskraft. Frankfurt am Main: Suhrkamp Verlag.

Bourdieu, Pierre (1983): Ökonomisches Kapital, kulturelles Kapital, soziales 
Kapital. In: Kreckel, Reinhard (Hrsg.): Soziale Ungleichheiten. Soziale Welt. Sonderband 2, Göttingen: Schwartz Verlag, S. 183-198.

Bourdieu, Pierre (1987): Der soziale Sinn. Kritik der theoretischen Vernunft. Frankfurt am Main: Suhrkamp Verlag.

Bourdieu, Pierre (1992a): Rede und Antwort. Frankfurt am Main: Suhrkamp Verlag.

Bourdieu, Pierre (1993a): Sozialer Sinn. Kritik der theoretischen Vernunft. Frankfurt am Main: Suhrkamp Verlag.

Bourdieu, Pierre (1993b). Narzißtische Reflexivität und wissenschaftliche Reflexivität. In: Berg, Eberhard/ Fuchs, Martin (Hrsg.): Kultur, soziale Praxis, Text. Die Krise der ethnographischen Repräsentation. Frankfurt am Main: Suhrkamp Verlag, S. 365-374.

Bourdieu, Pierre (1997a): Die männliche Herrschaft. In: Dölling, Irene/Beate, Kreis (Hrsg.): Ein alltägliches Spiel. Geschlechterkonstruktion in der sozialen Praxis. Frankfurt am Main: Suhrkamp Verlag, S. 153-217.

Bourdieu, Pierre (1997b): Eine sanfte Gewalt. Pierre Bourdieu im Gespräch mit Irene Dölling und Margareta Steinrücke. In: Dölling, Irene/Krais, Beate (Hrsg.): Ein alltägliches Spiel. Geschlechterkonstruktion in der sozialen Praxis. Frankfurt am Main: Suhrkamp Verlag, S. 218-230.

Bourdieu, Pierre (2005): Die männliche Herrschaft. Frankfurt am Main: Suhrkamp Verlag.

Bowen, A. Glenn (2006): Grounded Theory and Sensitizing Concepts. In: International Journal of Qualitative Methods. Jahrgang 5, Heft 4.

URL: ejournals.library.ualberta.ca/index.php/IJQM/article/view/4367 [Zugriff: 11.06.2012]

Brand, Ortrun (2013): Ich sehe was, was Du nicht siehst! Anmerkungen zu den Praktiken der Neutralisierung. In: Graf, Julia/Ideler, Kristin/Klinger, Sabine (Hrsg.): Geschlecht zwischen Struktur und Subjekt. Theorie, Praxis, Perspektiven. Opladen, Berlin \& Toronto: Verlag Barbara Budrich, S. 173186.

Breitenbach, Eva/Hagemann-White, Carol (1994): Von der Sozialisation zur Erziehung. Der Umgang mit geschlechterdifferenter Subjektivität in der feministischen Forschung. In: Beutler, Kurt (Hrsg.): Jahrbuch für Pädagogik 1994. Geschlechterverhältnisse und die Pädagogik. Frankfurt am Main: Peter Lang Verlag, S. 249-264.

Brehmer, Ilse (Hrsg.)(1990): Mütterlichkeit als Profession? Lebensläufe deutscher Pädagoginnen in der ersten Hälfte dieses Jahrhunderts. Band 1, Pfaffenweiler: Centaurus Verlagsgesellschaft.

Brück, Brigitte/Kahlert, Heike/Krüll, Marianne/Milz, Helga/Osterland, Ast- 
rid/ Wegehaupt Schneider, Ingeborg (1992): Feministische Soziologie. Eine Einführung. Frankfurt am Main: Campus Verlag.

Bundesministerium für Familie, Senioren und Jugend 2011 (Hrsg.): Erster Gleichstellungsbericht - Neue Wege-Gleiche Chancen - Gleichstellung von Frauen und Männern im Lebensverlauf.

URL: www.bmfsfj.de/RedaktionBMFSFJ/Broschuerenstelle/Pdf-Anlagen/ Erster-Gleichstellungsbericht-Neue-Wege-Gleiche-Chancen,property=pdf, bereich $=$ bmfsfj,sprache $=$ de,rwb=true.pdf [Zugriff: 11.12.2011].

Butler, Judith (1991): Das Unbehagen der Geschlechter. Frankfurt am Main: Suhrkamp Verlag.

Casale, Rita/Rendtorff, Barbara (2008): Vorwort. In: Casale, Rita/Rendtorff, Barbara (Hrsg.): Was kommt nach der Genderforschung? Zur Zukunft der feministischen Theoriebildung. Reihe Gender Studies. Bielefeld: TranscriptVerlag, S. 9-11.

Chebout, Lucy (2011): Wo ist Intersectionality in bundesdeutschen Intersektionalitätsdiskursen? - Exzerpte aus dem Reisetagebuch einer Traveling Theory. In: Smykalla, Sandra/Vinz, Dagmar (Hrsg): Intersektionalität zwischen Gender und Diversity. Theorien, Methoden und Politiken der Chancengleichheit. Münster: Westphälisches Dampfboot, S. 43-57.

Combe, Arno/Kolbe, Fritz-Ulrich (2004): Lehrerprofessionalität: Wissen, Können, Handeln. In: Helsper, Werner/Böhme, Jeanette (Hrsg.): Handbuch der Schulforschung. Wiesbaden: VS Verlag für Sozialwissenschaften, S. 833-851.

Connell, Raewyn (vormals Robert W.) (1999): Der gemachte Mann: Konstruktion und Krise von Männlichkeiten. Opladen: Leske \& Budrich Verlag.

Crenshaw, Kimberlè (1989): Demarginalizing the Intersection of Race and Sex: A Black Feminist Critique of Antidiscrimination Doctrine. In: The University of Chicago Legal Forum 139, S. 139-167.

Dausien, Bettina (1998): Die biographische Konstruktion von Geschlecht. In: Schneider, Noktar/Mall, R. A./Lohmar, Dieter (Hrsg.): Einheit und Vielfalt. Das Verstehen der Kulturen. Amsterdam/Atlanta: Rodopi B.V., S. 256-275.

Dausien, Bettina (2006): Geschlechterverhältnisse und ihre Subjekte. Zum Diskurs um Sozialisation und Geschlecht. In: Bilden, Helga/ Dausien, Barbara (Hrsg.): Sozialisation und Geschlecht. Theoretische und methodologische Perspektiven. Opladen \& Farmington Hills: Verlag Barbara Budrich, S. 1744.

Dausien, Bettina (2007): Reflexivität, Vertrauen, Professionalität. Was Studierende in einer gemeinsamen Praxis qualitativer Forschung lernen können. In: Diskussionsbeitrag zur FQS-Debatte „Lehren und Lernen der Methoden qualitativer Sozialforschung". Forum Qualitative Sozialforschung/Forum: Qualitative Social Research, 8 (1). 
URL: nbn-resolving.de/urn:nbn:de:0114-fqs0701D4Da3.

[Zugriff: 09.08.2011]

Dausien, Bettina (2009): Differenz und Selbst-Verortung. Die soziale Konstruktion von Geschlecht in Biographien als Forschungskonzept. In: Aulenbacher, Brigitte/Riegraf, Birgit (Hrsg.): Erkenntnis und Methode. Geschlechterforschung in Zeiten des Umbruchs. Wiesbaden: VS Verlag, S. 157-178.

Dausien, Bettina/Thon, Christine (2009): Gender. In: Andresen, Sabine/Casale, Rita/Gabriel, Thomas/Horlacher, Rebekka/Larcher-Klee, Sabina/Oelkers, Jürgen (Hrsg.): Handwörterbuch. Erziehungswissenschaft. Weinheim \& Basel: Beltz Verlag, S. 336-349.

Dausien, Bettina/Mecheril, Paul (2009): Forschungswerkstatt: Interpretation als Ko-Konstruktion.

URL: www.qualitative-forschung.de/methodentreffen/angebot/fw/2009.html [Zugriff: 16.04.2009].

Degele, Nina (2004): Sich schön machen. Zur Soziologie von Geschlecht und Schönheitshandeln. Wiesbaden: Verlag für Sozialwissenschaften.

Degele, Nina (2008): Einführung Gender/Queer Studies. München: Wilhelm Fink Verlag.

Degele, Nina/Winker, Gabriele (2009): Intersektionalität. Zur Analyse sozialer Ungleichheiten, Bielefeld: Transcript Verlag.

Dietze, Gabriele (2006): Critical Whiteness Theory und Kritischer Okzidentalismus. Zwei Figuren hegemonialer Selbstreflexion. In: Tißberger, Martina/Dietze, Gabriele/Hrzan, Daniela/Hausmann-Kastein, Jana (Hrsg.): Weiß - Weißsein - Whitness: Kritische Studien zu Gender und Rassismus. Frankfurt am Main: Peter Lang Verlag, S. 219-248.

Dietze, Gabriele (2008): Intersektionalität und Hegemoniale(selbst)kritik. In: Dietze, Gabriele/Brunner, Claudia/Wenzel, Edith (Hrsg): Kritik des Okzidentalismus. Transdizsplinäre Beiträge zu (Neo-)Orientalismus und Geschlecht. Bielefeld: Transkript Verlag, S. 27-44.

Dietze, Gabriele/Hornscheidt, Antje/Palm, Kerstin/Walgenbach, Katharina (2007): Einleitung. In: Walgenbach, Katharina/Dietze, Gabriele/Hornscheidt, Antje/Palm, Kerstin (Hrsg): Gender als interdependente Kategorie. Neue Perspektiven auf Intersektionalität, Diversität und Heterogenität. Opladen \& Farmington Hills: Verlag Barbara Budrich, S. 7-22.

Dölling, Irene (2003): Das Geschlechterwissen der Akteur/e/innen. In: Andresen, Sünne/Dölling, Irene/Kimmerle, Christoph (Hrsg.): Verwaltungsmodernisierung als soziale Praxis. Geschlechter-Wissen und Organisationsverständnis von Reformakteuren. Opladen: Leske \& Budrich Verlag, S. 113-165

Dölling, Irene (2005): ,Geschlechter-Wissen' - ein nützlicher Begriff für die ,verstehende' Analyse von Vergeschlechtlichungsprozessen? In: Zeitschrift 
für Frauenforschung und Geschlechterstudien. Jahrgang 23, Heft 1 und 2, S. 44-63.

Egloff, Birte (2002): Praktikum und Studium. Diplom-Pädagogik und Humanmedizin zwischen Studium, Beruf, Biographie und Lebenswelt. Opladen: Leske \& Budrich Verlag.

Engler, Steffanie (1993): Fachkultur, Geschlecht und soziale Reproduktion. Eine Untersuchung über Studentinnen und Studenten der Erziehungswissenschaft, Rechtswissenschaft, Elektrotechnik und des Maschinenbaus. Weinheim: Deutscher Studien Verlag.

Engler, Steffanie (2001): „In Einsamkeit und Freiheit?” Zur Konstruktion der wissenschaftlichen Persönlichkeit auf dem Weg zur Professur. Konstanz: UKV Verlag.

Engler, Steffanie (2010): Habitus und sozialer Raum: Zur Nutzung der Konzepte Pierre Bourdieus in der Frauen- und Geschlechterforschung. In: Becker, Ruth/Kortendiek, Beate (Hrsg.): Handbuch Frauen- und Geschlechterforschung. Theorie, Methoden, Empirie. 3. Auflage, Geschlecht und Gesellschaft Band 35. Wiesbaden: VS Verlag für Sozialwissenschaften, S. 257-268.

Faulstich-Wieland, Hannelore (2002): Frauen und Studium. Frauenstudium in der Erziehungswissenschaft. In: Otto, Hans-Uwe/Rauschenbach, Thomas/Vogel, Peter (Hrsg.): Erziehungswissenschaft: Lehre und Studium. Opladen: Lesk \& Budrich Verlag, S. 173-183.

Faulstich-Wieland, Hannelore (2005): Spielt das Geschlecht (k)eine Rolle im Schulalltag? Plädoyer für eine Entdramatisierung von Geschlecht. Vortrag in der Reihe Gender Lectures an der Humboldt-Universität Berlin am 11.07.2005. Berlin.

URL: www.genderkompetenz.info/veranstaltungen/genderlectures/faulstichwieland_manuskript_genderlecture.pdf [Zugriff: 20.09.2011].

Faulstich-Wieland, Hannelore/Horstkemper Marianne (2012): Geschlechterverhältnisse. In: Thole, Werner/Faulstich-Wieland, Hannelore/Horn, KlausPeter/Weishaupt, Horst/Züchner, Ivo (Hrsg.): Datenreport Erziehungswissenschaft 2012. Opladen \& Farmington Hills: Verlag Barbara Budrich, S. 194-211.

Fischer-Rosenthal, Wolfram (1995): Schweigen - Rechtfertigen - Umschreiben. In: Fischer-Rosenthal, Wolfram/Alheit, Peter (Hrsg.): Biographien in Deutschland. Soziologische Rekonstruktionen gelebter Gesellschaftsgeschichte. Opladen: Westdeutscher Verlag, S. 43-86.

Flick, Uwe (2004): Triangulation. Eine Einführung. Wiesbaden: VS Verlag für Sozialwissenschaften.

Foucault, Michel (1983): Der Wille zum Wissen. Sexualität und Wahrheit. Band 1, Frankfurt am Main: Suhrkamp Verlag.

Forster, Edgar (2005): Jungen- und Männerarbeit - ein Überlick über den 
Stand der Debatte. In: Krall, Hannes (Hrsg.): Jungen- und Männerarbeit. Bildung, Beratung und Begegung auf der "Baustelle Mann". Wiesbaden: VS Verlag für Sozialwissenschaft, S. 205-220.

Friebertshäuser, Barbara (1992): Übergangsphase Studienbeginn. Eine Feldstudie über Riten der Initiation in eine studentische Fachkultur. Weinheim und München: Juventa Verlag.

Friebertshäuser, Barbara (1997): Interviewtechniken - Ein Überblick. In: Friebertshäuser, Barbara/Prengel, Annedore (Hrsg.): Handbuch Qualitative Forschungsmethoden in der Erziehungswissenschaft. Weinheim, München: Juventa Verlag, S. 378-395.

Friebertshäuser, Barbara (2004): Ritualforschung in der Erziehungswissenschaft. Konzeptionelle und forschungsstrategische Überlegungen. In: Wulf, Christoph/Zirfas, Jörg (Hrsg.): Zeitschrift für Erziehungswissenschaft. Innovation und Ritual. Jugend, Geschlecht und Schule. Jahrgang 7, Beiheft 2, Wiesbaden: VS Verlag für Sozialwissenschaften, S. 29-45.

Friebertshäuser, Barbara (2006): StudentInnenforschung - Überblick. Bilanz und Perspektiven biographieanalytischer Zugänge. In: Krüger, HeinzHermann/Marotzki, Winfried (Hrsg.): Handbuch für erziehungswissenschaftliche Biographieforschung. Wiesbaden: VS Verlag für Sozialwissenschaften, S. 295-316.

Friebertshäuser, Barbara (2009): Verstehen als methodische Herausforderung für eine reflexive empirische Forschung. In: Friebertshäuser, Barbara/Rieger-Ladich, Markus/Wigger, Lothar (Hrsg.): Reflexive Erziehungswissenschaft. Forschungsperspektiven im Anschluss an Pierre Bourdieu. 2. Auflage, Wiesbaden: VS Verlag für Sozialwissenschaften, S. 229-249.

Friebertshäuser, Barbara (2012): Perspektiven erziehungswissenschaftlicher und ethnographischer Geschlechterforschung auf Körperinszenierungen im Jugendalter. In: Ackermann, Friedhelm/Ley, Thomas/Machold, Claudia/Schrödter, Mark (Hrsg.): Qualitatives Forschen in der Erziehungswissenschaft. Wiesbaden: Springer VS, S. 97-114.

Friebertshäuser, Barbara/Rieger-Ladich, Markus/Wigger, Lothar (2009): Reflexive Erziehungswissenschaft: Stichworte zu einem Programm. In: Friebertshäuser, Barbara/Rieger-Ladich, Markus/Wigger, Lothar (Hrsg.): Reflexive Erziehungswissenschaft. Forschungsperspektiven im Anschluss an Pierre Bourdieu. 2. Auflage, Wiesbaden: VS Verlag für Sozialwissenschaften, S. 9-19.

Fritzsche, Bettina/Hartmann, Jutta/Schmidt, Andrea/Tervooren, Anja (Hrsg.)(2001): Dekonstruktive Pädagogik. Erziehungswissenschaftliche Debatten unter poststrukturalistischen Perspektiven. Opladen: Leske \& Budrich Verlag.

Funk, Julika (2002): Transgender people. In: Kroll, Renate (Hrsg.): Metzler Le- 
xikon Gender Studies Geschlechterforschung. Ansätze - Personen - Grundbegriffe. Stuttgart/Weimar: J.B. Metzler Verlag, S. 391.

Garfinkel, Harold (1967): Studies in Ethnomethodology. Englewood Cliffs und New York: Prentice Hall.

Garz, Detlef (2012): Zum Stand interpretativer Forschung in den Erziehungswissenschaften - Standorte und Perspektiven. In: Ackermann, Friedhelm/Ley, Thomas/Machold, Claudia/Schrödter, Mark (Hrsg.): Qualitatives Forschen in der Erziehungswissenschaft. Wiesbaden: Springer VS, S. 2745.

Gerhard, Ute (2007): Feminismus heute? In: Feministische Studien. Zeitschrift für interdisziplinäre Frauen und Geschlechterforschung. Heft 1, Stuttgart: Lucius \& Lucius Verlag, S. 97-104.

Gildemeister, Regina/Wetterer, Angelika (1992): Wie Geschlechter gemacht werden. Die soziale Konstruktion der Zweigeschlechtlichkeit und ihre Reifizierung in der Frauenforschung. In: Knapp, Gudrun-Axeli/Wetterer, Angelika (Hrsg.): Traditionen Brüche. Entwicklungen feministischer Theorie. Freiburg im Breisgau: Kore Verlag, S. 201-254.

Gildemeister, Regine (2004): Geschlechterdifferenz - Geschlechterdifferenzierung: Beispiele und Folgen eines Blickwinkels in der empirischen Geschlechterforschung. In: Buchen, Sylvia/Helfferich, Cornelia/Maier, Maja S. (Hrsg.): Gender methodologisch. Empirische Forschung in der Informationsgesellschaft vor neuen Herausforderungen. Wiesbaden: Verlag für Sozialwissenschaften, S. 27-45.

Gildemeister, Regine (2007): Geschlechterforschung (gender studies). In: Flick, Uwe/Kardoff von, Ernst/Ines Steinke, (Hrsg.): Qualitative Forschung. Ein Handbuch. Hamburg: Rowohlt Verlag, S. 213-224.

Gilmore, David D. (1991): Mythos Mann. Rollen, Rituale, Leitbilder. München und Zürich: Artemis und Winkler Verlag.

Glaser, Edith/Andresen, Sabine (2009): Zur Einführung. Disziplingeschichte der Erziehungswissenschaft als Geschlechtergeschichte. In: Glaser, Edith/Andresen, Sabine (Hrsg.): Jahrbuch Frauen- und Geschlechterforschung in der Erziehungswissenschaft. Disziplingeschichte der Erziehungswissenschaft als Geschlechtergeschichte. Opladen \& Farmington Hills: Verlag Barbara Budrich, S. 7-11.

Glaser, Barney G./Strauss, Anselm L. (1967): The Discovery of Grounded Theory. Strategies for Qualitative Research. Chicago: Aldine Publishing Company.

Glaser, Barney G./Strauss, Anselm L. (1998): Grounded Theory. Strategien qualitativer Forschung. Bern, Göttingen, Toronto \& Seattle: Huber Verlag.

Gläser, Jochen/Laudel, Grit (2004): Experteninterviews und qualitative Inhaltsanalyse. Wiesbaden: VS Verlag für Sozialwissenschaft. 
Goffmann, Erving (1994): Das Arrangement der Geschlechter. In: Goffmann, Erving (Hrsg.): Interaktion und Geschlecht. Frankfurt am Main und New York: Campus Verlag, S. 105-158.

Götsch, Monika (2012): „n' Kerl mit sechzehn Jahren, der noch nie 'nem Weib an die Wäsche gegangen ist, gibt's ja eigentlich kaum noch". Sozialisation heteronormativen Wissens. Unveröffentlichte Dissertation an der AlbertLudwigs-Universität Freiburg im Breisgau.

Götsch, Monika/Klinger, Sabine/Thiesen, Andreas (2012): "Stars in der Manege?" Demokratietheoretische Überlegungen zur Dynamik partizipativer Forschung. In: Forum Qualitative Sozialforschung/Forum: Qualitative Social Research, 13 (1).

URL: www.qualitative-research.net/index.php/fqs/article/view/1780 [Zugriff: 24.10.2012].

Hagemann-White, Carol (1993): Die Konstrukteure des Geschlechts auf frischer Tat ertappen? Methodische Konsequenzen einer theoretischen Einsicht. In: Feministische Studien. Jahrgang 11, Heft 2, Weinheim: Deutscher Studien Verlag, S. 68-80.

Hall, Stuart (2003): New Labour's Double-shuffle. In: Soundings. Jahrgang 24, Heft 7, S. 10-24.

Hanschitz, Rudolf-Christian/Schmidt, Esther/Schwarz, Guido (2009): Transdisziplinarität in Forschung und Praxis: Chancen und Risiken partizipativer Prozesse. Wiesbaden: VS Verlag für Sozialwissenschaften.

Hark, Sabine (2008): Zwischen Aktivismus und Akademie. Die Zeiten feministischen Wissens. In: Casale, Rita/Rendtorff, Barbara (Hrsg.): Was kommt nach der Genderforschung? Zur Zukunft der feministischen Theoriebildung. Reihe Gender Studies. Bielefeld: Transcript-Verlag, S. 215-231.

Hausbichler, Beate (2008): Raum für_! In: dieStandard.

URL: http://diestandard.at/1224776349439/GenderSprache-Raum-fuer-_. [Zugriff: 24.04.2012].

Hasenjürgen, Brigitte (1996): Soziale Macht im Wissenschaftsspiel. SozialwissenschaftlerInnen und Frauenforscherinnen an der Hochschule. Münster: Verlag Westfälisches Dampfboot.

Heine, Christoph/Engel, Jürgen/Kerst, Christian/Müller, Elisabeth/Park, Sang-Min (2006): Bestimmungsgründe für die Wahl von ingenieur- und naturwissenschaftlichen Studiengängen. Ausgewählte Ergebnisse einer Schwerpunktstudie im Rahmen der Berichterstattung zur technologischen Leistungsfähigkeit Deutschlands. In: HIS-Kurzinformationen A. HochschulInformations-System, Heft 2, Hannover.

Heinrichs, Gesa (2001): Bildung, Identität, Geschlecht: Eine (postfeministische) Einführung. Königstein: Helmer Verlag.

Heintz, Bettina (1993): Die Auflösung der Geschlechterdifferenz. Entwicklungs- 
tendenzen in der Theorie der Geschlechter. In: Bühler, Elisabeth (Hrsg.): Ortssuche: Zur Geographie der Geschlechterdifferenz. Zürich \& Dortmund: eFeF Verlag, S. 17-48.

Heintz, Bettina/Nadai, Eva (1998): Geschlecht und Kontext. De-Institutionalisierungsprozesse und Geschlechtliche Differenzierung. In: Zeitschrift für Soziologie. Jahrgang 19, Heft 3, S. 573-588.

Helfferich, Cornelia (2011): Die Qualität qualitativer Daten. Manual für die Durchführung qualitativer Interviews. 4. Auflage, Wiesbaden: VS Verlag.

Hirschauer, Stefan (1994): Die soziale Fortpflanzung der Zweigeschlechtlichkeit. In: Kölner Zeitschrift für Soziologie und Sozialpsychologie. Jahrgang 46, Heft 4, S. 668-692.

Hirschauer, Stefan (1996): Wie sind Frauen, wie sind Männer? Zweigeschlechtlichkeit als Wissenssystem. In: Eifert, Christiane (Hrsg.): Was sind Frauen? Was sind Männer? Geschlechterkonstruktionen im historischen Wandel. Frankfurt am Main: Suhrkamp Verlag, S. 240-256.

Hirschauer, Stefan (2001): Das Vergessen der Geschlechter. Zur Praxeologie einer Kategorie sozialer Ordnung. In: Heintz, Bettina (Hrsg.): Geschlechtersoziologie. Kölner Zeitschrift für Soziologie und Sozialpsychologie. Sonderheft 41, S. 208-235.

Hirschauer, Stefan (2012): Die Praxis der Geschlechterdifferenz und ihre Infrastruktur. In: Graf, Julia/Ideler, Kristin/Klinger, Sabine (Hrsg.): Geschlecht zwischen Struktur und Subjekt. Theorie, Praxis, Perspektiven. Opladen, Berlin, Toronto: Verlag Barbara Budrich, S. 153-172.

Hirschauer, Stefan/Knapp, Gudrun-Axeli (2006): Wozu Geschlechterforschung? Ein Dialog über Politik und den Willen zum Wissen. In: Aulenbacher, Brigitte/Bereswill, Mechthild/Löw, Martina/Meuser, Michael/Mordt, Gabriele/Schäfer, Reinhild/Scholz, Sylka (Hrsg.): FrauenMännerGeschlechterforschung. State of the Art. Münster: Verlag Westfälisches Dampfboot, S. 22-63.

Hof, Renate (2005): Geschlechterverhältnis und Geschlechterforschung. Kontroversen und Perspektiven. In: Bußmann, Hadumond/Hof, Renate (Hrsg.): Genus. Geschlechterforschung/Gender Studies in der Kultur- und Sozialwissenschaft. Ein Handbuch. Stuttgart: Kröner Verlag, S. 2-41.

Hoffmann-Riem, Christa (1980): Die Sozialforschung einer interpretativen Soziologie. Der Datengewinn. Kölner Zeitschrift für Soziologie und Sozialpsychologie. Jahrgang 32, S. 339-372.

Honer, Anne (2007): Lebensweltanalyse in der Ethnographie. In: Flick, Uwe/ Kardoff, Ernst von/ Steinke, Ines (Hrsg.): Qualitative Forschung. Ein Handbuch. Hamburg: Rowohlt, S. 194-204.

Hornberg, Claudia/Schröttle, Monika/Khelaifat, Nadia/Pauli, Andrea (2008): Gesundheitliche Folgen von Gewalt unter Berücksichtigung von häuslicher 
Gewalt gegen Frauen. In: Gesundheitsberichterstattung des Bundes, Themenheft - Gesundheitliche Folgen, Heft 42, Robert Koch Institut Statistisches Bundesamt:

URL: www.gbe-bund.de/ [Zugriff: 07.12.2011]

Huck, Inga/Brosius, Hans-Bernd (2007): Der Third-Person-Effekt. Über den vermuteten Einfluss der Massenmedien. In: Publizistik. Jahrgang 52, Heft 3, S. 335-374.

Jacobi, Juliane (1990): Elisabeth Blochmann: First-Lady der akademischen Pädagogik. In: Brehmer, Ilse (Hrsg.): Mütterlichkeit als Profession? Lebensläufe deutscher Pädagoginnen in der ersten Hälfte dieses Jahrhunderts. Pfaffenweiler: Centaurus Verlagsgesellschaft, S. 256-264.

Jagose, Annamarie (2001): Queer Theory. Eine Einführung. Berlin: Querverlag.

Kaiser, Astrid/Oubaid, Monika (Hrsg.) (1986): Deutsche Pädagoginnen der Gegenwart. Köln und Wien: Böhlau Verlag.

Kelan, Elisabeth (2009): Performing Gender at Work. Palgrave: Macmillan Verlag.

Kelle, Udo (1996): Die Bedeutung theoretischen Vorwissens in der Methodologie der Grounded Theory. In: Strobl, Rainer/Böttger, Andreas (Hrsg.): Wahre Geschichten? Zur Theorie und Praxis qualitativer Interviews. Baden Baden: Nomos Verlag, S. 23-48.

Kelle, Helga (1999): Geschlechterunterschiede oder Geschlechterunterscheidung? Methodologische Reflexion eines ethnographischen Forschungsprozesses. In: Dausien, Bettina/Herrmann, Martina/Oechsle, Mechtild/Schmerl, Christiane/ Stein-Hilbers, Marlene (Hrsg.): Erkenntnisprojekt Geschlecht. Feministische Perspektiven verwandeln Wissenschaft. Opladen: Leske \& Budrich, S. 304-324.

Kelle, Udo/Kluge, Susann (2010): Vom Einzelfall zum Typus. Fallvergleich und Fallkonstrastierung in der qualitativen Sozialforschung. 2. Auflage. Wiesbaden: VS Verlag für Sozialwissenschaften.

King, Vera (2004): Die Entstehung des Neuen in der Adoleszenz. Individuation, Generativität und Geschlecht in modernisierten Gesellschaften. Wiesbaden: VS Verlag.

Klaus, Elisabeth (2008): Antifeminismus und Elitefeminismus. Eine Intervention. In: Feministische Studien. Zeitschrift für interdisziplinäre Frauen- und Geschlechterforschung. Jahrgang 26, Heft 2, Stuttgart: Lucius \& Lucius Verlag, S. 176-186.

Klees, Renate/Marburger, Helga/Schumacher, Michaela: Mädchenarbeit. Praxishandbuch für die Jugendarbeit. Teil 1, 5. Auflage, Weinheim und München: Juventa Verlag. 
Klinger, Sabine (2008): Selbstbewusste Mädchen. Das geschlechtssensible Selbstbehauptungs- und Verteidigungskonzept drehungen als zentraler Beitrag für die feministische Mädchenarbeit. München und Wien: Profil Verlag.

Klinger, Sabine (2011): Studierende Reden Geschlecht. Thematisierung von Geschlecht und Geschlechterverhältnissen an der Universität am Beispiel der Erziehungswissenschaft. Unveröffentlichte Masterarbeit an der KarlFranzens-Universität Graz.

Knapp, Gudrun-Axeli (1987): Arbeitsteilung und Sozialisation: Konstellationen von Arbeitsvermögen und Arbeitskraft im Lebenszusammenhang von Frauen. In: Beer, Ursula (Hrsg.): Klasse Geschlecht. Feministische Gesellschaftsanalyse und Wissenschaftskritik. Bielefeld: AJZ-Verlag/FF1.

Knapp, Gudrun-Axeli (2003): Konstruktion und Dekonstruktion von Geschlecht. In: Becker-Schmidt, Regina/Knapp, Gudrun-Axeli (Hrsg.): Feministische Theorie. Zur Einführung. 3. Auflage, Hamburg: Junius Verlag, S. 63-102.

Knapp, Gudrun-Axeli (2008): Achsen der Differenz. Aspekte und Perspektiven feministischer Grundlagenkritik. In: Wilz, Sylvia Marlene (Hrsg.): Geschlechterdifferenzen - Geschlechterdifferenzierungen. Ein Überblick über gesellschaftliche Entwicklungen und theoretische Positionen. Wiesbaden: VS Verlag, S. 291-322.

Knorr-Cetina, Karin (1989): Spielarten des Konstruktivismus. Einige Notizen und Anmerkungen. In: Soziale Welt. Jahrgang 40, Heft 1/2, S. 86-96.

Kolbe, Fritz-Ulrich/Combe, Arno (2008): Lehrerbildung. In: Helsper, Werner/Böme, Jeanette (Hrsg): Handbuch der Schulforschung. Wiesbaden: VS Verlag für Sozialwissenschaften, S. 877-904.

Koppetsch, Cornelia/Burkart, Günter (1999): Die Illusion der Emanzipation. Zur Wirksamkeit latenter Geschlechtsnormen im Milieuvergleich. Konstanz: UVK Verlag.

Krais, Beate (2001): Geschlechterverhältnis und symbolische Gewalt. In: Althoff, Martina/Bereswill, Mechthild/Riegraf, Birgit (Hrsg.): Feministische Methodologien und Methoden. Tradition, Konzepte, Erörterungen. Opladen: Leske \& Budrich, S. 245-252.

Krais, Beate/Gebauer, Gunter (2010): Habitus. 3. Auflage., Bielefeld: Transkript Verlag.

Krüger, Heinz-Hermann (2006): Einführung in die Theorien und Methoden der Erziehungswissenschaft. 4 Auflage. Opladen: Leske \& Budrich.

Kubisch, Sonja (2008): Habituelle Konstruktion sozialer Differenz. Eine rekonstruktive Studie am Beispiel von Organisationen der freien Wohlfahrtspflege. Wiesbaden: VS Verlag für Sozialwissenschaften.

Kutscher, Nadia (2010): Die Rekonstruktion moralischer Orientierungen 
von Professionellen auf der Basis von Gruppendiskussion. In: Bohnsack, Ralf/Przyborski, Aglaja/Schäffer, Burckhard (Hrsg.): Das Gruppendiskussionsverfahren in der Forschungspraxis. Opladen \& Farmington Hills: Verlag Barbara Budrich, S. 189-202.

Laqueur, Thomas (1992): Auf den Leib geschrieben. Die Inszenierung der Geschlechter von der Antike bis Freud. Frankfurt am Main \& New York: Campus Verlag.

Lemmermöhle, Doris/Klika, Dorle/Fischer, Dietlind/Schlüter, Anne (Hrsg.) (2000): Lesarten des Geschlechts: Zur De-Konstruktionsdebatte in der erziehungswissenschaftlichen Geschlechterforschung. Opladen: Leske \& Budrich.

Lenzen, Dieter/Krüger, Heinz-Hermann/Wulf,Christoph (2008): Editoral. In: Lenzen, Dieter/Krüger, Heinz-Hermann/Wulf,Christoph (Hrsg.): Zeitschrift für Erziehungswissenschaft. Schwerpunkt: Hochschulbildung. Jahrgang 11, Heft 4, Wiesbaden: VS Verlag für Sozialwissenschaften, S. 513-515.

Lenz, Ilse (2010): Intersektionalität: Zum Wechselverhältnis von Geschlecht und sozialer Ungleichheit. In: Becker, Ruth/Kortendieck, Beate (Hrsg.): Handbuch Frauen- und Geschlechterforschung. Theorie, Methoden, Empirie. 3. Auflage, Wiesbaden: VS Verlag, S. 158-165.

Liebig, Brigitte (2009): Gender Studies als Ausbildungsgang: Berufsqualifikation und Orientierungswissen. In: Liebig, Brigitte/Dupuis, Monique/Ballermer-Cao, Thanh-Huyen/Maihofer, Andrea (Hrsg.): Gender Studies in Ausbildung und Arbeitswelt. Das Beispiel Schweiz. Zürich: Seimso Verlag, S. 72-92.

Liebau, Eckert (1987): Gesellschaftliches Subjekt und Erziehung. Zur pädagogischen Bedeutung der Sozialisationstheorien von Pierre Bourdieu und Ulrich Oevermann. Weinheim und München: Juventa Verlag.

Lorber, Judith (1999): Gender-Paradoxien. Opladen: Leske \& Budrich Verlag.

Macha, Hildegard (2005): Grußwort. In: Casale, Rita/Rendtorff, Barbara/Andresen, Sabine/Moser, Vera/Prengel, Annedore (Hrsg.): Jahrbuch Frauen- und Geschlechterforschung. Geschlechterforschung in der Kritik. Opladen \& Bloomfield Hills: Verlag Barbara Budrich, S. 7-8.

Maihofer, Andrea (1994): Geschlecht als hegemonialer Diskurs. Ansätze zu einer kritischen Theorie des Geschlechts. In: Wobbe, Theresa/Lindemann, Gesa (Hrsg): Denkachsen. Zur theoretischen und institutionellen Rede vom Geschlecht. Frankfurt am Main: Suhrkamp Verlag, S. 236-259.

Maihofer, Andrea (1995): Geschlecht als Existenzweise. Macht, Moral, Recht und Geschlechterdifferenz. Frankfurt am Main: Ulrike Helmer Verlag.

Maihofer, Andrea (2002): Geschlecht und Sozialisation. Eine Problemskizze. In: Erwägung Wissen Ethik. Jahrgang 13, Heft 1, Stuttgart: Lucius \& Lucius, S. 13-26. 
Maihofer, Andrea (2004): Geschlecht als soziale Konstruktion - eine Zwischenbetrachtung. In: Pühl, Katharina/Paulitz, Tanja/Marx, Daniela/Helduser, Urte (Hrsg.): Under construction? Konstruktivistische Perspektiven in feministischer Theorie und Forschungspraxis. Frankfurt und New York: Campus Verlag, S. 33-43.

Maihofer, Andrea/König, Tomke (2004): „Es hat sich so ergeben”: Praktische Normen familialer Arbeitsteilung. In: Familiendynamik. Interdisziplinäre Zeitschrift für systemorientierte Praxis und Forschung. Jahrgang 29, Heft 3, Stuttgart: Klett-Cotta Verlag, S. 209-232.

Nadai, Eva (1999): Kontextualisierung der Geschlechterdifferenz? Geschlechtliche Grenzziehungen im Beruf. In: Honegger, Claudia/Hradil, Stefan/Taxler, Franz (Hrsg.): Grenzenlose Gesellschaft? Verhandlungen des 29. Kongresses der Deutschen Gesellschaft für Soziologie. Band 2, Opladen: Leske \& Budrich, S. 138-150.

Mangold, Werner (1960): Gegenstand und Methode des Gruppendiskussionsverfahrens. Fankfurt am Main: Europäische Verlagsanstalt.

Mannheim, Karl (1964a): Beiträge zur Theorie der WeltanschauungsInterpretation. In: Mannheim, Karl (Hrsg.): Wissenssoziologie. Berlin \& Neuwied: Schulte-Bulmke Verlag, S. 91-154.

Mannheim, Karl (1964b): Das Problem der Generationen. In: Mannheim, Karl (Hrsg.): Wissenssoziologie. Auswahl aus dem Werk. Berlin \& Neuwied: Schulte-Bulmke Verlag, S. 509-565.

Mannheim, Karl (1980): Strukturen des Denkens. Frankfurt am Main: Suhrkamp Verlag.

Marotzki, Winfried (2006): Forschungsmethoden und -methodologie der Erziehungswissenschaftlichen Biographieforschung. In: Krüger, HeinzHermann/Marotzki, Winfried (Hrsg.): Handbuch für erziehungswissenschaftliche Biographieforschung. Wiesbaden: VS Verlag für Sozialwissenschaften, S. 279-299.

Marshall, Catherine/Young, Michelle D. (2006): Gender and Methodology. In: Skelton, Christine/Francis, Becky/Smulyan, Lisa (Hrsg.): The SAGE Handbook of Gender and Education. London, Thousand Oaks \& New Delhi: SAGE Publications, S. 63-78.

Martin, Patricia Y. (2006): Practicing Gender at Work: Further Thoughts on Reflexivity. In: Gender, Work and Organization. Jahrgang 13, Heft 3, Hoboken \& New Jersey: Blackwell Publishing Ltd, S. 254-276.

Matthiesen, Ulf (1989): „Bourdieu” und „Konopka”. Imaginäres Rendezvous zwischen Habituskonstruktion und Deutungsmusterrekonstruktion. In: Eder, Klaus (Hrsg.): Klassenlage, Lebensstil und kulturelle Praxis: Beiträge zur Auseinandersetzung mit Pierre Bourdieus Klassentheorie. Frankfurt am Main: Suhrkamp Verlag, S. 221-299. 
Maurer, Susanne (1996): Zwischen Zuschreibung und Selbstgestaltung. Feministische Identitätspolitiken im Kräftefeld von Kritik, Norm und Utopie. Tübingen: Edition Diskord.

Maurer, Susanne (2006): Gouvernementalität, von unten her' denken. Soziale Arbeit und soziale Bewegungen als (kollektive) Akteure ,beweglicher Ordnungen'. In: Maurer, Susanne/Weber, Susanne (Hrsg.): Gouvernementalität und Erziehungswissenschaft: Wissen - Macht - Transformation. Wiesbaden: VS Verlag für Sozialwissenschaften, S. 233-252.

Maurer, Susanne (2008): Merkwürdige Selbstvergessenheit? Thematisierungsdynamiken im Kontext feministischer Theoriebildung. In: Casale, Rita/Rendtorff, Barbara (Hrsg.): Was kommt nach der Genderforschung? Zur Zukunft der feministischen Theoriebildung. Bielefeld: Transcript-Verlag, S. 101-119.

Maurer, Susanne (2012): ,Subjekt' als Widerstand? Einige Annäherungen aus feministischer Perspektive. In: Graf, Julia/Ideler, Kristin/Klinger, Sabine (Hrsg.): Geschlecht zwischen Struktur und Subjekt. Theorie, Praxis, Perspektiven. Opladen, Berlin, Toronto: Verlag Barbara Budrich, S. 131-152.

McRobbie, Angela/ Savier, Monika (Hrsg.) (1982): Autonomie aber wie! Mädchen Alltag Abenteuer. München: Verlag Frauenoffensive.

McRobbie, Angela (2010): Top Girls. Feminismus und der Aufstieg des neoliberalen Geschlechterregimes. In: Hark, Sabine/Villa, Paula Irena (Hrsg.): Geschlecht und Gesellschaft. Band 44, Wiesbaden: VS Verlag.

Mecheril, Paul/Rose, Nadine (2012): Qualitative Migrationsforschung - Standortbestimmungen zwischen Politik, Reflexion und (Selbst-)Kritik. In: Ackermann, Friedhelm/Ley, Thomas/Machold, Claudia/Schrödter, Mark (Hrsg.): Qualitatives Forschen in der Erziehungswissenschaft. Wiesbaden: Springer VS, S. 115-134.

Metz-Göckel, Sigrid (2008): Hochschulforschung und Frauen- und Geschlechterforschung - zwei Welten begegenen sich? In: Zimmermann, Karin/Kamphans, Marion/Metz-Glöckel, Sigrid (Hrsg.): Perspektiven der Hochschulforschung. Wiesbaden: VS Verlag für Sozialwissenschaften, S. 3763.

Meuser, Michael (1998): Geschlecht und Männlichkeit. Soziolgogische Theorie und kulturelle Deutungsmuster. Opladen: Leske \& Budrich.

Meuser, Michael (1999): Multioptionale Männlichkeiten? Handlungsspielräume und habituelle Dispositionen. In: Honegger, Claudia/Hradil, Stefan/Taxler, Franz (Hrsg.): Grenzenlose Gesellschaft? Verhandlungen des 29. Kongresses der Deutschen Gesellschaft für Soziologie. Band 2, Opladen: Leske \& Budrich, S. 181-197.

Meuser, Michael (2006): Riskante Praktiken. Zur Aneignung von Männlichkeit in den ernsten Spielen des Wettbewerbs. In: Bilden, Helga/Dausien, Bettina 
(Hrsg.): Sozialisation und Geschlecht. Theoretische und methodologische Aspekte. Opladen: Verlag Barbara Budrich, S. 163-178.

Meuser, Michael (2007): Repräsentation sozialer Strukturen im Wissen. Dokumentarische Methode und Habitusrekonstruktion. In: Bohnsack, Ralf/Nentwig-Gesemann, Iris/Nohl, Arnd-Michael (Hrsg.): Die dokumentarische Methode und ihre Forschungspraxis. Opladen: Leske \& Budrich, S. 207-224.

Meuser, Michael (2006): Riskante Praktiken. Zur Aneignung von Männlichkeit in den ernsten Spielen des Wettbewerbs. In: Bilden, Helga/Dausien, Bettina (Hrsg.): Sozialisation und Geschlecht. Theoretische und methodologische Aspekte. Opladen: Verlag Barbara Budrich, S. 163-178.

Mey, Günther/Mruck, Katja (2007): Qualitative Interviews. In: Naderer, Gabriele/Balzer, Eva (Hrsg.): Qualitative Marktforschung in Theorie und Praxis. Grundlagen, Methoden und Anwendungen. Wiesbaden: Gabler Verlag, S. $49-278$.

Micus-Loos, Christiane (2004): Gleichheit - Differenz - Konstruktion - Dekonstruktion. In: Glaser, Edith/Klika, Dorle/Prengel, Annedore (Hrsg.): Handbuch Gender und Erziehungswissenschaften. Bad Heilbrunn: Klinkhardt Verlag, S. 112-127.

Money, John/Hampson, Joan G./Hampson, John L. (1955): Hermaphroditism: Recommendations concerning assignment of sex, change of sex, and psychologic management. In: Bulletin of the Johns Hopkins Hospital. Jahrgang 97, Heft 4, S. 284-300.

Moser, Andrea (2010): Kampfzone Geschlechterwissen. Kritische Analyse populärwissenschaftlicher Konzepte von Männlichkeiten und Weiblichkeit. Wiesbaden: Springer Verlag.

Müller, Ursula (1999): Soziologie und Geschlechtergerechtigkeit am Beispiel der Forschung zu Frauen an Hochschulen. In: Dausien, Bettina/Herrmann, Martina/Oechsle, Mechtild/Schmerl, Christiane/ Stein-Hilbers, Marlene (Hrsg.): Erkenntnisprojekt Geschlecht. Feministische Perspektiven verwandeln Wissenschaft. Opladen: Leske \& Budrich, S. 144-166.

Müller, Ursula (2008): De-Institutionalisierung und gendered subtexts. Asymmetrische Geschlechterkultur an der Hochschule. In: Zimmermann, Karin/Kamphans, Marion/Metz-Glöckel, Sigrid (Hrsg.): Perspektiven der Hochschulforschung. Wiesbaden: VS Verlag für Sozialwissenschaften, S. 143156.

Münst, A. Senganata (2008): Hierarchie, Fachkompetenz und Geschlecht in Lehrveranstaltungen: Ergebnisse einer ethnographischen Teilnehmenden Beobachtung. In: Zimmermann, Karin/Kamphans, Marion/Metz-Glöckel, Sigrid (Hrsg.): Perspektiven der Hochschulforschung. Wiesbaden: VS Verlag für Sozialwissenschaften, S. 179-195. 
Naumann, Siglinde (2008): Heterogenität und Bildungsprozesse in bürgerschaftlichen Initiativen. Eine empirische Studie zur Transformation konjunktiver Orientierungen. Dissertation an der Humanistischen Fakultät der Universität Potsdam.

URL: http://opus.kobv.de/ubp/volltexte/2008/1978/pdf/naumann_diss.pdf [Zugriff: 05.11.2012]

Nestvogel, Renate (2004): Sozialisationstheorien: Traditionslinien, Debatten und Perspektiven. In: Becker, Ruth/Kortendiek, Beate (Hrsg): Handbuch Frauen- und Geschlechterforschung. Theorie, Methoden, Empirie. Wiesbaden: VS Verlag für Sozialwissenschaft, S. 153-164.

Nohl, Arnd-Michael (2007): Kulturelle Vielfalt als Herausforderung für pädagogische Organisationen. In: Zeitschrift für Erziehungswissenschaft. Jahrgang 10, Heft 1, Wiesbaden: VS Verlag für Sozialwissenschaften, S. 61-74.

Nohl, Arnd-Michael (2009): Interview und dokumentarische Methode. Anleitungen für die Forschungspraxis. 3. Auflage. Wiesbaden: VS Verlag für Sozialwissenschaften.

Oloff, Aline (2004): Doing Diplomacy und Gender Mainstreaming. Bourdieus Habitus-Feld-Konzept als Analyseinstrument für den Implementierungsprozess von Gender Mainstreaming in die Verwaltungspraxis am Beispiel des Auswärtigen Amts.

URL: www.gender.hu-berlin.de/w/files/ztgbulletintexte32/oloff_doing_diplomacy_und_gender_mainstreaming.htm [Zugrifff: 11.06.2009].

Otto, Hans-Uwe/Rauschenbach, Thomas/Vogel, Peter (2002): Zur Einführung. In: Otto, Hans-Uwe/Rauschenbach, Thomas/Vogel, Peter (Hrsg.): Erziehungswissenschaft: Lehre und Studium. Opladen: Leske \& Budrich, S. 710.

Pasero, Ursula (1995): Dethematisierung von Geschlecht. In: Pasero, Ursula/Braun, Friederike (Hrsg.): Konstruktion von Geschlecht. Pfaffenweiler: Centaurus Verlagsgesellschaft, S. 50-66.

Paulitz, Tanja (2010): Technikwissenschaften: Geschlecht in Strukturen, Praxen und Wissensformationen der Ingenieurdisziplinen und technischen Fachkulturen. In: Becker, Ruth/Kortendieck, Beate (Hrsg.): Handbuch Frauenund Geschlechterforschung. Theorie, Methoden, Empirie. 3. Auflage, Wiesbaden: VS Verlag, S. 787-798.

Plößer, Melanie (2005): Dekonstruktion - Feminismus - Pädagogik. Vermittlungsansätze zwischen Theorie und Praxis. Königstein: Helmer-Verlag.

Prengel, Anedore (1995): Pädagogik der Vielfalt: Verschiedenheit und Gleichberechtigung in Interkultureller, Feministischer und Integrativer Pädagogik. Opladen: Leske \& Budrich.

Prengel, Annedore (2007): Diversity Education - Grundlagen und Probleme der Pädagogik der Vielfalt. In: Krell, Gertraude/Riedmüller, Barba- 
ra/Sieben, Barbara/Vinz, Dagmar (Hrsg.): Diversity Studies. Grundlagen und disziplinäre Ansätze. Frankfurt am Main \& New York: Campus Verlag, S. 49-67.

Przyborski, Aglaja (2004): Gesprächsanalyse und dokumentarische Methode. Qualitative Auswertung von Gesprächen, Gruppendiskussionen und anderen Diskursen. Wiesbaden: VS Verlag für Sozialwissenschaften.

Przyborski, Aglaja/Wohlrab-Sahr, Monika (2009): Qualitative Sozialforschung. Ein Arbeitsbuch. 2. Auflage, München: Oldenbourg Verlag.

Pühl, Katharina/Paulitz, Tanja/Marx, Daniela/Helduser, Urte (Hrsg.) (2004): Under construction? Konstruktivistische Perspektiven in feministischer Theorie und Forschungspraxis. Frankfurt am Main und New York: Campus Verlag.

Rauschenbach, Thomas/Tillmann, Klaus-Jürgen/Tippelt, Rudolf/Weishaupt, Horst (2008): Datenreport 2008. Die Lage der Erziehungswissenschaft im Spiegel der Statistik. In: Zeitschrift Erziehungswissenschaft. Das Mitteilungsblatt der DGfE. Jahrgang 19, Heft 36, S. 75-84.

Rendtorff, Barbara/Moser, Vera (Hrsg.) (1999): Geschlecht und Geschlechterverhältnisse in der Erziehungswissenschaft. Eine Einführung. Opladen: Leske \& Budrich.

Rendtorff, Barbara (2005): Strukturprobleme der Frauen- und Geschlechterforschung in der Erziehungswissenschaft. In: Casale, Rita/Rendtorff, Barbara/Andresen, Sabine/Moser, Vera/Prengel, Annedore (Hrsg.): Jahrbuch Frauen- und Geschlechterforschung. Geschlechterforschung in der Kritik. Opladen und Bloomfield Hills: Verlag Barbara Budrich, S. 19-40.

Rendtorff, Barbara (2006): Erziehung und Geschlecht. Eine Einführung. Stuttgart: Kohlhammer Verlag.

Rendtorff, Barbara (2012): Warum Geschlecht doch etwas Besonderes ist. URL: www.portal-intersektionalität.de [Zugriff: 29.08.2012]

Rieger-Ladich, Markus (2005): Weder Determinismus noch Fatalismus: Pierre Bourdieus Habitustheorie im Licht neuerer Arbeiten. In: Zeitschrift für Soziologie der Erziehung und Sozialisation Jahrgang 25, Heft 3, Weinheim: Beltz Juventa Verlag, S. 281-296.

Rieger-Ladich, Markus (2009): Konturen einer machtkritischen Disziplingeschichte: Methodologische Überlegungen und leitende Forschungsfragen zur erziehungswissenschaftlichen Geschlechterforschung. In: Glaser, Edith/Andresen, Sabine (Hrsg.): Jahrbuch Frauen- und Geschlechterforschung in der Erziehungswissenschaft. Disziplingeschichte der Erziehungswissenschaft als Geschlechtergeschichte. Opladen \& Farmington Hills: Verlag Barbara Budrich, S. 15-28.

Rieger-Ladich, Markus (2010): Bedrohte Selbstachtung und Verlust der Zukunft. Habitustheoretische Analyse eines leitfadengestützten Interviews. In: 
Wigger, Lothar/Equit, Claudia (Hrsg.): Bildung, Biografie und Anerkennung. Interpretationen eines Interviews mit einem gewaltbereiten Mädchen. Opladen \& Farmington Hills: Verlag Barbara Budrich, S. 83-121.

Rieger-Ladich, Markus/Friebertshäuser, Barbara/Wigger, Lothar (2009): Reflexive Erziehungswissenschaft: Stichworte zu einem Programm. In: RiegerLadich, Markus/Friebertshäuser, Barbara/Wigger, Lother (Hrsg.): Reflexive Erziehungswissenschaft. Forschungsperspektiven im Anschluss an Pierre Bourdieu. Wiesbaden: VS Verlag für Sozialwissenschaften, S. 9-20.

Roer, C. Dorothee (1994): Weiblichkeit im Wandel. Einige Argumente aus tätigkeitstheoretischer Sicht. Einleitende Überlegungen. In: Beutler, Kurt (Hrsg.): Jahrbuch für Pädagogik 1994. Geschlechterverhältnisse und die Pädagogik. Frankfurt am Main: Peter Lang Verlag, S. 15-41.

Rosenberg von, Florian (2011): Bildung und Habitustransformation. Empirische Rekonstruktionen und bildungstheoretische Reflexion. Bielefeld: Transcript Verlag.

Rousseau, Jean-Jacques (2004 [1762]): Emile oder Über die Erziehung. Stuttgart: Reclam Verlag.

Rubin, Gayl S. (1975): The Traffic in Women: Notes on the 'Political Economy' of Sex. In: Reiter, Rayna (Hrsg.): Toward an Anthropology of Women. New York: Monthly Review Press, S. 157-210.

Schäffer, Burkhard (2012): Erziehungswissenschaftliche Medienforschung Medienpraxiskulturen im Generationenvergleich. In: Ackermann, Friedhelm/Ley, Thomas/Machold, Claudia/Schrödter, Mark (Hrsg.): Qualitatives Forschen in der Erziehungswissenschaft. Wiesbaden: Springer VS, S. 135156.

Schlüter, Anne (1999): Bildungserfolge. Eine Analyse der Wahrnehmungs- und Deutungsmuster und der Mechanismen für Mobilität in Bildungsbiographien. Opladen: Leske \& Budrich Verlag.

Schlüter, Anne/Faulstich-Wieland, Hannelore (2009): Geschlechterforschung in der Erziehungswissenschaft - Inspirationen und Modifikationen durch Pierre Bourdieu. In: Friebertshäuser, Barbara/Rieger-Ladich, Markus/Wigger, Lothar (Hrsg): Reflexive Erziehungswissenschaft. Forschungsperspektiven im Anschluss an Pierre Bourdieu. 2. Auflage, Wiesbaden: VS Verlag für Sozialwissenschaften, S. 211-227.

Schütz, Alfred (1971): Das Problem der sozialen Wirklichkeit. Gesammelte Aufsätze, Band 1, Den Haag: Martinus Nijhoff.

Schütze, Fritz (1987): Das narrative Interview in Interaktionsfoldstudien. Studienbrief der Fernuniversität Hagen. Teil 1, Hagen.

Schwiter, Karin/Wehner, Nina/Maihofer, Andrea/Huber, Evèline (2011): Zur Hartnäckigkeit geschlechtssegregierter Ausbildungs- \& Berufsverläufe. Konzeptionelle Überlegungen zu einer empirischen Untersuchung. In: Femina 
Politica. Zeitschrift für feministische Politikwissenschaft. Die Bildung der Geschlechter: Der Einfluss von Strukturen und aktuellen Reformen im Bildungswesen. Heft 2, Opladen \& Farmington Hills: Barbara Budrich Verlag, S. 20-32.

Siebert, Horst (2002): Der Konstruktivismus als pädagogische Weltanschauung - Entwurf einer konstruktivistischen Didaktik. Frankfurt am Main: VAS Verlag für akademische Schriften.

Stauber, Barbara (2004): Veränderte Generationenbeziehungen und ihre Konsequenzen für die Mädchenarbeit. In: Praxis. Zeitschrift für Sozialarbeit, Sozialpädagogik und Sozialpolitik. Jahrgang 34, Helft 1, Lahnstein: Verlag Neue Praxis, S. 30-39.

Stisser, Anna/Horn, Klaus-Peter (2012): Bachelors- und Masterstudiengänge in der Erziehungswissenschaft. In: Thole, Werner/Faulstich-Wieland, Hannelore/Horn, Klaus-Peter/Weishaupt, Horst/Züchner, Ivo (Hrsg.): Datenreport Erziehungswissenschaft 2012. Opladen \& Farmington Hills: Verlag Barbara Budrich, S. 19-48.

Stoller, Robert (1968): Sex and Gender: On the Development of Masculinity and Femininity. New York: Science House.

Suzuki, Shunryu (2006) [1970]. Zen mind, beginner's mind. In: Dixon, Trudy/Baker, Richard (Hrsg.): Zen mind, beginner's mind. New York: Weatherhill, S. 21-138.

Tübinger Institut für frauenpolitische Sozialforschung e.V. (Bitzan, Maria/Funk, Heide/Stauber, Barbara) (2000): Den Wechsel im Blick. Methodologische Ansichten feministischer Sozialforschung. Pfaffenweiler: Centaurus Verlagsgesellschaft.

Villa, Paula-Irene (2008): Post-Ismen: Geschlecht in Postmoderne und (De)Konstruktion. In: Wilz, Sylvia Marlene (Hrsg.): Geschlechterdifferenzen - Geschlechterdifferenzierungen. Ein Überblick über gesellschaftliche Entwicklungen und theoretische Positionen. Wiesbaden: VS Verlag für Sozialwissenschaften, S. 199-230.

Walgenbach, Katharina (2007): Gender als interdependente Kategorie. In: Walgenbach, Katharina/Dietze, Gabriele/Hornscheidt, Antje/Palm, Kerstin (Hrsg.): Gender als interdependente Kategorie. Neue Perspektiven auf Intersektionalität, Diversität und Heterogenität. Opladen \& Farmington Hills: Verlag Barbara Budrich, S. 23-64.

Walgenbach, Katharina (2012a): Intersektionalität - eine Einführung.

URL: www.portal-intersektionalität.de [Zugriff: 29.08.2012]

Walgenbach, Katharina (2012b): Intersektionalität als Analyseperspektive heterogener Stadträume. In: Scambor, Elli/Zimmer, Fränk (Hrg.): Die intersektionelle Stadt. Geschlechterforschung und Medien an den Achsen der Ungleichheit. Bielefeld: Transcript Verlag, S. 81-92. 
Weber, Martina (2003): Heterogenität im Schulalltag. Konstruktion ethnischer und geschlechtlicher Unterschiede. Opladen: Leske \& Budrich.

Wesely, Sabine (2000): Einführung in die Gender Studies. In: Wesely, Sabine (Hrsg.): Gender Studies in der Sozial- und Kulturwissenschaft. Einführung und neuere Erkenntnisse aus Forschung und Praxis. Wissenschaftliche Reihe. Band 33, Bielefeld: Kleine Verlag, S. 12-97.

Wetterer, Angelika (2002): Arbeitsteilung und Geschlechterkonstruktion. "Gender at Work" in theoretischer und historischer Perspektive. Konstanz: Universitätsverlag Konstanz.

Wetterer, Angelika (2003): Rhetorische Modernisierung: Das Verschwinden der Ungleichheit aus dem zeitgenössischen Differenzwissen. In: Knapp, GudrunAxeli/Wetterer, Angelika (Hrsg.): Achsen der Differenz. Gesellschaftstheorie und feministische Kritik II. Münster: Verlag Westfälisches Dampfboot, S. 286-319.

Wetterer, Angelika (2006): Ordentlich in Unordnung? Widersprüche im sozialen Wandel der Geschlechterverhältnisse. In: Österreichische Zeitschrift für Soziologie, Heft 4, S. 5-22.

Angelika Wetterer (Hrsg.) (2008): Geschlechterwissen \& soziale Praxis. Theoretische Zugänge - empirische Erträge. Königstein: Ulrike Helmer Verlag.

Wetterer, Angelika (2013): Das erfolgreiche Scheitern feministischer Kritik. Rhetorische Modernisierung, symbolische Gewalt und die Reproduktion männlicher Herrschaft. In: Appelt, Erna/Aulenbacher, Brigitte/ Wetterer, Angelika (Hrsg.): Gesellschaft. Feministische Krisendiagnosen. Münster: Verlag Westfälisches Dampfboot, S. 246-266.

West, Candance/Zimmerman, Don H. (1987): Doing Gender. In: Gender \& Society. Jahrgang 1, Heft 2, S. 125-151.

Zimmermann, Karin (2000): Spiele mit der Macht in der Wissenschaft. Passfähigkeit und Geschlecht als Kriterium für Berufungen. Berlin: Edition Sigma.

Zimmermann, Karin/Kamphans, Marion/Metz-Glöckel, Sigrid (2008): Hochschul- und Geschlechterforschung im Diskurs. In: Zimmermann, Karin/Kamphans, Marion/Metz-Glöckel, Sigrid (Hrsg.): Perspektiven der Hochschulforschung. Wiesbaden: VS Verlag für Sozialwissenschaften, S. 11-33. 


\section{Anhang: Richtlinien der Transkription}

TiQ: Talk in Qualitative Social Research (vgl. Przyborski/Wohlrab-Sahr 2009, S.166f. und vgl. Bohnsack 2008, S.235).

\begin{tabular}{|l|l|}
\hline\llcorner & $\begin{array}{l}\text { Das Häckchen markiert den Beginn einer Überlappung bzw. den } \\
\text { direkten Anschluss beim SprecherInnenwechsel }\end{array}$ \\
\hline$()$. & Pause bis zu einer Sekunde \\
\hline$(2)$ & $\begin{array}{l}\text { Anzahl der Sekunden, die eine Pause dauert. Ab 4 Sekunden } \\
\text { Pause erfolgt die Notation in einer Extrazeile. Auf diese Weise } \\
\text { wird beim Lesen des Transkripts das Schweigen allen an der } \\
\text { Interaktion Beteiligten zugeordnet. }\end{array}$ \\
\hline wirklich & Betonung \\
\hline Nein & $\begin{array}{l}\text { Laut (in Relation zur üblichen Lautstärke des Sprechers/der } \\
\text { Sprecherin) }\end{array}$ \\
\hline${ }^{\circ}$ nee ${ }^{\circ}$ & $\begin{array}{l}\text { Sehr leise (in Relation zur üblichen Lautstärke des Sprechers/der } \\
\text { Sprecherin) }\end{array}$ \\
\hline$;$ & Stark bzw. schwach sinkende Intonation \\
\hline$?$, & Stark bzw. schwach steigende Intonation \\
\hline Viellei- & Abbruch eines Wortes \\
\hline Oh=nee & $\begin{array}{l}\text { Wortverschleifung, Zwei oder mehrere Worte, die wie eines ge- } \\
\text { sprochen werden. }\end{array}$ \\
\hline Nei::n & $\begin{array}{l}\text { Dehnung, die Häufigkeit von : entspricht der Länge der Dehnun- } \\
\text { gen }\end{array}$ \\
\hline$($ doch $)$ & $\begin{array}{l}\text { Unsicherheit bei der Transkription, schwer verständliche Äuße- } \\
\text { rungen }\end{array}$ \\
\hline() & $\begin{array}{l}\text { Unverständliche Äußerung, die Länge der Klammer entspricht } \\
\text { etwa der Dauer der unverständlichen Äußerung }\end{array}$ \\
\hline$(($ stöhnt)) & $\begin{array}{l}\text { Kommentare bzw. Anmerkungen zu parasprachlichen, nicht- } \\
\text { verbalen oder gesprächsexternen Ergebnissen; die Länge der } \\
\text { Klammer entspricht im Falle der Kommentierung parasprach- } \\
\text { licher Äußerungen etwa der Dauer der Äußerung. }\end{array}$ \\
\hline @(Nein)@ & Lachend gesprochen \\
\hline Kurzes Auflachen \\
\hline$/ /$ mhm// & $\begin{array}{l}\text { Hörsignale, mhm der Interviewenden werden ohne Häckchen im } \\
\text { Text des/der Interviewten notiert. }\end{array}$ \\
\hline
\end{tabular}

Groß- und Kleinschreibung: Hauptwörter wurden groß geschrieben, und bei Neuansetzen eines/einer Sprecherin am Beginn eines 'Häckchens' wird das erste Wort mit Großbuchstaben begonnen.

Zeilennummerierung: Um das Auffinden und das Zitieren von Transkriptionsstellen zu ermöglichen, wird eine durchlaufende Zeilennummerierung verwendet. 


\section{Unsere Fachzeitschriften auf www.budrich-journals.de}

- Einzelbeiträge im Download

- $\quad$ Print + Online Abonnements

- Online-Freischaltung über IP - mit open access-Bereich

Als Abonnentln z.B. mit Kombi-Abo Print + Online bekommen Sie Ihr Heft

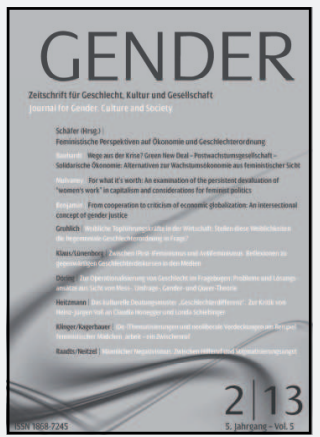
wie gewohnt bequem nach Hause geliefert. Zusätzlich haben Sie Zugriff auf das gesamte Online-Archiv.

Fragen Sie uns!
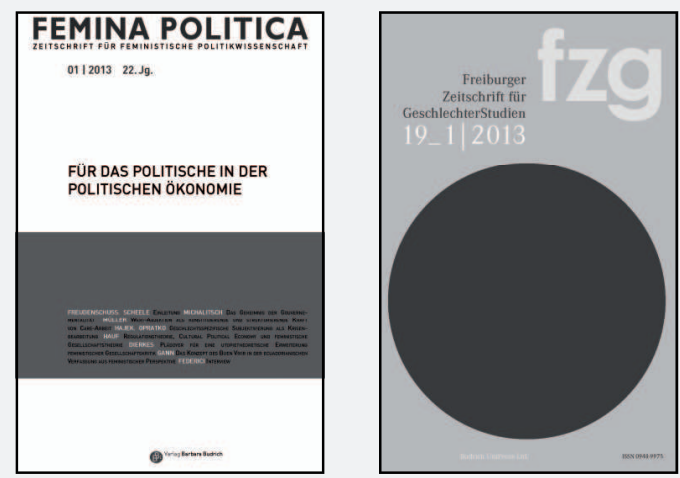

\section{Verlag Barbara Budrich •}

D: Barbara Budrich Publishers

Stauffenbergstr. 7. D-51379 Leverkusen Opladen

Tel +49 (0)2171.344.594 • Fax +49 (0)2171.344.693 •

info@budrich-journals.de

www.budrich-journals.de 